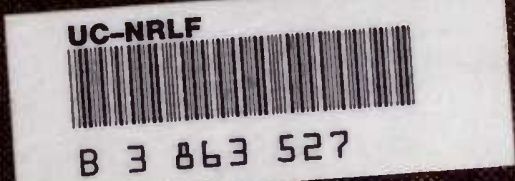

B 3863527
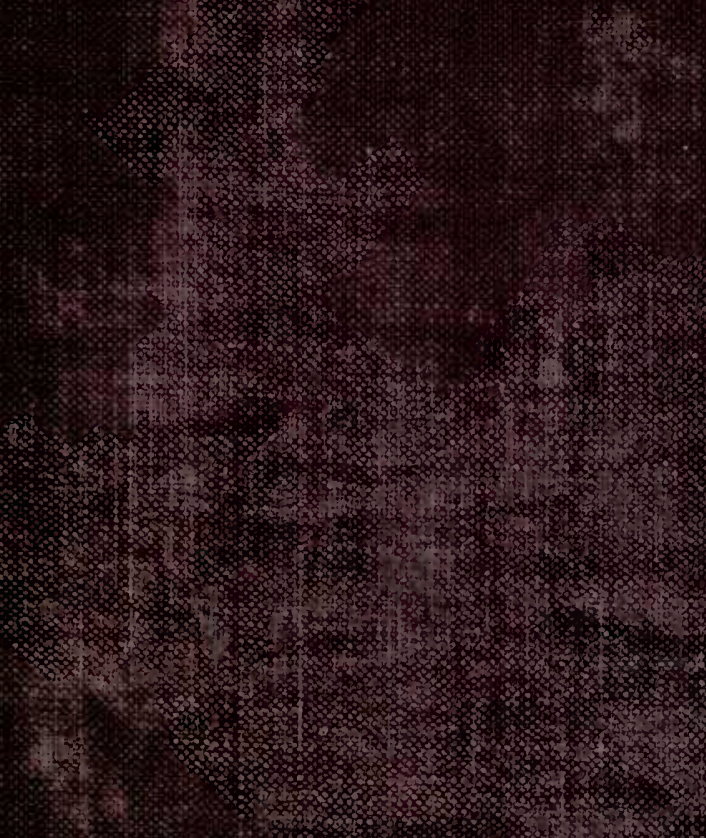


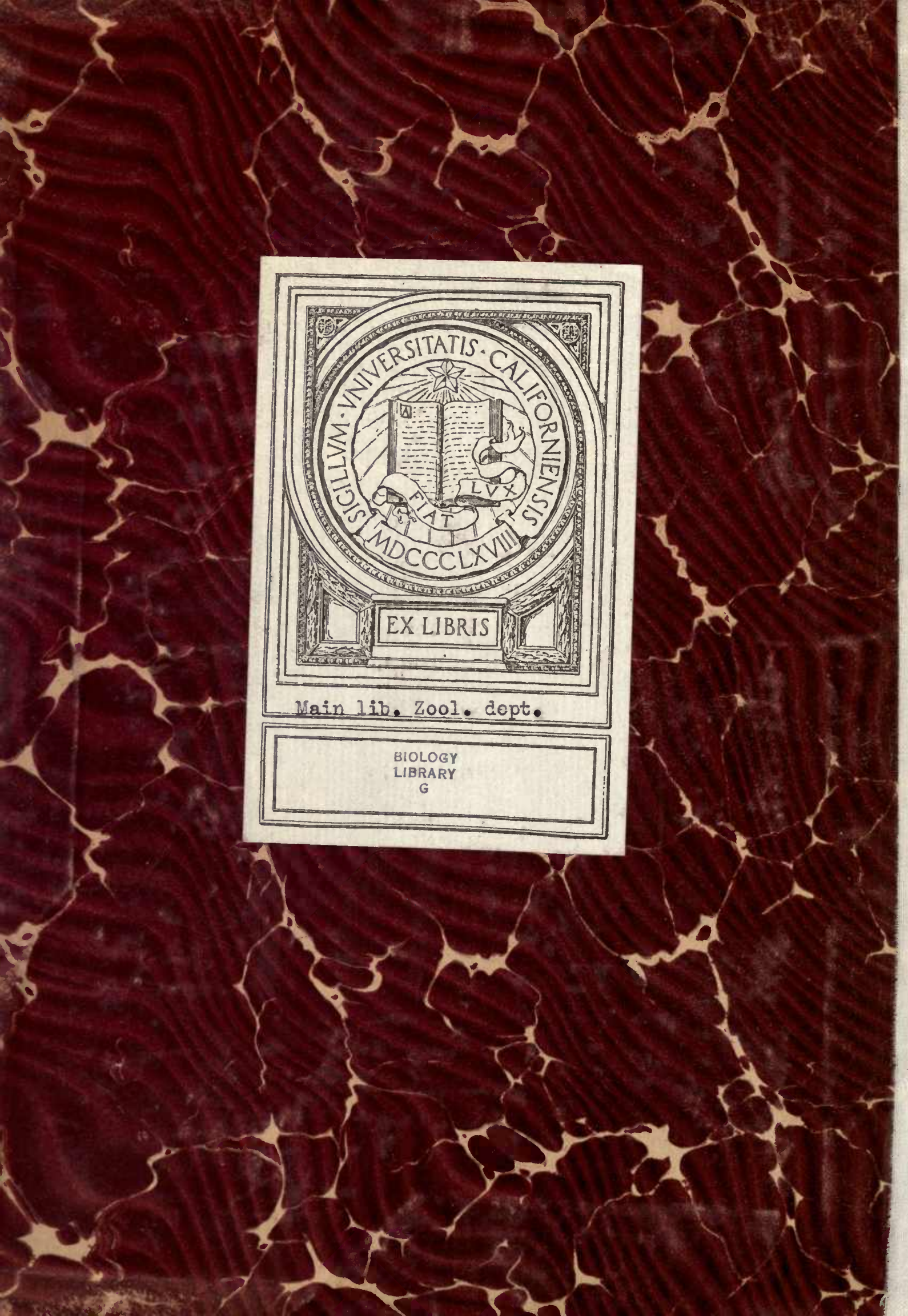




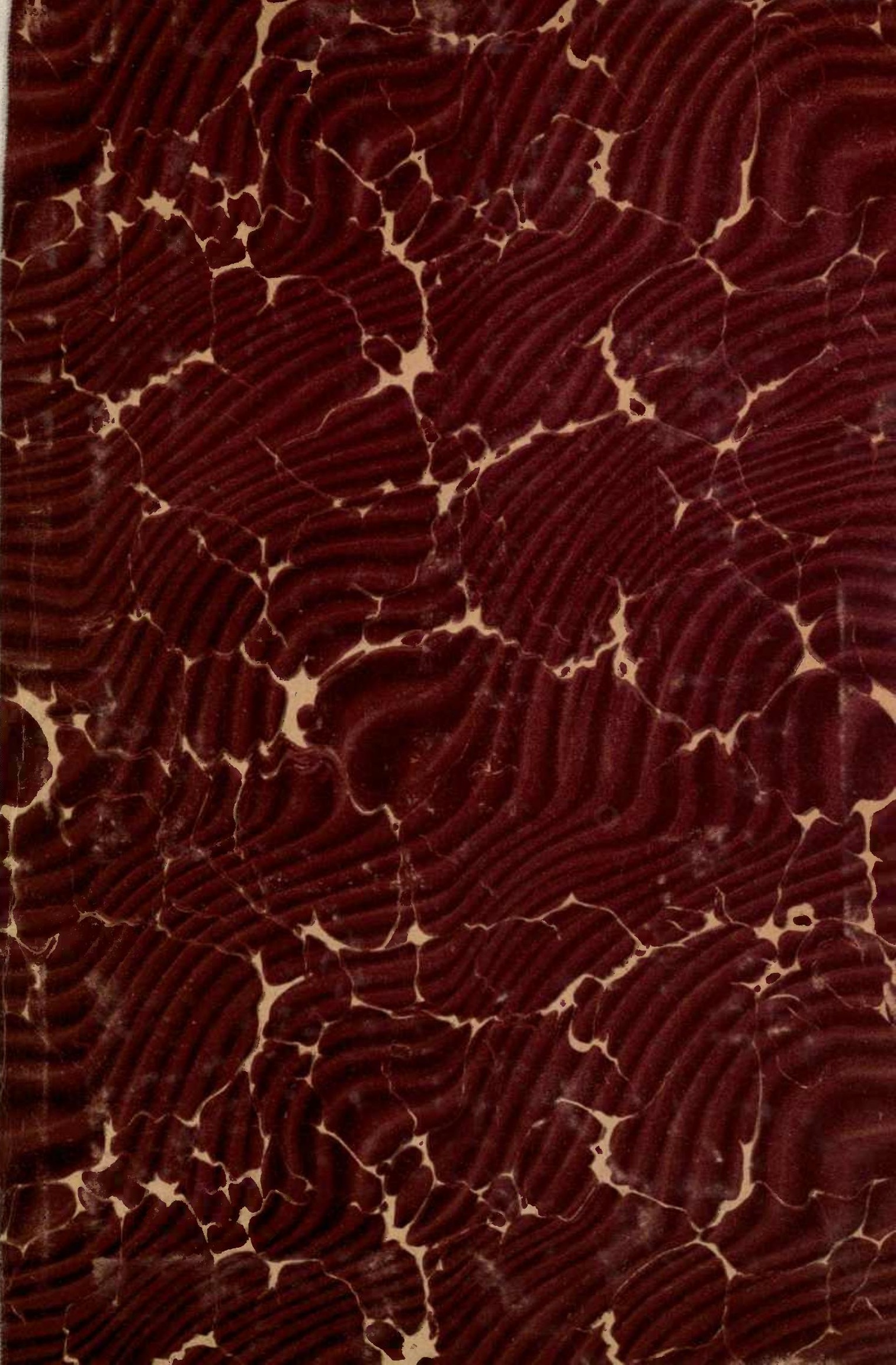






\title{
A
}

\section{LABORATORY TEXT-BOOK}

\author{
OF \\ EMBRYOLOGY
}

BY

CHARLES SEDGWICK MINOT, LL. D. (Yàle and Toronto), D. Sc. (Oxford) JAMES STILLMAN PROPESSOR OP COMPARATIVE ANATOMY IN THE HARVARD MEDICAL SCHOOL; PRESIDENT OP THE BOSTON SOCIETY OF NATURAL HISTORY

SECOND EDITION, REVISED

WITH 262 ILLUSTRATIONS, CHIEFLY ORIGINAL

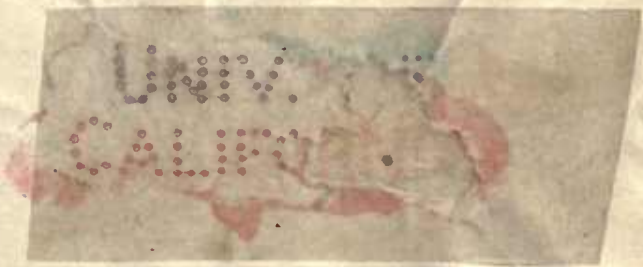

PHILADELPHIA

P. BLAKISTON'S SON \& CO.

1012 WALNUT STREET

1910 
Copyright, IgIo

By Charles Sedgwick Minot
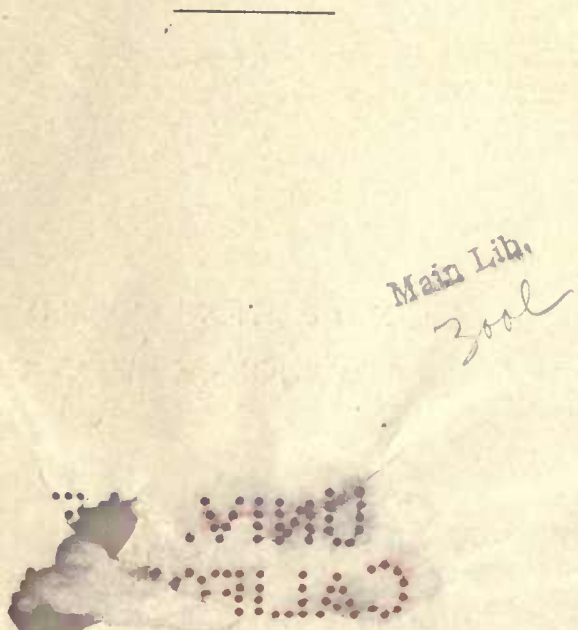

Prinled by

The Maple Press

lork, $P a$.

Sin. 
TO

\section{HENRY PICKERING BOWDITCH}

AS A TOKEN OF

ADMIRATION AND LONG FRIENDSHIP THIS VOLUME

IS DEDICATED BY THE

AUTHOR

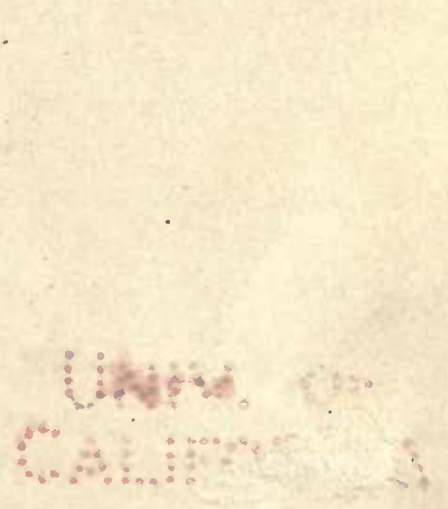





\section{PREFACE.}

The accompanying volume is designed primarily for the use of students taking a practical laboratory course in embryology. It is intended to direct the student's attention to actual, original observations, to be made by himself, and to aid him in drawing correct conclusions from those observations. By this plan, the student repeats and uses the actual methods by which embryological science has been built. If he pursues such a course diligently, he will be able at the end of it to say that he knows of his own knowledge. To attain this result is the ideal of laboratory education.

In preparing the new edition, advantage has been taken of the experience gained with the use of the book by the author's classes, and of valuable suggestions from many friends. The work has been so extensively revised that it may be described as almost a new book. Chapters III to VI have been entirely recast and rearranged so as to conform to the chronological order of development, an arrangement which it is believed most readers will prefer to that adopted in the original edition. Chapter II, on the early development of mammals, has been considerably expanded; not with the object of giving a comprehensive treatment of the subject, but rather with the intention of aiding the student to get, in connection with his laboratory work, a connected story in his mind of the development of the principal organs and systems of the body. Some new sections will be found also in Chapter I. A considerable number of the figures are replaced by new ones. The total number of illustrations has increased from 218 to 262 . With these changes it is hoped that the second edition will deserve a continuance of the favor shown to the original issue.

The author takes much pleasure in acknowledging gratefully the invaluable assistance afforded him by members of his laboratory staff, and wishes to call attention especially to the very admirable original illustrations which have been furnished especially for the book by Drs. J. L. Bremer, F. P. Johnson, F. T. Lewis, R. E. Scammon, and F. W. Thyng. Special mention must be made also of the figures from models made by Messrs. W. W. Behlow, G. C. Coe, L. M. Ferguson, C. A. Hedblom, and A. R. Kilgore, students in the embryological course at the Harvard Medical School.

A large majority of the illustrations are from the Harvard Embryological Collection, without which this work would not have been possible. The author requests those who use this book to communicate to him any suggestions, which their experience may lead to, for improving it.

Charles S. Minot. 



\section{CONTENTS.}

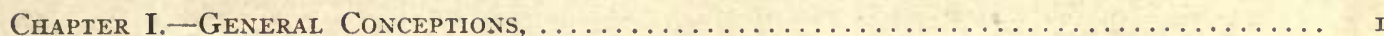

The Segmented Animals. Metamerism, ......................... 2

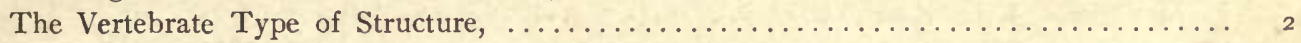

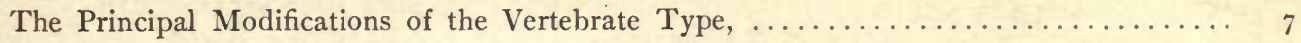

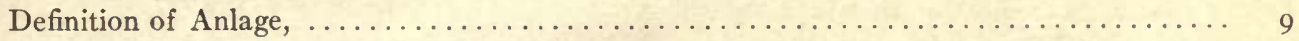

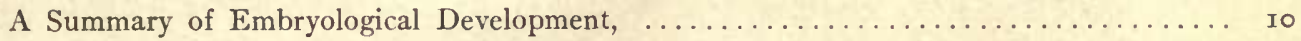

Cytomorphosis, ................................... II

Comparison of Larval and Embryonic Types of Development, ............. I6

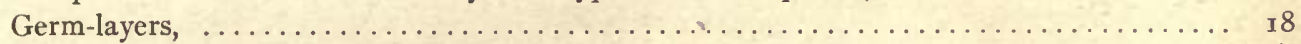

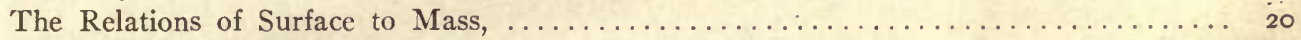

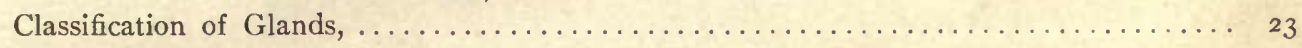

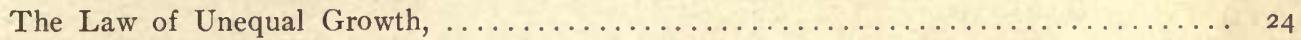

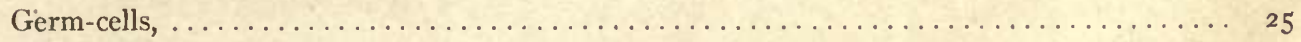

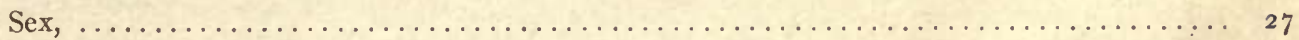

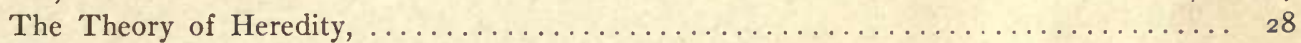

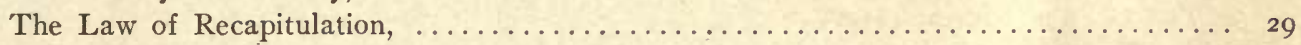

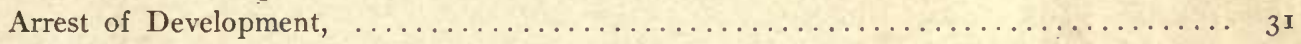

Chapter II.-The Early Development of Mammals, $\ldots \ldots \ldots \ldots \ldots \ldots \ldots \ldots \ldots \ldots \ldots \ldots \ldots \ldots \ldots$

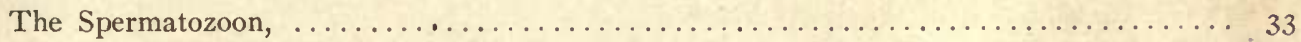

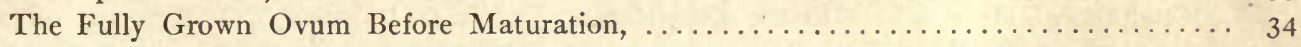

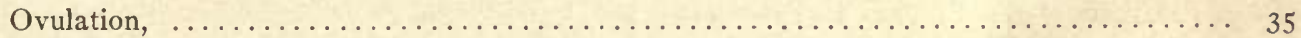

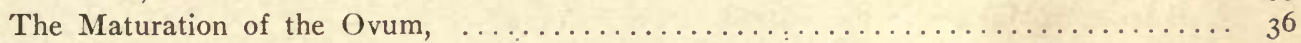

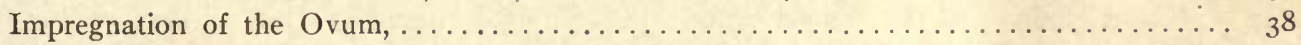

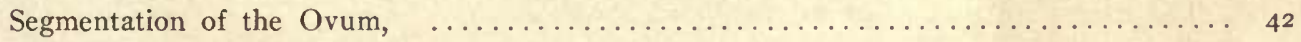

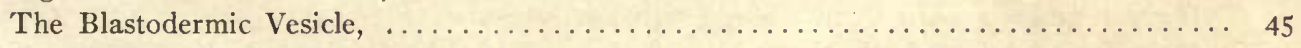

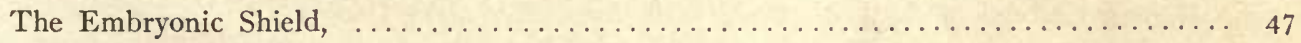

Growth of the Embryo and Separation of the Yolk, .................. 49

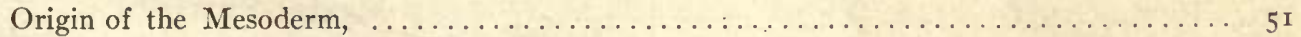

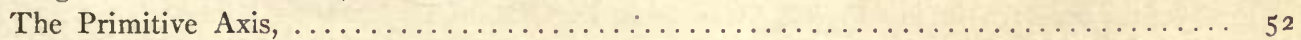

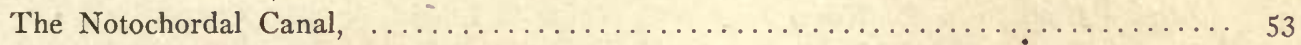

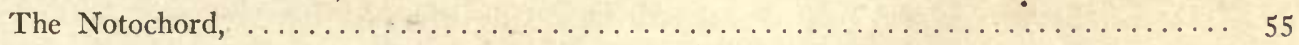

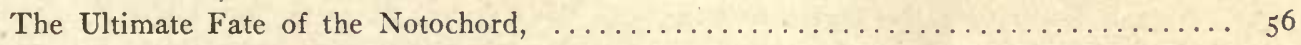

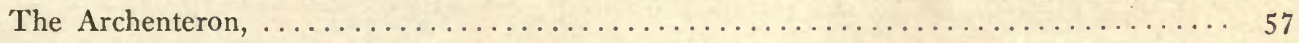

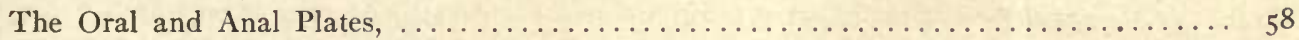

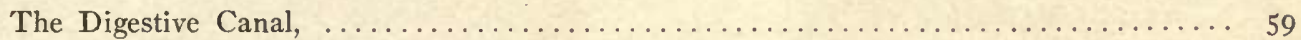

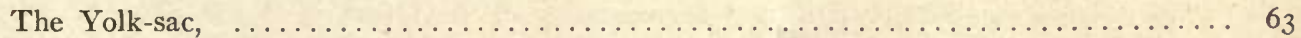

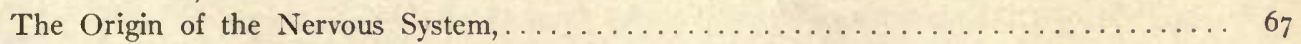

The Structure of the Medullary Canal, ............................ 69

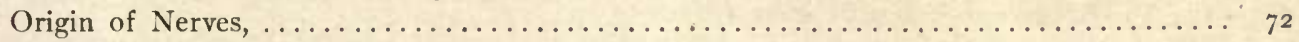


The Spinal Cord and Brain

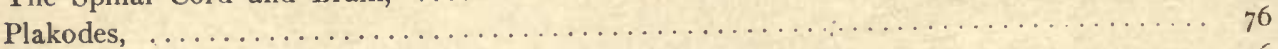

The Nasal Pits and Olfactory Nerves, .......................... 76

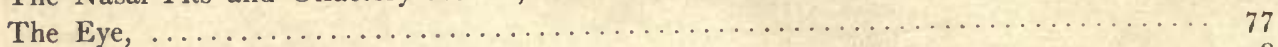

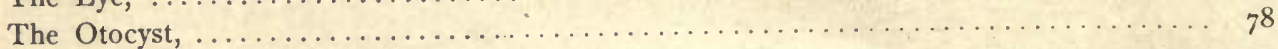

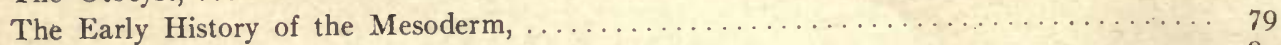

Somatopleure and Splanchnopleure, ............................. 82 .

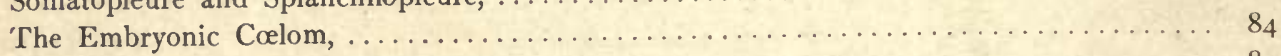

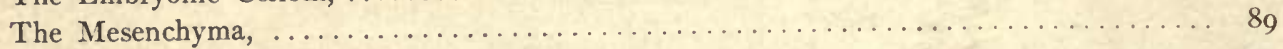

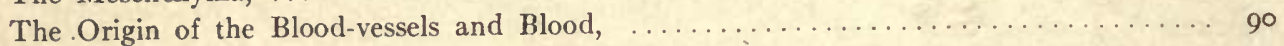

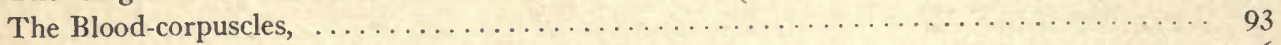

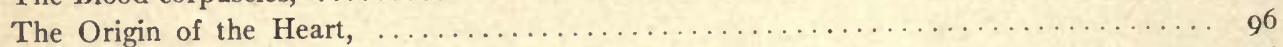

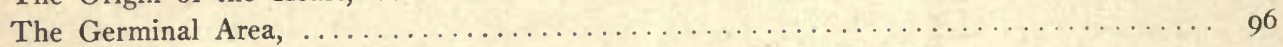

The Main Vessels of the Area Vasculosa, . . . . . . . . . . . . . . . . . .

The Aortic System, . . . . . . . . . . . . . . . . . . . . . . . . . . 99

The Venous System, ................................... 102

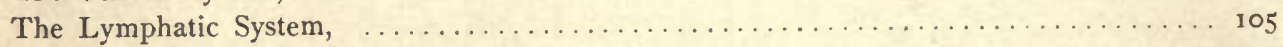

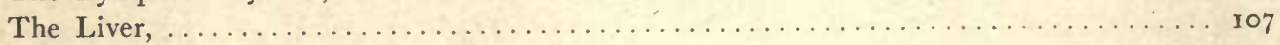

The Pancreas, . . . . . . . . . . . . . . . .

The Excretory Organs, . . . . . . . . . . . .

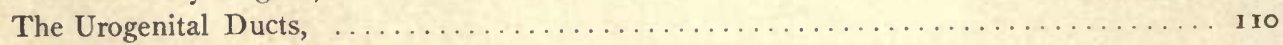

The Allantois, .....................................

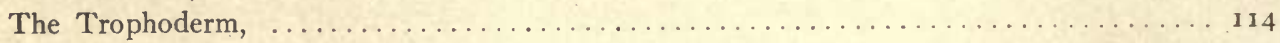

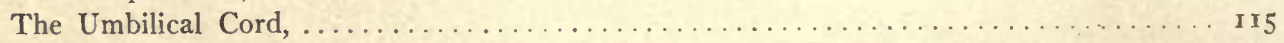

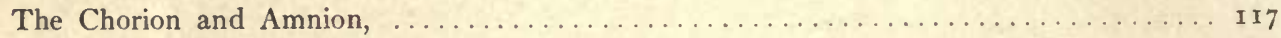

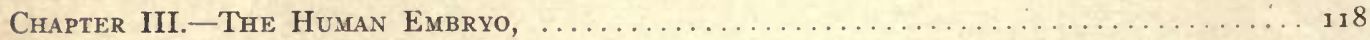

Calculation of the Age of the Human Embryo, $\ldots \ldots \ldots \ldots \ldots \ldots \ldots \ldots \ldots \ldots \ldots \ldots \ldots \ldots \ldots \ldots$

The Classification of the Early Stages, $\ldots \ldots \ldots \ldots \ldots \ldots \ldots \ldots \ldots \ldots \ldots \ldots \ldots \ldots \ldots \ldots \ldots \ldots$

Hypothetical Development of the Blastodermic Vesicle in Primates, . . . . . . . 122

Relations of the Embryo to the Uterus: the Two Stages, ................ I 24

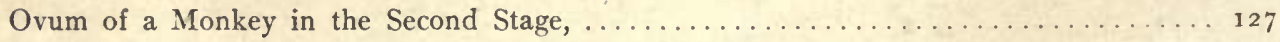

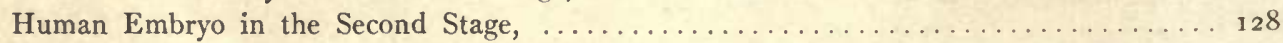

The Embryo of a Gibbon in the Third Stage; ..................... I

Human Embryo in th Fourth Stage with the Medullary Plate, ............. I34

Human Embryo in the Fifth Stage with Open Medullary Groove, ............ I 36

Human Embryo in the Sixth Stage with Medullary Canal, ............... I 37

Human Embryo in the Seventh Stage with One Gill-cleft Showing Externally,....... I 40

Human Embryo in the Eighth Stage with Two Gill-clefts Showing Externally,........ I 4 I

Human Embryo in the Ninth Stage with Three Gill-clefts Showing Externally, ....... I43

Human Embryo in the Tenth Stage with Four Gill-clefts Showing Externally, ...... 146

Human Embryo in the Eleventh Stage with the Cervical Sinus in Formation, ....... 147

Human Embryos of the Fourth Week to the Fourth Month, .............. 148

Chapter IV.-Study of the Segmentation of the Ovum and of the Blastodermic Vfici-

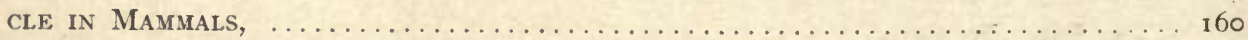

The Maturation, Fertilization, and Segmentation of the Ovum in White Mice, ..... I60

Method of Obtaining Blastodermic Vesicles from the Rabbit, .............. 66

Study of Rabbit Blastodermic Vesicles in Alcohol, ..................... I67

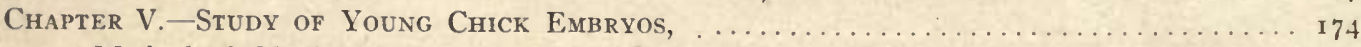

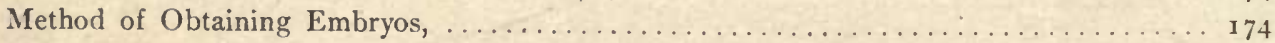


Embryo Chick with about Twenty-four Segments and Three Gill-clefts (About Forty-

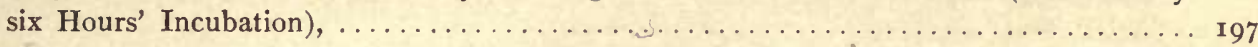

Embryo Chick with Twenty-eight Segments, ....................... I 99

The Study of Transverse Sections, .......................... I99

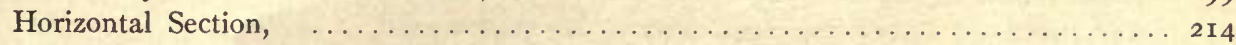

Histological Differentiation of the Chick Embryo with Three Gill-clefts, ........... 2 I6

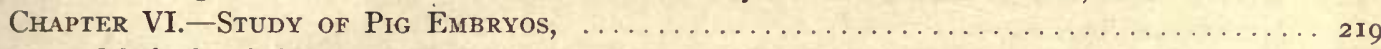

Methods of Obtaining Embryos, ......................... 2 I9

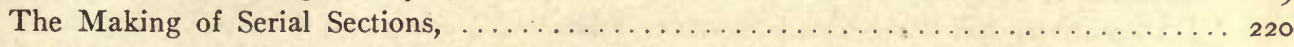

Selection of the Planes of Section and the Stages for Practical Study, ........... 220

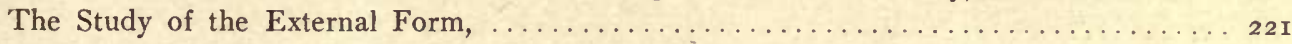

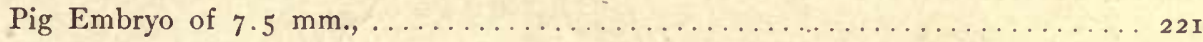

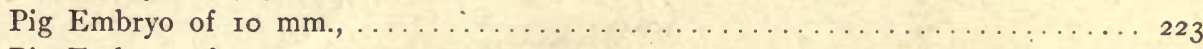

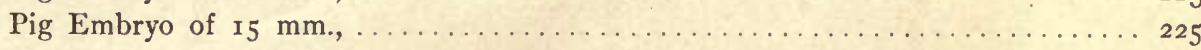

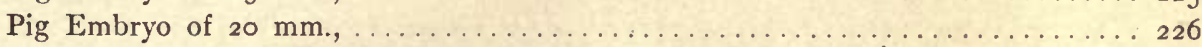

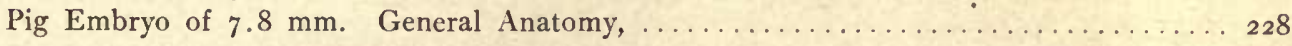

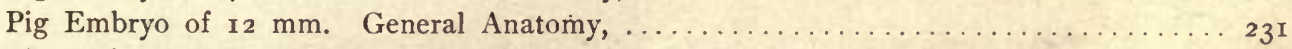

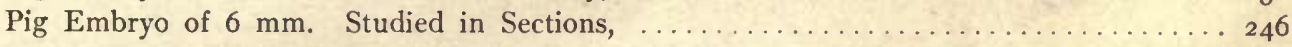

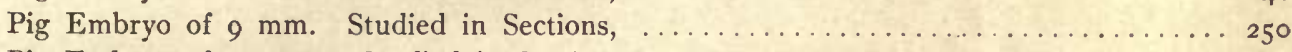

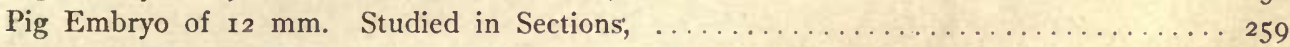

Study of Transverse Sections, ............................

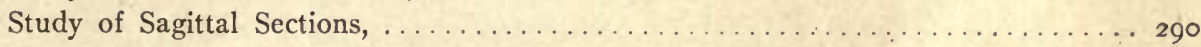

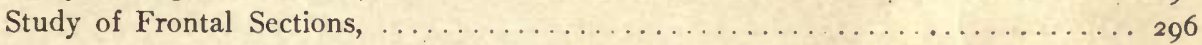

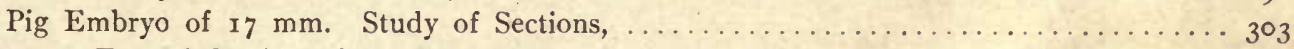

Frontal Section of the Umbilical Cord, ....................... 3 10

Pig Embryo of $20 \mathrm{~mm}$. Study of Sections, ....................

Transverse Sections . . . . . . . . . . . . . . . . . . . .

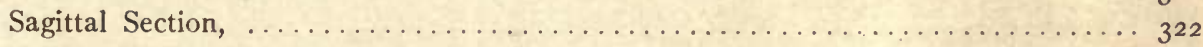

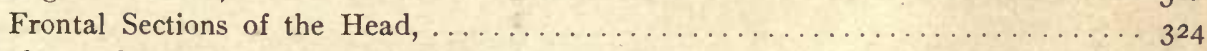

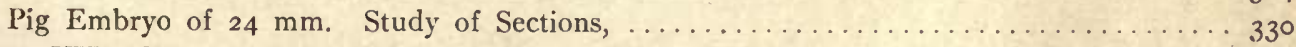

Chapter VII.-Study of the Uterus and the Fetal Appendages of Man, ......... 339

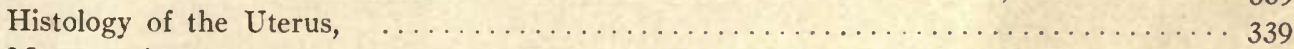

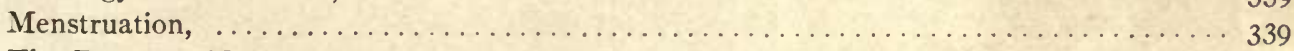

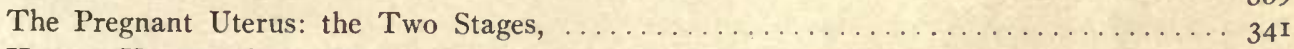

Human Uterus Three Months Pregnant, . . . . . . . . . . . . . . . . . . 343

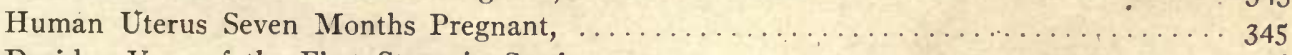

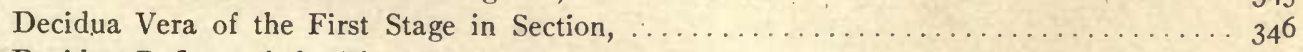

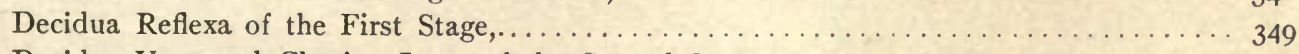

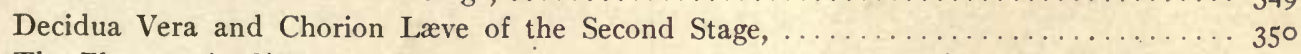

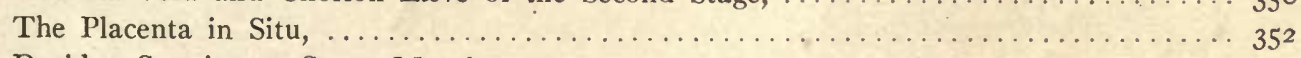

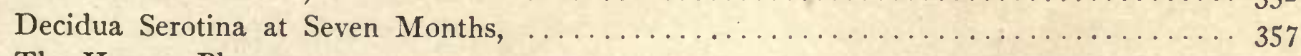

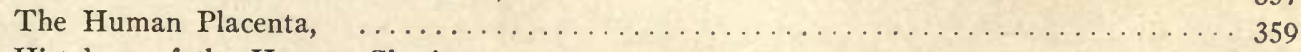

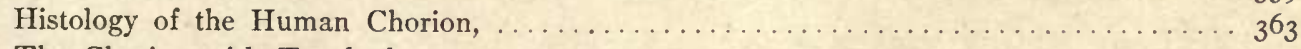

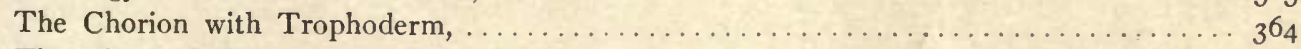

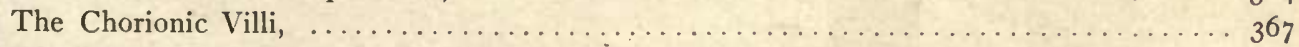

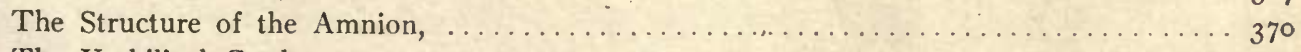

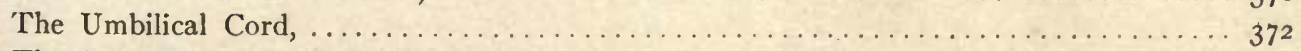

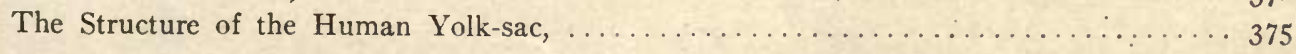




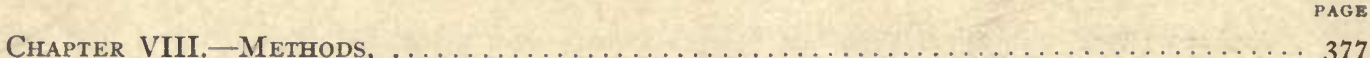

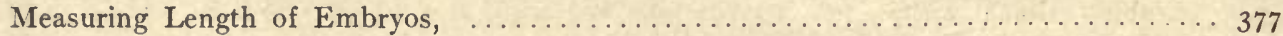

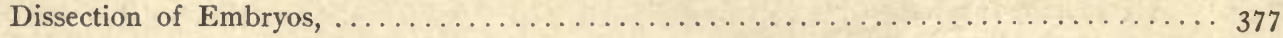

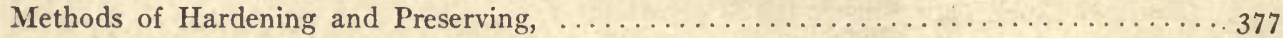

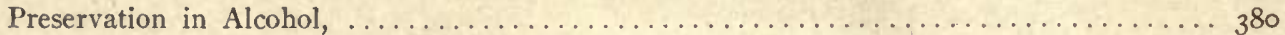

Directions for Imbedding Specimens to be Microtomed, $\ldots \ldots \ldots \ldots \ldots \ldots \ldots \ldots \ldots \ldots$

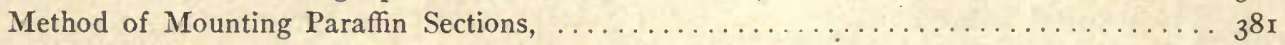

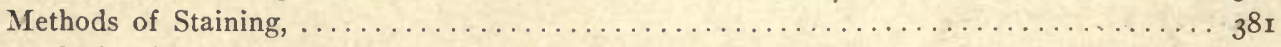

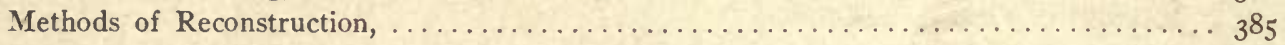

Directions for Orienting Serial Sections of Embryos, $\ldots \ldots \ldots \ldots \ldots \ldots \ldots \ldots \ldots \ldots \ldots \ldots$

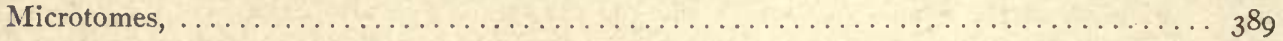

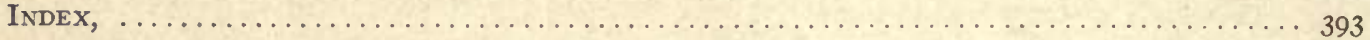




\section{TEXT-BOOK OF EMBRYOLOGY.}

\section{CHAPTER I.}

\section{GENERAL CONCEPTIONS.}

The student of embryology should start with as clear and definite a conception as possible of what he is to gain from his pursuit of that science. If he is a student of biology or of zoology, he must appreciate that knowledge of the laws of development is an indispensable part of what he must master in order to understand those sciences. He must appreciate that it is from the studies of the embryologist that are derived our conceptions of the nature of sex, of heredity, of variation, of differentiation, and many of our most important notions concerning evolution, both of the individual and of the race. He will learn further that the embryo illustrates to him with particular clearness the fundamental principles of morphology. If he be a medical student, he will find in embryology first of all the clue to the intelligent comprehension of the anatomy of the adult, a comprehension which he can obtain in no other way, but he will also gain much knowledge of direct practical value as to the embryo and as to the conditions in the adult, acquaintance with which is invaluable in medical practice. And, finally, he will find that it throws a vast light on pathology, both upon the problems of malformations and monstrosities, and also upon the whole question of pathological change in the tissues.

The best study of embryology, therefore, is that which continually passes beyond the direct observations to the conceptions which they justify and which underlie many important branches of science which are related to, and in a large part dependent upon, embryology.

The student ought to strive, accordingly, to pass from the direct observation of the specimen to the generalizations, and accustom himself to regard always each special preparation, which may be submitted, to his observation, as an illustration of some general principle. To facilitate his reaching this result this chapter offers a digest of some of the more important generalizations and fundamental laws of embryology. 


\section{The Segmented Animals. Metamerism.}

All vertebrates and certain invertebrates have-their bodies divided into a series of parts, which begins in the region of the head and extends to the caudal end of the body. These parts are called segments or metameres. In man each vertebra corresponds to one segment. In the earthworm and other annelids the segments are plainly marked on the outer surface of the body. Since all segmented animals are bilaterally symmetrical, each segment is bilaterally symmetrical and may therefore be described as a paired structure. Embryology has demonstrated that each segment arises as a pair of masses (somites) situated between the digestive canal and the outer surface of the body. Each pair of masses is formed from the middle germ-layer (mesoderm) exclusively (compare pages 84-85) and is known as "a primitive segment." The mesodermic somites are to be considered the essential primary morphological segments, and in the course of the development of the individual they produce adult metameric structures, among which may be muscles, skeletal elements, excretory organs, etc., the whole history of which depends upon their segmental origin. The nervous system and to a considerable extent the blood-vessels exhibit a segmental arrangement, which is usually regarded as a secondary correlation of these structures with the primary mesodermic metamerism. The spinal nerves exemplify the correlation. In brief, where the segmental organization exists, it dominates the anatomy alike of the embryo and the adult; therefore the student of embryology should pay special attention to the segmentation of the body in all its chief stages.

In regard to the bodily segmentation two general observations may be made: First, the development of segments begins at the cephalic end and progresses tailward; hence so long as the development of segments continues various stages of their differentiation may be found in a single embryo, the more advanced stages being always cephalad from the less advanced. Second, there is a fundamental difference between the metamerism of vertebrates and that of annelids and many other invertebrates, which consists in the unlike extent of the segments; for the primitive segments of vertebrates are confined to the dorsal region of the body, while in the other forms the segmentation extends from the start through the dorsal and ventral regions both. It is probable that the segments of vertebrates are homologous only with the dorsal part of the segments of annelids.

\section{The Vertebrate Type of Structure.}

When one traces the course of development of any vertebrate, one finds, speaking in general terms, that the fundamental characteristics, which are more or less common to all vertebrates, are those which first appear. Later, there come in the secondary characteristics which distinguish one class from another, and still later the subordinate characteristics by which the smaller subdivisions of the vertebrate type become differentiated one from another: This statement, however, is correct only if we add to it certain indispensable limitations. Every embryo at every stage of its development is an individual of the particular genus 
and species to which it belongs. It has at every stage peculiarities which distinguish it from every other species. The embryos of allied forms resemble one another more closely than do the embryos of forms which are only distantly related to one another. The specific qualities of an embryo are, however, more difficult to recognize than those of the adult, and the student will be far more impressed by the resemblances between embryos than by their differences. It is owing to this very fact that the distinctive peculiarities of the species are not accentuated in the embryo. We are able to derive from the embryos themselves a series of conceptions which render it comparatively easy to perceive the dominant morphological features of the vertebrate type.

It will be convenient to put down six fundamental characteristics of the vertebrate type as the most important, and to add to these six others which are also fundamental, but perhaps less distinctive. This enumeration is necessarily arbitrary, and can serve only to facilitate the work of the student. When his knowledge deepens, he will be able to free himself from the limitations which such a numerical classification may have put on his understanding of the matter.

A. The six most important characteristics are:

I. The pharynx and pharyngeal structures (gill-clefts, nerves, aortic arches, heart).

72. The notochord or structural axis.

3. Tubular central nervous system.

4. Limbs.

5. Position of mouth.

6. Division of the colom into:

(a) dorsal segmented part or cavities of the somites.

(b) ventral unsegmented part (splanchnocele), which is subdivided by the septum transversum into a thoracic and an abdominal portion.

B. Other fundamental but less distinctive characteristics are:

7. Stomach, intestine, and mesentery.

8. Position of liver, and its relation to veins.

9. Wolffian tubules and ovotestis (=urogenital ridge).

ro. Urogenital ducts (Wolffian and Müllerian).

Ir. Special sense-organs (nose, eye, and ear).

r2. Hypophysis.

The pig embryo illustrates all these characteristics, and we shall study the ways in which the typical mammalian modifications of the type are gradually evolved.

Let us now pass in review these twelve characteristics.

I. The pharynx is the cephalic portion of the digestive canal, and it acquires in all vertebrates a somewhat complicated structure. This complication depends primarily upon a series of lateral outgrowths from the pharynx which are known by the name of gill-pouches. They are symmetrically arranged and there- 
fore form pairs. They are designated by numbers, the pouch which lies nearest to the mouth being called the first, the next the second, and so on. Among the lower vertebrates the number of these gill-pouches varies from five to perhaps nine pairs. In mammals there are always four distinct pairs. In aquatic vertebrates the pouches acquire each an opening to the exterior at the side of the neck, and are then designated as gill-clefts or branchial clefts. We find that the position of the clefts determines the distribution of a series of the most important of the cephalic nerves and the primitive distribution of the branches of the aorta and of certain important muscles, hence the morphological features of the pharynx have a profound influence upon the entire anatomy of the body in that region. No similar pouches are formed from any other part of the digestive canal. The pharynx also gives rise to the thyroid gland, the anlage of which starts as an outgrowth from the median ventral side of the pharynx. The entoderm of the third pair of gill-pouches produces the anlages of thymus glands, and that of the fourth pair the anlages of the parathyroids.

2. The notochord is a rod of cells which extends nearly the entire length of the embryo. It lies in the median plane, a little below the ventral edge of the central nervous system. Its cephalic termination is always in the neighborhood of the pituitary body. It may be considered the primitive structural axis of the vertebrates. There are vertebrates in which it is the only structural axis ever produced, but in the great majority of vertebrates there is developed around the notochord a series of skeletal elements which we know as vertebræ, and which make a new structural axis in these forms. The notochord in these animals is found to run through the bodies of the vertebræ. The notochord diminishes in size as we ascend the vertebrate series. It is of very considerable diameter in the lowest fishes, smaller in amphibia and reptiles, and smallest of all in mammals. In the lower forms it persists throughout life as a continuous, rod. In the higher forms it tends to become attenuated in the vertebral, expanded in the intervertebral, regions, and in adult mammals persists only as a series of disconnected thickenings (nuclei pulposi) between the vertebræ.

3. The tubular central nervous system. This is found in vertebrates only, or in animals which are closely related to vertebrates, so closely that by many naturalists they are included in the same subkingdom. The hollow nervous system is enlarged in the region of the head, the enlargement constituting the brain. The rest of it is of smaller size and constitutes the spinal cord. That the brain and spinal cord form the wall of a tube is one of the fundamental conceptions of anatomy.

4. The limbs. There are two pairs, which are lateral extensions of the surface of the body and acquire in their interior a skeleton by which they are supported and muscles by which they are moved. No structures in any invertebrate animal are known to be homologous with vertebrate limbs.

5. The position of the mouth. The typical invertebrate mouth is surrounded 
by the nerrous system. For instance, in insects or in the jointed worms (annelids) there is a brain, so called, above the mouth, and a strand of nervous tissue running down on either side of the body past the mouth to join the ganglion on the lower side, thus completing a circumoral ring of nervous material through which the œsophagus passes. In vertebrates, on the other hand, the mouth is not enclosed by any œsophageal ring, and the entire nervous system is on one side of the body and dorsal to the mouth.

6. The division of the primitive body-cavity. The body-cavity in the embryo is known by the comprehensive name of the calom. It will not be possible to acquire a clear idea of its division until the embryos are actually studied. It forms many parts. Of these there are two dorsal series, one on each side of the central nervous system, which form cavities of what we designate as the somites of the body. There are also two large ventral divisions which extend from the region of the head to that of the future pelvis, one division for each side of the body. These two large parts are not divided into segments at all, though the cavities of all of the segments are primitively connected with these two main divisions. Comparatively early in the development the two main cavities become connected with one another so as to constitute a single cavity to which we apply the name of splanchnocele. The splanchnocele surrounds the heart of the embryo, where we recognize it as the pericardial cavity, and it extends through the future abdominal region, where we recognize it as the abdominal cavity. The pericardial and abdominal regions of the cavity are separated from one another in the embryo by a broad transverse partition which bears the name of septum transversum. This septum in mammals becomes in the adult the diaphragm. It is one of the most striking of all the morphological peculiarities by which vertebrates-are distinguished from invertebrates.

7. The stomach, intestine, and mesentery. The division of the digestive tract of vertebrates into two fundamental parts, stomach and intestine, is very characteristic.: The stomach is not only an enlargement of the digestive canal, but also may be distinguished from the intestine by its developing glands, which are specific to it and unlike those of the intestine proper. The elongated œsophagus occurs in the higher vertebrates only, and is not a general characteristic of the subkingdom. The mesentery by which the intestine is suspended to the dorsal wall of the abdomen is the survival of the original partition by which the two halves of the splanchnocele were separated from one another. The cavities in the abdominal region come into communication with one another by the very early disappearance of the partition on the ventral side of the intestine. But it should be noted at once that a portion of this primitive ventral partition, or, as we may call it, rentral mesentery, persists permanently in relation to the position of the liver.

8. The position of the liver. The primitive large veins of the embryo pass through the septum transversum, and it is by intercrescence with these veins, and 
as an appendage to the septum itself, that the liver is developed, although it is produced by a special local growth of the digestive canal.

9. The urogenital ridge. Out of a part of the primitive segments there are developed excretory organs, and these, as they increase in size, form two protuberances on the dorsal side of the splanchnocele. Each protuberance is what we know as the urogenital ridge, so named, first, on account of its form; and, secondly, on account of its producing not only the excretory organs proper, but also the genital glands.

Io. The urogenital ducts. There is primitively a single duct for each urogenital ridge. This duct is commonly known as the Wolffian duct. A little later in the history of the embryo there appears a second canal known as the Müllerian duct, which is closely parallel to the first, but which has no connection with any of the excretory apparatus, and is destined to serve later as the female genital duct. In no invertebrate have we found anything certainly homologous with these two ducts.

II. Special sense-organs. These are the olfactory, the visual, the so-called auditory organs, and the organs of the lateral line. We have to use the term "so-called" in speaking of the auditory organ because we now know that the ear in the lower vertebrates is not an organ of hearing, but an organ of balancing or orientation, and it is only in the higher vertebrates that there is added to this primitive function that of audition proper. It seems not improbable that many invertebrate animals have sense-organs which are homologous with those of vertebrates. Nevertheless, in the vertebrate type there are many peculiarities which are distinctive, and these we shall best learn from a 'study of the actual development. The sense-organs of the lateral line are highly developed important structures in the Ichthyopsida, but apparently are not represented in mammals at any stage of their development.

I2. The hypophysis. The hypophysis is the embryological name applied to the structure which we know in the adult as the anterior lobe of the pituitary body. The posterior or infundibular lobe is a portion of the brain, but the anterior lobe is an outgrowth from the cavity of the mouth of the embryo. Comparatively early in the development of the individual this outgrowth becomes entirely separated from the mouth-cavity (from the walls of which it arose), and forms a closed vesicle. It exists in every known vertebrate animal, has been much studied, but still remains an organ the significance of which we cannot explain. Its absolute persistency and the uniformity of its development indicate that it is an organ of importance, but beyond that we can hardly go.

To these conceptions, the student should add the following comprehensive morphological notions: The mammalian body. may be defined as two tubes of epithelium, one inside the other; the outer tube (epidermal or ectodermal) is very irregular in its form; the inner tube (entodermal) is much smaller in diameter, but much longer than' the outer and has a number of branches (lung, pancreas, etc.), and is placed within the ectodermal tube. Between these two tubes is the 
very bulky mesoderm, which is divided by large cavities (abdominal and thoracic) into two main layers, one of which is closely associated with the epidermis and forms the body wall, the somatopleure of embryologists; the other joins with the entoderm to complete the walls of the splanchnic viscera, and constitutes the splanchnopleure of embryologists. The mesoderm is permeated by two sets of cavities: (I) the heart and blood-vessels; (2) the lymphatic system. It is also differentiated into numerous tissues (muscles, tendon, bone, etc.), organs and the internal parts of the urogenital system. The nervous system, although developed from the ectoderm, is found separated from its site of origin, and completely encased in mesoderm.

\section{The Principal Modifications of the Vertebrate Type.}

Our knowledge of human development being at the present time incomplete, it is often necessary to supplement that knowledge by reference to facts of observation on the development of various vertebrates. Indeed, the best study of human embryology includes more or less comparative work. We shall, therefore, find frequent occasion to refer to the development of many vertebrate types. Accordingly, in this section there are given definitions of the principal subdivisions of the vertebrates to which we shall have occasion to refer.

From an embryological standpoint, vertebrates may be separated into two main divisions, which are commonly designated as the Amniota and Anamniota, distinguished by the presence or absence of the amnion, the amnion being a thin membrane, which immediately surrounds the embryo in the higher forms. It occurs in reptiles, birds, and mammals, which together constitute the Amniota. It is absent in the fishes and amphibians, which therefore constitute the Anamniota. These two divisions are also distinguished by other peculiarities. The higher forms referred to all have the organ known as the allantois, an appendage of the embryo, which is lacking in the lower forms. The comparative anatomist finds many points of resemblance between the various classes of fishes, on the one hand, and the amphibia, on the other, and indicates this relationship by the use of the term Ichthyopsida, which means "fish-like." In our present classification the term Ichthyopsida is synonymous with Anamniota. The comparative anatomist further recognizes a close relationship between birds and reptiles, and puts these together under the common designation of Sauropsida, or "reptile-like."

As regards the fishes, many classifications are more or less in vogue at the present time. For the purposes of this book, the following names for the classes have been adopted as names generally understood and sufficiently exact to meet our needs: The lowest fishes are the hag-fishes and lampreys, constituting the group of Marsipobranchs. Next comes the group comprising the sturgeon and its allies, for which we have retained the old term of Ganoids. To these fishes the central position in the system must be assigned, and it is probable that the higher fishes are more or less directly descended from Ganoid-like forms. They fall into three 
further classes, of which the largest and most varied is that of the bony fishes, or Teleosts. Another class, known as the Elasmobranchs, comprises the sharks, skates, rays, and electric fishes. The last class is known as the Dipnoi, or lung fishes, which comprise only three living forms, the Ceratodus, living in Australia, the Protopterus in Africa, and the Lepidosiren in South America.

The amphibia are divided into two classes, the Urodela, of which the newts and salamanders are familiar examples, and the Amura, of which the frogs and the toads are the best known representatives. The two types are easily distinguished by the presence or absence, respectively, of the tail in the adult.

As to the reptiles, it is unnecessary to consider their classification, as we shall not have much occasion to refer to them, our knowledge of their embryology being very fragmentary at the present time, save for a rather extended series of observations on the development of lizards. As regards birds, it may be noted that embryologists have worked chiefly upon the chick, which has been for a century the classic object of embryological study. There are comparatively few observations on the development of other species of birds.

Mammals are divided into three principal classes. Of these, the lowest is that of the Monotremes, of which the only living representatives are found in Australia and neighboring islands, a very few species concerning the development of which very little is as yet known, but which are of importance, as they resemble in certain respects the reptiles and assist us in drawing comparisons between the reptilian and the mammalian types. Of this class, the Australian duck-bill may be mentioned as typical.

The second class is that of the Marsupials, familiar to us in America through the common opossum. In Australia there are many genera and species of marsupials.

The third class comprises the majority of well-known mammals, and may be termed the Placentalia, and, for embryological purposes, it is convenient to consider the Placentalia as forming two principal subclasses, the animals with claws and the animals with hoofs, the Unguiculates and the Ungulates. Of the Unguiculates, we shall have occasion to refer to the Insectivora, of which the mole may serve as a type; the Cheiroptera, or bats; the Rodents, including the rats, guinea-pigs, rabbits, etc.; the Carnivora, cats, dogs, and allied animals; and, finally, the Primates, which include the lemurs, monkeys, apes, and man.

Of the Ungulates, we shall have occasion to refer chiefly to the pig and the sheep. The following table presents in their proper order those animals which we shall have occasion to consider.

Annelida

Atriozoa

Tunicata (Ascidia)

Cephalochorda

Amphioxus 
Vertebrata

Anamniota (Anallantoidea)

Ichthyopsida

Pisces

Marsipobranchia (lampreys, etc.)

Ganoidea (sturgeon, etc.)

Teleostea (bony fishes)

Elasmobranchia (sharks, skates, etc.)

Dipnoi (lung-fishes)

Amphibia

Urodela (newts, salamanders, etz.)

Ansura (frogs, toads)

Amniota (Allantoidea)

Sauropsida

Reptilia (lizards, crocodiles, snakes, turtles, etc.)

Aves

Mammalia

Montotremata (duck-bill, etc.)

Marsupialia (opossum, kangaroo, etc.)

Placentalia

Unguiculate series

Insectivora (moles, etc.)

Cheiroptera (bats)

Rodentia (rats, rabbits, guinea-pigs, etc.)

Carnivora (cats, dogs, etc.)

Primata (lemurs, monkeys, apes, man)

Ungulate series

Ungulata

Artiodactyla (even-toed) (cattle, sheep, pig, deer, etc.)

Perissodactyla (uneven-toed) (horse, rhinoceros, etc.)

Of the invertebrate animals there will be little to be said. There are two types of invertebrates which show relationship in their structure to true vertebrates. One of these is the class of jointed worms, or Annelids; the other is the class of Atriozoa, which comprises the subdivisions of Tunicata and of the Cephalochorda. All of our observations on the development of this last type are based on the one genus, Amphioxus, which will therefore be the name which. we shall use whenever we have to refer to these animals.

\section{Definition of Anlage.}

There will be frequent occasion to use this word in a strictly technical sense. It has been adopted from the German, as there is no satisfactory English equivalent for it. The French use the word "ébauche," and the Italians "abozzo." Primordium has been proposed as the Latin equivalent and is used by a few American authors, but anlage is generally employed by both American and English writers. "Anlage" may be defined as follows: The first accumulation of cells in the developing embryo recognizable as the commencement of a structure, organ, or part. 


\section{A Summary of Embryological Development.}

The following summary applies to what is known of vertebrates only. It would require some modifications to be applicable to the whole animal kingdom. Each individual arises from a single cell which is termed the impregnated or fertilized ovum. From this all embryological study starts. The fertilized ovum has its earlier history, since it is the product of the fusion of two sexual elements. It is a living cell, and therefore contains protoplasm and nucleus. It is also furnished with a certain amount of material known as yolk, which exists in the form of separate granules imbedded in the protoplasm. This yolk is the reserve food material, and by the assimilation thereof the protoplasm of the ovum can grow.

The first step in the development is thepeated division of the original cell so that there is produced an increasing number of cells. The earlier stages of this cell multiplication are designated as the segmentation of the orum. This name is due to the fact that the process was first observed in the eggs of amphibia in the early part of the last century, before the cell doctrine had been established. In default of a better name, the separate cells into which the ovum divided were called segments, for it was, of course, not known that they were cells. Although this term is no longer appropriate, it is still universally used because of its convenience. There are two principal types of ovum known: in one type we find only a small amount of yolk material; in the other a very large amount. There are ova known intermediate between these two types. When the ovum is of the first type, the whole of it undergoes segmentation at once, and to such an orum the term holoblastic is applied. In the second type, on the contrary, we find that the protoplasm tends to accumulate' at one pole of the cell and the yolk granules at the other. The protoplasmic portion exhibits a far more active cell division than the yolk-bearing portion, so that the segmentation seems to take place exclusively around one pole or part of the ovum, which is, therefore, designated as |meroblastic. After the segmentation of the ovum the multiplication of the cells continues, and they gradually arrange themselves in such a manner as to form three distinct sheets or laminæ, which are named "germ-layers." These layers are designated: the outermost as Ectoderm, the innermost as Entoderm, and the middle as Mesoderm.* From an embryological point of view the importance of these three primitive germ-layers cannot be over-emphasized. The principal occupation of the student will be to familiarize himself with the appearance of these layers and the modifications which they undergo, and the adult tissues which are produced from them. They dominate every phase of development, the form of every organ, the production of every tissue. Their importance is so great that embryology might almost be defined as the science of germ-layers.

* Some English and occasionally Continental authors use other terms for the germ-layers, namely, for ectoderm, epiblast; for entoderm, hypoblast; for mesoderm, mesoblast. I have preferred to maintain the older terms. which have been in almost universal use for a century. 
The primitive germ-layers consist of very simple cells, and are themselves at first extremely simple in their organization. The majority of the cells which they contain undergo a greater or less degree of modification as development progresses. This modification is termed differentiation, and is more fully considered in our next section, on Cytomorphosis. It is probable, however, that a certain number of the cells very early in the development are set apart, preserving the primitive character of their protoplasm and taking no share in the formation of the tissues of the body. These cells, comparatively unmodified, are known as the germ-cells; compare page 25 and the section on Heredity. As the remaining cells form part of the body of the individual, they may be designated as somatic cells. Besides the process of differentiation of the cells, we find that the production of organs is largely dependent upon the unequal growth of the germ-layers, one part growing rapidly, another more slowly, so that the layers acquire, as the embryo develops, a more or less complicated form, owing to the folding of the layers. The general principles which govern these important developments are considered in the section upon the Relations of Surface to Mass.

\section{Cytomorphosis.}

This term is used to designate comprehensively all the structural modifications which cells or successive generations of cells may undergo, from the earliest undifferentiated stage to their final destruction. It will be convenient, though somewhat arbitrary, to distinguish four fundamental successive stages of cytomorphosis. These stages are (I) the undifferentiated stage; (2) the stage of progressive differentiation, which itself often comprises many successive stages; (3) the regressive stage or that during which degeneration or necrobiosis occurs; (4) the stage of the removal of the dead material.

In the various parts of the body we find these stages to succeed one another at varying rates, and there are always to be found in every living vertebrate body a considerable number of cells which have passed through only a certain differentiation and do not present any of the phenomena of degeneration or of death. On the other hand, there are cells at every epoch of life after an early brief embryonic period which degenerate and die off, although the life of the individual is uninterrupted. At any given moment the body consists of cells which have made unequal progress through the cytomorphic cycle.

r. The Undifferentiated Stage.-A fertilized ovum is an undifferentiated being, although it has a very complex organization. As it has only one nucleus there can be no variety of nuclei. The term "undifferentiated" therefore applies especially to the protoplasm, which never has any special structures or formed parts, such as occur in the tissues and cells of the adult. It is, however, not uniform, but in many ova has distinct regional differences, which so far as hitherto noted depend upon peculiarities in the masses and strands of protoplasm, and upon the distribution of the yolk granules, of which there may be several kinds. 
The uneven distribution of the yolk granules in the ova of mammals (compare page 34 ) indicates that there are unlike regions, the morphological significance of which, however, is not known yet.

Two views as to the constitution of the ovum in relation to the structures which arise from it have been brought forward. According to one view, each part of the ovum is predestined to form a definite part of the adult and cannot form any other part. According to the other view, the ovum is homogeneous in its essential lack of true differentiation, and any part of it may form any part of the adult if given the requisite opportunity. The first view is known as the mosaic theory, the egg being compared to a mosaic, and was founded by Wilhelm Roux (I888). The second view is known as the theory of isotropism and was founded by Ernst Pflüger in I884.

Pflüger's theory of isotropism was based upon his experiments on frogs' eggs. Each egg has a small white area which normally lies underneath, the larger, darkly pigmented area of the egg alone showing from above. Out of the dark area the back, with the nervous system and other parts, takes its origin. If the eggs, freshly fertilized, are fastened with the white side up, then the white side produces an absolutely normal back and nervous system, normal as to form and function, though lacking the typical pigmentation. These observations were confirmed by Born, who further discovered that the segmentation nucleus always rises toward the upper side of the egg, and that the position of the nucleus determines which part of the orum shall become the dorsal side of the embryo. Another set of experiments by Oskar Schultze demonstrated that both the unpigmented and the pigmented sides of the same egg could be made to produce dorsal structures. Another class of experiments, which were first made by Hans Driesch, has revealed that the earliest cells (segmentation spheres, blastomeres, or cleavage cells, as they are variously called) produced by the ovum preserve the undifferentiated qualities of the parent egg, and may develop in one way or another according to circumstances. The egg of a sea-urchin divides into two cells, each of which multiplies and normally gives rise to half of the body of the animal. By somewhat violent shaking the two cells may be artificially separated; each cell may then develop into a complete larval sea-urchin, but of half the normal size only. Similar experiments have since been made by several investigators, who have obtained like results with other animals, vertebrate as well as invertebrate. Even more remarkable larvæ have been raised from blastomeres of the four-cell and eight-cell stages of segmentation, producing larvæ of one-fourth and one-eighth the normal size. Zoja claims to have repeated the experiment successfully on the eggs of Clytia and to have obtained one-sixteenth larvæ.

Roux's mosaic theory was based on W. His's principle of the organ-forming areas of the germ, or, as it has been also termed, the law of germinal localization. His pointed out that in the normal course of development every spot in the blastoderm corresponds to some future organ, and suggested that logically it is 
probable that every organ is represented by some region in the ovum itself. In other words, although the organs of course do not exist preformed in the egg, yet the material for them is there and prelocalized. Roux has developed this conception and has compared the egg to a mosaic; each member of the mosaic is assumed to undergo self-differentiation or its predestined development. Complete verification of this theory has not been secured, but it has been demonstrated that certain eggs of animals of several invertebrate orders contain substances, which have an exact distribution, and which have a definite fixed relation to adult structures. By putting some of these eggs in a centrifuge these substances may have their distribution artificially changed. In eggs thus altered the substances continue to transform themselves into their predestined structures, which consequently appear displaced. By these experiments the mosaic theory has received a limited confirmation.

At present it is impossible to recnncile the two theories of the constitution of the ovum, but since both are based apparently on facts it seems probable that they will, by wider knowledge, be fused into a single coherent conception.

Meanwhile the fact of most importance in cytomorphosis is, that the protoplasm of the ovum is undifferentiated and lacks completely any variations of its structure comparable to those which we observe as characteristic in the cells of adult tissues. The potentialities of the ovum, on the other hand, are of course very great. Experimental embryology is now endeavoring to ascertain what physiological causes render those potentialities active. From physiological embryology much is to be expected.

2. Differentiation.-This may be defined as a process by which the structure of the cells is modified, so that they become dissimilar by acquiring an organization which adapts them to special functions. The cells which arise during the segmentation of the ovum differ but slightly from one another. As development progresses we find the cells change, some in one way, some in another, so that many kinds of cells are produced, but of each kind we find a large number of cells. Each kind of cell may be said, roughly speaking, to form a tissue for itself. Cells of each tissue offer visible peculiarities by which they may be readily distinguished from one another under the microscope. It thus appears that the production of tissues is the main result of differentiation, so that this process of development may be fairly accurately defined as equivalent to histogenesis. As to the factors which cause differentiation, we have no satisfactory knowledge. 'We can, at present, only note the changes when they acquire such magnitude as to become microscopically visible. As to the physiological conditions which cause these changes we have almost no conceptions. It is probable that the nucleus has a leading rôle to play, but our knowledge of this rôle is too little advanced to permit a profitable discussion of the subject here.

The actual process of differentiation shows itself both in the protoplasm and in the nucleus of the cell. The changes in the former are the more conspicuous, and therefore the better known. The changes in the nucleus have still to be adequately 
studied. The changes in the protoplasm are twofold: First, in the intimate structure of the protoplasm itself and in the size and disposition of its strands and filaments; secondly, in the character of the various substances to be found imbedded in the protoplasm. These two kinds of change are well illustrated, the first, by the nerve-cells; the second, by the gland-cells, for instance, in the pancreas. The student can easily see that the character of the protoplasm in the adult nerve-cell differs profoundly from that of a cell from one of the embryonic germ-layers, having become visibly much more complex. In the secretory cells of the pancreas the zymogen granules are conspicuous; their distribution, uniform size, and refractile qualities demonstrate immediately their unlikeness to anything found in the embryonic cells. These granules are not protoplasm, but particles imbedded in the protoplasm or, as they may be called, enclosures.

The Law of Genetic Restriction.--Another fundamental idea, which it is most important for the student to grasp, is that differentiation acts as a progressive restriction upon the further development. Each successive stage of differentiation puts a narrower limitation upon the possibility of further advance.

The range of possible changes at any given time is determined not merely by the nature or kind, but also by the stage or degree of the previous differentiation. The law of genetic restriction governs the entire ontogeny. In order to illustrate it and to emphasize it, it will be profitable to consider a few illustrations from each of the germ-layers. First, then, the ectoderm. This layer early separates into two parts, one to form the nervous system, the second the epidermis; the nervous part thereafter never forms epidermal structures, the epidermal part never forms a medullary canal. The central nervous system retains in part a simple epithelial character (ependyma proper), but most of its walls become nervous tissue; its cells pass from the indifferent stage and become neuroglia cells or young nerve-cells (neuroblasts). Neuroglia cells never become anything else, and the nerve-cells are always nerve-cells to the end. Next, as to the entoderm. Wherever in it specialization takes place, as in the tonsil, thymus, thyroid, œsophagus, liver, or pancreas, each territory of cells keeps its characteristics and never assumes those of another territory. Finally, as to the mesoderm. It is found very early to include in vertebrate embryos four kinds of cells, of which the most numerous are the undifferentiated cells, the other three kinds being the endothelium of blood-vessels, red blood-cells, and germ-cells. All of these are precociously specialized; they are few in number, yet they are probably the parents of all the cells which are produced of their kind each throughout life. Passing on to a later stage, we note that when a striated muscle-fiber is produced a striated muscle-fiber it always remains, and it never becomes anything else.

Two Types of Differentiation.-There are two distinct types of cell differentiation which I think have not hitherto been clearly recognized or defined. For both types the starting-point is the same-the undifferentiated embryonic cell. In one type we find that, as the cells proliferate, a portion of them only undergoes differ- 
entiation, and another portion remains more or less undifferentiated and retains more or less fully the power of continued proliferation. The epidermis is a good representative of this type. Its basal layer consists of embryonic cells, which multiply; some of these cells move into the upper layers, enlarge, and differentiate themselves into horny cells; others remain in the basal layer and continue to multiply. The progeny of a given basal epidermal cell do not all have the same fate, but divide themselves into two kinds of cells, one kind retaining the ancestral character, the other becoming something new and unlike the parent cell. Differentiation according to the second type is characterized by its inclusion of all the cells. This type has its culminating and most perfect illustration in the central nervous system, where comparatively early in embryonic life all the cells become specialized, and with the acquisition of specialization they forfeit their power of multiplication - the neuroglia cells partly, the nerve-cells wholly.* The growth of the brain after early stages depends not on the proliferation of cells, but chiefly upon the increase in size of the individual cell. The correctness of this statement is not affected, in my belief, by the fact that epithelial portions of the medullary tube in comparatively late stages may be added to the nervous portion, the cells multiplying rapidly, as we see at the growing edge of the young cerebellum. The brain here grows by the addition of cells in the indifferent stage, but as soon as these cells are differentiated they conform to the general law and divide no more (neurones) or slowly (glia cells).

The importance to pathologists of a thorough knowledge of the genesis of the tissues from their germ-layers can hardly be emphasized too strongly, for it is more than probable that all pathological tissues are as strictly governed by the law of genetic restriction as are the normal tissues.

3. Regression.-The use of this term does not imply that a cell can move backward after differentiation into a stage of lower differentiation or into an undifferentiated condition. So far as we know at present, such a change does not occur, and we therefore look upon it as impossible. Regressive changes are very unlike the constructive changes which appear in differentiation, for they are destructive. They fall into three main groups: first, changes of direct cell death; second, necrobiosis or indirect cell death preceded by changes in cell structure; third, hypertrophic degeneration or indirect cell death preceded by growth and structural change of the cell, often with nuclear proliferation. Direct cell death implies that the cell loses its vitality, and, being dead, disintegrates; or, may be, is removed by some means, chemical or phagocytic, before disintegration occurs. Necrobiosis and hypertrophic degeneration are normal processes, which invariably occur in the normal body and play an important rôle in its development. Without their occurrence on a large scale the normal round of human life would be impossible. The student should free himself from the unfortunate tradition that these processes are exclusively pathological.

* With possibly very rare exceptions. 
Correct notions on this subject are so important that a few illustrations may be mentioned. Let us begin with necrobiosis. There are organs whose existence is limited in time, such as the thymus and fœtal kidney. These organs attain their full differentiation, and thereafter most of their elements die off and finally are resorbed, most of the organ disappearing. Another familiar illustration is offered by hairs, which die and are shed. Cell death on a large scale is a common phenomenon of the tissues. It occurs in the cartilage both when the cartilage is permanent and, even more conspicuously, when the cartilage gives way to bone, the disintegration of the cartilage cells preceding the irruption of the bone-forming tissues. It occurs among the gland-cells of the intestine, in the pregnant uterus, and in all the tissues of human decidua reflexa. Degeneration in the stricter sense of the ante-mortem and hypertrophic change of cell structure is also of widespread occurrence in the healthy body. Perhaps no instance of this is more familiar than the production of horny tissue in the epidermis or elsewhere. That fatty degeneration takes place normally has long been taught, while mucoid and colloid degeneration are so obviously normal that we commonly think of their pathological occurrence as merely an exaggeration of a normal state. Hypertrophic degeneration is an extremely common pathological process, but it also occurs as a normal process, as, for example, in epidermal cornification, as just mentioned, and very strikingly in the production of giant-cells (myeloplaxes, etc.), and on an astounding scale in the uterine tissues during pregnancy in many, perhaps all, mammals.

4. The Removal of Cells.-The sloughing off of cells is one of the most familiar phenomena, since it occurs incessantly over the epidermis and with hairs. Its part in menstruation and its colossal rôle in the after-birth are known to all, and every practitioner is accustomed to look for shed cells in urinary sediment. Large numbers of cells are lost by the intestinal epithelium. The destruction of bloodcorpuscles is incessant, and we might greatly extend the list of these illustrations. Owing to the enormous loss of cells to which the body is subject, there is provision to make good this loss. This provision is called "regeneration," and has been dealt with in an enormous number of investigations. During embryonic life regeneration plays a comparatively insignificant part, and we shall not have to deal with it further.

Of the four stages of cytomorphosis, the second, or stage of differentiation, is that which will principally claim our attention. But we cannot fully understand the developmental processes unless we also have constantly in mind the normal degeneration and death of cells, even in the embryo.

\section{Comparison of Larval and Embryonic Types of Development.}

We have seen in the preceding section that the first cells produced in development from the ovum are undifferentiated, and are capable of development in many and varied directions. The more they become specialized, the more their possibilities of further varied development are decreased. It is thus obvious that the 
greater the number of cells of the undifferentiated type that can be produced, the greater will be the number of elements which can be later differentiated. Hence, the more the period for the production of undifferentiated cells is prolonged and the commencement of differentiation postponed, the more complex may be the degree of organization ultimately attainable.

It is convenient to designate the undifferentiated cells as they arise from the segmentation of the ovum by the term "embryonic cells." The object of this section is to point out that the larval type of development is less favorable for the multiplication of embryonic cells than is the embryonic type; and, further, that the embryonic type becomes more and more marked as we ascend in the animal kingdom.

The Larval Type.-In the lower multicellular animals we encounter only larvæ; sponges, jellyfish, starfish, and worms all pass through their early stages as larvæ. Now, larvæ are animal forms which have to obtain their own food and to protect themselves against enemies. They are, therefore, provided with a variety of organs, or, as we may say, with differentiated tissues which enable them to perform the various physiological functions which are necessary for the maintenance of their existence. The differentiation of tissues comes in very early.

The Embryonic Type.-True embryos arise from eggs which contain a more or less considerable amount of yolk or nutritive material, the presence of which renders unnecessary any activity on the part of the embryo to obtain its food-supply; and we find, moreover, that these embryos are protected by hard shells or other devices from their enemies. Their only task is to pursue their own development. Under these circumstances it is possible for the embryos to continue for a long time the production of embryonic cells, and we observe that the beginning of the differentiation proper is correspondingly postponed. The transition from the larval to the embryonic type is very gradual. The yolk appears in the lower animals in small quantities, increasing in some of the higher types, and attaining its maximum in some of the highest. Since the embryo is dependent on the yolk, and since the yolk exists only in the higher forms in sufficient quantities, it follows that fully typical embryos can occur exclusively in the higher animal types.

In the mammalia the ovum contains a rather small quantity of yolk, yet the mammals are the highest animals and develop most perfectly according to the embryonic type. This peculiarity is due to the fact that two special physiological devices have been evolved in the mammals to supply food to the developing embryo. First, there is a special relation established between the embryo and the uterus by means of a complicated adjustment of embryonic and uterine tissues, which supplies nutrition to the embryo from the blood of the mother. Secondly, there are the mammary glands, which also serve the same function. By these two devices the embryo is even more completely freed from the necessity of seeking its food and protecting itself than is the case with those forms, such as the birds or elasmobranchs, in which the supply of food material is very large. 


\section{Germ-layers.}

The germ-layers are the first groups of cells to arise as the result of the segmentation of the ovum. They are three in number, and each forms a distinct sheet or lamina. As stated on page זo, these three primitive layers are termed "ectoderm," "mesoderm," and "entoderm." The ectoderm is the most external of the three, and upon the outside of the body parts of the ectoderm remain permanently to constitute the outside skin or epidermis. From its very position it necessarily is the part of the body to come into relation with the external world, and accordingly we find that its two great duties are to produce the protective covering of the body and the apparatus for receiving and utilizing sensations; in other words, the chief sensory organs and the nervous system. The entoderm, on the contrary, forms the internal cavity of the digestive canal and its appendages. It therefore is concerned chiefly with the production of the organs of digestion, and appears in the adult as the epithelium of the digestive and respiratory organs and of the glands appended to the digestive tract. The mesoderm, lying as it does between the other two layers, is shut off by them from direct relation with the external world or with foodmatter, and is accordingly restricted to a series of internal functions, of which four are especially important: (r) The function of circulation both of blood and lymph through definite channels; (2) of excretion; (3) of movement; (4) of supporting the body, especially the parts produced from the ectoderm and entoderm. It is from the middle germ-layer, therefore, that the connective and skeletal tissues arise, that the muscular tissues arise, that the excretory organs arise, and that the blood, blood-vessels, and lymphatics arise.

The inner and outer germ-layers are primarily simple epithelial structures, consisting each of a single layer of cells. This primitive characteristic is never wholly obliterated and really controls all of the modifications which these two layers undergo. The mesoderm, on the other hand, is primarily not epithelial, but mesenchymal. Mesenchyma consists of widely separated cells which form a contimuous network of protoplasm, the meshes of which are originally filled by a homogeneous intercellular substance or matrix. The student will have frequent occasion in his practical work to study it in its embryonic stages.

The Colom.-The cœlom is the primitive body-cavity of the embryo. It arises as a space in the mesoderm. Soon after this space has appeared we find that the cells of the mesoderm, which bound it, assume an epithelial character; consequently the mesoderm, after the cœlom has appeared, consists of mesenchyma and of an epithelial layer bounding the cœlom. This epithelial layer is called the mesothelium. The mesoderm, therefore, differs fundamentally from the ectoderm and entoderm by this peculiarity, that it comprises both an epithelial and a non-epithelial portion. Both portions play very important rôles in the production of the various tissues and organs of the body. There is another respect also in which the mesoderm differs from the other germ-layers, for we find that it increases in volume and in complexity as we ascend from the lower to the higher types of animals, or as we.pass 
from the embryo toward the adult condition, more than does either the outer or inner germ-layer.

The Specific Quality of the Germ-layers.-Each germ-layer has its specific and exclusive function in the production of tissues, giving rise only to the tissues which are proper to it and never to the tissues which are proper to either of the other layers. We must, indeed, so far as, our present knowledge goes, regard most, at least, of the cells in the germ-layers as originally wholly indifferent as individual cells. But we must, nevertheless, not forget that, as members of a germ-layer, their potential fate is already restricted. It is probable, if we could successfully transplant an undifferentiated cell from one germ-layer to another, that it could take part in the production of the tissues proper to that layer. But it is further probable that this would be impossible after the differentiation of the cells in any layer had fairly begun. After a cell has become definitively a member of one of the germ-layers, it probably never migrates to join another layer. The accompanying table presents the principal tissues classified according to the layers to which they belong. There have been classifications of organs on the germ-layer basis published before, but inasmuch as organs usually contain cells from two layers, we get a more correct presentation of the actual genetic relationship by confining our tabulation to the tissues. Blood-vessels and blood-cells arise very early, before the clear separation of the mesoderm and entoderm has occurred. It is possible that they are entodermal. With these two limitations the table presents our present knowledge.

CLASSIFICATION OF THE TISSUES.

(A) Ectoderial.

I. Epidermis.

$a$. epidermal appendages,

b. lens of eye.

2. Epithelium of

a. cornea,

b. olfactory chamber,

c. auditory organ,

d. mouth

(oral glands), (enamel organ), (hypophysis),

e. anus,

f. chorion, fetal placenta,

g. amnion.

3. Nervous system.

a. brain, optic nerve, retina,

b. spinal cord,

c. ganglia,

d. neuraxons,

$e$. chromaffine cells.
(B) Mesodermal.

I. Mesothelium.

a. epithelium of peritoneum, pericardium, pleura, urogenital organs,

b. striated muscles.

2. Mesenchyma.

a. blood-cells (red and white),

b. blood-vessels,

c. connective tissue, cellular reticulum, smooth muscle, pseudo-endothelium, fat cells, pigment cells,

d. lymphatics,

e. spleen,

$f$. supporting tissues, cartilage, bone,

g. marrow of bone.
(C) Entodermal.

I. Notochord.

2. Epithelium of

a. digestive tract, œesophagus, stomach, liver, pancreas, small intestine, yolk-sac, large intestine, cæcum, vermix, rectum, allantois (bladder),

b. pharynx,

Eustachian tube, tonsils,

thymus, parathyroids, thyroid,

c. respiratory tract, larynx, trachea,

- lungs. 
The Constitution of Organs.-Few organs are formed from a single germ-layer, for as we find organs in the vertebrate body they usually consist of two parts, one of which may be regarded as the part proper of the organ, upon which the performance of its special function directly depends, and the accessory part, which supplies the necessary physiological conditions for the functioning of the organ. For example: in a salivary gland the actual work of secretion is performed by the epithelial cells of the gland, but these cells cannot act unless they are supported by connective tissue and supplied with blood and lymph, three conditions which depend upon the mesoderm, and also supplied with nerves, a condition which depends upon the ectoderm. By far the majority of organs have their functional part produced from epithelium, and this epithelium may come either from the original outer or inner germ-layer, as the case may be, or from the mesothelial portion of the middle layer. But the organ, as a whole, requires for its completion the addition of other elements, as indicated in the example given. We find, therefore, that there are no adult organs which are constituted solely by either the ectoderm or entoderm, although there are organs the principal part of which may come from one or the other of these germ-layers, but to complete the organ the mesoderm must help. On the other hand, the mesoderm may form complete organs by itself, or at least with no other aid from the other germ-layers than is given by the supplying of nerve-fibers. Such purely mesodermal organs are illustrated by the spleen, the kidney, and the sexual glands.

\section{The Relations of Surface to Mass.}

However much the weight of an animal increases during its development, the ratio of the free surface to the mass alters but slightly from the ratio established when the embryo begins to take food from outside. It is only for convenience that I express this law in this precise form; in reality, about it our knowledge is scanty and our conceptions vague. According to a geometrical principle, when the bulk of a body bounded by a simple surface increases, the surface enlarges less than the mass - in the simplest case of a cube, the surface increases as the square, the mass as the cube, of the diameter. If in a cube of unit diameter one unit of surface bounds one unit of mass, then in a cube of three units diameter nine units of surface will bound twenty-seven units of mass; the proportion in the first cube is $\mathrm{I}: \mathrm{I}$, in the second $\mathrm{I}: 3$. To maintain the proper proportion in the embryo, simple enlargement is insufficient, therefore the surface increases by becoming more and more irregular. The irregularities are characteristic of each organ and part, and may be either large or microscopic. They may be conveniently grouped under two main heads-projections and invaginations.

Projections are illustrated by the limbs, filaments of the gills in fishes, the villi of the intestine, folds of the stomach in ruminants, etc. In every case the projection is covered by an epithelium and has a core of mesodermic tissue. 
Invaginations exist in much more varied form and play a principal part in the differentiation of the animal body. They may be classified under four principal heads: (I) dilatations; (2) diverticula; (3) glands; (4) vesicles. Dilatations have considerable importance in embryology: the stomach, lungs, bladder, and uterus arise as gradual dilatations of canals or tubes of originally nearly uniform diameters. Diverticula, in the sense of relatively large blind pouches, also form important organs, such as the cæcum and appendix vermiformis, or the gall-bladder; these structures arise each as a blind outgrowth of a canal, the walls of which at a certain point rapidly grow to form the pouch. Glands are, as first shown by Johannes Müller's classic researches, only small diverticula, which end blindly and appear in an immense variety of modifications; the manifold types of glands are discussed below in a separate paragraph; they constitute the largest class of organs with which we have to deal. The glands are developed from epithelium and push their way into the mesoderm upon which the epithelium rests, while in dilatations, and in diverticula, the epithelium and mesoderm expand together. Vesicles we call those epithelial sacs which develop somewhat like glands by growing into the mesoderm, but the mouth of the invagination closes by the coalescence of the epithelium, thus shutting the cavity. The closed sac separates from the epithelium from which it arose, and connective tissue grows between the two; the sac may then undergo various modifications. The membranous labyrinth of the ear is developed from the ectoderm in this way, as is also the lens of the eye. We might perhaps also class the medullary canal under this head (cf. Chapter V) if we choose to consider it as a vesicle so much lengthened that it has become a tube.

Glands.-A gland may be defined as a structure which produces material which is discharged from the gland and used elsewhere to meet a physiological need. According to the nature of this material, we distinguish two fundamentally different types of so-called glands. One of these we designate as the true glands, which produce chemical substances which are thrown off from the cells producing them to constitute the secretion of the gland, so that the cells themselves all remain in the gland. In the second type the cells themselves are multiplied, so that the structure yields, as it were, a crop of cells, which is removed from the site of origin and then utilized physiologically. Glands of this type may be called cytogenic. Of the true glands we may distinguish several sorts. The simplest kind consists of a single cell. Of unicellular glands, the goblet cells are the most familiar type known in man. Most true glands, however, comprise many cells and are classed as multicellular. The majority of these have an internal cavity, which may be simple or very complicated in its form, but is always bounded by a layer of epithelium. They have in addition a canal, which leads from the cavity of the gland to an external opening, and is called the duct. When the secretion is produced, the chemical substances formed by the epithelial cells are discharged into the cavity of the gland and thence How through the duct to the outlet. In certain cases a remarkable modification may occur by the obliteration of the duct, thus producing a so-called ductless epi- 
thelial gland. In such structures the secretion can escape only by transfusion into the blood or lymph.

The epithelial glands with ducts exhibit two main sorts of modification, for they are either small structures which occur in great numbers, or they are larger structures, each constituting a separate organ. Hence, we divide the glands into simple glands and compound or organic glands. The simple glands are always small and have one or several centers of growth, according as they are simple tubes or slightly branching. Those of each kind are always very numerous, and occur more or less together over considerable areas. Good illustrations of simple glands are offered by the sweat glands and the intestinal glands. The compound glands are of greater bulk. They are provided with a single main duct which is more or less branched, each branch connecting finally with the secretory portion proper of the organ, which portion may itself also be branched. Each gland falling in this division is a more or less complete organ by itself, receiving its special blood supply and its special innervation-it is, in short, a clearly marked physiological entity. A specially striking morphological modification in the structure of compound glands is offered by the liver. In this organ, branches of the glands unite and form an anastomosing gland structure, in connection with which we observe that the branches of the gland are not associated with a development of connective tissue and of blood capillaries between the epithelial elements of the organ, as in other compound glands; but are associated, on the contrary, with the presence of a sinusoidal circulation. We must therefore regard the liver as a type by itself.

Another class of secreting organs may be termed false glands, as they never have ducts at any stage of their development. Their chemical product is termed an internal secretion, and is removed by transfusion into the blood. One division of the false glands is derived from the growth of epithelium, while another division arises by modifications in mesenchymal cells. As regards the glands of the cytogenic class, we have to deal with those which produce the free wandering cells, of which the most familiar example is the white corpuscle of the blood; those which produce the red corpuscles of the blood; and, finally, those which produce the genital elements.

As the student proceeds in his study of embryology, he will have clear illustrations of the development and morphology of all the various sorts of glands. He will find it advantageous, as his acquaintance with glands increases, to consult the classification of glands as presented in the following table, based upon the very important morphological distinctions pointed out in the preceding paragraph. Formerly the classification of glands was based upon relatively unimportant details of their microscopic form, and not upon true morphological differences. Hence the classification here proposed differs radically from those in vogue up to the present time. 


\section{Classification of Glands.*}

Class A. Unicellular.

Class B. True Glands, always developed with ducts.

Division I. Simple Glands (unifollicular or single glands).

a. Ectodermal.

I. Sweat glands.

2. Sebaceous glands.

3. Buccal glands.

b. Entodermal.

- r. Esophageal.

2. Gastric.

3. Intestinal.

c. Mesothelial.

I. Uterine.

Division 2. Compound Glands (organic or true compound glands).

Type a, ductic epithelial branching (with capillary circulation).

r. Ectodermal.

Salivaries, tear gland, Harderian.

Mammary.

2. Entodermal.

Pancreas.

3. Mesothelial.

Appendicular glands of the urogenital system.

Type b, ductic anastomosing (with sinusoidal circulation).

r. Liver.

2. (Paraphysis ?)

Type c, ductless epithelial (with secondary obliteration of the duct).

r. Thyroid.

2. Hypophysal gland.

3. Infundibular gland.

4. Pineal gland (epiphysis).

Class C. False Glands, never developed with ducts.

Division I. Epithelioid Glands (? exclusively entodermal).

I. Parathyroid.

2. Islands of the pancreas.

3. Carotid.

4. Thymus.

Division 2. Mesenchymal ductless Glands.

I. Suprarenal cortex.

2. Coccygeal gland.

3. Interstitial cells of genital glands.

Class D. Cytogenic Glands.

Division I. Lymphceal structures; producing mesamoboids.

I. Lymph glands and follicles.

2. Hemolymph glands.

* The present table is a modification of the classification of glands proposed by the author in 1905 (Amer. Journ. Anat., iv, 256). The principal change is in putting the cytogenic glands in a class by themselves, as should have been done originally. 
3. Spleen.

4. (?) Tonsils and thymus.

Division 2. Sanguifactive organs.

I. Bone marrow.

Division 3. Genital glands.

I. Ovary.

2. Testis.

\section{The Law of Unequal Growth.}

The changing shapes of the embryo and the development of the irregularitiesprojections and invaginations - which preserve the proper proportion between the surface and the mass of the body, both depend upon the unequal growth of the germ-layers, especially in superficies. The expansion of a germ-layer having the epithelial type of structure* may take place by three means: (I) the multiplication of the cells; (2) the flattening out of the cells; (3) enlargement of the cells. In the early stages of development the influence of the first two factors predominates; during the later stages, especially after birth, the latter. Of the three factors, the first is the most important.

The unequal multiplication of the cells in all embryonic epithelia is the fundamental factor of development, and we see it shaping the embryo, its organs, and the parts of organs, before histological differentiation really begins. The distinct areas and centers of growth which are necessary to develop the human body out of the germ-layers are innumerable, and their distribution, limitations, and interactions make up a large part of the subject-matter of embryology. At every turn of our studies we encounter fresh illustrations. If in a limited area of a cellular membrane there occurs a growth of expansion more rapid than in the neighboring parts, then that area is, as it were, bounded by a fixed ring, and can, therefore, find room for its own expansion only by rising above the level of the membrane; thus, when in the embryonic region of the blastodermic vesicle the growth becomes more rapid, the embryo begins to rise above the level of the vesicle; thus, when, at a certain point of the surface of the embryo, a steady and long-continued growth occurs, the limb appears, gradually lengthening out, and enlarges from a small bud at first to a complete arm or leg. If the departure takes place the other way, we have an invagination produced; thus for every hair of the skin and for every gland of the intestine there is a separate center of growth.

The causes of the unequal growths are unknown. We have not even an hypothesis to offer as to why one group of cells multiplies or expands faster than another group of apparently similar cells close by in the same germ-layer. It is no real explanation to say that it is the result of heredity, for that leaves us as completely in the dark as ever as to the physiological factors at work in the developing individual.

The conception that the development of an animal depends fundamentally 
upon the unequal expansion and consequent foldings and bendings of the germlayers was first suggested by the researches of C. F. Wolff on the development of the intestine, and was more clearly recognized by Pander, who definitely asserted that the formation of the embryo is effected by foldings of the germ-layers, and the truth of Pander's view was conclusively demonstrated by C. E. von Baer in 1828 . In recent times His has studied the problem very intently, and in his memoir on the chick discussed it minutely. In this memoir is to be found most of what little we know of this aspect of embryological mechanics.

\section{Germ-cells.}

Recent investigations have made it probable that a few cells are set apart during the period of segmentation to form the germ-cells. Their number is small; they preserve for some time the appearance of segmentation spheres, as the cells which are formed during the segmentation of the ovum are sometimes called. They multiply very slowly during the earliest stages of development. A great majority of the cells produced during segmentation lose the character of segmentation spheres, and divide rapidly and repeatedly. They are termed somatic cells and form the various tissues of the body. The germ-cells, on the contrary, seem to multiply very slowly and never to become very numerous in the embryo. As they multiply they separate from one another and become more or less completely surrounded by tissue cells. They pursue their development, one is tempted to say, independently of tissue formation and somewhat like foreign members of the body. We put, accordingly, the germ-cells in a class by themselves in contrast to the body or somatic cells.

Our actual knowledge of the history of the germ-cells is very incomplete. The statements just made about them are based on observations on very few animals. Their exact origin has been traced only in five vertebrates, three fishes, the teleosts Cymatogaster and Micrometrus, the elasmobranch Squalus acanthias, and in the frog and turtle. In these five forms the germ-cells arise during segmentation, and remain more or less closely together, or segregated, during the earliest stages. They then separate from one another and gradually migrate into the epithelium, which covers the anlage of the genital gland and which thus becomes the so-called "germinal epithelium."

The most accurate information we have refers to their development in the dogfish. In this species the germ-cells are delaminated from the entoderm together with other cells of the mesoderm, and cannot, with our present knowledge, be distinguished from other mesodermic cells. They soon, however, become recognizable, because while the majority of the mesodermic cells are passing into the second stage (compare the section on Mesenchyma, page 89) these germ-cells change but little, if at all, so that they can be recognized as something distinct from the neighboring cells. For a short time they are found gathered into two compact groups (Fig. I, Germ-cells) symmetrically placed in the extra-embryonic region, but not far 
from the embryo. The cells then break apart from one another and gradually become separated, and migrate by unknown means, first over the wall of the intestine, which has meanwhile been differentiated, then over the surface of the mesentery into the anlage of the genital gland. During their entire migration they are lodged in the mesothelium, and when they have reached their final destination they are still in the mesothelium of the genital anlage, where they remain until finally differentiated in the adult. The epithelium, with the germ-cells in their definite position in it, is called the germinal epithelium (compare page 25). The germinal epithelium has been observed in all vertebrates, but the origin of the germ-cells in

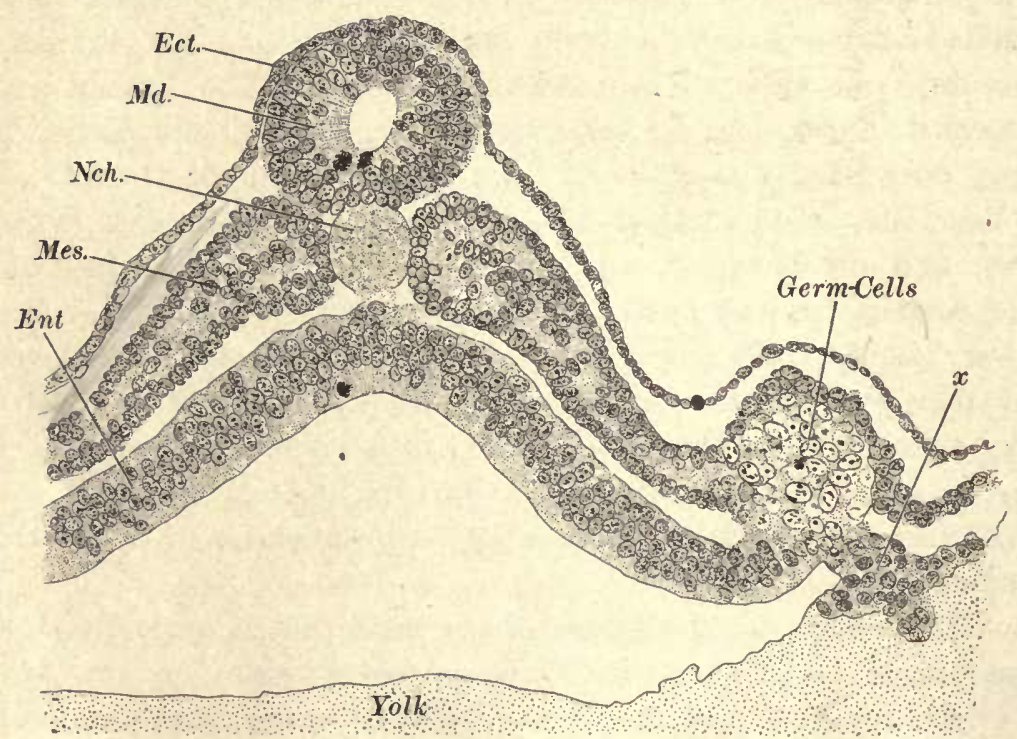

Fig. i.-Section across the Posterior Part of an Embryo Dog-fish (Squalus acanthias). Transierse SERIES 463 , SECTION I 47.

Ect. Ectoderm. Ent, Entoderm. Md, Medullary tube. Mes, Mesoderm. Nch, Notochord. $x$, Cellular strand connecting the germ-cell cluster with the yolk.

mammals is entirely unknown. The hypothesis may be accepted, that they arise in a manner essentially similar to that known in the dog-fish. For some of the theories based on the known development of the germ-cells, see page 28 .

The existence of the germinal epithelium has long been known, and its characteristics have been described in many text-books. The germ-cells in the germinal epithelium are known also by the names of "sex-cells" and "primitive ova." The transformation of these cells into true ova has been traced in a great many forms, so that the transformation may be considered as demonstrated conclusively for all vertebrate animals. It is further believed that the germ-cells also give rise to the male elements, playing in the formation of the testes a rôle similar to that which 
they play in the ovary. The proof that the germ-cells are the exclusive parents of the spermatozoa is difficult to obtain, but most embryologists regard the existing proof as sufficient.

When a germ-cell is transformed into an ovum, it undergoes great enlargement, its nucleus is modified, the protoplasm is changed in appearance and becomes loaded with yolk granules, and over the surface of the cell appear two membranes, an inner very thin one, called the vitelline membrane, and an outer much thicker one, known as the zona pellucida. (For a fuller description see page 34.) We thus learn that the germ-cells preserve their resemblance to segmentation spheres only during embryonic life. When they become ova, they pass through a series of important changes in their organization. Since germ-cells also give rise to the male elements, we must say further that in order to produce those elements the germ-cells pass through another series of profound changes.

It is further known that in order to evolve the sexual elements, both male and female, the cell which is to produce them divides twice, and in a special manner, which we designate by the term "reduction division." This process is described in all the recent text-books of cytology and histology. It does not fall within the scope of this work, which deals with embryology in the strict sense only.

\section{Sex.}

The sex of an individual depends primarily upon the nature of the sexual glands. The same two, right and left, parts produce the sexual glands in all vertebrates. Each part originally is a limited area of the surface of the cephalad end of the urogenital ridge (compare p. 6) and becomes either a testis or an ovary, and, since the two sides develop alike, the individual is wholly male or female as the case may be.

As an exceedingly rare anomaly, lateral hermaphroditism has been recorded. In this anomaly there is a testis upon one side, an ovary on the other. This is the only form of true hermaphroditism known to occur in the amniota.

Each sex is further distinguished by secondary sexual characteristics, in part such as are immediately concerned with reproduction, like the uterus, mammary glands, vas deferens, etc., in part such as are less directly connected with reproduction, such as size, distribution of hair, etc. In the course of development the sexual glands are clearly differentiated before the secondary sexual characteristics appear. Hence arises the question, have the glands a causal relation to the secondary characteristics?

The hormone theory is the only one, available at present, to explain such a causal relationship. It is known that various glands produce a so-called internal secretion, which is distributed through the body probably by the medium of the blood and acts upon structures quite remote from the organ producing the secretion. Similar chemical products arise also from organs, which cannot be regarded as glands in the usual sense. All of these secretions or products have received the 
comprehensive name of hormones (Starling). Now, it has become probable that the sexual glands produce hormones, which exert an effect upon other organs; for example, the mammalian corpus luteum is believed to yield a hormone, which so affects the uterus as to render it adapted to pregnancy. The hypothesis accordingly naturally suggests itself that hormones from the sexual glands occasion the development of the secondary sexual characteristics. It is well to bear in mind that our knowledge of hormones is still meager, and that the suggested hypothesis may or may not prove valid.

Concerning the cause of sex, i.e., why one individual is male and another female, we know very little. Nothing positive as to the cause of sex in vertebrates is known, though many speculations have been published. In certain insects, however, it has been discovered that the sexes are distinguished by the females having one more chromosome in each cell nucleus than the males.* This difference is explained by the fact that there are two kinds of spermatozoa, one of which contains an extra (accessory) chromosome. Those ova which at the time of fertilization receive an accessory chromosome become females, those that do not, males. At present we can add only that the important discoveries mentioned may furnish the clue to solve the problem of the cause of sex.

\section{The Theory of Heredity.}

We owe to Moritz Nussbaum the theory of germinal continuity-the only theory of heredity which seems tenable at the present time. According to this theory, the germ-cells are set aside during the segmentation of the ovum and preserve the essentially undifferentiated qualities of the protoplasm and nucleus of the orum, from the division of which they arise. Just as the cells formed during segmentation are capable of producing the various tissues of the body, so the germcells have and preserve this faculty. If we term the material of the original ovum germ-plasm, we may say that this germ-plasm gives rise to the various tissueforming cells which make up the body. And by this very conversion into tissue cells that germ-plasm is changed, and is no longer, as we have learned before, capable of the full range of development. The germ-cells, on the contrary, do remain so capable and it is precisely in order to preserve this capacity that they hold aloof from the formation of the body tissues and pursue their own independent career. A portion of the germ-plasm of the parent ovum is, so to speak, shortcircuited into the genital elements which produce the offspring.

If we accept this view, we are forced to make the supplementary hypothesis that the conspicuous complicated changes, by which the germ-cells are converted into sexual elements, do not involve differentiation in the true sense-i.e., strictly comparable to that which we observe in the somatic cells. Although this hypothe-

* In other insects more complicated relations have been discovered. The relation of the chromosomes to sex appears to be a complicated and difficult problem. 
sis seems a logical necessity of the theory of germinal continuity, we cannot at present verify it by any observed facts.

The only other theory of heredity which has ever been seriously considered is that of pangenesis, which was formulated by Darwin, whose words I quote: "But besides this means of increase I assume that cells before their conversion into completely passive or 'form-material' throw off minute granules or atoms, which circulate freely throughout the system, and when supplied with proper nutriment multiply by self-division, subsequently becoming developed into cells, like those from which they were derived. These granules, for the sake of distinctness, may be called cell-gemmules, or as the cellular theory is not fully established, simply gemmules. They are supposed to be transmitted from the parents to the offspring, and are generally developed in the generation which immediately succeeds, but are often transmitted in a dormant state during many generations, and are then developed."

Many modifications of this theory have been proposed by speculative writers, and many different names have been bestowed upon the gemmules of Darwin according to the fancy of each author and the particular set of qualities which he attributed to these imaginary particles. Such views attained their culmination in the set of elaborate and complicated hypotheses forming the doctrine of Weismann, or so-called Weismannism, which was for a time widely and actively discussed. All of these speculations have only an historical interest; having proved themselves, from a scientific standpoint, to be absolutely barren.

\section{The Law of Recapitulation.}

This law, as commonly formulated, is that the development of the individnal recapitulates the development of the race, or, in other words, the ontogeny recapitulates the phylogeny. This way of stating the law is in so far objectionable that it presents the theoretical interpretation of the law rather than the actual generalization of the facts. The essential datum upon which the law is based is, that the embryo of a given animal has striking morphological resemblances to the adult forms of lower allied types. Since the theory of evolution was established by Darwin this resemblance has been interpreted as due to the inheritance of ancestral characters appearing in the embryo. The embryo is looked upon as the representative of the actual ancestor by modification of which the adult form was evolved. It is further assumed that the change of the embryo into the adult type follows the same general course as the development of the remote ancestor into the particular species under consideration. Speaking broadly, this interpretation is undoubtedly justifiable. If it were exactly true, it would be necessary only to know the embryology of an animal in order to establish the evolution of the species. Experience, however, very quickly demonstrates that this procedure is by no means possible, because the embryo is not a correct or adequate record of the ancestral type. It is inadequate chiefly for three reasons: first, because the embryo has necessities of its 
own, and in the course of evolution embryos acquire special peculiarities by which they become adapted to the conditions of their life. Such changes in organization do not correspond to, but on the contrary diverge from, the inherited ancestral traits, and in so far as they are present they mask or alter those structural features of the embryo which represent the ancestral record. Second, because the embryos consist of undifferentiated cells (compare page II). Now, the adult ancestors representing lower types of organization of course had differentiated tissues, which enabled them to perform the functions of adult life. One of the first things which will impress itself upon the student of vertebrate embryology is, that, though he may find at the proper stage in the embryo the organs of the body clearly developed, yet, owing to the fact that they consist of relatively undifferentiated cells, they are incapable, in large part, of performing the functions which they are ultimately to assume, and the performance of which is the very object of their development. This change in histological structure brings about a marked unlikeness of the embryo to the assumed ancestral type. Third, the embryo at each stage of its development must be regarded as the mechanical cause of the next and of all following stages. It must necessarily, therefore, have in itself peculiarities by which it is distinguished from all other embryos. It is impossible, accordingly, that all embryos should be alike. It is only necessary for the student to compare embryos of various vertebrates one with another to satisfy himself that they have conspicuous distinctive characteristics. When our knowledge shall have grown sufficintely, we shall be able to classify vertebrates by their embryos as perfectly as or perhaps even more perfectly than we can by the consideration of the adult forms. Every embryo is modified from the very start away from the assumed ancestral organization, in order that its peculiarities may cause it mechanically to produce the new form which has been evolved.

In some of the invertebrate animals-as, for instance, among the hydroids and jellyfishes-the law of recapitulation can be much more easily verified than in the higher forms which have purely embryonic types of development. From what has been said, it will be recognized that the likeness of the embryo to the adult lower form is a general morphological resemblance only, not an exact one, and that therefore it is extremely difficult to infer from the embryonic organization what the ancestral type was. Hitherto all phylogenetic inferences drawn by embryologists have been largely speculative in character, and, it may be added, have been more remarkable for their number and variety than for their value.

The resemblance between embryos and lower adult forms has been known for a century past. It was first adequately asserted in I8I I by J. F. Meckel and since then has been constantly discussed. More, perhaps, was done to emphasize it by Louis Agassiz than by anyone else. Von Baer, the creator of modern scientific embryology, called attention in 1828 to the limitations which must necessarily be put upon Meckel's generalization. It is to be regretted that von Baer's wise thought on this subject has not been 'more appreciated. He put forth four 
generalizations: first, that which is common to a large group of animals develops in the embryo earlier than that which is special; second, from the most generalized stage structures less generalized are developed, and so on until finally the most special appears; third, the embryo of a given animal form, instead of passing through the other given forms, separates itself from them more and more; fourth, therefore, essentially the embryo of the higher forms is never like a lower form, but only like its embryo. The first to point out the possible phylogenetic significance of these facts with perfect clearness was Fritz Müller, in a little book entitled "Für Darwin," published in 1864. Ernst Haeckel took up this interpretation and secured wider attention for it. He termed the law of recapitulation the "biogenetic law." *

The student will encounter in his practical study many illustrations of the resemblances which we have been discussing, so that it is unnecessary here to do more than mention a few for the purpose of illustration. In the embryos of birds and mammals the pharynx forms a series of lateral pouches which we know as the gill-pouches, and which develop in the same way as, resemble strikingly, and are homologous with, the gill-pouches of fishes, which in the fishes give rise to the so-called gill-clefts. The heart of a young mammalian embryo is a simple tube with only a single continuous cavity resembling the heart of the lower fishes. The embryonic kidney or Wolffian body of man resembles, and is homologous with, the kidney of the frog, but it disappears almost completely before adult life. These few examples may suffice.

\section{Arrest of Development.}

This term is used to designate not the normal, but the abnormal, cessation of the ontogenetic process. It generally implies the persistence into adult life of an anatomical condition, normally present in the embryo, which is typically a temporary though essential phase of development. Usually there is no cessation of the histological differentiation. It is characteristic of these anomalies that they are more or less definite.

A few illustrations may. render the matter clear. The palate is formed as two shelves, which grow until they meet in the median line; sometimes they fail to meet and then the adult has a "cleft palate," the tissues of which, however, are as fully differentiated as those of the normal palate. In the young embryo a short blood-vessel (i.e., dorsal part of the left last aortic arch) connects the pulmonary artery with the dorsal aorta (compare page IOI), but it later becomes occluded and finally obliterated. Occasionally, however, it persists as an open vessel, which is termed the "ductus arteriosus" in the adult. It may grow in size and its walls become fully differentiated like those of an aorta. The external genitalia of the male may be arrested in their development, though they continue in such cases 
their growth and histogenesis. There results a "pseudo-hermaphrodite," a true male with external organs of the female type. In such cases the individual usually presents other female characteristics, such as a wide pelvis and enlarged mammæ.

The variations in adult structure due to arrest of development are the most frequent, important, and significant with which the student of anatomy has to deal. It is obvious that the possible variations are limited to anatomical conditions, which actually occur in normal embryos. 


\section{CHAPTER II. \\ THE EARLY DEVELOPMENT OF MAMMALS.}

\section{The Spermatozoon.}

The spermatozoa of mammals are filaments consisting of a short thick end called the head, and a very long, delicate thread called the tail. They are of minute size as compared with the ovum. The head varies greatly in shape according to the species. It contains chromatin, hence it stains darkly with those histological dyes which color nuclei. The tail consists of three parts: first, the middle piece, which is next the head, is short and the thickest of the three parts, contains an axial thread, and probably always has a very fine spiral thread running round it; second, the main piece, which is the longest part of the tail; and, third, the end piece, which is not more than a line, even as seen with very high microscopic powers.

The Human Spermatozoon.-The human spermatozoon is 0.055 $\mathrm{mm}$. long - the head being $0.005 \mathrm{~mm}$., the tail 0.050 , and the middle piece 0.009 . It is shown in two views in figure 2 . The head is flattened and pointed. Seen from the flat side, it appears oval (Fig. 2, $A$ ) with the front end generally tapering a little, but never pointed. The anterior half or two thirds has a brighter and more transparent part. Seen on edge (Fig. 2, B), the head has a pointed form with a posterior, thicker, round dark part. By adjustment of the focus it can be ascertained: that the sides near the point are depressed. Some writers maintain that there is a special tip projecting from the head as a cylinder thread, with a hook at its end. The middle piece, mi, is directly united with the head by a transverse joint. It is cylindrical and about as long as, or a little longer than, the head. Its surface is often granular or rough, and there cling to it a few shreds of protoplasm. It has a spiral thread, which is easily overlooked on account of its extreme fineness. The main piece, $m$, of the tail is about half as thick as the middle piece. It gradually

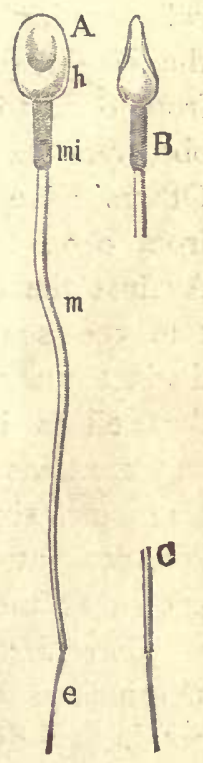

FIG. 2.- $\mathrm{HUMAN}$ SPERMatozoa. $A$, Complete spermatozoon. $B$, Head seen from the side. $C$, Extremity of the tail. $h$, Head. $m i$, Middle piece. $m$, Main piece. $e$, End piece. A.11 highly magnified. -(After Retzius.) tapers and ends abruptly at the beginning of the still finer and very short end piece, $e$. The tail probably contains an axial thread, as has been observed in other 
mammals. The head probably contains a minute body representing a centrosome, although it has not yet been satisfactorily demonstrated in man.

The spermatozoa, when free in the fluids in which they normally occur, are capable of active locomotion. This is achieved by means of the tail, which acts as the swimming organ by vibratory undulations which drive the spermatozoon along, head foremost. The tail has often been compared to the flagellum which serves as the locomotive organ for many of the unicellular organisms.

\section{The Fully Grown Ovum Before Maturation.}

The structure to be here described is not the true sexual element, but is only the modified germ-cell which has accomplished its period of growth and is ready to be transformed into the genuine female sexual element. This transformation is called the maturation, and is accomplished essentially by the expulsion of the socalled polar granules. The full-grown mammalian ovum is found in the ovary in the center of the discus proligerus of the Graafian follicle. It measures usually from 0.10 to $0.15 \mathrm{~mm}$. in diameter. It is approximately spherical. In some cases observers have found a very delicate vitelline membrane covering the protoplasm. Others have failed to observe this. Outside there is a thick envelope measuring from 0.02 to $0.03 \mathrm{~mm}$. in diameter and known as the zona pellucida or radiata. Against the outside of the zona rest the cells of the discus proligerus which constitute the so-called "corona radiata." The nucleus is large, spherical, contains a distinct nucleolus, and always occupies an eccentric position.* The protoplasm of the cell is large in amount, granular in appearance, forms a distinct reticulum, and contains a larger or smaller number of yolk granules which vary considerably in character, size, and distribution in different mammals. They are usually more or less concentrated in the central portion of the ovum, leaving the outer portion, known as the protoplasmic zone, more or less free.

The Human Ovum.-The full-grown human ovum is distinguished among mammalian ova for the clear development and ready visibility of all its parts, a peculiarity due chiefly to the small amount of the yolk and the fewness of the fat granules it contains. Figure 3 represents an ovum from a woman of thirty years. The specimen was obtained by ovariotomy, examined and drawn in the fresh state, being in the liquor folliculi. The specimen gave the following measures: The diameter of the whole ovum, including the zona radiata, $165^{-170} \%$; thickness of zona, $20-34 \mu$; perivitelline fissure, I. $3 \mu$; the clear outer zone of the yolk, 4-6 $\mu$; the protoplasmic zone, IO-2I $\mu$; the zone of yolk granules, $82-87 \mu$; nucleus, $25^{-27} \mu$.

The corona radiata, cor.r, consists of elongated radiating cells with rounded ends and oval nuclei. The zona pellucida shows a distinct radial striation. This is probably due to the presence of minute canals running through the zona. The ovum proper is separated from the zona by a narrow fissure, the perivitelline space,

\footnotetext{
* The nucleus was formerly termed "germinal vesicle"; the nucleolus, "germinal spot."
} 
within which it lies free and loose. Hence when a fresh specimen is examined, the same side of the ovum, that containing the nucleus and which is the lightest part, is always found uppermost. In this ovum no vitelline membrane was observed. The body of the ovum may be divided into an inner kernel containing the yolk granules, and an outer protoplasmal zone, of which the very thin outermost layer is clear and therefore more or less differentiated from the broader, deeper layer, which is granular and constitutes most of the zone, $P l$. The yolk grains are $I \mu$ or less in diameter. They are highly refringent and of various kinds. Their characteristics have not yet been accurately investigated. The nucleus is nearly spherical and has a conspicuous nucleolus. In fresh specimens the nucleolus shows amoboid movements, even at ordinary summer temperatures, for several hours after removal from the ovary. It is .only in hardened specimens that the reticulum of the nucleus can be clearly observed.

\section{Ovulation.}

The discharge of the ovum from the ovary is called ovulation. It results from structural changes in the Graafian follicle, and these changes continue after the departure of the ovum, transforming the Graafian follicle into a so-called corpus luteum. The exact history of these changes does not fall within the scope of this work.

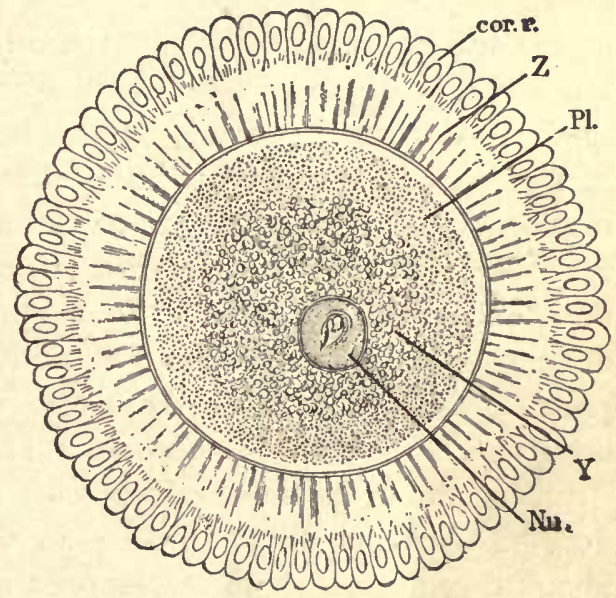

Fig. 3.-Full-grown human Ovum before Maturation.

cor.r, Part of corona radiata. $Z$, Zona pellucida. $\mathrm{Pl}$, Protoplasm. . $\mathrm{Y}$, Yolk. $\mathrm{Nu}$, Nucleus.(After W. Nagel.)

The essential steps in the process are the growth of the Graafian follicle and the thinning of its wall at a point at the surface of the ovary. The thin part is called the stigma. This breaks through and establishes an opening by which the ovum, surrounded by the corona radiata, together with the liquor of the follicle, can escape into the periovarial chamber, whence it makes its way into the Fallopian tube. The growth of tissue in the walls of the collapsed Graafian follicle fills up the space of the same, constituting a mass which is known as the corpus luteum on account of its yellow color. The most characteristic elements of this structure are the large cells which contain the pigment. Each cell has a rounded nucleus and a large protoplasmic body, which is also more or less rounded in form. The lutein granules are in these cells. The function of the corpus luteum was long entirely unknown. Recently the theory has been suggested by Born that these cells exert an influenca upon the uterus by which it is prepared to receive the ovum. This influence may be suggested to act by means of a chemical substance (hormone) produced by the lutein cells and added to the blood, which then affects 
the uterus. There are some experimental observations tending to prove the correctness of this theory.

The brilliant color of the corpus luteum is especially characteristic of man, and has determined the name of the structure. In sheep the pigment is pale brown, in the cow dark orange, in the mouse brick-red, in the rabbit and pig flesh-color.

\section{The Maturation of the Ovum.}

Maturation is the term applied to the series of changes by which the fully grown egg-cell is transformed into a true female sexual element. Viewed externally in the living ovum, the process manifests itself chiefly by the separating off of two small bodies of protoplasm, each of which contains some nuclear material. These small bodies are generally known by the name of polar globules. They take no further part in the development, ultimately disintegrate, and are lost. The remaining ovum is capable of impregnation. It is now known that the production of the polar globules is the result of a special form of cell division, which we term the "reduction division." When the first polar globule is formed, the egg-cell divides into one very large cell and a second very small one. When the second polar globule is formed, the larger of the cells again divides, producing a second small cell and a new large one. This large one is the true female element.

Maturation as a general process may be described as follows. For figures and detailed accounts of the process in the mouse, see Chapter V. When an ovum is about to mature, its nucleus moves nearer that point of the surface which may be regarded as the center of the so-called animal pole, the region of the ovum, which contains most of the protoplasm and less of the yolk material. During the migration of the nucleus, the cell as a whole usually contracts so that a space appears between it and the zona radiata. Concerning the force that moves the nucleus we have no definite knowledge. When near the surface, the nucleus as such disappears. Older writers supposed that it was lost altogether, but we now know that the disappearance of the nucleus is only apparent, not actual, being in reality a metamorphosis. It is probable that the first step is the discharge of the nuclear fluid into the surrounding protoplasm, causing the nucleus to become more or less shriveled. The second step is the dissolution of the membrane of the nucleus so that the nuclear contents are brought into direct contact and partly mixed with the protoplasm of the cells. The third step, which in time more or less accompanies the second, is the gathering of the chromatin of the nucleus into a definite number of separate granules or chromosomes (tetrads). These chromosomes are always conspicuous and are larger than those formed during ordinary cell division. Their number is also highly characteristic. As is now well known, there appear during the process of indirect cell division a fairly definite number of chromosomes, a number which is characteristic for each species. In numerous cases it has been observed that the number of chromosomes in the maturing egg-cell is exactly one half of that found during the ordinary cell divisions of the same species. 
The chromatin granules lie at first irregularly. Fourthly, there arises a characteristic spindle figure such as accompanies mitosis. The chromatin forms an equatorial plate, each granule being associated with one of the spindle threads. The shape of the spindle varies, as does also the distribution of the granules of the equatorial plate. In guinea-pigs the ends of the spindle are pointed and the threads are straight, the outline of the spindle being like a diamond; in the bat the spindles are barrel-shaped and the threads are curved. In many cases it is known, and it may be found to be true of all cases, that the axis of the spindle is at right angles to the radius of the ovum. The nuclear spindle now changes its position, becomes first oblique, and then radial. One end of the spindle lies close to the surface of the ovum. The first step is the division proper. The spindle, driven by an undiscovered power, moves centrifugally until it is partly extruded from the egg. The projecting end is enclosed in a distinct mass of protoplasm which gradually increases and soon becomes constricted around its base. The fragments of chromatin have each divided into two parts (dyads), and one half of each fragment moves toward one end, and the other half toward the other end of the spindle. The half fragments of each set move together, hence there seem to be two plates within the spindle. The translation of the groups of chromatin continues until they reach the end of the spindle. The achromatic threads then break through in the middle, and thus the original nucleus, or at least a part of it, has been divided. There are now two masses of nuclear substance, one in the ovum, the other in the polar globule. The result of the whole process is the formation of two cells extremely unequal in size, and each containing in its nuclear elements half the number of chromosomes characteristic of the body-cells. The number of chromosomes has, therefore, been reduced, hence the term reduction division. It will be noted that the actual reduction in the number of chromosomes took place when they were first formed in the maturing ovum, while the spindle or mitotic figure was developing.

The second polar globule is produced a short time after the first. When this occurs, the nuclear remnants in the ovum do not reconstitute themselves into a membranate nucleus, as occurs in ordinary cell division, but they change directly into a second spindle, which lies, as did the first, within the protoplasm of the animal pole. This second spindle occupies an oblique position, or may even be parallel with the surface. But it also soon takes up a radial position and produces a second polar globule in similar manner to the first. The dyads all divide, and the ovum receives the half number of chromosomes, each of which represents the fourth part of a tetrad (double chromosome). The second globule is usually smaller than the first.

It may also happen that the first polar globule may itself divide, so that three polar globules appear.

The Formation of the Female Pro-nucleus. - The nuclear material which remains in the main ovum after the separation of the polar globules is known as the 
female pro-nucleus. The nuclear remnant lies close to the animal pole and in clear protoplasm. The details of its further history vary according to the species of animal. Three tendencies are known to affect the pro-nucleus: viz., to move toward the central position in the ovum, to unite with the male pro-nucleus as soon as that is formed out of the spermatozoon which enters the ovum, and to assume the character of a membranate nucleus. As the time of the formation of the male pro-nucleus is variable, the other tendencies being more constant, the exact history of the female pro-nucleus may be said to depend principally on the appearance of the male pro-nucleus. The earlier that event, the less does the fermale pronucleus move centrifugally and the less does it assume the membranate form. Even among mammals there is variation.

Time of Maturation. - The time when the polar globules are formed varies according to the animal, and may be before or after the egg-cell leaves the ovary. In placental mammals maturation always begins, so far as known, in the ovary, and is said in some cases to be completed there. But in other cases it is certainly completed only after ovulation or when the ovum has passed into the Fallopian tube.

\section{Impregnation of the Ovum.}

Impregnation is the union of the male and female elements to form a single new cell, capable of initiating by its own division a rapid succession of generations of descendent cells. The process of union is commonly called the entrance of the spermatozoon into the ovum. The new cell is called the impregnated or fertilized ovum. The process of fertilization in the mouse is described and illustrated in Chapter V.

In all multicellular animals impregnation is effected by three successive steps: (I) The bringing together of the male and female elements; (2) the entrance of the spermatozoon into the ovum and the formation of the male pro-nucleus; (3) fusion of the pro-nuclei to form the segmentation nucleus.

Meeting of the Sexual Elements.-In all amniota the seminal fluid is transferred from the male to the female passages during coitus, and spermatozoa are thereafter, in mammals, found in the uterus. In default of actual knowledge it is commonly believed that the spermatozoa make their way by their own motions into the Fallopian tubes. The ovum, meanwhile (probably, in mammals, while completing its maturation), travels down the tube. The meeting-point, or site of impregnation, in placental mammals is about one-third way down from the fimbria to the uterus. The exact spot is remarkably constant for each species. Nothing is known by direct observation as to the site of impregnation in man, but there is no reason to suppose, as has unfortunately been often done, that the site is either variable or essentially different from that in other mammals.

The Entrance of the Spermatozoon into the Ovum.-It is probable, in mammals at least, that only one spermatozoon normally enters the yolk of the ovum and accomplishes its fertilization. It has been observed in those animals in which, as in 
the rabbit, there is formed a more or less considerable space between the yolk and the zona radiata, that a number of spermatozoa appear in this space, but apparently only one actually fuses with the substance of the ovum. The manner in which additional spermatozoa are excluded, after the first has entered, is still under discussion. The hypothesis has been suggested that the attractive power of the ovum is annulled or weakened by the formation of the male pro-nucleus from the spermatozoon which first enters. With our present knowledge the assumption appears unavoidable that the ovum exerts a specific attraction upon spermatozoa of the same animal species. Recent authorities incline to the view that this attraction is of a chemical nature, for it has been observed that certain chemical substances may attract very strongly unicellular organisms capable of free locomotion. The phenomenon is called chemotropism. According to this interpretation, the attraction of the ovum for the spermatozoon would be termed chemotropic.

At the time of fertilization the ovum in the Fallopian tube is surrounded by a number of spermatozoa; in the case of the rabbit, perhaps by a hundred, more or less. They are all, or nearly all, in active motion, for the most part pressing their heads against the zona radiata. Several of them may make their way through the zona into the interior. According to Hensen, only those spermatozoa which enter the zona along radial lines can make their way through. Those which take oblique courses remain caught in the zona. The female pro-nucleus is already present, and may be either with or without a membrane, according to the species. A single spermatozoon makes its way into the yolk proper, passing a short distance into the interior. It is uncertain whether the whole tail of the spermatozoon enters the ovum or not. In some of the lower vertebrates and in other animals it appears to do so. It is probably always the case that at least the main piece of the tail enters the yolk. The tail, as such, very soon disappears, while the head of the spermatozoon enlarges, probably by the imbibition of fluid from the surrounding yolk. The head of the spermatozoon is rich in chromatin, which forms a series of irregular masses as the head enlarges, producing a network appearance, and is thus converted into a nucleus-like body, the male pro-nucleus. At the same time in some animals the growing head surrounds itself by a membrane.

We now have a cell which contains two nucleus-like bodies, one derived from the head of the spermatozoon and the other from the nucleus of the egg-cell. They are termed respectively the male and female pro-nucleus. Each pro-nucleus, when it first appears, is small and gradually enlarges, probably in both cases by the imbibition of fluid. The relative size of the two pro-nuclei varies considerably in different species, and is probably a secondary and relatively unimportant relation. The proportion between the two probably depends upon the time when the male pro-nucleus is formed. If the spermatozoon enters early, while the female pronucleus is forming, it may make a pro-nucleus as large as that from the eggcell. If, on the other hand, the spermatozoon enters late, the female pro-nucleus enlarges, acquires a start, and the growing male pro-nucleus is, therefore, smaller. 
Concerning the fate of the middle piece of the spermatozoon and its share in the fertilization in the ovum of mammals, we possess no satisfactory information. It has been shown, however, in other animals that this middle piece produces a centrosome, and the only centrosome which appears in the fertilized ovum. The theory has been advanced that the ovum, after its maturation, has no centrosome, that a centrosome is always brought into the ovum by the spermatozoon in the manner just indicated. If we regard the centrosome as a permanent cell element, then we must further interpret the addition of the male centrosome as one of the most important phenomena of fertilization. Whether this hypothesis is correct or not, we are unable at present to decide.

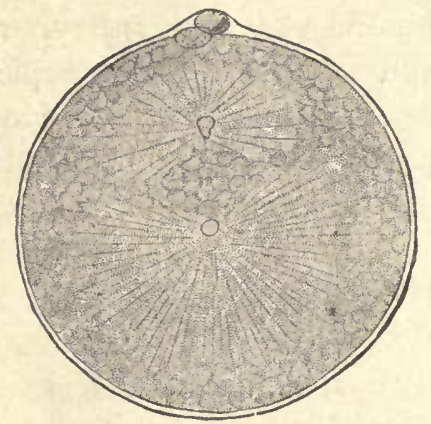

Fig. 4.-Ovum of a Worm (Sagitta) With Two Pro-nuclei, around Each Pro-nucleus is SHOWN THE Aster.-(After O. Hertwig.)

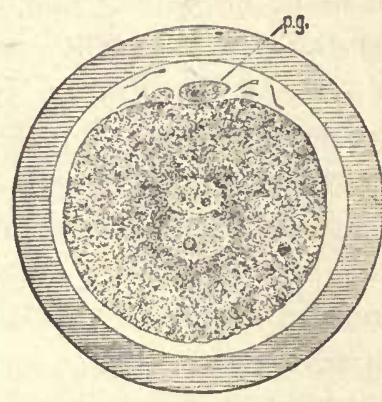

Fig. 5.-Ovum of a Rabbit, Seventeen Hours after Coltus, with the Pro-Nuclei about to Conjugate.

p.g, Polar globules.-(After Rein.)

Astral figures play a conspicuous part in the phenomenon of fertilization in many animals. Astral figures are produced in the protoplasm of the ovum by its assuming a special radiating structure. Astral figures may appear around both the male and female pro-nuclei (Fig. 4). In other cases the astral figure arises only in association with the head of the spermatozoon or male pro-nucleus. In mammals, so far as known, no astral figures are developed about either of the pro-nuclei. There is a clear space in the protoplasm around each nucleus, and such a clear space has often been noted also when the astral figure is present. It may possibly be interpreted as a rudimentary aster or center of astral formation.

The two pro-nuclei usually lie at some distance from one another. As soon as they are formed, or perhaps when they are fully differentiated, they tend to move toward one another and toward the center of the ovum. Concerning the path of the male pro-nucleus we possess interesting information from the study of the ova of the frog and axolotl. In these ova the spermatozoon leaves a trail of pigment, which consists of two limbs, one passing through the cortical layer of the ovum nearly perpendicular to the surface, and the other forming an angle with the first and leading directly to the female pro-nucleus. The female pro-nucleus tends 
always to move toward a central position. The force which draws the pro-nuclei together is unknown. The hypothesis that this force is chemotropic has met with favor.

The Fusion of the Pro-nuclei.-In the rabbit, as probably in all mammals, both pro-nuclei lie at first eccentrically, but both move toward each other and toward the center, meeting when the central position is attained. As they near one another both pro-nuclei perform active amœboid movements. After they meet they still continue their amœboid movements, and travel together to the center of the ovum (Fig. 5). One of the pro-nuclei assumes a crescent shape and embraces the other. In the mouse the history is similar. After the two pro-nuclei in this animal have met, they remain side by side, but they are without membranes. After the conjunction of the pro-nuclei the chromatin threads become distinct and draw closer together. Between them appears first a small spot or centrosome with a few radiating lines around it (Fig. II7). From the centrosome arises a spindle of achromatic threads (Fig. II8). The chromosomes, both male and female, attach themselves to the spindle, and therewith impregnation is completed and mitosis of the impregnated ovum initiated.

It is now believed that the pro-nuclei never unite to form a distinct membranate nucleus, the so-called segmentation nucleus of earlier writers, but that the fusion always takes place during the absence of the membranes of the pro-nuclei by the mingling of their contents. The time of mingling, however, varies as regards the formation of the chromosomes. It may take place before or after the chromosomes are developed. When, as in the mouse, the chromosomes appear as two distinct groups, it is possible sometimes to determine their number. In the mouse counting is difficult, but there seems little doubt that each pro-nucleus forms twelve chromosomes. Hence it results that there are twenty-four chromosomes in the segmentation spindle. This number, twenty-four, so far as has been determined, is the number which appears during later stages of segmentation and in all subsequent cell divisions of this animal. It is believed to be a general law that the male and female pro-nuclei each contribute the same number of chromosomes to the segmentation spindle except in those cases where an accessory chromosome is interpolated in the development. This number is identical with the number which appears during the reduction divisions which lead to the maturation of the ovum on the one hand and the development of the spermatozoon on the other; and, further, the number is one half the number of chromosomes which appear during ordinary cell divisions of the species. The most thorough study of the phenomenon which has yet been made is that by a succession of able investigators upon the large nematode Ascaris megalocephala. An admirable summary of the process of fertilization in Ascaris has been given by Oscar Hertwig.*

* "Lehrbuch der Entwicklungsgeschichte," eighth edition, I906. The large Ascaris is a particularly favorable object. The student who wishes to pursue, the practical study of impregnation further should select this form. 


\section{Segmentation of the Ovum.}

Shortly after the entrance of the spermatozoon into the ovum the segmentation spindle is developed by the union of the pro-nuclei, as described in the previous section. This spindle leads to a division of the ovum into two cells. These cells further rapidly divide. As stated on page Io, these early cell divisions are called the segmentation of the ovum.

The position of the first segmentation spindle is always eccentric, and appears to be approximately, if not exactly, the same as that of the egg-cell nucleus before maturation. The axis of the spindle varies greatly in its direction. It is sometimes at right angles to the radius of the ovum, which passes through the polar

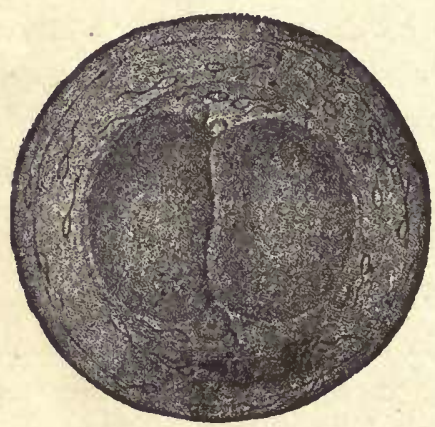

Fig. 6.-Ovum of a RabBit of Twenty-Four Hours.

The first cleavage has been completed; the two cells (segmentation spheres) are appressed; above the cells lie the polar globules; numerous spermatozoa lie in and within the zona pellucida.(After Coste.)

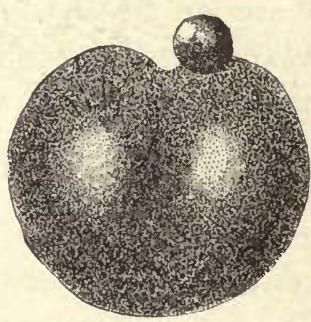

Fig. 7.-Ovum of a SNaIl (Limax CaMpestris) during the first Cleavage. The EnvelOPES OF THE OVUM ARE NOT DRAWN IN. $\times 200$ diams.-(After E. L. Mark.)

globules, but it is more usually oblique to this radius. It was at one time thought that the plane of division was always at right angles to the radius of the extrusion of the polar globules, but this view cannot be upheld. After the ovum has divided into two cells, the polar globules lie in the angle between these two cells (Fig. 6), because there the globules find room. It is to be noted that the globules accommodate themselves to the segmentation spheres, and that the formation of the spheres is not accommodated to the original position of the globules.

The degree of the eccentricity of the segmentation spindle varies in different ova, chiefly according to the amount of yolk; the greater the quantity of yolk in the ovum, the more marked is the eccentricity.

The actual first cell division (first cleavage or first segmentation) of a mammalian ovum has never been followed by direct observation, the practical difficulties not having hitherto been successfully overcome. Various phases of the division have, however, been seen, and the internal changes have been studied by means of sections. It accordingly seems expedient to interpolate the following ac- 
count of the external appearances of the first segmentation in the living ovum of the snail, Limax campestris. The eggs of this animal, by their size and in their mode of segmentation, have a certain resemblance to mammalian ova. The following description is taken from the account by E. L. Mark, published in I88I; it is nearly in his own words:

In Limax, after impregnation, the region of the segmentation nucleus remains more clear, but all that can be distinguished is a more or less circular, ill-defined area, which is less opaque than the surrounding portion of the vitellus. After a few moments this area grows less distinct. It finally appears elongated. Very soon this lengthening results in two light spots, which are inconspicuous at first, but which increase in size and distinctness, and presently become oval. If the outline of the egg be carefully watched, it is now seen to lengthen gradually in a direction corresponding to the line which joins the spots. As the latter enlarges the lengthening of the ovum increases, though not very conspicuously. Soon a slight flattening of the surface appears just under the polar globules; the flattening changes to a depression (Fig. 7), which grows deeper and becomes angular. A little later the furrow is seen to have extended around on the sides of the yolk as a shallow depression, reaching something more than halfway toward the vegetative or inferior pole, and in four or five minutes after its appearance the depression extends completely around the yolk. 'This annular constriction now deepens on all sides, but most rapidly at the animal pole; as it deepens it becomes narrower, almost a fissure. By the further deepening of the constriction on all sides there are formed two equal masses connected by only a slender thread of protoplasm, situated nearer the vegetative than the animal pole; the thread soon becomes more attenuated and finally parts. The first cleavage is now accomplished. Both segments undergo changes of form; they approach and flatten out against each other, and after a certain time themselves divide.

The succeeding cleavages of segmentation need to be followed out in greater detail than yet recorded. In many cases there appear to be three cells in the next stage, because one of the two primitive segmentation spheres divides sooner than the other. The more commonly received view is that four cells are produced next, but it may very well be that there is really a three-cell stage preceding the four-cell stage of which two figures are presented. The first of these (Fig. 8) represents the four-cell stage of the ovum of a bat, and the second (Fig. 9) represents the four-cell stage of the ovum of the Virginian opossum. That of the bat resembles the picture which we obtained from a number of animals, such as the rabbit, the guinea-pig, the dog, and others. That of the opossum differs so much from anything known in other mammals that it may be questioned whether it is entirely normal. In the mouse the zona is much thinner and assumes an irregular form, adapting itself to the pressure of the single spheres.

After the four-cell stage, the segmentation proceeds apparently with considerable irregularity, but we are soon able to see that the cells are grouping themselves 
into an uninterrupted external layer and an internal accumulation of cells. The outer layer is in contact, or nearly in contact, with the zona radiata, and may, therefore, be termed the subzonal layer (Fig. II, s.z).* The inner accumulation of cells is designated as the inner mass, i.m. Figure to represents a rabbit ovum of about seventy hours, according to the observations of van Beneden. He represents the subzonal layer, $E c$, as interrupted at one point, where one of the cells of the inner mass, i.m, is exposed. It is probable, however, that-van Beneden is in error in regard to this, and that the subzonal layer is really continuous. In the

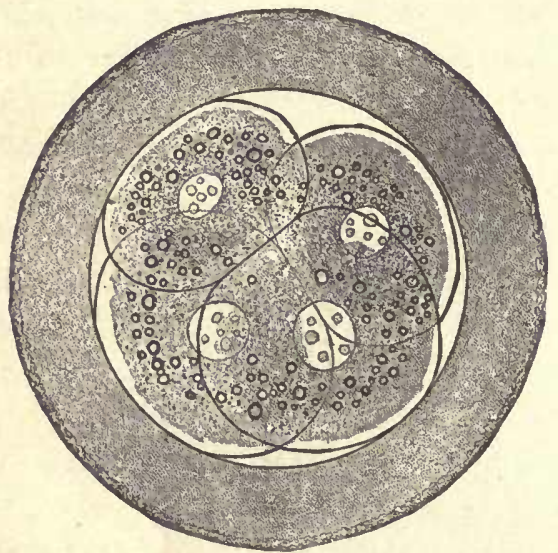

Fig. 8.-Ovum of a Bat (Vespertilio murina) with Four Segmentation Spheres.-(After van Beneden and Julin.)

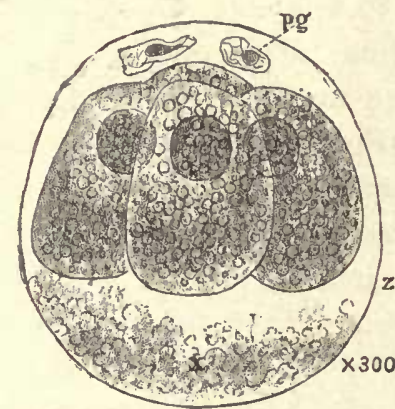

Fig. 9.-Ovum of a Virginlan Opossum, with Four SEGMENTS.

p.g, Polar globules. $x$, Coagulated material. $z$, Zona pellucida.-(After Emil Selenka.)

next stage (Fig. II) we find that the ovum has become larger by the appearance of a cavity in its interior. This cavity appears between the inner mass, $i . m$, and the subzonal layer, but at one side the inner mass remains adherent to, and closely connected with, the subzonal layer. We now have reached the stage in which the developing ovum may be designated as the blastodermic vesicle.

As to the interpretation of the parts, it is probable that the subzonal layer is ectoderm, and that the central cells of the inner mass are also ectodermal and share in forming the embryonic shield, and finally that the superficial cells of the inner mass (i.e., those next the cavity of the vesicle) are entodermal. At the stage we have now reached the blastodermic vesicle has a large part of its walls formed by the subzonal layer only, so that we call this the stage of the one-layered blastodermic vesicle.

* The subzonal layer is termed trophoblast by A. A. W. Hubrecht, and is held by him to be a special embryonic structure, developed in order fo establish special relations between the developing ovum and the walls of the uterus to secure the nutrition of the former. It has seemed best to present a purely objective account of the facts without entering into a discussion of the very interesting interpretations proposed by Hubrecht. 
Arrival in the Uterus.-During the stages described the ovum travels along the Fallopian tube and reaches the uterus in an early phase of the stage which we designate as the blastodermic vesicle. The transit requires about eighty hours in the mouse, about five days in the opossum, four days in the rabbit, and from eight to ten days in the dog. The time necessary in man is unknown. It may be supposed to be about one week.

Pro-chorion.-The ovum in many mammals becomes surrounded by a gelatinous covering, which is secreted by the glands of the uterus. It may be compared

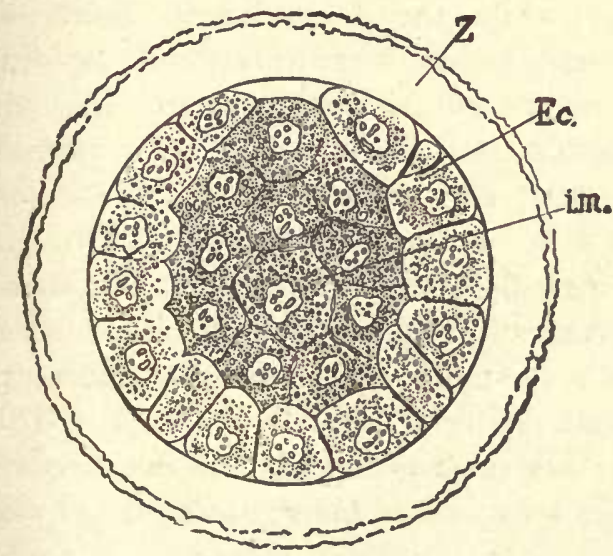

Fig. I0.-Rabbit's Ovum of about Seventy Hours.

Ec, Outer layer. $\quad i . m$, Inner mass of cells. $\quad Z$, Zona pellucida.-(After $E$, van Beneden.)

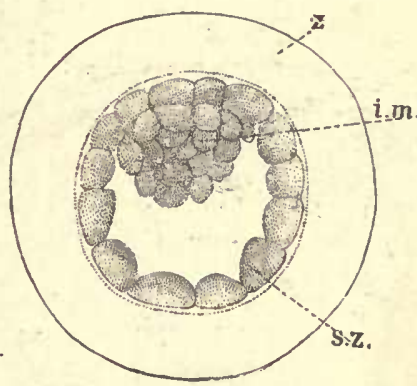

Fig. II.-Young Blastodermic Vesicle of a MOLE.

$i . m$, Inner mass of cells. s.z, Outer or subzonal layer. $z$, Zona pellucida.-(After W. Heape.)

to the white of the bird's egg. In the rabbit this envelope becomes enormously thick about the blastodermic vesicle and in other rodents is voluminous. In the dog it is less developed, but presents the further peculiarity that the secretion in the tubular glands may be hardened in connection with the envelope itself, which, therefore, appears, when the ovum is removed from the uterus, to be studded over with fine threads resembling villi. The gelatinous envelope has been termed by Hensen the pro-chorion. The thread-like projections seen in the dog were taken by Bischoff for true villi, and they have sometimes been referred to as the prochorionic villi. The term pro-chorion has been applied to other structures, as, for instance, to the subzonal layer of the blastodermic vesicle. The student needs to be warned against confusing the term pro-chorion in its various applications.

\section{The Blastodermic Vesicle.}

The blastodermic vesicle always consists at first of the subzonal layer and an inner cell mass attached at one point to the subzonal layer, and has a cavity between the inner mass and the subzonal layer; the vesicle itself is always enclosed 
in the zona radiata. The variations offered in different mammals are so great that a description less general than that given would hardly be applicable, even to the placental mammals.

The next step in development is the production of a complete second layer out of the cells of the inner mass. This layer extends completely around the vesicle and lies close against the subzonal layer, and encloses the main cavity of
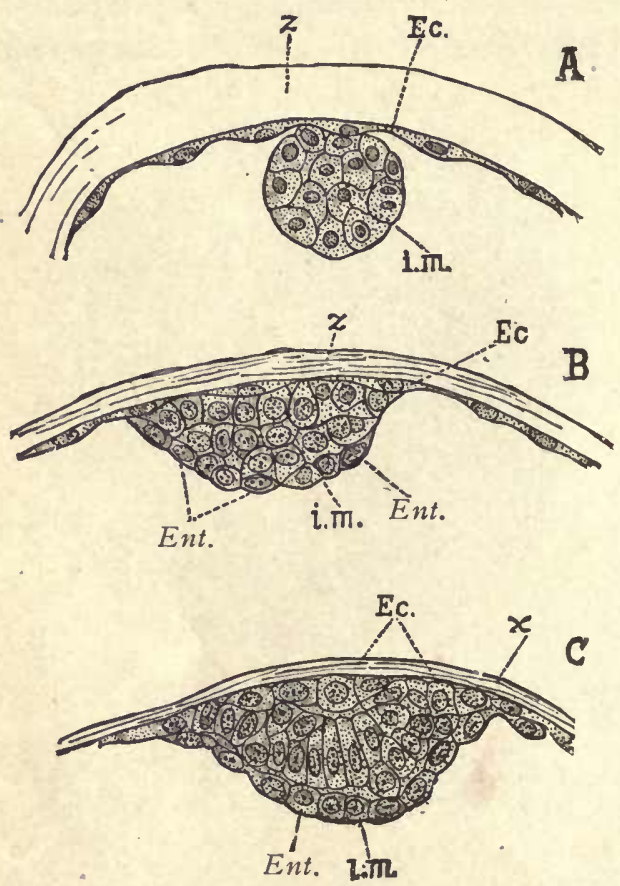

Fig. I 2.-Sections throvgh the InNer Mass of Blastodermic Vesicles of the Mole at Three Successive Stages.

$E c$, Outer or subzonal layer. $z, x$, Zona pellucida. i.m, Inner mass of cells. . Ent, Entoderm.(After W. Heape.) the vesicle. The way in which this inner vesicular layer is developed varies greatly. In the hedgehog it appears very precociously, while the blastodermic vesicle is very small, and afterward it expands rapidly, while the vesicle as a whole is growing. In the rabbit and in the mole it is formed much later, and the one-layered vesicle expands to a considerable diameter before the inner mass begins to spread out. The striking changes through which the inner mass passes in the mole are illustrated in figure I2. It forms at first a small globe, A. The inner mass subsequently flattens out, becoming lens-shaped, thinner, and larger in area, B. It continues spreading laterally and separates into three layers. The two outer layers enter into the formation of the true ectoderm, C. In the rabbit, and perhaps in the mole, the outer of the two layers is temporary only in existence. In some rodents it acquires a very great development and leads to the curious phenomenon known as the inversion of the germ-layers. The innermost of the layers; Ent, grows at its. edges, and its cells spread out gradually farther and farther under the subzonal layer until they extend completely around the vesicle and form, by meeting at the opposite pole of the ovum, a closed. vesicle. Very similar is the process in the rabbit. The cells at the expanding edge of the inner layer are found to spread rapidly, so that during the expansion they are more or less widely separated from one another. But they continue their expansion and multiplication until they form a complete inner epithelial layer.

The point where the inner mass and the subzonal layers are connected with one another marks the site of the future embryonic area.

The blastodermic vesicle grows rapidly in size, partly by the multiplication of its cells, partly by their becoming flattened out so as to cover a larger surface. 
The interior of the vesicle is filled with fluid. As the vesicle grows the fluid increases in amount, and is presumably derived by the ovum from the walls of the uterus. It is under pressure within the vesicle, as is shown by the manner in which it spurts out if the vesicle is broken. Nothing exact as to the composition of this fluid is known, though we may suppose it to resemble more or less the serous fluid of the adult body. The size and form of the vesicle offer characteristic variations in mammals. It starts as a more or less nearly spherical body. In the rabbit it assumes an oval shape, and by the seventh day measures about $4.0 \mathrm{~mm}$., and soon thereafter becomes attached to the wall of the uterus. In the hedgehog, the guineapig, and the mouse the ovum, while very small and more or less rounded in form, becomes imbedded in uterine tissue and develops into a special shape in adaptation to its new situation. In the ungulates the vesicle grows enormously, becoming a very long and slender sac. Thus, for example, in the sheep it may measure on the fourteenth day not less than $50 \mathrm{~cm}$. in length.

Another respect in which the blastodermic vesicles differ greatly from one another in various mammals is in regard to the early development of the subzonal layer, or, as we may call it, the ectoderm. In many cases the entire layer undergoes a precocious development, its cells multiply very rapidly, so that the layer becomes several cells thick. This thickened layer is known as the trophoderm. In other placental mammals this thickening is confined to a limited area of the ectoderm. For further description see Trophoderm, page II4.

\section{The Embryonic Shield.}

Sooner or later in the early history of every blastodermic vesicle, and always as the first indication of the development of the embryo proper, there appears a thickening of a small oval area of the outer layer in the region of the inner mass.

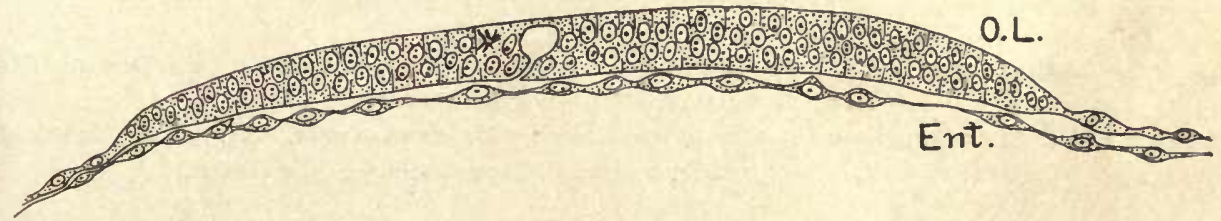

Fig. i3.-Transverse Section through the Embryonic Shield of the Blastodernic Vesicle of a Dog of Eleven or Fifteen Days (Preçise Age Unknown).

O.L, Outer layer. Ent, Entoderm. $\times 200$ diams.-(After Bonnet. $)$

This thickening is known as the embryonic shield. In the fresh specimen it marks itself by the greater opacity which it causes in the walls of the ovum where it lies. In those cases where the thickening of the ectoderm to form the trophoderm extends over the entire blastodermic vesicle, it is very difficult to follow the early history of the embryonic shield. In other cases, however, where the trophoderm occupies a special restricted area, the history of the embryonic shield may be more readily followed. The animals in which it has hitherto been chiefly studied are 
the rabbit, dog, cat, and sheep. In all of these the embryonic shield is simply a thickening of the outer layer (Fig. I3). The embryonic shield is at first small, but it rapidly expands and assumes a rounded or oval form. There next appears, in a more or less central position in the shield, a small, darker spot, which marks what is known as the primitive knot, a peculiarity of which is that it corresponds to an intimate union of the cells of the inner with those of the outer layer of the blastodermic vesicle. (Compare Fig. I26, B, page i7r.) Soon a linear shadow becomes visible extending from the primitive knot toward a point at the periphery of the embryonic shield (Fig. 14) which represents the embryonic shield of a dog

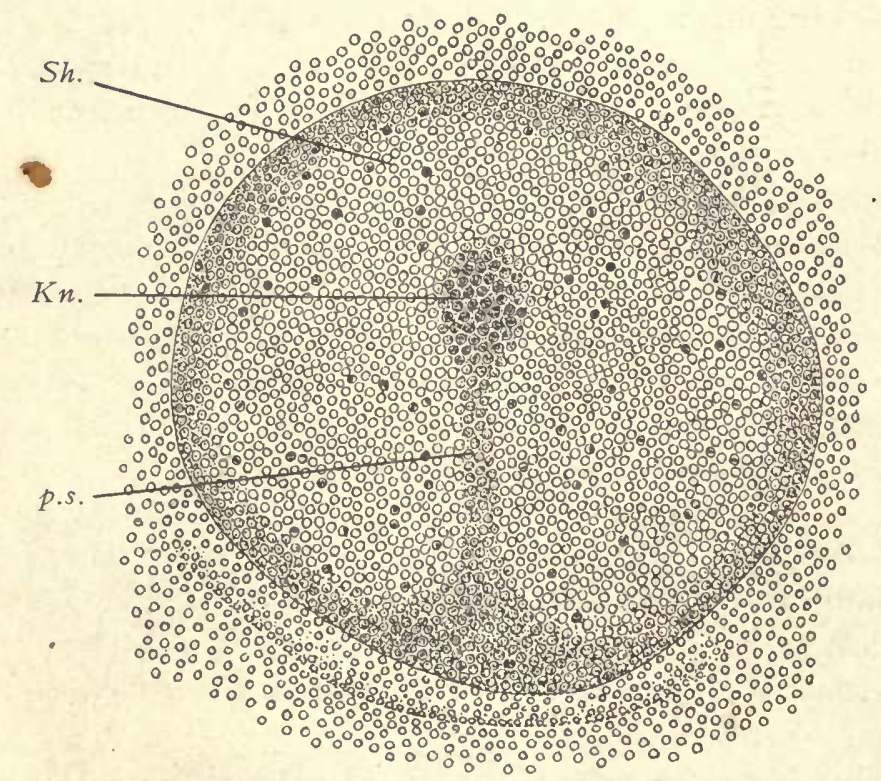

Fig. I4.-Surface View of the Embryonic Shield of the Blastodermic Vesicle of a Dog of Thirteen to Fifteen Days (Precise Age Unknown).

The specimen had been preserved with sublimate and stained with borax-carmin. Sh, Embryonic shield. $K n$, Hensen's knot. p.s, Primitive streak. $\quad \times$ roo diams.-(After Bonnet.)

at about two weeks. The shadow, p.s, from the primitive knot is termed the primitive streak, and it very soon becomes further characterized by the formation of a fine groove caused by a depression in the outer layer of cells. This is known as the primitive groove, and has been observed in all amniote embryos. Its exact significance has never been satisfactorily ascertained, and its interpretation is still a matter of scientific discussion. A transverse section through the primitive streak of a vesicle of a common European mole is shown in figure 15. At about the time the primitive streak appears the embryonic shield becomes oval in form. In those animals, such as the carnivora and ungulates, which have a large elongated blastodermic vesicle, we find that the long axis of the embryonic shield is nearly at 
right angles to the long axis of the vesicle. The size of the shield is about the same in all mammals which have been heretofore studied.

\section{Growth of the Embryo and Separation of the Yolk.}

In all vertebrates the development is strictly of the embryonic type, and accordingly there is made for the nutrition of the embryo some special provision,

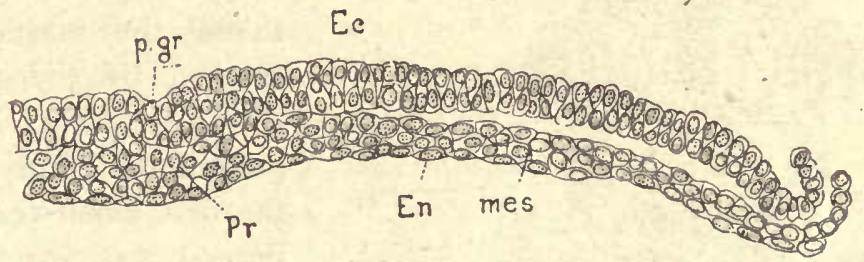

Fig. 15.-Transverse Section throvgh the Primitive Streak of an Embryo Mole.

Ec, Ectoderm. En, Entoderm. mes, Mesoderm. p.gr, Primitive groove. Pr, Primßive streak.-(After W. Heape.)

which in most cases consists of a stock of yolk material; but in the placental mammals the provision is made by means of the placenta for the transfer of nutriment directly from the mother. In either case the embryo has merely to assimilate the food already more or less prepared for it. It is perhaps owing to these provisions that the growth of the vertebrate embryo is extremely rapid. In the amniota there is a fundamental distinction between the embryo proper and its

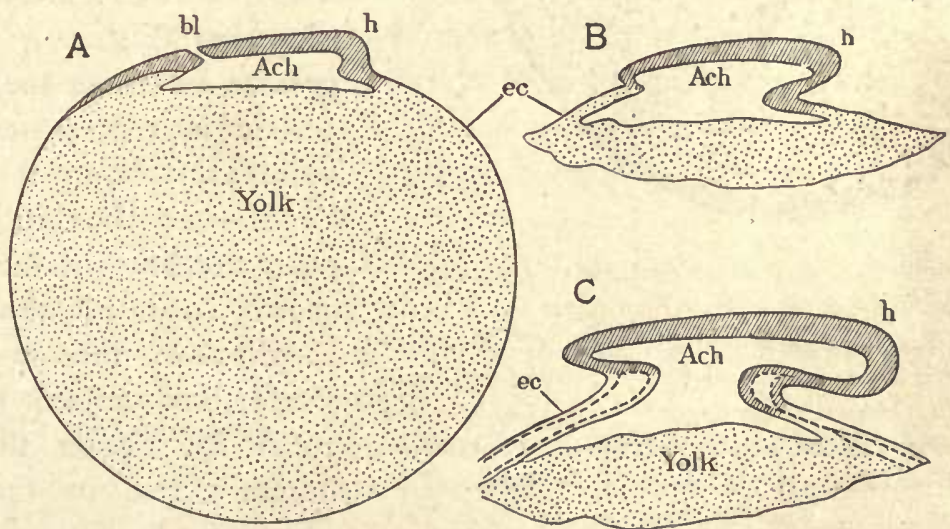

Fig. 16.-Diagrams to Illustrate the Separation of the Embryo from the Yolk. $b l$, Blastopore. $h$, Head of embryo. Ach, Archenteron or entodermal cavity. $e c$, Ectoderm.

so-called appendages - the yolk-sac, chorion, amnion, and allantois. The appendages are all finally sacrificed for the benefit of the embryo, and in mammals, except for a portion of the allantois retained in the body as the anlage of the bladder, the four appendages are ultimately cast off altogether and take no part in the construction of the child after birth. We note, in fact, as we ascend the verte- 
brate series, an increasing tendency to give the embryo prominence and to differentiate it more decisively from the embryonic appendages. This becomes so marked in the higher vertebrates that we speak of the growth of the embryo

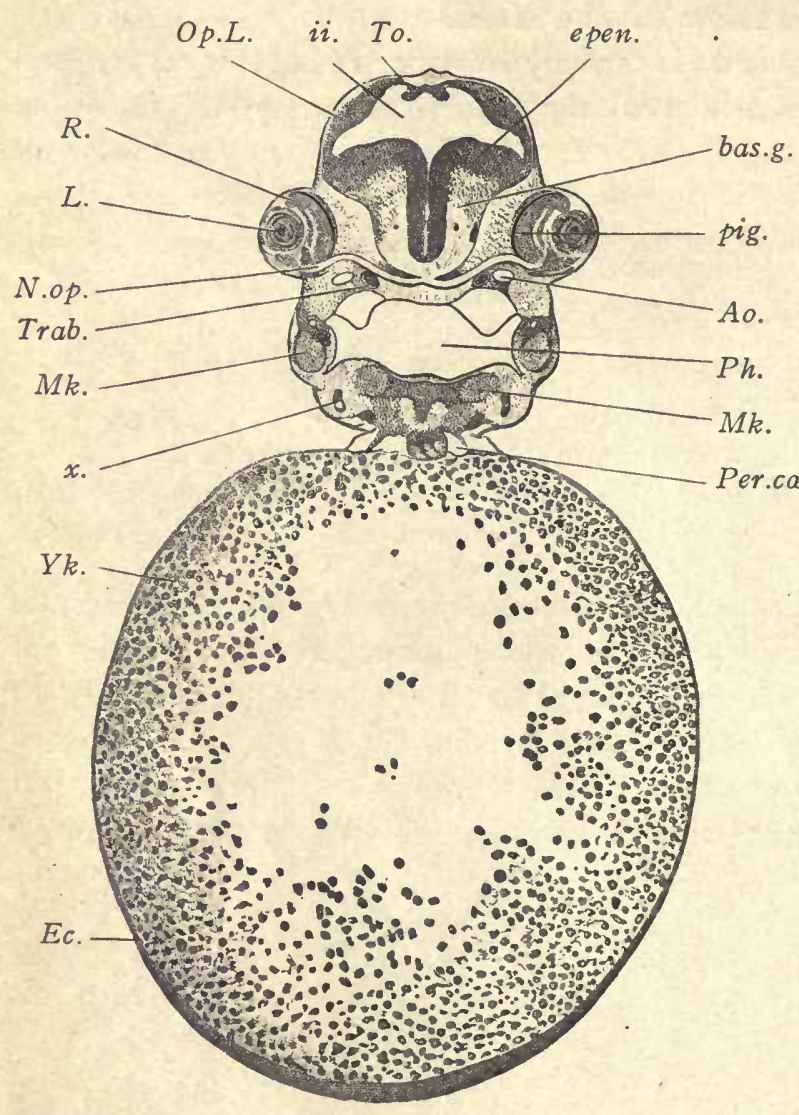

Fig. 17.-Transverse Section of an Embryo Catfish (Amiurus); Series 25, Section 43.

Ao, Aorta. bas.g, Basal ganglion of mid-brain. Ec, Ectoderm. epen, Ependymal layer of mid-brain. $i i$, Cavity of mid-brain. $L$, Lens. Mk, Meckel's cartilage. N.op, Optic nerve. Op. L, Optic lobe. Per.ca, Pericardial colom. Ph, Pharynx. pig, Pigment layer of the eye. $R$, Retina. To, Torus. Trab, Trabecula cranii. $x$, Undetermined organ. $Y k$, Yolk. $\times 40$ diams. show a similar expansion of the
embryo laterally (compare the three diagrams, Figs. $29,45, \mathrm{~A}$, and $45, \mathrm{~B}$ ). . Hence, though the connection between the embryo and the blastodermic vesicle may remain

* The separation of the embryo from the rest of the ovum has long been described as a process of the folding in of the germ layers on the under side of the body. The traditional perpetuation of this erroneous description is regrettable, for the separation of the embryo is really due to the expansion of the embryo, and in no sense to the constriction of its connection with the yolk. almost as a separate thing from the growth of the appendages.

The embryo is developed from the axial portion of the embryonic shield, the position of which is marked by the primitive streak (Fig. 14, p.s). In the territory around the embryo are developed the first blood-vessels, hence it is termed the area vasculosa (see page 66). About the time that the blood-vessels begin to appear, the separation of the embryo from the shield commences, and the extra-embryonic portion of the shield remains as part of the blastodermic vesicle, or yolk-sac. This separation is due wholly to the growth of the embryo.* The process is illustrated by the diagrams (Fig. I6), in which for. greater clearness the blastodermic vesicle is represented filled with yolk, as it is in the Sauropsida. Soon after the blood-vessels appear, the head of the embryo has grown so much that it not only rises above the surface of the shield, but projects forward (Fig. I6, A, h). Later the caudal end becomes free in the same way (Fig. I6, B, C). Cross sections 
unchanged, or even slightly increase in dimension, yet the growth of the embryo causes that connection to appear relatively small. A connection of I or $2 \mathrm{~mm}$. equals at first the entire length of the embryo, but a connection of 4 or $5 \mathrm{~mm}$. seems small when the embryo is 100 or $200 \mathrm{~mm}$. long.

The relations of the embryo to the yolk in the anamniota are illustrated by the accompanying figure $\mathrm{I} 7$, which represents a transverse section through a young stage of the catfish (Amiurus). The section passes through the head of the embryo and shows both eyes and the slender optic nerves, N.op, almost symmetrically cut on both sides. The yolk, $Y k$, is a a large mass heavily laden with yolkgranules. Between the tissues of the embryo proper and of the yolk-sac there is a direct continuity. Not only can the ectoderm, $E c$, be followed around from the embryo over the yolk-sac, but also a layer of mesoderm. The part of the yolk-sac which carries the yolk grains is, as above stated, a modification of the entoderm. There is no amnion.

\section{Origin of the Mesoderm.}

The development of the primitive streak and groove is accompanied by the appearance of the third or middle germ-layer, the mesoderm (Fig. 15, mes). As shown in the section there figured, the three germ-layers are fused together underneath the primitive groove, and are there thicker than elsewhere. As we pass laterally from the groove, the ectoderm and mesoderm both become thinner and are distinctly separated from one another. The entoderm consists of a single thin layer of cells very closely connected with the mesoderm. The mesoderm occupies at first only a small area in the immediate neighborhood of the primitive streak. It grows rapidly, so that its edge extends farther and farther over the blastodermic vesicle. The mesoderm is to be regarded as the product of the entoderm. Its exact origin in mammals has not yet been adequately traced. We know, however, that in birds, reptiles, and elasmobranchs the cells of the inner layer multiply rapidly, so that the inner layer becomes more than one cell thick. The upper cells soon split off from the lower and thus form themselves into the middle germlayer. The mesoderm therefore is said to be formed by delamination. It seems probable that in mammals the process is the same.

It may be mentioned that, according to Bonnet, the development of the mesoderm in the sheep is not quite as above described. It can be first distinguished at the stage when the primitive knot has appeared, and before the primitive streak is developed. In the fresh specimen it is seen as a slight turbidity of the vesicular walls just outside the edge of the shield (Fig. I8), while in the region of the shield there is no middle layer whatever. By the time the primitive streak has appeared in the sheep, the formation of the mesoderm has extended under the embryonic shield, and the relations between the germ-layers then become essentially as above described.

The cells of the mesoderm are at first quite closely packed, but as the layer 
grows they begin to move apart, though remaining connected with one another by protoplasmic processes. The cells separate least near the primitive streak, but their separation becomes gradually more and more marked toward the periphery of the layer, as shown in Fig. I9, which represents a part of the peripheral region of the mesoderm of a blastodermic vesicle of a rabbit of seven days.

In the details of its expansion the mesoderm varies greatly in different mammals. In some forms it develops very early and rapidly expands over the entire blasto-

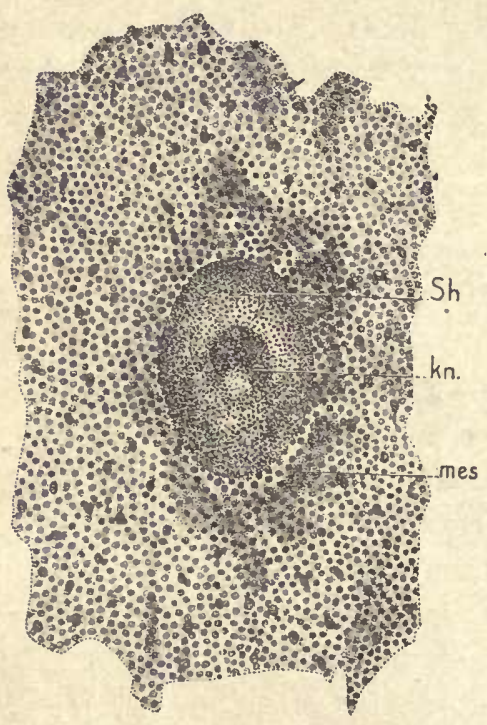

Fig. i8.-Central Portion of a Sheep's BlastoDERMiC VESICLE OF TWELVE to THIRTEEN DAYS.

$S h$, Embryonic shield. $k n$, Hensen's knot. mes, Shadow caused by mesoderm developing around the shield. $\times_{34}$ diams. $-($ After Bonnet. $)$

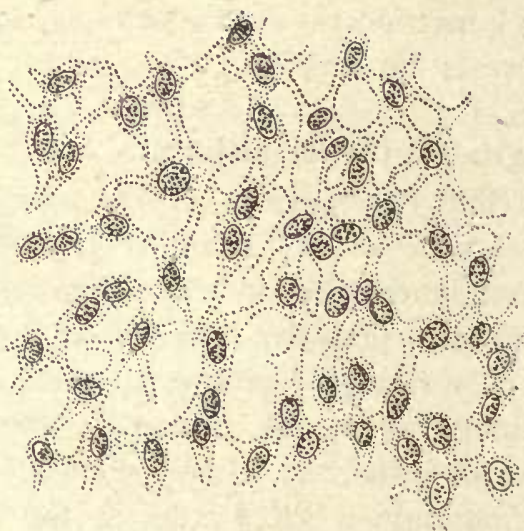

Fig. 19.-Blastodermic Vesicle of a Rabit of Seven Days. Portion of the Mesoderm of the AREa OpaCa. - (After Kölliker.)

dermic vesicle, which then becomes three-layered. This seems to be the method of its growth in man and other primates. In other cases, as in the dog and cat, it grows more slowly, but ultimately. encloses the entire entoderm. In the rabbit, on the contrary, it never expands more than about three fifths of the way over the blastodermic vesicle, one part of which, therefore-viz., that opposite the embryonever has any mesoderm whatever. This, however, is to be regarded as a special modification, since we must consider that primitively the mesoderm extended over the entire vesicle.

\section{The Primitive Axis.}

The next stage of development is characterized by the appearance of an accumulation of cells which extends forward from the primitive knot in the axial line. 
This thickening is termed the primitive axis. German writers commonly designate it as the "head process" (Kopffortsatz). The primitive axis may be easily distinguished in transverse sections from the primitive streak by the fact that in the former the thickening occurs in the mesoderm and entoderm, which are closely united, and it is separated from the outer layer; whereas in the latter the cells of the thickening are fused with both the entoderm and the ectoderm (compare Fig. I26, $\mathrm{A}$ and $\mathrm{C}$, page $\mathrm{I} 7 \mathrm{I}$ ).

The primitive axis corresponds to the region in which the body proper of the embryo develops, and represents the beginning of embryonic development in this restricted sense. It grows quite rapidly in length and width, and as it grows encroaches more and more upon the territory of the primitive streak, which is gradually obliterated by merging into the caudal end of the developing embryo, so that it can no longer be distinguished. The obliteration of the primitive streak is gradual, and there is a series of stages easily observed in amniota in which we find the embryonic developinent in the region of the primitive axis more or less advanced, while part of the primitive streak still presents to us, more or less clearly, its original condition.

\section{The Notochordal Canal.}

In regard to this canal our knowledge is imperfect. Any account of it which we can give may need correction. It is a very small canal which runs through the center of the primitive axis. It ends blindly in front, but opens through the ectoderm at its posterior end, at a point corresponding perhaps exactly to the position of the primitive knot. The first indication of the formation of the canal is an alteration in the form of the cells in the center of the primitive axis. These cells elongate in directions at right angles to the axis. Their nuclei become oval and are radially placed. The change begins posteriorly and progresses forward. The radial cells move apart, so that there arises a longitudinal canal. It may happen that in mammals, as in birds, the canal is not actually open at its posterior end. If that should be found to be the case in any instance, it would not alter our interpretation, for we should then consider that the walls had simply closed together. There are many instances of tubular structures being temporarily solid in embryonic stages. Such a condition, for example, has been observed in the œsophagus of elasmobranchs, in the large intestine of birds, and in other cases.

The opening of the notochordal canal is termed the blastopore, and is supposed to be identical with the blastopore of the anamniota.

After the notochordal canal is formed the blastodermic vesicle has, of course, two cavities: first, the small cavity of the canal; second, the large main cavity of the vesicle which is surrounded by entoderm. This larger space is designated as the yolk-cavity. After the canal has acquired a not inconsiderable length its lower wall develops a series of irregular openings (Fig. $20, n c h$ ) on its ventral side, by which it comes into communication with the large underlying yolk-cavity. These 
openings grow until the ventral wall of the notochordal canal is entirely lost. We then have the two cavities completely fused, making a single cavity bounded by a continuous layer of cells, the majority of which represents the lining of the yolkcavity, but the small minority represents the cells of the notochordal canal. The continuous layer of cells is known as the permanent entoderm, and the cavity itself, which is of double origin, is termed the archenteron. At about this time, probably sometimes earlier, sometimes later, according to the species, the blastopore becomes permanently closed and the entodermal cavity no longer has an opening to the exterior.

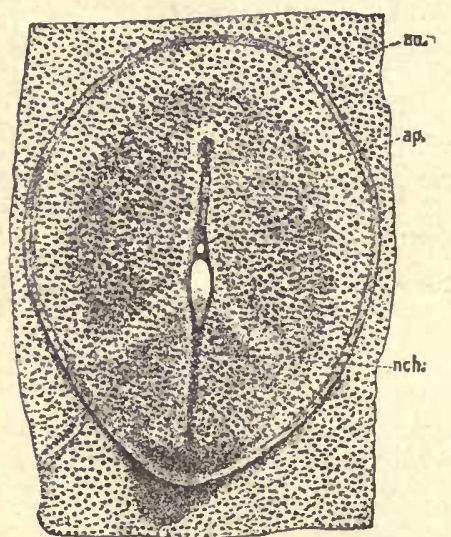

Fig. 20.-Germinal Area of a Guinea-pig at Thirteen Days and Twenty Hours, seen FROM THE UNDER (ENTODERMAL) SidE.

o.a, Area opaca. a.p, Area pellucida. nch, Notochordal canal with several irregular openings through the entoderm.-(After Lieberkiihn.)

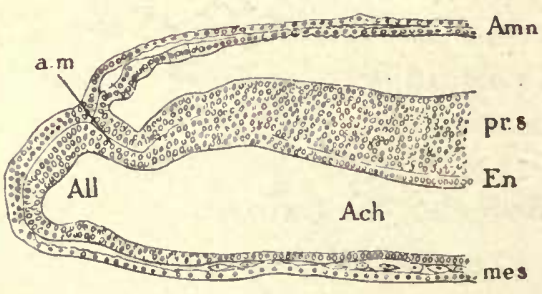

Fig. 21.-Longitudinal Section of the Posterior End of a Sheep Embryo of Srxteen Days.

A $m n$, Amnion. a.m, Anal membrane (or plate). pr.s, Primitive streak. En, Entoderm. Ach, Archenteron, or entodermal cavity of the embryo. All, Anlage of allantois. mes, Mesoderm.-(Afier R. Bonnet.)

In a number of vertebrates it has been demonstrated that the blastopore is soon divided into two parts: one anterior, which frequently remains open, and gives rise to the neurenteric canal, and one posterior, which gives rise to the anal opening. When the spinal cord (medullary canal) is developed it extends so far as to include the neurenteric canal and exclude the anus. The neurenteric canal is obliterated during early embryonic life, but so long as it remains open it constitutes a free communication between the archenteron and the medullary tube (spinal cord). The anal opening is early closed by a growth of the surrounding cells, which produces an occluding membrane known as the anal plate (Fig: 21, a.m). The plate includes a layer of ectodermal and of entodermal cells, but apparently no mesoderm. It persists for a long time and undergoes a considerable growth, but ultimately it is perforated to form the permanent anus.

The cells on the dorsal side of the notochordal canal have a different destination, for they become thickened to make the anlage of the future notochord. It is to this fact that the canal owes its name. 


\section{The Notochord.}

The notochord (chorda dorsalis) is a rod of peculiar tissue constituting the primitive axial skeleton of vertebrates. It begins in the embryo immediately behind the pituitary body and extends to the caudal extremity. It occurs as a permanent structure in all vertebrates, but undergoes much modification in the amniota. It appears very early in the course of development, being differentiated from the median dorsal wall of the notochordal canal, beginning at a time when the medullary groove (compare page 68) is not fully marked out posteriorly, and is nowhere closed. The notochordal anlage can be first detected as an axial band of cells, which at first is not well marked off from the mesoderm of the primitive axis. The anlage is thicker than the adjacent entoderm (Fig. 22, nch). The differentiation of the notochordal cells begins usually at the anterior end of the canal and progresses backward. It appears merely as a specialized part of the entoderm of the archenteron, but has a very sharp demarcation.

The notochordal anlage separates off and the entoderm proper closes across under it, so that the notochordal band lies between the entoderm and the overlying ectoderm (floor of the medullary groove or canal). The two primitive germ-

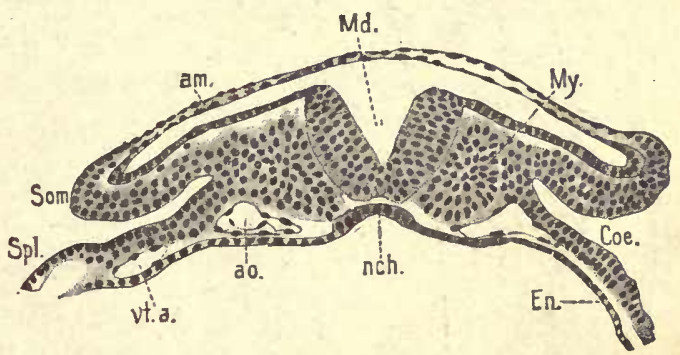

Fig. 22.-Transverse Section of a Mole Embryo (Heape's Stage H).

am, Amnion. $M d$, Medullary groove. $M y$, Primitive segment. Ce, Cœlom. En, Entoderm. nch, Notochord. as, Aorta. vt.a, Vitelline artery. Som, Somatic mesoderm. Spl, Splanchnic mesoderm.-(After W. Heape.)

layers come into actual contact in the median line, along which, therefore, when the notochord first separates from the entoderm, there is no middle germ-layer present. This condition exists in the chick with eight segments described in Chapter V. The separation of the anlage does not take place at the anterior extremity of the notochord until somewhat later, so for a considerable period the cephalic end of the notochord remains fused with the entoderm. The separation from the entoderm is effected in mammals by the entoderm proper shoving itself under the notochord toward the median line. When the cells from one side meet those of the other they unite with them and form a continuou's sheet of entoderm below the notochordal cells. The process of separation may be followed easily in the development of the frog and toad.

After its separation the notochord is a narrow band of cells, which starts anteriorly from the entoderm (the future lining of the alimentary tract), running backward to the blastopore. So long as the blastopore or neurenteric canal is open the notochord terminates in the epithelium lining it. For a certain period the notochord continues to grow tailward by accretion of cells from the walls of the blastoporic passage; and after the canal is permanently obliterated, the noto- 
chord may still continue to lengthen by acquisitions at its caudal end of additional cells from the primitive streak.

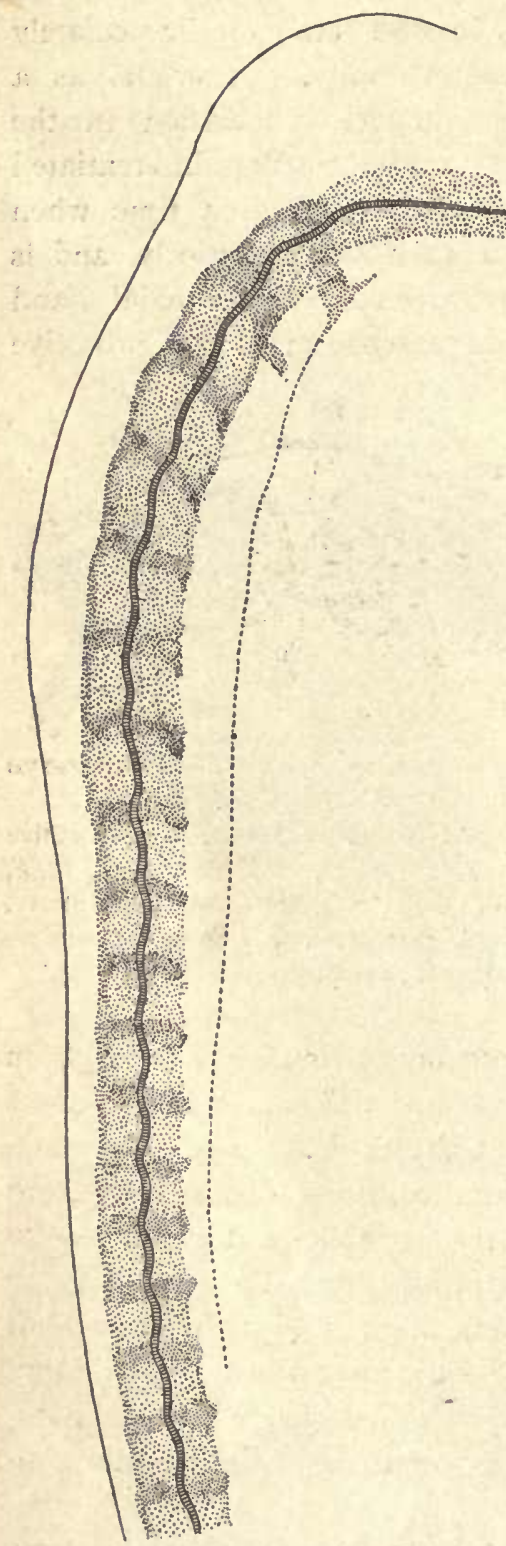

Fig. 23.-Notochord ANd ChondRostyle of a Sheep Embryo of I $4.6 \mathrm{mM}$. RECONSTRUCTION FROM SAgitTAL SERIES irog, Sections igo-r93.
After it is once formed as a band of cells, the notochord passes through various changes of form, but ultimately becomes a cylindrical rod with tapering extremities. It attains considerable size in the embryos of most vertebrates, but in those of placental mammals it is always small. It is probable that in mammals the notochord, when first separated from the entoderm, is a broad, flat band, and that this band subsequently draws together, diminishing its transverse and increasing its vertical diameter until it has acquired a rounded form. Finally its outline becomęs circular in cross-section. This series of changes begins near the anterior end of the notochord and progresses both forward and backward.

In later stages the mesoderm again grows across the median line of the embryo, completely surrounds the notochord, and forms a special sheath about it. Still later the mesoderm forms a broad envelope around the notochord, which we can soon recognize as the anlage of the chondrostyle, out of which the vertebral column and part of the base of the skull are to be differentiated. Very soon (Fig. 23) the chondrostylic anlage shows a series of transverse discs of denser tissue, the anlages of the intervertebral ligaments, the broader light spaces between the discs being the anlages of the vertebræ. In mammals, the notochord assumes an undulating course, which may be slightly irregular at first. The typical arrangement is shown in the figure-the dorsal summit of each flexure is intervertebral, the ventral hollow of each flexure is vertebral.

\section{The Ultimate Fate of the Notochord.}

As the vertebral column develops, the notochord slowly disappears in the regions of the vertebræ and even the space occupied by it is obliterated by the growth of the body of the vertebra. In the intervertebral discs, on the contrary, the notochord persists to form the nuclei pulposi of the adult. Each nucleus is 
enlarged, first, by the withdrawal of the notochordal cells from the vertebra into the adjacent intervertebral discs; second, by the growth of the tissue. The cavities occupied by the nuclei have distinct boundaries and present characteristic forms in different mammals. The sheath of the notochord is lost, the walls of the cells disappear, the tissue becomes a syncytium (Fig. 24) of granular appearance, and breaks up into multinucleated reticular masses, making an irregular network the meshes of which are filled with a more or less homogeneous substance resembling mucin, that does not, however, agree with mucin in its reactions. Tissue of this character may be easily observed in human embryos of the third and fourth month. It has been not infrequently stated that the notochord disappears in mammals, and that it contributes to the formation of cartilage. Both statements are now known to be erroneous. Owing to the persistence of the nucleus pulposus, the vertebral joint differs fundamentally from all other joints in the body of the adult.

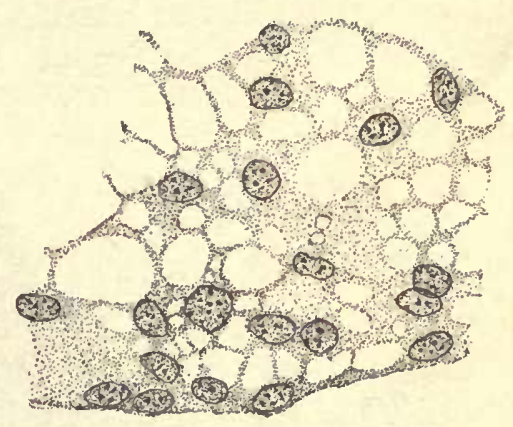

Fig. 24.-Pig Embryo of I50 MiM. Notochordal syncytium from nucleus pulposus. $\times 800$ diams. $-($ After $L$. W. Williams.)

\section{The Archenteron.}

The archenteron comprises the entire cavity bounded by the entoderm. At first it consists chiefly of the cavity of the yolk-sac (Fig. 25), but as it also includes the embryonic entodermal tract its development in the embryo greatly predominates as growth continues. As the head of the embryo protrudes, the archenteron forms a cephalic prolongation, known as the fore-gut (Figs. 25, Vd, and $\mathrm{I}_{3} 2, V d$ ), which ends blindly in front, but opens behind (caudad) into the general archenteric space, its opening being termed the fovea cardiaca, fo. Later as the caudal region becomes protuberant the archenteron sends into it a similar blind prolongation, known as the hind-gut (Fig. 25, H.g). As the embryo grows-compare the section on growth, page 49-the connection between the embryo and the yolk-sac, which seems so large in early stages (Fig. 25), increases very little, and therefore becomes relatively smaller. It never attains more than 3 or $4 \mathrm{~mm}$. The embryo, on the contrary, grows enormously (Fig. 34), and there is a corresponding enormous lengthening of the fore-gut and hind-gut. The former is the anlage of the pharynx, œsophagus, and stomach. The latter is the anlage of the large intestine and most of the ileum.

During embryonic life the archenteron is divided by the obliteration of the connection between the yolk-sac and the embryonic entoderm. For a time the original point of connection is marked by a small pouch (Meckel's diverticulum) of the ileum. The pouch normally disappears, but as an occasional anomaly (arrest of development) it persists in the adult. 


\section{The Oral and Anal Plates.}

These two structures resemble one another. Each occupies a small area and is formed by the intimate union of the entoderm with the ectoderm. When the union is first formed the two layers are distinct, but they soon fuse, so that no boundary can be recognized between them. Ultimately both plates break down, their cells

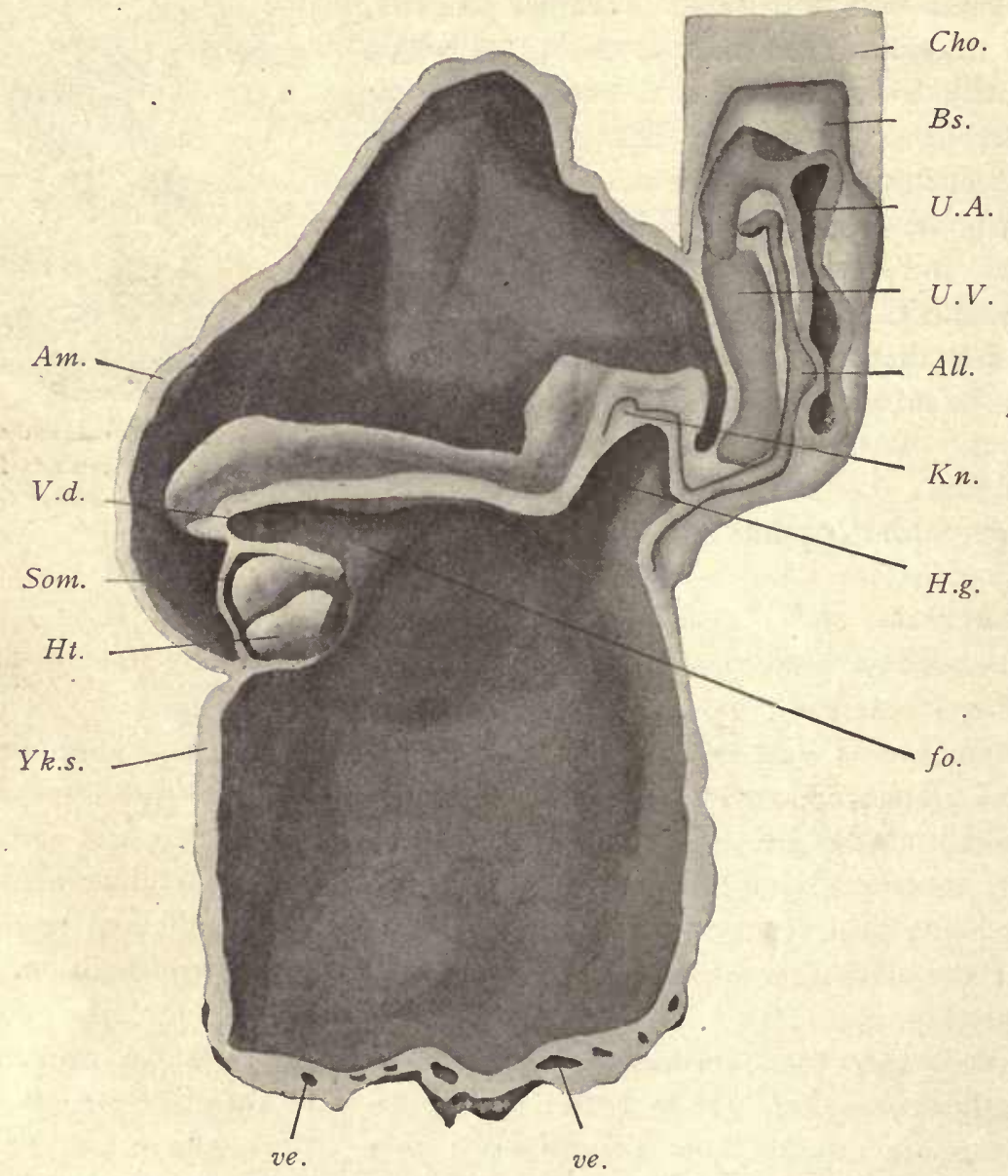

Fig. 25.-Wax Reconstruction of Dandy's Human Embryo with Seven Segments by Frederick T. LeWis. All, Allantois. Am, Amnion. Bs, Body stalk. Cho, Chorion. fo, Fovea cardiaca. H.g, Hind-gut. Ht, Heart. $K n$, Hensen's knot. Som, Somatopleure enclosing the pericardial cavity. U.A, Umbilical artery. U.V, Umbilical vein. $V d$, Fore-gut. ve, Blood-vessel. Yk.s, Wall of yolk-sac. $\times 40$ diams.

disappearing, and they are replaced by openings, that of the oral plate forming the opening between the mouth-cavity and the pharynx, that of the anal plate forming the primitive anal opening. The anal plate, before it breaks down, makes a considerable growth, forming an epithelial mass which plays an important part in the anatomical modeling of the region. The oral plate disappears very early; the anal plate much later. 
As soon as the head of the embryo has grown so much as to project as an independent part, we find that the oral plate lies on the under surface of the head, a little in front of the heart (Fig. 26). The pro-amnion, pro.am, arises from the somatopleure enclosing the heart, $h t$, so that, when the oral plate becomes perforate, the cavity of the entoderm, Ent, will communicate directly with the cavity enclosed by the pro-amnion, or, in other words, with the permanent amniotic cavity. Figure 72 , o.pl, shows the oral plate in a little later stage, shortly after which the plate ruptures.

A similar anal plate at the posterior end of the embryo also lies within the amnion (Fig. 21). This figure is taken from a sheep embryo in a very early stage, so that the anal plate appears to lie on the dorsal side. By the curling ventralward or the bending over of the tail end of the young embryo the anal plate is gradually transferred or rolled over on to the ventral side, where it permanently remains, For the relation of the anus to the blastopore see page 54 .

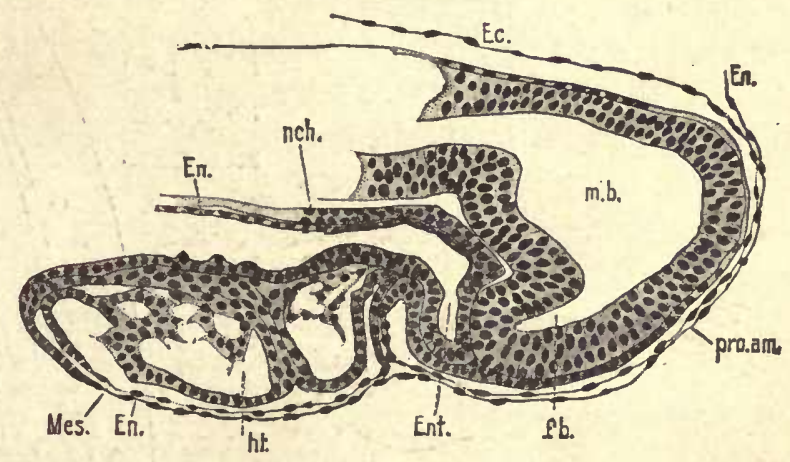

Fig. 26.-Longitudinal Section of the Head End of a Mole Embryo, Stage H.

Ec, Ectoderm. En, Entoderm. Ent, Anterior end of archenteric cavity with the oral plate on the cardiac side. $f b$, Forebrain. ht, Heart. m.b, Mid-brain. Mes, Mesoderm. nch, Notochord. pro.am,-Pro-amnion.-(After W. Heape.)

\section{The Digestive Canal.}

The digestive canal proper is developed by the growth and modifications of the fore-gut and hind-gut. The division between the two is a point in the ileum corresponding to the original connection with the yolk-sac, marked in the fetus by Meckel's diverticulum.

The fore-gut forms the pharynx (and lungs), the œsophagus, stomach, duodenum, and part of the ileum. It also produces, as appendages to the canal, the liver and pancreas.

The hind-gut forms most of the ileum and the entire large intestine, together with the cæcum and appendix.

The entoderm persists as the permanent epithelial lining, and produces all the glands of the digestive tract. It remains a thin layer throughout life. The mesoderm forms the greater part of the walls, furnishing the connective tissue, the smooth muscle layers, and the peritoneum, which last consists of the original mesothelium and a thin layer of chiefly fibrillar connective tissue.

The general course of the development is shown by figure 27 , which represents outlines of the entodermal canal in three human embryos, uniformly magnified twelve diameters. An earlier stage is shown in figure 25 . 
The fore-gut in the $4.2 \mathrm{~mm}$. embryo has lengthened. It communicates freely with the oral cavity proper, the limit of which is indicated by the hypophysis, $H y$, which is of ectodermal origin. The cephalic portion of the canal has undergone a
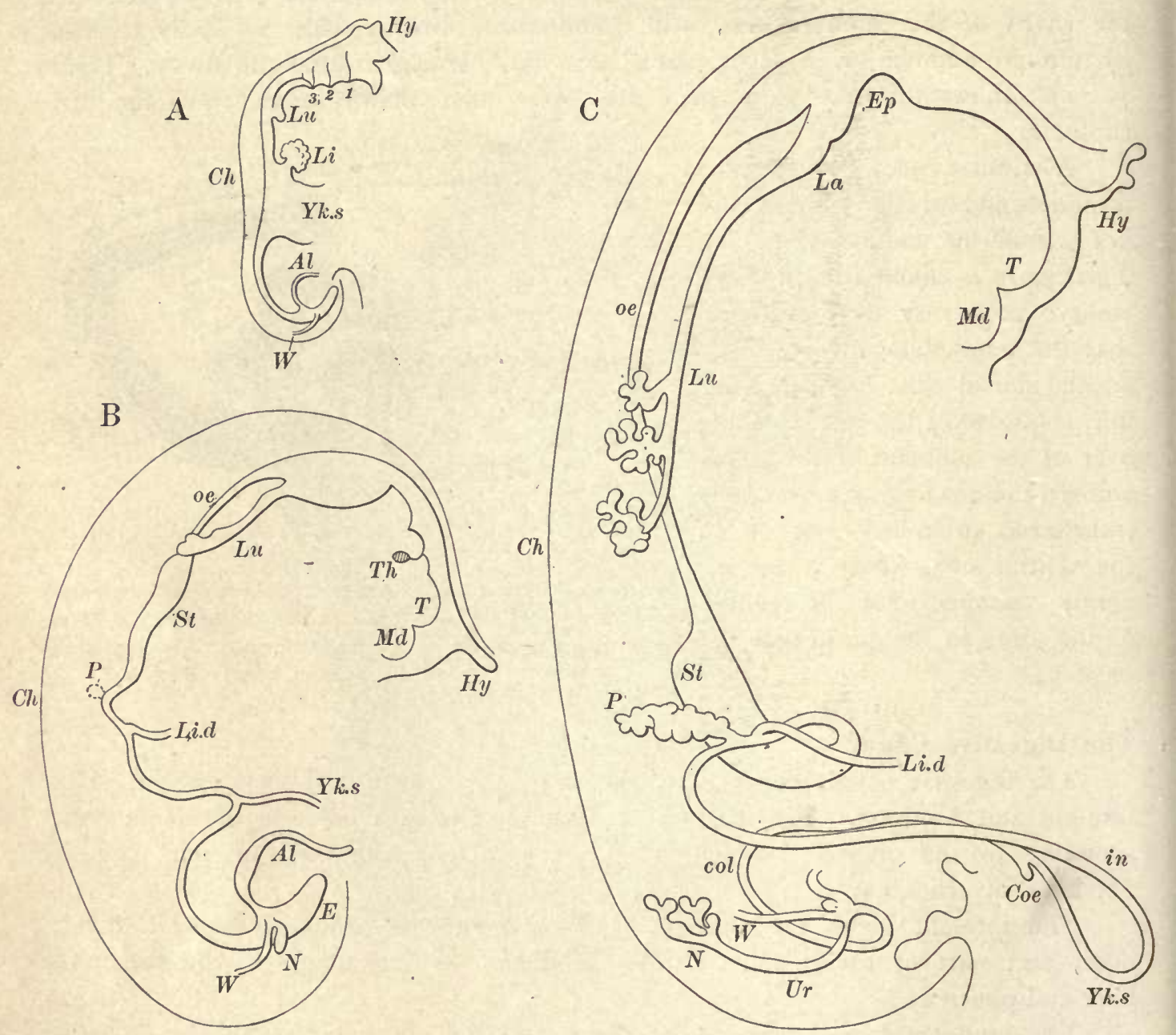

Fig. 27.-Outlines of the Notochord and Entodermal Canal of Three Human Embryos. A, 4.2 mi. B, 7.0 мM. C, $13.8 \mathrm{MM}$.

$A l$, Allantois. $C h$, Notochord. Col, Large intestine. E, Caudal intestine. Ep, Epiglottis. Hy, Hypophysis. in, Small intestine. La, Larynx. Li, Liver. Li.d, Hepatic duct. Lu, Lung. Md, Mandible. $N$, Renal anlage. $\propto$, Esophagus. $P$, Dorsal pancreas. St, Stomach. $T$, Tongue. Th, Thyroid gland. Ur, Ureter. W, Wolffian duct. Yk.s, Yolk-sac. I, 2, 3, first, second, and third gill-pouches. $X_{12}$ diams.(After $W: H$ is.)

great widening to form the pharynx, with its characteristic gill-pouches (Fig. 27, A, I, 2, 3)-compare below. From the caudad end of the pharynx, the anlage, $L u$, of the trachea and lungs has appeared on the ventral side. From the pulmonary an- 
lage, $L u$, to the hepatic, $L i$, extends a short tube which comprises the future oesophagus, stomach, and part of the duodenum. The liver, $\mathrm{Li}$, which arose as an outgrowth of the entoderm at the fovea cardiaca, has enlarged and become distinctly an appendage. Between the liver and the yolk-sac, $Y$ k.s, is a short broad tube, the beginning of part of the small intestine. In the $7.0 \mathrm{~mm}$. embryo, the fore-gut is much longer, and the differentiation of the osophagus, oe, stomach, $S t$, and duodenum, from which the anlage of the dorsal pancreas, $P$, has developed, is established. The liver is connected with the duodenum only by the narrow hepatic duct, Li.d, between which and the yolk-stalk, Yk.s, there is a considerable stretch of small intestine. In the $\mathrm{I} 3.8 \mathrm{~mm}$. embryo, the relations have been greatly altered by the growth and migration of the stomach (Fig. 27, C, St) which has descended from its original position into the abdomen, so that it is caudad of the diaphragm, and lies asymmetrically placed on the left side of the embryo. The stomach also turns so that its œsophageal end is toward the left, its duodenal end toward the right, and further revolves so that its left surface faces ventrally. In the $13.8 \mathrm{~mm}$. embryo, the migration and revolution of the stomach has not been completed. The descent of the stomach involves the elongation of the cesophagus (Fig. 27, C, oe) and the twisting of the duodenum.

The hind-gut has a simpler history. In the $4.2 \mathrm{~mm}$. embryo it has elongated and terminates blindly in the tail. Its caudal end is somewhat enlarged to form the cloaca, into which open also the Wolffian ducts and allantois (Fig. 27, A, W and $A l$ ). Between the cloaca and the yolk-sac, $Y k . s$, extends the cephalad portion of the hind-gut, nearly uniform in diameter. In the $7.0 \mathrm{~mm}$. embryo the conditions are similar, but the intestinal portion has lengthened and bent ventralward. The insertion of the yolk-stalk, $Y k . s$, marks the apex of the primitive intestinal loop. In the $\mathrm{I} 3.8 \mathrm{~mm}$. embryo, the loop has greatly lengthened and projects into the cavity of the umbilical cord (extra-embryonic cœlom), and a blind pouch, Coe, has appeared, the anlage of both the cæcum and the appendix. It marks the boundary between the large and small intestines, which as yet differ very little in diameter.

For some time a portion of the intestine lies in the umbilical cord, and may form several coils there, but gradually it is withdrawn so as to lie wholly within the abdomen proper.

The pharynx undergoes many modifications in form, and also produces an important series of accessory organs, including the thyroid gland, the tonsils, and the thymus. It comprises the cephalic portion of the fore-gut and originally overlies the heart (Figs. 25 and $\mathrm{I}_{32}$ ). The stretch of the fore-gut, which extends from the pharynx to the fovea cardiaca, remains at first short and narrow, most of the foregut being absorbed in the pharynx, which is produced by the expansion of the entodermal tube toward both sides of the neck; but the dorso-ventral diameter remains small. The expansion is greatest a short distance behind the mouth, and thence diminishes gradually toward the œesophagus, so that the pharynx of the em- 
bryo has a rhomboidal form which is complicated, however, by certain irregularities. These are due to the formation of the gill-pouches, of which there are four distinct pairs in mammals. Some authorities maintain that the ancestors of had five pairs, and that the pair lost was situated between the present third and fourth pairs (compare the remarks on "Zimmermann's arch," page ror). Each pouch is a lateral pocket of the fore-gut, having a tapering form, the apex of which comes into contact with the ectoderm. At the point of contact, entoderm and ectoderm fuse to constitute a closing plate, similar to the oral and anal plates. In aquatic vertebrates the closing plates are lost, and each gill-pouch becomes a true gill-cleft.

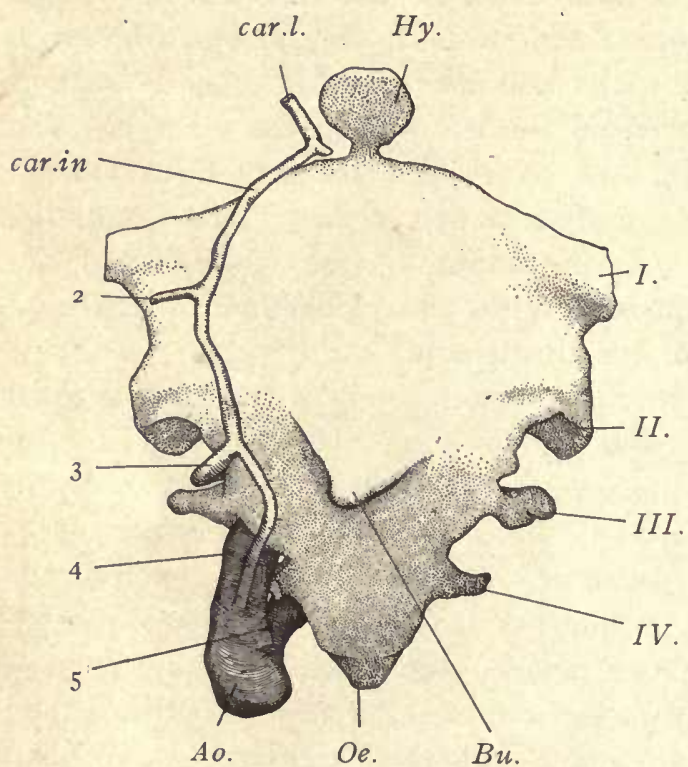

Fig. 28.-Pig Embryo of I 2 Mm. Series 5i8. OUtLINe of the Pharynx as SEen from the Dorsal Side, FROM A WAX MODEL BY A. R. KILGORE.

I, II, III, IV, Gill-pouches. 2, 3, 4, 5, Aortic arches. Ao, Aorta. $B u$, Bursa pharyngis. car. in, internal carotid. Car.l, Carotid loop to join intervertebral artery. $H y$, Hypophysis. Oe, Esophagus. $\times 22$ diams.

end by a prolongation downward and inward; the prolongation has a somewhat tubular form and extends far toward the aortic end of the heart; in the dorsal view of the model it does not show. The fourth pouch, IV, is much smaller than the others; it resembles the third pouch in having a ventral prolongation, but is quite variäble in form.

The entodermal epithelium of the second to fourth pouches exhibits certain specializations. One type is illustrated by the tonsil and thymus-the epithelium is thickened, assumes a reticular structure, and its meshes are invaded by leucocytes. 
Another type is illustrated by the epithelial bodies, which are small masses of compact cells resulting from a local epithelial growth, and penetrated by blood-vessels (sinusoids)-this type includes the parathyroid, nodulus thymicus, and postbranchial body.

The first gill-pouch becomes the Eustachian tube, the blind distal end being expanded into the tympanum.

The second pouch is partly obliterated, but its ventral part is converted into the tonsil.

The third pouch forms an epithelial body, the nodulus thymicus (Fig. I94, Nod) and its ventral cæcal prolongation is converted into the thymus. Its epithelium is said also to produce a parathyroid.

The fourth pouches give rise to a pair of parathyroids and to the post-branchial bodies, which develop from the ventral prolongations of the pouches.

The thyroid gland begins as a median evagination of the entoderm on the rentral side of the pharynx. It starts very early (human embryo of $3 \mathrm{~mm}$.). The blind end of the evagination becomes first bilobed, then branching - the branches are the anlages of the adult follicles. The duct of the gland is soon obliterated, but its point of origin is often permanently marked by the foramen crecum at the back of the tongue.

\section{The Yolk-sac.}

General Morphology. - The yolk-sac is the container of the nutritive yolk destined to be assimilated by the embryo. The principal factor in its morphological constitution is the entoderm, which, after the differentiation of the definitive germ-layers, contains nearly all of the yolk material. In the primitive vertebrates, as exemplified by the marsipobranchs, ganoids, dipnoi, and amphibia, we find this yolk material lodged in the walls of the primitive digestive tract. It is situated

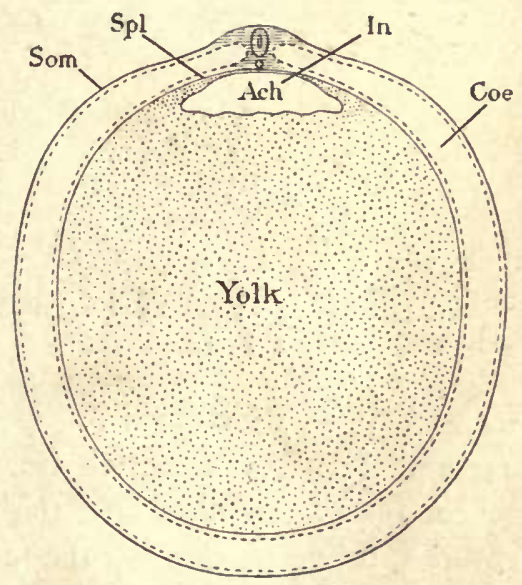

Fig. 29.-Diagramiatic Section of THE YolK OF a HeN'S Egg at aN EARLY STAGE TO SHOW THE RELATION of the Primitive Entodermal Cavity, Ach.

Cœ, Cœlom. In, Intestinal cavity. Som, Somatopleure. $S p l$, Splanchnopleure. chiefly on the ventral side of this tract and extends from the point where the heart is formed toward the tail of the embryo to the point where the allantois is formed. In other words, it is situated in a region-corresponding to the territory of the future abdominal cavity. In the primitive types just referred to, the yolk-bearing entoderm becomes divided into distinct cells which form a large mass. The condition may be understood from figure 44 , which represents a transverse section of the early stage of an axolotl embryo. The cavity of the entodermal canal (digestive tract) is small. It is bounded on its dorsal side by a single layer of cells distinctly epithelial in their development, and on the ventral side by a great mass 
of rounded cells heavily laden with yolk-granules, and containing conspicuously large nuclei. These large nuclei differ by their size and minute structure very much from the other nuclei in the embryo. The corresponding nuclei in higher animals are sometimes called parablast nuclei. Outside of the entoderm comes the second portion of the yolk-sac, the splanchnic leaf of the mesoderm. If we imagine the amount of yolk to be gradually increased, so that it would appear more distinct from the embryo proper, we should then apply to it the term extraembryonic. The yolk-sac of the higher forms differs from that of the lower forms only by its size, as is illustrated by figure 29 , which represents a diagrammatic transverse section of an early stage of the chick, before the formation of the amnion has begun. The essential relations may be seen by comparing figures 29 ,

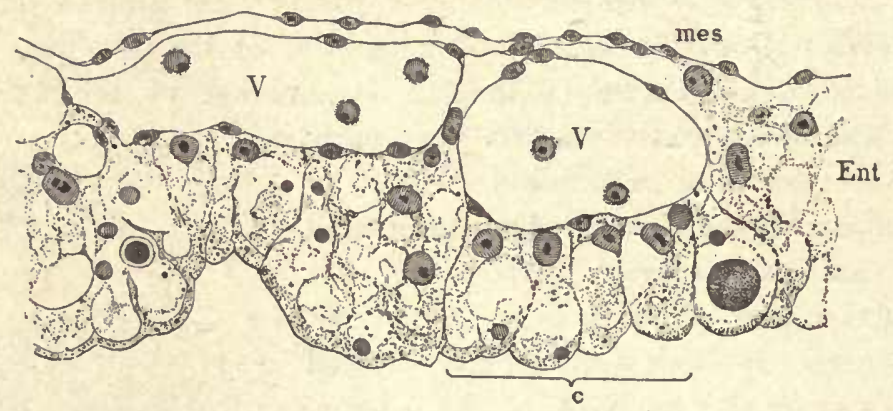

Fig. 30.-Wall of the Yolk-sac in the Region of the Area Opaca of a Chick of the Second Day. Mes, Mesoderm. V,V, Blood-vessels, containing a few young blood-cells. Ent, Entoderm. c , Four distinctly shown entodermal cells.

442 and 45. As shown in the section (Fig. 29), the yolk-sac, if we may so call it, is completely enclosed by the somatopleure of the embryo, and in the amniote embryo the condition is the same. The yolk-sac is surrounded by the somatopleure, which, however, in the amniota we call extra-embryonic. The extraembryonic somatopleure around the yolk-sac is called in birds the membrana serosa, and in mammals the chorion.

In amniota we can distinguish in the entoderm of the embryo, or yolk-sac, three distinct regions. The first of these includes the whole of the entoderm of the embryo and a certain territory around it. In this region, after the earliest stages are passed, the entoderm is found to be a very thin layer and to contain very few yolk-granules, and such few as it contains are small. This portion of the entoderm, therefore, seems translucent, an appearance which can easily be noted with the naked eye, and which has led to the name area pellucida, which has long been applied to this region. The region all around the area pellucida appears in the fresh specimen darker, and this is called the area opaca, the second region. The entoderm in this part consists of columnar cells (Fig. 30, c, and Fig. 3I). In the chick the cells are high cylinder cells of somewhat irregular shape, containing a loose network of granular protoplasm. The lower ends of the cells are rounded 
and projecting, and have a well-marked border of dense protoplasm. The nuclei are variable in size, but for the most part large, often three or four times greater in diameter than the neighboring mesodermic nuclei. They usually have one, sometimes two, conspicuous nucleoli. The nuclei always lie at the upper or basal ends of the cells, chiefly near one side of the cell. The cells contain yolk-grains which appear to be undergoing resorption. Toward the area pellucida the cells are smaller, the network of protoplasm closer, and the yolk-grains are either absent altogether or, if present, small in size and few in number. The transition to the thin entoderm of the area pellucida is quite abrupt. In the opposite direction the area opaca passes gradually, by changing its structure, into the general mass of the yolk, or area vitellina, the third of the regions of the yolk-sac, so called because it contains the bulk of the yolk material. The transition of the area opaca into the

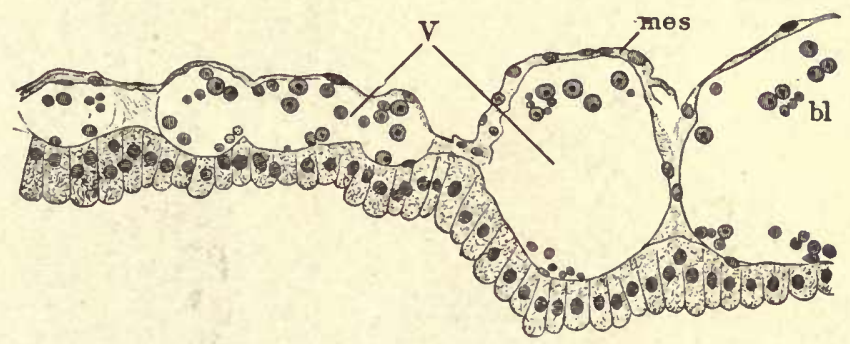

Fig. 31.-Wall of the YolK-Sac in the Region of the Area Opaca of a Rabbit Embryo of Thirteen Days. $V$, Blood-vessels containing young red blood-cells, $b l$. mes, Mesoderm.

area vitellina is marked by a considerable accumulation of cells which are arising from the yolk. This accumulation of cells is called the germinal wall. It is the connecting-link between the epithelium on the dorsal side of the entodermal cavity and the yolk or area vitellina, which forms the, ventral boundary of the cavity. If we follow successively the stages, we find that the area pellucida grows at the expense of the area opaca, and the area opaca at the expense of the area vitellina. These facts are to be interpreted as phases in the process of the assimilation of the nutritive yolk. The thin cells of the area pellucida are those in which the absorption of the yolk has been completed. The larger cells of the opaca are those in which the assimilation is going on, and it can be easily seen that it is most advanced in those cells which are nearest the embryo and least advanced in those cells which are nearest to the germinal wall. In mammals the area pellucida is well marked and resembles that of birds. The area opaca has well-defined cylinder cells (Fig. 3I) which have rounded ends, but are much smaller than in birds and contain very little yolk material. Cells of this character extend over also what we should call the area vitellina, which does not present the special features which it has in birds, for the reason that the yolk in mammals is so small in amount and the yolk-sac, therefore, is hollow. Later on the cells pass through degenerative changes, which need to be more exactly studied. In man the degen- 
erative change in the cells of the yolk-sac takes place very early. The mesoderm of the yolk-sac is at first a thin layer. Very early there appears an angioblast, or the anlage of the first blood-vessels and blood. In all cases in which the process has been accurately followed the angioblast makes its first appearance in the region of the area opaca, where it forms a network of primitive blood-vessels close against the surface of the yolk. The region occupied by these blood-vessels is called the area vasculosa. Its boundary in the direction away from the embryo is everywhere well defined. Gradually the development of blood-vessels progresses from the region of the area opaca into the region of the area pellucida and extends into the body of the embryo. We even have the embryo almost completely sur-

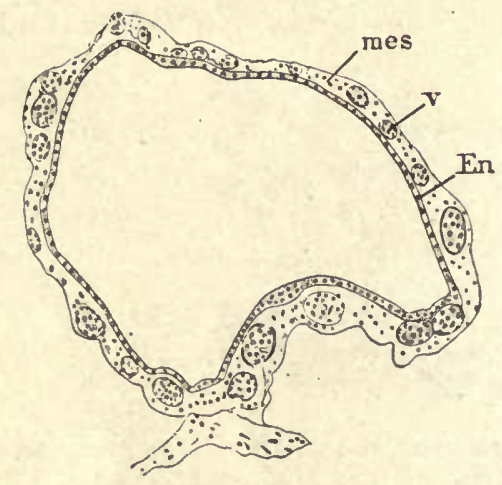

Fig. 32.- Section of the YolK-SAC of a Young Human Embryo.

En, Entoderm. mes, Mesoderm. v, Blood-vessels. - (After Keibel.)

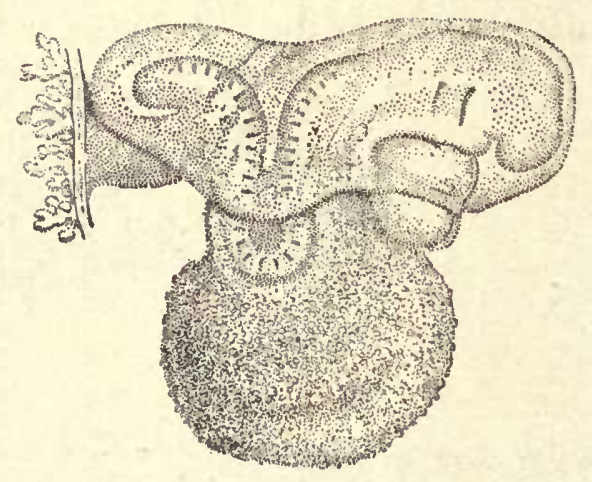

Fig. 33.- Human Embryo, 2.15 mar. Long.(After W. His.)

rounded by a region of extra-embryonic blood-vessels - the definitive area vasculosa. Now, it will be remembered that the area opaca is the territory in which the entodermal cells are actively assimilating the yolk, and we must believe that the blood-vessels which are thus early developed in close contact with the cells of this area are destined to take up food material digested by the entodermal cells and carry it to the embryo. Hence we interpret the early development of the extraembryonic vessels as due to physiological necessities.

The mesoderm at first forms a very thin layer over the angioblast. It next thickens by the multiplication of its cells, and we can then distinguish in it both the outer mesothelium and the inner mesenchyma. The mesothelium is the permanent external cover of the yolk-sac. The mesenchyma grows in between the primitive blood-vessels, and finally penetrates, at least in part, between the bloodvessels and the entoderm of the yolk-sac, a condition which is reached very early in the human embryo (Fig. 32).

The human yolk-sac is characterized by its small size and by the precocious expansion of the area vasculosa, so that in the very earliest stage known to us by 
observation blood-vessels are found over the entire sac. At the beginning of the third week the diameter of the yolk-sac is about equal to the length of the embryo (Fig. 25). By the end of the third week the sac has become distinctly pearshaped, its narrower pointed end being that by which it is connected with the intestinal canal of the embryo (Figs. 33, 34). The sac continues growing, up to the end of the fourth week, after which it enlarges very slightly, if at all. Its diameter is only from 7 to II $\mathrm{mm}$. It is then a pear-shaped vesicle attached by a long stalk to the intestine, the stalk having been formed by the lengthening of the neck of the yolk-sac. The cavity of the stalk early becomes obliterated and the entoderm in the stalk disappears altogether.

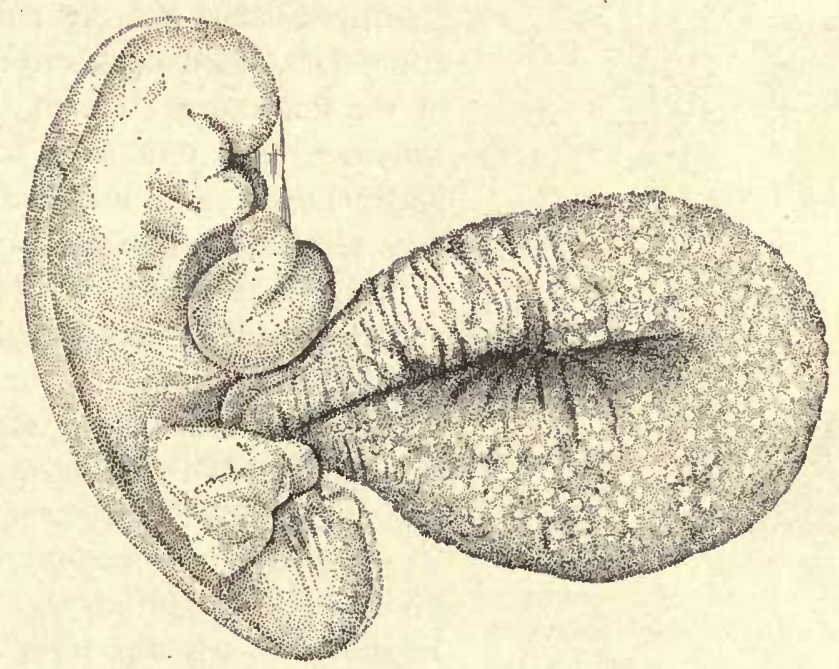

Fig. 34.-Human Embryo of 2.6 MM.-(After W. His.)

\section{The Origin of the Nervous System.}

It will be remembered that the ectoderm of the embryonic shield has at first a considerable thickness, for it consists of cuboidal or low cylindrical epithelial cells. The stage which follows next after the appearance of the primitive axis is characterized by the gradual thinning out of the ectoderm over the peripheral portions of the shield, while in the neighborhood of the axial line the full thickness of the outer germ-layer is not only retained, but is actually increased. For a time there is a gradual passage between thicker and thinner parts, but as development progresses the demarcation rapidly becomes sharper. By these steps the differentiation of the anlage of the central nervous system is accomplished. The thicker central portion of the ectoderm constitutes the medullary plate, which begins to appear shortly after the formation of the primitive streak. It extends over the primitive axis, the primitive knot, and the anterior end of the primitive streak (Fig. 35, Md), and also extends some distance to the right and left of the axial line. It is rounded 
in front, also behind, where, however, it gradually fades out. At the same time that the medullary plate is being thus differentiated, the central portionbe comes depressed, making the conspicuous furrow, $m d . F$, which begins just in front of the primitive knot and extends nearly to the anterior edge of the medullary plate. This axial depression is known as the dorsal furrow. Its appearance is shown in crosssection as illustrated by figure $36, f$. The furrow is narrow and deep. Its upper edge is rounded or curving. By the formation of the furrow the ectoderm of the

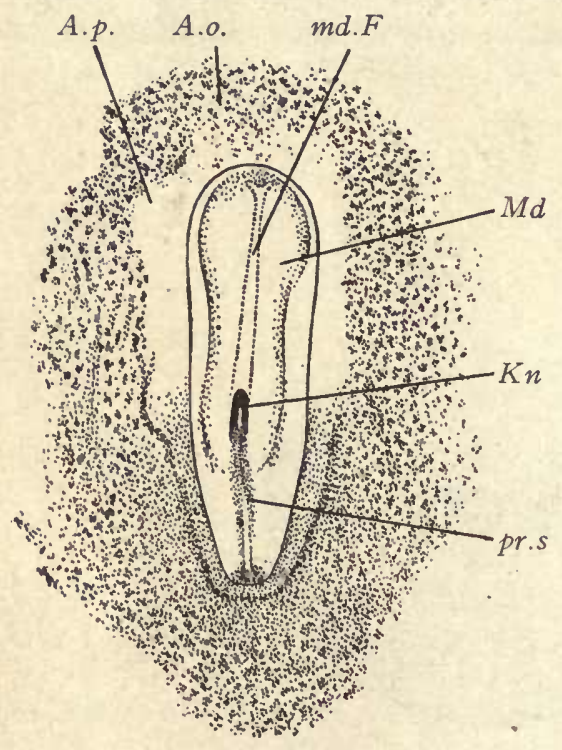

Fig. 35.-SURface View OF the EMbryonic Shield of a Dog Embryo, with Medullary Plate.

A.o, Area opaca. A.p, Area pellucida. $K n$, Hensen's knot. $M d$, Medullary plate. md.F, Medullary furrow. pr.s, Primitive streak. $\mathrm{X}$ I5 diams.

upon the territory of the primitive streak until this latter is obliterated.

The Medullary Groove.-Almost or quite as soon as the medullary plate is formed its lateral portions begin to arise on each side, so that the two halves of the plate together form a broad open trough known as the medullary groove, into which, of course, the dorsal groove is merged, so that it no longer can be recognized (compare Figs. 22 and I47). While the groove is being formed the medullary plate increases considerably in thickness. The nuclei multiply rapidly and lie irregularly scattered at various heights. The ectoderm alongside the medullary plate or groove thins out still further. The development is most rapid at a point corresponding to the posterior region of the future head. The farther from this point we go, the less advanced do we find the formation of the groove, 
so that at a certain stage there is a well-marked medullary groove in the cephalic region, the medullary plate behind that, and the primitive streak at the hind end of the embryo. But when the streak has disappeared, the medullary groove is found to extend the entire length of the embryo. Owing to this peculiarity, it is possible in a single embryo to follow all the principal stages of the formation of the medullary groove by the examination of a series of transverse sections. Such a stage is found in the rabbit at nine days, or in the chick at from thirty to forty hours of normal incubation (Figs. 129, 130, and I47).

The Medullary Canal.-The medullary groove gradually deepens, its sides rising higher and higher and arching more and more toward one another until the edges meet and coalesce, thus changing the groove into a tube - the medullary canal (Figs. 37 and $38, M d)$. The closure of the groove occurs in the cervical region first, and spreads from there in both directions. As the closure progresses forward it completes the canal in the region of the head. It occurs in such a manner that there is a very small opening, which is the last point to close. This opening seems to be a fixed point, occupying always the same relative position in all vertebrates. It is called the anterior neuropore. At this time the caudal end of the medullary groove may be still

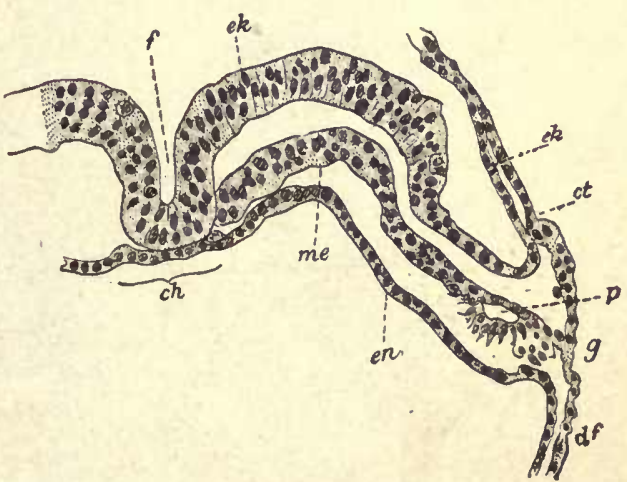

Fig. 36.-Cross-section of a Human Embryo of I. 54 MM.

$f$, Dorsal furrow. $e k$, Ectoderm. $c t$, Somatic mesoderm. $p$, Beginning of the embryonic cœlom. $g$, Junction of the extra-embryonic somatic and splanchnic mesoderm. $d f$, Splanchnic mesoderm. en, Entoderm. me, Mesoderm. ch, Notochord.-(After Count Spee.) open widely, forming the so-called rhom-

boidal sinus, compare figures $\mathrm{I} 29$ and $\mathrm{I} 3 \mathrm{O}$, and it is the last portion to close. Of the entire length of the primitive canal, about one half is the anlage of the brain, while the other half is the anlage of the spinal cord. In the subsequent development of the brain the transverse expansion of the canal is most conspicuous, while in the development of the spinal cord the elongation of the canal predominates. The dilatation of the brain begins very early.

The medullary canal produces the entire central nervous system. Some of the cells from its walls migrate out of the wall itself on either side. These cells produce the ganglia.

\section{The Structure of the Medullary Canal.}

When the medullary canal is first formed, it tends to present a rounded outline in transverse section. But its lateral walls being thicker than the wall on the dorsal and ventral sides of the canal, the internal cavity appears somewhat flattened (Fig. 37). On its ventral side it lies against the notochord. On its dorsal surface 


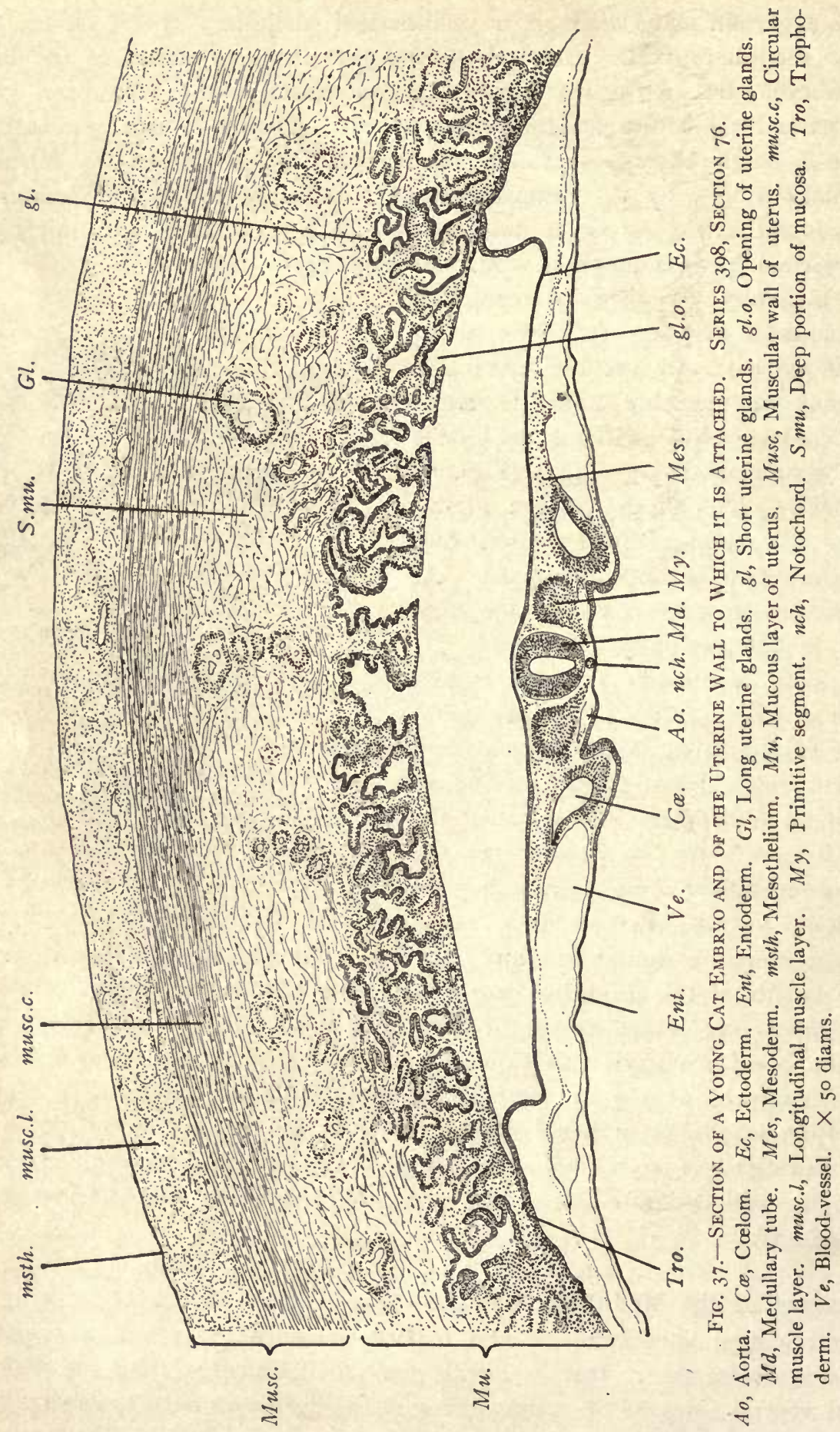


it is in contact with the overlying ectoderm, from which it has, however, completely separated, and it causes the overlying ectoderm to rise up somewhat. Its sides are in contact with the mesoderm, which is there developing into the primitive segments, page 84 . The nuclei in the wall of the canal are very numerous, oval in form, and usually with a single nucleolus. The nuclei are placed in the radial lines. For some time after the canal has become closed the nuclei multiply very rapidly by indirect division, but all of the mitotic figures are found close to the inner surface of the canal, which surface, it will be remembered, corresponds to the original outer surface of the ectoderm.

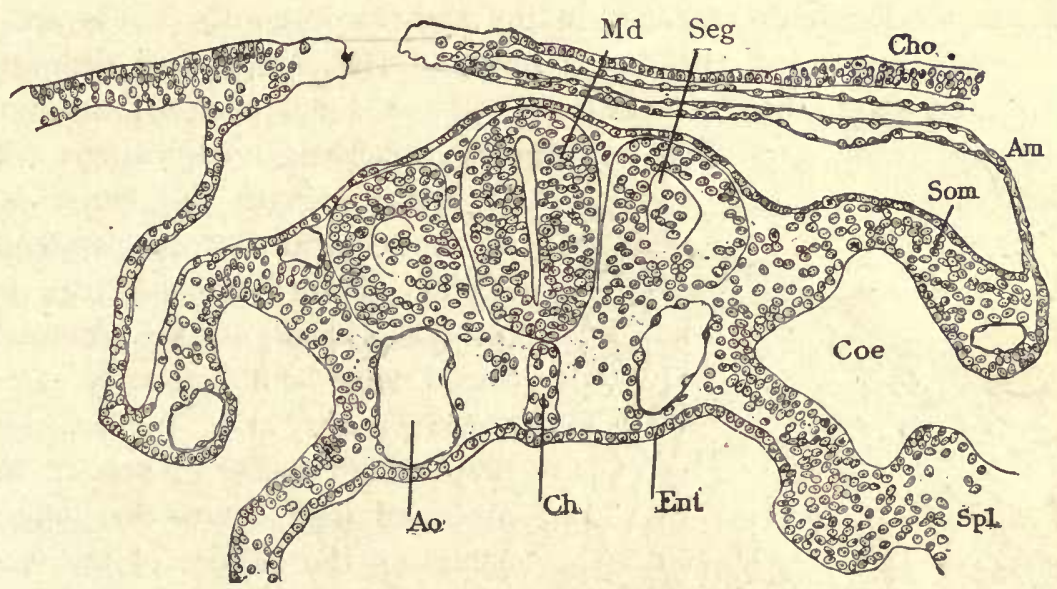

Fig. 38. - Transverse Section of a Rabbit Embryo of Eight Days and Two Hours.

$M d$, Medullary canal. Seg, Primitive segments. Cho, Chorion. Am, Amnion. Som, Somatopleure. Ce, Cœlom. Spl, Splanchnopleure. Ent, Entoderm. Ch, Notochord. Ao, Aorta.

The differentiation of the brain and spinal cord is indicated even during the stage of the medullary groove. The extreme anterior end of the groove is found to widen out so as to produce a pair of lateral expansions. As development progresses and the canal closes, these expansions become more marked and are themselves, of course, also closed over, so that when the canal is completed they appear as lateral diverticula or evaginations of the tube, which are known as the primary optic vesicles (Figs. I29 and I30). While the vesicles are developing the medullary tube expands in diameter throughout its cranial or anterior half without any noticeable change in the general histological structure of its walls. Very soon the expansion becomes unequal, and the inequalities are such that they produce three dilatations, which are known as the three primary cerebral vesicles (Fig. I3I). The first vesicle is in the region of the optic outgrowth, the second is just behind this, and the third is as long as the first and second combined and merges into the spinal cord. At the time these vesicles become recognizable they occupy about half the entire length of the medullary tube. Between the first and second vesicles there is a con- 
striction, and one also between the second and the third. The three vesicles are the anlages, respectively, of the fore-brain, mid-brain, and hind-brain.

In the region of the spinal cord the medullary tube soon becomes somewhat flattened from side to side, and therefore acquires a characteristic oval configuration as seen in cross-section (Fig. 38). We can now recognize in the cross-sections four regions: first, the two thick sides; second, in the median dorsal line the thin portion which we call the deck-plate, and in the median ventral line the thin portion which we call the floor-plate. Later on, each lateral portion becomes subdivided into two longitudinal bands, known as the zones of $H i$, and distinguished from one another as the dorsal and ventral zones (Fig. I16, D.Z, V.Z). After this stage there are six longitudinal zones in the embryonic cord. These are, first, the deck-plate; second and third, the dorsal zones of His; fourth and fifth, the ventral zones of His; and sixth, the floor-plate. These six zones also appear in the region of the brain, where, however, they undergo characteristic modifications. The zones

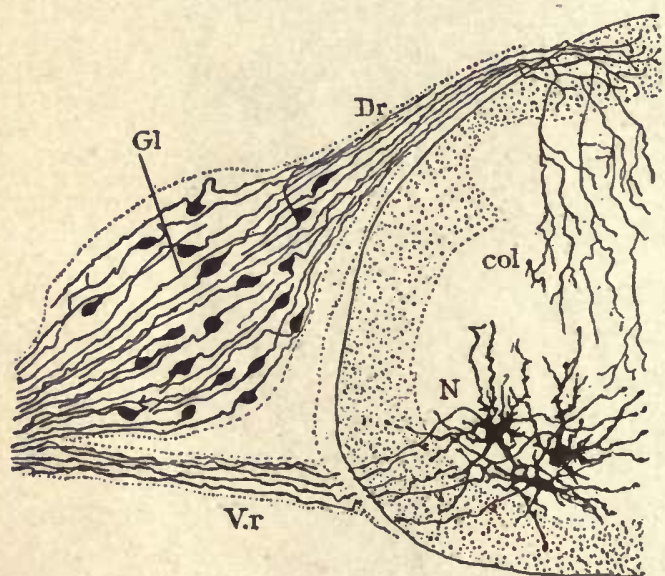

Fig. 39.-Transverse Section of the Dorsal Cord and Ganglion of a Chick of Nine Days.

V.r, Anterior root. Dr, Posterior root. Gl, Ganglion of dorsal root. col, Collateral, with its branches. $N$, Medullary neuroblasts with dendrites and axiscylinder. (The drawing is from a Golgi preparation. No sheath or neuroglia cells are represented.)-(After Cajal.)

of His dominate the entire morphology of the central nervous system, because all the sensory nerves enter the central nervous system at the lower edge of the dorsal zone and primarily ramify in the dorsal zone, and, further, because all efferent nerve-fibers arise in the ventral zone and pass out to the body at certain points on the surface of the ventral zone.

\section{Origin of Nerves.}

The essential constituents of a nerve are the neuraxons, each neuraxon being the prolongation of a nerve cell. In addition, the neuraxons are usually surrounded by medullary sheaths. The young cells from which the neuraxons grow out are called neuroblasts. The olfactory nerve has a special history (compare page 76). All the other nerves are produced by neuroblasts, which are developed from the cells of the medullary tube.

Shortly after the medullary groove has closed to form a tube, a number of cells migrate from the medullary wall, just at the junction of the dorsal zone and the deck-plate, and in sufficient numbers to form a longitudinal band, which is known as the ganglionic crest, which soon, however, breaks up into separate masses, the ganglia, which develop symmetrically. Some of the cells of each ganglion become neuroblasts (Fig. 39, Gl), each of which forms a centripetal process which 
enters the dorsal zone of the medullary tube, and a centrifugal process which passes downward to join the ventral root. Other ganglionic cells are converted into the medullary sheaths of the nerve-fibers, and still others migrate along the nervefibers and give rise to the peripheral or sympathetic ganglia.

The cells which are retained permanently in the medullary wall differentiate themselves into two classes: first, the so-called spongioblasts, which become the neuroglia of the adult, and second, the neuroblasts (Fig. 39, $N$ ). Each medullary neuroblast produces a single neuraxon and several dendrites, although a small number in certain positions remain always without dendrites. Most medullary neuraxons are distributed within the spinal cord or brain, but some of those, developed from cells in the ventral zone only, leave the medullary wall to produce the ventral (Fig. $39, V . r)$ and lateral nerve-roots. Ventral roots constitute the third, sixth, and twelfth cephalic nerves, and enter into the composition of all the spinal nerves. Lateral roots form part of the fifth, seventh, ninth, and tenth nerves, and the whole of the eleventh cephalic nerve, but probably take no part in the formation of any true spinal nerve.

The ganglionic crest of the chick is figured and described in Chapter V. The ganglia, roots, and nerves of the pig embryo are figured and described in Chapter VI.

\section{The Spinal Cord and Brain.}

The medullary tube, after giving off the cells which form the neural crest, becomes the definite anlage of the spinal cord and brain. The differentiation of the brain begins very early, and is marked by an enlargement of the medullary tube in the region of the future head (compare Fig. I3I). As seen there, the brain takes up nearly half the entire length of the medullary tube. The widening of the anterior portion of the medullary canal is not uniform, but tripartite. The brain is divided by two narrower parts into three wider divisions, which are termed the primary cerebral vesicles, and are named in their order-fore-brain, midbrain, and hind-brain. The fore-brain widens very rapidly so as to form two lateral projections, the optic vesicles (Fig. I3I, op.V). The division between the mid-brain and the hind-brain is at first indistinct, but soon becomes sharply marked off. The hind-brain then appears about as long as the other two vesicles combined, and tapers down toward the spinal cord, into which it merges without demarcation.

The walls of the medullary tube acquire throughout certain fundamental characteristics, which are best studied in transverse sections. The side walls become thickened, but the median, ventral, and median dorsal portions remain thin (Figs. $\left.\mathrm{I}_{57}, \mathrm{I}_{5} 8, S p . c\right)$ : The upper thin part is called the deck-plate; the lower thin part, the floor-plate. Soon each lateral wall is divided into two longitudinal bands, designated, respectively, the dorsal and the ventral zone (Fig. II6, D.Z, V.Z). In young embryos, as shown in the figure just cited, the two zones are separated from one another by an internal notch. Their morphological characteristics depend 
upon their relations to the nerve-roots. In the ventral zone are lodged all the neuroblasts which produce efferent nerve-fibers, which fibers constitute the ventral nerve-roots in the region of the spinal cord (Fig. 39), and in the region of the brain and the medulla oblongata produce both lateral and ventral roots. Neuroblasts are also differentiated in the dorsal zone, but the neuraxons which they send out are confined in their distribution to the central nervous' system itself, and never share in the formation of nerve-roots. The dorsal zone is further characterized by the fact that the afferent or sensory fibers from the ganglia enter its lower edge and have their first distribution within the dorsal zone. The stratification of the thickened portions of the medullary tube begins early. It is initiated by the appearance of a thin superficial layer, the ectoglia (Fig. 221, Ec.gl), which contains no nuclei. The thick nucleated portion of the medullary wall changes in appearance as the neuroblasts are differentiated. This occurs in such a way that there is always a layer of relatively undifferentiated cells next the cavity of the tube. This is known as the ependyma layer (Fig. I2I, Epen). It persists throughout life, remaining thin, and never containing nerve-cells. The layer between the ependyma and the ectoglia is called the gray layer, or cinerea (cin). This layer grows very rapidly, and the enlargement of the spinal cord and brain depends chiefly upon the expansion of the cinerea.

The three cerebral vesicles pass through numerous modifications in their form and cellular structure, yet their primary morphological characters are never obliterated. The brain always has a central cavity, the boundary walls of which constitute the organ. The fore-brain produces from its dorsal zone two lateral hollow outgrowths, the cerebral hemispheres, the cavities of which constitute the lateral ventricles of the adult. The median cavity of the fore-brain is the third ventricle; the communication between the third ventricle and the lateral ventricle is the foramen of Monro. The cavity of the mid-brain is always small, and its walls are greatly thickened; it is termed the iter in the adult. The cavity of the hind-brain is much enlarged and becomes the fourth ventricle. The part of the fore-brain from which the hemispheres arise lies farthest cephalad, and is termed the telencephalon. It is marked off on the dorsal side by a transverse fold, the velum transversum, which projects inward. The part of the fore-brain caudad from the velum is called the diencephalon. In the region of the fourth ventricle we can observe that the cerebellum and the pons arise from the region adjoining the midbrain. This region is called the metencephalon, and the part caudad from it-out of which the medulla oblongata is differentiated-is called the myelencephalon. The narrow connection between the mid-brain and the hind-brain is termed the isthmus. The following table indicates the relations of these embryonic divisions to the adult parts. In Chapter VI the essential facts of brain development are illustrated and described. 
TABLE OF BRAIN DEVELOPMENT.

I. Fore-brain $\ldots \ldots \ldots \ldots \ldots \ldots \ldots \ldots$.

(Lateral and third ventricles)

$1+2$
Telencephalon

Diencephalon

Mesencephalon

(Iter)

3. Hind-brain

(Fourth ventricle)
Optic vesicles.

Hemispheres.

Olfactory bulb.

Corpus striatum.

Lamina terminalis.

Infundibular gland.

Epiphysis.

Thalamus.

Tuber cinereum.

Pars mammilaris.

Corpora quadrigemina.

Cerebral peduncles.

Isthmus.

Cerebellum.

Pons.

Myelencephalon

Medulla oblongata.

The spinal cord develops in an essentially uniform manner except at its caudal extremity, the development of which is arrested before differentiation sets in. The undifferentiated extremity becomes the filum terminale of the adult. Typically, the three primary layers, ectoglia, cinerea, and ependyma, are early differentiated. The ectoglia increases in thickness and receives many nerve-fibers, which run for the most part lengthwise, and is thus transformed into the white matter of the adult cord. The cinerea becomes the gray matter and gradually assumes the characteristic adult outline in cross-sections (dorsal and ventral horns). The ependymal layer remains thin, its cells contributing to the formation of the neuroglia frame-work. During the growth of the cord, the ventral zones enlarge rapidly and each projects downward, leaving a notch between them (compare Fig. 213,Sp.c). The notch is closed on

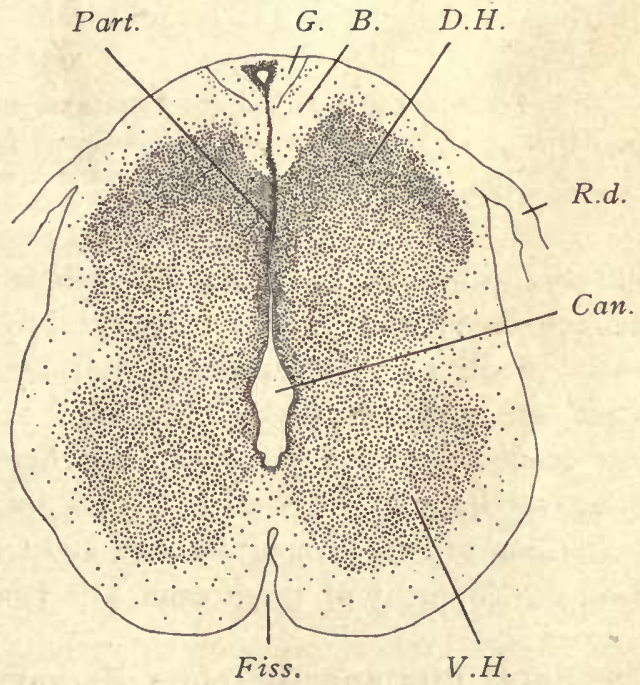

Fig. 40.-Transverse Section of Spinal Cord of a Human EMbryo of 32 MN.

$B$, Burdach's column. Can, Central canal. D.H, Dorsal horn. Fiss, Ventral fissure. $G$, Goll's column. Part, Dorsal partition. R.d, Dorsal nerve root. $\quad V: H$, Ventral horn.

its dorsal side by the thin floor-plate by which the two ventral zones are connected across. The ventral expansions increase and the notch is thus trans- 
formed into the ventral fissure, figure 40, Fiss., filled with mesenchyma or, in the adult, by vascularized connective tissue. Further, the inner surfaces of the dorsal zone meet and unite, thus obliterating the dorsal portion of the original central canal, and forming out of the fused ependymal layers the permanent posterior partition, figure 40 , Part. The central canal of the adult, figure 40, Can, corresponds, therefore, to the ventral part only of the original canal.

\section{Plakodes.}

Plakodes are small circumscribed thickenings of the ectoderm, which contribute to the development of the olfactory, visual, and auditory organs. There are accordingly three pairs of them. They resemble one another very closely in original appearance and their early history. A plakode, figure $4 \mathrm{r}, P l k$, is a rounded area of $\rightarrow$

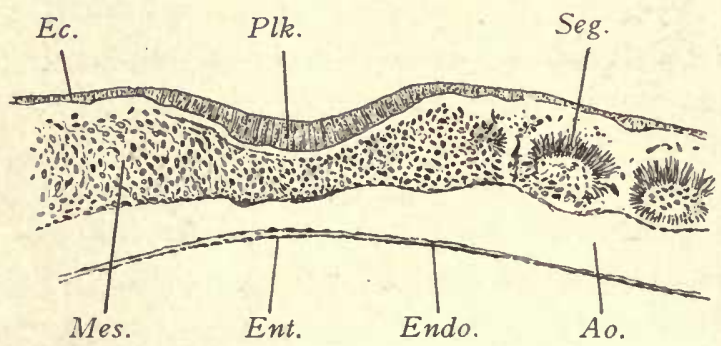

Fig. 4i.-Chick Embryo of Thirteen Segments. Sagittal Series 1452, Section 52.

Ao, Aorta. Ec, Ectoderm. Endo, Endothelium. Ent, Entoderm. Mes, Mesoderm. Plk, Auditory plakode. Seg, Mesodermic somite. $\times$ roo diams.

syncytial ectoderm of small dimensions, several times as thick as the surrounding ectoderm, with which it merges by a rapid transition in the diameter of the layer. Soon after its appearance it becomes invaginated. By preserving this condition the olfactory plakode forms the olfactory pit. In other cases the invagination deepens, then closes over, and the vesicle thus formed separates from the overlying ectoderm. The vesicle formed by the visual plakode becomes the lens of the eye. The vesicle formed by the auditory plakode becomes the otocyst. The ultimate development of these organs is considered in the three sections next following.

\section{The Nasal Pits and Olfactory Nerves.}

The olfactory plakodes arise as a symmetrical pair of thickenings underneath the fore-brain. They soon become invaginated, forming two shallow depressions just in front of the mouth, as is well shown in figure 165 . As development progresses, the depressions deepen and remain lined throughout by the thickened ectoderm (Fig. 194, Na). The orifices may be temporarily closed by the coalescence of the epithelium. The olfactory pits acquire a secondary opening into the oral cavity (Fig. 219). By the expansion of this cavity, the nasal chamber proper of the adult 
is produced. The form becomes complicated by the development of the turbinal folds (Fig. 212) and later by the addition of outgrowths from each nasal pit to form the accessory sinuses of the adult nasal cavity. The tissue between the two nasal pits forms the septum (Figs. 220 and 212, Sept). The epithelium of each pit sends a special invagination into the nasal septum to make the gland-like structure known as Jakobson's organ (Fig. 219, Jk.o). It will be seen from the above that the extent of the nasal epithelium becomes very great, but only a small area is concerned in the formation of the olfactory organ proper. This area underlies the olfactory bulb of the fore-brain, and is termed the olfactory epithelium. The cells of this become elongated and acquire boundaries from one another so as to make a cylinder epithelium. Some of the cells become the so-called olfactory cells, which are more or less isolated and separated from one another by the intervening supporting cells. The olfactory cells develop on their free ends a few small projecting hairs, and from their basal ends a single thread-like prolongation, which becomes a fiber of the olfactory nerve. This fiber penetrates the olfactory bulb, and there has its terminal arborization, which enters into special relations with the mitral cells of the bulb. All the fibers of the olfactory nerve arise in this way; hence the nerve-so far as its development is concerned-is unique in vertebrates. It differs permanently from all other nerves in that its fibers never acquire any medullary sheaths, because no cells migrate from the medullary wall or brain into this nerve as they do into all others.

Later, the complete separation of the nasal and oral cavities is accomplished by the development of the palate shelves which grow out from the walls of the oral cavity until they meet in the median line and unite with the lower edge of the nasal septum, as described and illustrated in Chapter VI.

\section{The Eye.}

The eye has a complicated history. The optic nerve and retina arise from the medullary tube (brain). The lens arises from the visual plakode. The remaining structures of the eye are of mesodermal origin.

Optic Vesicles. - The optic vesicles arise very early from the extreme cephalic end of the medullary tube, as two lateral outgrowths (Fig. r3I), each of which soon appears quite as large as the central portion of the medullary tube which produces it (see Fig. I33). The central portion of the tube, however, grows much more rapidly than the optic vesicles in order to form the brain. The distal portion of the optic vesicle expands, and we thus get the condition indicated in figure $\mathrm{r}_{54}, O p$. The vessel may now be said to be stalked. The stalk is the anlage of the optic nerve. The larger distal portion of the optic vesicle gives rise to the retina. The optic vesicle comes in contact with the ectoderm, and over the area of contact the visual plakode is differentiated. In the next stage the differentiation between the eyeball and optic nerve is clearly seen (Fig. 154). This is accomplished by modifications in the distal portion of the optic vesicle. Its outer wall becomes invagi- 
nated, producing a cup, the wall of which is necessarily double. The layer next the cavity of the cup increases in thickness and becomes the retina proper. The other layer remains thin and becomes charged with pigment, and is transformed into the so-called pigment layer of the retina. The opening of the cup is filled with the lens (compare also Fig. I7). In accordance with the fact that the optic nerve and retina are derived from the wall of the medullary tube, we find that their differentiation is essentially similar to that of the central nervous system. They develop neuroglia and nerve-cells with neuraxons. It is only by keeping in mind these facts that the histogenesis of the adult structure of the retina can be understood.

Lens.-The visual Plakode, which, it will be remembered, is at first in contact with the wall of the optic vesicle, is early invaginated (Fig. I53, L). The invagination closes over, making the lentic vesicle; this rapidly separates from the overlying ectoderm, which ultimately becomes the corneal epithelium. The wall of the vesicle which is nearest the retina and farthest from the epidermis rapidly thickens and forms the main substance of the lens, at the same time obliterating the cavity of the vesicle.

Mesoderm.-The mesoderm produces the choroid and sclerotic coats of the adult eyeball, the connective tissue of the iris and of the cornea, the lining epithelium of the anterior chamber of the eye, and the muscles which move the eye-ball.

For further details as to the history of the eye see page $33 \mathrm{I}$.

\section{The Otocyst.}

The otocyst arises by the invagination of the auditory plakode (Fig. 4I, Plk) to form the auditory pit "Fig. I52, Ot), which soon closes, forming an epithelial vesicle which quickly loses its connection with the overlying ectoderm. The vesicle or otocyst is the anlage of the membranous labyrinth of the ear. It lies close to the wall of the myelencephalon (Fig. I9I, Ot) between the ninth nerve and the ganglion complex of the seventh-eighth nerves. It becomes pear-shaped, the narrow end pointing dorsally. It soon develops a special prolongation (Fig. 42, D.endo) which extends dorsad near the brain-wall and is known as the ductus endolymphaticus. The ductus persists throughout life.

The ventral end (Coch) of the otocyst begins to elongate very early, and is converted gradually into a very long spiral epithelial tube, the scala media cochlece of the adult. The dorsal summit, S.C, of the otocyst is transformed into the semicircular canals. The middle part of the original vesicle also undergoes remarkable changes of form to produce the utriculus, which opens into the anterior and external semicircular canals; the canalis reuniens, which leads to the cochlea; and the sacculus, a blind pouch on the anterior side of the canalis reuniens.

The entire epithelial labyrinth becomes surrounded by a loose mesenchyma, which again is surrounded by denser tissue which forms the cartilaginous periotic 
capsule. The capsule enters into the formation of the cranium, and when it ossifies forms also the so-called bony labyrinth.

The auditory ganglion fuses very early with the cephalad wall of the otocyst, so that in sections no boundary between the ganglion and the epithelium can be distinguished. The upper part develops into the vestibular, the lower into the cochlear ganglion.

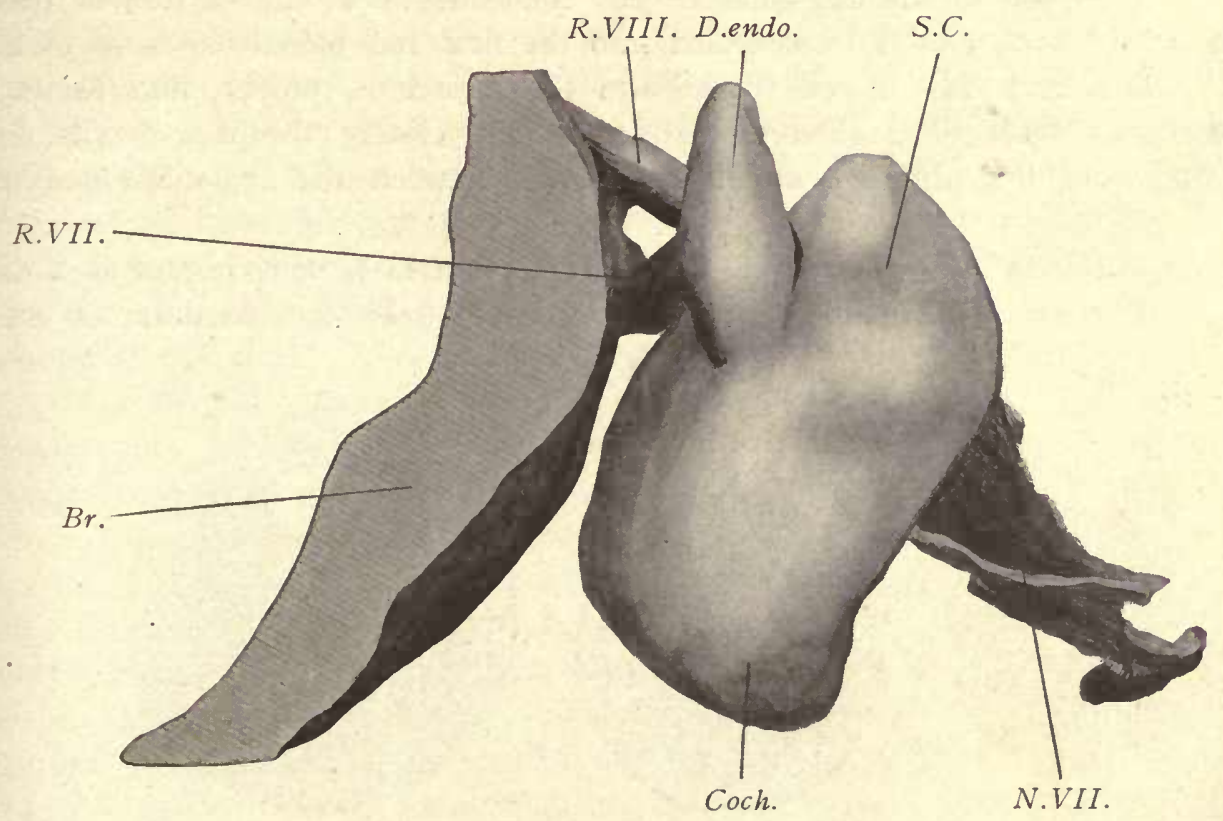

Fig 42.-Pig Embryo of I2 mm. Series 5. Reconstruction in Wax of the Otocyst, with Part of the Brain-wall and the Seventh and Eighth Nerves. By G. C. Coe and W. W. Behlow. View froui THE CAUdAD Side.

$\mathrm{Br}$, Brain-wall. Coch, Cochlear anlage. D.endo, Ductus endolymphaticus. N.VII, Seventh nerve. $R$. $V I I$, Root of seventh nerve. R. VIII, Root of eighth nerve. S.C, Region from which the semicircular canals are formed. $\times$ roo diams.

\section{The Early History of the Mesoderm.}

Concerning the precise origin and early development of the mesoderm authorities are by no means agreed, and in the interpretations offered there has been more of hypothesis than of observation. The most accurate observations have so far been made on the elasmobranchs, lizards, and chick. In these forms the entoderm (or segmenting yolk) in the neighborhood of the primitive streak produces cells which take their place so as to form a layer next to the entoderm. This layer gradually becomes more and more distinct until it can be definitely recognized as a separate layer, the mesoderm. It is probable that a similar process goes on in amphibia and in mammals, so that it is safe to say that the mesoderm probably arises by this process, which we call delamination, in all vertebrates. In its first 
stage the mesoderm has no distinct boundary against the underlying entoderm. It is thickest in the neighborhood of the primitive streak and thins out from that in all directions. It very early comprises two easily recognizable classes of cells. One of these forms a more or less distinct layer next to the yolk, and so distributes itself as to form a network of cavities of which these cells become the boundaries, thus developing the first blood-vessels. The cells which form them constitute the angioblast. A portion of the angioblastic cells comes to lie in the cavities of these primitive blood-vessels and is transformed into the first red blood-corpuscles of the embryo. The second class of cells constitutes the mesoderm proper, and forms a more continuous sheet of undifferentiated, somewhat closely compacted cells, extending out from the primitive streak and lying between the angioblast and the ectoderm.

The Expansion of the Mesoderm.-After the mesoderm is once formed as a distinct layer, it seems to have no longer any connection with the entoderm or ecto-
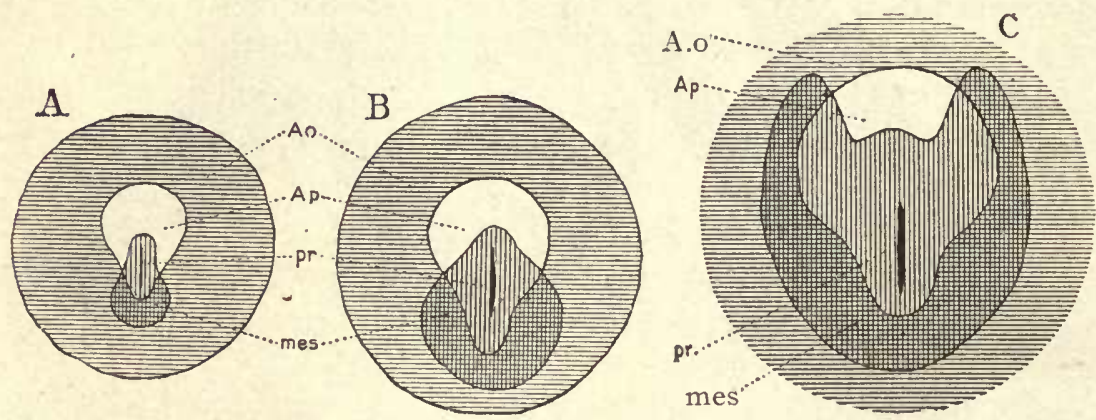

Fig. 43.-Three Diagrams of Embryonic Areas of Chicks to show the Growth of the Mesodern. The mesoderm is indicated by vertical shading, the area opaca by horizontal shading. A.o, Area opaca. A.p, Area pellucida. mes, Mesoderm. pr, Primitive streak.-(After Duval.)

derm, except in the axial line. Its further expansion is due to the proliferation of its own cells. During this early expansion the mesoderm assumes in all amniota a definite and characteristic series of outlines. It is at first pear-shaped (Fig. 43, A), the anterior end being pointed. It extends a short distance only in front of the primitive streak and is widest a little distance behind the area pellucida $(A p)$. (For a description of the area pellucida see Chapter V.) The condition in the chick at about the twentieth hour of incubation is indicated by figure $43, \mathrm{~B}$, drawn on the same scale as A, and at the close of the first day by figure $43, \mathrm{C}$. In the last stage figured it will be noticed that the mesoderm is expanding unequally in front, having sent out two lateral wings which leave a median space between them without mesoderm. These wings continue their growth, and finally meet in front, so that in the anterior part of the area pellucida there is a small tract without any mesoderm, although it is completely enclosed by mesoderm. This tract is the pro-amnion. The 
actual expanding edge of the mesoderm is quite irregular. The regularity shown in figure 43 is entirely diagrammatic.

The extent of the growth of the mesoderm over the extra-embryonic region of the mammalian blastodermic vesicle is very variable. Usually it extends completely around the vesicle, but in some cases, as in the rabbit, only part way (compare page $5^{2}$ ).

The Origin of the Colom.-The next-step in the differentiation of the middle germ-layer is the appearance of two slit-like cavities in it, one on each side. These cavities do not extend across the median line, for when they appear there is no mesoderm in the median line of the embryo. The cœlom is the anlage of the body-cavity, and in part persists in the adult as the pericardial, pleural, and abdominal cavities. Certain parts of its walls share in the production of muscles and of the excretory organs. The complete history of the cœlom is very complex. As the cœlomatic cavities appear, the cells bounding them take on a distinctly epithelial character. This limiting layer is termed the mesothelium.

The earliest phases in the development of the cœlom have been exactly followed only in a very few instances. In these it has been found that numerous fissures appear in the mesoderm and unite themselves so as to form a network of channels which grow, and produce by their fusion the cœlom. The fusion occurs so that two cavities are developed, one on either side, and parallel with the axis of the embryo. As the head of the embryo grows the two cavities grow into its cervical end, following the penetration of the mesoderm, and unite so as to form below the developing pharynx a single median cavity, the anlage of the future pericardial cavity. In the Sauropsida and in many mammals the pericardial coelom merges into two large expansions of the body-cavity which lie just alongside of the head of the embryo and are known as the amnio-cardiac vesicles (Fig. I 3 I, A.c.v). (Compare also the account of the splanchnocele, page 87.)

There are very great variations in the development of the cœlom in mammals. In some cases the cœlom grows so as to appear at an early stage in the body of the embryo (Fig. 37). In other cases it is developed in the entire extra-embryonic region of the blastodermic vesicle before it is developed in the embryo proper. This condition has been observed in primates, including man. It results in the formation of a layer of mesoderm surrounding the yolk-sac, and another layer underlying the extra-embryonic ectoderm, with a wide cœlomate space between the two mesodermic layers. This space we call the extra-embryonic cœlom. These relations are illustrated in figure 45 .

As soon as the colom has appeared the mesoderm is divided into two layers, an outer and an inner. The outer layer is in close contact with the ectoderm. It is called the somatic mesoderm. The inner layer is in close contact with the entoderm; it includes the entire angioblast, there being in early stages no blood-vessels or blood in the somatic mesoderm. The inner layer is called the splanchnic mesoderm. 


\section{Somatopleure and Splanchnopleure.}

The somatic mesoderm, together with the overlying ectoderm, constitutes the somatopleure or primitive body-wall. The splanchnic mesoderm, together with the underlying entoderm, constitutes the splanchnopleure. The somatopleure and splanchnopleure are, to a large degree, the elementary anatomical parts out of which the adult structure is produced. Although they each comprise cells belonging to two germ-layers, they nevertheless develop each almost as a unit, the cells of the two germ-layers entering into intimate co-operation with one another in the differen-

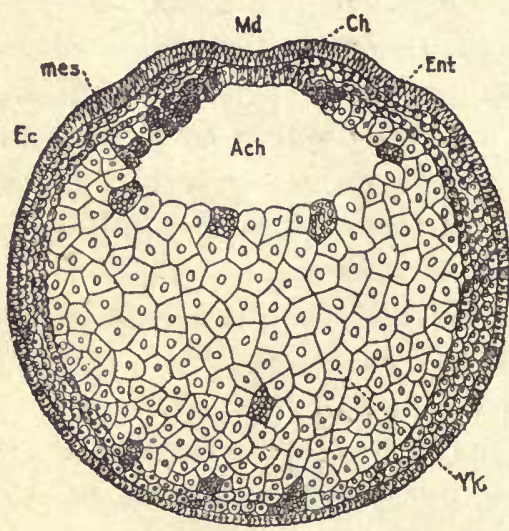

Fig. 44--Transverse Section of an Early Stage of an AXoloti.

Ec, Ectoderm. mes, Mesoderm. $M d$, Medullary groove. $\mathrm{Ch}$, Notochord. Ent, Entoderm. $Y k$, Yolk. Ach, Archenteron or primitive entodermal cavity.-(After Bellonci.) tiation of organs. In both somatopleure and splanchnopleure it is convenient to distinguish two main regions; namely, the embryonic, which enters into the constitution of the embryo proper, and the extra-embryonic, which enters into the formation of the so-called appendages of the embryo, that is to say, of parts which exist during embryonic life, but are lost at the time of birth, and take no share in the permanent body.

In the primitive type of vertebrate development there are no embryonic appendages. This condition is illustrated by figure 44 , which is a transverse section of a young stage of an axolotl. This may be readily compared with a blastodermic vesicle of a mammal, if we imagine the mass of yolk or entoderm reduced to a single layer of cells. We can then easily distinguish the ectoderm and the underlying somatic mesoderm, which together completely enclose the section. The splanchnic mesoderm lies close against the yolk and is separated from the somatic by the intervening coelom.

The general homologies of this primitive type of vertebrate embryos with the type which we find in the amniota may be readily grasped by the aid of the accompanying diagrams (Fig. 45), which are based somewhat on the processes as actually found in the chick. The embryonic structures properly so called are distinguished by shading. The yolk-sac is large and more or less a separate structure from the embryo. It is surrounded by a layer of mesoderm represented by a dotted line. In the direction of the embryo the mesoderm has continued to form part of the wall of the intestinal canal, In; hence we may say that the splanchnopleure forms the wall of the primitive intestinal canal and of the yolk-sac. The yolk-sac represents a lower portion of the splanchnopleure. It can be readily seen that we may compare it with the condition noted in the newt, and have to deal fundamentally with a question of relative proportions. The somatopleure, Som, enters into the formation of the embryo itself, but it also extends beyond. Its disposition be- 
comes complicated in the amniota by the formation of the amnion itself. We shall consider here only what is looked upon as the primitive method of the production of the amnion, and note only that the exact steps of the process are considerably modified in many mammals, in connection with the early modifications which the ovum undergoes in order to secure its attachment to the walls of the uterus (see the section on the trophoderm). The somatopleure forms two folds, one on each side of the embryo. These folds arch up over the back of the embryo. The inner leaf or part of each fold is the anlage of the amnion, $A m$. It consists of a layer of ectoderm next to the embryo, and.a layer of mesoderm, represented by the dotted line,
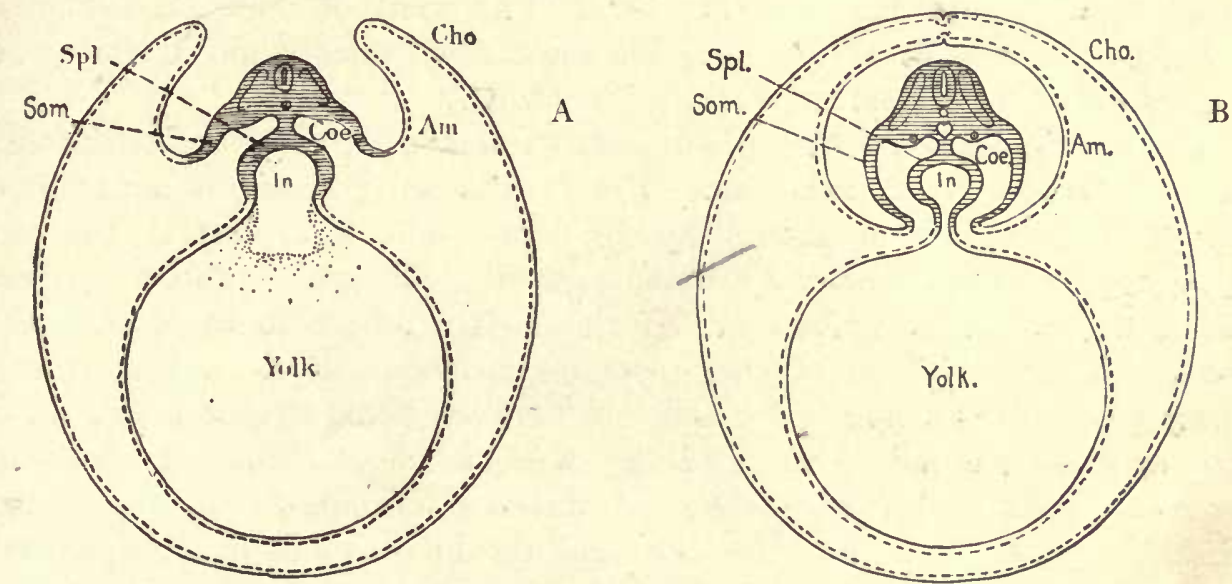

Fig. 45.-Generalized Diagram of an Auniote Vertebrate Embryo.

The first figure shows the condition before, the second after, the separation of the amnion from the chorion. $A m$, Amnion. Cho, Chorion. Ce, Cœlom. In, Intestinal canal. Som, Somatopleure. Spl, Splanchnopleure. Yolk, Yolk-sac.

turned away from the embryo. The remaining portion of the extra-embryonic somatopleure, Cho, extends around both the amnion and yolk-sac, forming a membrane called the chorion, which likewise consists, of course, of ectoderm, which, however, faces away from the embryo, and of mesoderm (dotted line), which is turned toward the embryo. As regards the embryo, therefore, the position of the two germ-layers in the amnion is reversed in the chorion. The two folds continue to grow until they meet above the back of the embryo and unite. The amnion (Fig. 45, Am) has thus become a closed membrane surrounding the embryo, and the chorion, Cho, has become a closed membrane surrounding the amnion, the embryo, and the yolk-sac.

By the processes indicated we have produced an embryo with its three primary appendages - the chorion, amnion, and yolk-sac. To these there is to be added a fourth appendage, the allantois, which also begins its development very early, and arises as a hollow outgrowth from the under side of the caudal end of the embryo and expands into the extra-embryonic colom or space between the yolk-sac and the chorion. 


\section{The Embryonic Cœlom.}

In the body of the embryo proper the colom acquires a very complicated disposition. It forms, first, a series of small cavities alongside of the medullary tube. The walls of these cavities are termed the somites. A pair of somites mark out a primitive segment. It forms, secondly, two large main cavities, which partially unite in later stages on the ventral side of the embryo, the primitive segments lying more on the dorsal side. These two large cœlom spaces constitute the splanchnocele, a term which has reference to the fact that this space surrounds the splanchnic viscera. Finally, it forms a series of so-called head-cavities, of which there are probably always three on each side of the head. The walls of these head-cavities in part produce the muscles of the eye. We must now consider the development of these divisions of the colom in the order indicated.

The Primitive Segments. - A segment consists of a pair of cavities symmetrically placed and bounded by mesothelium. The cavities are portions of the embryonic colom. For convenience of description the term somite is applied to one of the pair of structures which constitute a whole segment. The somites appear very early; the first pair can be recognized in the chick after twenty to twenty-two hours' incubation; in the rabbit, at the beginning of the eighth day. In both cases the medullary groove is still nowhere closed. In amniote embryos, just before the first segment appears, the mesoderm on either side of the axial line is considerably thicker than farther away from it. We can, therefore, distinguish two zones, namely, the thicker segmental zone near the axis, and the thinner, but much wider lateral or parietal zone (Figs. I29, I3I). The first step in the formation of the first segment is a loosening of the cells in the segmental zone, along a narrow transverse line. In the chick this occurs about $0.14 \mathrm{~mm}$. in front of the primitive streak, at a time when only a portion of the medullary groove is formed. Very soon there appears, close by, a second similar transverse loosening 'of the cells. The mesoderm of the segmental zone is thus cleft twice, the mesodermic cells between the two clefts constituting the first somite, which is somewhat cuboidal in form. The first segment appears in what later becomes the occipital region. All further segments are formed successively in a similar manner behind the first, the series growing by additions caudad. The segments differ somewhat one from another in the details of their development. The primitive somites, owing to their form and their proximity to the anlage of the central nervous system, were taken by early embryologists to be the beginnings of the vertebræ, and were, therefore, called the proto-vertebra. This name is still used, although the idea upon which it was based is known to be erroneous, because the primitive segments form much more than the vertebræ.

The association in time of the development of the medullary groove and primitive segments is important. By the formation of the groove the space between the ectoderm and entoderm alongside the groove is increased, and it is this space which gives the mesoderm the opportunity to grow in thickness so as to form the segmental zone next to the medullary groove. 
In the amniota when the somites are first formed they display usually no actual cavity, but we must consider that one is morphologically present, since we can easily observe the line of contact between the opposite walls of the segments. As observed in transverse sections, the somites are seen to become triangular in outline. The base of the triangle extends along the side of the medullary canal; the apex of the triangle lies next to the splanchnocele, and at the point of the triangle the somatic and splanchnic mesoderm separate widely from one another. Very soon the apex of the triangle forms a narrow piece (Fig. 46, N), which is known commonly as the nephrotome or intermediate cell-mass. While the nephrotome is being marked off the proximal portion of the segment enlarges, the cells assume a more distinctly epithelial character (Fig. 46, My), enclosing a considerable space, which, however, is completely filled by a mass of cells, $C$, which arise by a proliferation

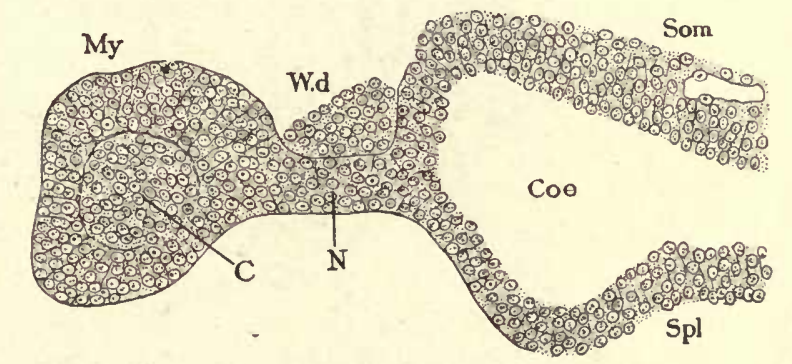

Fig. 46.-Transverse Section of the Mesoderm of a Chick Embryo with about Eighteen Segments. Only the mesoderm of one side has been drawn. The section passes through a recently formed segment. My, Secondary segment. C, Core of the segment. W.d, Wolffian duct. N, Nephrotome. Co, Colom. Som, Somatic mesoderm. Spl, Splanchnic mesoderm. $\times 227$ diams.

of the cells from the lower side of the segment. The line around this mass of cells marking it off from the other wall of the segment indicates the morphological cavity. In the sheep and the chick it has been observed that the cavities of the first four segments can be traced through the nephrotome to the splanchnocele. This represents a primitive condition, one which we find in all the segments of . some of the lower vertebrates. Did we know the development of the amniota only, we should not have been able to identify the cavity of the somite as morphologically a portion of the cœlom. The development in fishes shows conclusively that it must be so regarded.

The Separation of the Nephrotome.-The nephrotome early loses its connection on the one side with the enlarged central portion of the somite, and on the other with the mesodermic walls of the splanchnocele, so that each nephrotome forms a little mass of cells isolated from, but in definite topographical relation to, the other parts of the mesoderm. It may be noted that during these early stages one can always find the anlage of the Wolffian duct on the ectodermal side, and on the entodermal side the anlage of a blood-vessel. Very soon the nephrotome assumes 
a rounded form, and a cavity appears in its interior; it is then often called a segmental vesicle (Fig. 47, Nephr). The exact details of the process by which the nephrotome is separated from the other parts of the middle germ-layer have not yet been carefully studied. Each nephrotome is the anlage of one of the excretory tubules of the Wolffian body. It elongates into a tubule, which takes an S-shape, and extends in the transverse plane of the body. The lateral end of the tubule unites with, and acquires an opening into, the Wolffian duct. The median end expands and produces a nephric corpuscle, with the characteristic glomerulus and capsule.

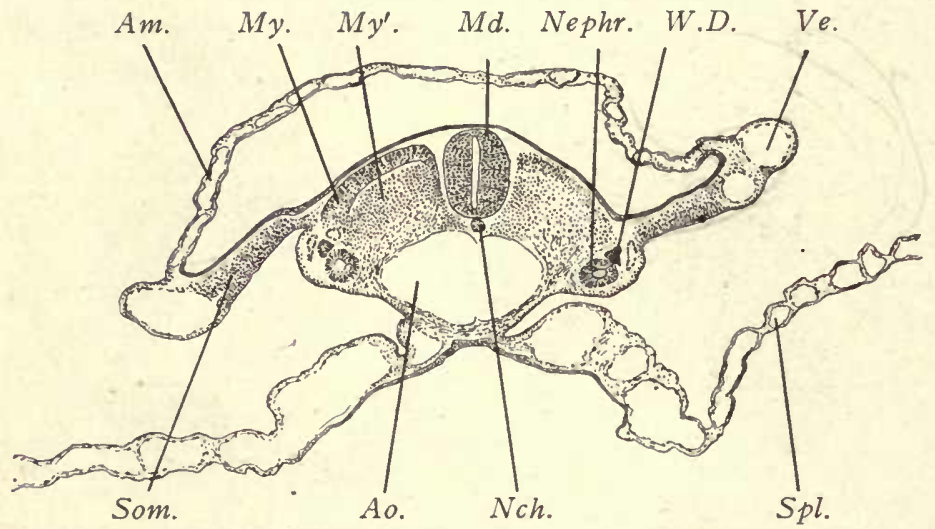

Fig. 47.-Section of a Very Young Cat Embryo. Transverse Series 4i3, Section i8i.

$A m$, Amnion. Ao, Aorta. $M d$, Medullary tube (spinal cord). $M y$, Outer, $M y^{\prime}$, inner wall of primitive segment

Nch, Notochord. Nephr, Nephrotome (segmental vesicle). Som, Somatopleure. Spl, Splanchnopleure.

$V e$, Blood-vessel. W.D, Wolffian duct. $\times 50$ diams.

The portion of the somite which is isolated by the formation of the nephrotome lies, of course, next to the medullary canal. The term primitive segment (as also proto-vertebra) is often applied to this structure as well as to the original somite before the separation of the nephrotome, but it would be better to refer to it as the secondary somite.* The secondary somite, when first formed, appears more or less nearly square in surface views, and triangular in cross-sections. As the medullary canal grows, so does the secondary somite, and it becomes, therefore, somewhat elongated in its dorso-ventral diameter. After this change in its shape we can distinguish in transverse sections of an embryo (Fig. 38) the outer wall, which lies under the ectoderm, and an inner wall, which lies toward the medullary canal and notochord. In the further history of the somite we can distinguish the following steps: first, the production of the dermatome (cutis plate) with the accompanying transformation of a portion of the cells of the inner wall of the-segment into the mesenchyma; next, the production of the true muscle-piate; thirdly, 
the breaking-up of the outer wall of the myotome. These portions are sufficiently described in the practical part, Chapter V.

The Splanchnocele.-The splanchnocele makes its first appearance in the parietal zone of the mesoderm in the manner above described (Figs. $45 \mathrm{~A}$ and B,Ce). It rapidly increases in size, so that a considerable space separates the somatic from the splanchnic mesoderm, as shown in figures 160 and 163 . When it first appears, it is a narrow fissure. It rapidly widens, extends toward the axis until it almost reaches the primitive segments, and also spreads out laterally into the socalled extra-embryonic region. As above stated, the rate and extent of its extraembryonic development vary greatly in different mammals. It develops in birds earlier and acquires distention first in the future cervical region, where it produces the amnio-cardiac vesicles (Fig. I36, A.c.v), in the median portion of whose united cavities the heart is lodged. The splanchnocele of the body proper appears after the primitive segments, and its expansion takes place at first only in the part of the mesoderm next to the primitive segments. Everywhere as the splanchnocele develops the mesodermal cells about it assume gradually more and more distinctly an epithelial character, so that it soon becomes proper to speak of the mesothelium or boundary epithelial wall of the cœlom.

The splanchnocele is also designated by several other names, and is sometimes called simply the body-cavity or somatic cavity. Others term it the ventral calom. By English embryologists it is usually called the pleuro-peritoneal space. Its future subdivisions become early indicated by a transverse ridge of tissue which is known as the septum transversum. This septum is situated at the posterior end of the heart, and is developed to allow the great veins to have access to the heart itself. It is the anlage of the future diaphragm. It separates the cœlom around the heart from that of the abdomen. It is a product of the splanchnopleure, so that it arises upon the ventral side of the colom. We have, as soon as this septum is present, the pericardial cavity on its cephalic side, the abdominal cavity on its caudal, and a small pleural cavity on its dorsal side.

The Crelom of the Head.- No adequate investigation of the early stages of the mesoderm in the head of amniota has yet been made. We know, however, that in the lower vertebrates there appear at least three distinct cavities resembling portions of the true coelom and bounded by epithelial cells, similar to the mesothelium in character. These cavities are generally regarded as portions of the true cœlom, and by many writers have been interpreted as true primitive segments. But this interpretation is not yet beyond doubt. The largest of these is called the mandibular cavity, because it has a prolongation which extends into the mandible of the young embryo. In front of it is the first or premandibular cavity, which is much smaller, and behind it is the third or hyoid cavity, which is intermediate in size between the first and second (Fig. 48). The head-cavities are best known in the elasmobranchs. They have also been found clearly developed in reptiles and certain birds. In mammals no actual cavities have been recorded. There are found 


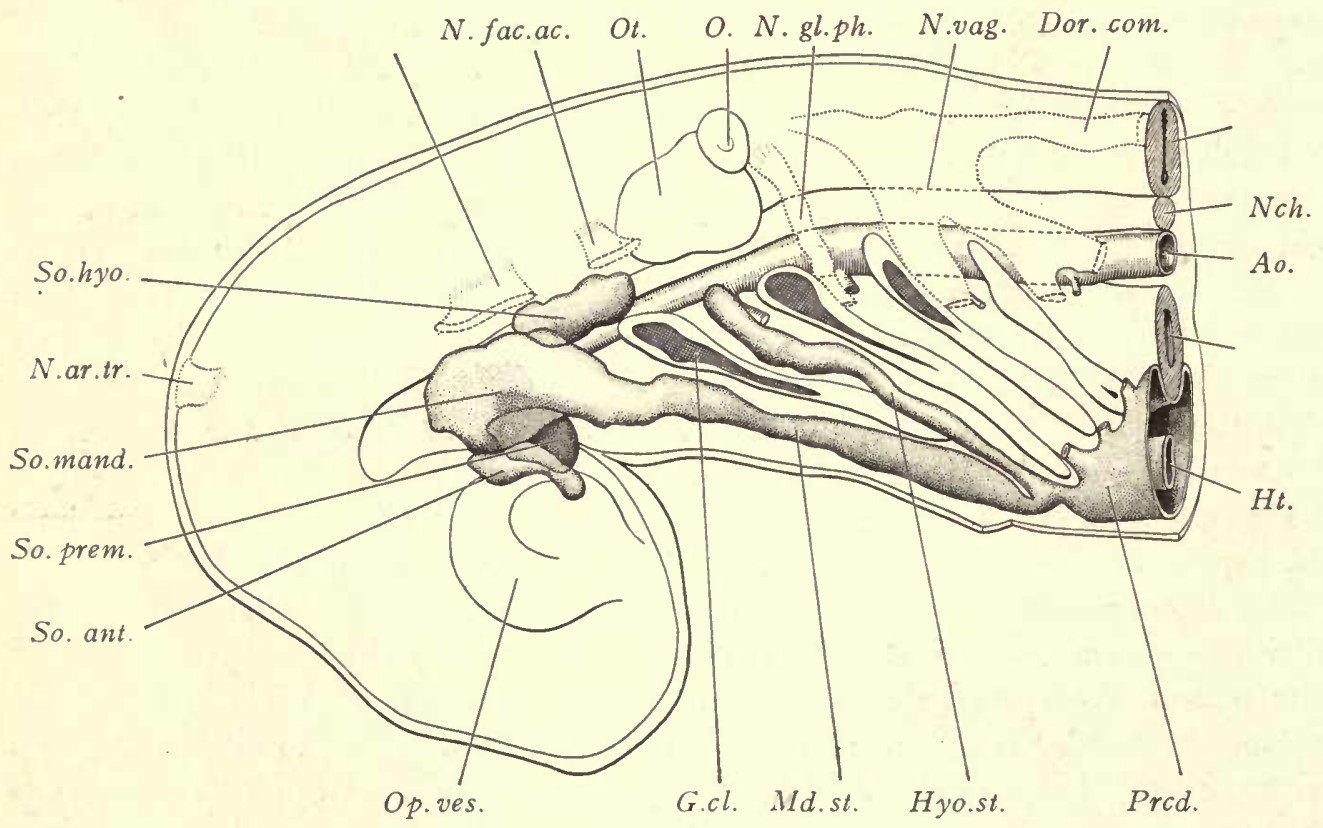

Fig. 48.-SQualus acanthias, 9.0 Mm. Series I495. Reconstruction to show the Head-Cavities.

By R. E. Scammon.

Ao, Dorsal aorta. Dor.com, Dorsal ganglionic commissure. G.cl, First gill-cleft; two others are open and marked by similar shading. Ht, Heart. Hyo. st, Stalk of mesoderm connecting the hyoid cavity with the pericardium. Md. st, Stalk of mesoderm connecting the mandibular cavity with the pericardium. $N c h$, Notochord. $N$. fac, ac, Facial-acoustic nerve trunk. N.gl.ph, Glossopharyngeal nerve. N. vag, Vagus nerve. N. ar. $t r$, Temporary ganglionic mass (Dohrn's "Urtrochlearis"). $O$, Orifice of the otocyst. Op.ves, Optic vesicle. Ot, Otocyst. Prcd, Pericardium. So.ant, Anterior head-cavity, a derivative of the premandibular cavity, and lacking in most animals. So.hyo, Hyoid head-cavity. So.mand, Mandibular head-cavity. So. prem, Premandibular headcavity. $\times 30$ diams. 
the anlages* of the muscles of the eye, and these are, by hypothesis, homologous with the cells of the walls of the head-cavities in the lower vertebrates, which cells produce the muscles of the eye.

\section{The Mesenchyma.}

By the term mesenchyma we designate the whole of the mesoderm of the embryo, except the mesothelial lining of the coelom. When fully differentiated histologically, it consists of more or less widely separated cells, connected with one another by intervening threads of protoplasm, which form a network between the cells (Fig. 49).. The remaining space is filled by a homogeneous structureless matrix or basal substance. It gives rise to a large number of adult tissues, as shown in the table on page I9.

In the early development, or histogenesis, of the mesoderm we can distinguish four stages: first, that of distinct cells; second, the formation of the cellular network; third, the formation of the mesothelium; and, fourth, the differentiation of the mesenchyma. The first stage is known chiefly through observations on the early stages of elasmobranchs, reptiles, and birds. In these types the first cells which are delaminated from the entoderm to form the anlage of the meso-

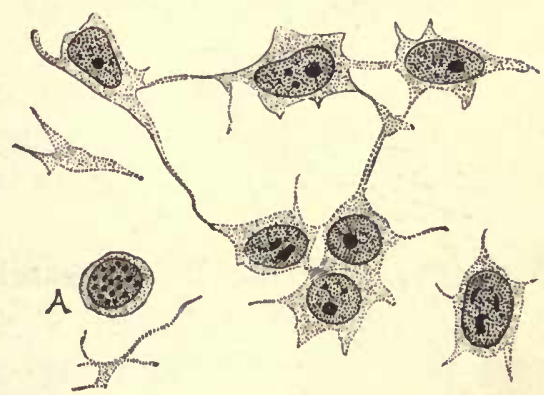

Fig. 49.-Chick Embryo of the Third DAY.

Mesenchyma from near the otocyst. $A$, cell in mitosis. derm, are of quite large size and lie between the entoderm, or yolk, and ectoderm, and are without connection with one another. The number of mesodermic cells increases both by the multiplication of the cells already delaminated and by the addition of others from the entoderm. Whether this stage occurs in mammals or not, we do not know at present. In the second stage the primitive cells are found to have acquired connection with one another, the protoplasm of one cell uniting by a process, or prolongation, with the protoplasm of another cell, and so on until the whole tissue becomes a network. When the primitive streak has been formed in the mammalian blastodermic vesicle we find the mesoderm in this condition. The third stage is brought about by the development of the cœlom as above described. The colom is bounded by the undifferentiated mesoderm. To produce the fourth stage, single cells leave the primitive mesodermic layer by migrating out of it on the side away from the cœlom. The cells left behind are ultimately reduced to a single layer, the permanent mesothelium. The emigrant cells constitute the mesenchyma and are found to be connected both with one another and with the mesothelial cells by protoplasmatic processes, but they do not lie close together, as in the epithelium, so that there is a considerable, though variable, amount of inter-

* The anlages may be seen in a pig embryo of $10 \mathrm{~mm}$. between the jugular vein and the internal carotid artery as a group of embryonic cells quite distinct from the surrounding mesenehyma. 
cellular space. By the migration of the cells and their multiplication, the mesenchyma is produced. It fills up all the room between the mesothelium and the two primary germ-layers so far as it is not occupied by the developing blood-vessels and, later, by lymph vessels.

Apparently the entire mesothelium may participate in the production of the mesenchymal cells. Its different regions, however, do not so participate all to an equal degree, or at the same time. The throwing off of mesenchymal cells may be observed in certain parts of the embryo in somewhat advanced stages of development, and it seems not impossible that the process may be found to occur even in adult life.

The mesoderm, by the formation of mesenchyma, becomes very early unlike the other germ-layers. Both ectoderm and entoderm are epithelial membranes. The mesoderm is partly epithelial, partly mesenchymal, and from the mesenchyma arise special kinds of tissue which are characteristic of the middle germ-layer, and never are produced from either the outer or inner germ-layers.

\section{The Origin of the Blood-vessels and Blood.}

As stated above (pages 66 and 8o), the angioblast and first blood-vessels appear in the circumscribed region in the mesoderm of the yolk-sac and lie close against the entodermal cells of the area opaca. The region which they occupy is termed the area vasculosa. From the area vasculosa the development of bloodvessels extends, as stated, across the area pellucida into the embryo.* During these early stages the only blood-vessels are in the splanchnopleure. After their formation has extended into the body of the embryo, it spreads into the somatopleure also, which, therefore, acquires its blood-vessels at a later stage. It should be noted, however, that the development of the blood-vessels begins before the cœlom has been developed over the area vasculosa. While they are forming, the cœlom expands; and after it has appeared, the primitive blood-vessels are found always exclusively in the splanchnic mesoderm.

Definition.-The essential part of a blood-vessel is its endothelial wall. In early stages all the blood-vessels consist only of endothelium. Arteries and reins differ but little, if at all, in histological structure during early embryonic stages, and are distinguished chiefly by the direction of blood-currents passing through them. Capillary blood-vessels and sinusoids have, as a rule, throughout life merely an endothelial wall. Arteries and veins become strengthened by the development of special coats around the endothelium which arise by transformations of the mesenchymal cells in the immediate neighborhood of the vessels.

The Development in the Chick. - The first indication of the blood-vessels is a reticulate appearance, which can be recognized in the mesoderm in surface views of the fresh or hardened embryo at the end of the-first day. The reticulate structure increases rapidly in extent and distinctness during the second day of incubation.

* It has been recorded that in lizards the vascular anlages appear first in the area pellucida. 
It is confined to the region of the mesoderm surrounding the embryo proper, and which is, therefore, known as the area vasculosa, as above stated (compare Fig. I3I). As soon as there are several primitive segments in the embryo, the network in the mesoderm shows traces of coloration in irregularly shaped reddish yellow spots, which are largest and most numerous around the caudal end of the embryo. These spots are called blood-islands (Fig. I3I, Bl.is) because the central cells in them are transformed into the first blood-corpuscles. The network appearance is due to the development of the angioblast, which is a set of cells delaminated from the entoderm or the yolk, and intervening between the mesoderm proper and the entoderm. The angioblast at first assumes the form of more or less solid cords. The meshes

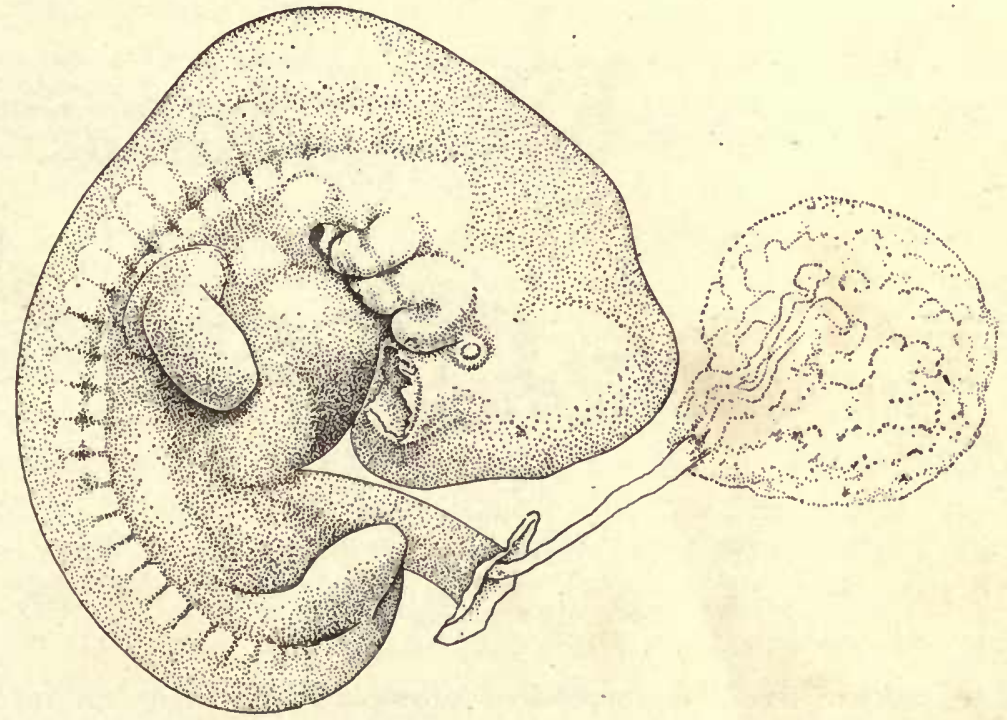

Fig. 50.-Human Embryo of 9.4 Mm. Probable Age Thirty Days. $\times 8$ diams.

of the angioblast are partly or wholly filled by mesodermic cells. The cœlom now appears in the extra-embryonic area, and thereafter the anlages of the blood-vessels are connected with the splanchnic mesoderm only. The anlages of the bloodvessel at this stage form a thick network without distinction of stem or branch, except that the edge of the area, bounded by a broad band of angioblast, gives rise to a single large vessel, which is known as the simus terminalis. The anlages are all in one layer, none overlying the others, and up to this stage they are all solid. The terminal sinus becomes connected with the venous system.

The blood-islands are spots where there is a cluster of cells, which remain attached to one another and to the walls of the vessels. The cells develop hemoglobin in their interior, hence the clusters have a reddish color which renders all the islands very conspicuous in surface views of fresh specimens. Blood-islands appear first in the area opaca, but almost immediately after in the pellucida also. They 
have at first a rounded or branching form. In the inner part of the pellucida they are small and stand alone. Toward the periphery they are larger, more closely set, and more united with one another. Their development is greater around the caudal end of the embryo.

In the next stage the vascular anlages become hollow, and then may be called true blood-vessels. When they acquire a lumen, the blood-islands are found to remain attached usually to the upper side of the vessel like a thickening of its wall (Fig. 5I, bl.is). Very soon after the vessels have become hollow the cells of the blood-islands break apart and lie free in the cavity of the vessel, thus forming the first blood-corpuscles. They are characterized by having a rounded nucleus with a very distinct nucleolus, and a minimal covering of protoplasm only. After
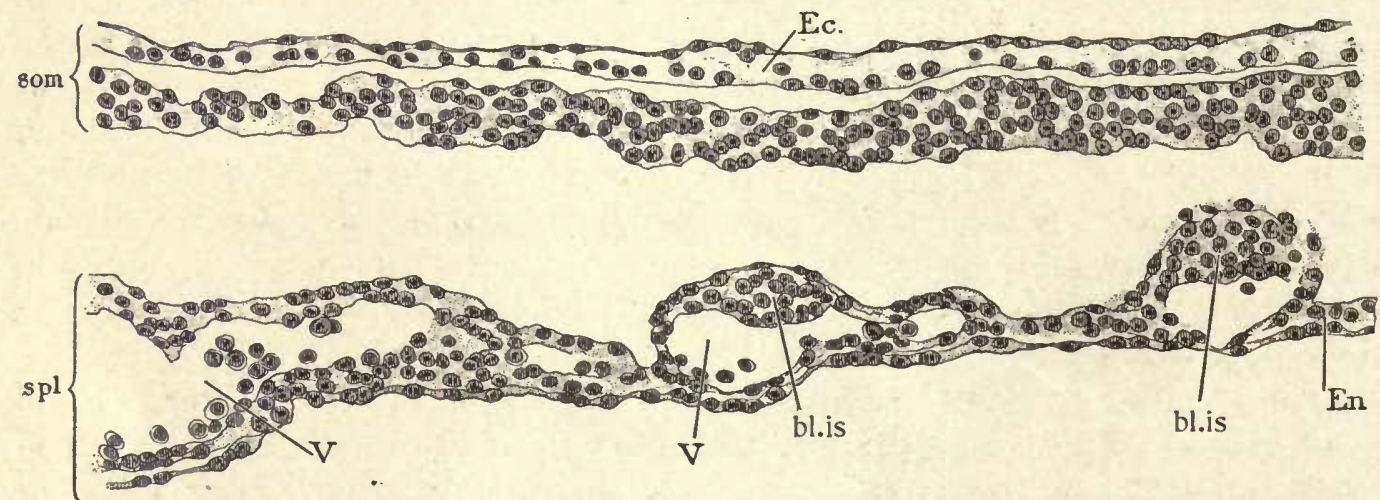

Fig. 51.- Section of the Area Vasculosa of a Chick Embryo of the Second Day.

Som, Somatopleure. Spl, Splanchnopleure. Ec, Ectoderm. En, Entoderm. bl.is, Blood-isands. V, V, Bloodvessels. $\times 227$ diams.

the cells have become free the amount of protoplasm in each cell increases. The cells multiply rapidly by mitotic division. It is believed that all the bloodcorpuscles, both red and white, are descendants of these cells derived from the blood-islands.

The angioblast continues growing by the development of buds from the vessels already formed. These buds are rounded or pointed, forming, as it were, spurs. They often end by meeting one another and uniting. They are usually hollow from the first, and after they meet one another or an adjacent vessel, the cavities become continuous, and thus the vascular network is extended.

The Development in Mammals.-The origin of the blood-vessels in mammals is not adequately known. The solid primary anlages appear in the extra-embryonic area vasculosa and extend later into the embryo. They present well-marked bloodislands, which make their first appearance in rabbit embryos of the eighth day, just before the appearance of the first primitive segments. It is characteristic of most mammals that the entire yolk-sac, probably owing to its small size, becomes, very early indeed, vascularized throughout. 
The Growth of the Vessels into the Embryo.-The entrance of the vessels into the embryo chick begins toward the end of the second day. The buds which form the extra-embryonic angioblast grow first toward, then into, the embryo. The penetrating vessels follow certain prescribed paths. Part of the vessels run along the posterior edge of the amnio-cardiac vesicles, and enter into connection with the posterior end of the heart, which has meanwhile been progressing, and which-owing to the early separation of the head end of the embryo from the yolk-is the only part of the heart which the vessels can reach directly. While the vessels are approaching the heart their differentiation into various sizes is going on, the smallest ones to remain as capillaries, the larger ones to become arteries or veins. The only two veins in the first stage are those above mentioned, which are called the omphalo-mesaraic. Another set of vessels penetrates along the splanchnopleure of the body on each side until they attain the small space between the notochord and somite and the entoderm, where they fuse so as to form a longitudinal vessel, the anlage of the descending aorta. (Fig. I43, Ao.D). It should be noted that this anlage is primitively double. The aorta appears first in the region toward the head. It grows forward above the pharynx, bends ventrally just behind the mouth, dividing as it bends, one branch going around each side of the future pharynx, and uniting again on the ventral side of the pharynx in the median ventral line, in order to join the anterior end of the tubular heart. The heart begins to beat before the vessels unite with it. The first blood-cells have already been formed; hence as soon as union is accomplished the blood circulation starts up, the blood passing through the aorta to the body, thence by numerous lateral branches to the area vasculosa, and returning by the two omphalo-mesaraic veins to the heart. It will thus be seen that almost the entire circulation is extra-embryonic.

The other embryonic blood-vessels are developed by buds from the walls of the vessels already present in the embryo, in the same general manner as new vessels are formed in the area vasculosa. These buds give rise to the endothelium only of the embryonic vessels. When a vessel becomes an artery or a vein, the media and adventitia are added, as above stated, by differentiation of the surrounding mesenchyma.

During further development many small blood-vessels abort, and often appear as disconnected bits, closed at both ends and containing corpuscles. Such structures were at one time supposed to be developing blood-vessels and were accordingly termed "vaso-formative cells." The blood-corpuscles in them are of course not developing, but degenerating.

\section{The Blood-corpuscles.}

The first blood-corpuscles are free cells of an indifferent character and capable of wandering through the walls of the blood-vessels, which in early stages are easily permeable, as if of a merely gelatinous consistency. The primitive blood-cells, as they may be called, give rise not only to the permanent blood-corpuscles, both red 
and white, but various other cells outside of the vessels, of which two classes are especially important-the free wandering cells in the mesenchyma or connective tissue and the giant cells. The latter, however, contribute to the blood, for they form in the spleen, bone-marrow, and other organs, long processes, like pseudopodia, which break up into fragments. These fragments are the blood-plates. It is probable that all blood-cells and wandering cells are exclusively descendents of the primitive blood-cells, although some writers maintain that their number is increased in both the embryo and the adult by transformation of cells of the mesenchyma.

When the circulation begins, the number of corpuscles is small, but it rapidly increases by mitotic division of the cells. At the very start, like all cells produced by segmentation of the ovum, the blood-cells are quite large, but they rapidly decrease in size until they reach the "first-stage;" in which they appear as small round cells (in the chick 8.3 to $I 2.5 \mu$ in diameter) with a rounded granular reticulate nucleus and a minimal amount of protoplasm. In the next stage the amount of protoplasm increases. We have next to consider separately the cytomorphoses of the red and white corpuscles.

Red Corpuscles.-By examining the blood of chick embryos of successive ages we can trace the-differentiation of the red cells. We find that the protoplasm enlarges for several days, and that during the same time there is a progressive diminution in the size of the nucleus, which, however, is completed before the area of protoplasm reaches its ultimate size. The nucleus is at first granular, and its nucleolus or nucleoli stand out clearly. As the nucleus shrinks, it becomes round and is colored darkly, and almost uniformly, by the usual nuclear stains. This change is called pyknosis. The blood-cells of mammals pass through the same metamorphosis as those of birds. For example, in rabbit embryos of eight days (Fig. 52, A) the cells have reached the stage with a granular nucleus and welldeveloped cell-body. Corpuscles of this kind are characteristic of fishes and amphibia, and they may, therefore, be designated as the ichthyoid cells. Two days later the nucleus is already smaller, and by the thirteenth day has shrunk to its final dimensions. The cells in this condition are characteristic of the reptiles and birds, and may be designated, therefore, as sauroid cells. The nucleated stage of the cells is typical of embryonic life only in mammals. During the fetal period the nuclei of the red cells gradually disappear and the cells are transformed into the non-nucleated corpuscles, which occur only in mammals, so that this last may be designated as the mammalian stage. The nuclei disappear by extrusion from the cells. Usually they break into fragments, which are then expelled. Sometimes, though rarely, the nucleus goes out intact. The successive stages of the blood-corpuscles in mammals illustrate the law of recapitulation (page 29). When the nucleus disappears, the corpuscle becomes smaller. In the human embryo at one month the red cells are the predominant blood-corpuscles. At two months they are still the most numerous, although the non-nucleated corpuscles have begun 

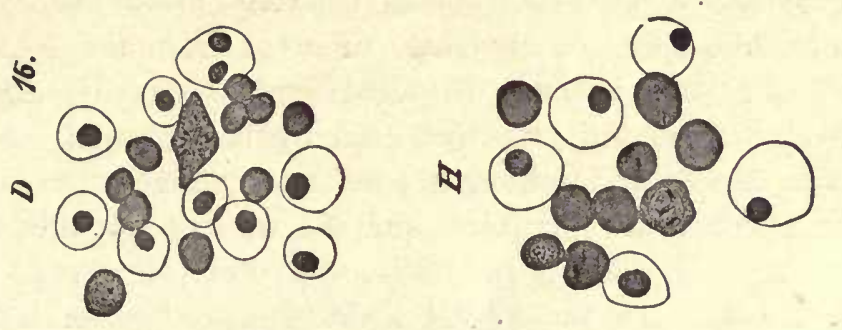

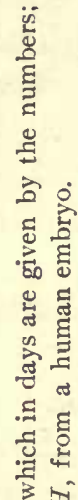

(3)

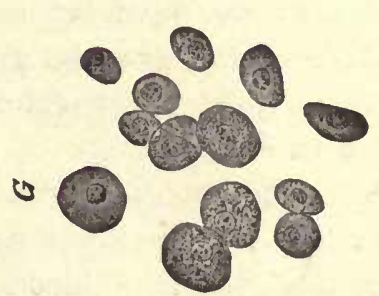

瓷岁

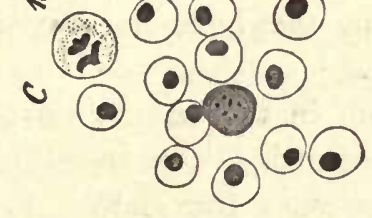

(-)

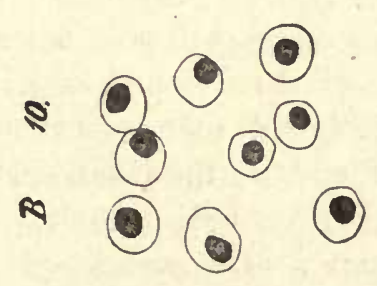

(-)

ค 00

$+000$

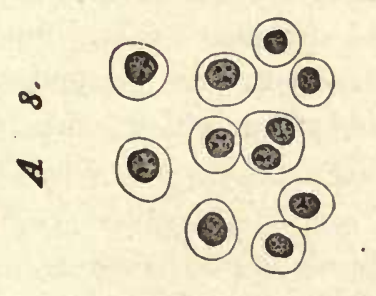

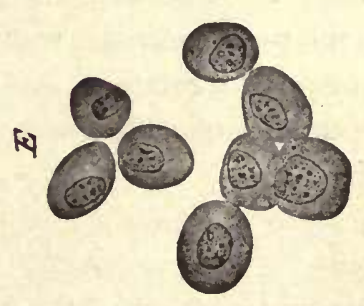

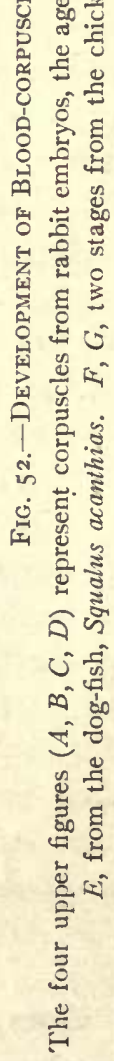


to appear. At three months the non-nucleated corpuscles constitute by far the majority of all corpuscles in the blood.

Leucocytes. - The primitive blood-cells, being colorless, have been termed leuco. cytes by some authors, but they are obviously different from the leucocytes of the adult blood. Some of them become so-called lymphocytes (young leucocytes), which are distinguishable from the primitive cells by the internal structure of the nucleus. Others grow in size and follow at least two cytomorphic paths. In one series the protoplasm develops fine granules, and the nucleus becomes first elongate, then reniform, and finally beaded. In this form they appear as polymorphonuclear neutrophile leucocytes. In the second series the protoplasm acquires coarser granules, which are really phagocyted morsels of red blood-cells, and the nucleus becomes reniform. These cells are termed the eosinophile leucocytes.

The term leucocyte properly embraces all the white corpuscles of the adult blood, but has been erroneously restricted by some recent authors to the granular forms. The young stages of the granular leucocytes are sometimes termed myelocytes, because in the adult they occur chiefly in the bone-marrow, which is the chief sanguifactive tissue in the adult.

It is doubtful whether leucocytes ever develop in the circulating blood. They appear abundantly after the lymph-glands are formed. The usual explanation is that some of the wandering primitive blood-cells enter the glands, there multiply, and in part become leucocytes.

\section{The Origin of the Heart.}

The manner in which the head of the embryo becomes free is described on page 49 (compare also Figs. I6 and I32). The origin of the cœlom is described on page 8r. When the head becomes free, the colom is found soon to extend across the median line. This takes place at the cervical end of the head just where the tissues of the embryo bend over to join the yolk (Fig. I32, p. I83). This median colom is the beginning of the pericardial cavity. In connection with it the development of the heart occurs. The formation of this organ is probably initiated by an ingrowth of the cells of the angioblast, which give rise to the endothelium of the heart (Fig. I38, Endo). The mesothelium of the dorsal side of the primitive pericardial colom produces the muscular walls of the heart (Fig. I29, m.ht). The early development and primitive relations of this organ can be understood by the account given in Chapter $\mathrm{V}$ of the structure of a chick embryo with eight segments.

\section{The Germinal Area.}

The germinal area is that portion of the amniote ovum (mammalian blastodermic vesicle) in the center of which the embryo is differentiated. It comprises, therefore, both the embryo proper and the region immediately surrounding it. In mammals it corresponds in extent with the embryonic shield (p. 47). In its center we find 
the anlages of the embryonic structures proper. In its extra-embryonic part we find the three primitive germ-layers. Underneath the entoderm is the cavity of the yolk-sac. In the mesoderm we have occurring the development of the cœlom, and in the splanchnic mesoderm the differentiation of the primitive blood-vessels. These primitive vessels occupy the sharply defined territory, the edge of which is marked by the simus terminalis (Fig. I3I, V.t). The first differentiation in the germinal area, which can be clearly recognized by the naked eye, is the appearance of the area pellucida, which is due to the thinning of the entoderm over the central area. Next ensues the differentiation of the primitive streak (Fig. I4). Further progress results in the gradual differentiation of the embryo, in the sharp demarcation of the area pellucida, which becomes pear-shaped, and in the appearance of the blood-vessels and the resulting differentiation of the area vasculosa or opaca. Figure I3I, on page I82, represents the embryonic area of a hen's ovum after about twenty-seven hours' incubation. The embryo is well advanced in development, for, although the primitive streak, pr.s, still remains in part and the medullary groove is still open behind, the brain is already marked out and the head has become partly free. Alongside the medullary canal lie eight pairs of segments. Around the embryo one easily recognizes the somewhat pear-shaped area pellucida, $A . p$, and the darker area opaca, by which it is enclosed. The area vasculosa stands out conspicuously and is bounded by the already distinguishable sinus terminalis V.t. Around and underneath is the translucent pro-amnion, proam, from which the mesoderm is altogether absent, and which, therefore, cannot contain any bloodvessels. Nor are there at this state any vessels in front of the pro-amnion. The general topographical arrangement is the same in mammals (compare page I79 and Fig. I30).

\section{The Main Vessels of the Area Vasculosa.}

Soon after the capillary network of the areas opaca and pellucida has penetrated the embryo, certain lines of the network begin to widen, and soon distinctly assume the size and functions of main trunks; some of these unite with the posterior venous end of the heart (Fig. 59, Ve.ht), which has meanwhile been formed in the embryo, and others become connected with the anterior or aortic end. Even before this the heart has begun to beat, so that, as soon as all connections are made, the primitive circulation starts up. The arrangement of the vessels is not the same in birds and mammals. The disposition in birds is indicated by the diagram shown in figure 53, in which, it should be remembered, the embryo and the capillary network are drawn many times too large in proportion to the area vasculosa. The area is bounded by a broad circular vessel, the sinus terminalis, S.T, which constitutes a portion of the venous system in birds, for in front of the head of the embryo the sinus leaves a gap, and is reflected back along the sides of the body of the embryo to make two large veins, which, after uniting with the other venous channels coming from various parts of the area vasculosa on each side, enter the 
embryo as two large trunks, Om.V, known as the omphalo-mesaraic veins; these two veins unite in a median vessel, the sinus venosus, S.V, which runs straight forward and enters the posterior end of the heart. The sinus venosus also receives the veins from the body of the embryo, namely, the anterior cardinals, Jug, and posterior cardinals, card. The two cardinals of each side unite, making a short transverse trunk known as the common cardinal, D.C, which in turn empties into the sinus venosus. The entire venous current is thus brought to the heart in a united stream; it passes out through the aorta, the greater part ascends the aortic

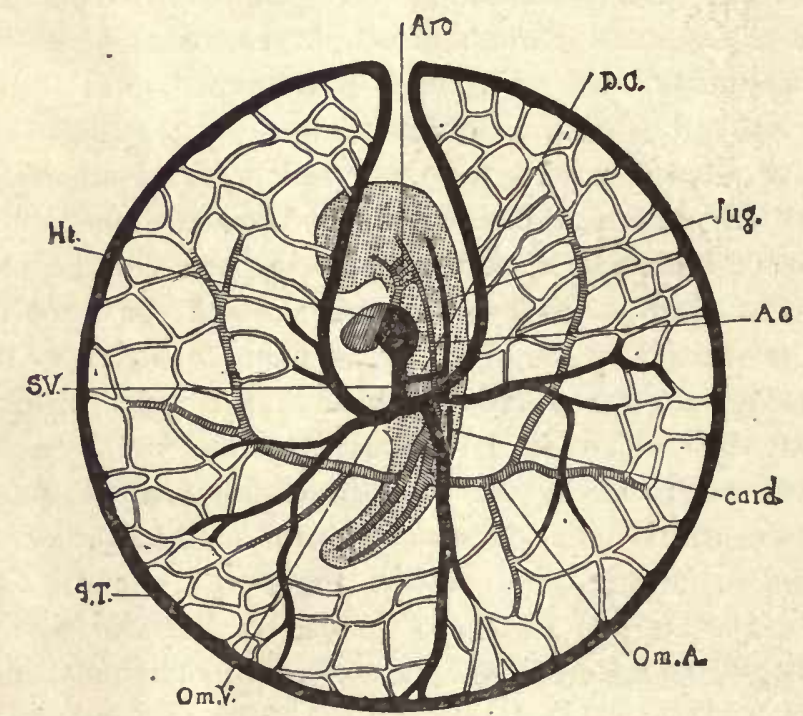

Fig, 53.-Diagrair of the Circulation in a Chick at the End of the Third Day, as seen from the Under (ENTODERMAL) Side.

The embryo, with the exception of the heart, is dotted; the veins are black. Ao, Aorta. Arc, Aortic arches. card, Posterior cardinal vein. D.C, Common cardinal vein. Ht, Heart. Jug, Anterior cardinal vein. Om.A, Omphalo-mesaraic or vitelline artery. Om.V, Omphalo-mesaraic or vitelline vein. S.T, Sinus terminalis. S.V, Sinus venosus.

arches and passes back through the main aorta, $A o$, and divides at the posterior fork of the aorta, the bulk of the two currents passing out through omphalic arteries, Om.A, and thence to the capillaries of the area vasculosa and so on to the venous trunks again. As shown in the figure, which presents the under side of the area, the left omphalo-mesaraic vein preponderates, and in the latter stages this difference becomes more marked, until finally the right stem is very inconsiderable in comparison with the great left vein. The time at which the disparity commences is extremely variable, as is also the degree of inequality between the two veins.

The following description probably represents what was the primitive condition of vessels in the mammalian area vasculosa. It applies to an early stage in the rabbit. An essentially similar arrangement of the vessels exists also at a correspond- 
ing stage in the dog. The veins are much more symmetrical than in the chick, and have the same general plan; the sinus terminalis belongs to the venous system, so that the connection with the arterial circulation, found later, is secondary; the aorta of the embryo is double, and gives off on each side (segmentally arranged?) transverse branches, one of which develops into the large trunk shown in figure 54; the network of small vessels forms two layers, of which the upper is connected with the arteries, the lower with the veins. The change from the earlier condition to the later has still to be followed.

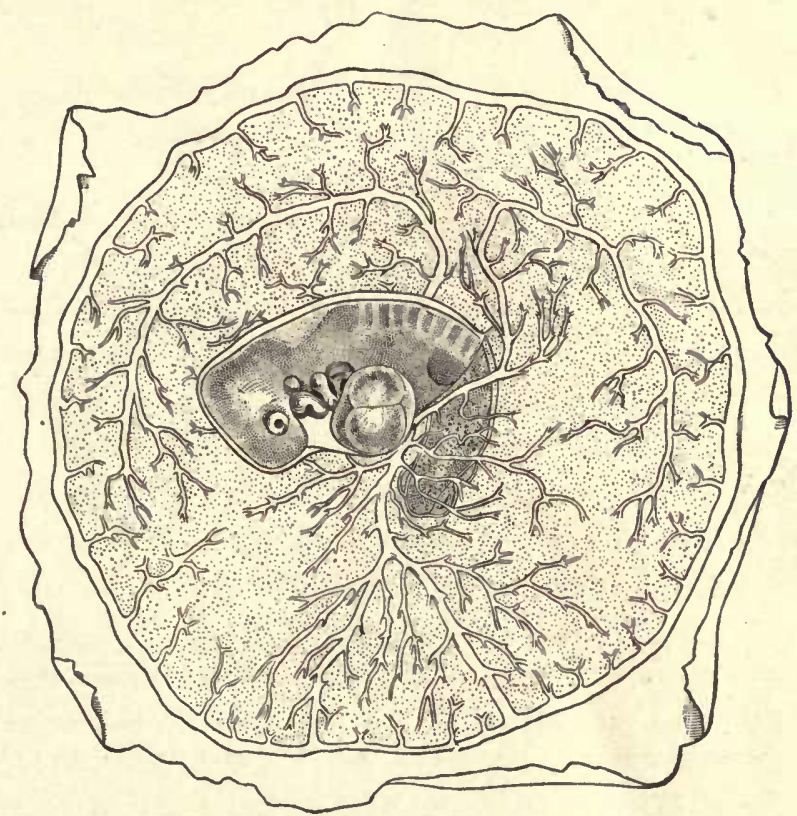

Fig. 54.-Area Vasculosa of a Rabbit, Presumably of about Twelve Days.--(After Van Beneden and Julin.)

The arrangement of the main vessels in the area vasculosa at a later stage in the rabbit is quite different. The sinus terminalis forms a complete ring (Fig. 54), and is connected with the arterial system by a single trunk, which corresponds to the left omphalic artery of the bird. For some time the connection between the embryonic arteries and the area vasculosa is entirely through capillaries, and the arterial trunk on the vascular area does not appear in the rabbit for several days. There are two veins, one arising from each side of the body and passing out on to the area vasculosa over the back of the embryo; they are the two large upper vessels in the figure.

\section{The Aortic System.}

In early stages the aortic end of the heart terminates under the ventral floor of the pharynx. The endothelial heart continues as the aorta, which almost at 
once branches to the right and left. Each branch forms five vessels, the so-called aortic arches (Figs. 55, 92, and 93). The first arch is the first formed, the other four are formed in succession behind it. The arches show a constant relation to the pharyngeal gill-pouches, there being one pouch between every two arches. The arches pass dorsalward around the broad pharynx (Fig. 28), and those of each side become united by a single dorsal longitudinal vessel (Fig. 56, Ao.D), the dorsal or descending aorta. The two dorsal aortæ pass caudad until they meet and unite in the median line to form the main aorta. (Compare Figs. 196, Ao.S;

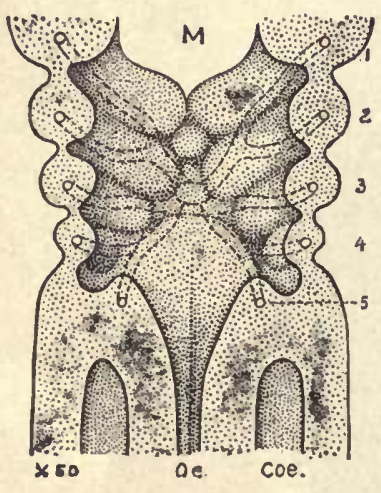

Fig. 55. - Anterior Wall of the Phar-
ynX of a Human Embryo of 3.2 Mm.

I-5, Gill-arches; the arches are separated from one another by the entodermal and the corresponding ectodermal gillpouches; the aortic arches are drawn in dotted lines and arise from the median cardiac aorta. $M$, Mouth. $\mathrm{Oe}$, CEsophagus. $\mathrm{Ca}$, Colom. $\times 5^{\circ}$ diams.-(After W. His.)

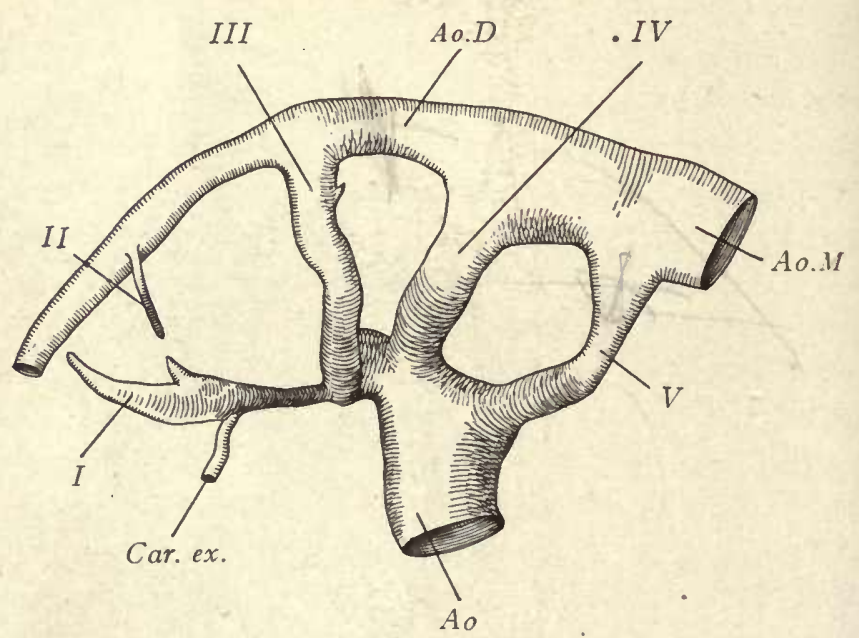

Fig. 56.-Pig Embryo of 6.0 mim. Series 9. Aortic Arches of Left Side. Froma Wax Reconstruction by L. M. Ferguson.

I, II, III, IV, V, Aortic arches. Car.ex, External carotid. Ao, Cardiac aorta. Ao. D, Dorsal aorta. Ao.M Median aorta.

197, Ao, and 198, Ao.) In a pig of $7.8 \mathrm{~mm}$. the five aortic arches can be still traced, but the first arch has begun to disappear, and the condition illustrated in figure 169 is established. Its ventral portion, $I$, persists, however, and together with its own vascular prolongations into the lower jaw gives rise to the external carotid (Fig. Ior, car.ex). The descending aorta on the dorsal side between the tops of the first and second arches also persists and is prolonged into the head to constitute the internal carotid (Fig. I72, car.i). Presently the second arch also disappears, and both carotids are, as it were, thereby lengthened. This is the condition which we find in our embryo of $12 \mathrm{~mm}$. (Fig. I72). The third, fourth, and fifth arches are still present. From the base of the third arch runs forward the external carotid, and from the summit of the third arch runs forward the 
internal carotid. The dorsal ends of the third and fourth arches are still connected, but this connection, instead of being a large aortic vessel, as in earlier stages, has now contracted and almost disappeared, and will soon be lost altogether, so that in the adult there will be no connection between the dorsal ends of the third and fourth arches. The fifth arch is still connected with the dorsal end of the fourth. It gives off the small pulmonary artery to the lungs. On the side toward the heart the relations of the arches are also changed. The main aortic vessel which springs from the heart is, in the $12 \mathrm{~mm}$. pig, divided into two vessels - the pulmonary aorta on the ventral side and the true aorta in a more dorsal position. The division has so taken place that the third and fourth arches are connected only with the true aorta, while the fifth arch is connected only with the pulmonary aorta. The part of the fifth arch on the left side between the origin of the pulmonary artery proper and the main descending aorta offers at this stage an open communication between the pathways of the pulmonary and of the main body circulation. This dorsal half of the fifth aortic arch is known as the ductus arteriosus. It remains throughout the fetal period as an open channel, so that the blood from the right ventricle flows in part to the lungs, in part into the dorsal aorta. The lumen of the ductus arteriosus disappears in man soon after birth. As an anomaly it occasionally persists throughout life, involving serious modifications of the normal circulation. The dorsal part of the fifth aortic arch of the right side has a different history, for it aborts early in embryonic life, and there also occurs an abortion of the entire descending aorta from the end of the fourth arch on the right side downward to the level of the diaphragm. When this abortion has taken place, the entire aortic stream flows from the heart to the left side of the embryo. The aortic branches on the right side appear as follows in the adult: The main stem, from which the five arches originally sprang, is the arteria innominata, which gives off a stem, the common carotid, from which spring the two carotids of the right side. Next, a vessel which represents the persistent fourth right arch, which no longer has any direct communication with the aorta, but at its end gives off the subclavian and vertebral arteries. The vessel which corresponds to the right fourth arch is usually described as a portion of the stem of the subclavian artery in the adult. The aortic branches on the left side appear as follows in the adult: first, the common carotid, the stem of the original first to third arches; second, the subclavian, which includes a part of the fourth arch, but consists chiefly of what was originally only a branch of the arch. The pulmonary artery has as its stem a portion of the fifth arch, in man on the left side only, but the vessel arises as a separate branch from the fifth arch.

There often appear irregular vessels in early stages between the fourth and fifth arches, and these have been held by some writers to represent an additional partially aborted aortic arch. If this is the case, then the arch here called the fifth would be more correctly termed the sixth. The arch supposed to be lost is sometimes distinguished as Zimmermann's arch. 
The descending and median aortæ give off intersegmental vessels, some of which persist in the adult. The main aorta produces three main branches on its ventral side, the histories of which are somewhat complicated. They are the cœliac axis, the superior mesenteric, and the inferior mesenteric.

\section{The Venous System.}

The veins do not for the most part, if at all, arise as independent vessels, but by the transformation of channels in a network of small vessels. Arteries develop in the same way, but with them the growth of the main stem as such plays a

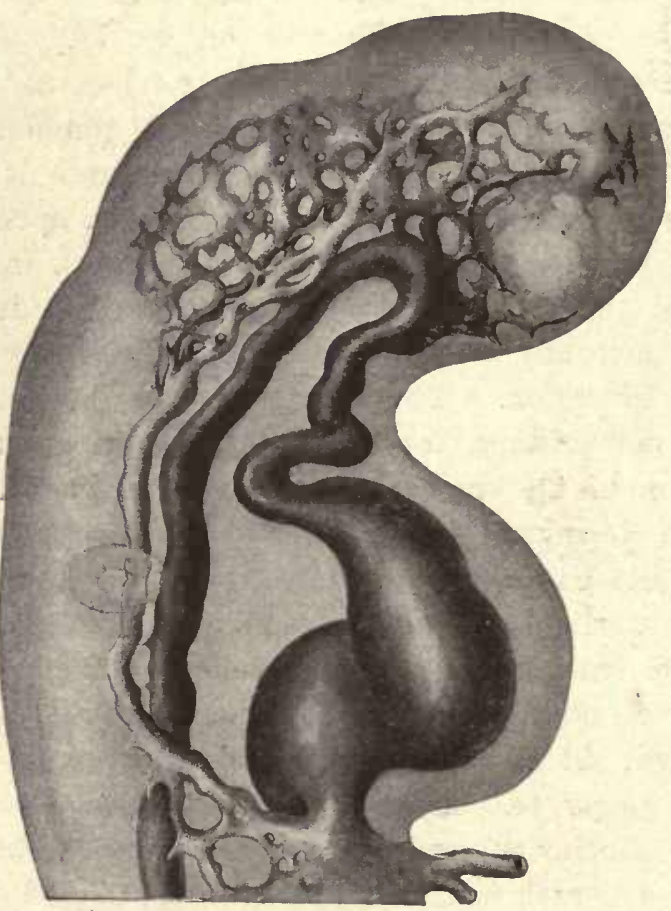

Fig. 57.-Chick Embryo with Seventeen Segments. Drawn from a Specimen which had been AutoINJECTED WITH INDIA-INK.-(Afier H. M. Evans.)

greater rôle. The method of development is illustrated by figures 57 and 58 . From the first aortic arch a network of small vessels spreads into the head at the sides of the fore-brain and mid-brain, gradually occupying an increasing territory until the whole head is supplied. The plexus forms a single vascular layer between the brain and epidermis and is at first without any main channels (Fig. 57). Soon some of the capillaries enlarge, forming a branching system (Fig. 58), the branches leading into a main stem which extends from the head to the posterior or venous end of the heart. This stem, which rapidly increases in size, is the anterior cardinal vein, 
which is matched by a similar vein on the opposite side of the head. The remaining primitive veins and the later secondary veins are all formed in a like manner.

The first veins to be formed are the omphalo-mesaraic, which are evolved from the vascular network of the area vasculosa, and extend into the body of the embryo, running in the splanchnopleure (Fig. I 58, Om.S, Om.D). They approach one another in the median line (Fig. 59), unite, and are prolonged forward to make the endothelial heart.

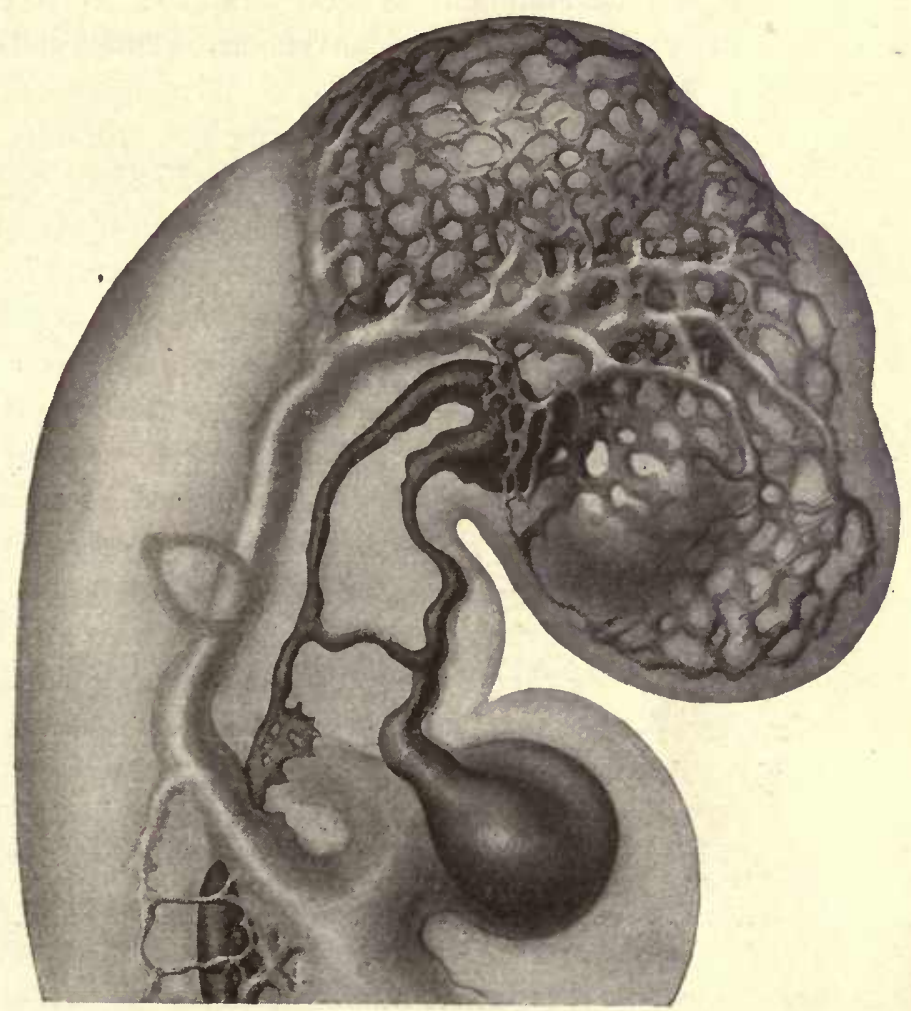

Fig. 58.-Chick Embryo with Twenty-five Segments. Drawn from a Specimen which had beex AutoINJECTED WITH INDIA-INK.-(After H. M. Evans.)

There are three other pairs of main primitive veins, all developed entirely within the body of the embryo: I, the anterior cardinals, which drain the head (compare Fig. I54, card, and Fig. I55, Ve); 2, the posterior cardinals, which drain the body from the tail to the heart and occupy each a characteristic position laterad from the aorta, and dorsad from the splanchnocele (Fig. 158 , card, and Fig. 159, card, card.s); the two cardinal veins unite at the level of the venous end of the heart and form thus on each side a short transverse stem, the common cardinal, which opens into the heart and was. formerly named the ductus Cuvieri; 3 , the 
umbilical veins, which are differentiated a little later from the vascular plexus of the somatopleure. (Compare Figs. 90, 93, and the description of the veins in the human embryo of the ninth stage, p. I43.)

The pulmonary veins represent a new system of vessels added to those already mentioned. Their exact origin has not been traced, but about the time the branching of the entodermal lung begins, they appear and open into the left auricle of the heart.

The eight pairs of primitive veins pass through complicated metamorphoses owing principally, first, to the development of new branches to receive the blood from the organs as they arise; second, to the conversion of small collateral channels

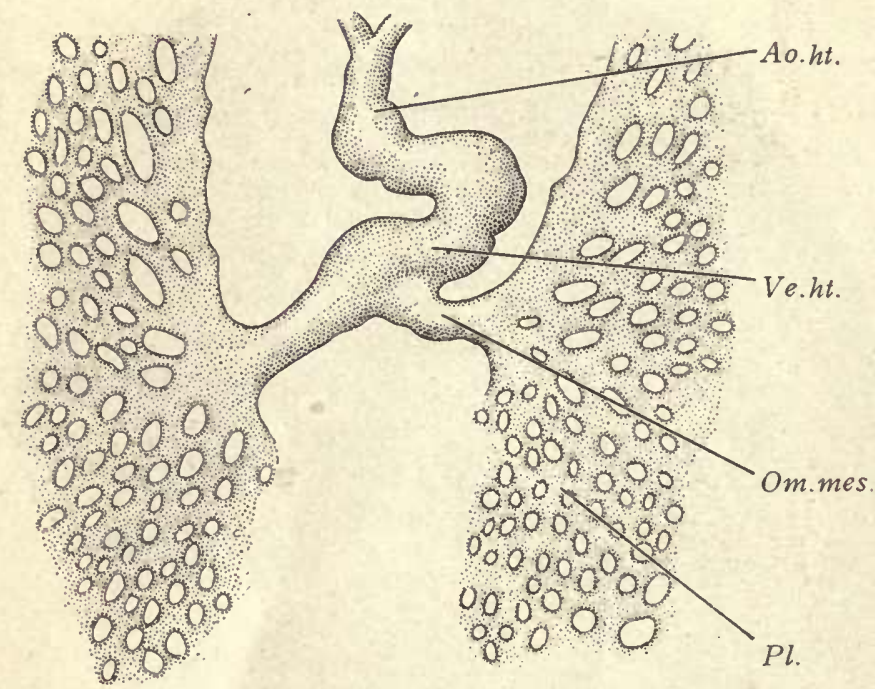

Fig. 59.-Chick of about 40 Hours. View from underneath of Part of the Vascular System. Ao.ht, Aortic limb of endothelial heart. Om.mes, Omphalo-mesaraic vein. $P l$, Part of the vascular plexus of the area vasculosa. Ve.ht, Venous end of the endothelial heart. $X_{25}$ diams.

into main vessels; and, third, to the obliteration of parts of the original vessels. The first process is illustrated by the history of the veins of the limbs; the second by the formation of the lateral vein of the head and of the subcardinal vein of the Wolffian body; the third, by the disappearance of the right umbilical vein and of portions of the omphalo-mesaraic veins.

The vena cava inferior is a new pathway formed by utilizing portions of several originally distinct and separated venous channels. At first the blood from the abdominal viscera must return to the heart chiefly through the posterior cardinal veins (Fig. 53, card), but the vena cava inferior offers a direct course. Its development depends primarily upon the union of the cephalad end of the right Wolffian body with the liver, followed by a vascular fusion of the two organs which renders it possible for the blood of the right subcardinal vein to pass through the 
blood-spaces of the liver directly to the heart. This makes a very direct channel, a more direct one than existed previously when the blood from the subcardinal came to join that of the cardinal, passing up to the common cardinal and then back to the heart. The new channel through the liver rapidly enlarges and becomes recognizable as the vena cava inferior. This important venous trunk is a combined vessel, comprising, first, a part of the hepatic vein; second, a large channel developed from the sinusoids of the liver; third, the upper part of the right subcardinal vein, and, fourth, the lower part of the right cardinal.

\section{The Lymphatic System.}

The lymph vessels arise in connection with the veins and are probably outgrowths of the vascular endothelium, although some authorities state that they may begin as sub-endothelial spaces. They may be recognized by their very delicate but distinct endothelial walls, thus differing from the mesenchymal spaces, and by the complete or almost complete absence of blood-corpuscles within them. They probably end blindly. They appear relatively late, for they do not develop until after the limb buds are well advanced (pigs of $\mathrm{I} 4 \mathrm{~mm}$.). The first lymph-vessels develop from the anterior cardinals in the cervical region, and rapidly fuse to make a pair of very large vesicles, the jugular lymph-sacs (Fig. 60, S.l.j), which are closely applied to the veins. Each jugular lymph-sac empties into the cardinal vein near its junction with the subclavian through a valve-like orifice. Whether this connection is primary or secondary is still uncertain. From each sac, narrow vessels bud out into the mesenchyma, anastomose with one another, and, by spreading more and more, produce the lymphatic system of the neck and head. Subsequently, the jugular sac is resolved into the deep cervical plexus' of lymphatics. Similar lymph-sprouts in slightly older embryos produce a more irregular median mesenteric sac (Fig. 6o, S.l.m) just below the renal anastomosis, R.A; and also another sac, $\mathrm{Cis}$, dorsal to the first. The mesenteric sac sends branches into the mesentery to drain the intestine, and at the same time joins the dorsal sac which enlarges, becoming the cisterna chyli. Still later there are formed in a similar manner two smaller sacs of dense plexuses, termed the sciatic, for they develop in connection with the root of the sciatic vein (Fig. 6o, Sci). The sciatic sacs are produced later than the other four. Like the jugular, the four later formed sacs serve as centers of growth for the lymph-vessels. In addition, other lymphatics develop from other veins. Especially notable are the sprouts from the azygos vein, which unite and ultimately give origin to the main lymphatic trunk, the ductus thoracicus, which joins tailward the cisterna, headward the left jugular sac.

The lymph-glands make their first appearance considerably later than the vessels (rabbits, $25^{-} 30 \mathrm{~mm}$; human embryos, 30-45 $\mathrm{mm}$.). Each appears between a vein and a lymph-vessel and is recognizable as mesenchyma crowded with young leucocytes. The glands are very small at first, but are quite sharply circumscribed. 


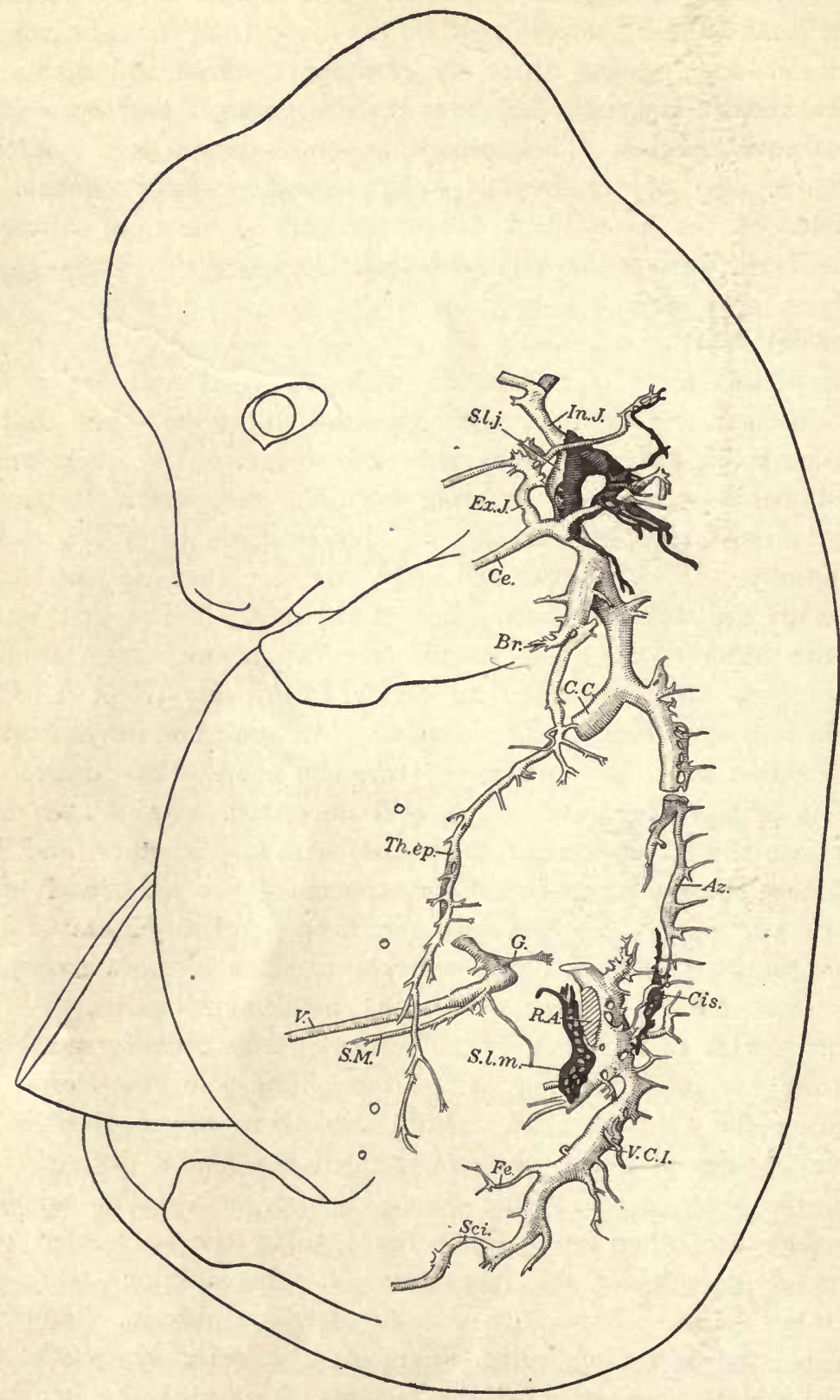

Fig. 60.-Pig of 20 mm. Series 59. Reconstruction of the' Lymph-vessels and Part of the Venous System. By F. T. Lewis.

$A z$, Azygos vein (remnant of the posterior cardinal). Br, Brachial vein. C.C, Common cardinal vein. Ce, Cephalic vein. Cis, Cisterna chyli. Ex.J, External jugular vein. $F e$, Femoral vein. $G$, Gastric vein. In.J, Internal jugular vein. R.A, Renal anastomosis between the subcardinal veins. Sci, Sciatic vein. S.l.j, Jugular lymph-sac. S.l.m, Mesenteric lymph-sac. S.M, Superior mesenteric vein. Th.ep, Thoraco-epigastric vein. V, Omphalo-mesaraic or vitelline vein. V.C.I, Vena cava inferior. $\times 8$ diams. 


\section{The Liver.}

When the omphalo-mesaraic veins, the first large veins to appear, are developed, they are situated in the splanchnopleure and join the heart. They are of such large size as to cause a projection into the cœlom. This projection is the septum transversum (p. 87). As shown in the diagram (Fig. I6), the entoderm of the digestive canal of the head of the embryo passes over behind the pericardial cavity and behind the septum transversum into the yolk-sac. Out of the entoderm covering the septum transversum on its caudal side, the anlage of the liver is developed (Fig. $25, f o$ ). "This anlage is produced by a rapid proliferation of the entodermal cells, and they grow toward the space occupied by the omphalo-mesaraic veins (Fig. 157). An intergrowth of the liver cells and of the endothelium of the veins takes place. The cavity of the veins becomes subdivided into smaller blood channels, which we call sinusoids to distinguish them from capillary vessels. The liver cells arrange themselves in the form of cords which are termed the hepatic cylinders. Each hepatic cylinder is closely invested by the venous endothelium. The liver consists at first only of hepatic and endothelial cells and is situated in the septum transversum.

When the liver becomes larger, it protrudes from the septum transversum, but does not separate from it, so that in the adult the liver is always found attached to the diaphragm, which is merely the modified septum transversum.

\section{The Pancreas.}

The pancreas is a double organ, for it arises from two distinct anlages: first, the dorsal pancreas, which appears as an entodermal evagination on the dorsal side of the duodenum (Fig. 6r, Panc.d), soon branches, and, continuing to grow, rapidly develops into the body and tail of the adult organ. Its connection with the intestine becomes the dorsal pancreatic duct (ductus Santorini). The ventral pancreas appears a little later and grows more slowly than the dorsal. It arises as an outgrowth (Fig. 6I, Panc.v) from the entodermal ductus choledochus. It develops

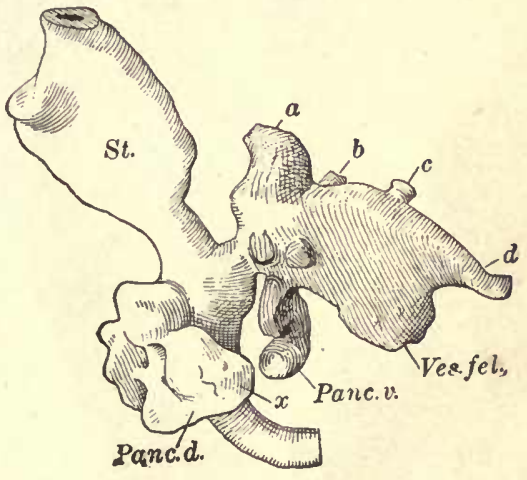

Fig. 61.-Pig Embryo of 5.5 ma. Series 915. WaX ReCONSTRUCTION OF the Stomach and Pancreatic Anlages By F. W. Thyng.

$a, b, c, d$, Cords of hepatic cells. Panc.d, Dorsal pancreas. Panc.v, Ventral pancreas. St, Stomach. Ves.fel, Gallbladder. $x$, Ventral process of the dorsal pancreas (situated on the right of the portal vein). $\times 55$ diams.

into the head of the pancreas and dorsal pancreatic duct (ductus Wirsungi). The two anlages expand, meet (Fig. 62), and unite. In the pig, the dorsal anlage is farther from the stomach than the ventral, but in man it is nearer the stomach.

In the pig, the ventral pancreatic duct is obliterated and the dorsal duct alone is normally persistent in the adult. In man, on the contrary, the dorsal duct is normally obliterated and the ventral duct persists. Occasionally both ducts are 
found in human adults, and in such cases the dorsal duct has been commonly termed "accessory."

The cords of pancreatic cells are at first solid, but they for the most part acquire lumina, thus becoming epithelial gland-tubes. The areas of Langerhans are small patches of pancreatic cell cords found in the adult without any lumina.

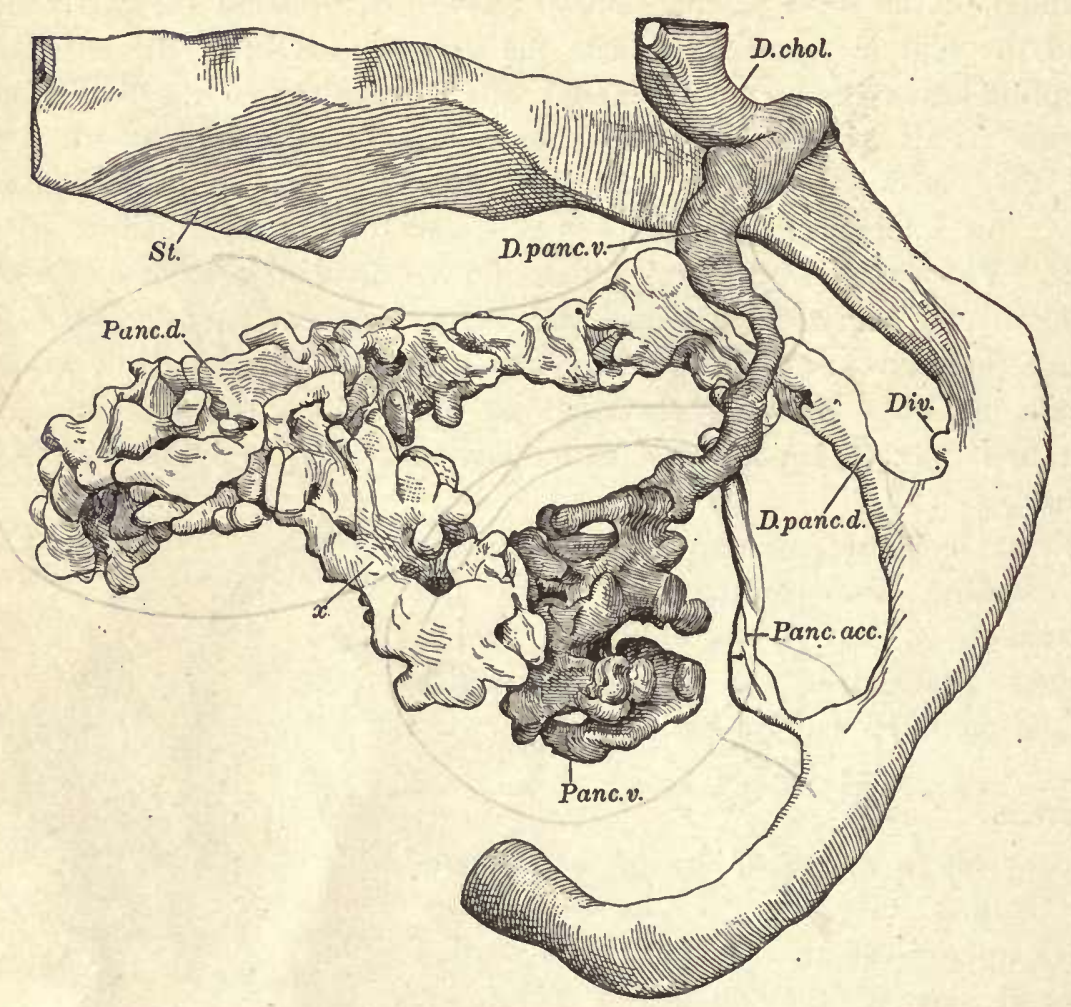

Fig. 62.-Pig Embryo of 20 mim. Series 6o. Wax Reconstruction of the Duodenum and Pancreatic Anlages by F. W. Thyng.

Div, Duodenal diverticulum. D.chol, Ductus choledochus. D.panc.d, Ductus pancreatis dorsalis. D.panc.v, Ductus pancreatis ventralis. Panc.acc, Pancreas accessorium (anomaly). Panc.d, Pancreas dorsale. Panc.v, Pancreas ventrale. St, Stomach. $x$, Ventral process of the dorsal pancreas, on the right of the portal vein. $\times 55$ diams.

\section{The Excretory Organs.}

No less than three distinct excretory organs are known to occur in vertebrates.

Of these, the first is termed the pronephros, or head kidney, on account of its position toward the head and in the neighborhood of the heart. It is well developed and the only excretory organ in certain fishes and in the early larval stages of amphibia. In elasmobranchs, which occupy in this respect an exceptional position, and in amniota it exists in a rudimentary form only, except as to its duct, which plays an important rôle in the further development. The pronephros consists of 
a few epithelial tubes which take a somewhat twisting course, but may be said to run, in general terms, transversely. Each tube begins with a ciliated funnel-shaped opening (Fig. 63, $f$ ) not far from the median line of the embryo, and ends, after a more or less contorted course, in a longitudinal duct, which, after receiving all of the tubules, runs toward the posterior end of the embryo and opens into the extremity of the entodermal or digestive canal. Opposite the funnels, and separate from the pronephros proper, there is a so-called glomus (Fig. 63, gl), which is a projection of not inconsiderable size from the mesentery. When fully developed the glomus contains a rich network of blood-capillaries, so that it somewhat resembles the glomerulus of the kidney. The circulation of the pronephros is sinusoidal.

The second of the excretory organs is termed the mesonephros, Wolffian body, or fetal kidney. It is the only excretory organ in elasmobranchs. In adult am-

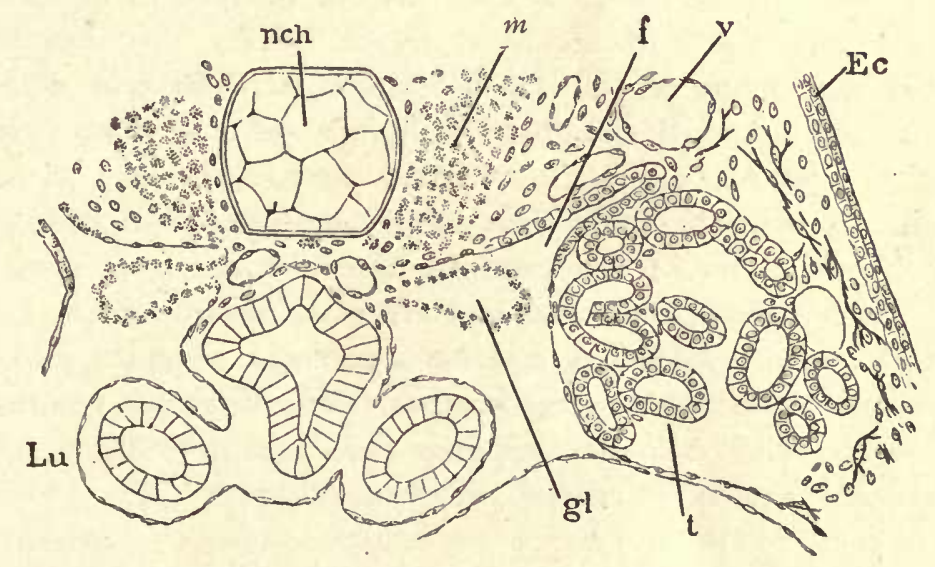

Fig. 63.-Frog (Rana temporaria) Tadpole of I 2.o m. Cross-section of the Pronephric Region. $n c h$, Notochord. $m$, Muscles. $f$, Pronephric funnel. $v$, Blood-vessel. Ec, Ectoderm. $t$, Pronephric tubule. $g l$, Glomus. $L u$, Lung. $\times 90$ diams.-(After M. Fürbringer.

phibians it replaces the pronephros, which is purely a larval structure. It is present in the embryos of all amniota, but undergoes a partial degeneration before adult life, being itself replaced in adult amniota by the true kidney. The mesonephros resembles somewhat the pronephros, especially as found in the ichthyopsida. It occupies a much larger region of the body than the pronephros. It has no glomus associated with it, but each tubule contains a glomerulus very similar in its general structure to the glomerulus of a true kidney. In the ichthyopsida each tubule begins with a ciliated funnel, and, after making several coils, opens into the pronephric duct. In the amniota the mesonephros, or, as it is more commonly called in these animals, the Wolffian body, is essentially an embryonic structure. Its tubules, however, do not have at any stage the ciliated funnels to be found in amphibia and fishes, but they have glomeruli and they open into the pronephric 
duct, which, on account of its relations to the organs is in this type more commonly spoken of as the Wolffian duct. The circulation of the organ is sinusoidal. Further details are given in the practical part in connection with study of the pig embryos, pages $2 \dot{5}^{2}$ and 306 .

The third of the excretory organs is termed the metanephros, or true kidney. In the mammalian embryo, after the Wolffian body has acquired a considerable development, there appears a small outgrowth of the Wolffian duct at a point near the junction of the duct with the allantois. It extends dorsad and cephalad (Fig. I $72, k i$ ), and may be termed the renal evagination. Its blind end expands to become the pelvis of the kidney, while its stalk remains narrower and is converted into the ureter, by which the urine is conveyed from the pelvis to the bladder (allantois). By outgrowths of the epithelium of the pelvis the collecting tubules are developed. Around the pelvis appears an envelope of special cells, easily recognized by their darker staining (Fig. 210). These cells are thought to be derived from the nephrotomes of the segments of the renal region, which have lost their epithelial arrangement and have migrated to form the envelope. The cells gather themselves gradually into small clumps, the number of clumps continuing to increase during a long period. Each clump assumes an epithelial arrangement and acquires a lumen and elongates into a tubular form. The tubule elongates, one end joins a collecting tubule, and the lumina of the two structures become continuous. The other end of the tubule remains closed and is converted into the renal corpuscle. The tubule now grows rapidly in length and becomes very irregular, in its course; the part which joins the collecting tubule becomes the proximal convoluted tubule; the part which joins the renal corpuscle becomes the distal convoluted tubule, and the middle part between these two becomes the loop of Henle.

\section{The Urogenital Ducts.}

The genital and excretory organs always develop together and constitute the urogenital system. The genital glands are always distinct, but the primary excretory or Wolffian duct, after producing the outgrowth to form the renal anlage (p. 309), is transformed into the permanent male duct. The other primary canal is termed the Müllerian duct. It is exclusively genital, for with its mate it develops the uterine tubes, the uterus, and the vagina, but in the male it becomes vestigial.

The Wolffian duct is the excretory canal of the mesonephros (p. Iog). It extends along the ventral surface of the organ; the mesonephric or Wolffian tubules acquire openings into it, and it itself opens at its caudal end into the base of the allantois. It serves for a time as a true excretory duct, but loses this function when the mesonephros degenerates. It meanwhile acquires a second cony connection with the testis by means of some of the Wolffian tubules in the neigitborhood of the genital gland. The tubules in question acquire a connection with the sexual cords of the testis and, when the cords become seminiferous tubules, the Wolffian tubules are ready to conduct the semen to the Wolffian duct (vas deferens). The 
course of the Wolffian duct is changed during fetal life by the migration of the testis from its original abdominal position into the scrotum. In the female the Wolffian duct becomes vestigial.

The Müllerian ducts develop much later. They may be found in the I $2 \mathrm{~mm}$. pig as two short funnels formed by the mesothelium and situated on the ventral side of the mesonephros near the septum transversum (diaphragm). The funnels point backward and grow into tubes, which run on the ventral side of the Wolffian duct and presently connect with and open into the base of the allantois. The pelvic portions of the two Müllerian ducts approach one another in the median line and in the female they fuse, making a median epithelial canal, the anlage of the uterus and vagina. The original entrance to the canal persists as the fimbriate opening and the stretch of the original canal between the funnel and the uterus becomes the uterine tube.

\section{The Allantois.}

The allantois is a diverticulum of the entodermal canal, and is, therefore, lined by entodermal epithelium (Fig.,2r). It arises on the ventral side of the caudal end of the embryo in proximity to the anal plate. In its development we can distinguish two main types. The first type is illustrated by the sauropsida and the ungulates. In them it grows out and rapidly enlarges so as to form a vesicle of considerable size and connected with the embryo by means of a narrow hollow stalk. When the allantois develops according to this type, it is spoken of as free, because it has no connection with the extra-embryonic somatopleure (chorion and amnion). This form of the allantois may be readily observed in chick embryos, for by the fourth day it has become a considerable rounded vesicle which lies in the extra-embryonic colom between the yolk-sac and the extra-embryonic somatopleure or membrana serosa. During the fifth day it rapidly enlarges, and at the beginning of the sixth day is nearly or quite as large as the head of the embryo. In ungulates the growth of the free allantois begins very early and becomes enormous. Its principal expansion is sideways, that is to say, at right angles to the axis of the embryo, and it becomes a large sac, very much larger, indeed, than the entire embryo.

The second type of allantois occurs in the placental mammals of unguiculate series and is not known to occur in any species of the ungulate type. In probably all unguiculates the posterior end of the body has a prolongation which is known as the body-stalk (Fig. 69, b.s). Into this body-stalk the diverticulum constituting the allantois extends (Fig. 80, All, and Fig. 25, All). The entoderm of the allantois is surrounded by mesoderm, which is present in the body-stalk in considerable volume. On the outer surface there extends a layer of ectoderm, so that the three germ-layers enter into the formation of the body-stalk as they do into the formation of the embryo. These relations are illustrated by the diagram (Fig. 64). By means of the body-stalk a connection is established between the embryo and 
the extra-embryonic somatopleure or primitive chorion, Cho. Later, when the formation of the amnion is completed, the essential relations are found to be as illustrated by the diagram (Fig. 64, B). The amnion arises from the distal end of the body-stalk, but the body-stalk retains its connection with the chorion. When the allantois becomes free, the connection with the chorion is entirely lost. The maintenance of that primitive connection in the unguiculates is to be regarded as a new modification of the relations of the embryonic appendages, evolved only in the higher animals. The maintenance of

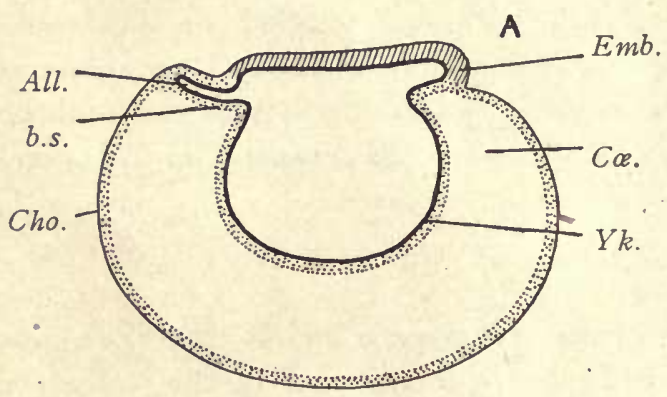
that connection makes possible the modification in the structure of the chorion, which is of the greatest morphological importance. This modification is the development of the blood-vessels in the chorion. The anlages of these bloodvessels are outgrowths of the embryonic angioblast. They appear so as to form four vessels which grow through the length of the body-stalk in the neighbor-

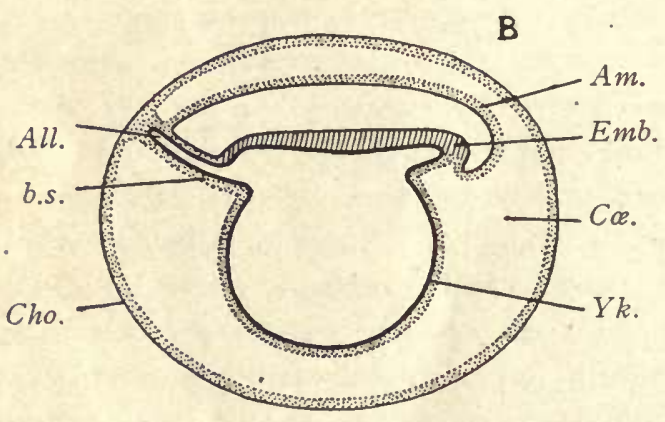

Fig. 64.-Diagrams Illustrating the Relations of the Allantois in Unguiculate Mammals.

A, Before, B, after the formation of the amnion. All, Entodermal allantois. Am, Amnion. b.s, Bodystalk. Cho, Chorion. $\mathrm{C} \mathfrak{B}$, Extra-embryonic coelom. Emb, Anterior end of embryo. $Y k$, Yolk-sac. hood of the allantoic diverticulum. Two of these vessels are veins and two are arteries. They are termed the umbilical vessels. The umbilical veins at the embryonic end of the body-stalk enter the somatopleure of the embryo (Fig. I86, V.U.S., V.U.D), through which they make their way toward the heart (Fig. 93, Alv). The umbilical arteries enter the body of the embryo, pass caudad alongside the allantois (Fig. 210 , A.um), curve past the cloaca onto the dorsal side of the body (Fig. I69, A.um), and join the caudal end of the aorta, so that they may be termed the terminal branches of the embryonic aorta. In early stages they are the largest branches which the aorta has. At the distal end of the body-stalk the four vessels enter the mesoderm of the chorion, there branch abundantly, and produce a rich network of blood-vessels throughout the entire membrane. The unguiculate mammals, therefore, are characterized by this special feature, the possession of the body-stalk which contains the allantoic diverticulum and gives access for the blood-vessels, and therefore also, of course, for the blood, to the chorion, which thus becomes vascular. In all other amniota the chorion is without blood-vessels. 
The size of the allantoic cavity in unguiculates varies considerably. In man it is minimal, forming only a long and very narrow tube (compare Fig. 66, All). In rodents it expands somewhat, but it never becomes free in the sense that it is separated from the body-stalk, although it may acquire a partial independence. In this case it may also become more or less vascular by the development of branches from the umbilical arteries and veins around the allantois.

In those animals in which the allantois is free, the umbilical arteries and veins have all their branches in the allantois, there being no body-stalk. The embryo is without connection with the chorion, and, therefore, these vessels in their ramifications are restricted to the allantois.

Relations of the Allantois to the Chorion in Ungulates.-Since the true chorion is the outermost of the fetal envelopes, it alone can come in contact with the walls of the uterus. All placental developments, therefore, necessarily depend upon the chorion. Now, in ungulates, where the chorion is without blood-vessels, there is no circulatory apparatus to transfer any nutritive material, which may be taken up by the chorion from the uterus to the embryo, until a second union takes place between the vascularized allantois and the chorion. The inner surface of the chorion and the outer surface of the allantois are both mesodermic. The two mesodermic layers come into contact with one another and unite loosely. The vessels of the allantoic mesoderm are thus brought into physiological union with the chorion, but, being allantoic vessels, they are, of course, morphologically different from the chorionic vessels of unguiculate mammals. These considerations demonstrate that the ungulate placenta is allantoic rather than chorionic, and is, morphologically speaking, essentially different from the true chorionic placenta, which can be developed only in those animals and embryos which have a permanent body-stalk.

The simple relations of the chorion in the Ungulata to the uterine wall is illustrated by the accompanying figure 65 , which shows a portion of the chorion of a pig embryo of $\mathrm{I}_{5} \mathrm{~mm}$., together with the surface of the uterus to which it was fitted. The two membranes were accidentally separated in the preparation. The chorion consists of a layer of cylinder epithelial cells, $E c$, each of which can be distinctly made out, and of a layer of mesoderm, Mes, containing only few cells and blood-vessels, two of which, $V e$, are shown in the section; the mesodermic cells are a little more crowded near the epithelium. Each ectodermal cell is distinctly marked off from its neighbors by a line. The protoplasm stains somewhat; the nuclei are slightly oval and granular, and are situated near the middle of the cells. The top of each cell is concave. The uterine epithelium, Ut.Ep, resembles in the general form of its cells and in the character of its protoplasm the chorionic ectoderm, but differs from it in that each cell has a convex free end, and, further, in that the nuclei of the cells are situated near the top of the layer. When the relations of the two epithelia have not been disturbed, it is readily observed that the concavity of each chorionic ectodermal, cell receives the convex 
end of the uterine epithelial cell, so that the two layers are closely fitted together, cell for cell.

The Bladder. - The allantois extends from the cloaca to the umbilicus, and beyond the umbilicus into the umbilical cord. It comprises, therefore, an embryonic and an extra-embryonic portion. The former is the anlage of the urogenital sinus, the urethra, and the bladder. The embryonic portion is always united to the abdominal wall (Fig. 210), the mesenchyma, which surrounds the entodermal allantois, $A l l$, and the umbilical arteries, S.um, being fused in the mid-ventral line
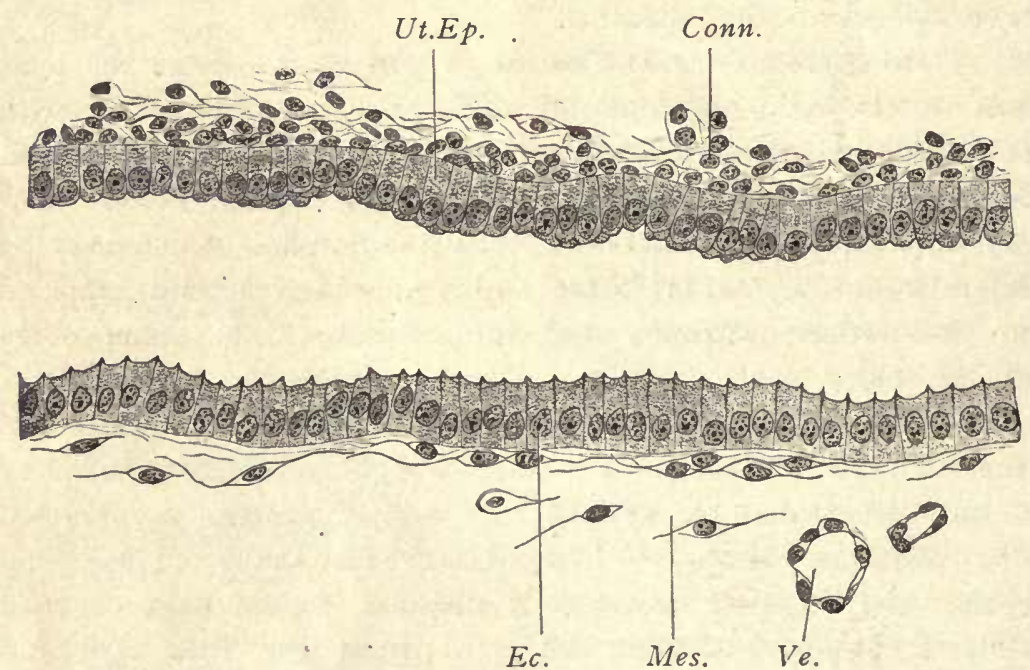

Fig. 65.-Pig, i5.o mar., Series i35, Section 58, to Show the Relations of the Chorion to the Uterus. Conn, Connective tissue of the uterus. Ec, Chorionic ectoderm. Mes, Chorionic mesoderm. Ut.Ep, Uterine epithelium. Ve, Chorionic blood-vessel. $\times 350$ diams.

with the mesenchyma of the somatopleure. This connection is retained throughout life. The opening of the Wolffian ducts into the allantois is established very early. The ureters at first open into the Wolffian ducts, but they soon migrate so as to open separately directly into the allantois (bladder) above the ducts.

\section{The Trophoderm.}

Trophoderm is the name applied to the special layer of cells developed on the outer surface of the ectoderm of the mammalian blastodermic vesicle. It has as yet been observed only in unguiculates. The trophoderm layer may be developed over the entire surface of the ovum, as in man, or over only a portion thereof, as in the rabbit and cat. Its principal known function is to destroy the tissues of the uterus of the mother with which it comes in contact. The destruction of the tissue is supposed to serve two purposes: First, to supply nutrition to the embryo. It is from this supposed function that the layer derives its name of 
trophoderm. Second, to secure the attachment of the ovum to the wall of the uterus. This preliminary attachment is called the implantation of the ovum. In some cases the trophoderm is developed very early over the surface of the ovum (Fig. 74), appearing almost as soon as the stage of the blastodermic vesicle is reached, and while the vesicle is very small. In such cases the ovum creates a space for itself by dissolving away the epithelium and connective tissue at a small spot on the uterine surface, making a cavity in which the ovum lodges. In other cases the trophoderm is developed later and does not appear over the whole of the blastodermic vesicle. The area over which it exists in such cases is called the placental area (compare pages $\mathrm{I} 27$ and $\mathrm{179}$ ). The trophoderm in these forms unites very closely indeed with the surface of the uterus (Fig. 37, Tro) and the uterine tissues undergo degeneration and resorption. We may regard as the first step toward the production of the placenta proper the disappearance of the trophoderm. Our knowledge of its disappearance is incomplete, but it is probable that it is due to a transformation of the cells of the trophoderm, associated with contemporaneous modifications of the chorionic membrane, of which the general result may be said to be formation of the chorionic villi which constitute the fetal protion of the placenta. The modified trophoderm cells are supposed to enter into the formation of the ectodermal covering of these villi.

\section{The Umbilical Cord.}

The umbilical cord may be best defined as the tissues connecting the body proper of the embryo with the amnion. It accordingly includes a portion of the body-stalk and a certain extent of the body-wall or somatopleure. In early stages we can hardly speak of an umbilical cord, because the amnion arises close to the embryo (Fig. 83). As development progresses the body-stalk lengthens out and the amnion arising from it recedes farther and farther from the embryo, this recession being assisted by a growth of the somatopleure which leads to the formation of the umbilical cord proper. By this means a tubular structure is produced, the cavity of the tube being a prolongation of the cœlom of the embryo. During the first develfopment of the umbilical cord the neck of the yolk-sac becomes constricted and very much lengthened out, forming the yolk or vitelline stalk. The yolk-stalk springs within the embryo from the wall of the intestine, runs through the cœlom of the umbilical cord, and makes its exit beyond the amnion, as shown in figure 102. The yolk-sac proper still occupies its original position between the amnion and chorion. The student should note carefully that the umbilical cord is never covered by the amnion, for it has unfortunately been often stated that it is so covered. An idea of the relations can be gathered from cross-sections of the cord (Fig. 66). The colom, $C \propto$, is a large cavity and contains the yolk-stalk, $Y$, with two blood-vessels, but with its entodermal cavity entirely obliterated. Above the body-cavity is the duct of the allantois, All, lined by entodermal epithelium, and in 
its neighborhod are two arteries and a single vein. In yet earlier stages there are two veins. The outer surface of the section is bounded by ectoderm. The further development of the cord depends upon the growth of the connective tissue and blood-vessels, the abortion first of the cœlom, later of the yolk-stalk, and lastly of the allantoic duct. Remnants of the allantoic epithelium are, however, often found in the umbilical cord even at birth. There occurs also a further differentiation of the connective tissue and of the entoderm.

The umbilical cord is characteristic of mammals. It varies greatly in length. In the pig it is very short. In man it attains great length and size, becoming at full term about $55 \mathrm{~cm}$. in length and $12 \mathrm{~mm}$. in thickness. When fully developed the human cord has a whitish color and presents a twisted appearance somewhat
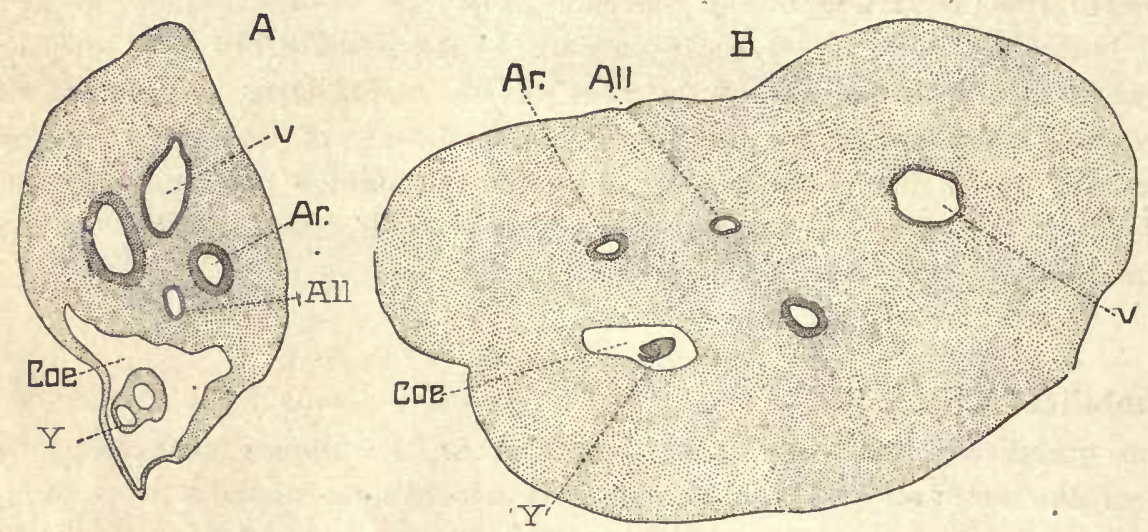

Fig. 66.-Sections of Two Human Umbilical Cords.

A, From an embryo of $2 \mathrm{r} \mathrm{mm}$.; B, from an embryo of sixty-four to sixty-nine days. All, Allantois. Ar, Umbilical artery. C $\propto$, Colom. v, Umbilical vein. $Y$, Yolk-stalk.

like a rope. Its surface is smooth and glistening. The attachment of the cord to the embryo is known as the umbilicus. Its attachment to the chorion is in the placental region (chorion frondosum).

The twisting of the cord is well marked externally at the time of birth by the spiral ridges, within each of which a large blood-vessel runs. The number of spirals varies from 3 to 32 , the turns beginning at the embryo, and, though usually from left to right, are sometimes from right to left. The twisting begins about the middle of the second month. Its cause is unknown, but there is no reason to assume that it is due to revolutions of the embryo. The cord is covered by a layer of epithelium which is continuous at the distal end with the epithelium of the amnion, and at the proximal end with the epidermis of the embryo. The cord contains typically no capillaries, and, except in the immediate neighborhood of the embryo, no nervefibers. 


\section{The Chorion and Amnion.}

These are two membranes which always surround the embryo, the chorion being the outer, the amnion the inner, membrane of the two. Morphologically, they are modifications of the extra-embryonic somatopleure. The accompanying diagrams render this clear. In figure 29 , we see that the cavity of the mesoderm, coe, has extended completely around the yolk. There is a layer of mesoderm, represented by a dotted line, on the outside of this cavity, which joins with the overlying ectoderm, represented in the diagram by a continuous line, to constitute the somatopleure, Som. In figure 45, we see the somatopleure folded up on the dorsal side of the embryo; the leaf of the fold nearest the embryo is the anlage of the amnion, am; the rest of the extra-embryonic somatopleure is the anlage of the chorion- In the second figure of the diagram, the two folds have met above the embryo and united, thus making a closed inner amniotic and a closed outer chorionic envelope. The actual appearances of two such stages as in the diagram are illustrated by figures 38 and 47 . By this account we learn that the two envelopes are produced by a folding of the somatopleure.

When we come to study the development of mammals in detail, we discover that there are many remarkable variations in the early development of the amnion of which no general explanation is yet possible; but inasmuch as the folding process is the only one in Sauropsida, and also occurs in many mammals of different classes, it is generally assumed to be the primitive method.

In man the development of the chorion and amnion differs extremely from the scheme given above. It is described as accurately as our present knowledge permits in Chapter III.

However developed, the fetal envelopes present certain constant characteristics: both consist of ectoderm and mesoderm, but in the case of the amnion the ectoderm is turned toward the embryo, whereas the chorionic ectoderm faces the outside. The cavity between the amnion and the embryo becomes filled with the amniotic fluid, which serves as an important mechanical protection to the developing embryo. It is through the chorionic ectoderm only that the ovum can come into actual contact with the walls of the uterus. It is the chorion alone which is concerned in the formation of the true placenta (compare Chapter VII).

The amnion is a thin, pellucid, non-vascular membrane; the chorion is thicker, more nearly opaque, and has in man and all nearly related animals a highly developed vascular system. 
CHAPTER III.

\section{THE HUMAN EMBRYO.}

Our knowledge of the early stages of human development is very imperfect. Upon the fertilization and segmentation of the ovum in man there are no observations whatever at present. It is not even known exactly how long the ovum requires for its passage through the Fallopian tube. The earliest stages of which we have comparatively adequate accounts are those represented by Peters's ovum (I899) and Herzog's (I909). A number of human embryos in various early stages after the formation of the medullary canal and up to the stage with four aortic arches have now been reported and studied, some few of them thoroughly and carefully.

\section{Calculation of the Age of the Human Embryo.}

The age of the embryo must be reckoned from the date of the fertilization of the ovum, which presumably occurs in man in the upper third of the Fallopian tube. It may be that ova become fertilized at various epochs, but fail to continue their development except when the fertilization occurs at the beginning of a menstrual period. Ovulation occurs at all periods, but most frequently about the time of menstruation, which is the expression of structural changes in the uterus which enable the ovum to implant itself in the uterine wall. Hence only when fertilization coincides with the beginning of menstruation can conception follow with the result that the menstrual flow is stopped. Accordingly, the age of the embryo is usually to be reckoned from the date of the beginning of the first menstrual period which has lapsed.

Experience, however, shows that sometimes conception occurs without stopping the menstrual change at the time, but eliminating only the subsequent periods, and in such cases the age must be estimated from the beginning of the last menstruation. In the two cases the age of the embryo would differ by a month (twenty-eight days), and this difference is so great that it obviates errors of estimate.

Up to the end of the ninth week the form and size of the embryo exhibit a correlated development, so that generally an embryo at a given stage of development in form will agree with its fellows in size; but to this rule there are not infrequently exceptions, and sometimes an embryo is found much larger than others 
at the same stage. Moreover, the variability of embryos is very great, for in specimens otherwise alike we find this or that organ advanced or retarded in its development as compared with the embryo as a whole. Nevertheless it is possible with the information at command to determine with tolerable certainty the age of an embryo within two days plus or minus, up to the end of the ninth week. For the course of development during the third month we possess as yet no satisfactory data, but embryos of full three months are quite frequently obtained, and are very characteristic in size and configuration (see page 156 ).

F. P. Mall's formula for calculating the age of human embryos is

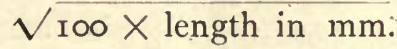

The length is measured from the vertex to the breech.

\section{The Classification of the Early Stages.}

Any attempt to divide embryos into stages must necessarily establish artificial groups, for in nature there is no demarcation. Division into stages is for convenience, and ought, therefore, to be made by natural and obvious characteristics. It seems to me that eleven stages may be conveniently discriminated, as follows:

First Stage.-Segmentation of the Ovum: The general process is described on pages 42 to 45 . There are no observations upon this stage in man, or any primate, except one monkey's ovum in the four-cell s age described by Selenka.

- Second Stage.-Blastodermic Vesicle: The general development of the blastodermic vesicle in mammals is described on page 45. Its development in man is unknown. During this stage the embryonic shield is differentiated. An ovum of a monkey in this stage is described on page 127 , and one of the very few known human ova is described on page 128 .

Third Stage.-Primitive Streak: Two human ova with a primitive streak before the formation of the medullary plate have been observed. ${ }^{1}$ In one of these, Frassi's embryo, the diameter of the entire ovum was $\mathrm{r} 3 \times 5 \mathrm{~mm}$. The diameter of the yolk-sac $1.9 \times 0.9 \mathrm{~mm}$. The embryonic shield was $1.17 \mathrm{~mm}$. long by 0.6 mm. wide. The primitive groove is shallow and occupies about half the length of the shield. The anterior end of the groove marks the position of the future neurenteric canal; its posterior end, the position of the anal plate.

Fourth Stage.--The Medullary Plate: In this stage there are several embryos known. In all of them the amnion and chorion are already differentiated. There is a large extra-embryonic cœlom. The chorionic vesicle is rounded and somewhat flattened. In its greatest diameter it measures from 8 to $10 \mathrm{~mm}$. It is beset with short branching villi which are present over the entire surface. The general relations are indicated in the accompanying diagram (Fig. 69). The chorion has a distinct epidermal and mesodermal layer. To its inner surface is attached the body-stalk wihch unites the embryo and chorion. From it springs the amnion

\footnotetext{
${ }^{1}$ The Harvard Embryological Collection has an embryo, Series 825 , in fine preservation. It is a little younger than Frassi's. It is hoped to publish an account of it soon.
} 
covering the embryo, which measures only I.O to $1.5 \mathrm{~mm}$., and from the ventral surface of the embryo arises the yolk-sac, which is of rounded form and about equal in diameter to the length of the embryo.

Fifth Stage.-The Medullary Groove: The general relations of the embryo and its appendages are the same as in the previous stage (compare Figs. 82 and 25). In the cases recorded the chorionic vesicle varied greatly in size. It bore villi over its entire surface, and the villi were considerably branched. The embryos of this stage vary in length, but measure about $2.0 \mathrm{~mm}$. The medullary ridges are

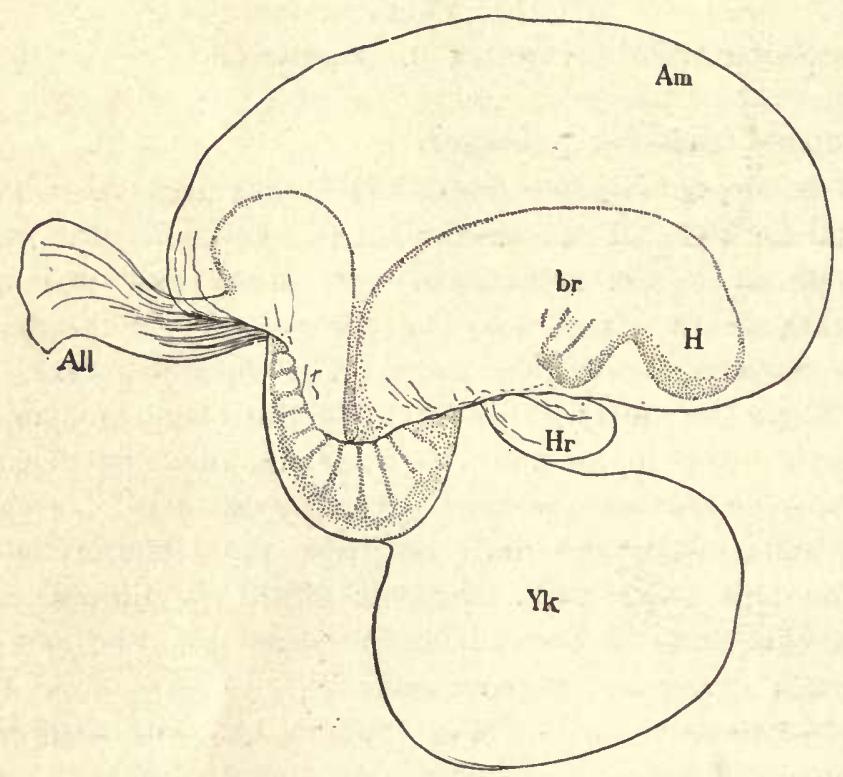

Fig. 67.-Human Embryo at the Beginning of the Third Week.-Eighth Stage. $A l l$, Allantois. Am, Amnion..$b r$, Branchial region. $H$, Head. $H r$, Heart. $Y k$, Yolk-sac.

very characteristic, rising high above the yolk-sac and enclosing a deep medullary groove between them. During this stage the formation of the segments is progressing. Thus one of the embryos described had seven segments.

Sixth Stage.-Medullary Tube: In this stage the medullary groove is partly closed and the heart is clearly differentiated. It must be remembered that the closure of the medullary groove progresses slowly and is not completed until the ninth or tenth stage. The embryo measures from 2.2 to $2.5 \mathrm{~mm}$. in length. The head projects well in front of the yolk. The primitive segments are partly developed. In one case seven, in another thirteen, were found to have been formed. The caudal end of the embryo also projects beyond the yolk, but less than does the head (compare Fig. 83 ). The auditory invagination is probably not yet formed. There are no gill-clefts showing externally. 
Seventh Stage.-One Gill-cleft Showing Externally: Not known by observation. Eighth Stage.-Two Gill-clefts Showing Externally: Several embryos in this stage have been found and some of them accurately studied. They usually have a remarkable bend in the back (Fig. 67), which imparts to the embryo a very singular appearance. Nothing similar to this bend or dorsal flexure has been observed in any other embryos. It has been held by $\mathrm{His}$ and others to be a normal condition, and not the accidental result of a mechanical strain exerted by the yolk-sac. If the condition is normal, it must exist for only a very brief period, as it is not encountered in older or younger stages. We may suppose if it is normal that the change from the concave to the convex position of the embryo, as found in the next stage, is very abrupt. The head of the embryo (Fig. 67) shows the characteristic head-bend, and the tail end of the embryo is also bent over ventralward. The heart is large and very protuberant. It is bent so that we can clearly distinguish the auricular, ventricular, and aortic limbs. It shows distinctly its inner endothelial portion and outer mesoderm. The yolk-sac extends from the heart backward to where the body of the embryo turns to make the dorsal flexure. Between the heart and the head the oral invagination has been formed, but is still separated by the oral plate from the entodermic canal. Above the heart on either side is an open invagination of the ectoderm, the anlage of the so-called otocyst, which in its turn is the anlage of the epithelial labyrinth of the adult ear. In one embryo of this stage there were found twenty-nine primitive segments.

Ninth Stage.-Three Gill-clefts Showing Externally: This is, on the whole, the best known of the early stages of human development. The embryos described as belonging to it vary from 2.6 to $4.2 \mathrm{~mm}$. in length. In one of them, in which the embryo measured $3.2 \mathrm{~mm}$., the chorionic vesicle measured II by $\mathrm{I} 4 \mathrm{~mm}$., and its supposed age was from twenty to twenty-one days. The general shape of these embryos is indicated by figure 89 .

The head is bent down and the back is very convex. In figure 89 the tail is rolled up and turned to the left. Usually, however, the tail turns to the right and the head is twisted to the left, so that the long axis of the body describes a large segment of a spiral revolution; the spiral form is marked in embryos a little older.

Tenth Stage.-Four Gill-clefts Showing Externally: The internal gill-pouches reach the ectoderm, and for each there arises a corresponding external depressionthat of the fourth arch is often indistinct; hence this stage is more easily recognized by the beginning of the limb-buds. A good embryo near the end of this stage has been carefully studied by Broman.

Eleventh Stage.-Open Cervical Simus: The cervical sinus is formed by the invagination of the ectodermal area of the fourth and fifth and later also of the third gill-arches. The deep depression thus formed lasts for some time, but closes over ultimately (embryos of $10 \mathrm{~mm}$.). The eleventh stage comprises a relatively long period. 


\section{Hypothetical Development of the Blastodermic Vesicle in Primates.}

As there exist no direct observations on the earliest stages of man, we can only surmise what those stages may be. It is evident that there is a very precocious development of the mesoderm, of the extra-embryonic cœlom, of the amnion, and of the trophoderm, because these four features are found very marked in the earliest known stages alike of man, apes, and monkeys. There are certain rodents and insectivora in which these same peculiarities occur more or less emphasized in the earliest stages of which we possess knowledge. If we utilize these data as a basis, we can reconstruct the following hypothetical scheme of the earliest stages in man.

The accompanying diagrams (Figs. 68 and 69) represent three successive purely hypothetical stages of the human ovum. They are all conceived to represent longi-

A

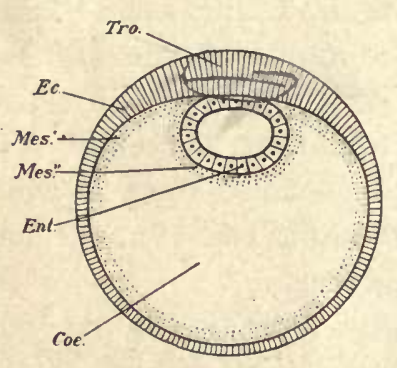

B

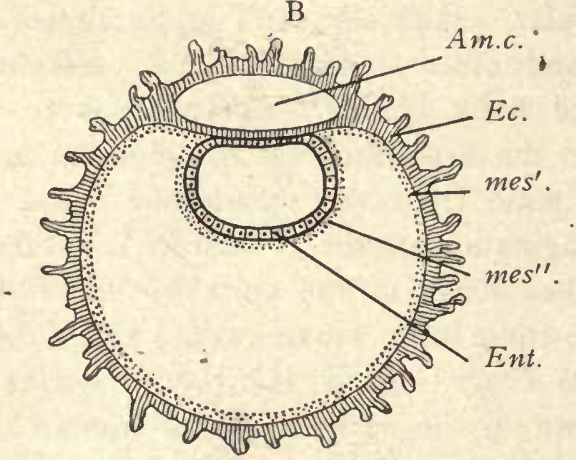

Fig. 68.- Two Diagrams to Illustrate the Hypothetical Early Development of Primates.

Am.c, Amniotic cavity. Ce, Cœlom. Ec, Ectoderm, in B, bearing the anlages of villi. Ent, Entoderm. Mes', Somatic mesoderm. Mes", Splanchnic mesoderm. Tro, Trophoderm.

tudinal sections. In the first stage the ectoderm, $E c$, forms a moderate sized vesicle and is already thickened. It should probably be conceived as consisting of an inner distinctly cellular layer and an outer much thicker trophodermic layer which is thickest over what corresponds to the embryonic region. This special thickening is marked Tro in diagram A. The entoderm, Ent, forms a small vesicle underlying the thickened portion of the trophoderm. The mesoderm, Mes, is well advanced in its development and already contains the large extra-embryonic cœlom, Coe, and is therefore divided into one layer which surrounds the entoderm, and a second layer which underlies the ectoderm. In other words, the splanchnopleure and somatopleure are already differentiated. In the next stage (Fig. 68, B) there has been a growth, the ovum has become larger, the trophoderm has increased in thickness, and in the mass of thickened ectoderm overlying the yolk-sac there has appeared a cavity - the future amniotic cavity-which is, of course, entirely surrounded by ectoderm. The portion of the ectoderm on the under side of this cavity consists of a single layer of cells which by assuming a cylindrical form constitutes the thickened area- 
which we can identify as the embryonic shield (compare Fig. I3 and Fig. 68, B). The solid mass of ectoderm above the amniotic cavity is later to form a part of the amnion and part of the chorion. At the posterior end of the embryo there appears a considerable accumulation of mesoderm (Fig. 69, b.s), which is the anlage of the body-stalk. Into this the entoderm has grown in the form of a cylindrical tubular prolongation, the anlage of. the allantois. As a consequence of the growth of the trophoderm and of the formation of the amniotic cavity, the embryo or embryonic shield, $E m b$, together with the yolk-sac, $Y k$, attached to it, has been forced down into the interior of the chorionic vesicle. This phenomenon is very marked in certain rodents and leads to the so-called inversion of the germ-layers. In the next stage the amnion is formed. This is accomplished by the penetration of the mesoderm with accompanying extension of the extra-embryonic cœlom into
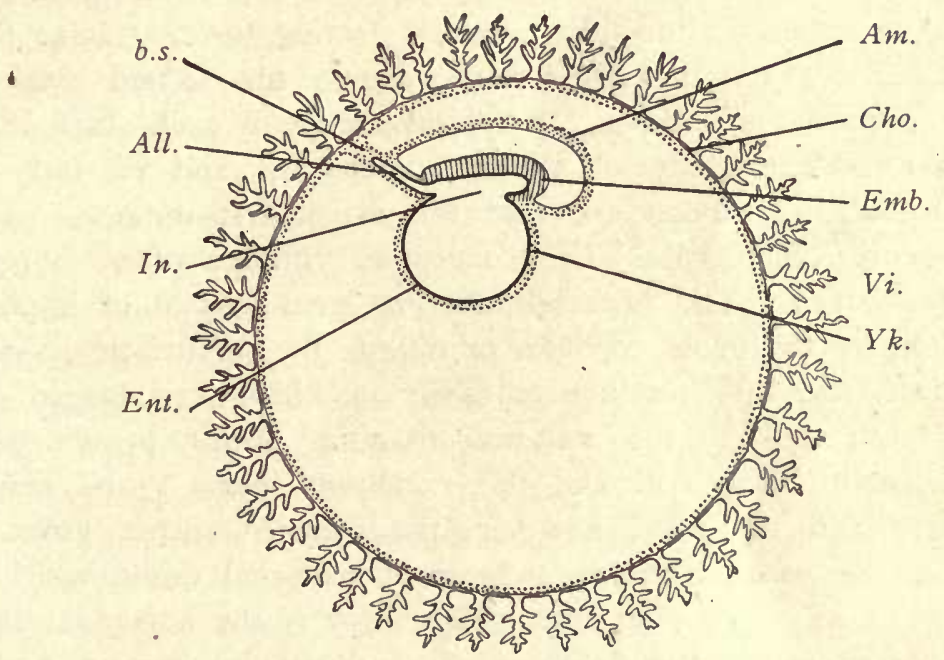

Fig. 69.-Diagram of an Early Stage of a Primate Embryo.

All, Allantois. Am, Amnion. b.s, Body-stalk. Cho, Chorion. Emb, Embryo. Ent, Entoderm. In, Entodermal cavity of embryo. Vi, Villi of chorion. $Y k$, Yolk-sac.

the mass of the ectoderm overlying the amniotic cavity (compare Figs. 68, B, and 69 ) until the condition shown in figure 69 is brought about. This stage is known by observation (compare Fig. 80). The amnion, $A m$, is now completely separated from the chorion, Cho, which forms a relatively large vesicle and consists of a thin layer of mesoderm, and a very thick layer of ectoderm, which has an inner cellular stratum and an outer very much thicker trophodermic stratum. The trophoderm is now very much altered by the appearance of numerous spaces or channels in it which develop so that each of these spaces ends blindly toward the interior of the chorion, but many of them are open upon the surface of the trophoderm. As the ovum at this stage is already embedded in the uterine mucosa, the channels in the trophoderm can receive maternal blood, and such is their original 
function. The embryo and yolk-sac, as compared with the chorionic vesicle, are very small in size. The body-stalk, b.s, is well developed and contains a wellmarked allantoic anlage, $A l l$, formed by the entoderm. The embryo includes as yet very little, if any, mesoderm. Probably a neurenteric canal exists at this stage. During the transition of stage B (Fig. 68) to stage C (Fig. 69), the blood-vessels appear in the mesoderm of the yolk-sac.

\section{Relations of the Embryo to the Uterus: the Two Stages.}

The Two Stages.-During the first half or perhaps five months of pregnancy the decidua reflexa is present. This period is called the first stage, to distinguish it from the remaining period, or second stage, during which there is no decidua reflexa. The reflexa during the first stage grows very thin and at the same time degenerates. It is finally resorbed. The exact date of its disappearance is not known, but falls somewhere in the fifth month. During the first stage the chorion læve is in contact with the decidua reflexa, during the second stage with the decidua vera. On pages 343 and 345 a typical uterus of each stage is described.

The First Stage.- The study of young human ova and of early stages of various primates leads us to conceive that the ovum first implants itself in the mucous membrane of the uterus. "The conception "implantation" is the outcome of very recent researches. The essential idea we have formed of implantation is that the trophoderm of the ovum corrodes or digests the uterine tissues with which it comes in contact, and thus produces a cavity in which it is lodged and where it attaches itself intimately to the maternal tissues. Owing to this process the ovum is at first partly uncovered, and this condition seems to be permanent in monkeys. In man and the apes, however, the uterine mucosa grows over the exposed portion of the ovum, forming a layer of maternal tissue which separates the ovum from the cavity of the uterus. This layer is the anlage of the decidua reflexa. As the ovum grows, the decidua reflexa must also expand, and we soon reach a condition in which the primitive relations of the parts can be easily followed.

When the uterus becomes pregnant, the mucous membrane of the organ undergoes changes in structure, and it is then commonly no longer termed the mucosa, but the decidua or caduca. The decidual membrane is histologically characterized by, first, modifications in the glands, the epithelium of which in large part degenerates; second, the transformation of a large number of the connectivetissue cells into cells of large size, which, on account of their being so extremely characteristic, are called the decidual cells, and, third, by a growth of its bloodvessels.

The decidual membrane of the uterus is divided into three regions: first, the decidua serotina, the area (Fig. $70, s, s$ ) to which the ovum is attached; second, the decidua vera, comprising all the remaining portions of the mucosa forming part of the walls of the body of the uterus; third, the decidua reflexa, the arching dome of 
maternal tissue, $r, r$, which rises from the walls of the uterus and completely encapsules the ovum. The arrangement of the parts is illustrated in figure 70 , which represents a median section of a uterus about five weeks pregnant. The whole uterus is considerably enlarged. The mucous lining of the uterus is very greatly thickened. The ovum is attached on the dorsal side of the uterus. This is the normal position. The diagrams so commonly met with which represent the insertion of the ovum at other points should not be accepted by the student. The reflexa rises around the ovum, completely covering it in so as to make a closed bag. The ovum itself is a sac known as the chorionic vesicle. The trophoderm has now quite disappeared, except so far as it persists to cover the villi. The villi themselves are shaggy and more or less branched. Their tips are united either with the surface of the decidua serotina or with that of the decidua reflexa. In the interior of the chorion is lodged the embryo with its yolksac and surrounded by the amnion.

If the walls of the uterus are cut through and simply reflected, leaving the bag of the decidua reflexa intact, the appearances will be found essentially as in figure 7I. The mucosa is enormously hypertrophied and contains a great many dilated irregular blood-sinuses. From the dorsal side of the organ is suspended a large closed bag or sac, the decidua reflexa; D.ref, nearly filling the cavity of the uterus. The reflexa presents in the stage figured the same general appearance as the surface of the uterus. If the reflexa be open, we

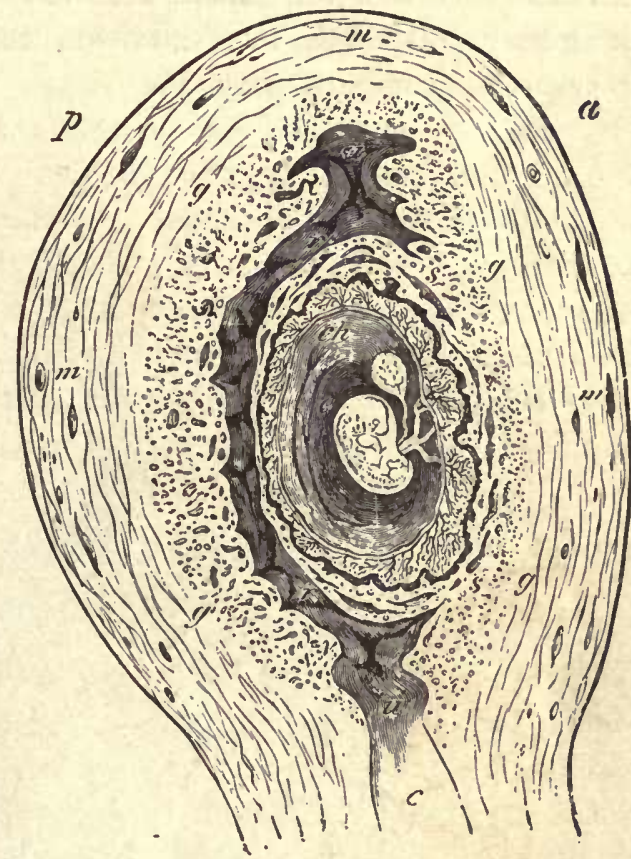

Fig. 7o,-Semi-diagramiatic Outline of an ANTERo-posterior SECTION OF a HuMaN Uterus Containing an Embryo of about Five Weeks.

$a$, Anterior, $p$, posterior surface. $g$, Outer limit of the decidua. $s, s$, Limits of the decidua serotina. $c h$, Chorion, within which is the embryo enclosed by the amnion, and attached to the chorion by the umbilical cord; from the cord hangs the pedunculate yolk-sac, $r, r$, Decidua reflexa. c, Cervical canal.-(After Allen Thompson.) come, of course, upon the villous chorion of the ovum, and find, as above stated, that only the tips of the villi are united with the surface of the reflexa. In the fresh state the decidua is reddish gray, spongy or pulpy, soft, and moist. After the fourth month it acquires, especially in the superficial layers, a duller brownish color, which subsequently becomes more marked. This coloration is due to the decidual cells. During the first two or three months the scattered openings of the uterine glands can still be distinguished over the surface of the serotina and vera. The surfaces themselves of the vera and reflexa, though somewhat irregular, remain 
more or less smooth. The inner surface of the reflexa is more irregular and has protuberant parts united with the tips of the chorionic villi. The surface of the decidua serotina, on the contrary, becomes very irregular during the progress of pregnancy, forming little mounds which may become so high as to resemble columns or so broad as to constitute septa. In later stages the septa become very well developed, attaining a height of from 5 to $\mathrm{I}_{5} \mathrm{~mm}$. "They are irregularly disposed, but subdivide the placenta of later stages into the so-called cotyledons (compare page 362 ).

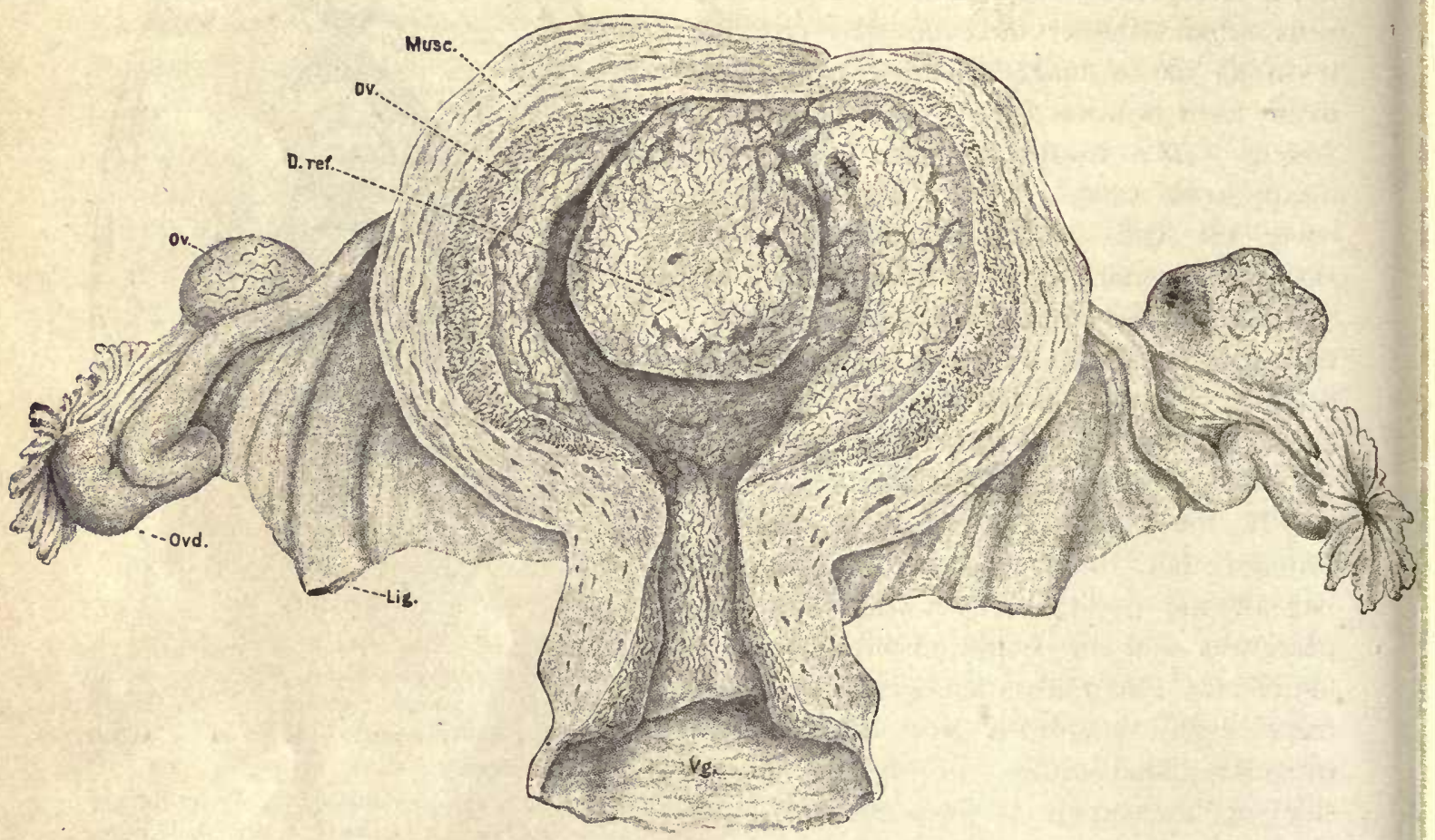

Fig. 7 1.-Human Uterus, about Forty Days Advanced in Pregnancy.

Musc, Muscularis. Dv, Decidua vera. D.ref, Decidua reflexa. Ov, Ovary. Ovd, Oviduct (Fallopian tube). $\mathrm{Lig}$, Round ligament. $\mathrm{Vg}$, Vagina. The uterus has been opened by cutting through the anterior walls and reflecting the sides. - (After Coste. $)$

The Second Stage.-The body-stalk becomes converted into the umbilical cord. This cord runs from the body of the embryo to the chorion (Figs. 70 and 87). It is always connected with that portion of the chorion which is adjacent to the decidua serotina. It carries the arteries and veins from the body of the embryo to the chorion. From the end of the umbilical cord the blood-vessels branch out over the chorion and into the chorionic villi. Thus the chorionic circulation of the embryo centers about the chorionic end of the umbilical cord, and, as this end is in the part of the chorion overlying the decidua serotina, we have here established from 
the very start an important factor in the further differentiation. From what has been said it is evident that the portion of the chorion underlying the decidua reflexa is more remote from the center of the embryonic circulation. In the same way we find that the decidua reflexa is remote from the blood supply in the uterus, and, as a matter of fact, we may observe that during the second month of pregnancy the blood-vessels, both in the decidua reflexa and in the portion of the chorion near it, begin to disappear and ultimately are completely atrophied. After this atrophy has been accomplished the circulation of the chorion is restricted to that portion overlying the decidua serotina. When the blood-vessels of the chorion under the decidua reflexa abort, the villi also abort, so that this part of the chorion becomes smooth, and is, therefore, called the chorion lave. Over the serotina the villi continue to grow, hence the corresponding region of the chorion becomes known as the chorion frondosum. The chorion frondosum constitutes the fetal portion, the decidua serotina the maternal portion, of the permanent placenta. The maternal blood circulates in the intervillous spaces, which are bounded by fetal ectoderm. The fetal blood circulates in the fetal blood-vessels of the chorionic villi. The circulatory channels of mother and fetus are always distinct, and no mingling of the maternal and fetal blood is possible under normal conditions.

\section{Ovum of a Monkey in the Second Stage.*}

This embryo was obtained from a Semnopithecus nasicus in Borneo by Selenka, who has also described an almost identical stage of $S$. pruinosus. It rested against the wall of the uterus and was uncovered, there being no decidua reflexa developed in monkeys. It measured about $2 \mathrm{~mm}$. in its greatest diameter. Figure $7^{2}$ represents a section through the ovum and adjacent tissues of the uterus. The chorionic , vesicle is very large, but the embryo, $S h$, and yolk-sac, $Y k$, are relatively very small. The chorion on one side is quite smooth; on the opposite side it has developed numerous outgrowths, most of which are formed exclusively of the ectoderm, but a few contain an ingrowth of mesoderm in their interior. The ectoderm on the side toward the uterus has two layers, an inner cellular layer with relatively small nuclei, and an outer syncytial or trophodermic layer with larger. nuclei of variable size. The ovum occupies a depression on the surface of the uterus from which the uterine tissues have disappeared, with the result of breaking through the walls of some of the blood-vessels, bl.lac, so that now the maternal blood may escape from these vessels into the spaces left between the irregular outgrowths and the embryonic chorion. We must assume that the trophoderm of the embryo has actually dissolved away or digested the tissues of the uterus, thus providing an attachment for the ovum, securing its embedding in the wall of the uterus, and establishing an opportunity for the maternal blood to flow into the intervillous spaces. In later stages of the primates the trophoderm is very much reduced, and therefore

* Compare Classification of Stages, p. r19. 
fulfills its functions in the very earliest stages by establishing these primitive conditions of blood-supply.

A section of the embryo on a larger scale is shown in figure 73. There appears only the embryonic shield, $S h$, which is remarkable for its small area and great thickness. The yolk-sac is also very small and is lined by a distinct layer of entoderm, Ent. Above the embryonic shield is the amniotic cavity, which is, of course, bounded by ectoderm which is continuous with the ectoderm of the embryonic shield. The amniotic cavity has a curious extension into the body-stalk; b.s,

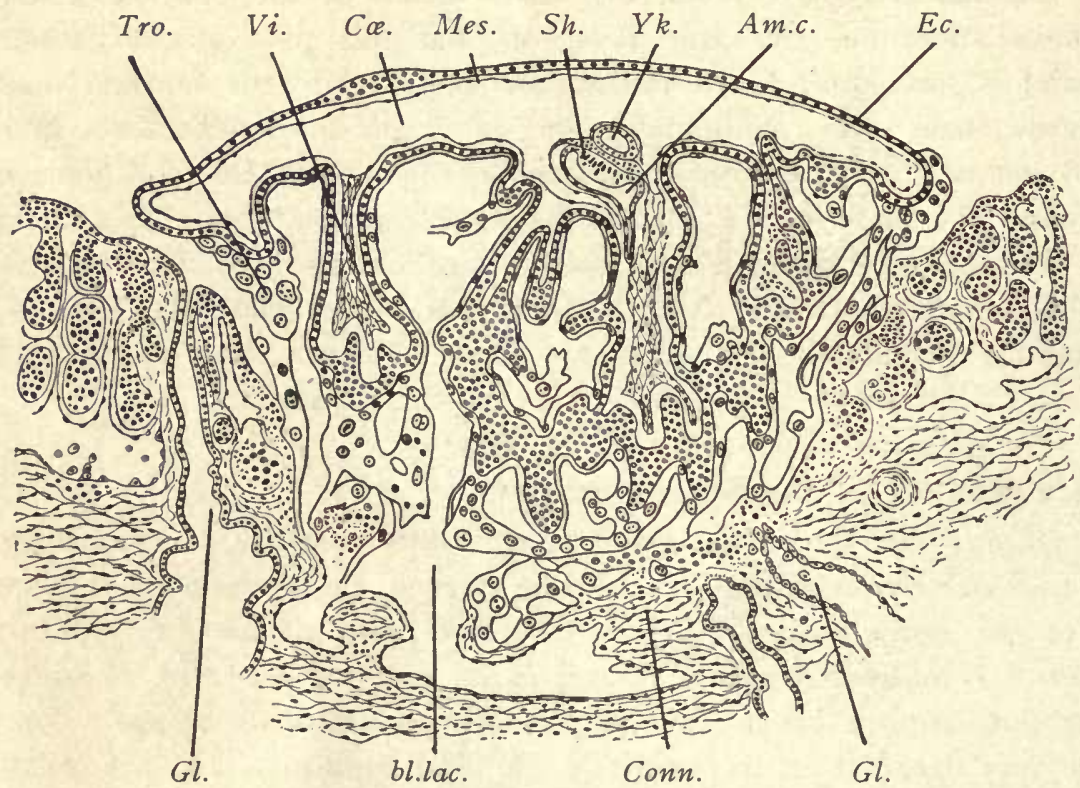

Fig. 72.-Blastodermic Vesicle of a Monkey (Semnopithecus nasicus) Attached to the Uterus; Vertical. SECTION.

Am.c, Amniotic cavity. bl.lac, Blood-lacuna. Cre, Extra-embryonic coelom. Conn, Connective tissue of the uterus. Ec, Ectoderm. 'Gl, Gl, Uterine glands. Mes, Mesoderm of embryonic chorion. Sh, Embryonic shield. Tro, Trophoblast. $V i$, Mesodermic core of a chorionic villus. $Y k$, Yolk-sac.-(After E. Selenka.)

by which the embryo is connected with the chorion. The mesoderm is chiefly developed over the chorion, as shown in figure 72 . It is very slightly developed in the embryo (Fig. 73, mes), but forms a layer over the yolk-sac and over the amnion, and forms a considerable mass of tissue to constitute the body-stalk, b.s.

\section{Human Embryo in the Second Stage.}

The embryo to be described was investigated by $\mathrm{H}$. Peters. It was found attached to the dorsal wall of a uterus almost completely embedded in the mucosa, but it was not wholly covered thereby, so that there was no decidua reflexa yet present. A blood-clot overlay what would have been otherwise the exposed portion of the ovum. The trophoderm formed an enormously thick layer of very 
irregular outline and contained many large spaces filled with maternal blood (Fig. 74). The exact external diameter of the ovum could not, therefore, be determined. It measured, however, approximately $2.4 \mathrm{~mm}$. by $1.2 \mathrm{~mm}$. The internal diameter of the chorionic vesicle was about $\mathrm{r} .6$ by $0.8 \mathrm{~mm}$. The trophoderm is everywhere intimately united with the uterine tissue. The embryo, $S h$, is represented by an embryonic shield consisting of cylinder cells. It is small and lies on the side of the ovum away from the cavity of the uterus. It rests upon the small yolk-sac, $Y k$, and is overlain by the amniotic cavity, $A m$. $c$, which is bounded everywhere by ectoderm - on one side, of course, that of the embryonic shield; on the

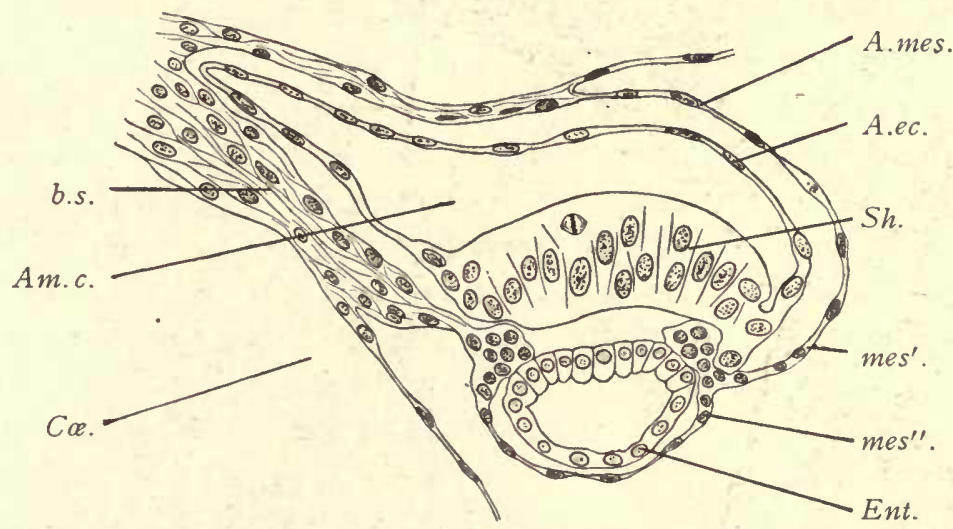

Fig. 73.-Embryo of the Preceding Figure More Highly Magnified.

Am.c, Amniotic cavity. A.ec, Amniotic ectoderm. A.mes, Amniotic mesoderm. b.s, Body-stalk. Ce, Extraembryonic cœlom. Ent, Entoderm. mes', Somatic, mes", splanchnic, mesoderm. Sh, Embryonic shield.(After E. Selenka.)

other the thin amniotic ectoderm proper. The mesoderm extends around the ovum, forming a layer underneath the chorionic ectoderm over the yolk-sac and above the amnion. At one point, close to the embryo and yolk-sac, it encloses a triangular space the meaning of which is not known. As indicated in the figure, the mesoderm was found to have shrunken somewhat, and the appearance of the embryo and yolk-sac also suggests a somewhat imperfect preservation, histologically speaking, of the tissues. As regards the condition of the uterus, the following points may be noted. In the neighborhood of the ovum the decidua vera had acquired a thickness of about $8 \mathrm{~mm}$., while on the opposite or anterior side it was only from 5 to $6 \mathrm{~mm}$. in diameter. Only in the immediate neighborhood of the ovum could there be seen any differentiation of the mucous membrane into an upper, more compact.layer, and a deeper, looser cavernous layer. The epithelium of the glands and the tissues of the uterus were well preserved, except in the immediate neighborhood of the ovum. The picture produces the impression that the ovum, in order to secure a place for itself, has completely destroyed the uterine tissues with which it has been in contact, thus implanting itself in the maternal tissue. As a 


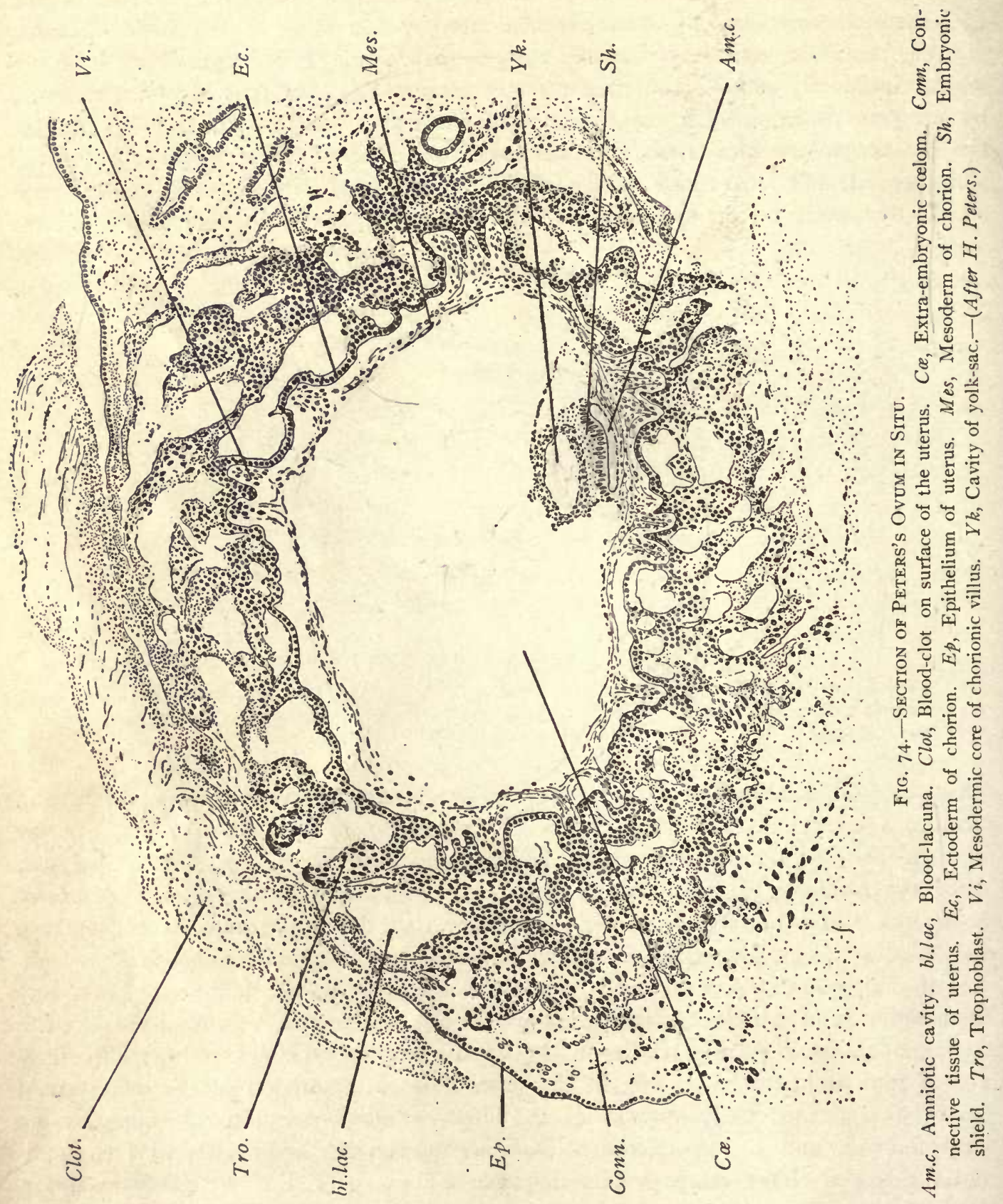


consequence of the destruction of the maternal tissues the walls of some of the blood-vessels have been broken through, and this has allowed the blood to escape from those vessels into the lacunæ of the trophoderm.

The trophoderm of the ovum offers a very complex picture, owing chiefly to the changes which it is undergoing. The changes are due apparently to hypertrophic degeneration. The layer of the chorionic ectoderm next to the mesoderm retains more or less evidently a cellular character. The remaining portions tend to form a syncytium in which the nuclei become enlarged and the cell-boundaries obliterated, while the protoplasm of the cells also changes in character and becomes more homogeneous in texture and much denser. The syncytium disappears by resorption, and its disappearance causes the formation of spaces in the trophoderm. Many different pictures occur in connection with these processes, for in some places the nuclei tend to gather in groups, in others they disappear; in some instances strands of degenerative material are left, while nearby some of the trophoderm may retain its more primitive appearance and be but slightly altered. Finally, it should be noted that at various points the chorionic mesoderm is growing out into the trophoderm. Each of these mesodermic outgrowths is to be interpreted as the anlage of the central portion of a chorionic villus, and out of the neighboring chorionic ectoderm will be differentiated the ectodermal covering of the villus. It seems, from a comparison of later stages, that the trophoderm degeneration never goes so far as to leave any of the chorionic villi without an ectodermal covering. But this covering varies extremely in its exact character as we find it in later stages, even in adjacent parts of the same villus, for it may be either a single layer of cells or a layer of cells covered by a thin coat of syncytium or merely a syncytial layer (compare page 354). The disappearance of all of the trophoderm, except so much as remains to share in forming the ectodermal covering of the villi, produces the so-called intervillous spaces of later stages, in which, as above stated, maternal blood circulates.

An ovum in situ slightly more advanced than Peters's has been described carefully by Herzog. The specimen is now in the Harvard Embryological Collection, Series I 500. It differs from Peters's ovum in having a prolongation of the entoderm into the body-stalk to make the anlage of the allantois.

\section{The Embryo of a Gibbon in the Third Stage.}

The embryo to be described was obtained from a female Hylobates concolor in Borneo by Selenka. It is a little more advanced than Frassi's human embryo, mentioned on page IIg. It still had traces of the primitive streak, at the anterior end of which was an open neurenteric canal. The medullary plate was partially differentiated from the embryonic shield. It was thoroughly studied by Selenka, and is one of the best known very early ova of any primate. The entire ovum is represented in figure 75 . The figure was reconstructed from the sections. It shows the chorionic membrane studded with villi. The diameter of the chorion was about 8.5 
$\mathrm{mm}$. The number of villi was about one hundred, of which some seventy are clustered about the region where the embryo was found. The others are scattered over the surface of the membrane. They are considerably branched. Each one is covered by ectoderm which consists of two layers, an inner distinctly cellular, and an outer one in which the cell-boundaries are indistinct and which is known, therefore, as a syncytium and represents the remains of the original trophoderm. Each

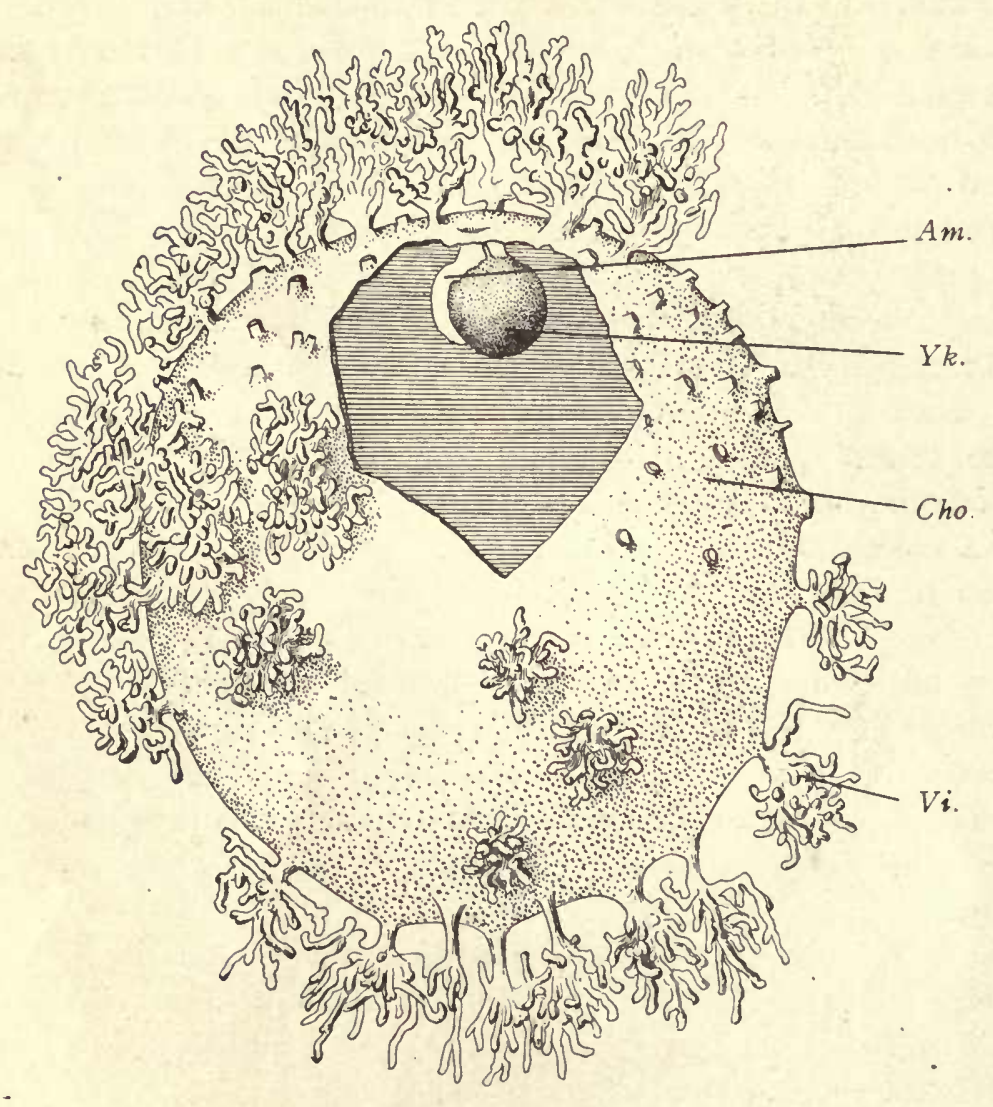

Fig. 75.-Embryo of a Gibbon (Hylobates concolor) in the Third Stage. Am, Amnion. $Y k$, Yolk-sac. Cho, Chorion. Vi, Villi.-(After E. Selenka.)

villus contains a core of mesodermic tissue. The chorionic membrane is represented as open in order to show the size and position of the yolk-sac, $Y k$, and of the amnion, $A m$, which encloses the embryo as it rests upon the yolk-sac. The embryo itself is not shown in the illustration. Both the yolk-sac and the amnion are, of course, covered by a layer of mesoderm. The entire space between these two inner structures and the chorion corresponds to the extra-embryonic cœlom, the very precocious and enormous development of which is a special characteristic of 
primates, including man, and is not at present known to be paralleled by the conditions in the early stages in any other mammals.

A side view of the embryo on a larger scale is represented in figure 76 . The embryo is connected with the chorion by a well-marked body-stalk, b.s, is covered by the arching amnion, $A m$, and rests upon the yolk-sac, which in comparison to the chorionic sac seems very small. The yolk-sac, $Y k$, already has developed from it a network of blood-vessels, $V e$, which contain blood-corpuscles but have not yet developed into the embryo itself. The disposition of the vessels is best illustrated by the section (Fig. 77). The yolk-sac is, of course, lined in its interior by entoderm. It has formed already a prolongation, $A l l$, into the body-stalk. This prolongation is the anlage of the future allantois. Figure 78 represents a surface view of the

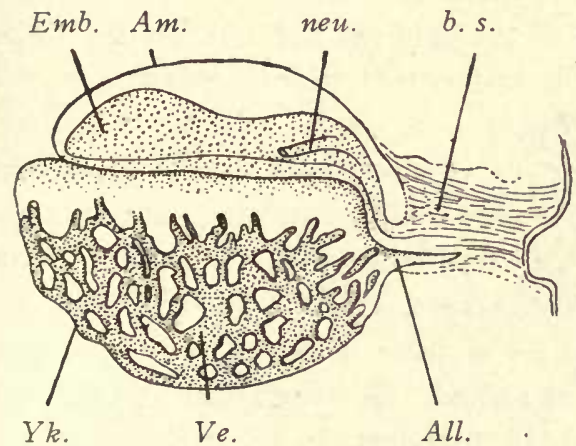

Fig. 76.-Embryo of a Gibbon, Side View of THE EMbryo of FigURE 75.

$E m b$, Embryo. Am, Amnion. neu, Neurenteric canal. b.s, Body-stalk. $Y k$, Yolk-sac. Ve, Blood-vessels. All, Allantois.-(Afier $E$. Selenka.)

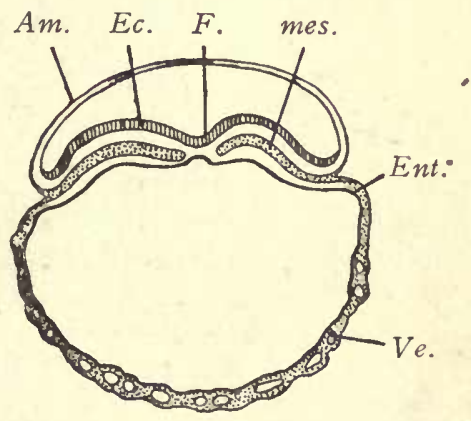

Fig. 77.-Transverse Section of the Embryo of the Preceding Figure.

$A m$, Amnion. Ec, Ectoderm. F, Dorsal furrow. mes, Mesoderm. Ent, Entoderm. Ve, Bloodvessel.-(After $E$. Selenka.)

same embryo, or perhaps one should say, rather, of the embryonic shield. At the posterior end there is the short primitive streak, the anterior limit of which is marked by the opening of the neurenteric canal, neu, which passes obliquely downward and forward, as shown also in figure 76 . From the end of the neurenteric canal there extends forward a slight thickening of the entoderm which can be recognized as the anlage of the notochord, nch. Figure 77 represents a transverse section through the region of the notochord. It shows the amnion, $A m$, arching over the embryo, the thickened ectoderm of the embryonic shield, and the anlage of the notochord. The mesoderm, mes, of the embryo no longer extends across the median line and is without any cœlom. At the edge of the embryo the mesoderm splits and one layer passes over on to the amnion, the other on to the yolksac. In the wall of the yolk-sac, $D$, one can easily distinguish a layer of the entoderm, Ent, and also in the mesodermic portion the young blood-vessels, Ve. Comparison with a section of a somewhat older embryo of another gibbon, Hylobates 
Rafflesi, also described by Selenka, will be found instructive. The relations are here similar to those shown in the section just described, although the stage is somewhat more advanced, for we see that the amniotic cavity is larger, that the form-

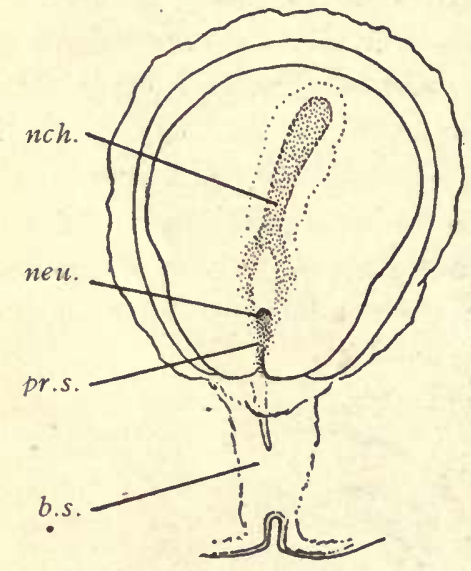

Fig. 78.-Surface View of the EMbryonic Area of the Ovum SHOWN IN FigURE 77.

b.s, Body-stalk. neu, Neurenteric canal. nch, Notochord. pr.s, Primitive streak. ation of the medullary groove has begun, that the cœlom is beginning to appear in the embryonic mesoderm, and that the blood-vessels of the yolk-sac have increased greatly in size. In this embryo there were traces of the formation of three segments a little in front of the neurenteric canal which was still present and open. This embryo was found to be attached to the wall of the uterus and to be enclosed in a decidua reflexa. In later stages the decidua reflexa of the gibbon unites with the decidua vera, and is then lost completely by resorption. The general character of the ovum and its relations to the uterus justify us in the belief that it is extremely similar to the human embryo at the same stage.

The Harvard Embryological Collection contains one very well preserved embryo in the third stage, Series 825. It is a little younger than the gibbon embryo above described. A monograph of this valuable specimen is in preparation.

\section{Human Embryo in the Fourth Stage with the Medullary Plate.}

The general relations in this stage have been indicated by the diagram (Fig. 69). A more exact idea of the embryonic structures may be gathered from figure 79 , a dorsal view of the embryo, from figure 80 , which represents a median section of the embryo taken from a wax model reconstructed from the sections, and figure $8 \mathrm{I}$, a transverse section through the neuropore. The general disposition of the parts agrees very closely with the previous stage as described for primates. The embryo and yolk-sac are very small in comparison with the entire ovum, and they are connected by means of the body-stalk, b.s, with the chorion, Cho. The bodystalk contains the entodermal anlage, $A l l$, of the allantois. The embryo is covered by the amnion, $A m$, which arises in front of the head of the embryo, now becoming marked off, and runs above the embryo to join the distal end of the body-stalk. The opening of the yolk-sac, $Y k$, is about equal to the length of the embryo. The yolk-sac is, of course, lined by entoderm and has a thick layer of mesoderm supplied already with relatively large blood-vessels containing blood-corpuscles; the vessels are developed chiefly upon the inferior hemisphere of the yolk-sac. The embryo measured I.54 mm. in length. Its dorsal surface is represented in figure 79. This surface is occupied by the very broad medullary plate of thickened ectoderm. Toward the middle of its length the medullary plate is somewhat narrower 
than elsewhere. Along its median line runs the deep, narrow, dorsal groove which at its caudal end widens out and disappears. Just behind it is the opening of the relatively large neurenteric canal, behind which again follows a remnant of the primitive groove. A transverse section a little in front of the middle of the embryo is shown in figure 36 . The ectoderm, $e k$, is very much thickened to constitute the medullary plate; the narrow central longitudinal furrow, $f$, mentioned

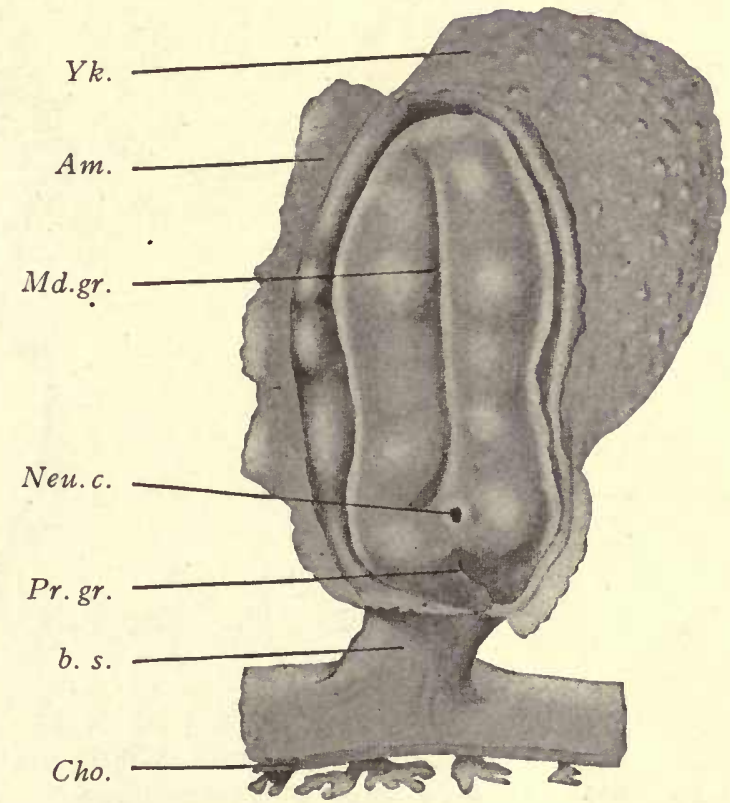

Fig. 79.-Reconstruction of a Human Embryo i.54 mm. Long. The amion has been opened TO SHOW THE DORSAL SURFACE OF THE EMBRYO.

$Y k$, Yolk-sac. Am, Amnion. Md.gr, Medullary groove. Neu.c, Neurenteric canal. Pr.gr, Primitive groove. b.s, Body-stalk. Cho, Chorion.-(After Count Spee.)

above is very noticeable. Outside of the embryo the ectoderm is reflected on to the amnion, $c t$, over the back of the embryo. The entoderm is a thin layer of cells in the center of which the notochordal band ch can be distinguished. In sections near the neurenteric canal the notochord is better marked, being there much thicker than the remaining entoderm, The mesoderm, me, is a distinct layer, although, as other sections show, it is fused in the median line of the primitive streak behind the neurenteric canal with both ectoderm and entoderm. Although the extra-embryonic cœlom is fully developed, that of the embryo is present as a small fissure, $p$, only. Figure $8 \mathrm{I}$ is a section passing through the neurenteric canal, and shows, therefore, the amnion, am, the thickened medullary plate, $e$, of the embryo, and the large yolk-sac, $d$. The yolk-sac is formed, of course, of splanchno- 
pleure. The thickening of the mesodermic layer in the lower part of the yolk-sac in order to allow space for the developing blood-vessels, $b, b, b$, is well shown in the figure.

Eternod has studied an embryo in this stage. He finds that the heart is already present underneath the slightly projecting head. From its anterior end it sends out two aortic branches which run on either side near the notochord, pass in a gentle curve around the neurenteric canal, come nearer together in the region

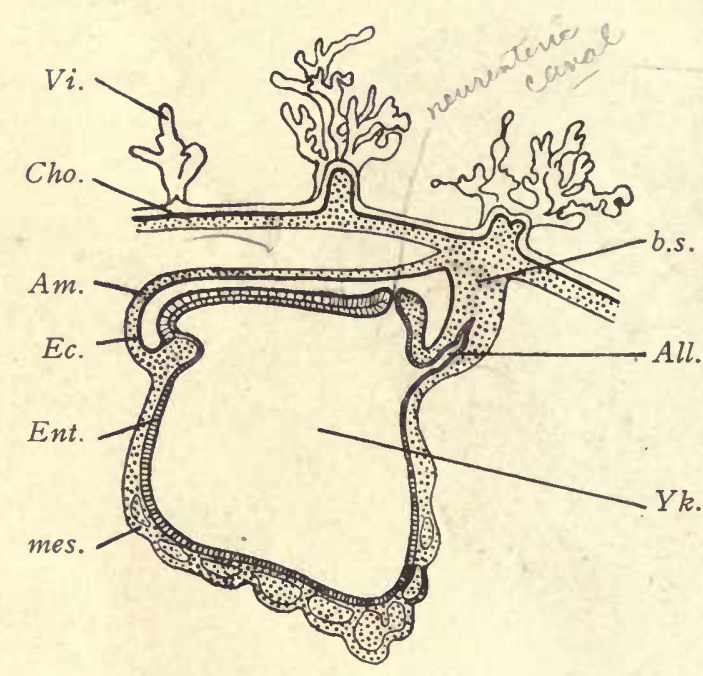

Fig. 80.-Human Embryo of I.54 mm. Median SecTION FROM A WAX MODEL RECONSTRUCTED FROM Sections.

All, Allantois. Am, Amnion. b.s, Body-stalk. Cho, Chorion. Ec, Ectoderm. Ent, Entoderm. mes, Mesoderm. $V i$, Chorionic villus. $Y k$, Cavity of yolk-sac.-(After Count Spee.)

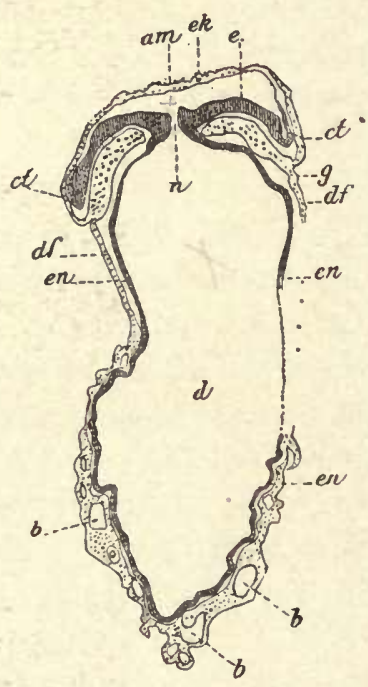

Fig. 81.-Human Embryo of I.54 Mm.

Transverse section passing through the neurenteric canal and yolk-sac. am, Amnion. $e k$, Ectoderm. ct, Amniotic mesoderm. $g$, Meeting-point of somatopleure and splanchnopleure. $d f$, Mesoderm of yolksac. $b, b, b$, Blood-vessels. en, Entoderm. $n$, Neurenteric canal. $d$, Cavity of yolksac. $e$, Medullary plate.-(After Count Spee.)

of the primitive groove, and enter the body-stalk, through which they run parallel to the allantois and form ramifications in the chorion. He finds also two veins in the body-stalk which, when they reach the embryo, unite to a single median trunk, which quickly divides into two vessels which run in the mesoderm of the yolk-sac near the embryo proper until they reach the venous end of the heart, into which they open. They each receive a venous branch from the caudal side of the yolk-sac.

\section{Human Embryo in the Fifth Stage with Open Medullary Groove.}

Several embryos in this stage have been studied. Two have been studied by W. His; one he designates as "E" and the other as "SR" (Fig. 82). The chorionic vesicle of "E" measured $8.5 \times 5.5 \mathrm{~mm}$.; of "SR," $9 \times 8 \mathrm{~mm}$. More 
satisfactory is the embryo described by Dandy, a median section of which is given in figure 25. The embryo in " $E$ " measured (?) $2.1 \mathrm{~mm}$; in "SR," $2.2 \mathrm{~mm}$. (Fig. 82). It will be noticed at once that the condition is very similar to that shown in figure 8o, but the embryo is somewhat more advanced. The most important changes in the embryo at this stage are its general growth, so that it rises above the yolk and has both projecting head and projecting tail. The medullary groove is very deep and extends the entire length of the embryo. Toward its caudal end it probably has an open neurenteric canal. The dorsal outline of

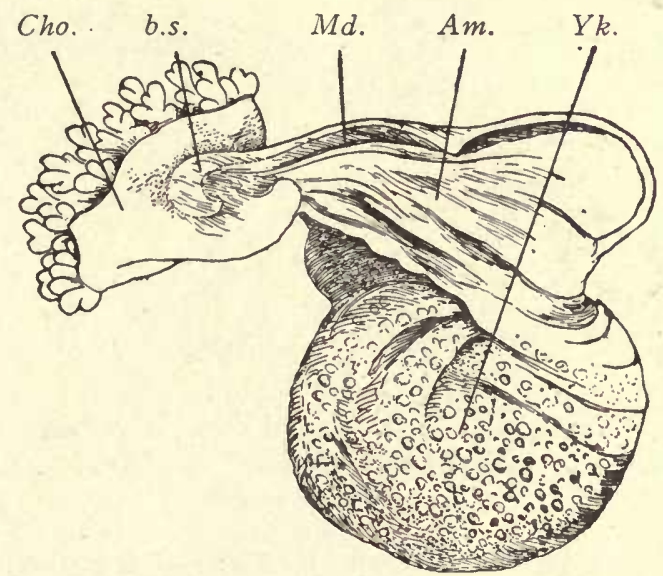

Fig. 82,-Human Embryo with Open Medullary Groove.

Am, Amnion. b.s, Body-stalk. Cho, Chorion. Md, Medullary folds. Yk, Yolk-sac.-(After W. His.)

the embryo is somewhat concave. On the under side of the projecting head, between it and the anterior limit of the yolk-sac, the anlage of the heart has appeared, and its cavity may be supposed to be in connection with the bloodvessels of the yolk-sac. The development of segments has begun; Dandy's embryo had seven. From the under side of the projecting tail end springs the body-stalk, to the distal end of which the chorion is attached. The chorion is completely covered by short branching villi. The yolk-sac has still a very broad connection with the embryo, and contains blood-vessels throughout its entire extent. The space between it and the chorion, the extra-embryonic cœlom, is very large.

\section{Human Embryo in the Sixth Stage with Medullary Canal.}

This stage does not include the whole period from the beginning to the completion of the closure of the medullary groove to form the medullary canal, but only the first part of this period. The best-known specimen of this stage was described by Kollmann. It measured $2.2 \mathrm{~mm}$. in length and had the medullary groove open through the anterior two thirds of its length, but closed along the caudal third. The embryo had thirteen segments (Fig. 83). The yolk-sac was attached to the embryo for a distance of $\mathrm{r} .5 \mathrm{~mm}$., leaving the head to project 
$0.5^{8} \mathrm{~mm}$. and the tail to project $0.3 \mathrm{~mm}$. The head is already somewhat enlarged and slightly bent over toward the ventral side. It forms at least one third of the whole embryo. The dorsal outline of the embryo is concave in the region where the segments have developed. The caudal end is slightly curved over and is connected on its under side with the body-stalk, $A l$, by which the embryo is attached to the chorion. Between the yolk-sac, Yk.s, and the head, the heart, $H t$, is prominent. By analogy with other vertebrates we assume that the hearttube, when it first appears in man, is straight and occupies a longitudinal median

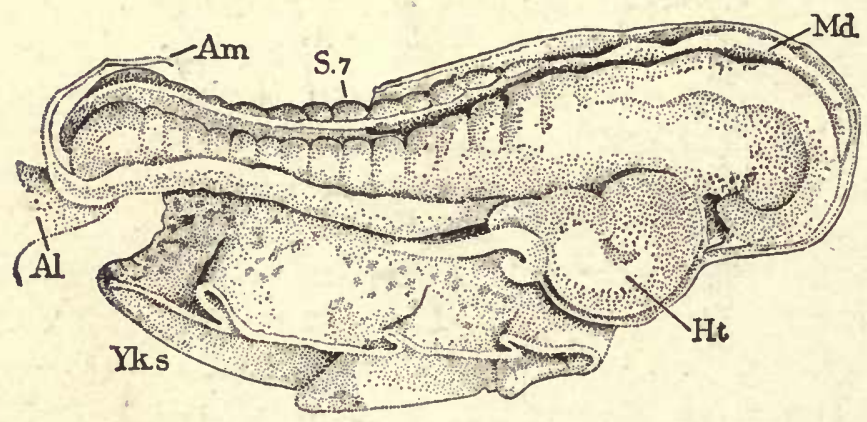

Fig: 83.-Human Embryo of from Thirteen to Fourteen Days.

Am, Amnion. S.7, Seventh segment. Md, Medullary groove. Ht, Heart. Yk.s, Yolk-sac. Al, Body-stalk -(After J. Kollmann.)

position. In this embryo it has already become a relatively large organ and the tube itself is strongly bent. No anlage of the eye or ear was distinguished. The amnion was a thin, transparent membrane enveloping the embryo quite closely. The closeness of the amnion to the embryo was probably accidental (compare Figs. 84 and 85 ). The chorion was covered externally by branching villi; its diameter, including the villi, was $18 \mathrm{~mm}$.

Another embryo, the position of which in the series of known stages has long been a matter of dispute, I feel, after renewed study, must be assigned to a place very close to Kollmann's embryo just described. The specimen in question was figured by Coste in his.monumental "Atlas of Embryology."* The embryo was enclosed in a villous chorion (Fig. 84) and was provided with a large vitelline sac,

* The greatest difficulty comes from Coste's statement as to the magnification of his drawings, according to which the embryo must have been about $4.4 \mathrm{~mm}$. long, or nearly double the length which we now know to be normal for embryos in the stage in which this one seemş to be. Other difficulties arise because Coste has given no further description of this embryo than that which appears in the explanation of his plate. Neither that explanation nor the figures themselves afford any information concerning the dorsal side of the embryo or as to whether it had a partially open medullary groove or not. Coste's figures indicate that thirteen or fourteen segments were visible externally. The shape of the head, the size and curvature of the heart, the form of the tail, and the concavity of the dorsal outline in the segmented region of the embryo all indicate an extremely close resemblance to Kollmann's embryo. As Coste's figures were all made from fresh specimens freehand, we shall probably commit no error if we assume that the magnification was not correctly given. By making this assumption I think the difficulties as to placing Coste's embryo vanish.

Coste's private collection was said to be at the College of France, but upon search this specimen could not be found, so that attempts to ascertain its actual length were without result. 


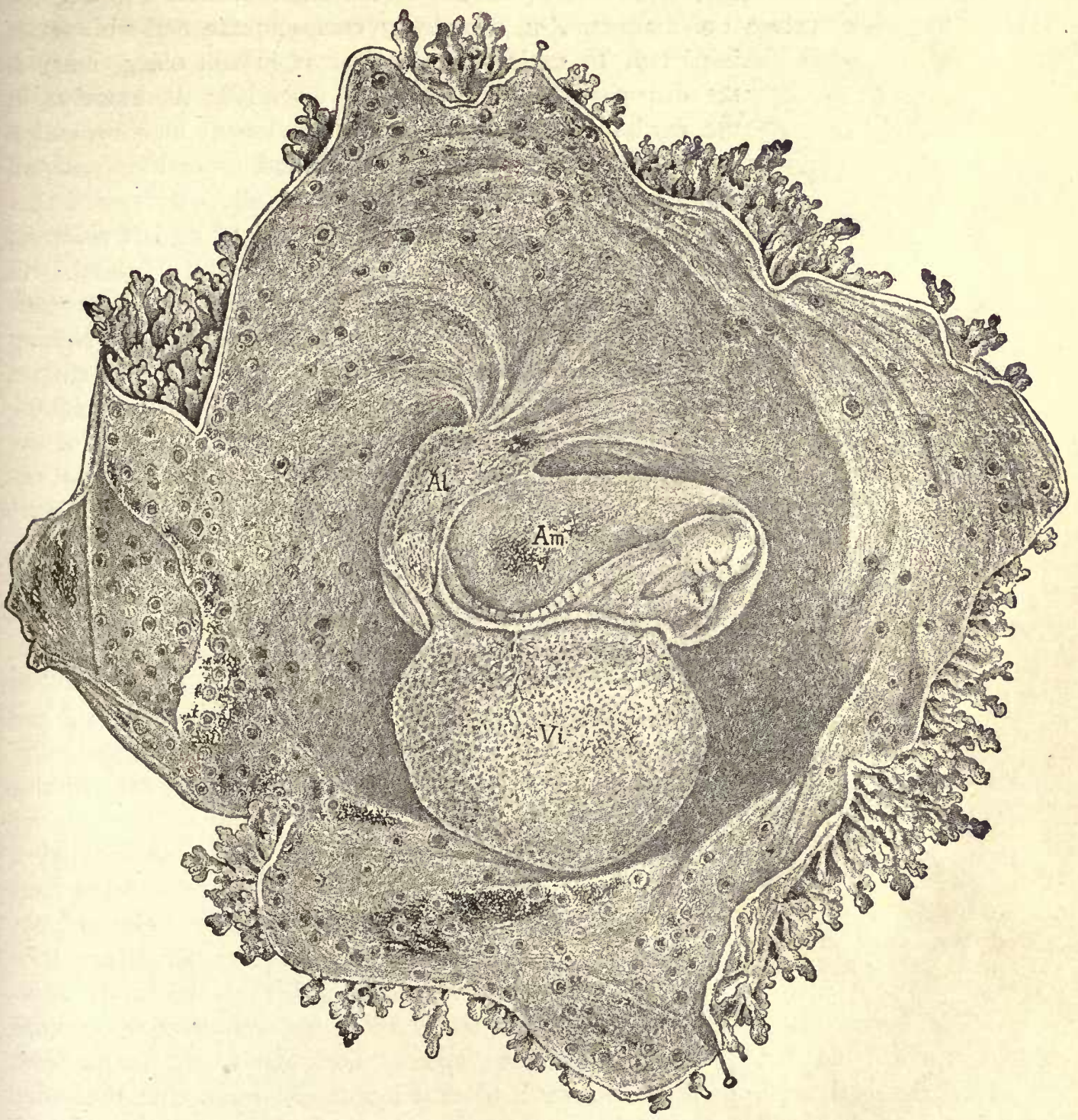

Fig. 84.-Human Ovum, said to be from Fifteen to Eighteen Days Old, (Compare footnote, page I38.) $^{8}$. The chorion has been opened and spread out to show the embryo and its adnexa. Al, Body-stalk containing the allantoic diverticulum. Am, Amnion surrounding the embryo. Vi, Yolk-sac. (After Coste.) 
$V i$, having a very broad connection with the embryo and covered with a network of vessels, in which was a fluid not yet red. A thick body-stalk, $A l$, can be seen running from the under side of the embryo's tail to the chorion; from the anterior side of the stalk springs the amnion, $A m$, completely enclosing the embryo. It is

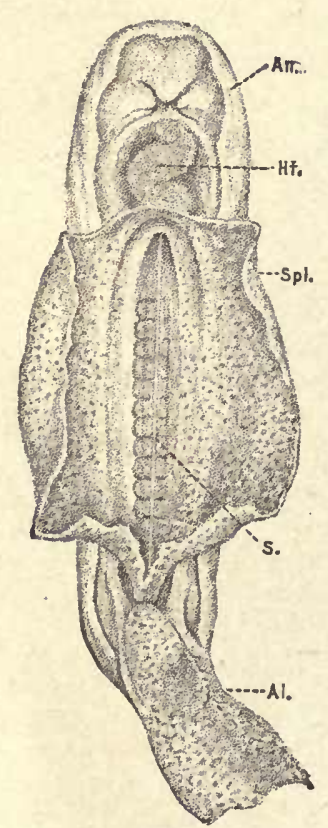

FIG. 85.-EMBRYo OF Figure 84, SEPARATED FROM THE YOLK-SAC AND VIEWED FROM THE UNDER SIDE.

Am, Amnion. $H t$, Heart. Spl, Splanchnopleure extending beyond the embryo to form the yolk-sac. $s$, Notochord with a row of primitive segments on each side. Al, Body-stalk. (After Coste.) important to notice that in this, as in still older embryos, the disposition of the amnion is essentially the same as in the earliest stages; the line of attachment of the amnion is down the sides of the allantois and around the embryo about on a line with the top of the yolk. As regards the embryo, it is drawn slightly canted on to its left side; its back is concave; the head end is thickest; behind and below it can be seen the heart, already a bent tube, shining through; and on the dorsal side, the light-looking œsophagus is distinguishable; in the figure a wedge-shaped shadow intervenes between the straight œsophagus and the bent heart; the heart causes a conspicuous bulging of the body between the head and the yolk-sac; the caudal extremity is thick and rounded and curves upward. Figure 85 is a ventral view of the same embryo after most of the yolk-sac has been cut off; its walls, $S p l$ (splanchnopleure), are seen to pass over without any break into those of the intestinal cavity. In the central line the notochord, $s$, can be perceived through the translucent dorsal wall of the intestinal cavity; it is flanked on each side by the row of square segments. Behind, we see the large bodystalk, $A l$, and in front the tubular heart, $H t$, with a decided flexure to the right of the embryo; the anterior end of the heart makes an opposite bend, separating off a limb which becomes the bulbus aorta. The chorion consists of two layers, one of which forms the uninterrupted inner surface of the chorion, while the outer layer alone forms the hollow villi (Figs. 84 and 245); hence, in looking at the inside of the chorion, we seen numerous round openings which do not penetrate the inner layer. Fortunately, we learn from Kölliker, who had an opportunity in I86I to examine the chorion, that the outer layer was epithelial, with cells of the same character as in the epithelium of older vascularized villi, and that the inner layer consisted of developing connective tissue, and carried fine blood-vessels. It thus appears that Coste was the first to observe the rôle of the epithelium in the growth of the villi.

\section{Human Embryo in the Seventh Stage with One Gill-cleft Showing Externally.}

No human embryo with only one gill-cleft showing externally is known. 


\section{Human Embryo in the Eighth Stage with Two Gill-clefts Showing Externally.}

Several embryos in this stage have been described and some of them studied anatomically. Those which are best preserved and which we have best reason to think are normal present a very singular appearance, owing to the deep bend in the segmented region of the body so as to constitute at the dorsal outline of the embryo at that point a U-shaped curve (Fig. 86).

This bend is known as the dorsal flexure. Embryos of earlier stages have an indication of this flexure, as shown in figure 84 . Until we have intermediate stages we cannot be sure that the assumption which seems natural is also correct; namely, that the deep dorsal flexure of figure 86 is merely an accentuation of the cavity on the dorsal side of the embryo in earlier stages. In older embryos the dorsal flexure is normally absent (compare Fig. 88 and the following figures). It is possible that the change from the concave to the convex position is very abrupt, and it is not improbable that the time of the occurrence of this change is variable. The head of the embryo and the tail both project far beyond the yolk-sac, which, however, still shows a broad attachment to the embryo. The right-angled head-bend is well marked and the region of the fore-brain pro-

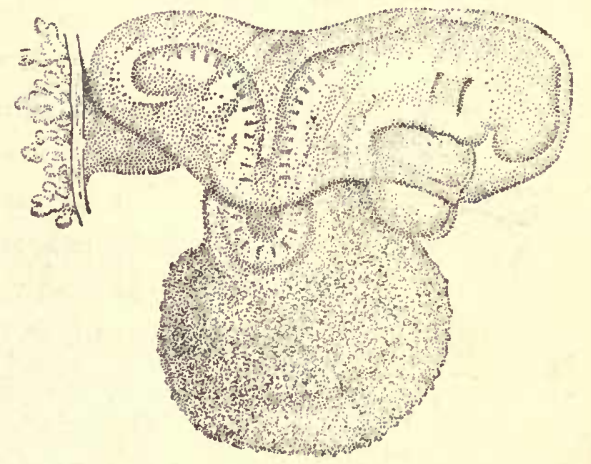

Fig. 86.-Human Embryo, 2.I5 MM. Long.(After W. His.) jects "downward so as to leave a depressed area between the head and the heart. This depression corresponds to the position of the oral cavity. The heart is large, protuberant, and considerably bent, so that we can distinguish its three primary limbs. From the under side of the caudal end of the embryo springs the stout body-stalk by which the embryo is united with the villous chorion. In another embryo of this stage there were twenty-nine segments present. Above the heart on the side of the pharyngeal region two external depressions are visible corresponding to the first two gill-clefts. They are elongated in a dorso-ventral direction and are narrow. This position of the amnion is well shown in figure 86. It arises from the body-stalk at the side of the embryo along the yolk-sac and cardiac region, and extends around the embryo, but is not yet fitted closely.

The anatomy of this stage is known to us chiefly through the observations of His upon two embryos designated by him as Lg. and Sch. I. Lg. measured 2.I5 $\mathrm{mm}$; Sch. I, $2.20 \mathrm{~mm}$. The two embryos resemble one another closely. The following description applies especially to Lg. The anatomy can be understood from the accompanying figure 87 . The medullary tube extends the entire length of the embryo and is the principal component of the head. From the region of the fore-brain has been formed an outgrowth to constitute the optic vesicle, $O p$. At the side of the hind-brain and on the dorsal side of the pharynx is situated the 
anlage of the ear, $O t$, which at this stage is merely an open invagination of the ectoderm. The region of the mid-brain is marked by the head-bend, so that the axis of the fore-brain is approximately at right angles to the axis of the hind-brain. Another consequence of the head-bend is that the lower process of the head is brought very close to the pericardial chamber enclosing the heart, $H t$. Between the head and the pericardial sac is situated the oral invagination or future mouth-

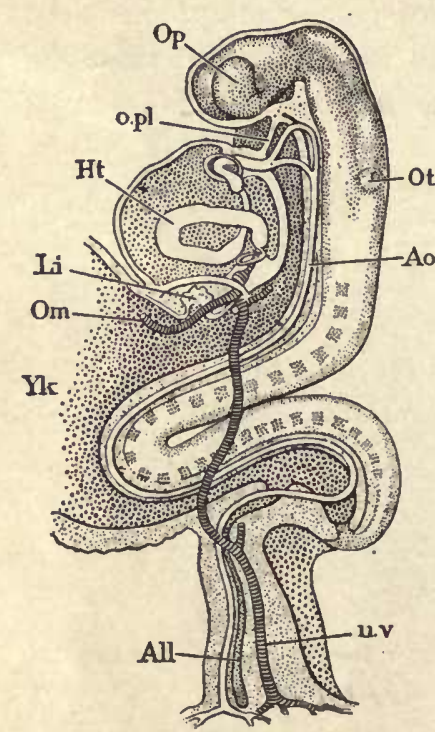

Fig. 87.-Reconstruction of the ANATOMY OF THE EMBryo SHown in Figure 86.

$O p$, Optic vesicle. o.pl, Oral plate. $H$, Endothelial heart. $L i$, Liver. $O m$, Omphalo-mesaraic vein. $Y k$, Yolk-sac. All, Allantoic diverticulum formed by the entoderm. $u . v$, Umbilical vein. Ao, Aorta. Ot, Otocyst.-(After W. His.) cavity, which is still separated from the entodermal canal by the oral plate, O.pl, which consists merely of a thin layer of cells belonging to the ectoderm and entoderm (compare page 58). The pericardial chamber is large; in the figure only the endothelial portion of the heart, $\mathrm{Ht}$, is represented. Around this endothelial tube is a second and-more bulky one from which arises the muscular wall of the heart. The volume of the heart is, therefore, much greater than indicated by the figure, hence the large size of the pericardial chamber. On the dorsal side of the heart, between it and the hind-brain, lies the entodermal canal, which is here the anlage of the pharynx. It has two diverticula or gillpouches which are not indicated in the figure. On the side toward the mouth the endothelial part is continued beyond the pericardial chamber and gives off two vessels on each side, the first and second aortic arches, which pass around the pharyn $x$ and unite again upon its dorsal side, and then, as the aortæ, $A o$, descend along the ventral side of the nervous system, soon uniting in the median line to form the single dorsal aorta which runs along nearly to the tail of the embryo, where it forks; and its branches, passing one on each side of the intestinal canal, enter the body-stalk and run to the chorion, where they branch out. Behind the pharynx the entodermal canal merges into the cavity of the yolk-sac, $Y k$, and then beyond the yolk-sac extends again into the tail of the embryo, forming an expansion there which is known as the cloaca. From the under side of the cloaca runs out the allantoic diverticulum, $A l l$, which extends as a narrow tube of entoderm through the allantoic stalk to the level of the chorion, where it ends blindly. The pericardial chamber on its caudal side is bounded by the septum transversum, in which we find the anlage of the liver, $L i$, already present, and through which, on either side, the great vein from the yolk-sac, the omphalo-mesaraic or vitelline vein, $O m$, passes to the heart. Of the veins of the embryo only the umbilical, $u v$, is shown in the figure. This vein gathers the vessels from the chorion, passes through the body-stalk, then runs in the somato- 
pleure of the embryo to join the omphalo-mesaraic vein and enter the heart. In the figure only the general course of the vein is indicated. The fact that it is situated in the somatopleure could not. well be shown.

\section{Human Embryo in the Ninth Stage with Three Gill-clefts Showing Externally.}

Our knowledge of this stage is quite good. The described embryos vary in length from 2.6 to $4.2 \mathrm{~mm}$. The chorionic vesicles are about ro $\mathrm{mm}$. in diameter, varying according to the size of the embryo. Figures 88 and 89 represent two embryos of this stage, the latter being the more advanced. The back of the embryo is normally (or at least usually) convex. The head is bent

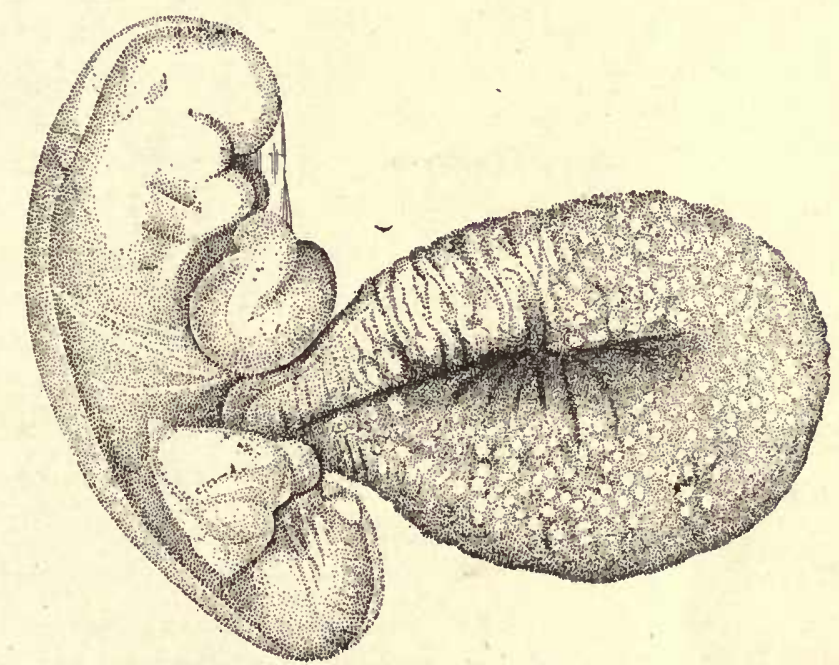

Fig. 88.-Human Embryo of 2.6 m. Length.-(After W. His.)

to one side, usually to the right, and the tail to the other, the whole embryo having a slight spiral twist. The embryo has become quite large in proportion to the yolk-sac. The three gill-clefts are readily seen, the first being the largest, the third the smallest. The column of tissue between the first cleft and the mouth is the mandibular process. Between it and the fore-brain lies the shorter rounded maxillary process. The segments are clearly marked externally along the back (Fig. 89). The origin of the amnion is shown in figure 89 also. The heart has grown and something of its more complicated form is indicated in the external modeling of the embryo. The anlage of the future ear is now a closed vesicle or otocyst (Fig. 9o, ot). From the region over the heart almost to the caudal extremity the segments of the body are distinctly marked externally.

The general anatomy of this stage will be understood by the aid of the accompanying figures 90 to 93 , which are all reconstructions from sections. The position of the notochord, $\mathrm{Ch}$, is indicated by a line (Fig. 9I). The pharynx is large and 
wide. It has three lateral outgrowths on each side, I, 2, 3, the gill-pouches. In front and near the cephalic end of the notochord there is a small median outgrowth, the anlage of the hypophysis, $H y$. Toward the neck-bend the pharynx becomes narrower and passes over into the small entodermal tube, from which we can detect the outgrowth, $L u$, which represents the commencing formation of the lungs. This narrow tube leads to the space above the yolk-sac, Yk.s. Just

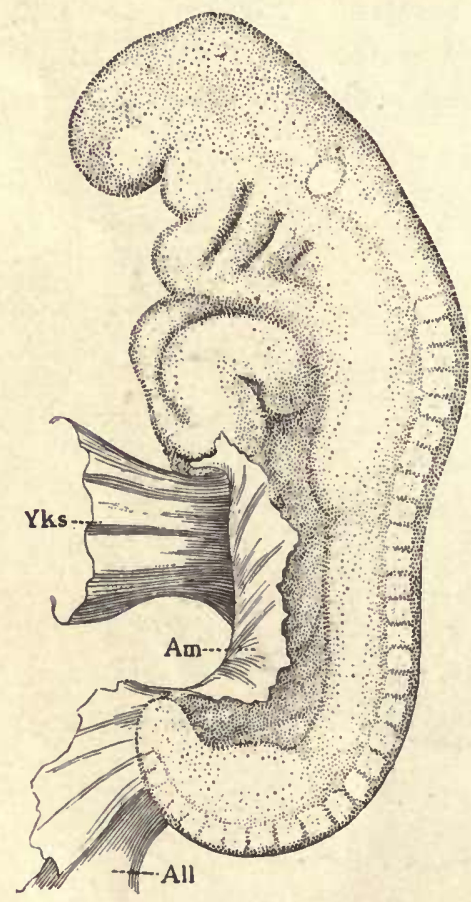

Fig. 89.-Human Embryo 4.2 Mm. $Y k s$, Yolk-sac. Am, Amnion. All, Body-stalk.-(After W. His.)

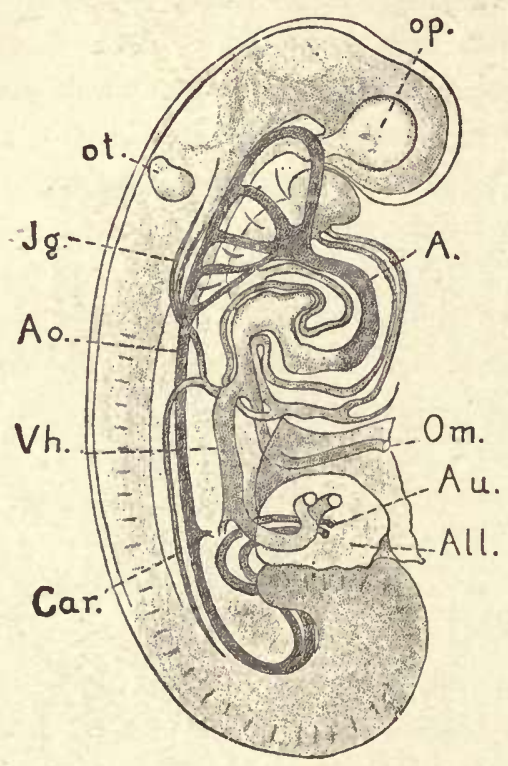

Fig. 90.-Reconstruction OF the ANATOMY OF THE EMBRYo OF 2.6 Mu. IN FIGURE 88.

$A$, Aortic limb of heart. All, Body-stalk. Ao, Dorsal aorta. $A u$, Umbilical arteries. Car, Posterior cardinal vein. $J g$, Anterior cardinal vein. $O m$, Omphalo-mesaraic vein. op, Optic vesicle. ot, Otocyst. $V h$, right umbilical vein.-(After $W$. His.)

where it passes into the yolk-sac the entoderm has formed the rudiment of the liver, $L i$. Figure 55 gives a view of the anterior wall of the pharynx of another embryo. In front is the large opening of the mouth, $M$, the oral plate between the mouth-cavity and the entodermal canal having disappeared. This embryo being a little older, the traces of the four gill-clefts can already be seen, and there are four entodermal gill-pouches. The aortic vessels are indicated by dotted lines. The cardiac aorta reaches the pharynx between the bases of the second and third gill-arches, and divides into two branches, one on each side. The anterior branch 
forks and runs through the first and second arches. The posterior branch forks, one fork going to the third, and the other, after again forking, supplies the fourth and fifth branchial arches. This arrangement of the aortic branches is typical. Between the bases of the first and second arches is a small protuberance which is the anlage of the tongue and is named by His the tuberculum impar. Studies of the sections demonstrate that the cavity of the abdominal region (splanchnocele) has on each side of its dorsal surface a longitudinal ridge, the commencement of the Wolffian body: The ridge already contains traces of the canals of the Wolffian

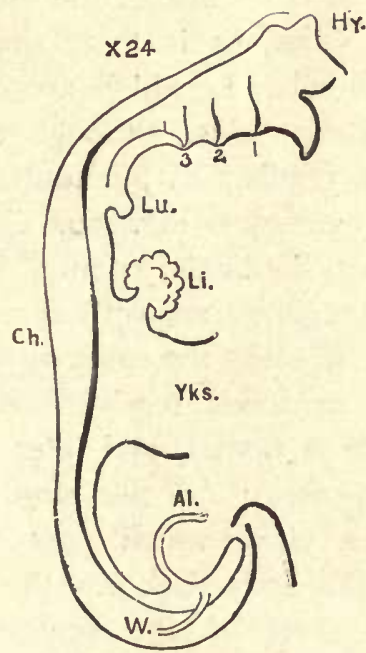

Fig. 91.-Outline of the Entodermal Canal of A Human EMBRyo of 4.2 MM.

$H y$, Hypophysis. I, 2, 3, Lines marking the position of the pharyngeal gill-pouches. $L u$, Lungs. $L i$, Liver. $Y k s$, Yolk-sac. $A l$, Allantois. $W$, Wolffian duct. $C h$, notochord.(After W. His.)

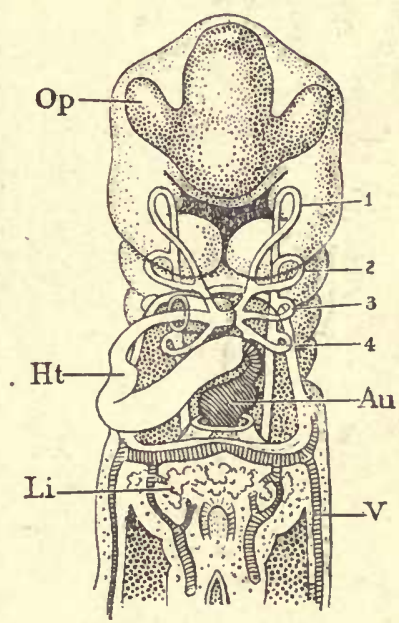

Fig. 92.-Reconstruction OF THE ANATOMY OF A Human Embryo, 3.2 MM. Long, SHowing the ANTerior ENd Vitewed from the Ventral Side.

$O p$, Optic vesicle. $H t$, Heart. $L i$, Liver. $V$, Allantoic vein. $A u$, Auricle of the heart. I, 2, 3, 4, Aortic arches.

body. Of especial interest is the arrangement of the circulatory apparatus (Figs. 88 and 92). In the first figure the arteries are shaded dark; the heart is an S-shaped tube which is really double, consisting of an inner endothelial tube continuous with the arteries and veins at either end of the heart, and an outer mesodermic tube which is confined to the heart and is unconnected with the blood-vessels. The venous end of the heart lies near the yolk-sac. It is convex toward the head. The arterial end of the heart is convex toward the tail. When viewed from the ventral side, the venous process of the heart (Fig. 92, $A u$ ) is seen on the left and the arterial process, $H t$, is seen on the right. The heart is continued forward by the large aorta (Fig. 90, A), which gives off five branches on each side of the neck. These branches again unite on the dorsal side and run backward to unite with 
the fellow-stem, and so form the single median dorsal aorta, $A 0$, which runs way back and terminates in two branches, $A u$, which, curving round, pass out through the body-stalk and supply the circulation of the chorion. The five branches in the neck are known as the aortic arches. The column around each branch con-

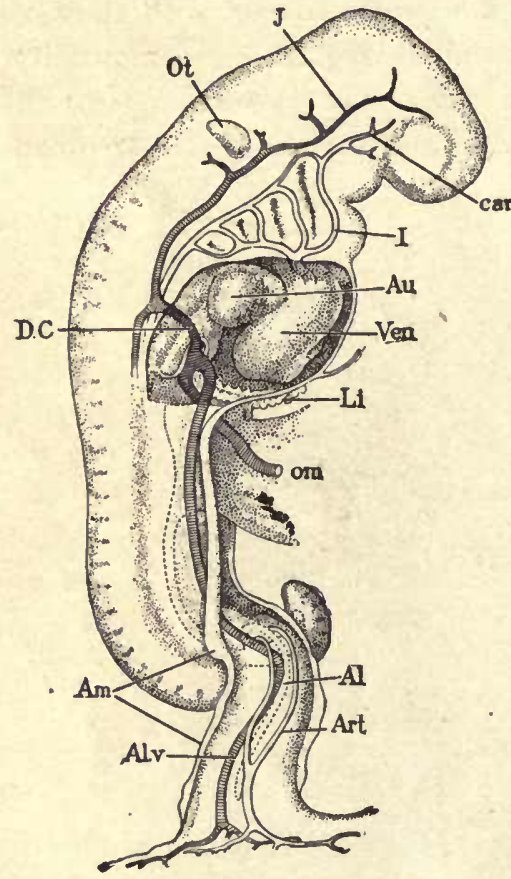

Fig. 93.-RECONSTRUCTION OF THE ANATOMy of the Human Embryo of 4. 2 MM. Shown in Figure 89.

$O t$, Otocyst. $J$, Anterior cardinal vein. car, Carotid artery. I, First aortic arch. $A u$, Auricle. Ven, Ventricle. $L i$, Liver. om, Omphalo-mesaraic vein. $A l$, Allantoic diverticulum. Art, Allantoic artery. Al.v, Allantoic vein. $A m$, Origin of the amnion. D.C, Common cardinal.- (After $W$. His.) stitutes the so-called branchial arch. Each branchial arch is further marked out by the gill-cleft in front of it and behind it, as shown in figure 90. The reconstruction of the third embryo in the side view (Fig. 93) affords further information concerning the disposition of the heart and the large blood-vessels. The veins, as is there shown, are (I) the anterior cardinals, J, which are often referred to as the jugular veins, although they are not identical with the jugulars of the adult; (2) the posterior cardinals (compare Fig. 90, Car); the posterior and anterior cardinals, coming from the caudal and cephalic regions, respectively, unite to form a single transverse stem, the common cardinal, D.C (the posterior cardinals receive their blood chiefly from the Wolffian bodies, and later undergo complicated metamorphoses); (3) the large umbilical or allantoic veins, $A l . v$, which pass up from the chorion through the body-stalk into the somatopleure until at the level of the septum transversum, above the liver, $L i$, they empty into the common cardinal; (4) the omphalo-mesaraic or vitelline veins, om, which come up from the yolksac on either side and meet the common cardinals at the venous end of the heart. This figure also shows the disposition of the aortic arches and an early stage of the primitive internal carotid artery, car. The muscular, but not the endothelial, heart is represented in the reconstruction.

\section{Human Embryo in the Tenth Stage with Four Gill-clefts Showing Externally.}

Few embryos belonging to this stage have been obtained. The one shown in figure 94 was carefully studied and described by W. His. Its probable age is twenty-three days. The embryo forms almost a complete circle, the tail being close to the head. The limb-buds have appeared. The heart is large and causes a marked swelling of the body beneath the branchial arches, I, 2, 3, 4, all four of which show clearly on the surface. The entodermal canal has attained nearly the condition shown in figure $27, \mathrm{~B}$. 
Human Embryo in the Eleventh Stage with the Cervical Sinus in Formation.

The embryo figured (Fig. 95) was described by Mall, and one almost identical has been studied by H. Piper. Its age is probably twenty-six days. At this stage the embryo is flexed so as to describe almost a circle, the tail being almost in contact with the head, yet comparison with figure 94 reveals that the straightening of the back of the embryo has begun. Although the limbs, A.l and P.l, have increased in size, they are still only rounded buds. The head, which is bent to the right, partly conceals the cardiac region. The nasal pit, $N a$, is a broad,

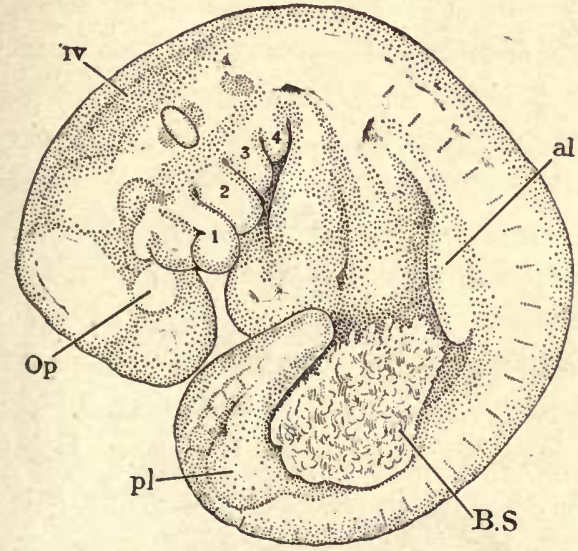

Fig. 94.-Human Embryo of about TwentyTHREE DAYS, 4.O MM. $\times$ I5 diams.- (After W. His, Embryo a.)

$a l$, Anterior limb-bud. B.S, Body-stalk. $O p$, Optic vesicle. pl, Posterior limb-bud. iv, Fourth ventricle. I, Mandibular process. 2, Hyoid arch. 3, 4, Third and fourth gillarches.

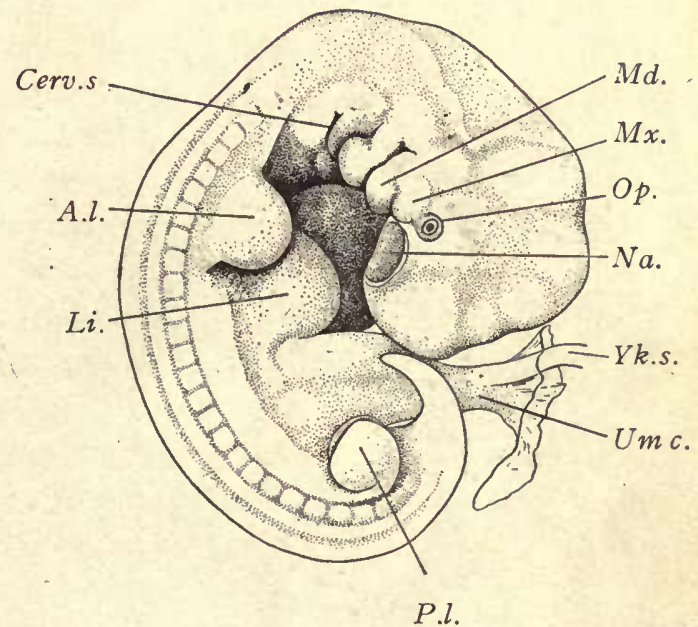

Fig. 95.-Human Embryo of 7.0 Mм. $\times 8$ diams. -(After F. P. Mall.)

$A . l$, Anterior limb. Cerv.s, Cervical sinus. Li, Liver. $M d$, Mandibular process. $M x$, Maxillary process. $N a$, Nasal pit. $O p$, Eye. P.l, Posterior limb. Um.c, Umbilical cord. Yk.s, Yolk-stalk.

shallow fossa. The eye, $O p$, consists of the small optic vesicle and overlying lens. The maxillary process, $M x$, is well developed. Behind the mandibular process, $M d$, is the first cleft, or anlage of the external auditory meatus. The cervical sinus, Cerv.s, is in process of development, but on the left side is not so deep as on the right side, which is figured. The ventral ends of the branchial arches are continuous with the cardiac region of the body. As shown in the figure, twentyfour segments are clearly marked externally. The large cardiac region fills out the space between the anterior limb, $A . l$, and the tip of the head. The ventral surface of the abdomen is prolonged to form the umbilical cord, Um.c, from which projects the slender yolk-stalk, Yk.s. The position of the liver is indicated by a distinct protuberance below the foreleg. 


\section{Human Embryos of the Fourth Week to the Fourth Month.}

The following series of illustrations (Figs. 96-II3 inclusive) are from specimens in the Harvard Embryological Collection, all normal or nearly so. To facilitate comparison figures $97-107$ are uniformly magnified five diameters, while figures $108-\mathrm{II}_{3}$ are life size.

Embryos of Four Weeks, 7.5 to $8.0 \mathrm{~mm}$.- They are characterized especially by the extreme development of the neck-bend. The fourth and fifth branchial arches are entirely buried in the cervical sinus, and the third arch is turning in. In other words, the process of invagination of the sinus, though far advanced,

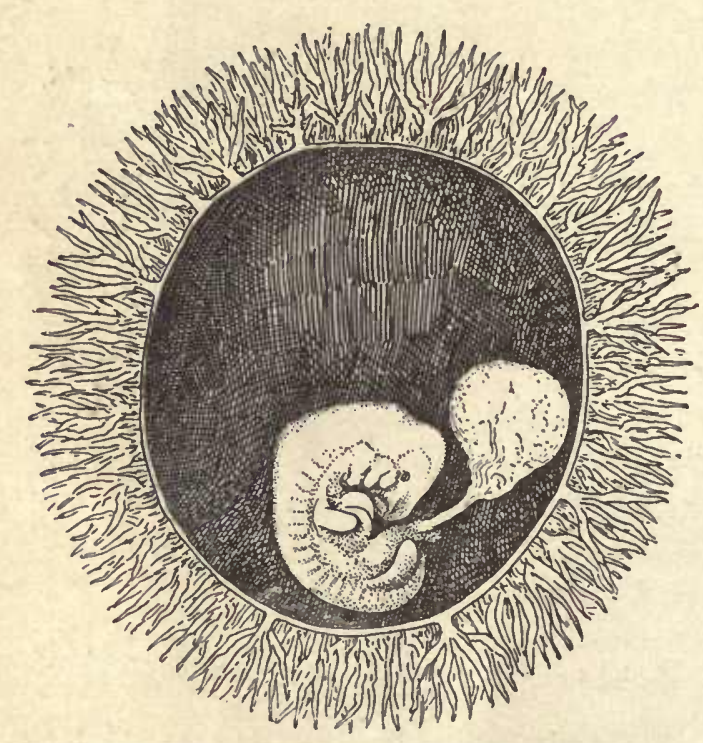

Fig. 96.-Human Ovum With Embryo of 9.4 MM. The Chorion has Been Partly Removed to Show the Embryo. $\times 3$ diams. $-($ Minot Collection, 275.)

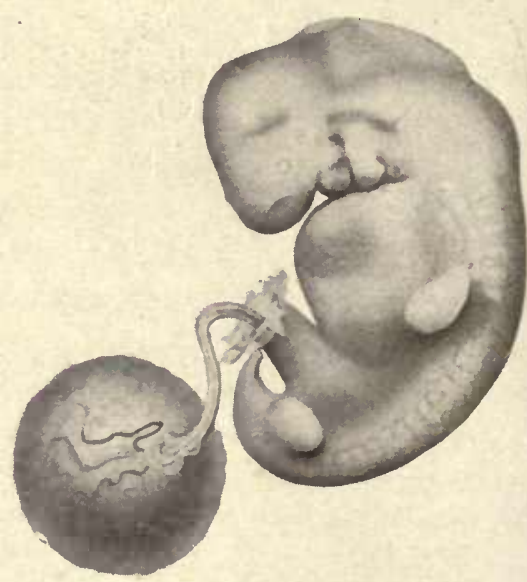

Fig. 97.-Human Embryo of 9.6 мm. Series roor. $\times 5$ diams.

is not completed. The invagination of the ectoderm to form the lens is still open, though about to close. The back of the embryo is partly straightened. The limb buds are beginning to expand at their distal ends to make the anlages of the hands and feet.

Embryos of Twenty-eight to Thirty Days, 8.0 to $10.0 \mathrm{~mm}$.- The form of human embryos at the end of the first month is very variable, and it has not been possible hitherto to establish with certainty a typical normal shape. Their length varies because the head begins to rise with accompanying diminution of the neckbend, hence the length may be increased by a change of form without a corresponding growth of the embryo as a whole or advance in structure. Figure 96 illustrates the proportions of the embryo, yolk-sac, and chorion at this stage. Figure 97 shows an embryo of $9.6 \mathrm{~mm}$. with the yolk-sac and stalk. In this specimen the oblitera- 
tion of the neck-bend, the growth of the limbs, the narrowness of the opening of the sinus cervicalis, the elongation of the umbilical cord, and the expansion of the hind-brain are all evidences of advancing development (compare Fig. 95). From the distal end of the umbilical cord springs the amnion, beyond which there passes out from the cord the narrow stalk of the yolk-sac. The cavity in the interior of the cord is a continuation of the cœlom of the embryo and through it the yolkstalk takes its course.

Figure 98 is very instructive, for it represents an embryo which, although $0.2 \mathrm{~mm}$. shorter than the one shown in figure 97 , yet is much more advanced in development, as is evidenced strikingly by the enlargement of the whole head and the elongation of the limbs and the demarcation of the hand from the rest of the anterior limb. The orifice of the cervical sinus is narrow. On the ventral side of the anterior limb, the body shows three rounded eminences corresponding to the auricle of the heart, the ventricle of the heart, and the liver.

Embryos of Thirty-one to Thirty-two Days, io to I2 $\mathrm{mm}$.-As typical specimens of this stage we may take two embryos, one of $10.0 \mathrm{~mm}$. (Figs. 99 and 100), the other of II. $5 \mathrm{~mm}$. (Fig. IOI). Figure 99 shows the embryo, Series 1000, the chorion and amnion having been opened; the embryo lies somewhat obliquely on its left side, therefore figure too has been added to give a correct profile compara-

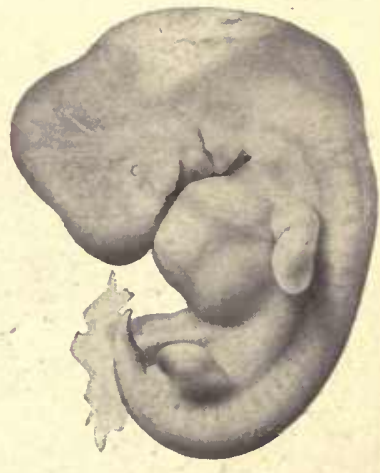

Fig. 98.-Human Embryo of 9.4 MM. SERIES IOO5. $\times 5$ diams. ble with the other figures of the series. As compared with the previous stage (Fig. 97), the back has straightened out somewhat, though the lower end of the body is still rolled over. The head has risen and increased considerably in size. Between the end of the region of the hind-brain and the level of the arm the dorsal outline has become slightly concave. This concavity His designated the "Nackengrube." The first gill-cleft, owing to the completed closure of the cervical sinus, is the only one visible externally. It is the anlage of the external auditory meatus. It is separated from the mouth by a prominent mandibular arch. On the cephalic side of the mouth the maxillary process has become more prominent, but the two portions of the maxilla do not yet meet in the median line. The primitive segments are still marked externally. The limbs show indications of their tripartite division, the fore-limb being more advanced than the hind-limb. The division of the digits of the hand is just indicated. The abdomen bulges out, owing to the growth of the liver. There is a true tail, which is now near its maximum development. The umbilical cord has lengthened and shows the commencement of its spiral twisting. The amnion springs from the end of the cord, leaving only a short stretch of the body-stalk between the cord proper and the chorion. The amnion envelops the embryo closely. In embryos slightly older than these the changes in form above mentioned have progressed further. The body is straighter, the head 
is larger, and has risen so as to be about at right angles to the body. The concavity (Nackengrube) below the hind-brain in the outline of the neck is more marked. The limbs are longer, the fingers more distinct. Where the mandibles meet in the median line, the separation of lip and chin has begun.

Embryos of Thirty-six Days, $14 \mathrm{~mm}$. - The correlation of age and size for this stage cannot be recorded as absolute, but we may say that embryos of this length

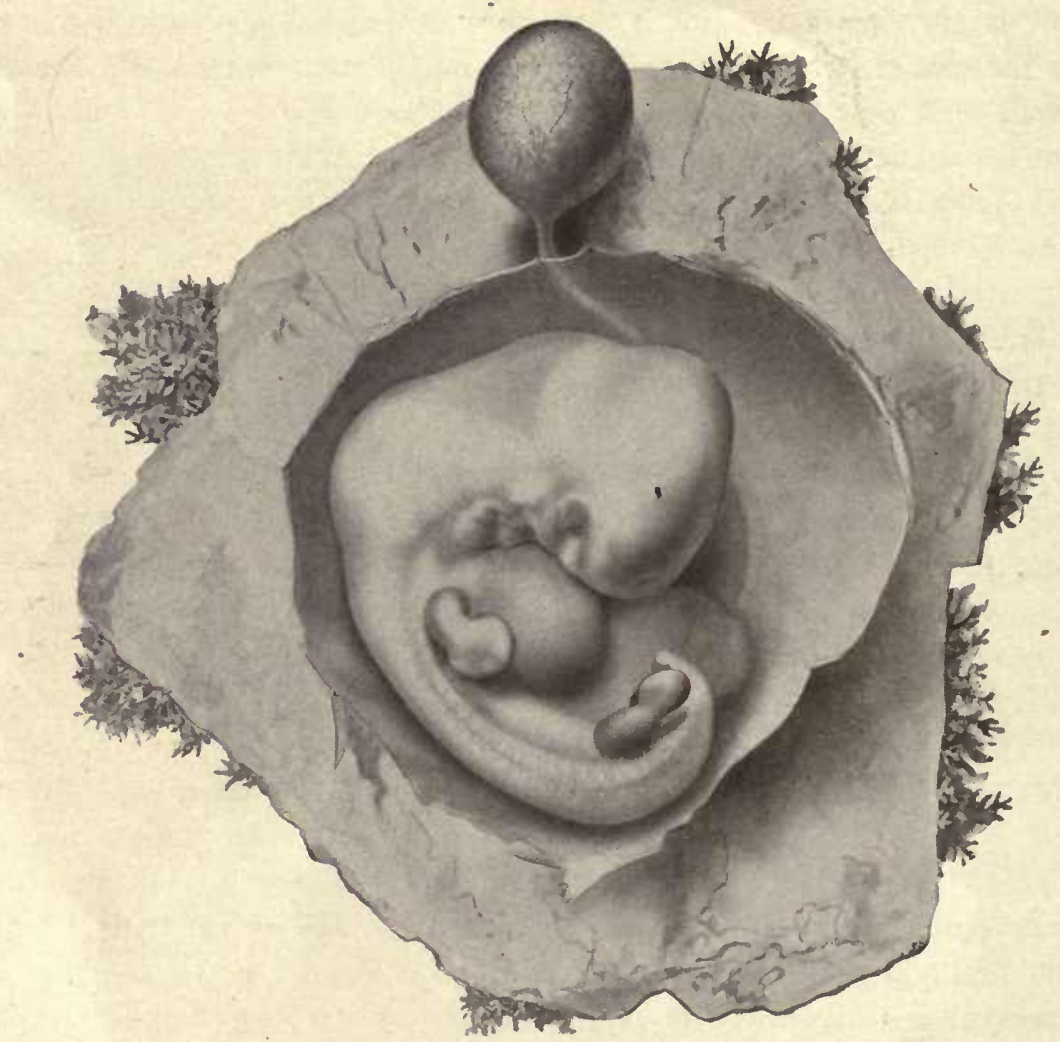

Fig. 99.-Human Embryo of ro.o mm. with the Amnion, Chorion, and Yolk-sac. Series rooo. $\times 5$ diams. (Compare Fig. 100.)

are about five weeks old. The body is now nearly straight (Fig. 102). The lower limbs project beyond the outline of the body in profile views. The bulging of the outline at the neck-bend is characteristic of this stage, but in the specimen figured the protuberance is unusually great. The ventral outline, owing to the large size of the heart and liver, is very protuberant, and at this stage we find that the portion of the umbilical cord adjoining the embryo is greatly enlarged, owing to the distention of its colom, so that a large cavity is furnished in which there are always found, as indicated in figure 84 , several coils of intestine. This protrusion of a portion of the intestinal canal, and sometimes even of a small portion of the 
liver, into the extra-embryonic cœlom of the umbilical cord is a constant phenomenon. It begins at a somewhat earlier stage and continues for a considerable period. This curious condition has been observed in many different kinds of mammals in the corresponding stage. Later on, the viscera are entirely withdrawn from the umbilical cord and the cavity itself is wholly obliterated. The umbilical cord is a hollow prolongation of the body-wall or somatopleure of the embryo, and the amnion springs from its distal end. The yolk-stalk is very long and narrow. Its entodermal cavity is obliterated. It is the representative of the original broad connection between the yolk-sac and the entodermal cavity of the embryo, although it is now only a small appendage of a loop of the intestine. It bears the blood-

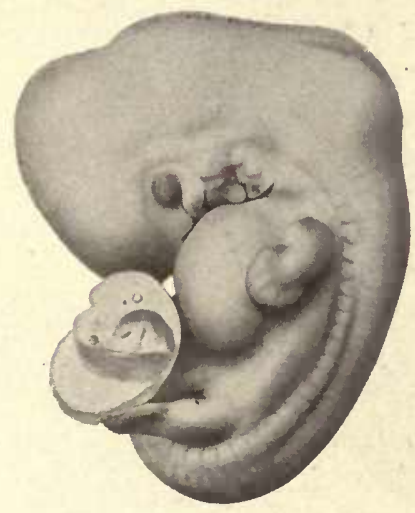

Fig. 100.-Human Embryo of ro.o mM. Series 1000. $\times 5$ diams. (Compare Fig. 99.)

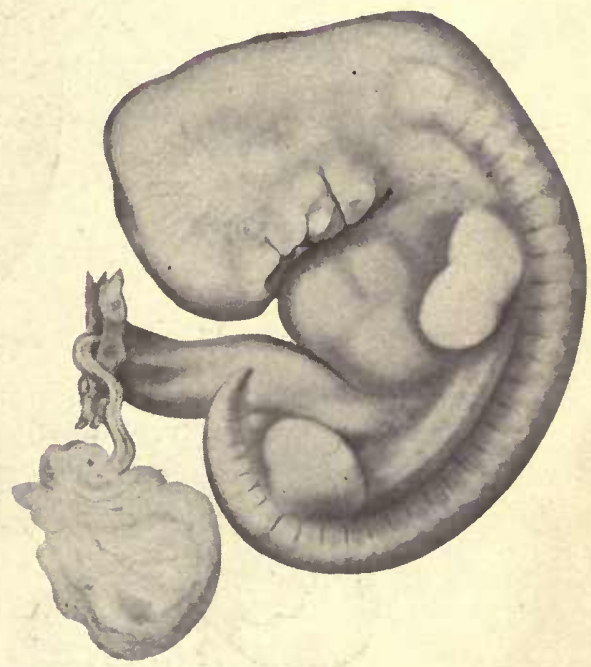

Fig. ioi.-Human Embryo of II . 5 MM. SERIES I006. $\times 5$ diams.

vessels which run from the embryo and ramify upon the yolk-sac. On the caudal side of the umbilical cord we find the tissue of the original body-stalk in which run the allantoic vein and the two allantoic arteries which ramify upon the chorion.

Embryos of Thirty-eight Days, $16 \mathrm{~mm}$. in a chorionic vesicle of 45 by $40 \mathrm{~mm}$.The age of this specimen (Fig. I03) is known by estimate only. This stage represents the transition of the embryo to the fetus, because after the fortieth day the form is distinctly human. The head has risen considerably, and the back has straightened still more, the rectangular neck-bend thus becoming emphasized. The body has become still more protuberant on the ventral side, and in side views the arms reach to the outline of the body. In the anterior limb we note the first indications of the five digits and of the separation of the upper and lower arms. To 
illustrate the variations in the proportions of embryos and to show a slightly more advanced stage, figure 104, of a $17.8 \mathrm{~mm}$. embryo is given and also figure 105, $\mathrm{A}, \mathrm{B}$, giving two views of an embryo of $\mathrm{I} 8$. I $\mathrm{mm}$. All three specimens are probably normal, for it is known that variation is much greater during development than in the adult, a fact which is to be explained in large part by the temporary accelerations or retardations of the development of single organs or regions, which are subsequently compensated for.

Embryos of Forty Days, $19 \mathrm{~mm}$. - The head has risen far toward its definite position, with the result of a very rapid apparent increase in the total length of

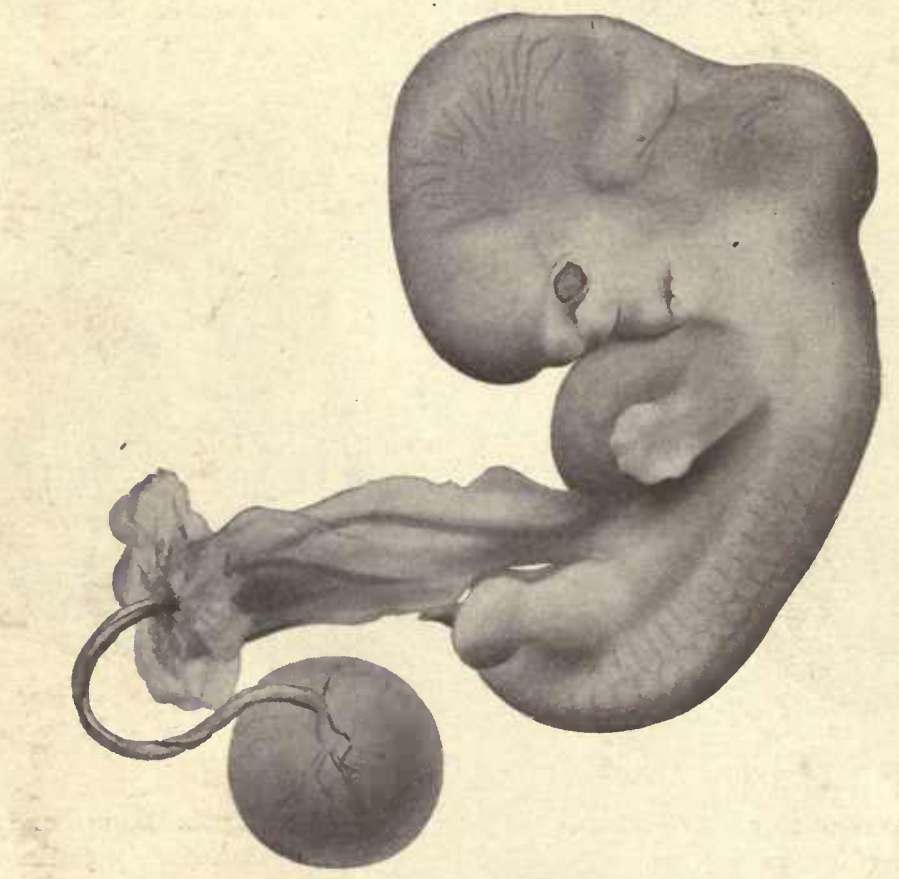

Fig. 102.-Human Embryo of I4.5 Mm. Series IOO3. $\times 5$ diams.

the embryo. The change of position of the head results in bringing the mid-brain finally directly above the hind-brain, the embryo being conceived as having the body vertical. During the elevation of the head the concavity (Nackengrube) at the back of the neck is gradually obliterated. In both head and rump the external modeling, which in earlier stages indicated more or less the position of the internal organs, has become blurred, and in the next stage is found to have nearly or quite disappeared. The maxillary processes have met and united in the median line. The anlages of the eyelids have developed. The concha of the ear is indicated. The arm reaches beyond the heart; the fingers appear as separate outgrowths.

Embryos of Fifty Days, 2I mm.-The author has a fair specimen which came into his possession with no history whatever, but it agrees very closely with His's 


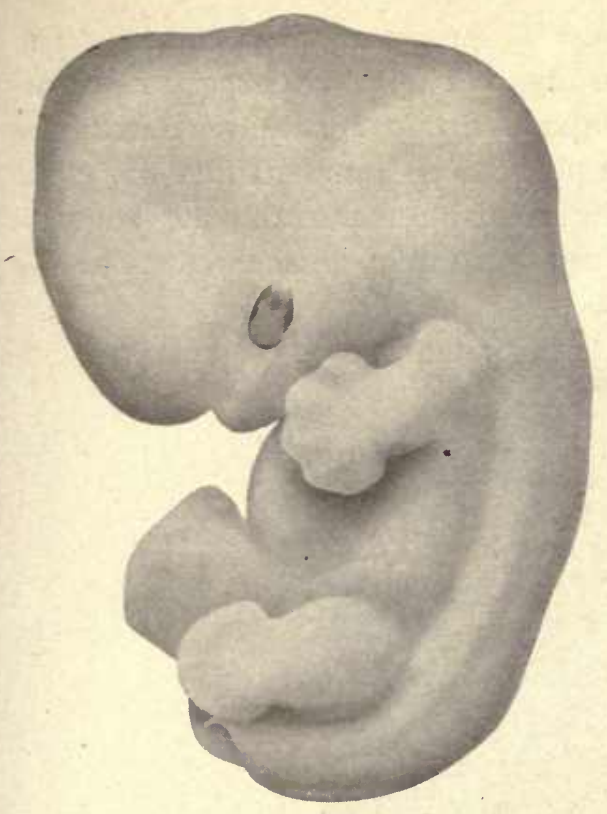

Fig. io3.-Human Embryo of i6.0 mir. SERIES Ii $28 . \times 5$ diams.

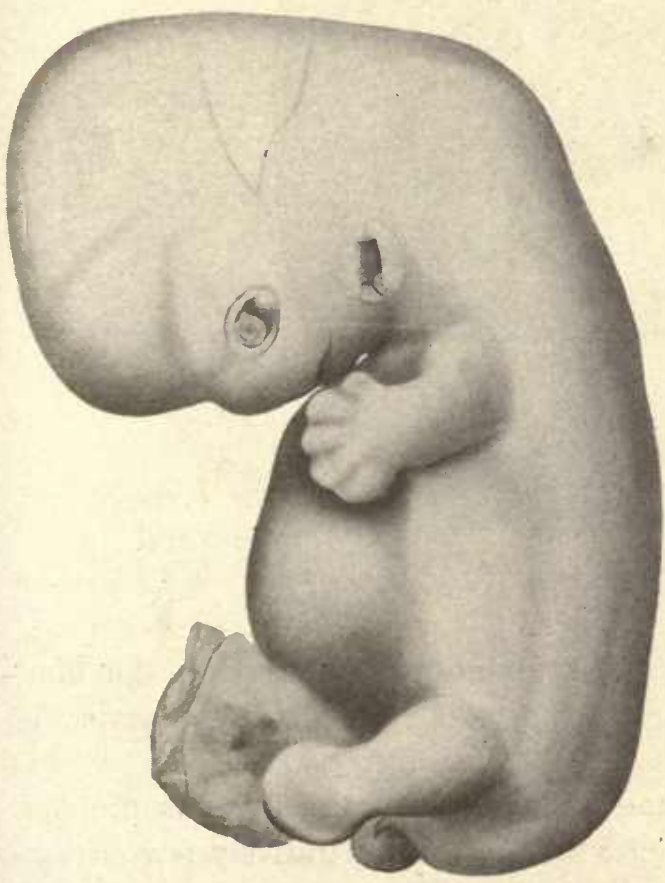

A

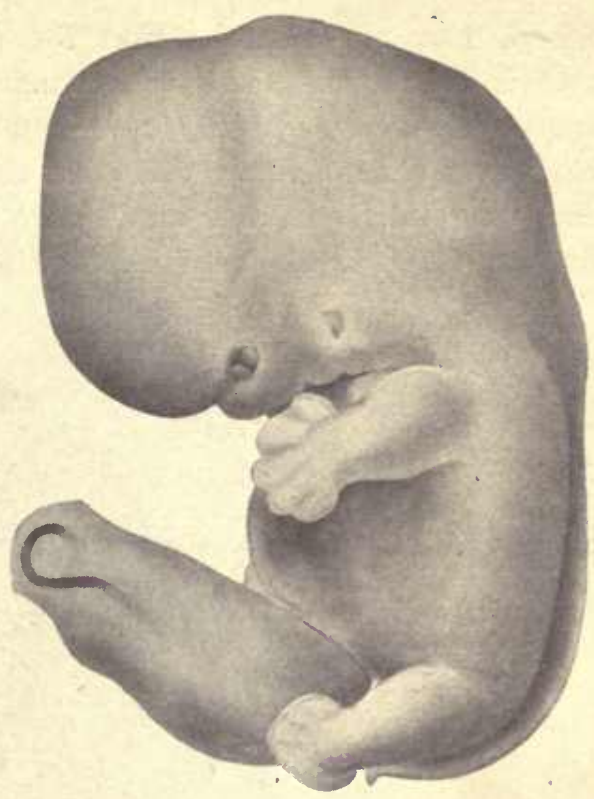

Fig, 104.-Human Embryo of i 7.8 ma. SERIES $839 . \times 5$ diams.

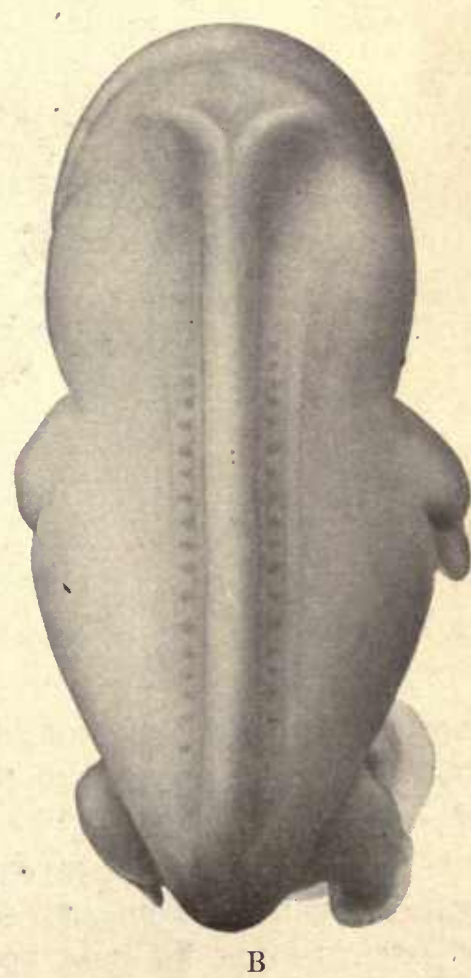

Fig. 105.-Human Embryo of i8. 1 mm. Series it 29. $\times 5$ diams. 
embryo Ltz, of which he fixes the probable age as just over seven weeks. The head is nearer its final position than in figure 103, and relatively larger in proportion to the body, In the eye, cornea and conjunctiva are clearly separated; the face has the fetal form, the nose, mouth, and chin being fully marked off. The arms are clearly divided into upper and lower segments; the five digits are well developed; the hands rest over the heart and nearly touch one another. The leg

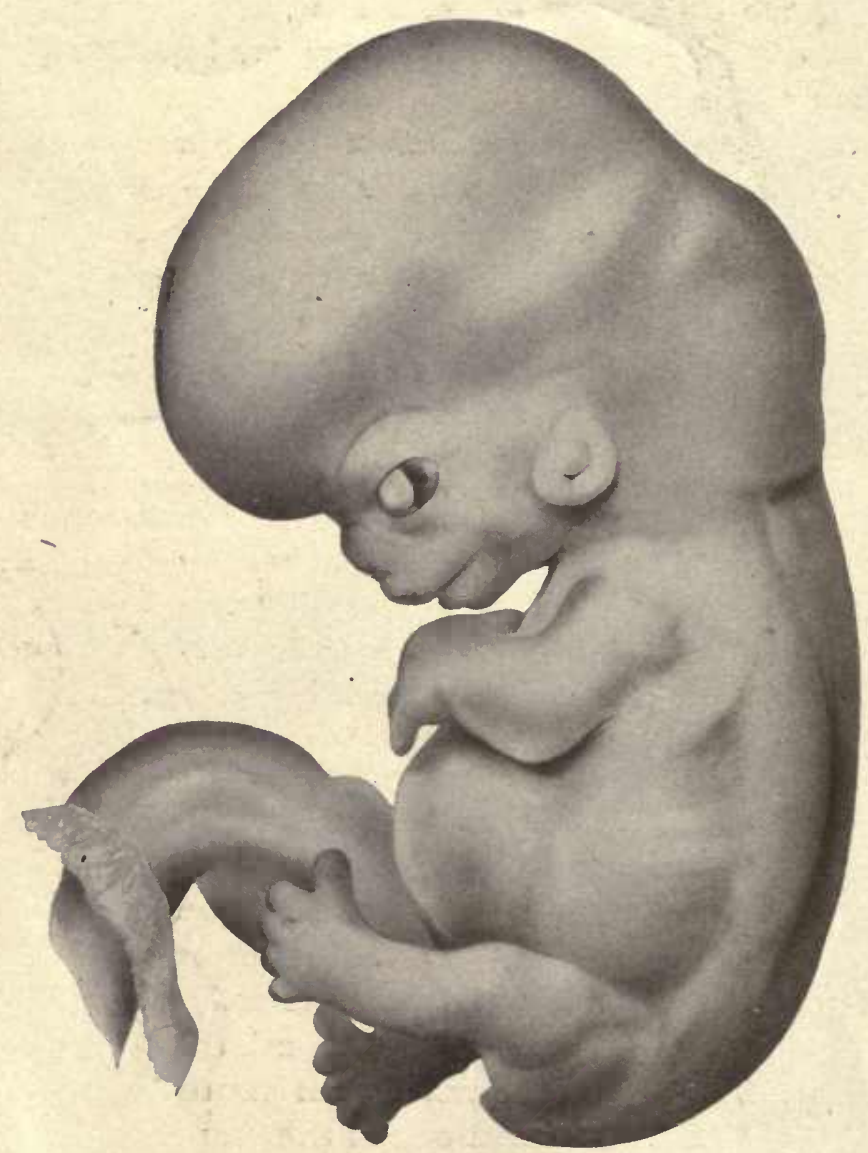

Fig. 106.-Human Embryo of 22.8 mm. Series 87 r. $\times 5$ diams.

shows the triparite division; the toes are just beginning to be free, but the hindlimb is much less advanced than the fore-limb. The tail is still a freely projecting appendage.

Embryos of Fifty-three Days, 22-23 mm.-The specimen (Fig. Io6) is probably quite normal. As compared with the last stage, there are comparatively few changes of external form; the most noteworthy are perhaps the increased development of the legs and feet and the commencing disappearance of the free tail. At this time the 
EMBRYO OF SIXTY-TWO DAYS.

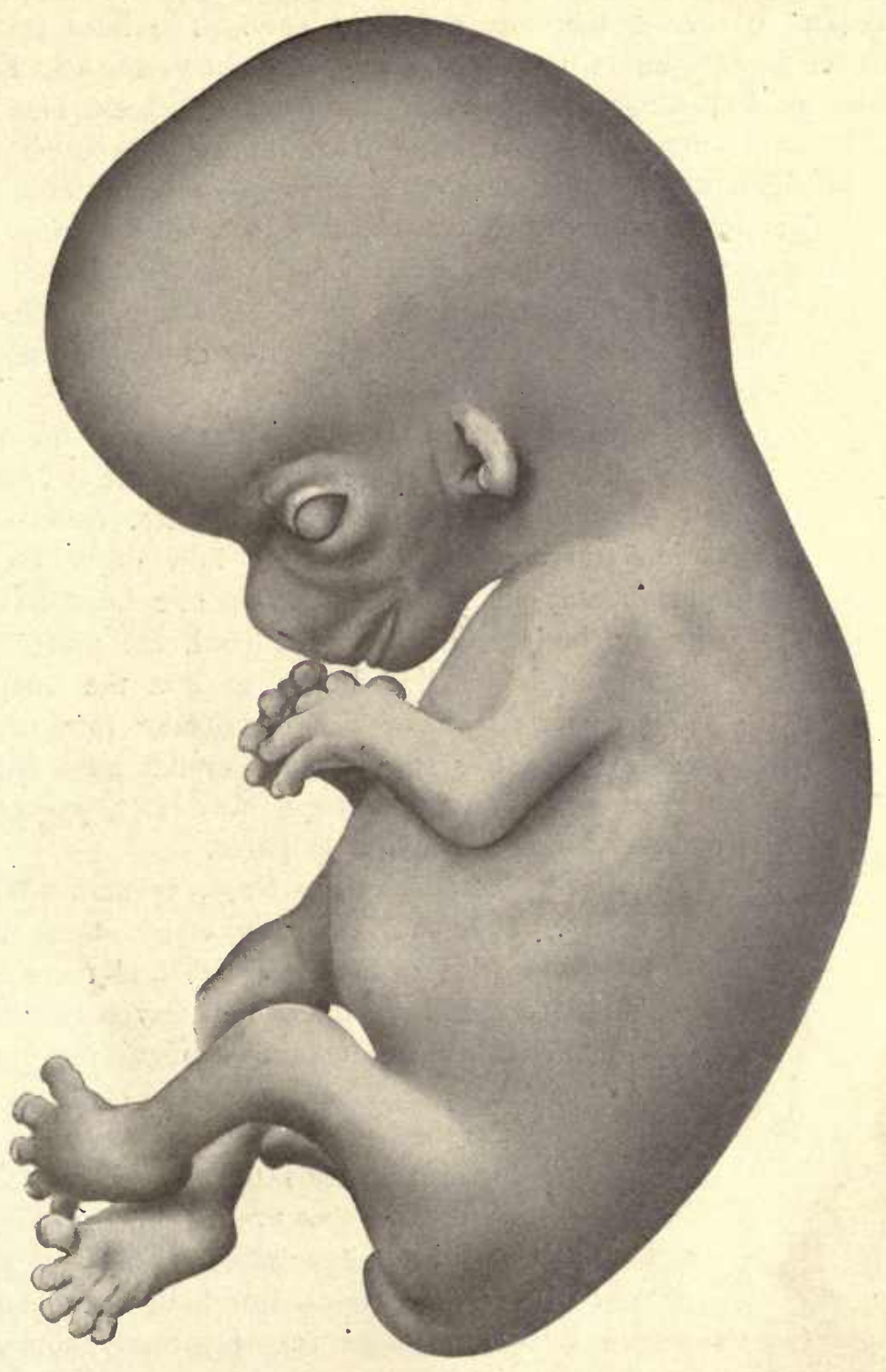

Fig. 107.-Human Embryo of 30 mm. Series 9i3. $\times 5$ diams. 
protrusion of the coils of the intestine into the cœlom of the umbilical cord is about at its maximum.

Embryos of Sixty-two Days, $30 \mathrm{~mm}$.- The present specimen (Fig. 107) came with no data and its age is therefore estimated only. The head is still larger in proportion to the body than in figure ro6. The face shows the two lines which, as seen in profile, mark the two ridges which run over the cheek, one alongside the nose to the corner of the mouth, the other from the eye; these ridges are highly characteristic of the ninth week, and traces of them not rarely persist in the adult physiognomy. The limbs have grown considerably, the hands being lifted toward the face; at the elbow there is a considerable bend; the toes are all free, and the soles of the feet are turned each toward the other. The tail has disappeared as a free appendage. The external genitalia are considerably developed; the clitoris-penis projects some distance.

Embryo of Sixty-four Days, $32 \mathrm{~mm}$.-A specimen came with the following history: "January 4, I886, last flow began; March I3, 1886, abortion"; between these two dates are sixty-eight days; but as the flow took place, conception probably occurred after menstruation, therefore if we deduct four days, making the age sixty-four days, we shall probably be not far wrong. The head has not yet assumed its final angle with the body. On the other hand, the protuberance of the

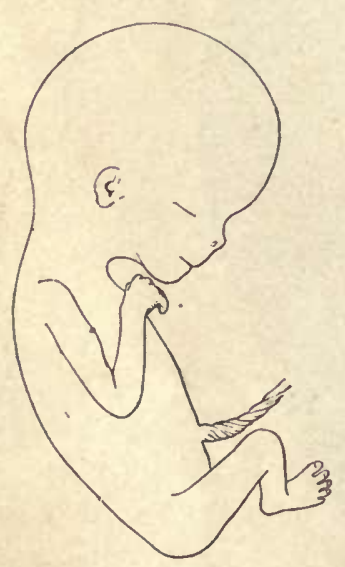

Fig. 108.-Human Embryo of 55 MM. SEVENTY-FIVE Days. Natural Size. abdomen is much reduced, so that the body as a whole has begun to have a more slender form than in earlier stages. In this specimen the eyelids have not even begun to meet; in another they have met, except just in the center, where is still a loophole.

Embryo of Seventy-five Days, $55 \mathrm{~mm}$.-We figure next (Fig. 108), a fetus concerning which there are no data. Comparison with embryos of two and three months leads us to place it a little under half way between them. The specimen has essentially the configuration of the young child; but the head is very large and the body slender; the position of the limbs is typical; the upper arm is bent down, the forearm extends toward the chin; the knee is bent so as to throw the foot toward the median line; the soles of the feet are placed obliquely facing one another; the anlages of the nails can be recognized on both the fingers and toes.

Embryos of the eleventh and twelfth weeks are very rarely obtained. I have never had a normal one of this period with data to determine the age.

Embryos of Three Months, 78 to $80 \mathrm{~mm}$. - In my experience there is no other age at which abortion of normal embryos occurs so frequently as at three months, and I possess a number of specimens of this age, which agree very, closely with one another in size and form. The fetus drawn in figure rog may be taken to represent accurately the appearance of the human embryo at three months. The 
position of the limbs is typical for this age, but there are slight variations, in that the hands, one or both, may project more and be higher or lower; usually the right foot lies in front of the left, but not always. Figure IIo gives the front view of the face of the same embryo to show the closed eyelids, the broad triangular nose, the thick lips, and the pointed chin.

Embryos of Three and One-half Months, 108 to I Io mm.-I have several speci-

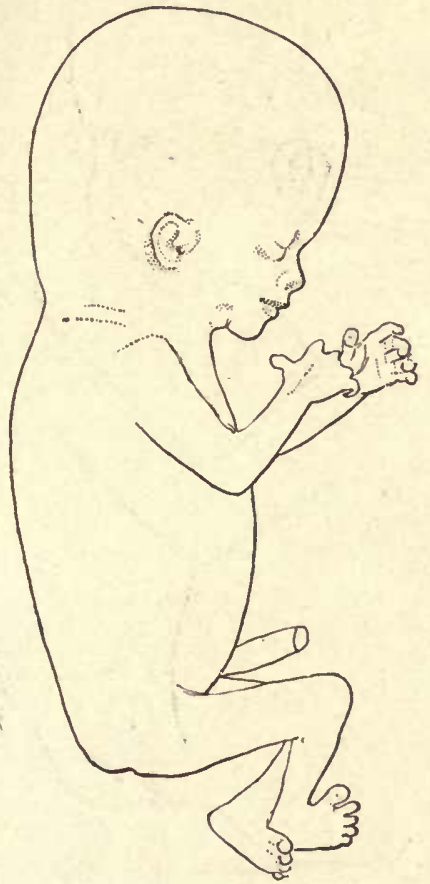

Fig. 109.-Human EMbryo of 78 ma. Three Months. Natural Size. mens which represent this age. Two of them are figured, one to show the natural attitude (Fig. III) in utero, the other (Fig. II2) to show the natural attitude assumed by the embryo when released from its membranes. The first specimen came to me with no history, but as it is certainly a little larger than several other fetuses of about one hundred and six days, it is probably a little older.

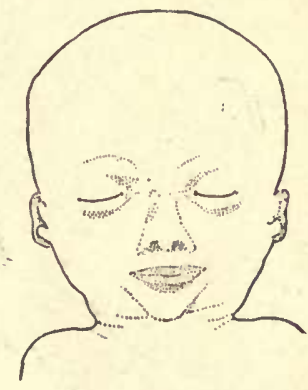

- Fig. IIO.-Front View of. THE FACE of the EMbryo Shown in Figure iog. Natural Size.

The head is bent forward (Fig. III); the back is curved; the arms and legs are both raised toware the face; the right leg is nearly straight, so that the toes are brought against the forehead, while the left leg is bent at the knee, bringing the left foot against the right thigh. In this attitude the embryo fills out as perfectly as possible an oval space, and fits, therefore, the cavity of the uterus. The second specimen (Fig. II2) shows the attitude assumed by the embryo when free, and proves that the position in utero (Fig. III) is a constrained one. This fetus was received November 30,1883 . The delivery took place on the morning of that day, and the last menstruation had ceased one hundred and six days previously; the remarkably fresh condition of the fetus indicated that it had been dead only a very short time, so that we cannot be far wrong in putting its exact age at one hundred and six days.

Embryo of Four Months, I55 mm.-The fetus shown in figure II3 came in a 
very fresh condition, January 2, I887, with the statement: "Conception said to have taken place September I, I886; fetus came away January 2, about noon." The embryo is typical in size and development for four months, except that the

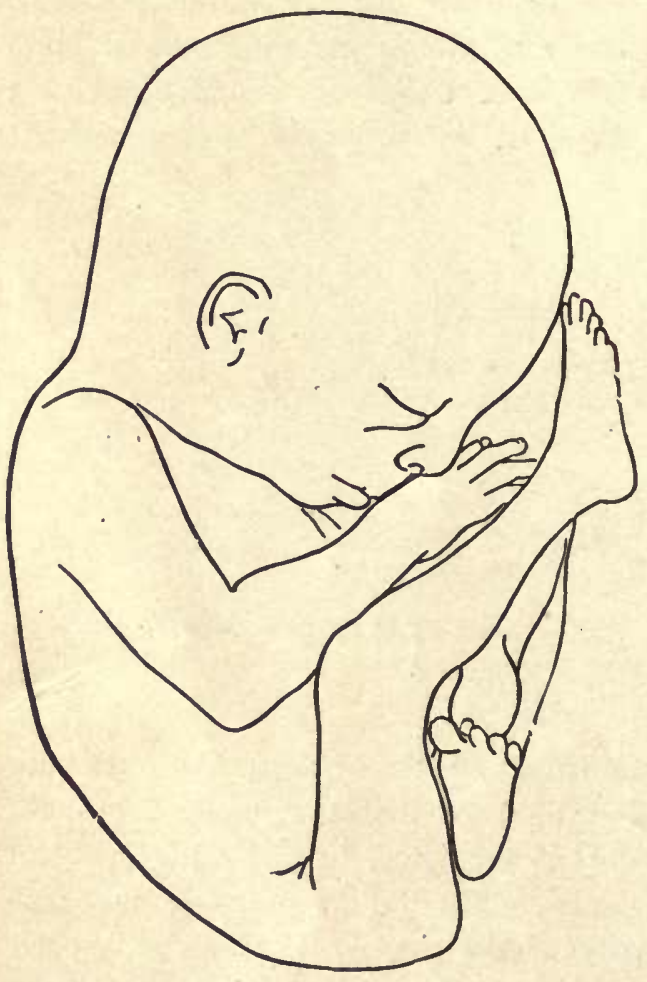

Fig. III.-Human Earbyo of I 20 MM. (? ONE Hundred and Ten Days.) Natural Size.

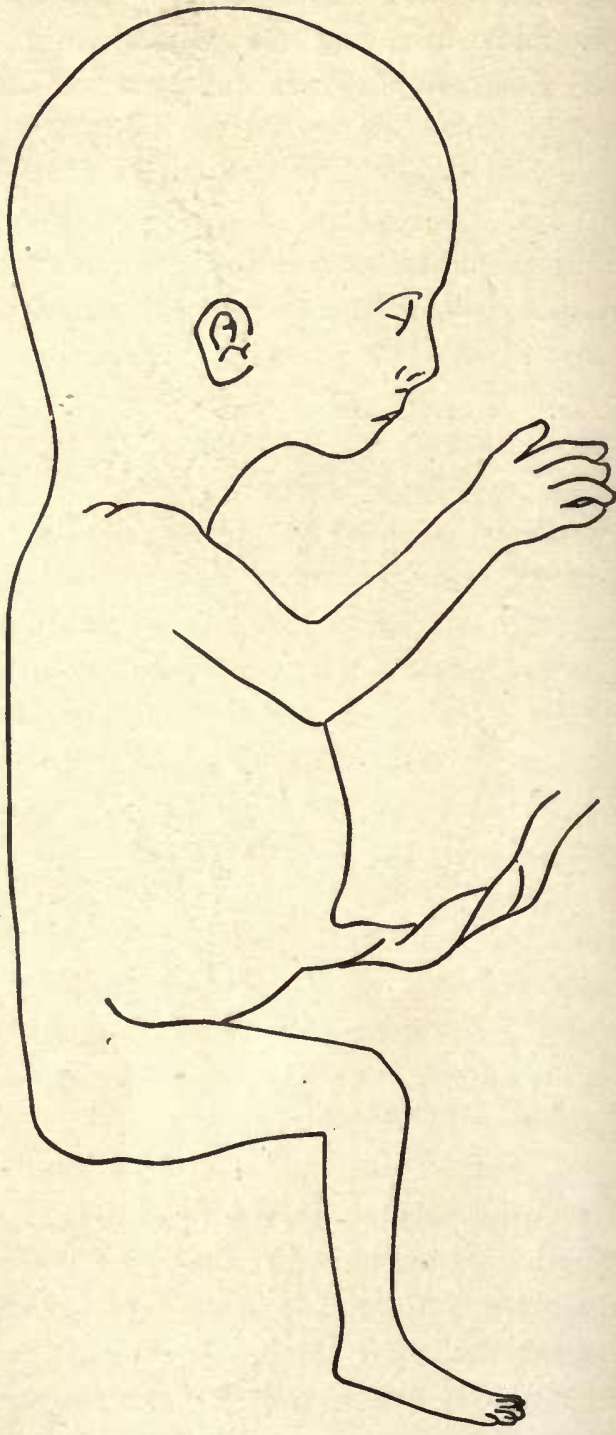

Fig. II 2.- Human Embryo of il8 mim. ONe Hundred and Six Days. Natural Size.

crown is higher than usual, and the antero-posterior diameter of the head somewhat below the average.

The natural attitude in utero is similar to that of figure III; the attitude shown is that-assumed by the fetus when released from the membranes. 


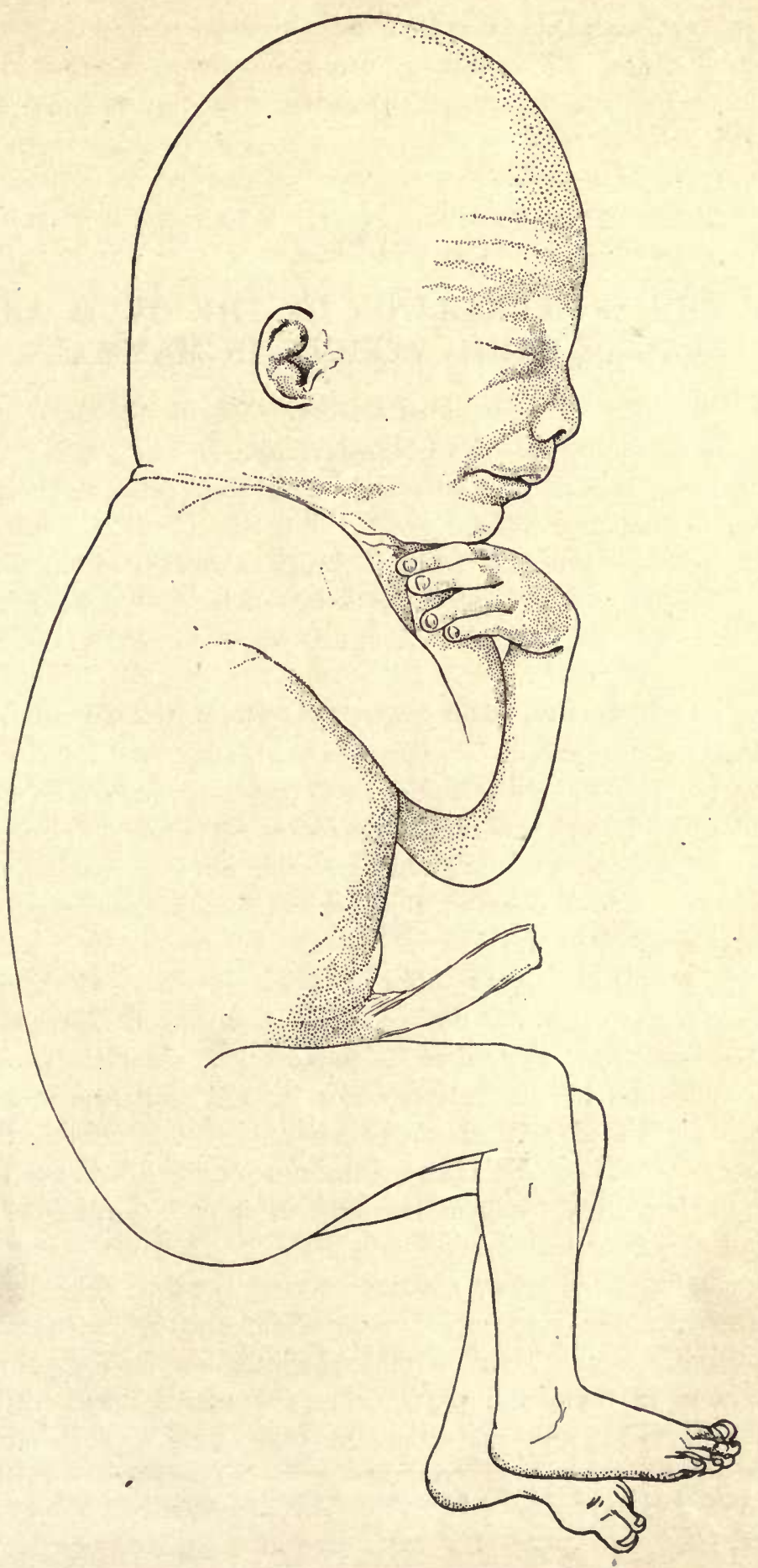

Fig. ir3.-Human, Embryo of 55 mim. One Hundred and Twenty-three Days. Natural Size. 


\section{CHAPTER IV.}

\section{STUDY OF THE SEGMENTATION OF THE OVUM AND OF THE BLASTODERMIC VESICLE IN MAMMALS.}

In selecting material for general laboratory work on the early stages of mammals, we are governed by practical considerations. The white mouse and the rabbit are both easily kept in the laboratory and their brecding may be accurately determined. Up to the present time the earliest phases of the development of the mammalian embryo have been far more thoroughly studied in the white mouse than in any other mammal. For the next following stages the same remark applies to the rabbit. Hence these two forms have been chosen for the practical study.

\section{The Maturation, Fertilization, and Segmentation of the Ovum in White Mice.}

These animals are selected for the practical study of the earliest stages of development for two reasons: first, because the processes have been more thoroughly studied in them than in any other mammals; and, second, because they are easily kcpt and breed freely, so that abundant material may be secured with comparatively little trouble. Those desiring further information are referred to Sobotta's and Kirkham's original memoirs.*

Heat occurs twenty-one days after littering, a fact which may be taken advantage of to secure ova of the desired age. Coitus can take place only during heat, for it is then only that the vagina is found open; at other times its epithelium concresces to a solid mass. The spermatozoa do not penetrate into the tube until some time after the coitus. After the discharge of the semen, the contents of the large seminal vesicle are ejaculated into the vagina, completely filling it and hardening into a white plug (bouchon vaginal), as in guinea-pigs. From twenty to thirty hours later the plug softens and falls out.

The uterine tubes are narrow, much contorted canals. The fimbriate opening of the tube penetrates the connective tissue about the ovum so that the fimbrix lie in the periovarial space. There is ciliated epithelium in the proximal region of the tube only, none in the distal parts or in the uterus itself. During heat the periovarial space is filled with an abundant clear fluid. This also distends the

* Sobotta, "Die Befruchtung und Furchung des Eies der Maus," Arch. \%. mikrosk. Anat., vol. xLv, I5-93, Pl. II-IV (I895).

Kirkham, "The Maturation of the Egg of the White Mouse." Trans. Connecticut Academy, xiII, 65-87, I'l. I-VIII. (Corrects several important errors of the preceding paper.) Also, Biol. Bull., (r9Io) XVIII, 245. 
proximal part of the tube, forming, as it were, a special sac, with a distended epithelial lining. At the time of coitus ovulation has generally taken place; the ovum, still surrounded by the cells of the corona radiata, is found in the fluid of the distended proximal section of the tube: It is probable that the ova are carried from the periovarial space not only by the currents created by the cilia of the fimbriate opening, but also by a sort of pumping action of the tube itself. For at the beginning of the period of heat we find that the periovarial space contains much fluid, but later, when the ova are in the tube, this space is empty and the tube contains fluid. The ovum of the mouse measures only $80 \mu$ or less in diameter, and is therefore the smallest known mammalian ovum. (The ovum of the cat measures $200 \mu$, of the rabbit $16 \mathrm{r} \mu$.) It is surrounded by a very thin zona pellucida $(16-36 \mu)$, and contains only a few yolk grains, a portion of which may be blackened by osmic acid. These ova offer the further special peculiarity that the first polar globule, which is always formed in the ovary, is lost in $80-90$ per cent of the ova, probably by extrusion through the zona pellucida, so that even after the formation of the second globule, they still often have only a single globule within the zona. The second globule is produced only after the ovum has been transferred to the uterine. tube, and then only after a spermatozoon has entered. The process for formation of the first and second globules is not the same, although there is a general similarity.

The First Polar Globule.-The first polar globule is formed, as stated, while the ovum is still in the unruptured Graafian follicle of the ovary. The nucleus moves toward one side of the ovum and is there transformed into a mitotic spindle, the axis of which is more or less nearly at right angles to the radius of the ovum (Fig. II4). The spindle itself is large, pointed at the ends, with curving achromatic threads. The chromosomes are probably twelve in number, but they vary in size and shape, and even in number, which has been explained as the result of precocious division of some of them. They gather themselves into an equatorial plate. They are elongated, pointed at the ends, with irregular sides, and are very large. Minute centrioles have been observed at the end of the spindle, but there are no astral rays extending from the ends of the spindle into the protoplasm. The chromosomes become somewhat $\mathrm{V}$-shaped. They divide by a trans-

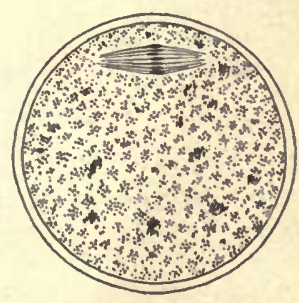

Fig. II4.-OVuII OF White MOUSE, WITH THE FIRST POLAR Spindle in TangenTLAL Position. $\times 500$ diams.- (After $J$. Sobotta.) verse separation at the apex of the $\mathrm{V}$. Chromosome halves migrate toward the end of the spindle. The stages occur probably about twenty-four hours before the rupture of the follicle. The spindle now assumes a radial position, and one of its poles lies close to the surface of the ovum, which has meanwhile diminished in size so that there is a considerable space between the yolk and the zona pellucida. Division occurs and the first polar globule is formed, and lies in the perivitelline space. In the mouse it is remarkable, as is also the second, but smaller, polar 
globule, for its large size. It is usually spherical in fresh, oval in preserved specimens, and measures in the living state from $22-28 \mu$ in diameter. It has a distinct cell-membrane, a protoplasm which resembles that of the ovum, and may even contain granules of yolk. Soon after its separation from the ovum its nucleus becomes well developed and membranate. Except, therefore, that the number of chromosomes which enter into its formation is half the normal number, we might say that it differs little from an ordinary cell.

The Second Polar Globule.-After the formation of the first polar globule ovulation takes place, and during the next changes the ovum is situated in the ampulla of the Fallopian tubes. In the mouse, unless the ovum is fertilized, it forms no second polar globule, but instead undergoes autolysis either in the ovary or in the uterine tube. The nucleus of the ovum does not enter into a condition of repose, but at once transforms itself, as in other animals, into the second polar spindle. After the constricting off of the first polar globule, twelve half chromosomes (dyads) are left in the ovum. They are drawn into the equator of a new spindle and split longitudinally.

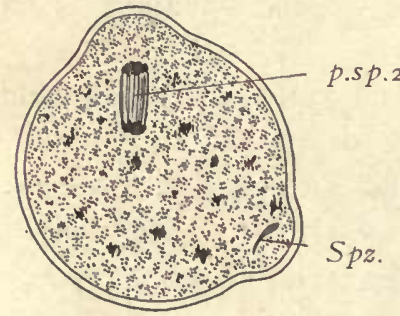

Fig. I15.-Ovum of White Mouse, Dividing to Produce the Polar Globule.

P.sp.2, Second polar spindle. Spz, Head of spermatozoon. $\times 500$ diams. (After $J$. Sobotta.)

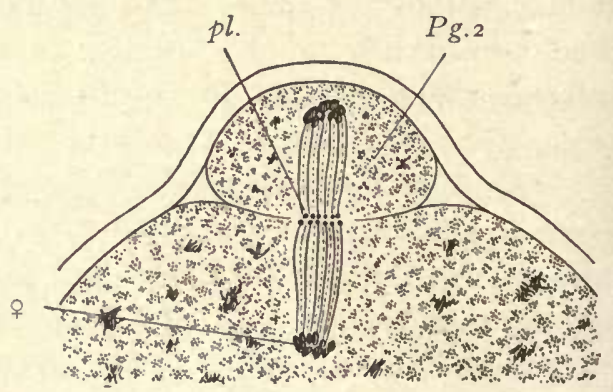

Fig, ir6.-Ovum of White Mouse, Showing the Metaphase of the Division Producing the First Polar Globule.

$P g$. 2, Second polar globule. $p l$, Cell-plate. $q_{\text {, }}$ Female pro-nucleus. $\times \mathrm{I}_{500}$ diams.-(After J. Sobotta.)

The second polar spindle is smaller than the first. It lies at right angles to the axis of the ovum and quite close to the surface. It contains twelve thick achromatic fibers, which do not unite at the poles with one another, but end parallel, so that the tip of the spindle is blunted. The chromosomes, when the membrane first disappears, lie irregularly, but shortly after the formation of the spindle they collect together to form an equatorial plate, somewhat as in the figure. They are irregular and of uneven size, twelve in number, or possibly the number may vary somewhat. The chromosomes then divide transversely, and the halves move rapidly toward the ends of the spindle, which during this change passes into the radial position (Fig. II5). The twenty-four univalent chromosomes lengthen into filaments of various sizes, and by their form the second spindle can 
be readily identified. The surface of the ovum or the apex of the spindle forms a protuberance. Division of the achromatic fibers takes place, and there is formed a well-marked cell-plate (Fig. II6), and presently the polar globule becomes constricted off. The second body is smaller than the first, measuring from $7-12 \mu$ in diameter, and in the majority of cases is the only one to be found inside the zona after fertilization. Its twelve chromosomes soon form a resting membranate nucleus. The cell-plate appears with unusual distinctness. It is at about this stage that the spermatozoon is found to have entered the ovum (Fig. II 7, B) and to have formed there the male pro-nucleus. During all these stages no centrosome appears at the poles of the spindle, but centrioles are said to have been observed at the spindle apices. No astral rays appear in the protoplasm, although in many
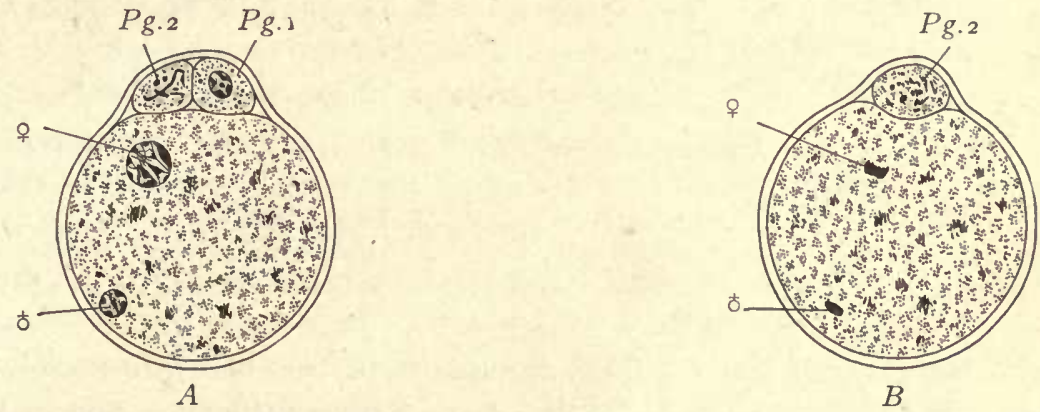

Fig. il 7 .-Two Ova of White Mouse. A, with two Polar Globules. B, with the Second Polar Globule ONLY.

Pg. I, First polar globule. Pg. 2, Second polar globule. t, Male pro-nucleus. $\&$ Female pro-nucleus. $\times 500$ diams. $-($ After J.Sobotta. $)$

eggs these astral figures are extremely conspicuous. The female pro-nuclear elements appear at first as a dense cluster of chromatin granules (Fig. II $7, B$ \%), and fuse apparently into a compact mass, which grows rapidly in size, presumably by the absorption of fluid from the yolk, and, as it enlarges, acquires a more distinct outline, and presently shows a network structure in its interior (Fig. II $7, A$ ), with irregular chromatin masses. It continues to grow more and more, and develops at the same time a series of nucleoli more or less uniform in size. This stage may be regarded as that of the completed female pro-nucleus.

Fertilization occurs in the ampulla of the uterine tube about 6-ro hours after the coitus. Unless it occurs the development of the second polar globule does not take place. It is accomplished, normally, by the penetration of a single spermatozoon into the yolk. The tail of the spermatozoon usually enters the egg at least in part. The head of the spermatozoon can be recognized at first by its shape (Fig. II6, o). In. position it is typically more or less remote from the polar spindle. While the second polar globule is forming the head assumes a rounded form, and becomes the male pro-nucleus (Fig. Ir7, ¿). The group of twelve chromosomes left 
in the ovum after the division of the polar spindle becomes the female pro-nucleus (Fig. II $7, \%$ ). Both pro-nuclei now enlarge, the female most, and assume a nearly spherical form, but have no membrane (Fig. II $7, A$ ). They approach one another, drawing also toward the center of the ovum, until they come to lie side by side,

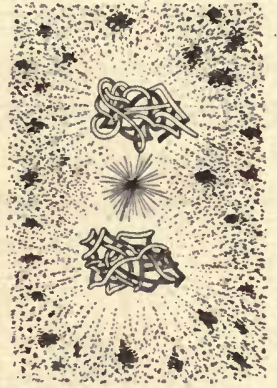

FIG. II8,-OVuM OF WHITE Mouse. Beginning OF THF, Conjugation of the ProNUCLEI. $\times 1500$ diams. $-($ After Sobotta.)

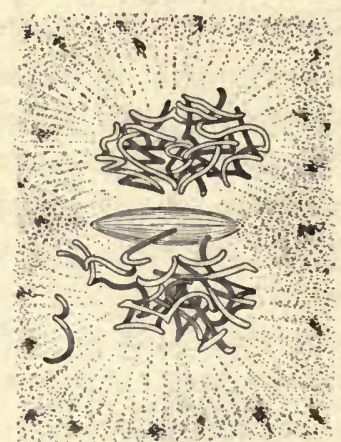

Fig. irg.-Ovul of IVhite Mouse. Conjugation of the PRO-NUCLEI, AND FoRMATION OF the Segmentation Spindle. $\times$ I500 diams.-(After Sobotta.)

yet separated by a small space. The chromatin of the' two pro-nuclei forms distinct threads. Next there appears in the space between them a centrosome with a few radiating lines around it (Fig. II8). From the centrosome arises, just how is not clear, a spindle of achromatic threads (Fig. II9). The chromatin of each pro-

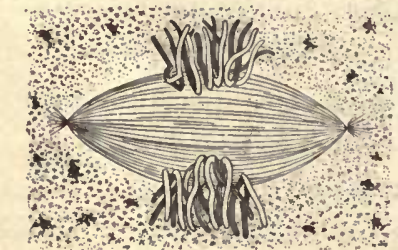

Fig. I20.-OVUM OF WhItE Mouse. First Segmentation SPINDLE WITH THE CHROMOSOMES OF THE PRO-NUCLEI STILL FORMING TWO DISTINCT Groups. $\times{ }_{500}$ diams.(After Sobotta.)

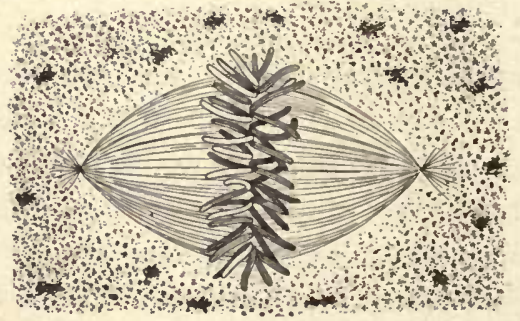

Fig. I21.-Ovum of White Mouse. First Segmentation Spindle with Equatorial Plate of Chromosomes, $\times$ I $_{500}$ diams.-(After Sobotta.)

nucleus now forms a group of well-defined, elongated, somewhat crooked chromosomes. The two groups of chromosomes are quite distinct, and are separated from one another by the intervening spindle (Fig. IIg). The spindle continues to grow, and the chromosomes of the male pro-nucleus on the one side and the female pro- 
nucleus on the other attach themselves to the equatorial region of the spindle (Fig. I20). The spindle continues to grow; the chromosomes become $\mathrm{V}$-shaped and arrange themselves as the so-called equatorial plate, in which the chromosomes of the two pro-nuclei can no longer be distinguished from one another (Fig. I20). At each end of the spindle is a distinct centrosome with a very faint, small astral radiation in the neighboring protoplasm. This spindle is the beginning of the division of what we may call the segmentation nucleus. In the mouse the two pro-nuclei do not actually fuse into a single nucleus before the formation of the spindle, which initiates the first division of the fertilized ovum, so that, strictly speaking, there is no fusion of the pro-nuclei to make a segmentation nucleus. There is, nevertheless, a true fusion of the pro-nuclei accomplished, although it is somewhat masked by the early commencement of the first segmentation spindle, which develops at the same time that the fusion of the pro-nuclei is being completed.

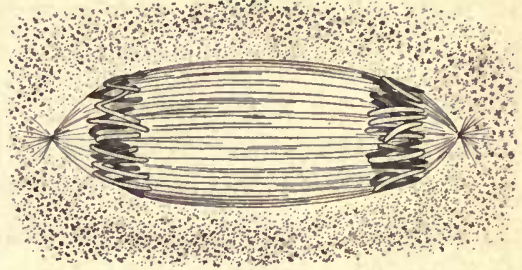

Fig. I 22.-Ovum of White Mouse. First Segmentation Spindle.

The chromosomes have divided and have migrated toward the poles of the spindle, forming two groups. $\times$ I500 diams.-(After Sobotta.)

The chromosomes of the equatorial plate now divide, probably by splitting longitudinally, so that the number of chromosomes is doubled. During the splitting the chromosomes tend to draw apart from one another. At the same time the spindle, without changing its length, becomes somewhat narrower. The chromosomes now move apart from the equator toward the two poles, forming two groups, each group
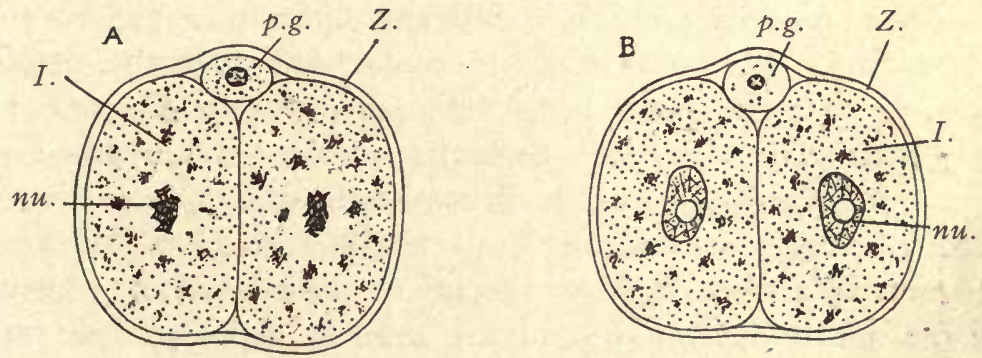

Fig. I23.-Ova of White Mouse with Two Segmentation Spheres or Cells.

$A$, Telophase of the division; the chromosomes are reconstituting the nucleus. $B$, Membranate nucleus reconstituted. I, First cell of segmentation. $m u$, Nucleus. p.g, Polar globules. Z, Zona pellucida. $\times 500$ diams.-(After Sobotta.)

containing half of the total number of chromosomes (Fig. 122), and at the same time the whole ovum becomes somewhat elongated in the direction corresponding with the axis of the spindle. The chromatin granules accumulate at the two poles of the spindle. The achromatic threads between the poles break through. Then the actual cleavage of the elongated ovum into two cells becomes marked in the 
protoplasm, and the line of separation of the two cells passes through the equator of the spindle (Fig. I23, A). The accumulated granules of chromatin then reconstitute the resting membranate nucleus (Fig. 123, B). In brief, the segmentation of the ovum is a typical indirect or mitotic cell-division. In the mouse the first cleavage is completed about twenty-six hours after the coitus. The second cleavage is not completed until twenty-four hours later. When first formed, the two segmentation spheres are oval and entirely separated from one another, but subsequently they flatten against one another and become appressed, a phenomenon of which we have no explanation. In most mammals which have been studied there is more or less space between the segmenting ovum and the zona (see Fig. 6), but in the mouse this space is reduced to a minimum and the zona is often stretched into irregular forms during the changes of the ovum.

\section{Method of Obtaining Blastodermic Vesicles from the Rabbit.}

The does should be allowed to become pregnant and be isolated until they have littered; the date of littering should be noted, and thirty days thereafter the buck be admitted and the exact time of the covering recorded. At the proper number of days thereafter the animal should be killed and the uterus removed at once. It may be opened with two pairs of forceps used to split the outer muscular walls of the organ, beginning the operation at the lower end of the uterus. With a little care this can be done without rupturing the mucous membrane, which is to be afterward also opened in a similar manner with the forceps and the blastodermic vesicles exposed. They are small bodies of rounded form and with a brilliant pearly luster, and are easily observed. During the earlier stages, which occur in the Fallopian tubes, the ova are very small and difficult to find, but by the time the ovum has reached the uterus it has become a blastodermic vesicle measuring about $0.6 \mathrm{~mm}$. in diameter, and, therefore, easily seen by the naked eye. From the fourth day after coitus until the beginning of the seventh day the vesicles lie free in the uterus. Usually early in the seventh day the vesicles, which then measure about 4.5 by $3.5 \mathrm{~mm}$, , begin to attach themselves to the wall of the uterus, and thereafter are much more difficult to remove. At the beginning of the fifth day the ova measure about 0.6 to $0.9 \mathrm{~mm}$. in diameter, but vary greatly in size, and are found more or less near together in the upper portion of the oviduct. By the end of the sixth day they measure about $4.0 \mathrm{~mm}$. and are distributed throughout the entire length of the uterus.

The most useful stages are the vesicles from the beginning of the sixth and seventh days. To preserve the vesicles they must be gently removed from the uterus, great care being necessary not to injure them, and dropped into Zenker's or Hermann's fluid. In either of these they may be left for about an hour and then washed and preserved in the usual manner. Specimens should be examined in the fresh state, just after they have been preserved, and after they have been stained, before they are imbedded. For staining, alum cochineal or borax carmine 
is recommended. Finally, the specimens are to be imbedded in paraffin and cut in series in the usual manner; sections of from 6 to $8 \mu$ are desirable. Unfortunately, no method has yet been devised by which these delicate vesicles may be imbedded without distortion of their form, so that, when the sections are finally obtained, the blastodermic walls are wrinkled and more or less out of shape. But fortunately, owing apparently to its greater thickness, the embryonic area usually escapes distortion and appears in the sections of normal form, or nearly so.

\section{Study of Rabbit Blastodermic Vesicles in Alcohol.}

All of the most important points in the structure of the blastodermic vesicles of the rabbit from the fourth to the seventh day may be fairly well observed by examining the hardened vesicles in alcohol under the microscope. For such examinations the so-called live-box, such as was formerly much used by microscopists for the study of living creatures, will be found very convenient. Care must be taken to have plenty of alcohol around the specimen and not to lower the cover so much as to exert any pressure upon the vesicle. It is not difficult to place the vesicles so that any part of their surface may be examined with a No. 7 objective. In the uncolored specimen the nuclei and even many of the boundaries of the cells can be clearly made out.

In the following descriptions ages have been chosen at which the important characteristics can usually be observed. The variation is so great in range during early stages that the development described below for a given age is often found in older or younger specimens, and specimens of a given age may exhibit a less or a more advanced stage of the embryonic formation than is here put down for that age. In general the correspondence of the stage of development to the size of the vesicle is more exact than to its age.

Vesicles at Five Days $(5 \times 24$ hours).- At this age the vesicles are always found in the upper portion of the uterus. Sometimes all of those in one uterus are quite close together, at other times' somewhat scattered and lying singly. The vesicles are extremely variable in size, for they measure from 0.6 to $0.9 \mathrm{~mm}$. They are spherical or nearly so, and are surrounded by a thin membrane, which in reality corresponds to both the zona pellucida and the outer albuminous envelope, which in the rabbit ovum during segmentation is very thick and conspicuous, but which is always extremely thin when the stage of the blastodermic vesicle is reached. Upon the outside of this really double membrane appear a certain number of small villus-like projections, which are highly refringent. They are probably identical in character with the villi which have been observed upon the ovum of the dog (page 45), but are smaller in all of their dimensions. Immediately underneath the external membrane there is a continuous layer of cells belonging to the ectoderm and extending completely around the ovum. The layer is sometimes designated specifically as the "outer layer" or as the "subzonal layer." It also extends over the embryonic shield; the portion upon the shield is often termed 
Rauber's layer, it having been first observed by that investigator. The cells of the outer layer are quite large and their boundaries are easily recognizable in surface views. Their sides may number four, five, or six, six being perhaps the more usual number, and are variously disposed, so that the cells differ in shape and size. During the next two days of development the cells become, if anything, more irregular in outline and somewhat smaller. The boundaries between the cells are very fine lines; the nuclei are rather large and oval in form, and contain from three to four or five highly refringent granules. Each nucleus is surrounded by a denser court of protoplasm in which there are many granules, some of which are highly refringent. The peripheral portion of the cell is of a loosely reticulate structure with comparatively wide meshes between the threads of the protoplasm. Occasionally there appear in the protoplasm of these cells narrow, elongated, highly refringent bodies somewhat resembling bacilli in appearance, and therefore they are termed the bacilliform bodies. Their nature is unknown; they are more apt to be found in older vesicles. The outer or subzonal layer can be made out over the embryonic shield only by very careful observation. In the shield the cells are several layers thick. The inner cells are very much smaller in size than the cells of the outer layer, are more granular, and contain smaller nuclei which take up a relatively large place in the cell in proportion to its apparent area. Closer observation, utilizing the fine adjustment of the microscope, will show that there are two kinds of cells in the inner part: first, those which, like the cells of the subzonal layer, belong to the ectoderm; and, second, an inner layer of cells, which apparently belongs entirely to the entoderm. In the region of the embryonic shield the ectoderm is, therefore, made up of two distinct layers of cells. The outer or subzonal (Rauber's layer) disappears during the sixth day of development as a distinct layer. The cells of the entoderm form a very thin continuous layer on the under side of the embryonic shield. They may be recognized by the very granular, and therefore dark,* appearance of their protoplasm, and by the rounded form and small size of their nuclei. Similar cells may be observed also extending beyond the limits of the embryonic shield, though not there forming a continuous layer, except perhaps for a very short distance, but rather lying scattered about in patches or isolated. As the cuboidal cells of the ectoderm are confined to the region of the embryonic shield, the cells of the entoderm outside of the shield lie close against the subzonal layer. Here they may be more easily studied than in the shield itself. They are very much smaller than the cells of the outer layer and contain each a nucleus with highly refringent granules, which are now numerous and smaller than the somewhat similar granules in the overlying nuclei of the ectoderm. The farther away we proceed from the edge of the embryonic shield, the fewer we find the entodermal cells. The extent of their distribution varies greatly, and apparently more or less in relation to the size of the blastodermic vesicle, since 
in the smallest vesicles of this age we find the cells only a short distance beyond the edge of the shield, yet in the largest vesicles they have expanded even past the equator.

Vesicles at Six Days. - At this age the vesicles are found more or less scattered and isolated in position from one another through the upper half of the uterus. They are nearly spherical and measure from 1.0 to $\mathrm{r} .6 \mathrm{~mm}$; their walls are very transparent and the somewhat more opaque, round or oval embryonic shield can be readily distinguished with a hand lens (Fig. I24). Its size varies with the diameter of the vesicle, being larger in the larger vesicles; but the proportions are not exact, for a vesicle of given diameter may have an embryonic shield of either larger or smaller dimensions than other vesicles of the same size. Hence, vesicles of different sizes may have embryonic shields of similar dimensions. The actual diameter of the shield is between 0.2 and $0.35 \mathrm{~mm}$. The general structure of the vesicles is the same as at five days, but certain differences may be noted. In preserved specimens the external membrane is very apt to be wrinkled. The subzonal layer has very much the same appearance as before, though the cells are somewhat smaller and it has almost disappeared over the region of the embryonic shield. The manner of its disappearance has not been definitely settled. There is no evidence that the cells degenerate or are cast off, hence one inclines to the hypothesis that the cells of the subzonal layer become incorporated in the inner layer of the cuboidal ectodermal cells, for in sections shown at

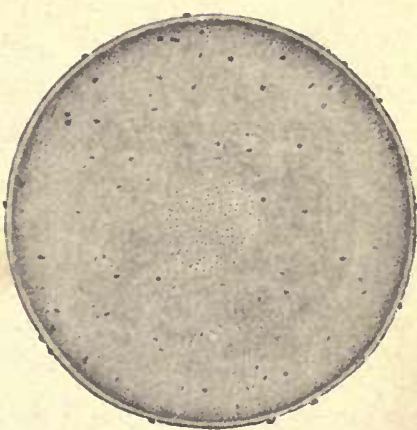

Fig. 124.-Blastodermic VesiCLE of a Rabbit of Six Days and ONe ANd One-half Hours. From an Alcoholic Specimen. $\times 20$ diams. this stage the ectoderm is one-layered in the region of the shield. The entodermal cells also have essentially the same appearance as at five days, but they extend considerably farther around the vesicle, are more numerous, and form a more continuous layer. Sections show that the subzonal layer outside of the shield is very thin, but its outer surface is fitted to the inner surface of the zona pellucida. The center of each cell is somewhat thicker, projecting toward the interior of the vesicle. It is in this thicker projecting portion that the nucleus is situated. Along the borders of the cells the layer is of course thinner, and it is under these thinner parts that the thicker nucleated portions of the entodermal cells are lodged. Hence, in surface views, the nuclei of the two layers are seen to alternate more or less with one another. This characteristic disposition is not kept everywhere, but is subject to considerable variations. In the very most advanced ova of six days a small spot sometimes can be observed in the embryonic shield which is noticeable from its greater opacity. This spot corresponds to Hensen's knot, but it does not usually show itself distinctly until considerably later.

Vesicles at Seven Days. - Vesicles at this age vary greatly in size, and the stage 
of development varies with the size-how exactly, we do not yet know. Preliminarily we may fix on the normal size as being that of vesicles the greatest diameter of which is about $4 \mathrm{~mm}$. Such vesicles are somewhat oval in shape and slightly flattened on the side bearing the embryonic shield. The membrane enclosing them is very thin; the albuminoid layer can scarcely be distinguished, but the zona pellucida is very distinct. The shield (Fig. I25, Sh) is. somewhat elongated and distinctly pear-shaped. Its long axis is parallel with that of the vesicle. It varies greatly in its dimensions. Shields I mm. wide and from I. 3 to $\mathrm{I} .4 \mathrm{~mm}$. long are not uncommon. The student will be likely to encounter other dimensions. The most striking addition is the appearance of a darker area, mes, at the posterior or pointed end of the shield. This darker area is also somewhat pear-shaped, but

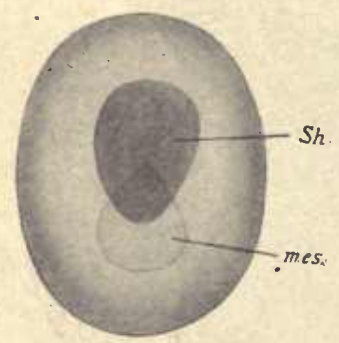

Fig. I 25--BLASTODERMIC VESICLE OF A RABBIT at SEVEn Days.

$S h$, Embryonic shield. mes, Mesoderm. (Semi-diagrammatic.) $\times 6$ diams. its pointed end is near the center of the shield, its rounded end a little distance behind the point of the shield. The darker area owes its formation to the appearance of a new layer of cells between the ectoderm and entoderm. This layer consists of loosely connected cells with rounded nuclei easily distinguishable in surface views from those of the subzonal layer. The greater part of these cells are certainly mesodermic, but a portion of them share in the formation of the primitive streak and notochordal canal, and perhaps do not belong to the mesoderm. In the region outside the embryonic shield the outer layer is easily distinguished; its cells have marked outlines, but are of smaller dimensions than in earlier stages, their nuclei are large, for the most part oval, and contain several highly refringent and conspicuous granules. The number of granules varies; when there are only two or three, they are apt to be elongated as if several small granules had united. The entodermal cells have spread well past the equator of the vesicle and present, for the most part, a distinctly epithelial arrangement, although at the edge of the expanding layer the cells are still more or less scattered. The entodermal cells are easily distinguished by changing the focus of the microscope, when their darker protoplasm and smaller size, together with their smaller darker nuclei, make them readily recognizable. The granules in the entodermic nuclei are smaller and more numerous than in the overlying ectodermal nuclei.

During the next few hours further changes ensue. At the apex of the pearshaped mesodermic area there appears a small spot, which is known as Hensen's knot. At first Hensen's knot consists of a little thickening accompanied by a union of the cells of the middle layer with those of the overlying ectoderm. Next occurs the development of the primitive streak, which runs from Hensen's knot backward toward the apex of the embryonic shield, and very soon thereafter along the line of the primitive streak there develops the external and shallow primitive groove. At Hensen's knot the three layers now are found to be intimately united, so that, 
though they may everywhere else, when fresh, be separated from one another, the germ-layers at this point cannot be separated, except by tearing. Finally, in the next stage there grows out in front of Hensen's knot the so-called head-process, an axial band of cells in which the notochordal canal develops.

\section{A}

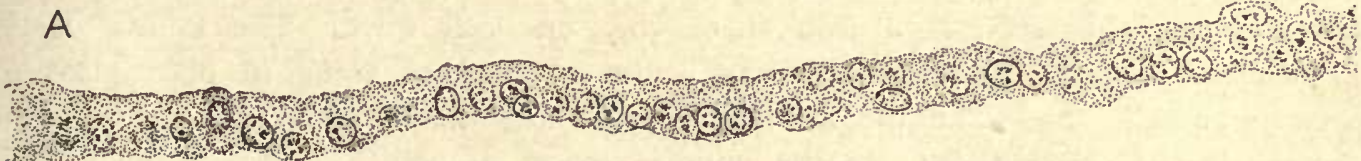
1. i

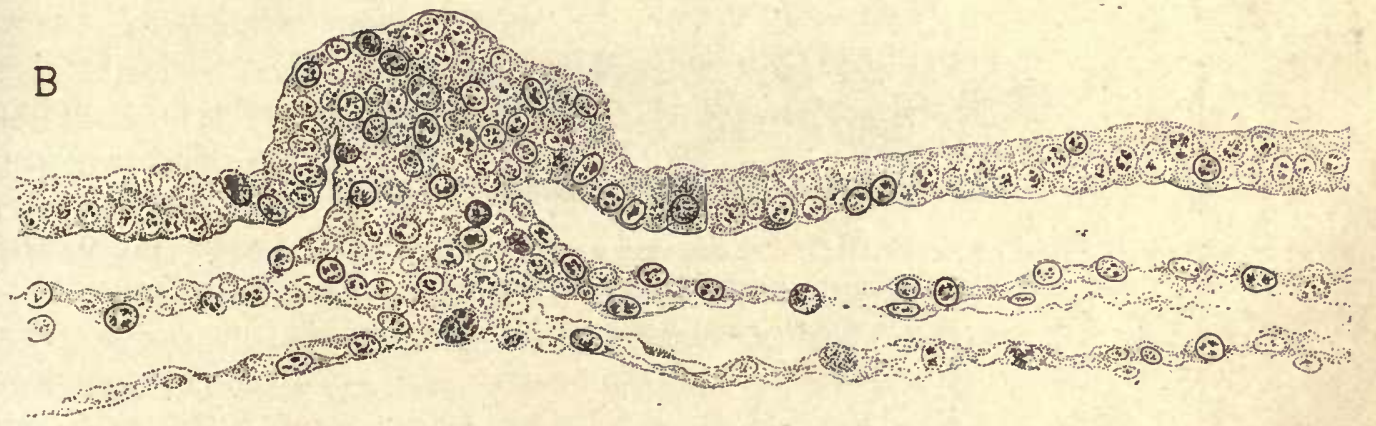

C

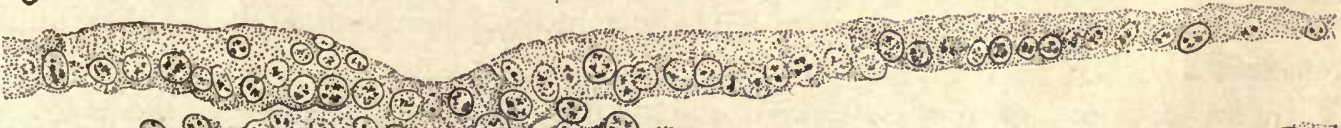

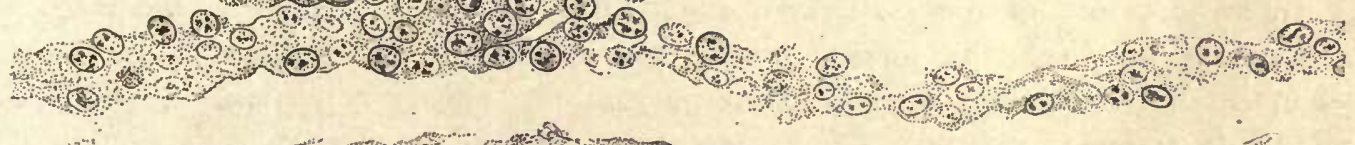

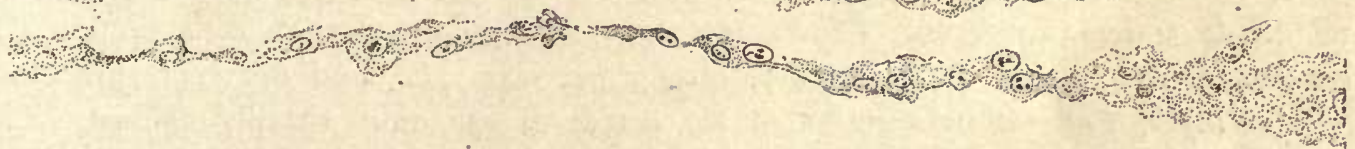

Fig. I26. - Three Transverse Sections of a Rabitt Embryo of Seven and One-half Days. Series 622, SeCTIONS $247,260,38 \mathrm{r} . \quad \times 300$ diams.

Examination of Cross-sections.-The structure of the embryonic shield is well shown by cross-sections. Figure 126 represents three sections through the embryonic shield at seven and one-half days: A, through the head process; $B$, through Hen- 
sen's knot; C, through the primitive groove. The three primitive germ-layers are easily recognized in each section. They are somewhat separated from one another, but in life probably lay close together. The upper layer or ectoderm is the thickest and consists of low columnar cells; it is characteristic of the embryonic shield, and at the edge (not included in the figure) of the shield changes to a thin sheet of cells, which forms the outer layer of the rest of the blastodermic vesicle. The lowest layer, entoderm, is a thin sheet only one cell thick. The middle layer, mesoderm, is more irregular, and has begun to thicken, being in places two or even three cells thick. In the median line the mesoderm enters into special relations with the other layers. In the middle section, B, which passes through Hensen's knot, it forms a considerable axial thickening, which fuses with the entoderm below and ectoderm above, and builds with the latter a dome-like projection. The axial thickening extends forward from Hensen's knot, constituting the so-called head-process, and in this region, $\mathrm{A}$, the mesoderm is united only with the entoderm.

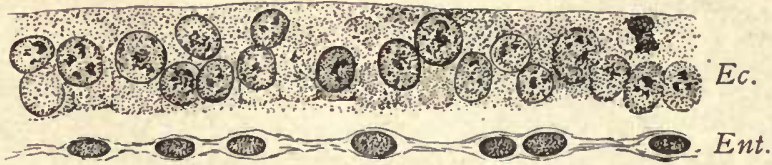

Fig. 127.-Rabit Embryo of Seven Days. Transverse Series 12, Section 216, through the Anterior Portion of the Embryonic Shield.

Ec, Ectoderm. Ent, Entoderm. $\times 350$ diams.

Behind Hensen's knot the thickening extends backward, making the primitive streak, C, which is characterized by the union of the mesoderm with the ectoderm only, and by the primitive groove, a shallow median longitudinal depression of the ectoderm.

The structure of the embryonic shield at seven days is further illustrated by figures 127 and 128 , the former passing across the anterior portion of the shield, where it is two-layered, and the latter across the posterior portion, in which the middle layer has appeared. Figure 176 shows the middle portion of a section. It consists merely of the outer, thicker, ectodermal layer, $E_{c}$, and the very thin entodermal layer, Ent. Both surfaces of the ectoderm are quite sharply defined. The nuclei are rather large and show several large, deeply stained nucleoli in each. The outline of the nucleus is sharp, and, in addition to the larger granules, there are many smaller ones less deeply stained scattered through the nucleus. The nuclei vary considerably in size, shape, and position. The protoplasm of the ectodermal cells is lightly stained, and granular in appearance. The boundaries between adjacent cells are indicated by delicate lines, which extend through the entire thickness of the ectoderm, which is now but a single layer of cells. The original outer 
(Rauber's) layer has disappeared. The entoderm is very thin, but is thickened a little where each nucleus is lodged. The nuclei are smaller than those of the ectoderm, more darkly stained, and the granules in them less coarse than those in the nuclei of the ectoderm. Between the two layers is a narrow space; whether an artefact or not is difficult to say. Figure I 28 represents a transverse section through the posterior part of the embryonic shield where the primitive streak, pr.s, is just forming. The position of the median plane is approximately indicated by the vertical line $M$. About this plane there is a considerable accumulation of cells which merges without boundary into the superficial cells of the shield. A short distance from the median line the outer layer of the shield becomes a distinct epithelium, $E c$, consisting of a single layer of cells. The edge of the shield is marked by a rather abrupt transition to the thin outer layer of the extra-embryonic region. On the under side of the section extends the thin entoderm as a continuous layer, which is only loosely connected with the central mass of cells overlying it near the median plane. Finally, from the median mass of cells extends laterally the sheet of mesoderm, Mes, between the outer and inner germ-layers. The mesodermic cells are somewhat loosely distributed, and have round nuclei with distinct chromatin granules and well-marked protoplasmic bodies, which give off strands by which the cells are united to one another. The middle germ-layer is the least compact of the three.

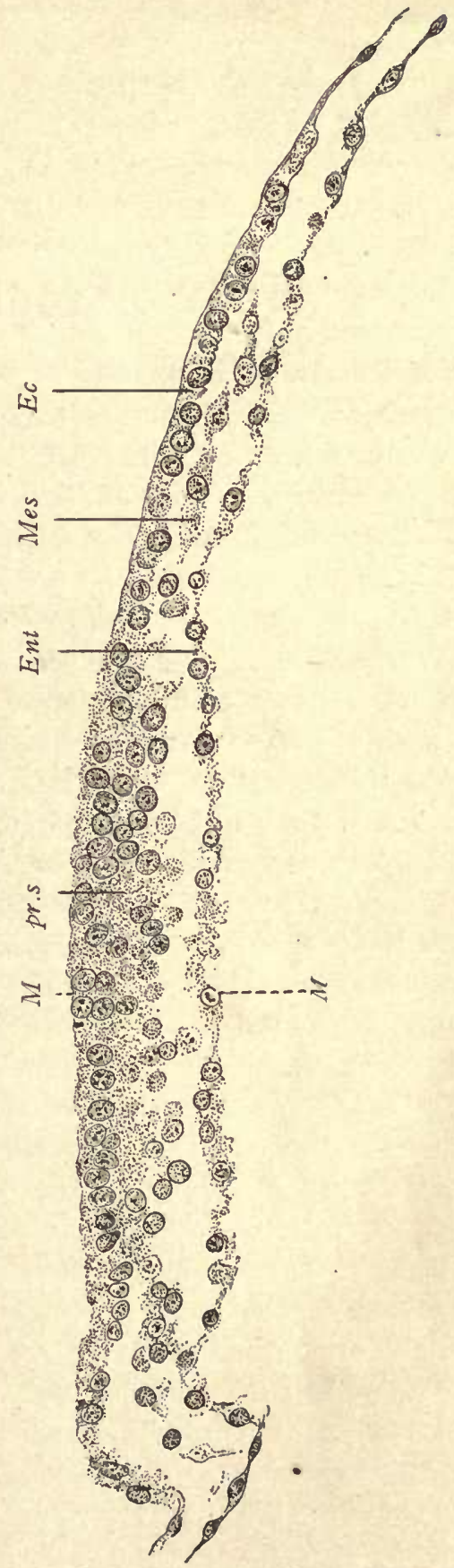




\section{CHAPTER V. \\ STUDY OF YOUNG CHICK EMBRYOS.}

\section{Method of Obtaining Embryos.}

Fertile eggs can usually be obtained from dealers, who can supply them in quantities as needed, or hens may be kept with little trouble especially for the purpose. In that case the hen herself will be found the best incubator, for the number of eggs which develop normally under a hen is larger than in an artificial incubator, and abnormalities of development are less frequent. A good setter will remain upon the eggs, even though some are removed and replaced by fresh ones, for about a month. She should be plentifully supplied with water and soft food, which is best kept at a little distance off, so that she will be obliged to leave the eggs to feed. A box that is somewhat secluded, and affords some protection, warmth, and shelter from the light, should be provided. In order to obtain the most accurate results it is desirable to place the eggs as soon as laid immediately under the hen. Only by this means can an approximate correlation between the stage of development and the duration of incubation be secured.

Artificial incubators are now made to work satisfactorily.* The temperature of an incubator should be maintained at about $38^{\circ} \mathrm{C}$. (100.4 $4^{\circ} \mathrm{F}$.). It should on no account be allowed to rise above $40^{\circ} \mathrm{C}$. ( $\left(104^{\circ} \mathrm{F}\right.$.), for that destroys a portion of the eggs and causes the production of many abnormalities in the remainder; and, if possible, a fall to a lower temperature should be avoided, although the results of a lower temperature are less disastrous. No incubator should be used which does not permit a constant supply of fresh air and of moisture. The date should always be marked on each egg when it is placed in the incubator. If a number of eggs from a dealer are artificially incubated the same length of time, they are pretty sure to cover a considerable range of stages, as, of course, eggs so supplied are of varying ages, the exact time of laying not being recorded.

In this work two stages of the chick are especially studied. The first stage studied is that of a chick with seven segments, which is normal after about twentyseven hours' incubation. The second is normally produced after about forty-six hours' incubation. The embryo should have about twenty-eight segments and three

* The one used at the Harvard Medical School is heated by a kerosene lamp and has a capacity of roo eggs. It is called the New Method Incubator, and was purchased from M. A. Coffin, Burlington, Mass. In the market other incubators may be found, doubtless equally good, among them patterns adapted for the use of gas where that is preferred. 
gill-clefts showing externally. Embryos a little less or a little more developed are almost equally serviceable.

Removing the Embryo.-Before the egg is opened a basin should be prepared and filled with normal salt solution warmed to about $40^{\circ} \mathrm{C}$. ( $104^{\circ} \mathrm{F}$.). The basin should be large enough to permit the entire egg to be submerged in it.

Take the egg warm from the incubator or the hen; allow it to rest quietly in one position for two or three minutes before opening it. This is in order to insure that the side of the yolk which contains the embryo is turned uppermost. After an egg is disturbed the yolk will turn and resume its normal position, for which but a short time is necessary. The egg may now be held in one hand, the shell cracked, and the pieces of the shell above the yolk be removed with forceps, making a hole about an inch in diameter. The inner egg membrane may be removed with the shell. If any of the white of the egg tends to overflow, it should be immediately snipped off with a pair of scissors, otherwise it will cause the yolk to roll over, thus concealing the embryo.

The embryo and germinal area are now to be examined with the naked eye or, better, with a hand lens. The student will detect very easily the area pellucida, which lies at right angles to the long axis of the egg, and also see in the middle of the area a long whitish streak, which marks the anlage of the embryo. Around the area pellucida can be seen the mottled vascular area which will vary in appearance according as the development of the blood-vessels and blood-islands is more or less advanced. The area vasculosa is a portion of the larger area opaca which merges at its periphery into the general yolk. In embryos of the second half of the second day, thirty-six to forty-eight hours, the contraction of the heart can be readily seen, and usually the outlines of the head of the embryo may be made out. The germinal area is now to be separated from the rest of the yolk. To accomplish this, plunge one blade of a sharp pair of scissors into the yolk a little beyond the edge of the vascular area, and cut rapidly around until a circular incision has been completed; then take a flat spatula and plunge it boldly into the yolk at a depth of perhaps an eighth of an inch underneath the embryo. Next lift out the embryo together with the yolk and the overlying white of the egg, steady it a little if necessary on the spatula with a pair of forceps or needle, and transfer it rapidly to the dish of warm salt solution. With a pair of fine forceps the edge of the germinal area may be seized, and by gentle motion it may be separated from the mass of yolk and also from the thin, whitish, overlying membrane of the yolk, and at the same time from so much of the white of the egg as may have been carried along. As one becomes more practised in these operations, it is not difficult to remove the germinal area without taking much yolk along with it.

The operation may be modified as follows: After the shell is opened the egg may be tilted so as to allow the white to run off, and as it runs over the edge it is snipped through with the scissors, and as much of the white removed as is 
possible in this way. The whole egg is then submerged in the warm salt solution, an incision around the germinal area made as above, and the embryo floated off from the yolk.

Preservation of the Embryo.- The next step, after the embryo has been removed from the yolk and lies in the salt solution, is to put a glass slide in the salt solution and carefully float the embryo and germinal area upon it, and then remove them together. The slide is now to be laid flat on the table and the germinal area spread out carefully upon it. In this operation good results may often be obtained by allowing a few drops of warm salt solution to fall upon the center of the germinal area. The currents produced by the falling drops will be sufficient to spread out the blastoderm in its natural form,* and at the same time to wash away any superfluous yolk grains that may be adherent to the preparation. At this stage the preparation should be examined by the student with a low power of the microscope, as described below. To preserve the specimen, four or five drops of Zenker's fluid are allowed to fall upon the specimen gently and quietly as it lies upon the glass slide. The specimen is allowed to stand for about ten minutes and is then transferred to a dish containing a larger quantity of Zenker's fluid. The transfer should be made by submerging one end of the slide in the dish and floating the specimen off. In from two to four hours the hardening of the specimens will be completed. They must then be washed thoroughly by decanting off the Zenker's fluid and replacing it with water, and this water must itself be replaced several times during the next twenty-four hours. Further treatment of the specimen is as described on page 378 .

The Making of Serial Sections.-Specimens are best colored with alum cochineal in toto. They are then imbedded in paraffin and cut into series. The most useful sections are those which are transverse to the axis of the spinal cord. They should not exceed Io $\mu$ in thickness.

\section{Embryo Chick with Eight Segments. (About twenty-eight hours' incubation.)}

The following description is almost equally applicable to embryos with six or ten segments.

Examination in the Fresh State. - The embryo when first removed from the yolk should be placed in a staining-dish with a small quantity of normal salt solution and examined with a low power of the microscope as a transparent object. The specimen as a whole has a grayish or brownish gray tint. Most of the germinal area is dark, the transmission of light being stopped by the numerous yolkgrains contained in the entodermal cells (compare page 64). In the center of the germinal area the transparent area pellucida is very conspicuous, and has an edge which is quite sharply defined, more so than after the specimen has been preserved. It is shaped somewhat like an elongated pear, the broad end of which surrounds

* The student will observe that the fresh blastoderm is very easily distorted. 
the cephalic end of the embryo (Fig. 129). It should be noted that this figure was drawn from a hardened, and not from a fresh specimen. The head of the embryo lies toward the large end of the area pellucida and projects freely above the surface of the germinal area. Underneath the projecting head is a very clear area with distinct lateral boundaries. It is called the pro-amnion and contains no mesoderm whatever. Near the head are two characteristic areas, one on each side, easily recognized by the fact that the surface of the germinal area rises like a dome over

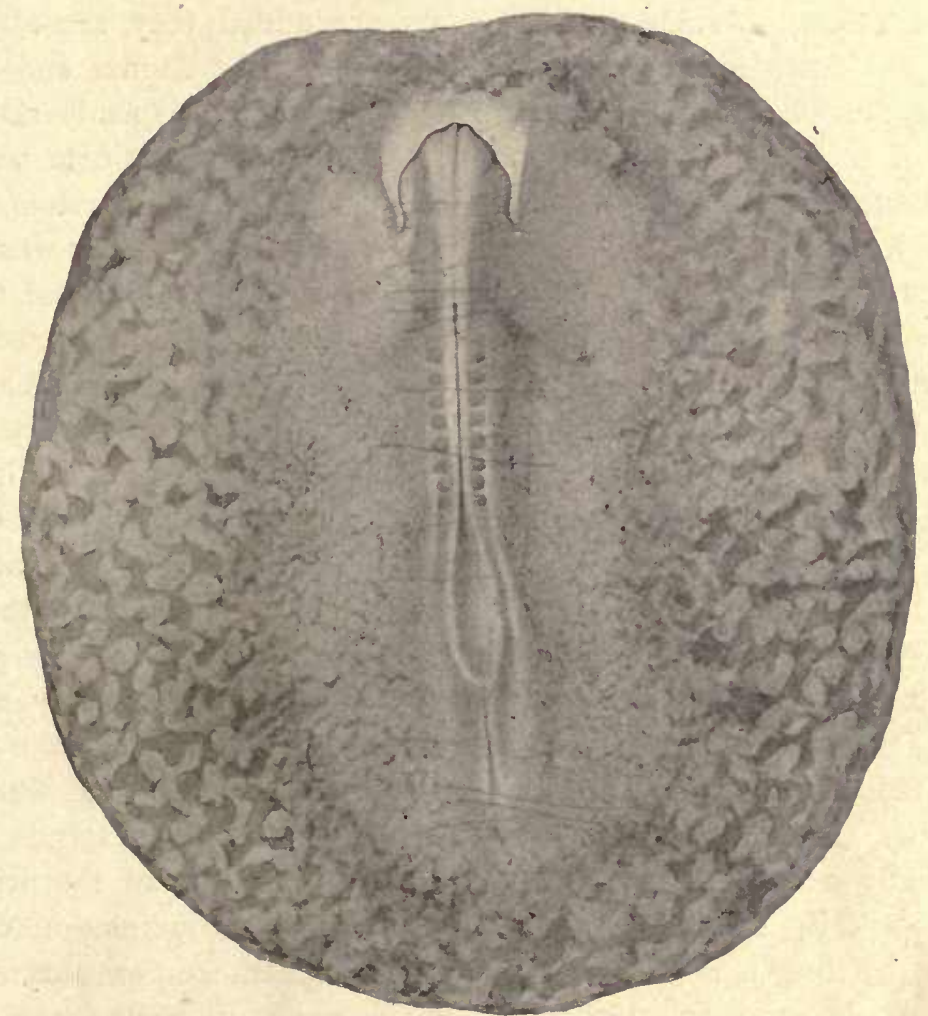

Fig. 129.-Chick Embryo after Twenty-Seven Hours' Incubation, with Eight Primitive Segmexts. Alcoholic Specimen. $\times$ I5 diams.

each space. The spaces are termed the amnio-cardiac vesicles. They are in reality local expansions of the cœlom which cause the somatopleure, or upper leaf of the germinal area, to arch upward on either side of the embryo. By the study of crosssections (Fig. 136) the relations may be clearly understood. The posterior limit of the head is marked by a curving line (to see this sharply the focus must be lowered) the cavity of which faces the caudal end of the embryo. This line marks the position of the fovea cardiaca and from it the fore-gut extends into the head of the embryo. On the cephalic side of the fovea and underneath the foregut the heart will be developed. On the sides of the fovea, running forward 
toward the median line of the embryo, one can distinguish two darker bands which represent the beginning of the formation of the blood-vessels, growing in from the extra-embryonic region to meet in the median line of the embryo and participate in the formation of the heart. These bands are the anlages of the omphalo-mesariac veins. Behind the fovea appear eight pairs of rather opaque blocks of tissue, symmetrically placed right and left. These are the primitive segments and are formed exclusively by the mesoderm. The first pair of blocks lie a short distance behind the fovea and the last pair a short distance in front of the rhomboidal sinus (compare below). When new segments are added they are about the same size as those previously formed, and always arise at the caudal end of the series. The growth of the embryo in length during these stages depends rather upon the multiplication of the segments than upon the growth of the single segments. The principal axial structure is the anlage of the central nervous system, the so-called medullary groove, already partly converted into a medullary canal; for at this stage it is closed from the anterior limit of the head to a variable point of the segmented region of the embryo. For a general account of the origin of the medullary groove from the ectoderm see page 67 . Chicks with eight segments vary extremely as to the extent of the closure of the groove. The line of closure can be readily seen. It is somewhat wavy and irregular in its course, and the closure itself is somewhat irregular, so that we may find one or several points where the closure is not yet completed although it is complete behind and in front of these points. At the anterior extremity of the head the closure is always incomplete, there. being an opening there which persists for some time and is known as the anterior neuropore. Above the primitive segments, where it is not closed, the medullary groove has its edges close together, but a short distance behind the last segment the groove widens abruptly and fades out gradually. This widening is termed the rhamboidal simus. The sinus marks the caudal limit of the nervous system and extends so as to embrace the cephalic end of the primitive streak. The region of the primitive streak appears quite dark by transmitted light, owing to the accumulation of 'cells which belong chiefly to the mesoderm. This dark appearance extends forward and merges into a dark band on either side, which runs up to the row of segments. The dark band is the segmental zone, out of which new segments are differentiated. On the surface of the primitive streak is a longitudinal furrow, the primitive groove, which begins just within the rhomboidal sinus and extends backward, often bending to one side or the other, usually to the left. The groove is shallow in front, deeper behind, and ends quite abruptly. More careful examination of the area opaca shows that it already possesses a well-defined area vasculosa, the peripheral boundary of which is more or less definitely marked. In the fresh specimen only traces of the formation of the blood-vessels and blood-islands can be made out.

Examination of the Specimen after Hardening.-The specimen, after it has been hardened, should be examined under the microscope in water or alcohol; and, again, after it has been stained it should be cleared in oil and further'examined. 
This will enable the student to make out the blood-islands and something of the blood-vessels in the area vasculosa, and also the shape of the brain which (Fig. I3I) has expanded widely just behind the neuropore; the lateral expansions are the anlages of the optic vesicles (Fig. 133). The remainder of the brain extends from the optic enlargement to a point a little behind the fovea, fov. It is much wider than the remaining portion of the medullary canal; it tapers from the optic

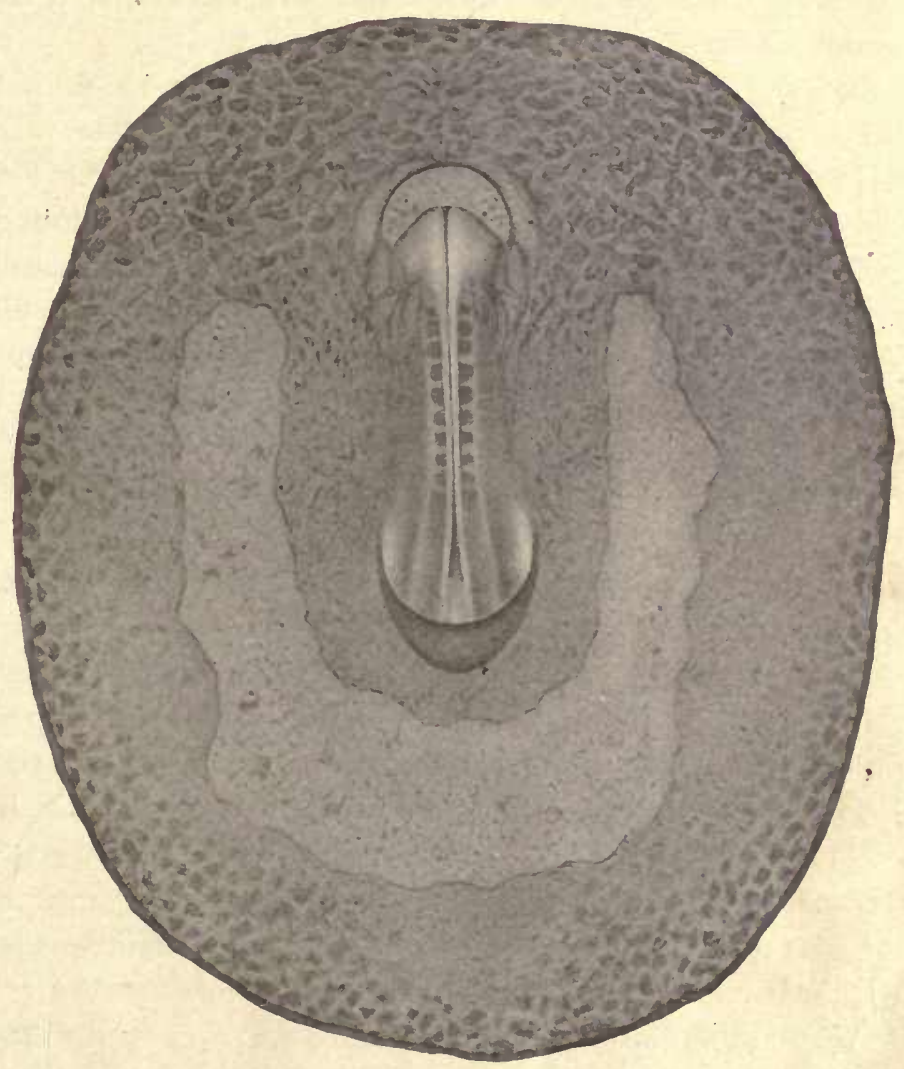

Fig. 130,-Embryonic Area of a Rabbit with eight segments, with the Placental area Partly Torn off. $\times \mathrm{I}_{5}$ diams. (Drawn by T. H. Emerton.)

vesicle and extends backward. One cannot yet distinguish in it positively any subdivision into mid-brain and hind-brain. On the contrary, its walls are often somewhat irregularly sinuous and vary considerably from specimen to specimen.

Comparison with a Rabbit Embryo.-In the ovum of the mammalia the ectoderm presents a modification known as the trophoderm. In the rabbit this trophoderm is developed over a limited region which is called the placental area (Fig. I 30 , a.pl), by which the embryo is attached to the wall of the uterus. When the embryo figured was removed, a portion of the placental area remained attached to the uterus, hence the defect shown in the specimen. The vascular area is 
nearly circular; its boundary is marked by a well-defined terminal vessel, v.t. The nearly straight embryo lies in the center and exhibits plainly the medullary canal and primitive segments. The optic evaginations are already present. The head is free; on its under side the heart is forming, and beneath it is a relatively large and conspicuous pro-amnion, pr.a. Blood-vessels are present over the area vasculosa, but not yet in the embryo. It will be seen, therefore, that though the proportions differ greatly from those in the chick, the fundamental relations in the rabbit are the same as in the bird.

Examination of the Specimen after Staining.-After the chick has been stained in toto it should be cleared in oil of cloves, or other suitable fluid, and further examined in surface views with low powers of the microscope. For this purpose it may be placed in a small shallow staining dish. It will be found advantageous to have also whole chicks with their areæ vasculosæ permanently mounted in Damar. Embryos up to about forty-eight hours' incubation are readily prepared in this way. The germinative area with the embryo is treated like an ordinary section. Specimens thus mounted must be protected from the pressure of the cover-glass by putting under two opposite edges strips of paper or, better, of glass to support the cover. Strips of glass as needed can be cut from broken cover-glasses with a writing diamond.

Figure I ${ }^{\mathrm{I}}$ represents a chick with eight fully formed segments stained with alum carmine and viewed as a transparent object.

The distribution of the blood-islands and various details of the structure of the embryo, which in the fresh specimen are obscure, can be readily observed in the cleared preparation. The blood-islands, Bl.is, contain crowded young blood-cells and are conspicuous owing to the intensity with which they are stained. They are largest and most numerous in the posterior part of the area opaca, and on either side become gradually smaller and farther apart toward the anterior end and are absent altogether at the level of the head. A few small islands appear in the area pellucida around the caudal end of the embryo. The aninio-cardiac vesicles, A.c.v, are marked by the arching up of the surface of the area pellucida on both sides of the head. In the embryo, the segments, Som.3, the walls of the medullary tube (brain, $\mathrm{Br}$, and spinal cord, $S p . c$ ), and the omphalo-mesaraic veins, $V . o m$, are sharply defined. The first segment is imperfectly formed, and never acquires a full development; it, together with segments two, three, and four, are called the occipital segments because they enter into the formation of the occipital region of the head and never undergo full differentiation like the other segments. In mammals also there are (probably four) occipital segments. The fifth segment of our chick becomes the first cervical segment of the adult. The medullary tube, $M d$, has sharply defined, walls. It is completely closed through the brain region, except at the anterior neuropore. At its cephalad extremity the tube has expanded laterally to form the optic vesicles, $O p . V$, each of which is the anlage of a retina and optic nerve. The middle region of the tube, between the optic vesicles, is the first 
cerebral vesicle, fore-brain or prosencephalon. The second cerebral vesicle, mid-brain or mesencephalon, Br.2, comprises the part of the medullary tube immediately behind the optic vesicles and includes a little more of the length of the tube than the vesicles. In older embryos (I4-20 segments) it becomes clearly marked off by constrictions from both the fore- and hind-brain. The third cerebral

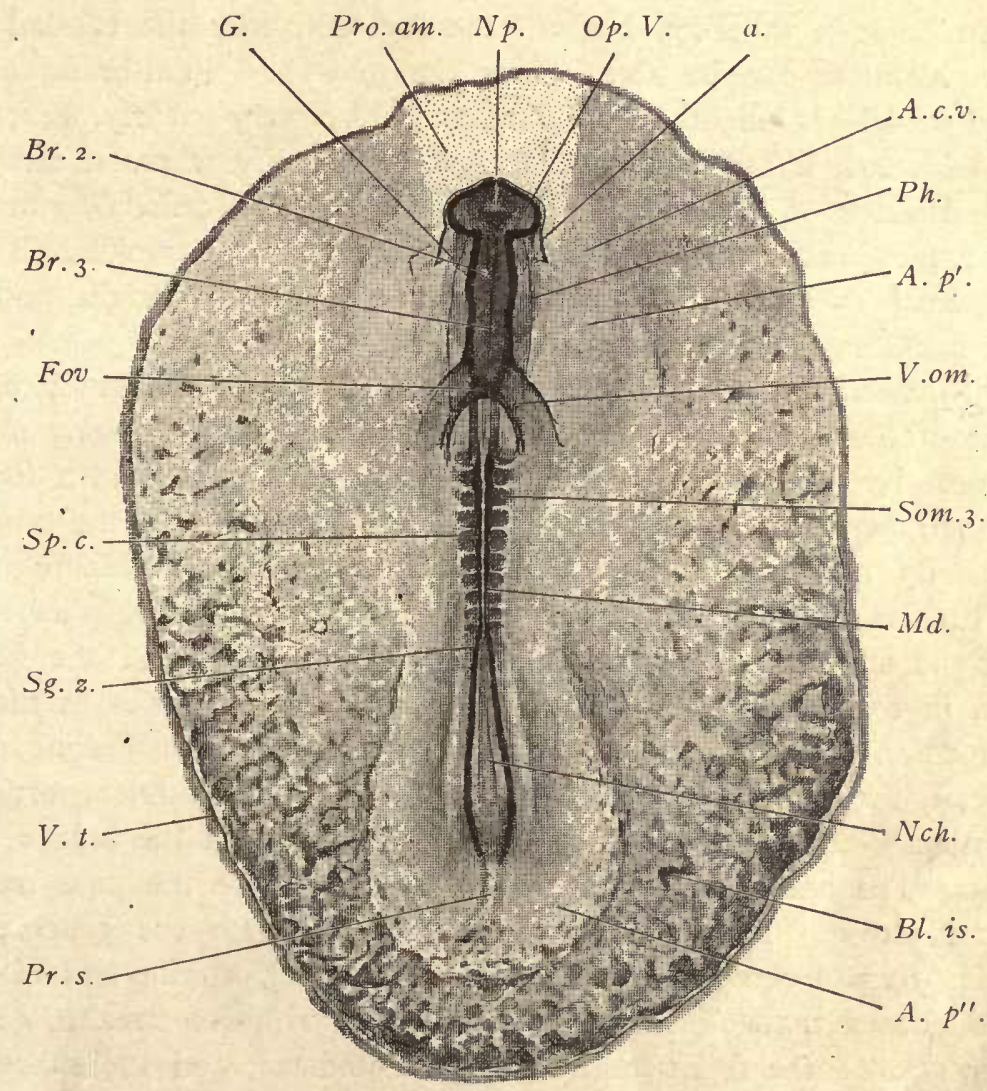

Fig. I3I.-Chick with Eight Fully Formed Segments, Stained with Alum Cochineal and Mounted in DAMAR.

$a$, Outline of the free portion of the head. A.c.v, Amnio-cardiac vesicle. A.p', A.p", Area pellucida. Bl.is, Blood-island. Br.2, Second cerebral vesicle. Br.3, Third cerebral vesicle. Fov, Fovea cardiaca. $G$, Post-optic ganglionic crest. $M d$, Medullary groove. $N c h$, Notochord. $N p$, Anterior neuropore. $O p . V$, Optic vesicle. Ph, Outline of pharynx. Pro.,am, Pro-amniotic area. Pr.s, Primitive'streak. Sg.z, Segmental zone or band of mesoderm, from which new segments arise. Som.3, Third somite. Sp.c, Spinal cord. V.om, Vena omphalo-mesaraica. V.t, Vena terminalis. $\times$ I5 diams.

vesicle, hind-brain or rhombencephalon, $\mathrm{Br} \cdot 3$, is as long as the other two and has a tapering form; it becomes the cerebellum, pons, and medulla oblongata of the adult. The cavity of the brain is wide, but in the region of the hindbrain it tapers caudad and so passes over into the narrow cavity of the spinal 
cord, Sp.c. In this specimen the open medullary groove, $M d$, begins at the level of the fourth segment. The outline, $a$, of the free portion of the head is sharply marked, as is also the outline, $P h$, of the pharynx or fore-gut, which opens at the fovea cardiaca into the general sub-germinal entodermic cavity. The omphalo-mesaraic veins, V.om, can be traced peripherally to their junctions with the vascular network of the area pellucida, and if the embryo be viewed by its ventral surface, the two veins can be seen to unite, beneath the fore-gut, with the caudad end of the heart. The notochord can be seen under the mid- and hind-brain, as a narrow median band which is slightly irregular, and also very clearly, nch, underneath the rhomboidal sinus; it fades out at its caudal extremity where it merges into the undifferentiated tissue of the primitive streak. A band of cells, $G$, can be seen on either side of the head, extending tailward from the optic vesicle. This band is usually designated as part of the ganglionic crest; but its origin and fate have not yet been satisfactorily elucidated.

Longitudinal Section of a Chick.-In order to facilitate the study of the transverse sections of this stage, figure $\mathrm{I}_{32}$ is inserted, which is a nearly median longitudinal section. In consequence of the head end, $H$, having grown forward above the pro-amnion, pro.a, it has become free on all sides, and at the same time the entodermal cavity has been carried forward with the head, making the so-called fore-gut of English authors. This fore-gut is the anlage of the pharynx, the oesophagus, and the stomach. Underneath the posterior portion of the fore-gut there has appeared in the mesoderm 'a cœlomic cavity, $p$, which serves as the connection across the median line with the amnio-cardiac vesicles just described in surface views. We can, therefore, distinguish in the fore-gut the anterior portion from the posterior portion which overlies the cœlom. This cœlom is the anlage of the pericardial cavity. The anterior division of the fore-gut forms the pharynx.proper. It ends blindly in front. The opening of the fore-gut into the general entodermic cavity, Ach, is termed the forea cardiaca, fo. At the posterior end of the embryo we have a thickened mass of cells constituting the primitive streak, Pr.s. The line on the under side of the figure represents the entoderm, and the space underneath it is a portion of the primitive entodermic cavity.

Study of Transverse Sections. - Attention should be directed, first, to the three germ-layers, their composition and their rôles in the production of organs; second, to the exact topographical relations of the various organic anlages, because these relations are fundamental and determine the anatomical dispositions in the adult. Before beginning the detailed study of the sections, the student should have a clear conception of the manner in which the free head of the embryo merges into the embryonic body and germinative area. Fifteen figures represent as many crosssections of an embryo chick with eight fully formed segments, and the ninth segment beginning. The drawings are uniformly magnified soo diameters. There are interpolated figures $\mathrm{I}_{3} 2, \mathrm{I}_{3} 8, \mathrm{I} 39, \mathrm{I} 47, \mathrm{I} 49$ from other embryos to illustrate certain details with higher magnifications. 
Section through the Optic Vesicles (Fig. I33).-The section is oval, the head being flattened dorso-ventrally: Its outer boundary is a layer of cells, Ec, constituting the ectoderm. The inner and outer surfaces of the ectoderm are marked in the section by distinct lines. With higher powers the ectodermal nuclei are readily seen; there are no cell boundaries, although the protoplasm is gathered into columns and strands with clear spaces between. We have in fact to deal rather with a syncytium than with a layer of cells. On the dorsal side the ectoderm shows a

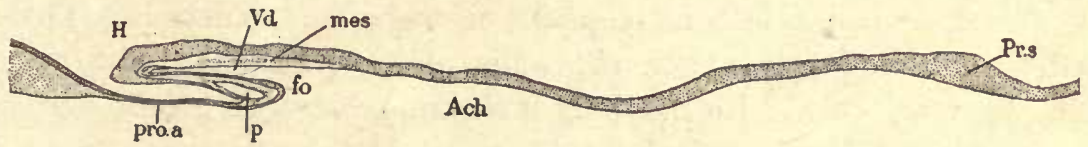

Fig. I32.-Longitudinal Section of a Young Chick Embryo.

$H$, Head. $V d$, Anterior portion of digestive canal (Vorderdarm). mes, Mesoderm. fo, Fovea cardiaca. $p$, Pericardial cœlom. pro.a, Pro-amnion. Ach, Entodermal cavity, in life bounded below by the yolk. Pr.s, Primitive streak.

thickening, $G$. If this be followed back in the series of sections it will be found to be continuous "both with the ectoderm and with an internal group of cells alongside the mid-brain (Fig. I34, G). We shall return to the consideration of the group in question in connection with the description of the next figure. In the mid-dorsal line the ectoderm from each side reaches the anterior neuropore, $N p$, which is still open, and is reflected inward to form the thicker wall, $M d$, of the medullary tube, here widely expanded to form the optic vesicles, $O p$. The outer ectoderm, Ec, and

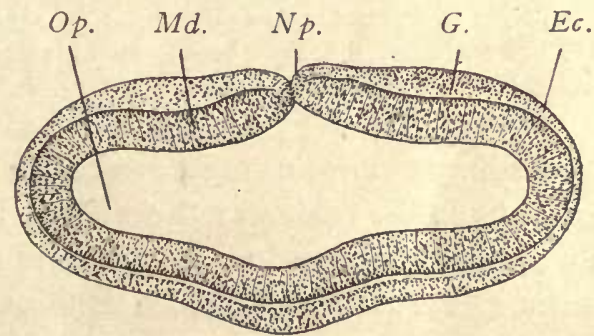

Fig. I33.-Section of Chick Embryo with Eight Segments. Transverse Series 642, Section 2 i. $E c$, Ectoderm. $G$, Ganglionic thickening. $M d$, Wall of medullary tube. $N p$, Neuropore. $O p$, Optic resicle, $\times$ roo diameters.

inner ectoderm, $M d$, are everywhere in contact with one another, so that in the whole section there is but a single germ-layer, the outer. Soon the middle germlayer will penetrate between the two sheets of ectoderm, and permanently obliterate the primitive relations.

Section through the Mid-brain (Fig. I34). - The section of the head is oval, and bounded everywhere by the ectoderm, $E c$, or as it may now be called, the epidermis. The head is completely free, but underneath lie the layers of the germinal 
area. Immediately below the head is the pro-amniotic area (Pro.am) which consists of only two very thin layers of cells, the upper ectoderm, the inner entoderm. By following through the series of sections it is easy to satisfy oneself that the two layers of the pro-amnion are directly continuous with the like-named layers of the embryo proper. At a little distance from the head, the lateral limit of the proamnion appears, being marked by the appearance of the mesoderm between the other two germ-layers. The edge of the mesoderm is sharply defined. The ectoderm has formed also the thick wall, $M d$, of the medullary tube, which at this point is completely closed and has lost its connection with the epidermis. There are no distinct cell boundaries anywhere in the walls of the medullary tube at this stage. The nuclei are oval, each with its long axis more or less nearly vertical to the surface of the tube. Mitotic figures are frequent and occur always near the inner

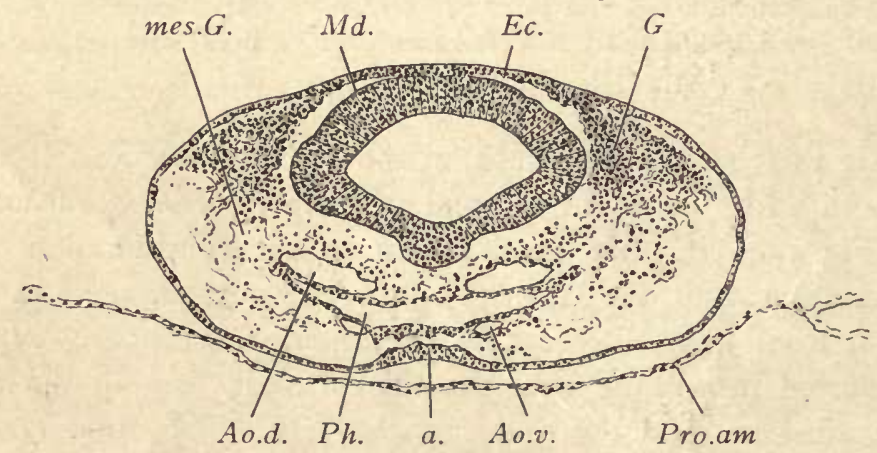

Fig. 134--Section of Chick Embryo with Eight Segments. Transverse Series 642, Section 86. $a$, Ventral thickening of ectoderm (part of oral plate). Ao.d, Dorsal aorta. Ao.v, Ventral aorta. Ec, Ectoderm.

$G$, Ganglionic crest. $M d$, Wall of medullary tube, mid-brain. mes, Mesenchyma. Ph, Fore-gut. Pro.am,

Pro-amniotic area. $\times$ too diams.

or free surface of the medullary wall; in other words, next the cerebral cavity. The microscopic structure of the tube is similar throughout its whole extent. Underneath the brain is the fore-gut, $P h$, somewhat crescentic in cross-section, and formed of a single layer of epithelium, the entoderm, which is thinner on the dorsal, thicker on the ventral side of the fore-gut, a difference which becomes more marked in later stages. In the median ventral area the entoderm is somewhat thickened and adjoins a similar thickening, $a$, of the underlying ectoderm. The two thickenings are beginning to unite at present, but are still distinct and easily break apart. Very soon, however, they fuse into a single lamina, which is known as the oral plate and in which all trace of the double origin is lost. The ectodermal thickening, $a$, is depressed below the level of the ventral surface of the head. By the upgrowth of the tissues around it, the depression is increased, until in later stages it appears as a deep invagination, lined by ectoderm, and the floor of which is formed by the oral plate. The invagination is termed the stomodaum, and is destined to form a large part of the mouth. The oral plate soon undergoes autolysis, and by 
its own disappearance creates the oral opening of the fore-gut. Close to the fore-gut lie four blood-vessels, two above and two below, the dorsal, Ao.d, and ventral, Ao.v, aortæ, respectively. The dorsal vessels are much the larger. If the series of sections be followed through cephalad the ventral aorta will be found, before the tip of the fore-gut is reached, to bend dorsalward and join the dorsal aorta of the same side. If the series be followed through in the caudad direction, it will be observed that the two ventral aortæ draw toward the median line until they meet and unite in a single trunk, the main aorta, which is continuous with the heart (Fig. I35, Ht). It is thus learned that the blood leaves the heart at its cephalic end by a single channel, which soon divides; the branches curve upward and pass to the dorsal side of the pharynx, forming two dorsal channels conducting the blood-stream backward. The blood-vessels consist each of a very thin layer of cells, epithelial in character and termed endothelium; the nuclei are flattened and therefore appear oval in section. All the remainder of the section is occupied by loosely scattered cells, which are of two sorts: first, those marked mes, which fill the ventral and lateral regions, and constitute the true mesenchyma; the mesenchymal cells have nuclei with small amounts of protoplasm around them, and strands of protoplasm connect the cells together; there are no cell boundaries; the t.ssue might be described as an irregular reticulum with nucleated nodes; second, those cells marked $G$, which form two lateral groups on the dorsal side adjoining the mid-brain; these groups have been named the ganglionic crests by some writers, mesectoderm by others. The cells in question resemble those of the true mesenchyma, but have more protoplasm around the nuclei and appear therefore more deeply stained than the mesenchyma proper. If the cells of the crest be followed dorsally they will be seen to form a narrow band which joins the ectoderm near the median line, and by following the sections headward, the crests will be found to merge with ectodermal thickenings (Fig. ${ }^{3} 33, G$ ). From these relations it has been inferred that the crest on each side arises from a local proliferation of the ectoderm. The crest is easily. seen in surface views of stained chicks (Fig. I3I $G$ ). Two principal views as to the future of the crest cells of the mid-brain region have been brought forward: first, that they are true ganglionic anlages, which disappear by autolysis; second, that they are converted into true mesenchyma.

Section through the Hind-brain (Fig. 135 ). - The head is no longer free, but fuses laterally with the layers of the germinal area; hence the ectoderm, $E c$, instead of bending over on to the ventral side, bends in the opposite direction-away from the embryo. The mesoderm stretches across the median line under the embryo. There is a large space, Coe, in the mesoderm; the space is part of the primitive body-cavity or ccelom; it extends completely across the embryo and out into the germinal area on each side. The cœlom is everywhere bounded by a thin epithelial layer, msth, the mesothelium, which at this stage resembles an endothelium as seen in section; it forms one part of the mesoderm, the bulky mesenchyma forming the other part. 
Below the colom is another cavity, Pro.am, that of the pro-amnion, lined by ectoderm and opening anteriorly. This structure is not further dealt with here, partly because its history is complicated, partly because it does not occur in the human embryo. The ventral aortæ (Fig. I34, Ao.v) have united into a single blood-channel, $H t$, which we can identify as the blood-channel of the heart; it is called the endothelial heart. The mesothelium, msth, on the dorsal side of the cœlom forms a protuberant fold, the mesothelial heart, which surrounds the inner vascular. space. The two heart-walls are some distance apart. The inner heart produces only the lining endothelium of the adult organ, the mesothelial heart produces all its muscular and connective-tissue components, and also the pericardium. The difference

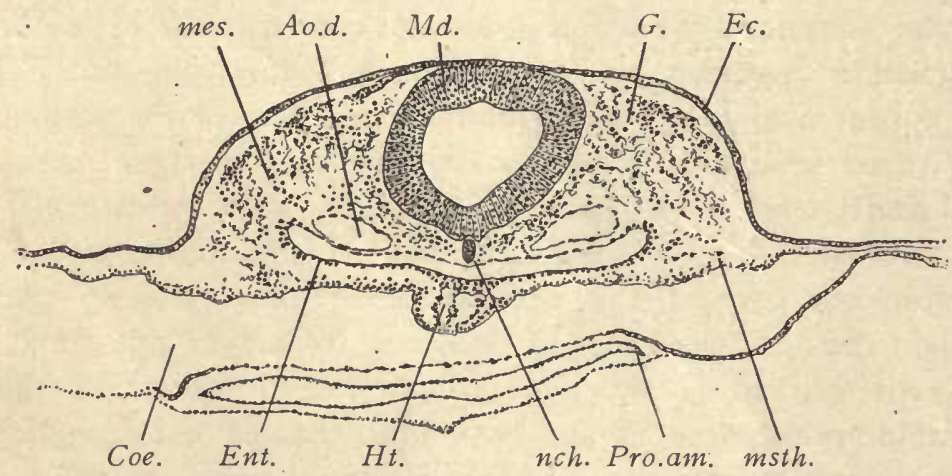

Fig. I35.-Section of Chick with Eight Segments. Transverse Series 86, Section 86.

Ao.d, Dorsal aorta. Coe, Cœlom. Ec, Ectoderm. Ent, Entoderm. G. Ganglionic crest. Ht, Aortic end of the endothelial heart. nch, Notochord. Md, Hind-brain. mes, Mesenchyma. msth, Mesothelium. $P h$, Fore-gut. Pro.am, Pro-amnion. $\times$ roo diams.

in the form of the cross-sections of the hind-brain, $M d$, mid-brain, and fore-brain should be noted. Between the brain and fore-gut, and touching both, lies the small notochord, nch, with a sharp outline. The ganglionic crest, $G$, of the mid-brain is still traceable, but occupies a much smaller area than in figure 134 . In the middorsal line the crest, $G$, the epidermis, $E c$, and the medullary tube, $M d$, are fused together. In correspondence with the reduction of the crest, the area occupied by the true mesenchyma, mes, is increased.

Section through the Cephalic (Aortic) End of the Heart (Fig. I36).- The general topography is similar to that of figure I35. The most striking differences are, that in the present section the heart is very much larger, is bent to the right of the embryo (the left of the figure), and has a narrow connection (mesocardium) with the floor of the pharynx; that the colom is much expanded to form the amniocardiac vesicles, A.c.v, one on each side, which are continuous with one another on the ventral side of the heart; that the lips of the medullary tube are in contact at $c$, but have not actually fused; and that there is no pro-amnion, because it does not extend so far back under the embryo. The following details should be ob- 
served: The epidermis, $E c$, of the embryo is thickened and fits closely against the dorsal portion of the hind-brain, with which it is actually fused in the median line. The ganglionic crest. is represented only by a few more lightly stained cells at the junction of the epidermis with the medullary wall, $M d$, but is much more developed in nearby sections, both cephalad and caudad. The fore-gut, $P h$, is very wide, the entoderm on its dorsal side is very thin, but grows thicker toward the lateral boundaries of the gut, and is thickest in the mid-ventral line, where it forms a shallow median groove; the nuclei in this groove are all next the external surface of the entoderm. The mesothelium, $m s t h$, is a thin layer, which above the amnio-cardiac vesicles enters into the formation of the somatopleure or true body

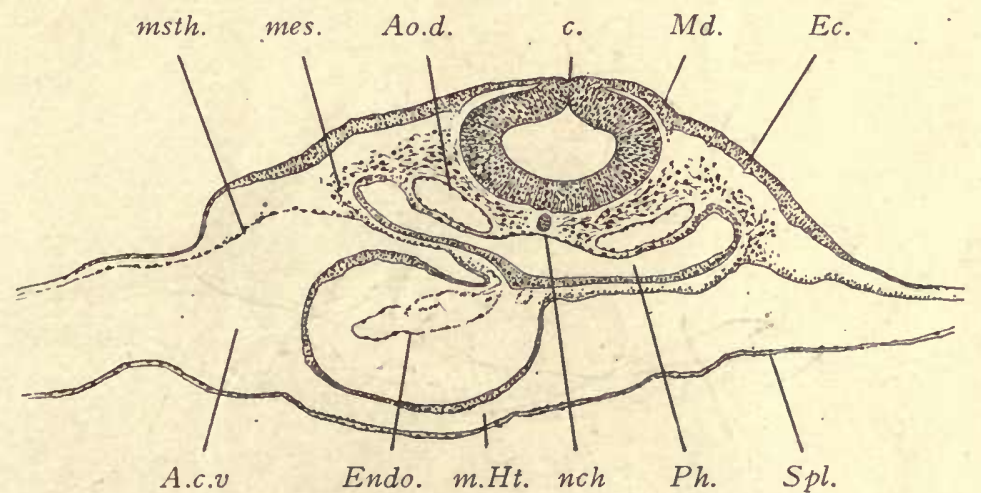

Fig. I36.-Section of a Chick Embryo with Eight Segments. Transverse Series 642, Section ii 4. A.c.v, Amnio-cardiac vesicle. Ao.d, Dorsal aorta. $c$, Line of Closure of the medullary canal. Ec, Ectoderm. .

Endo, Endothelial heart. Md, Wall of hind-brain. mes, Mesenchyma. m.Ht, Mesothelial heart. msth,

Mesothelium. nch, Notochord. Ph, Fore-gut. Spl, Splanchnopleure. $\times$ roo diams.

wall. It is not sharply separated from the mesenchyma, mes, as can be very well seen in the part of the layer underneath the pharynx. When the heart is reached the mesoderm forms a wide duplicature, $m . H t$, the mesothelial heart, which is a layer of much greater thickness than the mesothelium proper, and which offers the important characteristic that it shows no differentiation into mesothelium and mesenchyma. Between the mesothelial heart-tube and the endothelial, Endo, there is a wide space which contains no visible structures, hence we assume that the two cardiac tubes are kept apart by fluid only. Beneath the colom (amnio-cardiac vesicles, A.c.v) is the splanchnopleure, $S p l$, which has two thin layers: the upper is mesoderm, the lower entoderm. The mesoderm has numerous nuclei, and if followed out laterally to the area opaca will be found affixed to blood-vessels and blood-islands, which together constitute the angioblast or anlage of the vascular system. It can be observed in most places readily that the angioblast lies beneath the mesoderm proper and is distinct from it. The entoderm has few nuclei and in the area pellucida is very thin, but where it passes to the area opaca it gradually but rapidly thickens, and is composed of very large columnar cells (compare Fig. 30) 
with large vacuoles, left by yolk masses which the cells have digested; toward the periphery vacuoles with partly absorbed yolk may be found.

Section through the Venous End of the Heart (Fig. I37).-The relations differ but little from those in figure ${ }^{3} 6$ except for the heart and the ganglionic crest. The heart shows its bend toward the left side (the right in the figure), and in this bend both the endothelial, Endo, and the mesothelial portions, m.Ht, participate. The mesocardium, $x$, is clearly recognizable, and comprises two mesodermic lamina. By it the heart is suspended throughout its length from the ventral wall of the fore-gut. The mesocardium soon disappears, and the mesothelial heart thereupon becomes closed dorsally, and is attached only by its aortic and venous ends to the neighboring tissues. A strand of cells from the endothelial heart passes

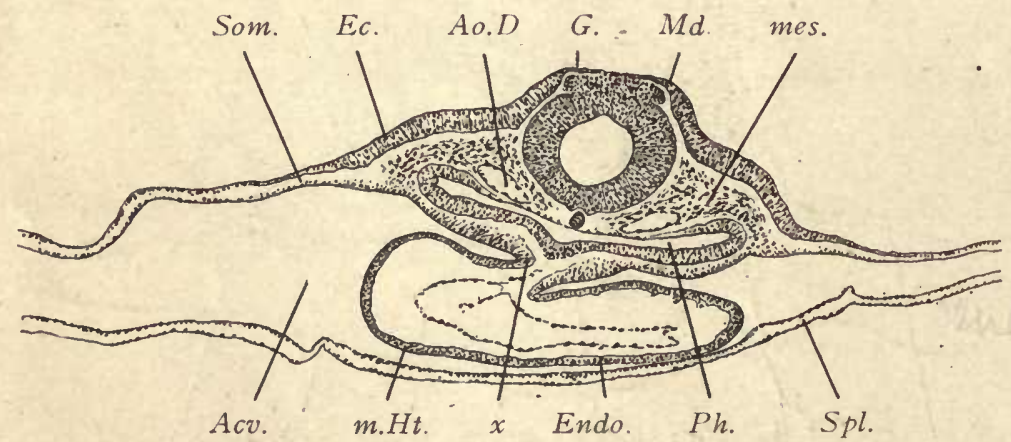

Fig. 137.-Section of a Chick Embryo with Eight Segments. Transverse Series 642, Section izo. $A c v$, Amnio-cardiac vesicles. Ao.D, Dorsal aorta. Ec, Epidermis. Endo, Endothelial heart. G, Ganglionic crest. Md, Medullary tube (Hind-brain). mes, Mesenchyma. m.Ht, Mesothelial heart. Ph, Fore-gut. Som, Somatopleure. Spl, Splanchnopleure. $x$, Mesocardium. $\times$ 100 diams.

through the mesocardium and joins the entoderm. The significance of this junction is not clear. The ganglionic crest, $G$, is very distinct; it is overlaid by a thin lamina of the epidermis, and is in texture quite unlike the brain-wall proper, $M d$. The cells composing it are considerably individualized and somewhat separated from one another by clear spaces.

Figure ${ }_{3} 38$ represents a section somewhat more highly magnified through the heart anlage of a slightly younger embryo. The medullary groove, $M d$, is not closed. The colom does not yet extend across the median line, but there is only a thin partition separating the amnio-cardiac vesicles, Am.ves, from one another. The mesothelial heart, msth, is a relatively thick layer, thrown into irregular folds. The endothelial heart is represented only by a few scattered angioblastic cells, Endo, which as yet show no definite order.

The further development of the heart may be understood by the examination of a somewhat older stage (Fig. I39). As shown in the illustration, the mesothelium has become very protuberant, $m . h t$, in the median line underneath the foregut, $P h$. On either side it rapidly thins out, $m s t h$. In the protuberant fold we 
can recognize the future muscular heart, as it is sometimes called. The few cells above described (Fig. I38, Endo) have increased considerably in number and have joined themselves together in such a manner as to indicate clearly the formation of

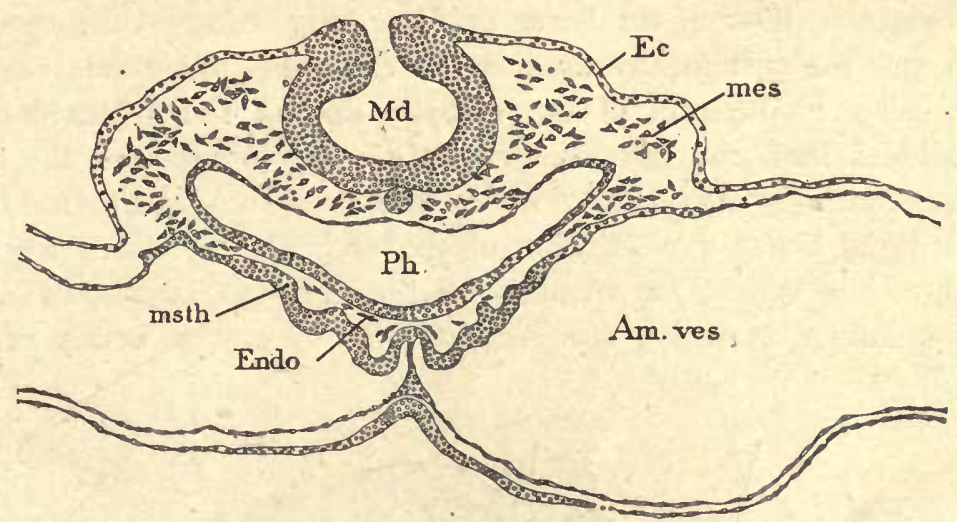

Fig. 138.-Section of a Chick Embryo with Seven Segments. Transverse Series 5 io, Section i8 4. A m.ves, Amnio-cardiac vesicle. Ec, Ectoderm. Endo, Cells forming the anlage of the endothelial heart. Md, Hind-brain. mes, Mesenchyma. msth, Anlage of mesothelial heart. Ph, Fore-gut.
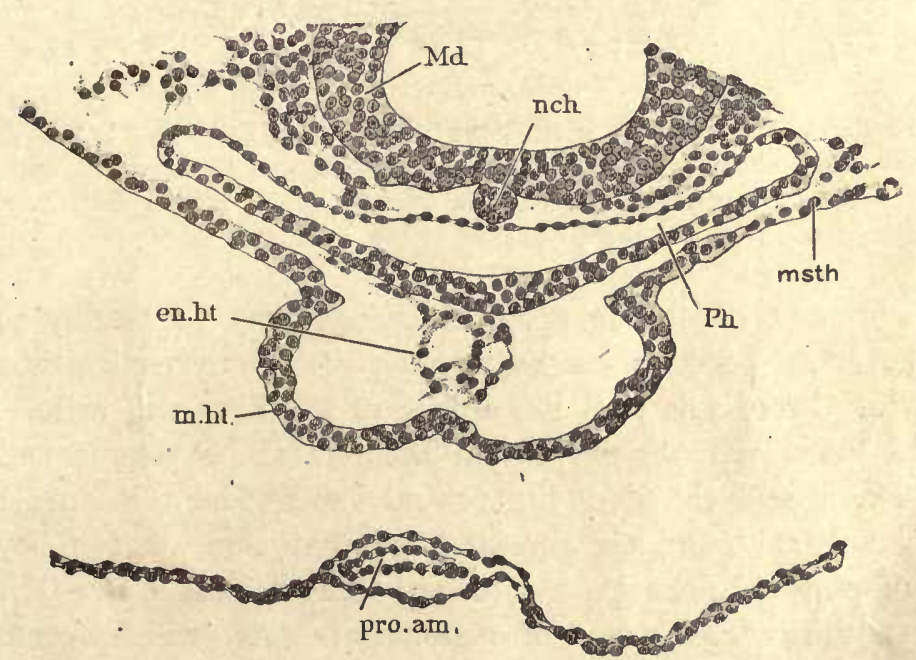

Fig. 139.-Chick Embryo, Transverse Section across the Anlage of the Heart in a Stage slightly more AdVANCED THAN Figure 138.

$M d$, Wall of medullary tube. nch, Notochord. msth, Mesothelium. Ph, Pharynx. pro.am., Tip of pro-amnion. en.ht, Endothelial heart. m.ht, Muscular heart.

the endothelial heart (Fig. I39, en.ht). At first the cells are irregularly disposed and have several irregular cavities between them, which soon fuse so as to form two main cavities running longitudinally. As the two cavities enlarge they meet in the median line and-remain separated at first by a wall of two layers of endothelium. 
This wall soon-breaks through, and there results a single median tube of endothelium which presently appears to be connected with the mesothelium, m.ht, by long cell-processes across the wide intervening space. The heart is now a double tube connected by the mesothelium with the tissues above.

Section through the Wall of the Fovea cardiaca (Fig. 140).- -Underneath the whole of the embryo and the germinative area is the extensive archenteric cavity, bounded above by the cellular entoderm of the embryo and of the splanchnopleure. The archenteron includes both the intestinal cavity of the embryo and the cavity of the yolk-sac, and accordingly its lower floor is the mass of yolk. Into the head of the embryo runs the closed prolongation of the archenteron, which we have studied as the fore-gut. The posterior opening of the fore-gut is known as the fovea cardiaca. The manner in which the entoderm at the fovea bends ventralward to

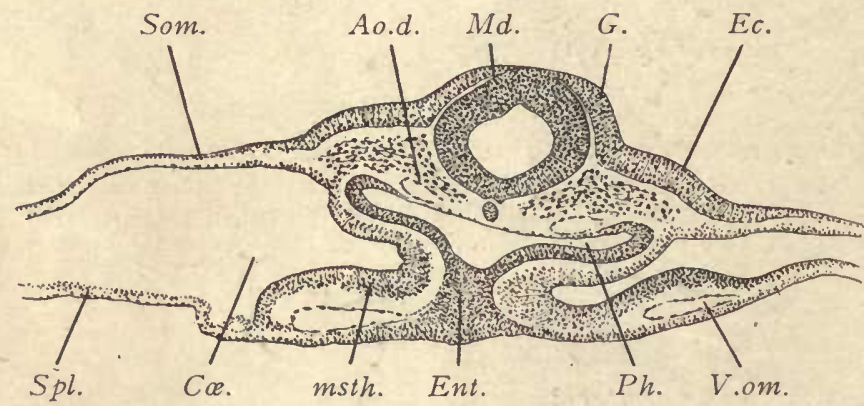

Fig. 140.-Section of a Chick Embryo with Eight Segments. Transverse Series 642, Section i 49 Ao.D, Dorsal aorta. Ce, Cœlom. Ec, Epidermis. Ent, Entoderm. G, Ganglionic crest. Md, Hind-brain. $m s t h$, Mesoderm of the septum transversum. Ph, Fore-gut. Som, Somatopleure. Spl, Splanchnopleure.

$V . o m$, Omphalo-mesaraic veins, $\times$ roo diams.

pass from the fore-gut on to the splanchnopleure may be readily understood from figure I39. The present section passes through the nearly vertical wall of entoderm, Ent, at the fovea. From this wall the anlage of the liver will arise. The omphalomesaraic veins, V.om, pass by it to join the caudal or venous end of the heart. The fore-gut, $P h$, is still closed on its ventral side. The veins are in the splanchnopleure, and, being cut obliquely, appear somewhat elongated. They each cause a protuberance of the splanchnopleure toward the cœlom, Coe. The protuberance is covered by a thick dense layer of mesoderm, Msth, which forms an arch over the vein, so as to leave a clear space between it and the endothelium of the vein. The two protuberances constitute the anlage of the septum transversum, which is itself the anlage of the diaphragm. The region cephalad of the septum is the cervico-thorax; the region caudad, the future abdomen. If the series of sections be followed headward, the veins can be traced to their union with the heart in the median line. If the series be followed tailward, the veins can be traced out into the area pellucida, where they branch. The hind-brain, $M d$, is of smaller diameter than in the previous sections. The epidermis, $E c$, is closely fitted against the dorsal 
half of the medullary tube and fuses in the mid-dorsal line with the ganglionic crest, $G$, which in its turn fuses with the medullary wall. The crest, though not large or conspicuous, can be distinguished readily. The mesenchyma, is clearly differentiated only above the fore-gut, and on either side just beyond the lateral boundary of the fore-gut it fuses with the mesothelium.

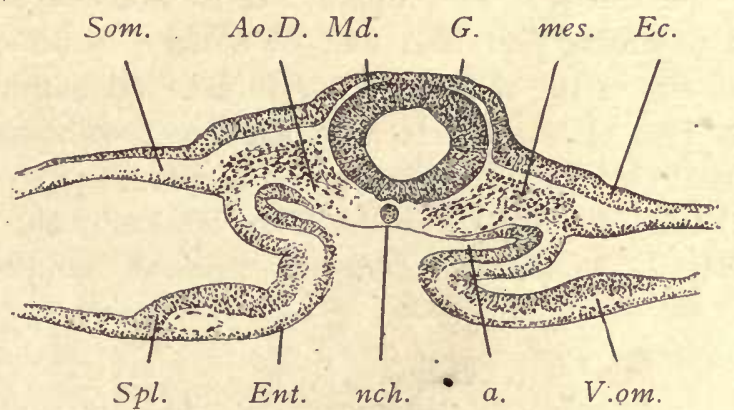

Fig. i4i.-Section of a Chick Embryo with Eight Segments. Transverse Series 642, Section i44. $a$, Groove corresponding to the prolongation of the lateral portion of the fore-gut. Ao.D, Dorsal aorta. Ec,

Epidermis. Ent, Entoderm. $G$, Ganglionic crest. $M d$, Wall of Hind-brain. mes, Mesenchyma. $n c h$, Notochord. Som, Somatopleure. Spl, Splanchnopleure. V.om, Omphalo-mesaraic veins. $X$ ioo diams.

Section. behind the Fovea cardiaca and in. Front of the First Segment (Fig. I4I).The closed entodermal cavity of the embryo has become open and communicates freely with the yolk-cavity. The omphalo-mesaraic veins occupy a more lateral position. Otherwise the section differs so little from figure I40, that it does not call for special description.

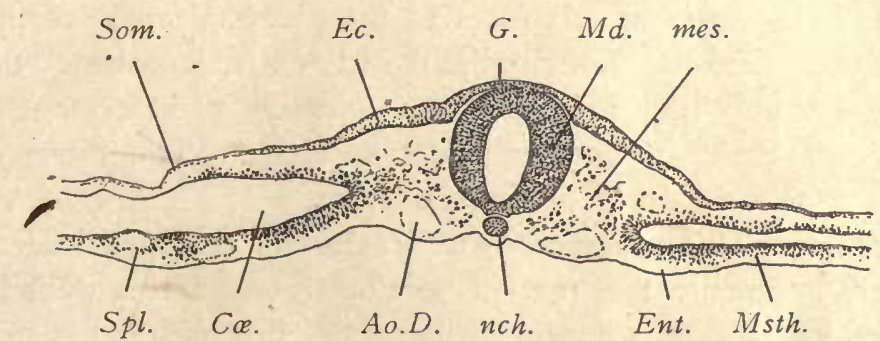

. Fig. 142.- Section of a Chick Embryo with Eight Segments. Transverse Series 642, Section i62. Ao.D, Dorsal aorta. Cre, Cœlom. Ec, Ectoderm. Ent, Entoderm. G, Anlage of Ganglionic crest. mes, Mesenchyma of the intersegmental cleft. Msth, Mesodermic lining of the coelom. $n c h$, Notochord. Som, Somatopleure. $S p l$, Splanchnopleure; $\times$ roo diams.

Section between the First and Second Segments (Fig. 142).-We are still in the region of the hind-brain, which extends to the fourth or last occipital segment. There is no distinction or limit between the embryonic and the vitelline divisions of the archenteron. The hind-brain, $M d$, is oval in section; on its dorsal side it fuses with the ganglionic crest, $G$, which seems now rather a part of the brain-wall 
than a separate structure. The mid-ventral wall or floor of the hind-brain is relatively thin, a feature which marks the transition to the spinal cord, which always has a thin floor-plate. The notochord, $n c h$, is large and transversely oval in section. The two dorsal aortæ, Ao.D, occupy the same relative positions as in the previous sections. The coelom, $\mathrm{Coe}$, is a comparatively narrow fissure, but can be followed laterally far out into the area opaca. It is bounded above and below by mesoderm, Msth, for the most part thin and of a loose texture; but on the lower side in the embryonic region the mesoderm forms a broad band, the cells of which are densely packed. The thick mesodermic band is continuous with that which forms the covering of the septum transversum (Fig. I40, $M$ sth). 'The morphological significance of the band is undetermined. The mesenchyma, mes, occupies the space between the hind-brain and ectoderm on the one hand and the aorta and

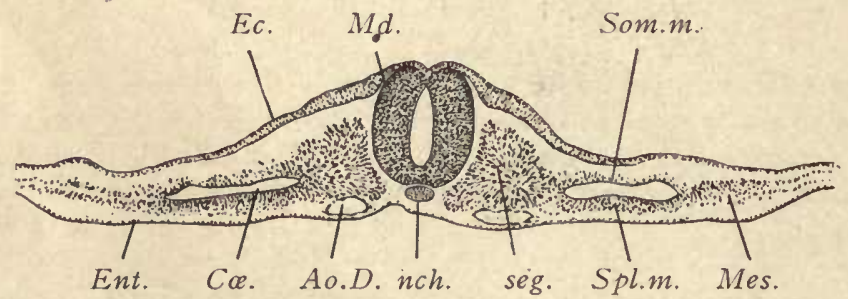

Fig. i43.-Section of a Chick Embryo with Eight Segments. Transverse Series 642, Section i8o. Ao.D, Dorsal aorta. Ce, Coelom. Ec, Ectoderm. Ent, Entoderm. Md, Hind-brain. Mes, Mesoderm. nch,

Notochord. seg, Mesodermic segment or somite. -Som.m, Somatic mesoderm. Spl.m, Splanchnic mesoderm. $\times$ roo diams.

cœlom on the other. It consists of loosely scattered cells, connected with one another by strands of protoplasm. It fuses at the proximal angle of the coelom with the lining mesoderm thereof. Longitudinal sections demonstrate that the loose mesenchyma occupies only the narrow space between two segments, and that it fuses with the denser tissue of both adjacent segments. The intersegmental space is not a cleft but a partition of loose mesenchyma.

Sections through the Third Segment (Fig. I43).-As the hind-brain ends at the level of the fourth segment, the present section is near the transition from the cephalic to the cervical region, segment 5 being the first cervical segment. Accordingly we find that the medullary tubè, $M d$, although not yet closed, has in crosssection a form resembling that of the cord at this stage. The ectoderm, $E c$, runs from the lips of the medullary groove as a layer which is somewhat thickened over the embryo, but becomes very thin over the area pellucida. The colom, Coe, is of small dimensions, although irregular clefts in the mesoderm of the germinative area indicate its extension. The somatic mesoderm, Som.m, is a thin layer above the cœlom; the splanchnic mesoderm is a much thicker layer, Spl.m, below the cœlom. Both mesodermic layers extend beyond the colom toward the medullary tube to form the mesodermic somite, seg, which with its fellow of the opposite side constitutes 
a complete segment. The mesoderm does not extend across the median line, being blocked by the notochord, $n c h$. The somite is bounded mesially by the medullary tube and notochord, dorsally by the ectoderm, ventrally by the aorta and entoderm. Its cells are so arranged as to make a dorsal, a mesial, and a ventral wall, and a core of cells more loosely grouped. Careful study of the segments in various stages has led to the conclusion that the core belongs to the ventral wall. The line of contact between the dorsal wall and the core is accordingly the potential prolongation of the cœlom, and in certain embryos there is a cœlomatic space present in the position indicated. The somite has a broader part toward the medullary wall and a narrower part toward the main cœlom. When a segment undergoes its full development, the narrow part forms a separate structure, the nephrotome.

Section through the Segmental Zones (Fig. I44).-The medullary groove, $M d$, is not closed, but is deep and narrow, its dorsal lips nearly in contact with one another. The embryonic ectoderm, $E c$, is slightly thickened. The entoderm is

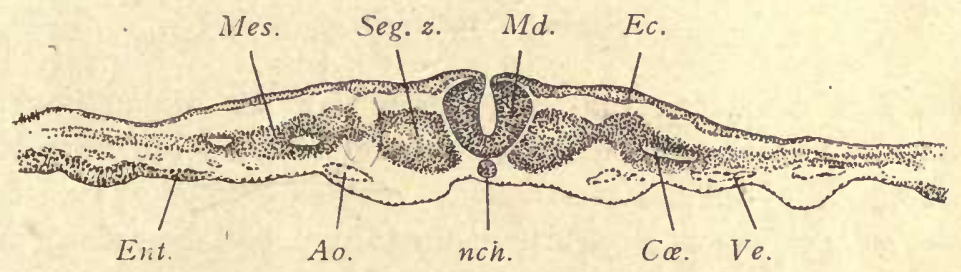

Fig. 144.- Section of a Chick Embryo with Eight Segments. Transverse Series 642, Section 267. $A o$, Dorsal aorta passing from the embryo to the area vasculosa. Ce, Beginning of cœelomatic cavity. Ec, Ectoderm. Ent, Entoderm. Md, Medullary groove. Mes, Mesoderm. nch, Notochord. Seg. z, Segmental zone of the mesoderm. Ve, Blood-vessel of the area vasculosa lying belorv the mesoderm proper. $\times$ roo diams.

a thin layer which on one side, Ent, shows the beginning of the thickening characteristic of the area opaca. The notochord, nch, is nearly circular in section and is larger here than nearer the head. The dorsal aortæ, Ao, have left their position and are passing outward to ramify upon the area pellucida. It is thus evident that the distribution of the blood from the heart to the area vasculosa takes place considerably caudad of the veins which collect the blood and return it to the heart. The blood-vessels, Ao.Ve, lie between the mesoderm proper and the entoderm, and constitute with the associated blood-islands the angioblast. The situation of the angioblast in early stages is typical for all birds and also for mammals. The mesoderm, Mes, has only irregular spaces, Coe, which by their expansion and fusion will give rise to the continuous cœlom. On either side of the medullary groove, the mesoderm forms a thickened mass, the segmental zone, Seg.z, which is markedly constricted where it joins the lateral mesoderm. Out of the constricted area the nephrotomes are differentiated. The somites arise by transverse cleavage of the segmental zone, each new somite being formed immediately caudad to the last-formed somite. The so-called cleavage depends upon a great loosening of the 
mesenchyma, as shown in figure 142 , and not upon the development of an actual fissure or cell-less space. If the series of sections be followed toward the tail, the segmental zone will be found to fade out gradually.

Section through the Open Medullary Groove (Fig. 145).- As we have seen in . following the series of sections, the farther tailward we pass the less advanced is the development. In the present section (Fig. I45), we find that only the notochord, $n c h$, is separated from its germ-layer. The three germ-layers, the ectoderm, Ec,

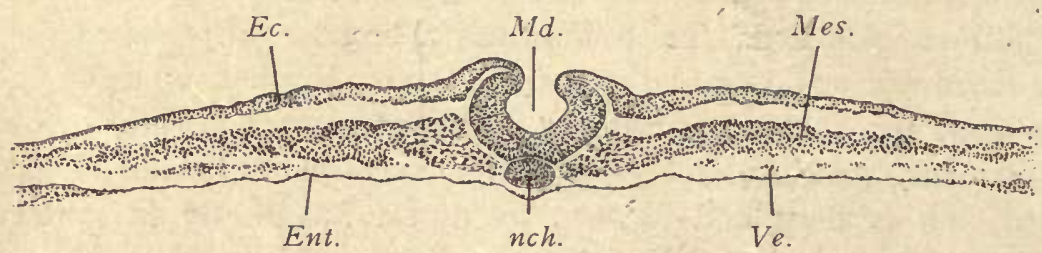

Fig. 145. - Section of a Chick Embryo with Eight Segments. Transverse Series 642, Section 314. Ec, Ectoderm. Ent, Entoderm. Md, Medullary groove.-Mes, Mesoderm. nch, Notochord. Ve, Bloodvessels. $\times$ 100 diams.

mesoderm, Mes, and entoderm, Ent, are merely laminæ of undifferentiated cells, the ectoderm alone showing a modification where it forms the wall of the medullary groove, $M d$. The angioblast or layer of blood-vessels, $V e$, forms a welldefined separate anlage, which is clearly distinct from both mesoderm'and entoderm. The notochord, $n c h$, is of large size, and in part occupies a notch on the under side of the medullary groove. A little farther caudad the notochord fuses with the wall of the overlying medullary groove, and still farther on the united structures fuse also with the entoderm (Fig. 146).

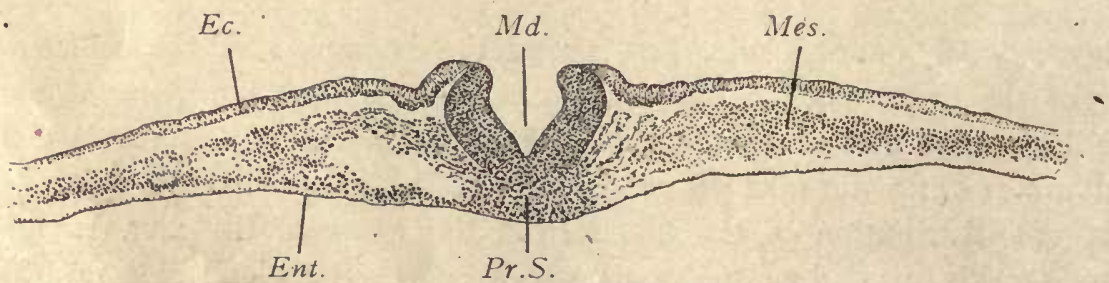

Fig. 146.- Section of Chick Embryo with Eight Segments. Series 642, Section 350.

Ec, Ectoderm. Ent, Entoderm. Md, Medullary groove. Mes, Mesoderm. Pr.S, Primitive streak. X.10o diams.

Section through the Medullary Groore, near its Caudal End (Fig. I46).-The disposition of the parts is somewhat similar to that in figure 145 , but the following. differences are to be noted: The medullary groove, $M d$, is deeper and more troughlike in section and its floor merges into the primitive streak, Pr.S, or axial cord of cells, which merges below with the entoderm and laterally with the mesoderm. The fusion with all three germ-layers is the essential characteristic of the primitive streak. The mesoderm, Mes, of the present section is voluminous, and shows in 
the embryo-region no trace of a cœlomatic fissure. Over the area pellucida there is no angioblast between the mesoderm and entoderm, it not having yet penetrated from the area opaca.

Figure 147 represents, under a considerably higher magnification, three sec-
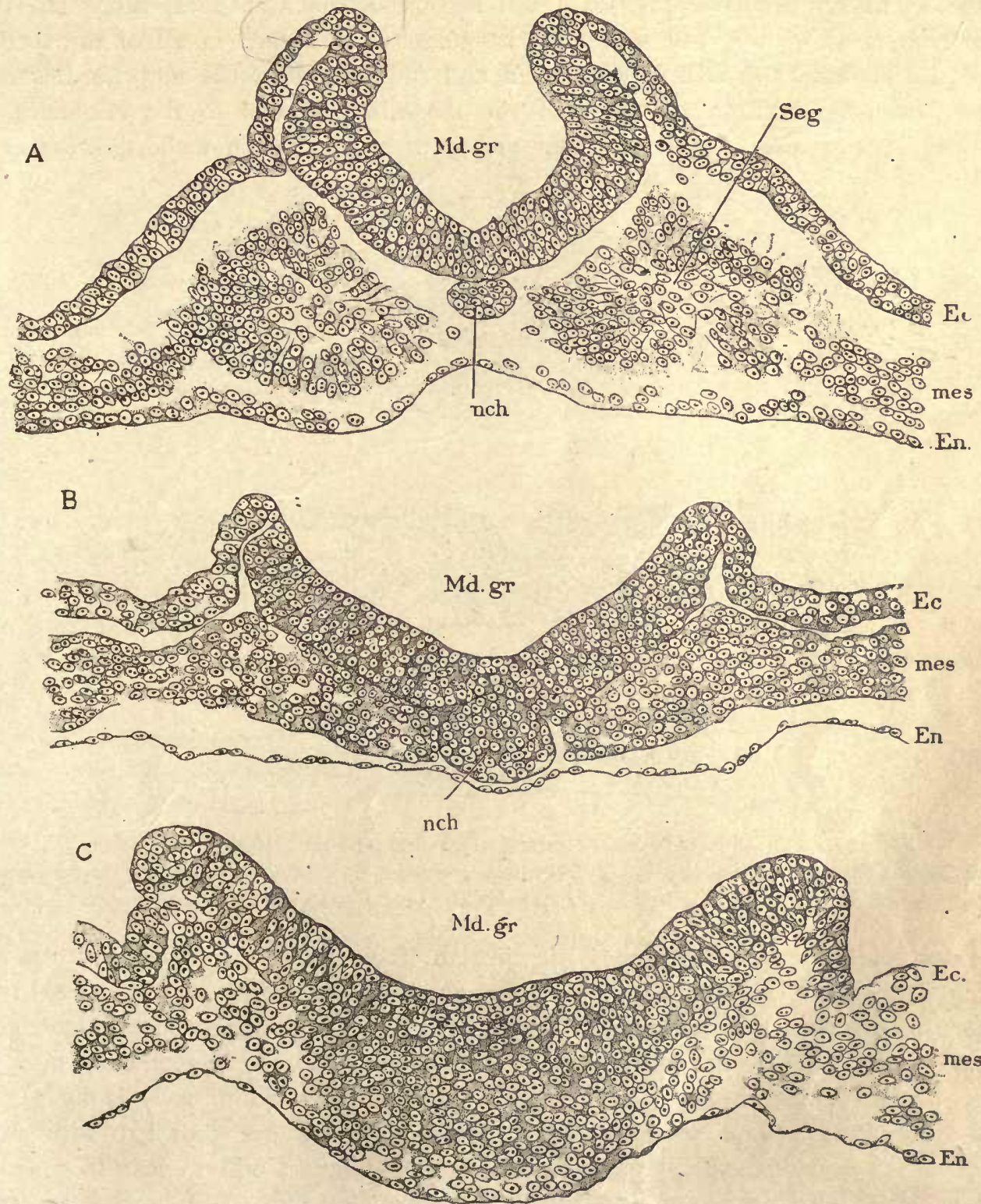

Fig. I47.-Chick Embryo with Seven Segments. Transverse Series 5io, Sections 3 I I, 2 I 2, I 72.

Three transverse sections across the caudal end of the medullary groove. $A$, Section through one of the segments.

B, Section posterior to the segments. C, Section just in front of the primitive streak. Md.gr, Medullary groove. nch, Notochord. Ec, Ectoderm. mes, Mesoderm. En, Entoderm. $\times 230$ diams. 
tions at different levels through the open groove of a slightly younger chick. In the first, A, the groove is quite deep and the young primitive segment is shown. At the edge of the groove its thick walls pass over continuously, but quite abruptly, into the general ectoderm, $E c$, covering the embryo. Close under the median line of the medullary groove appears an oval section of the notochord, $n c h$. The entoderm, En, is quite thin and somewhat irregular, as is shown in all of the sections. In $B$ the medullary groove is wide open and quite shallow, the notochord is much larger and extends from the floor of the medullary, groove to the entoderm and occupies in part a deep notch in the medullary wall. The notochord prevents the

$M d . g r$.
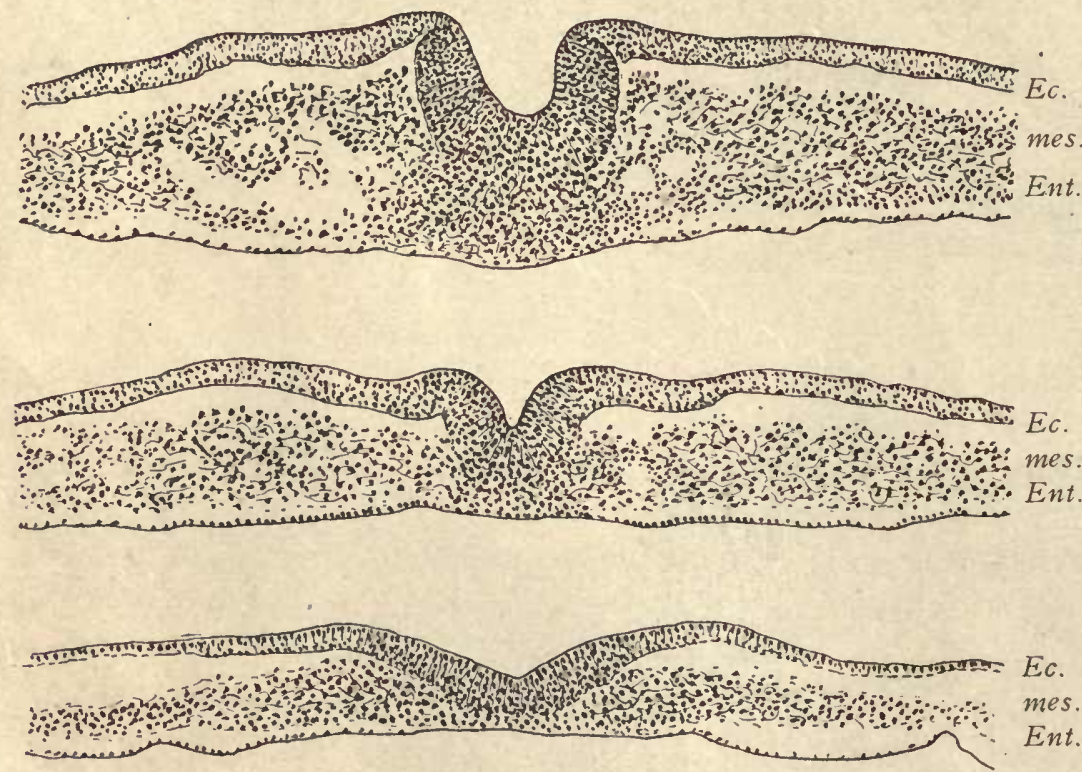

Fig. 148.-Three Sections of a Chick Embryo with Eight Segments. Series 642.

A. Section 366 , through the cephalic end of the primitive groove. B. Section 400 , through the middle of the primitive groove. C. Section 424 , through the caudal end of the primitive groove.

extension of the mesoderm across the median line. In $\mathrm{C}$ the medullary groove is fading out and merging into the beginning of the primitive streak, which forms a large mass of cells in the median line in which the boundaries between the germlayers cannot be determined. Laterally this mass of tissue passes over into perfectly distinct germ-layers, of which the middle or mesoderm, mes, is by far the most, voluminous. The walls of the medullary groove are crowded with nuclei which lie at every possible level, some close to the inner, others close to the outer surface, and also in every possible intervening position. The nuclei are much crowded, there being but little protoplasm. No distinct cell boundaries can be made out. The nuclei are further remarkable on account of their very conspicuous nucleoli. 
Sections through the Primitive Groove (Fig. I48). - In all of these we find merely the three germ-layers, which are all united in the median line with the axial band of cells, constituting the primitive streak. The ectoderm has the deep furrow which toward the head runs into the medullary groove. Caudad, the primitive groove widens out and is gradually lost. The thinning out of the mesoderm should also be noticed, as the series is followed in the caudad direction. Under a higher power the character and arrangement of the cells comes out more clearly (Fig. I49).

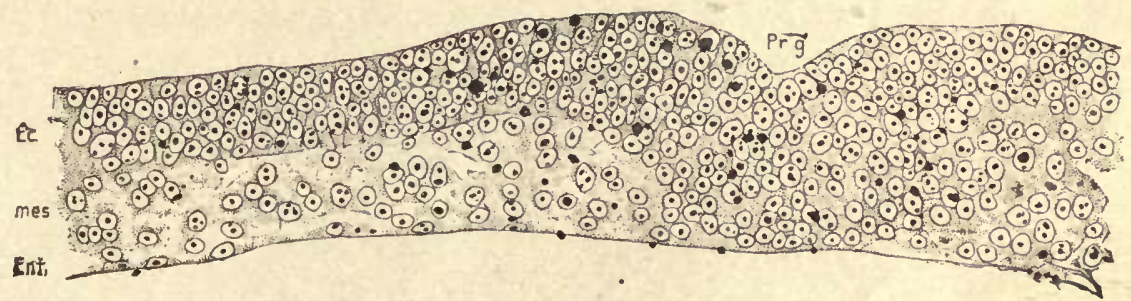

Fig. I49.-Chick Embryo with Seven Seg̈ments. Transverse Section across the Primitive Groove. Ec, Eetoderm. mes. Mesoderm. Ent, Entoderm. Pr.g, Primitive groove. The large black dots represent yolk-grains. $\times 230$ diams.

\section{Embryo Chick with about Twenty-four Segments and Three Gill-clefts (about} Forty-six Hours' Incubation).

The following description will apply almost equally well to embryos with from twenty-six to twenty-nine segments.

Examination in toto.-The specimen as a whole, as in the fresh state, has a grayish tint when viewed by transmitted light. As soon as it is hardened the opacity of all the tissues is greatly increased. In the center of the germinal area is the very conspicuous area pellucida, which is somewhat pear-shaped. The portion around the anterior end of the embryo (Fig. ${ }_{5} 5, A . p$ ) is very wide. In the center of the area vasculosa appears the embryo, the head end of which is twisted over so that the left side of the head lies against the yolk. This twisting of the neck and head so that they become asymmetrical in position is very characteristic of birds. Below the head and somewhat to the right may be seen the tubular heart, $H t$, which, in the fresh specimen, pulsates regularly. Around the area pellucida comes the dark area opaca, in which we readily distinguish the outer boundary or terminal sinus of the area vasculosa. In this there is already a well-developed network of blood-vessels through which the blood is circulating, being driven by the heart. The blood moves out from the embryo by two large vessels, A.vi, which lie symmetrically, the vitelline or omphalo-mesaraic arteries. These arteries arise from the dorsal aorta of the embryo and pass out to the area vasculosa, over which they ramify. The blood returns to the heart by means of a network of small vessels, across the posterior part of the area pellucida; the network close to the embryo fuses into two larger short trunks, one each side. The two trunks 
are the anlages of the omphalo-mesaraic veins, which gradually grow out and branch in the extra-embryonic region, enlarging at the same time (compare Fig. 53). The general form of the embryo is indicated by figure $\mathrm{I}_{52}$. In the region of the head we notice the very well-marked head-bend which is established in the region of the mid-brain, M.b. The medullary tube in the region of the head is

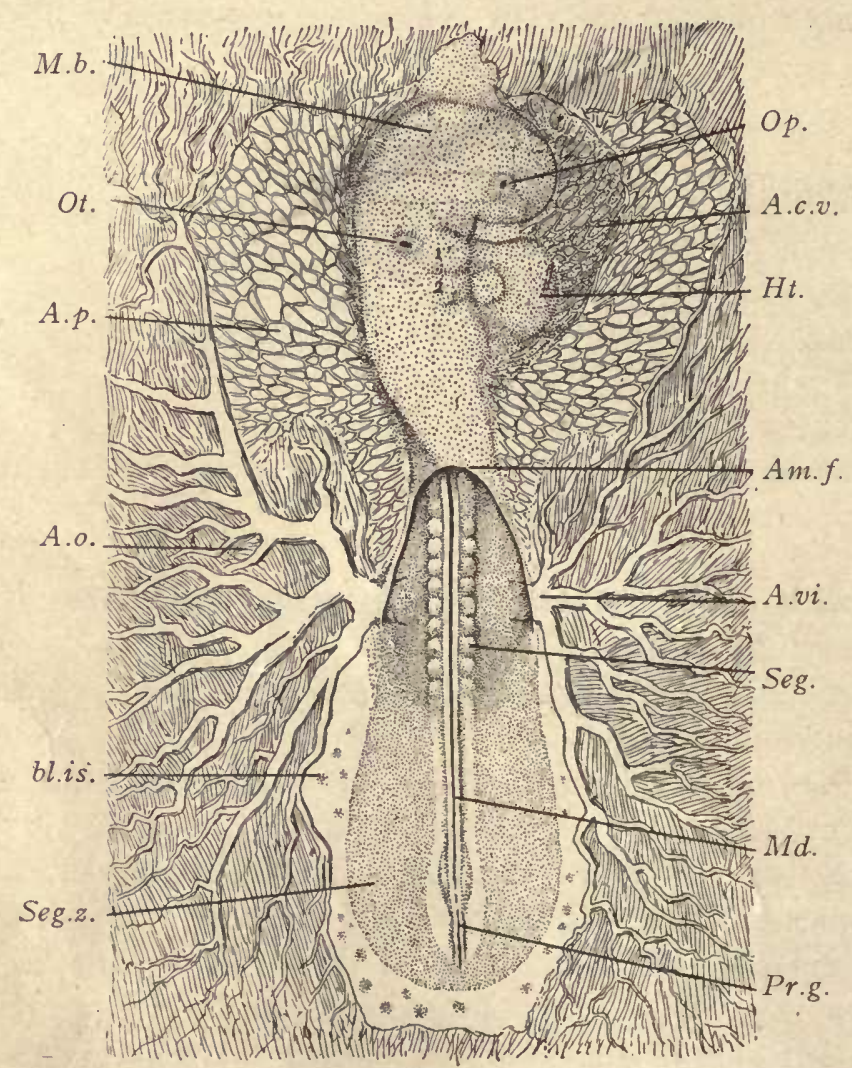

Fig. 150.-Embryo Chick with about Twenty-four Segments. Surface View from the Dorsal Side. A.c.v, Amnio-cardiac vesicle. Am.f, Posterior edge of the amniotic fold. A.o, Area opaca. A.p, Area pellucida. A.vi, Arteria vitellina (or omphalo-mesaraica). bl.is, Blood-island in the area pellucida. Ht, Heart. M.. , Mid-brain. $M d$, Medullary canal or spinal cord. $O p$, Optic vesicle. $O t$, Otocyst. Pr.g, Primitive groove. Seg, Primitive segment. Seg.z, Segmental zone. I, 2, Gill-arches. $\times$ I 5 diams.

very much enlarged and is divided into three well-marked primary cerebral resicles, which appear distinctly in specimens that have been stained and cleared. The first of these is quite large, and at its side lies the anlage of the eye, $O p$, in the center of which one readily distinguishes the commencement of the lens as a small invagination of the ectoderm with its orifice still open. The second cerebral resicle is much smaller than the first in every dimension. It occupies the region of the head-bend and is separated from the first vesicle by a constriction, and from the 
third, vesicle by another constriction. The third vesicle in length more than equals the first and second combined, and at its widest part is nearly equal in diameter to the second vesicle. It tapers out toward the caudal end of the embryo and passes over into the much smaller portion of the medullary canal, which represents the anlage of the spinal cord. At the side of the third vesicle we can see an open pit, the anlage of the inner ear or otocyst, $O t$. On the side of the neck between the third cerebral vesicle and the heart there are three external depressions which bound the first and second branchial arches, I, 2, of the embryo. Behind each arch the depression marks the site of a gill-cleft. The first is the longer, the second the shorter. Between the projecting head and the first branchial arch the outline of the embryo makes a depression which marks the position of the developing. oral cavity. The heart is a large tube, $H t$, in a still larger pericardial cavity (cœlom), the membranous covering of which is somatopleuric. The omphalomesaraic veins join the venous or posterior end of the heart. The heart is very much bent; its anterior end turns toward the gill-clefts and there gives off the primitive aortic branches, which finally join again so as to form the median dorsal aorta which sends off the two vitelline arteries, A.vi. On either side of the medullary canal can be seen the primitive segments, Seg. The first of these which is distinct lies close behind the otocyst. At the. posterior end of the embryo additional segments are still forming, and the precise number of segments varies from embryo to embryo. The medullary canal, $M d$, is closed, but beyond its extreme limit traces of the primitive groove, Pr.g, can still be seen. The network of blood-vessels over the area vasculosa is very distinct and characteristic. The network, however, does not yet extend into the body of the embryo proper. The limit of the body of the embryo is suggested by the darker tissue, Seg.z, surrounding the spinal cord, $M d$, on either side. About the hinder end of the embryo, both in the pellucida and in the opaca, appear. a number of small spots, the bloodislands, bl.is, many of which have in the fresh specimen a reddish color. In hardened specimens the opacity of the blood-islands renders them conspicuous, especially in the area pellucida.

\section{Embryo Chick with Twenty-eight Segments.}

The Study of Transverse Sections.-A series of figures from transverse sections of an embryo of this stage is herewith presented. They have been selected so as to show the principal typical structures. The position of the sections can be followed more easily by comparing each transverse section with figure I66, to determine its place and the organs through which it must pass.

Section through the Right Auditory Invagination (Fig. $\mathrm{I}_{5} \mathrm{I}$ ). - Owing to the curvature of the neck-bend, the section of the head is not symmetrical. It passes through both the hind-brain, $h . b$, and the fore-brain, f.b. Underneath the former appears. a small structure, $n c h$, the notochord, and on one side can be seen the auditory invagination, $\mathrm{O} t$, which is formed wholly by the locally thickened ectoderm, 
which is elsewhere quite thin. The ectoderm, $E c$, covering the dorsal side of the hind-brain is very thin, but the portion in front of the auditory invagination is somewhat thicker. The ectoderm of the invagination is very much thickened and contains numerous somewhat crowded nuclei at all levels. These nuclei are rounded in form and have one or two very distinct nucleoli. On the posterior side of the otocyst there is very little mesoderm; on the anterior side, much more. Between the

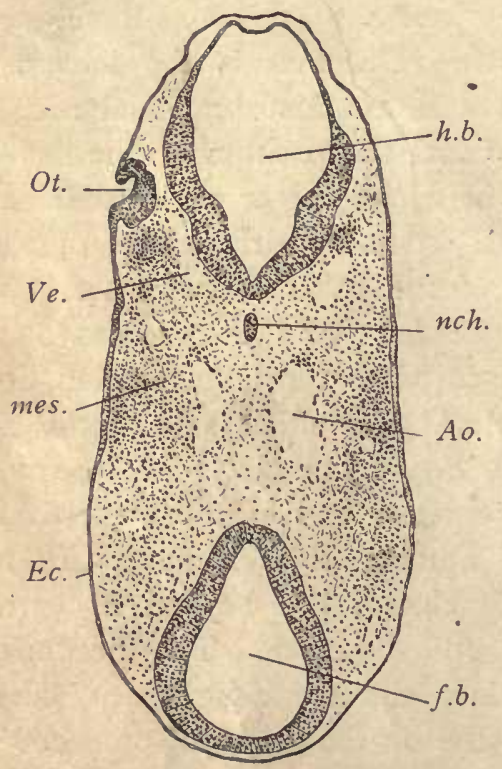

Fig. I5I.-Section of Chick Embryo with about TWEnTY-eight SEgments. TransVerse Series 92, Section 73.

Ao, Aorta. Ec, Ectoderm. f.b, Fore-brain. h.b, Hind-brain. mes, Mesoderm, nch, Notochord. Ot, Otocyst. $V c$, Vein. $\times 50$ diams.

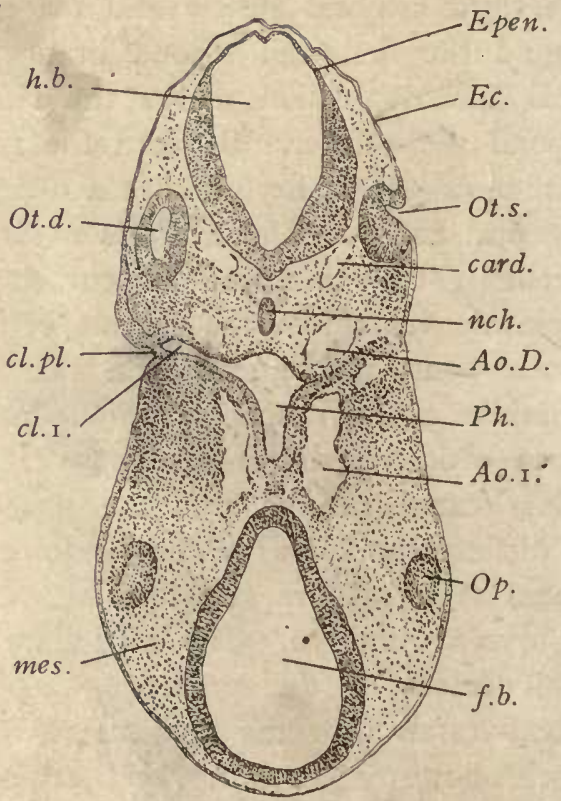

Fig. 152,-Section of Chick Embryo with about TWenty-eight Segments. Transverse Series 92, Section 83.

Ao.1, Prolongation toward the fore-brain of the first aortic arch. Ao.D, Descending aorta. card, Anterior cardinal vein. cl.pl, Closing plate. cl.r, First gill-pouch. Ec, Ectoderm. Eper. Roof of hind-brain. $f . b, \cdot$ Fore-brain. h.b, Hind-brain. "mes, Mesoderm. nch, Notochord $O p$, Optic vesicle. Ot.d, Right otocyst. Ot.s, Left otocyst. $P h$, Pharynx. $\times 50$ diams.

developing otocyst and the notochord there is a blood-vessel, Ve, with merely endothelial walls, a branch of the cardinal vein. Between the hind-brain and forebrain near the notochord, the two aortæ, $A o$, are cut. In their interior there can usually be seen a certain number of nucleated blood-cells varying somewhat in size and appearance, but generally having a rounded form with distinct outline and a well-defined nucleolated nucleus.

Section through the Left Auditory Invagination (Fig. 152).- Owing to the irregular form of the embryo the sections through the otocyst are not symmetrical. The 
present section shows the opening of the left otocyst, Ot.s, and a closed section of the right otocyst, Ot.d. At its lower inner edge the outer boundary of the wall of the otocyst is indistinct, this appearance being due to the union of the cells of the acoustic ganglion with the wall of the otocyst. The section also passes through the first gill-cleft, $c l . I$, of the right side, and shows very distinctly indeed the closing plate, $c l . p l$, which is formed by a fusion of the ectodermal and entodermal cells. On the opposite side of the section the same cleft is imperfectly shown. On the posterior side of the cleft is the dorsal aorta, Ao.D, and on the anterior side of. the cleft, extending toward the fore-brain, $f . b$, appear the sections of the two prolongations of the first arches, Ao.I, toward the fore-brain. In this specimen each prolongation forms a loop, which rejoins its arch dorsally. In the region of the forebrain appears a shaving from the edge of the optic evagination, $O p$. The anterior cardinal veins, card, appear just inside of the otocyst close to the ventral wall of the hind-brain, h.b.

Section through the Invagination of the Optic Lens (Fig. 153).-This section also passes through the hind-brain, $h . b$, forebrain, f.b, and through the openings of both invaginations to form the anlages, $L$, of the lenses of the eye. These invaginations bear a striking resemblance to those which form the otocysts. The ectoderm, $E c$, over the roof of the fore-brain is very thin and passes abruptly into the thickened layer which forms the wall of the invagination. On the ventral side the ectoderm is somewhat thicker. The wall of the lentic

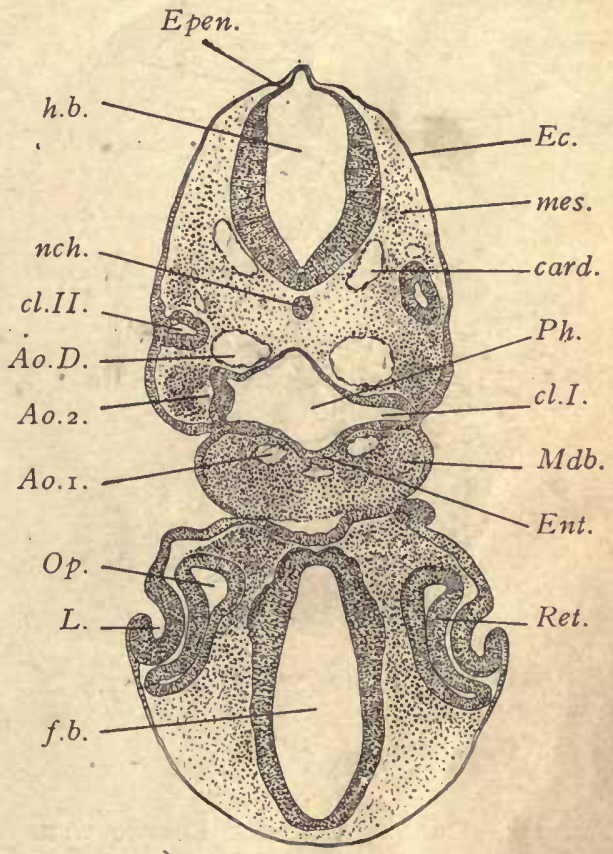

Fig. 153.-Section of Chick Embryo with about Twenty-eight Segments. Transverse SERIES 92, SECTION 96.

Ao.D, Descending aorta. Ao.I, First aortic arch: Ao.2, Second aortic arch. card, Anterior cardinal vein. cl.I, First gill-cleft. cl.II, Second entodèrmal gill-cleft. Ec, Ectoderm. Ent, Entoderm. Epen, Roof of hind-brain. f.b, Fore-brain. $h . b$, Hind-brain. $L$, Invagination of lens. $M d b$, Mandibular arch. mes, Mesoderm: nch, Notochord. $O p$, Optic vesicle. $P h$, Pharynx. Ret, Retina. $\times 50$ diams. vesicle is quite thick; its nuclei are numerous, but are situated chiefly on the mesodermal side of the layer, so that toward its outer surface the layer is comparatively free from nuclei. The invagination of the lens rests against the optic vesicle, the wall of which, Ret, next to the lens is thicker than the posterior or inner wall of the optic vesicle. The thickened outer portion is the anlage of the retina, the thinner inner portion is the anlage of the pigment layer covering the retina. The fore-brain, $f . b$, has an elongated form with quite thick walls crowded with nucle-Between 
it and the hind-brain appears the cavity of the pharynx, $P h$, which on the left side of the embryo shows a prolongation, cl.I, which extends almost to the surface of the embryo. This prolongation is the first gill-pouch. On the dorsal side of the pharynx appear the two large aortic trunks, $A o . D$, and on its ventral side the two smaller first aortic arches, Ao.r. These are situated in the mandibular

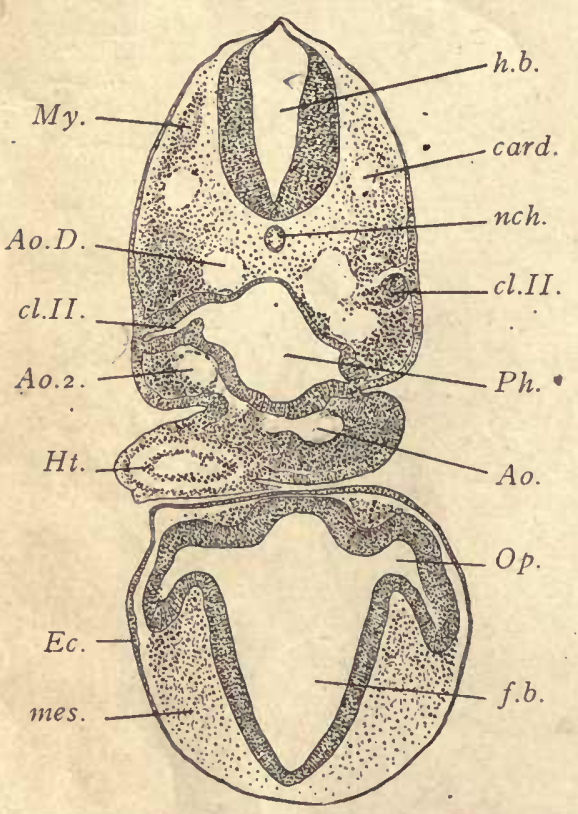

Fig. I54.-Section of Chick Embryo with about Twenty-eight Segments. TransVERSE SERIES 92, SECTION IO4.

$A o$, Trunk of the aorta. Ao.D, Descending aorta. Ao.2, Second aortic arch. card, Anterior cardinal vein. $c l . I I$, Second entodermal gill-pouch, $E_{c}$, Ectoderm. f.b, Forebrain. $h . b$, Hind-brain. $H t$, Heart. mes, Mesoderm. $M y$, Muscle plate. nch, Notochord. Op, Optic vesicle. $P h$, Pharynx. $\times$ 5o diams. branchial arch, $M d b$, which is well marked externally by a rounded protuberance. The distal end of the second gill-pouch is shown on the right side of the section, $c l . I I$.

Section through the Optic Stalks. (Fig. I54). - The head of the embryo now appears quite isolated from the body. It is bounded by a distinct layer of ectoderm, $E c$, and contains the very large fore-brain, $f . b$, which gives off on either side an optic evagination, $O p$, the walls of which are quite thick, about the same as those of the fore-brain proper. Each optic evagination is widest toward the side of the head and is constricted toward the brain, with which, therefore, it is connected by a stalk in which we can already recognize the anlage of the optic nerve. Between the two optic stalks on the side toward the pharynx the floor of the fore-brain bends downward and almost joins the superficial ectoderm. All of the space between the walls of the fore-brain and the optic evagination on the one hand, and of the superficial ectoderm of the head on the other, is filled with undifferentiated mesenchyma. In this tissue blood-vessels. nerves, lymphatics, and muscles will grow, and the tissue itself is to produce the cutis, the subcutaneous tissue, the skull, the dura mater, arachnoid membrane, and pia mater. We have in the present undifferentiated stage of this mesenchyma a most striking contrast with the complicated histological conditions of the adult. The opposite part of the embryo represents the cervical region. At one side-we see a small piece of the heart appearing, $H t$, and higher up is the wide pharynx, $P h$, underneath which is a blood-vessel, $A o$, the main aorta. To the left appears another blood-vessel, Ao.2, a portion of the second aortic arch. 'The pharynx shows on one side the prolongation of its cavity which constitutes the second gill-pouch, cl.II. On the dorsal side of the pharynx Mreaz descending aortæ, Ao.D, that on the right of the figure being joined 
by the second aortic arch, near which appears an accumulation of more deeply colored cells, cl.II, part of the entodermal wall of the second gill-pouch. Between the pharynx and the hind-brain we have a round section of the small notochord which appears quite deeply stained, and therefore stands out conspicuously from the very loose mesenchyma by which it is surrounded. It is not until later stages that the mesenchymal cells begin to crowd around the notochord to constitute the anlage of the future vertebral column. At the present stage the differentiation of the axial skeleton around the notochord has not begun. As. regards the hind-brain, $h . b$, we observe that its sides are already considerably thickened, but its dorsal wall is quite thin and has already expanded considerably, thus initiating the formation of the thin ependymal roof of the fourth ventricle. On either side of the hind-brain appears a blood-vessel, card, the anterior cardinal, which by transformation and migration is to lead to the formation of the jugular veins of the adult.

Section through the Aortic End of the Heart (Fig. 155). - The cervical region of the head and the tip end of the region of the fore-brain are cut separately. On the lower side of the pharynx is attached the double heart-tube, of which the endothelial portion, endo, is in actual contact with the thick entoderm, En, which forms the floor of the pharynx. The heart-tube shows its bend toward the right of the embryo. There is a considerable space between the endothelial heart and the muscular heart, $m . h t$, and this space is almost wholly free of tissue, except in the immediate neighborhood of the pharynx itself. Close to the connection of the heart-tube with the pharyngeal floor there runs off on either-side the-membrane of the amnion. Where it starts from the embryo the amnion has considerable thickness and appears somewhat folded in the section; but as it turns to cover the embryo it becomes very thin. It consists only of two very delicate layers, mesodermic and entodermic, both one cell thick. The two layers lie close together, but are easily distinguished. On the right-hand side of the embryo the raphe of the amnion may be observed, raph, and in this section it is constituted by only two strands of mesoderm which pass over from the amnion on to the chorion, Cho, or membrana serosa, as it has been called by many embryologists. The arrange-ment of the envelopes of the head is somewhat more complicated. Underneath the left side of the section of the cervical portion of the head runs the splanchnopleure, $S p l$, in which one can readily distinguish numerous sections of blood-vessels, which, on the side toward the embryo, are covered by mesoderm, and on the side away from the embryo are covered by entoderm. If we follow along the splanchnopleure to a point near the section of the region of the fore-brain, we find that it encounters a circle of ectoderm, $E c$, which surrounds that portion of the head. When the splanchnopleure reaches this ectoderm, its two layers divide or split apart. The mesoderm bends off toward the right* side of the embryo and forms, together with a portion of the ectoderm, a part of the true amnion, $A m^{\prime}$, of the head. The 
entoderm, Ent, on the contrary continues in the same direction as before, until it joins the ectoderm on the left side of the head to form the pro-amnion, Pro.am. Beyond the head the entoderm and mesoderm again unite and we have a continuation of the splanchnopleure, Spl. Owing to the development of the pro-amnion, the relations of the fetal envelopes surrounding the head are complicated. The

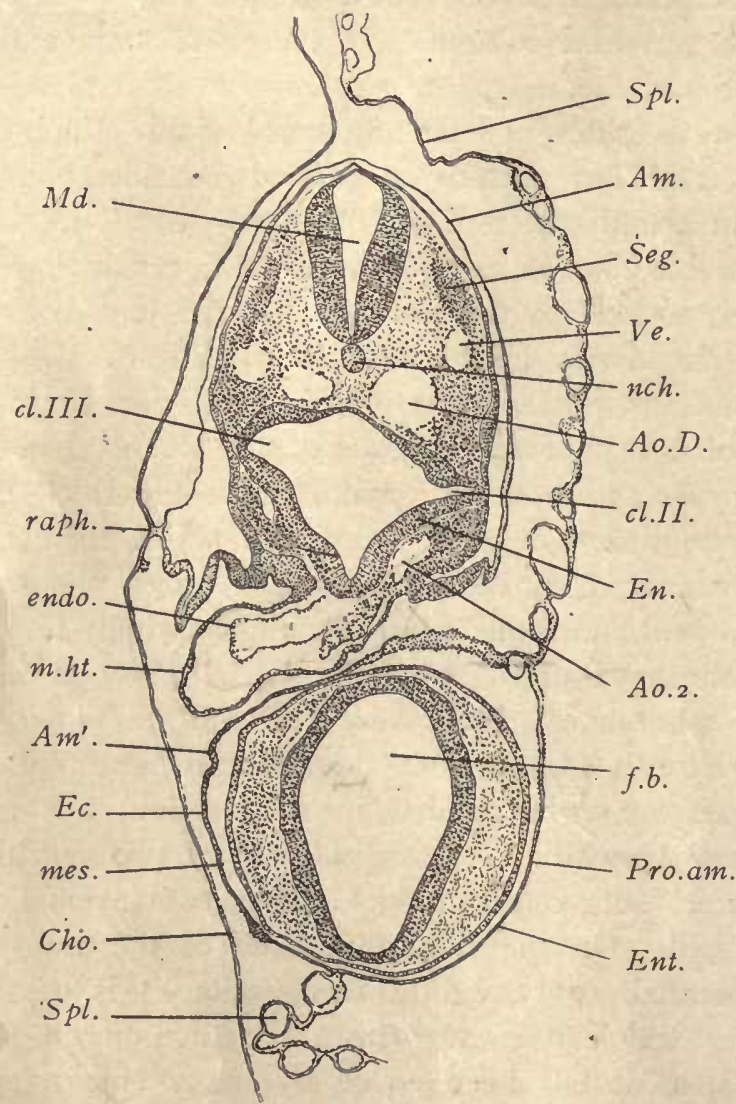

Fig. 155.-Section of Chick Embryo with about Twenty-eight Segments. Transverse Series 92, SECTION II 4 .

$A m, A m^{\prime}$, Amnion. Ao.D, Descending aorta. Ao.2, Second aortic arch of the left side. Cho, Chorion. $c l .1 I$, Second entodermal gill-pouch. cl.III, Third entodermal gill-pouch. Ec, Ectoderm. En, Entoderm of pharynx. endo, Endothelial heart. Ent, Entoderm of pro-amnion. f.b, Fore-brain. $M d$, Medulla oblongata. mes, Mesoderm of amnion. m.ht, Muscular heart. nch, Notochord. Pro.am, Pro-amnion. raph, Raphe of amnion. Seg, Segment. Spl, Splanchnopleure. Ve, Anterior cardinal vein. $\times 50$ diams.

student may, however, easily satisfy himself that the layer, $E c$, in figure 157 , is really ectoderm by following it through in the series of sections, for he will then find that it becomes continuous in other regions, on the one hand, with the ectoderm of the true amnion, and, on the other, with the epidermis of the body proper. In the cervical region we have a transverse section of the lower portion of the 
hind-brain, $M d$, corresponding to the part of the future medulla oblongata near its junction to the spinal cord. Underneath it is the section of the notochord, nch, and on either side sections of a secondary somite, Seg. Just below each somite is $a^{\circ}$ cardinal vein, $V e$, and below the vein, but nearer to the median line, lies the dorsal aorta, Ao.D. The pharynx expands on each side; the prolongation on the left of the embryo is the second gill-pouch, cl.II, that on the right is the third gill-pouch, cl.III. The pharynx itself is lined by entoderm, En, which is very thin in the median dorsal line, but immediately. below the dorsal aortæ it thickens abruptly and continues as a quite thick layer on to the ventral side. In the median ventral line it forms a deep groove, and in the walls of this groove we find that the nuclei are. not distributed through the whole thickness of the entoderm, but occupy chiefly its outer or basal portions, so that the portion of the layer next the cavity of the groove is formed almost wholly of protoplasm. At the tip of the gill-pouch the entoderm has come into actual contact with the ectoderm, and the cells of the two germ-layers have there united, without distinguishable boundary being kept between the layers. The fused ectoderm and entoderm constitute the closing plate of the gill-cleft, and such a plate is formed at the tip of every gill-pouch. On the left side of the ventral surface of the pharynx appears the section of the second aortic arch, Ao.2. Opposite but higher up is the section of the right third aortic arch. By following along through a few sections (in the series here studied, from four to six) the junction of these arches with the endothelial tube of the heart may be observed. The student should verify this connection and satisfy himself that the endothelium of the blood-vessels is a continuation of the endothelium of the heart. This fact is of great morphological and physiological importance. Of the section of the region of the fore-brain little need be said. The ectoderm has begun to thicken somewhat. The walls of the fore-brain, $f . b$, itself have not begun to show any differentiation into layers. There is a considerable development of mesenchyma between the brain and the superficial ectoderm.

Section through the Venous End of the Heart (Fig. I56).-We have now passed in our series beyond the level of the head, so that no part of that is included in the section. The general topography of the part is similar to that of the preceding section (Fig. I55), but there are many important differences of detail. We are now in the region of the spinal cord, proper, Sp.c, which here offers to us its characteristic early embryonic form. It is oval in section, its walls are thickened on each side, but are thinned on the dorsal side, where they constitute the deckplate, and on the ventral side, where they form the floor-plate; the cavity is narrow and slit-like. The notochord lie close under the ventral side of the medullary tube and below it is the mediar dorsal aorta, Ao, a single and very large vessel, which is formed by the union of the two dorsal aortæ shown in figure 157 , Ao.D. Immediately below the aorta follows the pharynx, $P h$, which is now, more rounded in form and does not extend har laterally. Its entodermal lining is mod- 
erately thick, but it is somewhat thinner near the median dorsal line. On either side of the pharynx the mesodermal layer, mes, is very thick and stands out conspicuously, owing to its dark staining. Above the pharynx it thins out and passes over on to the somatopleure, $S o m$, and so on to the amnion, $A m$. On the ventral side of the pharynx the mesodermal layer passes over into the muscular wall of

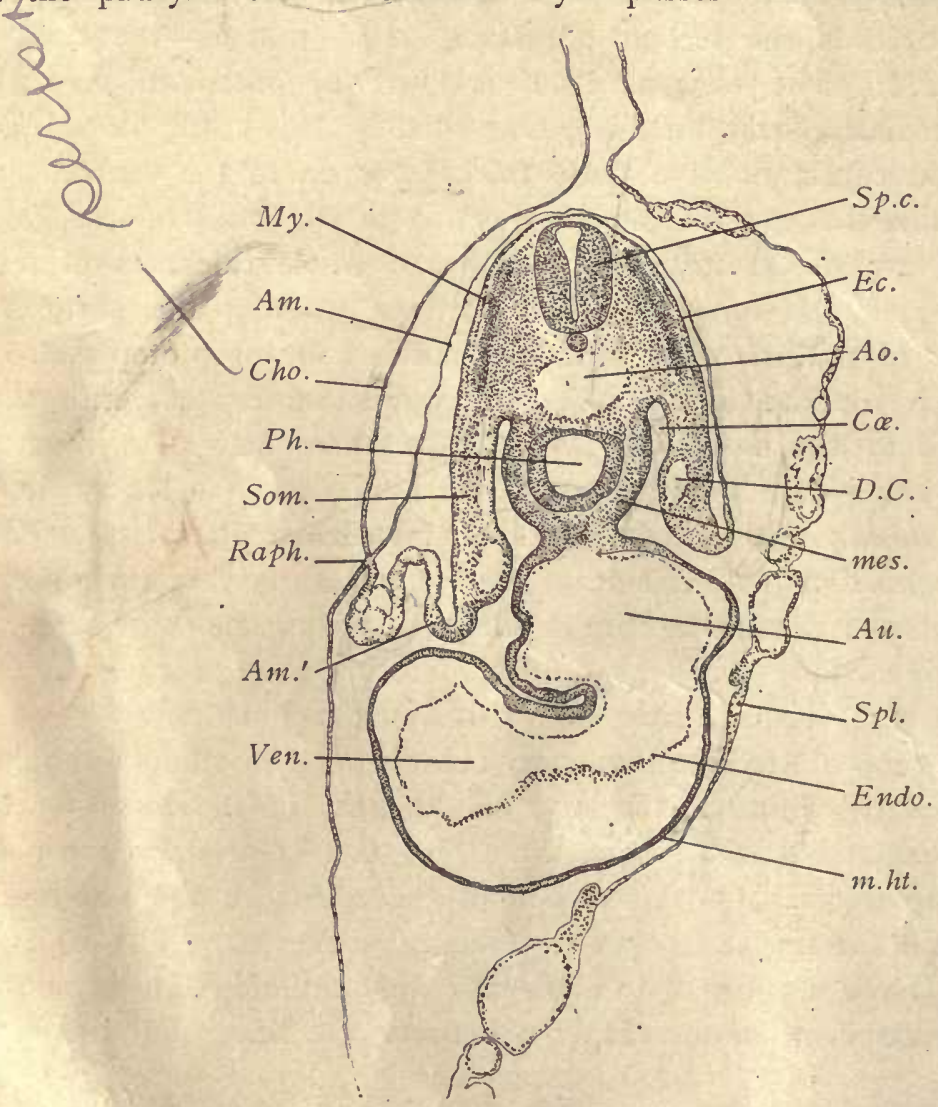

Fig. 156.-Section of Chick Embryo with about Twenty-eight Segments. Transverse Serifs 92, SECTION I 44.

Am, Am', Amnion. Ao, Aorta. Au, Cardiac auricle. Cho, Chorion. Co, Cøelom, D.C, Duct of Cuyier. Ec, Ectoderm. Endo, Endothelial heart. mes, Mesoderm. mi.ht, Muscular heart. My; Primitive segment. Ph, Pharynx. Raph, Raphe of amnion. Som, Somatopleure. Sp.c, Spinal cord. Spl, Splanchnopleure. Ven, Ventricle of heart. $\times 50$ diams.

the heart, $m . h t$. The heart itself is very large; it has two tubes, the endothelial, endo, and the muscular, $m . h t$, which are very distinct. The endothelial cavity is very large. It is especially expanded immediately underneath the pharynx to form the auricular end of the heart, which receives the veins. Throughout a large part of the auricle the endothelium is closely fitted against the muscular wall. Farthes ventralward the double heart-tube bends to the right of the embryo to form the ventricular limb. Ven, in which the entothelial cavity is also enlarged. 
The heart as a whole occupies about one-half the area of the entire section of the embryo, being of relatively enormous proportions. The cardinal veins, D.C, have moved down as compared with the previous section, and are now found to lie in the somatopleure, in which there also appear several sections of smaller blood spaces above the main cardinal vessel. The path of the cardinal through the somatopleure carries it toward the heart. The vertical part of the vessel, which effects a union with the heart, is known as the common cardinal. The common cardinals also deliver the blood from the posterior cardinals to the heart. They are at somewhat different levels on the two sides of the embryo, that on the right side being lower and occupying a sort of prominence on the mesothelial side of the somatopleure. If the cardinal veins are traced along through successive sections, it will be found that they open directly into the auricles of the heart, crossing over the colom, $C o$. The crossing is accomplished by a growth of the somatopleure which unites with the, wall of the heart. The openings of these veins are at this stage morphologically symmetrical and are entirely distinct from the openings of the omphalo-mesaraic veins, which enter the heart farther tailward. If sections in the series between the present one and that through the aortic end of the heart (Fig. 155) be examined, it will be found that the heart in the middle part of its course is entirely detached from the pharynx, so that the heart-tube is suspended by its two ends from the ventral side of the pharynx. By the crossing of the cardinal veins the portion of the cœlom, $C o$, on either side of the pharynx is shut off from the portion of the colom around the heart. At the raphe, raph, of the amnion the ectoderm of the amnion joins that of the chorion, Cho. In the portion of the somatopleure, $A m^{\prime}$, which runs from the raphe to the embryo there are a number of spaces of rounded form which appear like so many vesicles. The nature of these vesicles is uncertain.*

The secondary somites, $M y$, are very characteristic, and should be studied with a higher power. The somite consists of an outer layer and an inner layer of about equal thickness, and these two layers pass over into one another at the dorsal and ventral edges of the segment. They are closely pressed against one another, so that there is no space between them. The outer layer is more deeply stained than the inner; its nuclei are somewhat less distinct and are rounded in form. Those of the inner layer are elongated in form, as may be easily observed by raising and lowering the focus. The outer layer is quite close to the ectoderm, and the inner layer rests against the large mass of mesenchymal tissue which surrounds the spinal cord, notochord, and aorta.

Section through the Anlage of the Liver (Fig. 157). - In this section the general topography is similar to that of the last, so that we need describe only the new

* They seem to be bounded on one side by ectoderm, on the other by mosoderm; but as the significance of these vesicles, I cannot express any opinion. The separate onening front of, and independently of, the omphalo-mesaraic veins, so far as I am

It is a condities of morphological importance. 
structures and relations which appear. A little piece of the ventricular limb of the heart with its double walls, m.ht,endo, still appears. The section is, strictly speaking, beyond the venous end of the heart and passes through the sinus venosus Si.V, which is formed by the union of the omphalo-mesaraic veins entering the body of the embryo from the splanchnopleure of the yolk-sac, or, in other words,

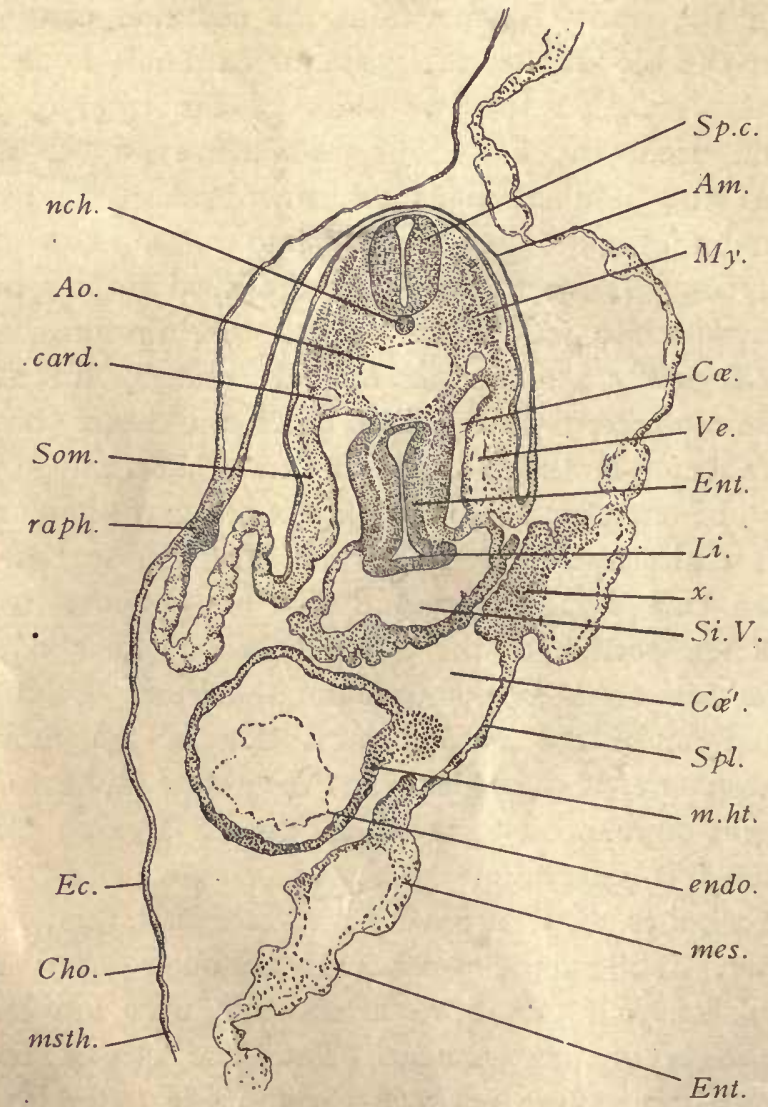

Fig. 157.-Section of Chick Embryo with about Twenty-eight Segments. Transverse Series 92 , SeCtion 165.

Am, Amnion. Ao, Aorta. card, Cardinal vein. Cho, Chorion. Co, Co', Colom. Ec, Ectoderm. endo Endothelial heart. Ent, Entoderm. Li, Liver. mes, Mesoderm. m.ht, Muscular heart.' msth, Mesothelium. $M y$, Primitive segment. nch, Notochord, raph, Raphe of amnion. Si.V, Sinus venosus of heart. Som, Somatopleure. Sp.c, Spinal cord. Spl, Splanchnopleure. Ve, Vein. $x$, Accumulation of mesodermic tissue about the omphalo-mesaraic vein. $\times 50$ diams.

from the area vasculosa. In the splanchnopleure, $S p l$, there is a thickening, $x$, ai we mes oderm which narks the crossing of the veins from the yolk-sac to the part of the hear. The entoderm of the embryo forms a tube, Ent, which karthan wo-ventral diameter. The entoderm itself is quite nortion. From the ventral side of the entoder- 
mal canal spring two small pouches or diverticula, the anlages of the liver. The left diverticulum is well shown in the figure; the right diverticulum appears a few sections farther on. It is especially important to note that the entodermal epithelium of the hepatic diverticulum comes into immediate contact with the endothelium' of the blood spaces. During the later development this relation is preserved, and there is a complicated intercrescence of the entodermal cells constituting the liver and of the vascular endothelium.* The intercrescence leads to the forma-

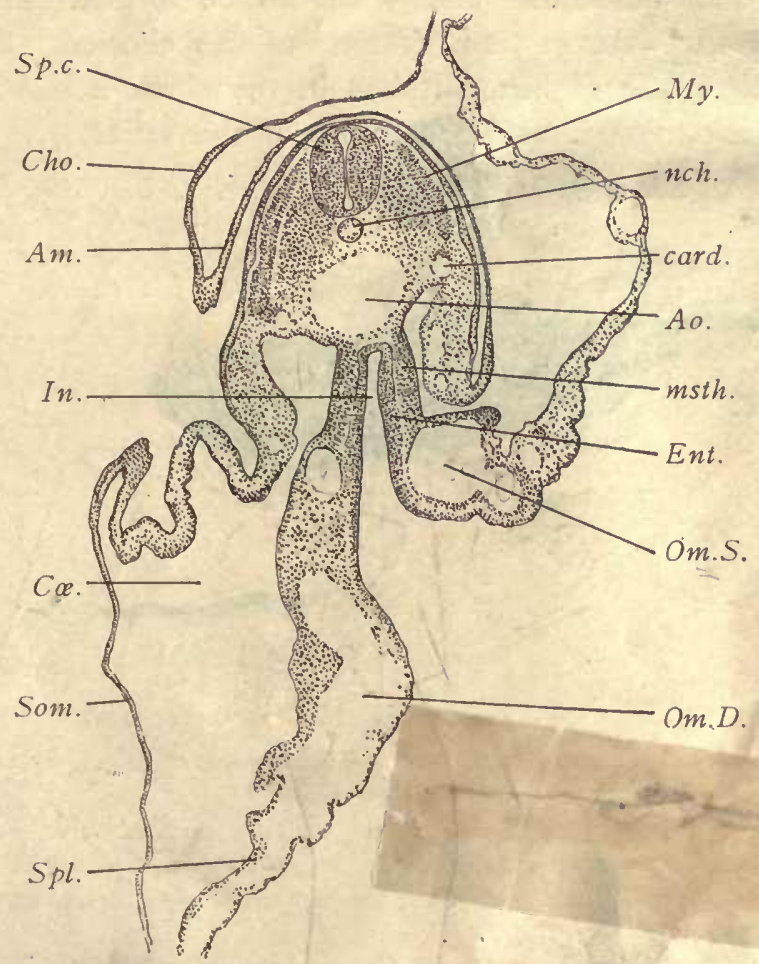

Fig. 158.-Section of Chick Eubryo with about Twenty-eight Segments. Transverse Series 92 , SECTION I79.

Am, Amnion. Ao, Aorta, card, Cardinal vein. Cho, Chorion. Co, Coelom. Ent, Entoderm. In, Intestine. msth, Mesothelium. $M y$, Muscle plate. nch, Notochord. Om.D, Right omphalo-mesaraic vein. Om.S, Left omphalo-mesaraic vein. Som, Somatopleure. Sp.c, Spinal cord. $S p l$, Splanchnopleure. $\times 50$ diams.

tion of the sinusoids, which are highly characteristic of the liver and which give rise to the so-called capillaries of the hepatic lobules of the adult liver. These "capillaries" are, however, 'always true sinusoids, and morphologically not capillaries at all. Owing to the junction of the veins and liver, a portion of the body cavity, $C \alpha^{\prime}$, at the side of the pharynx is shut off from direct fonnection with the pericardial cavity. The ridge of tissue dividing the two cavities from one-another is the septum transversum. If the series of sections be followed through tail-

* A few sections anterior to this the beginning of the intercrescen is obscrvable. 
ward, it will be found that at this stage further back the septum transversum is formed also upon the right side of the body of the embryo. The mesothelium between the upper division of the cœlom, $C \propto$, and the sides of the entodermal canal is very much thickened and deeply stained. On either side of the very large median aorta, $A o$, and just above the cœlom, appear the right and left posterior cardinal veins, card. Concerning the fetal envelopes little need be said, except to call attention to the large raphe, raph, of the amnion, which is now a rather conspicuous ectodermal thickening and seems to be formed rather at the expense of. the ectoderm of the amnion than of that of the chorion. Such an ectodermal raphe is very characteristic of birds; it has in the chick a considerable extent and therefore appears in many successive sections of the series.

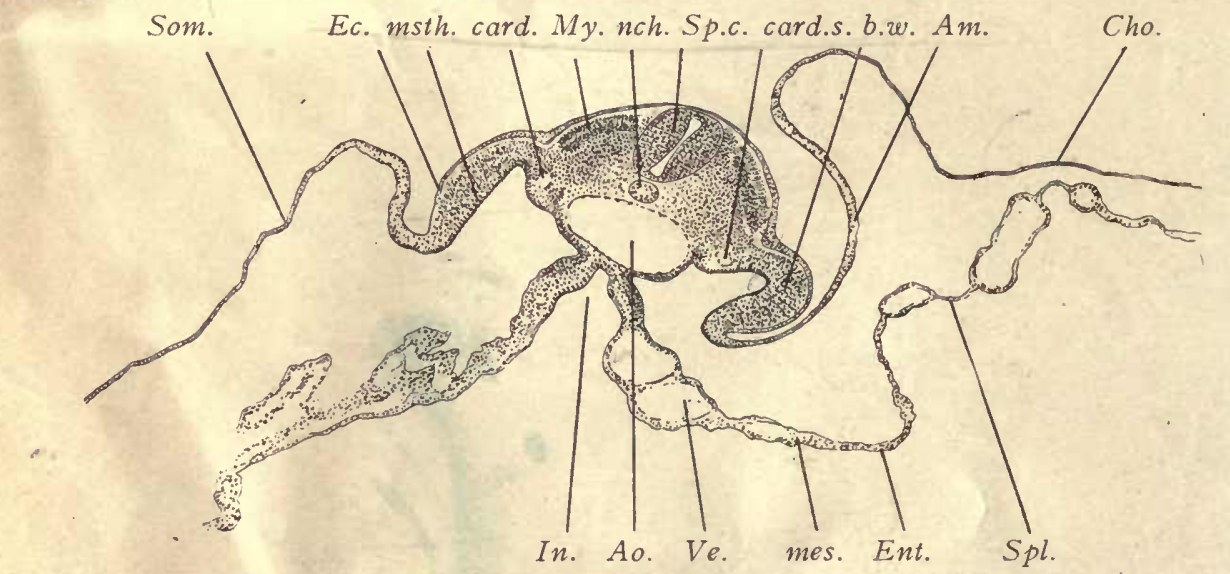

Fig. 159.-Section of a Chick Embryo with about Twenty-eight Segments. Transverse Series 92 , SEction 220.

Am, Amnion. An 1. u,u, ,ody-wall. card, Right posterior cardinal vein. card.s, Left cardinal vein. Cho, Cheison. Fc, Ectoderm. Ent, Entoderm. In, Intestine. mes, Splanchnic mesoderm. msth, Mesothelium. My, Myotome. nch, Notochord. Som, Somatopleure. Sp.c, Spinal cord. Spl, Splanchnopleure. $V e$, Vein. $\quad \times 50$ diams.

Section through the Omphalo-mesaraic Veins (Fig. I58). - This section is intermediate in structure between figure ${ }_{57} 7$ and figure ${ }_{559}$, here described. We are now beyond the region of the heart and liver. The cavity of the intestine is open on the ventral side, so that the walls of the intestine pass over directly into the extra-embryonic splanchnopleure, $S p l$, in which are lodged the very wide omphalomesaraic veins, Om.D and Om.S, which are entering the body of the embryo to run forward past the liver anlage (Fig. I57) to join the posterior or venous end of the heart. It will also be noticed that the amniotic fold does not join its fellow, and therefore has no raphe. In this condition the amnion is said to be "open."

Section through the Anterior Portion of the Open Intestine (Fig. I59).- In this section the intestinal cavity, In, being without a ventral wall, opens directly into the general entodermal cavity under the germinal area and above the yolk-mass (compare the diagrams Figs. 29 and 45 ). The median plane of the emlsryo is still 
inclined to the left. The extra-embryonic somatopleure, Som, rises in two high folds, one on each side of the embryo; the inner portion of each fold, $A m$, belongs to the amnion, the outer portion, Cho, to the chorion. The splanchnopleure, $S p l$, passes without demarcation into the wall of the intestinal cavity, In. The entoderm, Ent, of the extra-embryonic splanchnopleure is very thin, but where it passes into the embryonic region toward the median line, it thickens a little. The splanchnic mesoderm is a thin layer of mesothelium which, of course, bounds the cœlom everywhere and can be followed continuously over on to the somatopleure. The splanchnic mesenchyma is loose in texture and surrounds the large blood-vessels. The splanchnic mesoderm on either side of the intestinal groove appears quite dark, owing to the condensation of the tissue. Whether this condensation is developed from the mesothelium or from the mesenchyma it is very difficult to say.

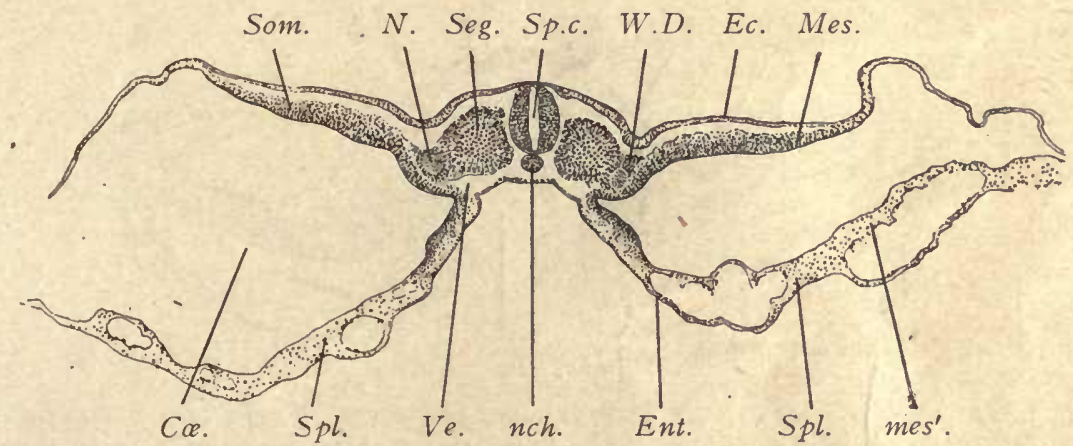

Fig. i6o.-Section of a Chick Embryo with about Twenty-eight -Segments. Transverse Series 92. SECTION 356.

$C_{a}$, Cœlom. Ec, Ectoderm. Ent, Entoderm. Mes, Somatic mesoderm. mes', Splanchnic mesoderm. $N$, Nephrotome. nch, Notochord. Seg, Segment. Som, Somatopleure. Sp.c, Spinal cord. Spl, Splanchnopleure. Ve, Blood-vessel. W.D, Wolffian duct. $\times 50$ diams.

The somatopleure, Som, where it becomes embryonic, increases greatly in thickness and forms an arch, b.w, which is the beginning of the formation of the ventral body-wall of the chick. The form of the arch indicates the commencing closure of the embryonic somatopleure on the ventral side, by which the body of the embryo will ultimately become shut off from the underlying layers of the blastoderm. In the median plane of the embryo we find the spinal cord, cut somewhat obliquely, the notochord, $n c h$, and the very large section of the aorta, Ao. The great transverse width of the aorta is due to its approaching division toward the caudal end of the body to form the two branches which run out to the area vasculosa and are known as the omphalo-mesaraic or vitelline arteries. Before they leave the body of the embryo each of these arteries gives off a branch which continues in the body of the embryo not far from the notochord and close to the entoderm. These branches subsequently become the allantoic arteries. On either side of the spinal cord lie the secondary somites, $M y$. A short distance from the aorta. 
on either side appear sections of two rather small blood-vessels, the cardinal veins, card. Between the vein on each side and the aorta there is a little accumulation of denser tissue. If a series of sections is followed through, the Wolffian duct may be traced into this condensed tissue, and when the duct is differentiated, it will take the place of this tissue between the aorta and the vein.

Section through the Middle Portion of the Open Intestine (Fig. r60).-Comparison of this section with the preceding is instructive as an illustration of the fact that the differentiation of structures is found less advanced as we proceed toward the caudal end of the embryo. In the present section the amniotic folds can hardly be said to have appeared at all, although the colom, $C \propto$, is very wide indeed, and there is little differentiation in either the somatopleure, Som, or splanchnopleure, $S p l$, between the embryonic and extra-embryonic regions. The ento-

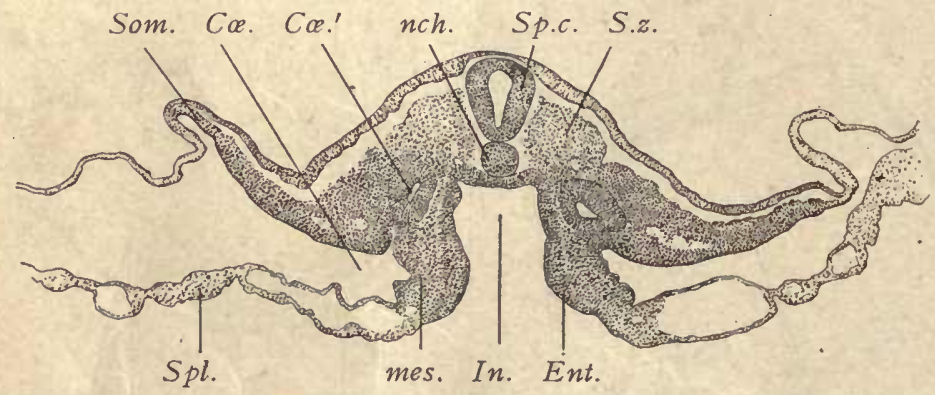

Fig. 161.-Section of a Chick Embryo with Twenty-eight Segments. Transverse Series 92, Sectiox 4 i9. $C a$, Colom. $C a^{\prime}$, Diverticulum of the cœlom. Ent, Entoderm. In, Intestinal cavity. mes, Mesoderm. nch, Notochord. Som, Somatopleure. Sp.c, Spinal cord. Spl, Splanchnopleure. S.z, Segmental zone. $\times 5^{\circ}$ diams.

derm is a little thicker in the embryo than in the extra-embryonic territory. A similar difference may be observed in the ectoderm. The embryonic mesoderm in both somatopleure and splanchnopleure is considerably more developed and much denser than in the extra-embryonic parts. The axial structures of the embryonamely, the spinal cord, Sp.c, and notochord, nch-are about the same as further forward, but the mesoderm is much less advanced than further headward as is evidenced by the small amount of mesenchyma above the axial structures and by the slight differentiation of the mesothelium. The condition of the segments and their relations to the somatic and splanchnic mesoderm are closely similar to those represented in figure 46. Each somite consists of a larger part, Seg, of rounded outline, close to the medullary tube, and of a narrower part, the nephrotome, $N$, which connects the inner portion of the somite with the lateral mesoderm. The secondary somite consists of a distinctly marked wall which extends around underneath the ectoderm and against the side of the medullary tube, and of a thick inferior wall which fills up also the center of the somite. Between the nephrotome and the entoderm are small blood-vessels, $V$ e.

Section throngh the Postcrior Portion of the Open Intaine (Fig. 16r). - This 
section is similar to the last, but we may note especially the following differences: The spinal cord, Sp.c, shows a comparatively large cavity, which is widest on the dorsal side, so as to be somewhat triangular in section. In place of the segments we have only the mass of cells, S.z, which constitutes the segmental zone, out of which later segments will be differentiated. The segmental zone, S.z, is of a rather loose texture and merges without boundary into the somewhat denser mesenchyma of the somatopleure and splanchnopleure of the embryo. The dense tissue of the somatopleure extends much farther laterally than the corresponding tissue in the splanchnopleure. The notochord, $n \mathrm{ch}$, is very large and fills out the entire space between the ventral boundary of the spinal cord and the entoderm, and though the mesoderm comes in contact with the notochord, it does not surround it, the relations here representing an earlier stage of development than any

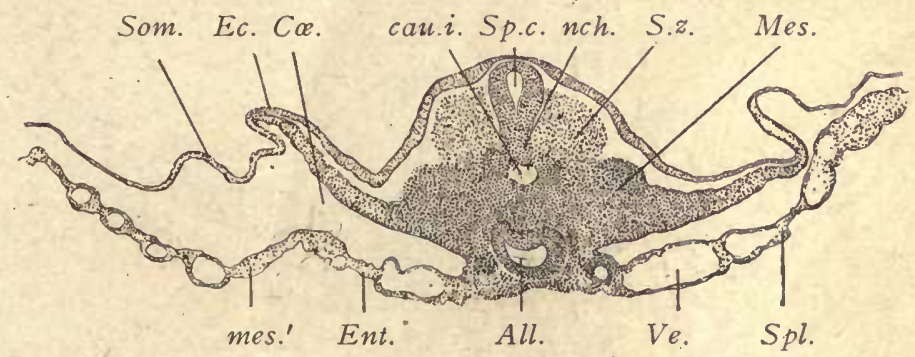

Fig. i62.-Section of a Chick Embryo with Twenty-eight Segments. Transverse Series 92, Section 424. All, Allantois. cau.i, Caudal intestine. Co, Cœelom. Ec, Ectoderm. Ent, Entoderm. Mes, Mesoderm. mes', Splanchnic leaf of mesoderm. nch, Notochord. Som, Somatopleure. Sp.c, Spinal cord. Spl, Splanchnopleure. S.z, Segmental zone of mesoderm. Ve, Blood-vessel. $\times 50$ diams.

which we find further headward. The entoderm, Ent, of the embryonic region is considerably thickened and forms an intestinal channel, In, of very characteristic form; for the top of this channel is nearly horizontal, while the sides are vertical and form a distinct angle with the top. In the midst of the mesoderm, on either side of the intestine, there is a small cavity, $C e^{\prime}$, which in two or three sections further forward is found to unite with the general cavity of the colom. The morphological meaning of this special pocket of the body-cavity is unknown.

From this point onward in the series changes in the appearance of the sections take place very rapidly. The two sections next to be described are quite close in the series to the present one.

Section through the Caudal Intestine (Fig. 162). - In this section we encounter the singular fusion of the germ-layers which is characteristic of the caudal extremity of all vertebrate embryos during early stages. In the median line we see three distinct cavities. The dorsal of these may be readily identified as the continuation of the cavity of the spinal cord. The middle and ventral cavities are entodermal; the upper of the two entodermal cavities, cau.i, represents a prolongation of the entodermal cavity into the developing tail of the embryo (compare Fig. I6). 
The lower cavity is the anlage of the allantois, $A l l$, which is destined to grow out during the next few days into a relatively large round vesicle. The tissue on the ventral side of the spinal-cord, Sp.c, is connected by a band of cells with the wall of the caudal intestine, cau.i. If the sections just in front are studied care-fully, it can be easily observed that the notochord also passes over without boundary into the same band of cells, which is a mass representing the fusion of the walls of the medullary canal of the intestine and of the tissue of the notochord. In this fused tissue we can, with our present means, detect no signs of the coming differentiation. Just as the walls of the caudal intestine are fused with the tissues on the dorsal side, so also are they fused on the ventral side with the tissue of the allantois. If we follow the tissues laterally, we see that they merge into the mesoderm proper. From the mesoderm there has been a distinct upgrowth of

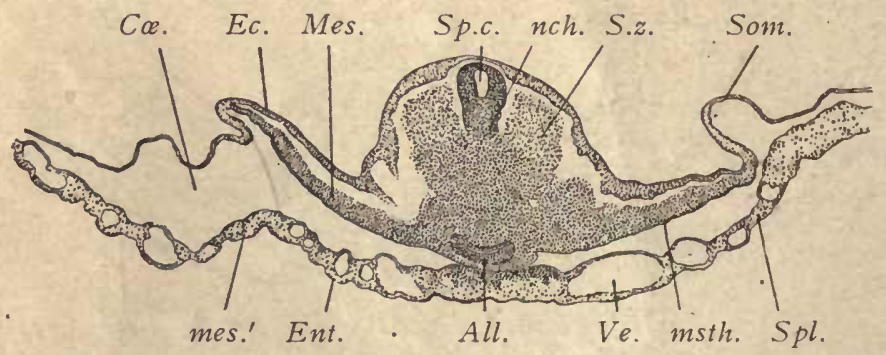

Fig. 163.-Section of a Chick Embryo with Twenty-eight Segments. Transverse Series 92, Section $42 \%$. All, Allantois. Ca, Cœlom. Ec, Ectoderm. Ent, Entoderm. Mes, Mesoderm. mes', Splanchnic mesoderm. $m s t h$, Mesothelium. nch, Notochord. Som, Somatopleure. Sp.c, Spinal cord. Spl, Splanchnopleure. $S . z$, Segmental zone of mesoderm. $V e$, Blood-vessel. $\times$ 5o diams.

tissue of rather loose texture on either side of the medullary canal to form the segmental zone, $S . z$.

Section through the Allantois behind the Intestine (Fig. I63).-This section is only three in the series beyond that last described, yet it is posterior to the caudal intestine and shows, therefore, more completely the fusion of the structures in the axial region. Except for the absence of the caudal intestine, the description of the last section might apply also to this. The shape of the spinal cord, Sp.c, is somewhat different, and its merging on the ventral side with the underlying tissues is more marked. The cavity of the allantois is smaller and almost slitlike. The other differences do not call for special description.

Horizontal Section (Fig. i64).- The student will find it profitable to make a series of sections in the horizontal plane, trying to cut them as nearly as possible parallel with the median plane of the fore-brain and mid-brain.

The accompanying figure $\mathrm{I} 64$ is from a section of such a series. It shows very clearly the general form of the embryo, the curvature of the neck, the sharp angle of the head-bend, and the almost straight body. In the section represented the long stretch of the cavity of the fourth ventricle or hind-brain, Ven.IV, is 


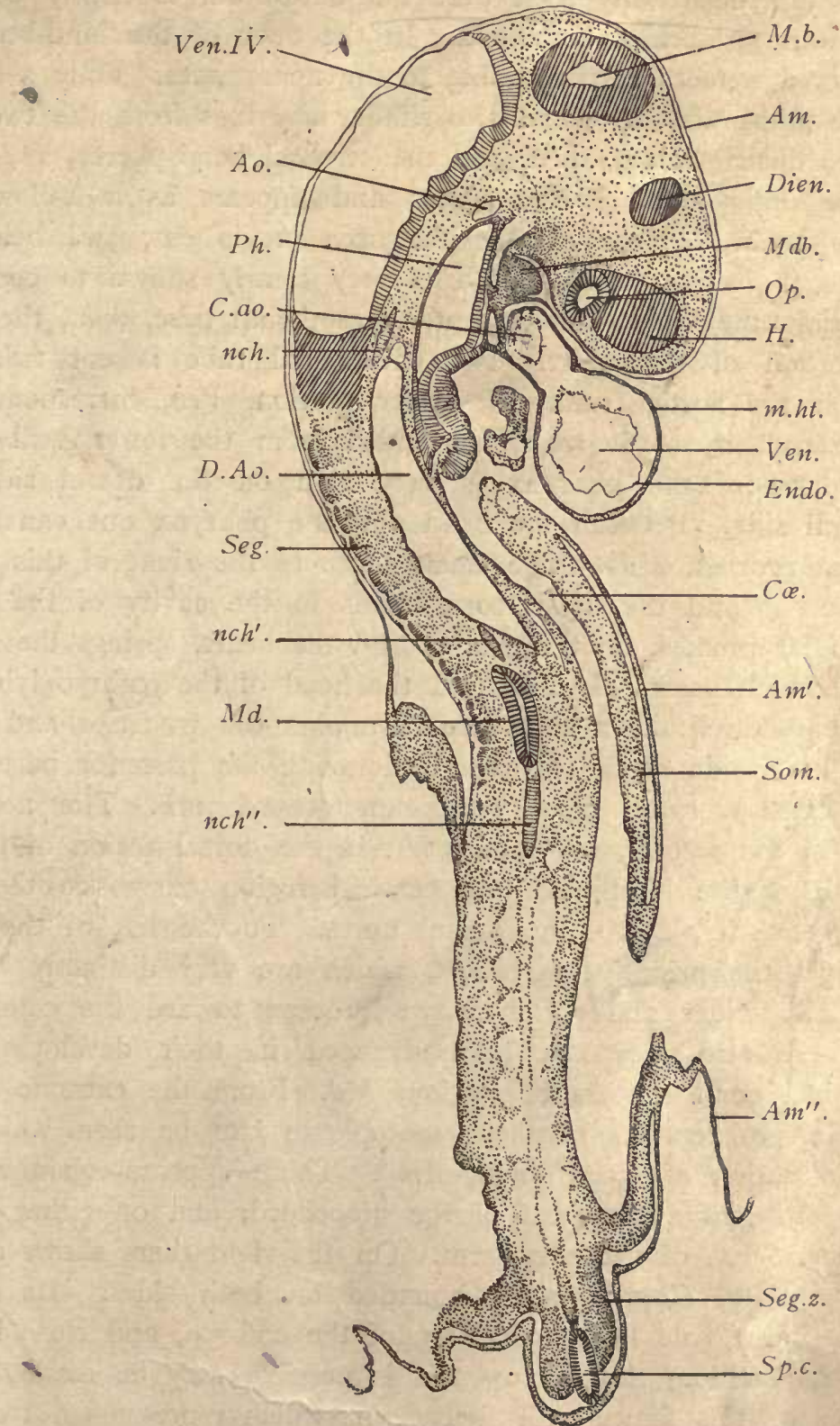

Fig. i64.-Horizontal Section of a Chick Embryo with about Twenty-eight Segaents.

$A m, A m^{\prime}, A m^{\prime \prime}$ Amnion. Ao, Aorta. C.ao, Cardiac aorta. C $\propto$, Colom. D.Ao, Dorsal aorta. Dien, Diencephalon. Endo, Endothelial heart. $H$, Cerebral hemisphere. M.b, Mid-brain. Md, Medullary tube. $M d b$, Mandible. $m . h t$, Muscular heart. $n c h, n c h^{\prime}, n c h^{\prime \prime}$, Notochord. $O p$, Optic resicle. Ph, Pharynx. Seg, Segment. Seg.z, Segmental zone of mesoderm. Som, Somatopleure. Sp.c, Spinal cord. I'cn, Yentricle. Ven. IV, Fourth ventricle or cavity of the hind-brain. $\times 30$ diams. 
well shown, and it can be readily seen that the hind-brain is nearly equal in length to the mid- and fore-brains combined. In the floor of the hind-brain appears a series of curved notches corresponding to the neuromeres. Only a shaving from the side of the mid-brain, $M . b$, and two similar shavings from the two parts of the fore-brain, the diencephalon, Dien, and the cerebral hemispheres, $H$, appear in the section. The optic nerve is cut across and appears as a hollow tube. Underneath the hind-brain a piece of the pharynx,.Ph, is cut, and below the pharynx is the large projecting heart, which is very clearly shown to consist of an inner or endothelial tube, Endo, and an outer mesothelial tube, $m . h t$, the anlage of the muscular portion of the heart. The endothelial tube is cut twice; the upper portion, $A o$, is the aortic trunk, the lower portion, Ven, corresponding to the ventricle. The heart is, as it were, suspended from the lower wall of the pharynx. The entoderm of the pharynx is very thin on the dorsal side, and thicker on the ventral side. Between the head and the pharynx one can see the projecting mandibular process, $M d b$. The small space to the right of this process in the figure, between it and the head, corresponds to the cavity of the mouth. Close to the mandibular process, on the side toward the heart, springs the amnion of the embryo, $A m$, which passes close around the head of the embryo lying very near it, and can be followed down to where it rejoins the posterior end of the embryo, on the left-hand side of the figure. Underneath the posterior part of the hind-brain can be seen a small piece of the notochord, $n c h$. The notochord appears twice more in the section, $n c h^{\prime}$ and $n c h^{\prime \prime}$, in the dorsal region of the embryo. From the end of the hind-brain the cervical region curves to the right. In it there is a large cavity, D.Ao, the dorsal aorta. To the left of the dorsal aorta we begin to get the primitive segments, which are very distinctly marked. They become gradually wider and wider as we proceed toward the caudal end of the embryo. There also they are less advanced in their development. A small bit of the spinal cord appears in section, $M d$. From the extreme inferior end of the section a prolongation of the somatopleure can be scen which also leads off into the formation of the amnion, $A m^{\prime \prime}$. There appears again a piece, Sp.c, of the spinal cord and a fragment of the notochord, and on either side of this a segmental zone, Seg.z, of the mesoderm. On the right there shows a small portion of the body-cavity, $C \propto$, distinctly bounded on both sides. Its exterior boundary is a piece of the true body-wall, Som, of the embryo, and close by it is another portion of the amnion, $A m^{\prime}$. How this is possible may be readily understood by comparison of this figure with figure $\mathrm{I} 6 \mathrm{I}$, which represents a transverse section of a similar embryo in this region.

\section{Histological Differentiation of the Chick Embryo with Three Gill-clefts.}

It is important that the student make a thorough examination and study with a high power of all the cells and tissues of the embryo at this stage so as to familiarize himself with the embryonic characteristics of the germ-layers. The 
cellular homogeneity of the embryo is strikingly evidenced by the nuclei, which in all parts of the embryo are very similar in size, shape, and structure. They are all rounded in form, varying between spherical and slightly oval outlines, which are seldom quite regular. The outline of the nucleus is always well marked, there being a superficial layer of nuclear substance, which gives a darker appearance to the edge of the nucleus. In the interior there is a single or sometimes two, very rarely three, nucleoli, which are quite large and stain deeply. The strands of substance between the nucleolus and the outer part of the nucleus are very slight, and the space around the nucleolus, therefore, appears light. The protoplasm of the cells is never large in amount, so that the cell-body about each nucleus is not conspicuous, except in the case of the blood-corpuscles, which are, in this respect, somewhat more advanced than the other cells of the embryo.

The ectoderm offers chiefly variations in its thickness, being very much attenuated in some parts, as, for instance, in the posterior portion of the head, where the outer ectoderm overlies the hind-brain. Most of the epidermal parts have begun to increase in thickness, and contain nuclei in two or even. three layers. There are several special thickenings of the epidermal layer, for which the name of plakodes has been proposed (compare page 76). At the present stage three pairs of plakodes are seen. The first is the pair of areas which are to be invaginated to form the olfactory pits; the second is the pair which are already invaginated to form the anlages of the lenses of the eyes, and the third pair is also invaginated to form the otocysts. The portion of the ectoderm which forms the medullary tube is also very much thickened, except, of course, so far as the floorplate and deck-plate have been differentiated. In both the plakodes and in the thickened portions of the medullary wall the nuclei occupy nearly the whole thickness of the layer, being themselves several layers deep. They are, however, partially absent from that portion of the ectoderm which is near the original external or free surface. Close to this surface there are, however, a certain number of nuclei, the large majority of which are in various phases of division, as shown by the numerous mitotic figures. No mitoses appear, except in the superficial portion of the layer. Over the greater part of the amnion the ectoderm is so very thin as to resemble almost an adult endothelium, but over the chorion or serous membrane it is a little thicker.

The entoderm appears in three distinct forms: first, the large, long, columnar cells of the area opaca; second, the very thin cells of the area pellucida; and, third, the somewhat thicker cell-layer in the embryo proper. For an account of the cells of the area opaca and area pellucida see page 64. The entoderm in the embryo presents considerable variations in thickness which have been pointed out in the descriptions of the sections. Where it is thick enough to permit it, the nuclei are disposed in several layers, and in such places we find that the nuclear divisions take place only in the superficial portion of the entoderm, the phenomenon here being similar to that which we have already noted in the ectoderm. The 
notochord has a sharply defined outline, as if bounded by a distinct membrane. It contains nuclei which are quite closely placed, but it does not show, at least in ordinary preparations, any recognizable division into separate cells.

The mesoderm offers several varieties, not so much in the character of the single cells as in their methods of grouping. We notice, first, that there are parts of the mesoderm which are quite thick, and in which we cannot perceive any division into mesothelium and mesenchyma. Such a thick layer of mesoderm may be observed at either side of the pharynx (Figs. 156, Ph, and 157), or, again, toward the caudal end of the embryo in both the somatopleure and splanchnopleure, occupying a larger territory in the former than in the latter (Fig. I63). But for the most part the mesoderm has progressed beyond this stage and shows clearly the differentiation of a thin mesothelial layer lining the cœlom and the scattered mesenchymal cells. The mesothelium is quite thin in some parts, almost or quite as thin as adult endothelium. The mesenchyma consists of cells with small protoplasmic bodies connected together by fine threads of protoplasm and with a transparent homogeneous matrix between the cells. It varies greatly in appearance according as the cells are more or less closely crowded together, or widely separated from one another. These differences we designate as varying degrees of condensation in the mesenchyma. The variations occur in a perfectly definite and constant manner, though we are far from understanding yet either the cause or the morphological significance of these variations. The secondary somites vary greatly in structure, because they are in unlike stages of differentiation, those toward the tail being least, and those in the cervical region most, advanced. We can, therefore, in a single embryo observe several phases of the breaking-up of the inner wall of the somite to form mesenchyma about the medullary tube and notochord. The transformation is accomplished by a spreading out and moving asunder of the cells, and we can also trace a gradual differentiation of the muscle-plate, out of the inner portion of the somite. The external layer, or so-called cutis-plate, offers an apparently more or less epithelioid structure in all of the somites. The Wolffian duct is differentiated only through a part of the embryo. It is - a small cord of cells that has as yet no central cavity. The blood-vessels are formed solely by the endothelium (angioblast). There is nowhere any condensation of the mesenchyma about the blood-vessels as yet. There are no capillaries whatever in the embryo. One of the most important vascular modifications has, however, been initiated in the anlage of the liver, where we find the vascular endothelium coming into close contact with the entodermal cells of the liver, preparatory to the later complete differentiation of the hepatic sinusoids. The blood-corpuscles are round in form with fairly distinct outlines. Their protoplasmic bodies are much larger than those of any other cells of the embryo at this stage, but their nuclei resemble in size and structure those of other tissues. 


\section{CHAPTER VI. \\ STUDY OF PIG EMBRYOS.}

\section{Method of Obtaining Embryos.}

The pig is recommended for embryological study because specimens of the embryos in sufficiently early stages can be obtained at the larger packing establishments in considerable numbers and with little trouble or expense. When this material is not obtainable, rabbit embryos may be substituted, as these animals are easily kept and breed freely (compare page i66). The enormous precocious development of the chorionic vesicle in pigs produces an enlargement of the uterus which is usually sufficient, by the time the embryo has attained a length of $6 \mathrm{~mm}$., to be observable to the untrained eye. It is, therefore, only necessary to ask the man who removes the viscera from the pigs to lay aside for examination all of the uteri which appear distended. These should not be turned about violently, but handled carefully and should be opened immediately. As soon as the ovum is exposed it will probably be ruptured, and there will occur a free outflow of opalescent fluid, amniotic and allantoic. With the aid of fine forceps and a horn spoon the embryo may be lifted up-and it should on no account be directly touched-and transferred to a dish containing Müller's fluid, in which the specimen should remain for five or ten minutes. It is then transferred with the help of the horn spoon to Zenker's fluid. Metal instruments cannot be used on account of the corrosive sublimate in the Zenker solution. In one or two hours the embryo should be transferred to fresh Zenker solution and left therein a varying length of time, according to the size of the specimen. In general it. may be said for-

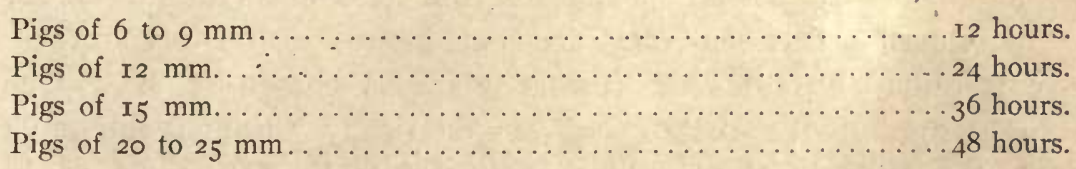

It is undesirable to leave any specimen in the Zenker solution more than forty-eight hours. The Müller's fluid is used for cleaning the specimen. It causes a granular, non-adherent coagulum to form from the fetal fluids. If the specimen is put directly into Zenker's fluid, a fibrous coagulum is formed which often adheres closely to the embryo so as to obscure its shape. Such a fibrous co- 
agulum cannot be removed without injuring the embryo. After having remained a proper length of time in the Zenker solution, specimens are further washed for twenty-four hours in running water, and then treated with alcohol and iodine in the usual manner.

\section{The Making of Serial Sections.}

Specimens should be colored with alum cochineal in toto, then imbedded in paraffin and cut into serial sections according to the directions given in Chapter VIII. It is advantageous to apply a counterstain-orange $\mathrm{G}$ is recommended.

\section{Selection of the Planes of Section and the Stages for Practical Study.}

It is customary to distinguish three fundamental planes-the transverse, the sagittal, and the frontal. It is impossible to so define these planes that the definition shall be exact for all stages. But in general it may be said, reference being had to the entire embryo, that the transverse plane is one which will be at right angles to the notochord and medullary tube at the level of the heart; that the frontal plane will be one. at right angles to this, passing symmetrically through the limbs of the embryo; and, finally, that the sagittal plane is one parallel to the median plane of the body. As in younger embryos the form is very asymmetrical, both the head and caudal end of the embryo being twisted to one side, the planes which would be true for the body of the embryo in the region of the heart would not be true elsewhere. For the practical use of the student, therefore, in these younger stages it is better to determine the direction of the plane by the floor of the fourth ventricle, so that by "transverse" will be understood a plane of section which cuts the head of the embryo symmetrically, no matter how it may cut the body, and which runs parallel to the floor of the fourth ventricle (medulla oblongata). The frontal plane should be perpendicular to this and also cut the head of the embryo symmetrically. The sagittal plane in these cases is also that of the head and not of the body. Such planes are recommended because in the study of the sections more is gained by having the planes readily understood in the region of the head than in the region of the body. In later stages, when the body has become straighter, the difference in planes for the head and the body may be practically left out of consideration, except that for the heads of older pigs when they are cut alone-as on account of the size of the body is often desirable - the frontal plane is chosen so as to run at right angles to the plane of the palate and symmetrically through the embryo. Sections through the head at right angles to this may be designated as horizontal.* Students will find that it is very much easier to study transverse and frontal sections when they are symmetrical. No pains, therefore, should be spared to orient the embryo properly in the microtome before the sections are cut.

* The system of planes here described is that adopted for the Harvard Embryological Collection, and has been found convenient in practice. 
Selection of the Stages. - The most profitable stage to study is that of an embryo of from II to I3 $\mathrm{mm}$. in length. Each student should have three specimens of this stage, and it is advantageous that the specimens given each student be approximately of the same size. The embryos ought to be first studied carefully as to their external form and then cut into serial sections in the transverse, sagittal, and frontal planes. Of these, the transverse series forms the principal basis of study, and the other series are to be used principally to clear up the student's conception of the relation of parts. Embryo pigs of the size specified have the typical class characteristics of mammalian embryos, and may readily be distinguished from the embryos of any other class of vertebrates. The differentiation of the anlages of all the important organs is accomplished, so that these anlages can be identified with certainty and their genetic relations to the adult structures can be clearly grasped by the student. At the same time, although the anatomical differentiation is well advanced, the histological differentiation has made very little progress, hence the embryos in question are particularly instructive to beginners. The anatomy of the pig at this stage is, therefore, readily understood by the student who knows the general anatomy of the adult. Older embryos are more complicated and yield such long series of sections that the beginner is apt to be discouraged. Younger embryos, owing to their spiral twisting, are exceedingly difficult for students to understand when sectioned. After having thoroughly mastered the structure of the pig embryo of from in to $\mathrm{r}_{3} \mathrm{~mm}$., the student may advantageously extend his study of embryos to other sizes. If, as is done in this work, the principal study is made with embryos of $\mathrm{I} 2 \mathrm{~mm}$., the student may proceed to make sections of other stages as follows:

Pig embryo of $6 \mathrm{~mm}$., transverse.

Pig embryc of $9 \mathrm{~mm}$., transverse and sagittal series.

Pig embryo of $\mathrm{I} 7 \mathrm{~mm}$., transverse series.

Pig embryo of $20 \mathrm{~mm}$., transverse and sagittal series.

(Of the head alone, the frontal series.)

Pig embryo of $24 \mathrm{~mm}$, of the head alone, frontal series.

\section{The Study of the External Form.}

The student should make a careful and thorough study of the external form of every embryo, and make, with the aid of the camera lucida, an exact drawing of every embryo before he cuts it into sections. He will soon learn that such a drawing is almost indispensable for the interpretation of the sections.

In the following paragraphs, embryos of $7.5,10, \mathrm{I}_{5}$, and $20 \mathrm{~mm}$. are figured and described from specimens which have been hardened in Zenker's fluid and preserved in alcohol. The description of these stages will be sufficient to enable the student to understand any of the embryos he is required to study.

Pig Embryo of $7: 5 \mathrm{~mm}$. (Fig. 165).-The student may be helped in the identification of parts by comparison with figure I66, which has explanatory lettering. 
The length of the embryo measured in a vertical line as the embryo is placed in the figure is $7.5 \mathrm{~mm}$., but its greatest length in any direction is $8.0 \mathrm{~mm}$.

The head is somewhat triangular in form, being broadest toward the front (the left in the figure) and narrowing posteriorly to join the rest of the body. The upper boundary of the head is a nearly straight line, the extent of which marks approximately the territory of the hind-brain.

Toward the left the outline forms a rounded curve which marks the territory of the mid-brain, and then continues obliquely downward in a straighter course until it curves over on to the under side where it forms three notches. The first

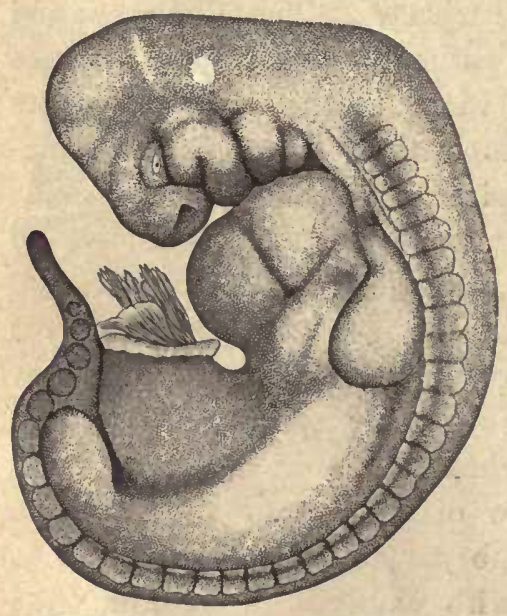

Fig. 165.-Pig EMbryo of 7.5 MM. $\times 8$ diams. notch indicates the position of the mouth, the second marks the boundary between the first and second branchial arches, the third the boundary between the second and third arches. On the tip of the head, just in front of the mouth, is a shallow depression, the anlage of the nasal pit, and above is the small eye. From the eye to the mouth runs a shallow furrow, the lachrymal groove. The first branchial arch is called the mandibular; it is broad and separated by a furrow from the second. Between it and the eye lies the maxillary process: The second branchial arch is termed the hyoid. The third is smaller and somewhat drawn inward, while the fourth and fifth have sunken so far as to produce a deep pit with a triangular outline, which has been named the cervical sinus.

The body has a long curving dorsal outline terminating in the recurved tail. Near this outline thirty-seven segments show externally, because each one creates a protuberance of the ectoderm. The least developed segments are in the tail. From there toward the head they show a progressive advance in the stage of development attained. The two limbs are rounded buds, the anterior being the larger, and offer no trace of their future articulation. Between them stretches a long protuberance, which is due to the Wolffian body or mesonephros, the precocious development of which is characteristic of ungulates. In man at a corresponding stage the mesonephros is relatively less voluminous. Immediately ventrad from the fore-limb the two lobes of the liver can be discerned through the translucent bodywalls. Between the liver and the head is the very large heart. The division between its auricle above and its ventricle below can be seen clearly. The abdominal region of the body is prolonged outward between the tail and the heart, and so forms the commencement of the umbilical cord, the end of which is marked by a thin membrane, the amnion, which has been almost completely removed. In life the amnion forms a closed sac around the embryo, and is distended by the amniotic fluid. From the end of the umbilical cord project remnants of the yolk- 
sac, and the allantois, both of which pass through the cord to join internal structures of the embryo.

Pig Embryo of $10 \mathrm{~mm}$. (Fig. I66). - The form of the embryo has undergone notable changes, as comparison with figure 165 will show. The head is larger, the expansion in the regions of the mid- and fore-brains being particularly noticeable. The limb-buds have lengthened, as has also the umbilical cord. The third branchial arch has disappeared from the surface into the cervical sinus. The head as a whole lies nearly at right angles with the back, so that the dorsal outline of the

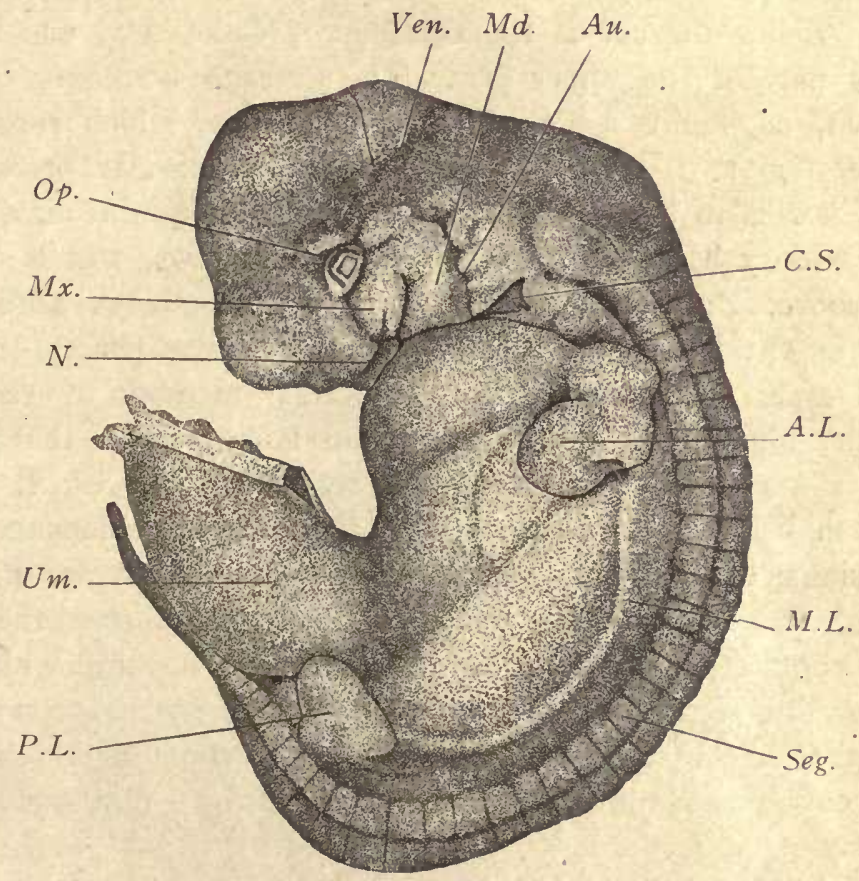

Fig. I66.-PIg Evibryo of Io Mu.

A.L, Anterior limb. Au, Auditory, or first gill-cleft. C.S, Cervical sinus. Md, Mandibular process. M.L, Milk-line. $M x$, Maxillary process. $N$, Nasal pit. Op, Eye. P.L, Posterior limb. Seg, Muscular segment. Um, Umbilical cord. Ven, Floor of fourth ventricle (medulla oblongata). $\times 8$ diams.

head forms a distinct though rounded angle with that of the back. This angle marks the position of the neck-bend, and also the junction of the brain with the spinal cord. The very distinct neck-bend is characteristic of the mammalian embryo. It is less evident in birds and reptiles; absent in amphibians and fishes. Its development probably causes the cramping of the ventral cervical region, which leads to the formation of the cervical sinus, C.S, and to the disappearance from the surface of the second, third, and fourth gill-clefts. Another consequence of the neck-bend is the approximation of the nasal regions, $N$, of the head to the cardiac region of the body. The cephalic region has a second flexure, the head-bend 
proper, which occurs at the level of the mid-brain, the nature and significance of which become clearer when the disposition of the nervous system is studied (compare Fig. 178). From the mid-brain one axis extends backward through the region of the hind-brain, Ven, to the neck-bend; the other axis extends vertically downward to the region of the fore-brain. On the surface of the head we find the nasal pit, $N$, distinctly marked. The eye, $O p$, shows clearly the outlines of the optic vesicle and of the lens in the center. It is entirely without lids. The small size of the eye is a characteristic of the mammalian embryo by which it differs from all sauropsidian forms; but, as previously stated, the embryonic eye is slightly larger in certain other mammals. Below the eye is the maxillary process, $M x$, which is destined to form the greater part of the upper jaw. The anterior boundary of the maxillary process is marked, as before, by the lachrymal groove, which runs now from the angle of the eye, $O p$, to the nasal pit, $N$. The mandibular process, $M d$, out of which the lower jaw is to be developed, is separated from the maxillary process by a groove, the boundary between the upper and lower jaws, and is bounded . behind by a second groove, $A u$, the anlage of the future meatus auditorius externus. This groove marks the boundary between the mandibular process and the first, or hyoid, branchial arch, and is itself the ectodermal member of the first gill-cleft. The cavity of the hind-brain is very large and is known as the fourth ventricle, Ven; as it has a very thin roof it can be readily distinguished. The thickened floor of the fourth ventricle is the anlage of the medulla oblongata. The opening of the cervical sinus, C.S., is triangular, as before; within it are hidden the third, fourth, and fifth branchial arches. In slightly older embryos the orifice of the sinus is further contracted, becoming a small rounded opening which finally closes over completely. The territory of the mandibular process and cervical sinus corresponds to the pharyngeal region. It is the site of some of the most important, interesting, and complicated developments by which the embryonic is changed into the adult anatomy.

The dorsal outline of the body forms a long sweeping curve, ending in the tail. Comparison with figure ${ }^{6} 65$ shows at once that the straightening out of the dorsal region is begun, yet at this stage the dorsal side of the embryo is nearly three times as long as the ventral. The umbilical cord has grown in length, and is constricted in diameter as it joins the abdomen, yet its connection with the body occupies practically the entire length of the ventral median line. The position and number of the segments, Seg, is still shown by the external modeling. Both limbs are well advanced, the anterior, A.L., more so than the posterior. From the base of the brain to the base of the hind limb extends the milk-line, M.L, curving so as to be nearly parallel to the dorsal outline of the body. Along it the mammary glands are ultimately developed. Extending across the body are several shadowy lines shimmering through the translucent body-walls. One marks the position of the embryonic diaphragm; it extends from the upper edge of the anterior limb obliquely downward toward the edge of the umbilical cord. Another, which extends 
in a nearly straight line from limb to limb, marks the ventral edge of the large Wolffian body, or mesonephros, the dorsal limit of which is approximately indicated by the milk-line, M.L. The outlines of the smaller left dorsal lobe of the liver are distinct, and map out a pointed area immediately below the fore limb, A.L. Above the diaphragm lies the protuberant cardiac region, the outlines of which pass from the umbilical cord below the nasal and pharyngeal regions toward the cervical sinus. The long tapering tail extends alongside the umbilical cord.

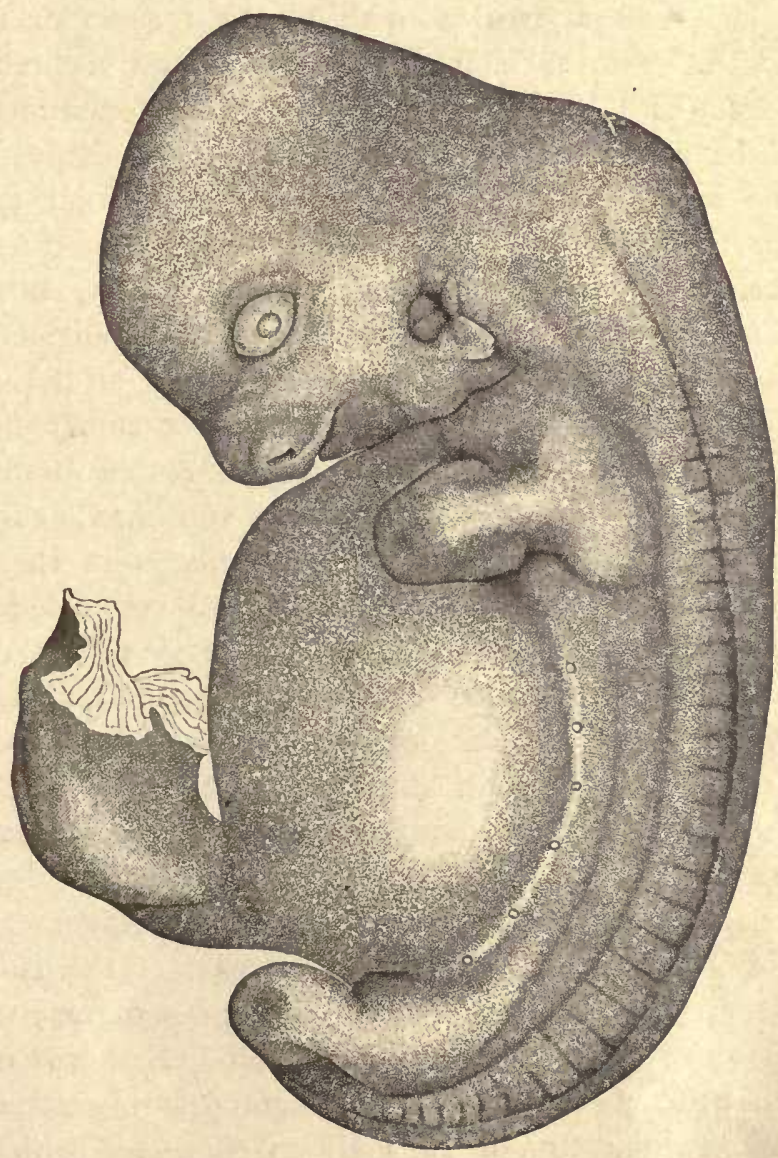

Fig. 167 - Pig EMbryo of 15 MM. $\times 8$ diams.

Pig Embryo of $15 \mathrm{~mm}$. (Fig. I67).-As compared with figure I66, the present embryo (Fig. I67) has not only grown in all its dimensions, but has also changed in form. Unlike the embryo proper, the umbilical cord has grown very little. We notice at once that the outline of the back is less curved than before, that the ventral side of the body has acquired a convex outline, and that the head has become considerably larger, both absolutely and relatively to the body of the embryo, and 
has, moreover, risen so that the neck-bend is diminished. The limbs are beginning to show the differentiation of the feet. Examined more carefully, the embryo offers the following details: the eye, which is characteristically small, has become almondshaped, and the circular lens can be seen in the midst of it. In the embryos of rodents, carnivores, and primates the eye is relatively larger than in the pig. By the growth of the facial region the development of the snout has been initiated, and the opening of the nasal pit now appears as the external nares toward the end of the short snout. The lower jaw is clearly differentiated and the slit of the mouth is distinct. There has been a great growth of the regions of the fore-brain and mid-brain, and it is this growth chiefly which has caused the relative expansion of the head as compared with the rest of the body. The auditory groove now appears distinctly as the anlage of the external meatus of the ear, behind which a protuberance can be seen which is the anlage of the external concha of the ear. The cervical sinus has wholly disappeared. Along the line of the back the primitive segments are scarcely recognizable in the cervical region, but near the upper limb they still show distinctly and from there are indicated with increasing clearness as we pass toward the lower limb. The marks of the segmental divisions do not extend so far on the dorsal side as in the earlier embryos, but are restricted to what may be called the segmental ridge. Along the milk-line a series of small, white, circular spots can be seen. In the specimen figured there were six of these; their number is variable. They are the anlages of the mammary glands, and are at this stage merely local thickenings of the ectoderm or epidermis. There has been a considerable growth of the dorsal region of the body, and this is perhaps most clearly indicated by the position of the milk-line, which is much farther away from the median dorsal line than in the $10 \mathrm{~mm}$. pig. Both limbs are paddle-shaped, and, though still very short, have a broad terminal expansion, which is the anlage of the foot. The front foot has somewhat the outline of a truncated pyramid, while the hind foot is more rounded. In the anterior limb the differentiation into upper and lower divisions is suggested.

Pig Embryo of $20 \mathrm{~mm}$. (Fig. 168).-Comparison of this stage with figure ${ }^{6} 67$ reveals a general progress, but no such striking changes of external form as distinguished the embryo of $15 \mathrm{~mm}$. from that of $10 \mathrm{~mm}$. Embryos of this length vary considerably in their proportions, but the one figured is characteristic of the stage. The enormous transverse diameter of the body as compared with its length is very striking, and the very large size of the head in proportion to the body is almost equally remarkable. In the head the growth of the regions of the fore-brain and mid-brain has continued, and the divisions between the mid-brain and hind-brain are marked by concavities in the outline of the head. The eye is both absolutely and relatively larger. Above it can be distinguished readily the anlages of the great bristles which develop over the eye, corresponding to the human eyebrow. These anlages appear as whitish spots, for they are thickenings of the ectoderm. The snout has increased in length; the external ear has grown longer and has begun to 


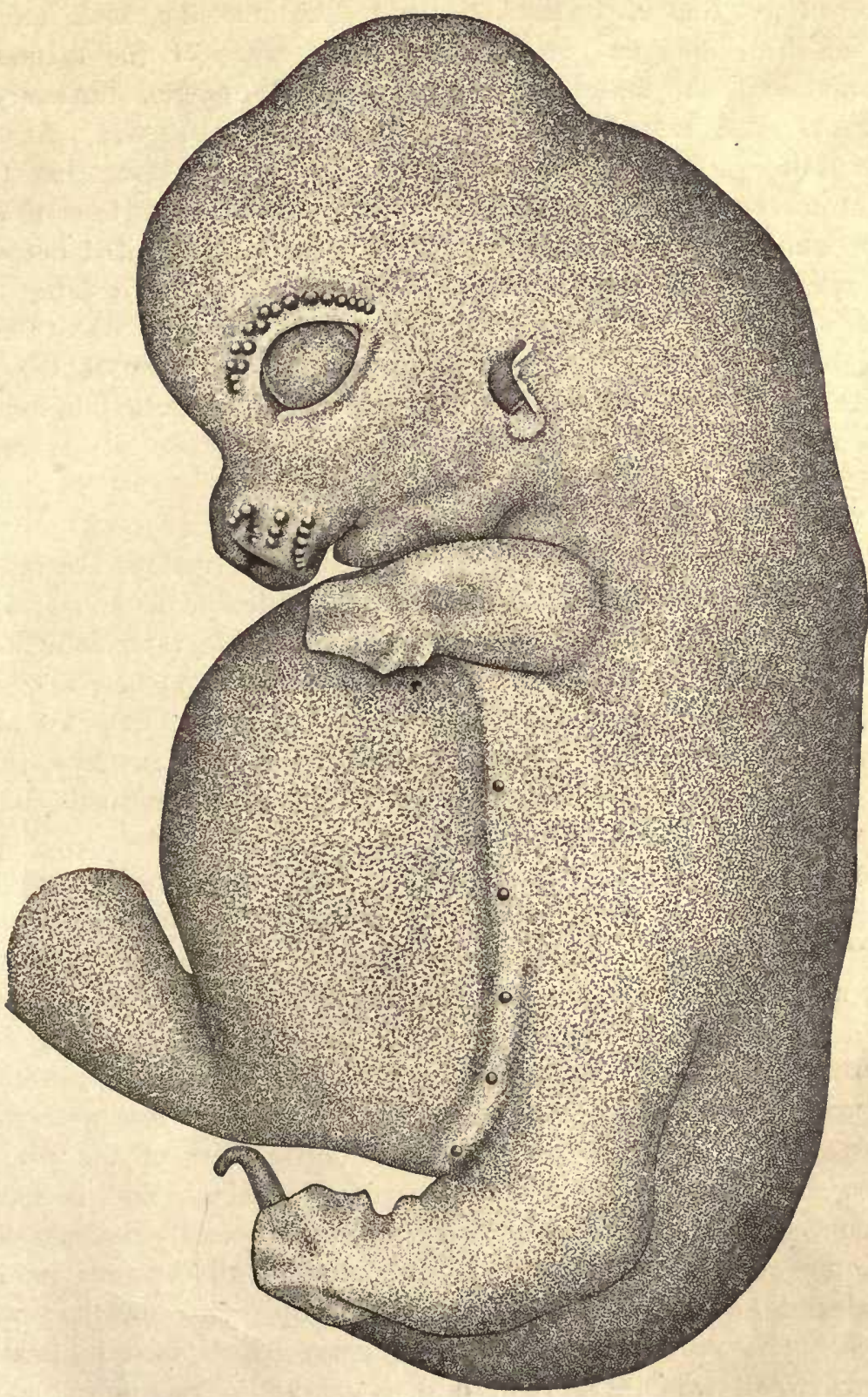

Fig. I68.-Pig EMbryo or 20 mMr. $\times 8$ diams. 
assume its permanent pointed form. The limbs have increased considerably in length, but not yet enough to project beyond the abdomen. In both feet the differentiation of five toes is clearly indicated. The milk-line, as a line, has almost completely vanished, but the row of dots, the anlages of the mammary glands, which develop along the milk-line, persists ${ }^{*}$ and will undergo further development in later stages. The number in the specimen figured is five. As the row of anlages marks the position of the milk-line, it is readily seen that the line has migrated ventralward as compared with earlier stages. Comparison of the embryos of 15 and $20 \mathrm{~mm}$. demonstrates that, during the period comprised between the two stages, the growth of the dorsal part of the embryo is far greater than of the ventral part. Comparison of figures 167 and 168 shows at once that the area occupied in both figures by the region on the ventral side of the milk-line is about the same. In the pig of $20 \mathrm{~mm}$. there is no indication of the segmental structures recognizable in the surface modeling.

\section{Pig Embryo of $7.8 \mathrm{~mm}$. General Anatomy.}

Anatomical Reconstructions from the Sections.-Rerconstructions are of the greatest assistance in the study of sections, and much facilitate the identification of all the parts. Students using this book should, while examining their sections, constantly refer to the reconstructions. It is unnecessary to give elaborate descriptions of each of them, since the explanations of the lettering of the figures will suffice for the identification of all the parts shown. Certain brief explanations as to each of the figures are, however, desirable. The chief value of reconstructions is to render clear the topographical distribution of the organs.

The reconstruction* presented in figure 169 shows the general topography of the embryo, and illustrates chiefly the digestive, vascular, and central nervous systems. The digestive tract is represented by the outline of its epithelial portion only. In the actual specimen the walls are thicker, because they include the mesenchyma immediately surrounding the epithelium. The mouth is an opening between the head and mandible, $M d$, and leads directly into the pharynx, $P h$. From the dorsal side of the mouth springs the hypophysis, $H y$, which lies close against the wall of the fore-brain and is destined to form the anterior lobe of the pituitary body. The pharynx, $P h$, is narrow in its dorso-ventral diameter, and is represented in median section. From its dorsal surface arises the small conical diverticulum, S.P, situated near the hypophysis. If followed backward, the pharynx is found to bend tailward and to form two median branches, the more dorsal of which is the œsophagus, $E$. The ventral branch is the trachea which soon bifurcates to form. the main bronchi, of which the right only is shown in the figure, at Bro. The lateral gill-pouches are not shown in this figure. The œsophagus has lengthened, and leads to the stomach, St, which has already descended and has so revolved

* Through the kindness of Dr. Thyng, it is possible to use this illustration in advance of its publication by the author, whose paper on the anatomy of the $7.8 \mathrm{~mm}$. pig is soon to appear in full. 


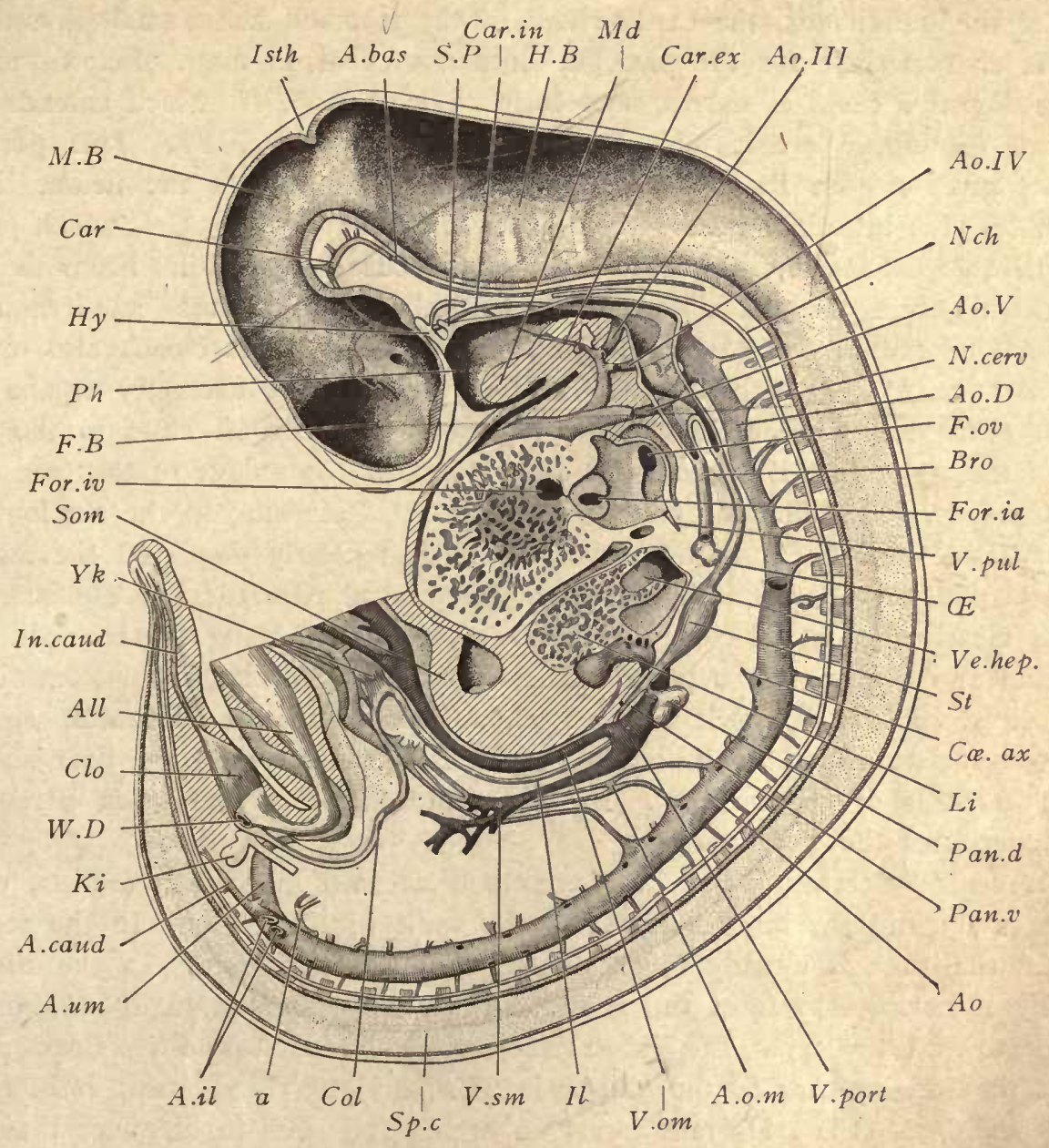

Fig. 169.-Pig Enbryo of 7.8 Mm. Transverse Series 1358 . Reconstruction by Frederick W. Thyng. a, Artery. A.bas, Arteria basilaris. A.caud, Caudal artery. A.il, Double openings of left common iliac, artery into the aorta. All, Allantois. Ao, Main aorta. Ao.D, Descending aorta. Ao.III, Right third aortic arch. Ao.IV, Right fourth aortic arch. Ao.V, Right fifth aortic arch. A.o.m, Omphalo-mesaraic artery. A.um, Umbilical artery. Bro, Bronchus. Car, Carotid loop. Car.ex, External carotid anlage. Car.in, Internal carotid. Co.ax, Coliac axis. Clo, Cloaca. Col, Colon, entodermal part. F.B, Fore-brain. For.ia, Interatrial foramen. For .iv, Foramen interventriculare. F.ov, Foramen ovale. H.B, Hind-brain. $H y$, Hypophysis. In.caud, Caudal intestine. Il, Small intestine, entodermal part. Isth, Isthmus. $K i$, Kidney. $L i$, Liver. M.B, Mid-brain. Md, Mandible. Nch, Notochord. N.cerv, Cervical nerve. $\Subset$, Esophagus. Pan.d, Dorsal pancreas. Pan.v, Ventral pancreas. (The line does not quite reach the organ, which lies ventrad from the intestine.) $P h$, Pharynx. Som, Somatopleure. S.P, Seessel's pocket. Sp.c, Spinal cord. St, Stomach. Ve.hep, Hepatic vein. V.om, Omphalo-mesaraic vein. V.port, Portal vein. V.pul, Pulmonary vein. V.s.m, Superior mesenteric vein. W.D, Wolffian duct. Yk, Yolk-sac. $X$ I4 diams. 
on its own axis that its dorsal border nearly faces the left. Just below the entrance of the œesophagus, the cardiac end of the stomach has a small diverticulum, which is characteristic for the pig, but does not occur in man. Below the stomach the digestive canal is narrow and forms a long loop, $I l$, which extends to the umbilical opening, where it joins the neck of the yolk-sac, $Y k$. This portion of the tract gives rise to the duodenum, jejunum, and most of the ileum. Beyond the yolk-sac the intestinal tract is continued as a narrow tube, Col, which leads to a considerable expansion, the cloaca, $\mathrm{Clo}$, at the base of the tail. From the cloaca there extends into the tail a very narrow prolongation of the entodermal canal known as the tail-gut, or caudal intestine, In.caud. Into the cloaca also open the Wolffian ducts, W.D, which are the ducts of the primitive excretory organs of the embryo. The duct on the left side is represented as cut off close to the cloaca. More of the right duct is included in order to show the anlage of the true or permanent kidney, $K i$, which is budding off from it. Returning to the portion of the intestine (duodenum) next to the stomach, we find clearly displayed the anlage of the dorsal pancreas, Pan.d. The anlage of the ventral pancreas also appears, Pan.v, but less clearly. It is an elongated mass of entoderm, lying to the right of the duodenum and ventral to the portal vein, V.port. It takes its origin from the duct of the liver. The liver itself, $L i$, has already acquired a considerable size. On its ventral surface lies the gall-bladder, which is connected with the liver substance by several cords of hepatic cells. In the adult, only one connection between the gall-bladder and the liver persists.

The central nervous system is represented as seen in median section, displaying the cavity and the inner surfaces of the walls of the cavity. In the region of the head the tube is already much dilated to make the brain. In the region of the body it narrows to form the spinal cord, Sp.c, which gradually diminishes in diameter toward the tail. The brain is clearly subdivided into its three primary vesicles: the fore-brain, F.B; the mid-brain $M . B$; and the hind-brain, H.B. Owing to the head-bend, the mid-brain forms an arch. It is less in diameter than either mid-brain or hind-brain. The demarcation between the mid-brain and hind-brain, known as the isthmus, Isth, is strongly marked. The hind-brain, H.B, is longer than the mid- and fore-brain combined. It diminishes in diameter posteriorly, and passes without definite demarcation into the spinal cord. Owing to the head-bend, the fore-brain, $F . B$, is brought to underlie, as it were, the anterior portion of the hind-brain, and to overlie the heart. The heart is a very large organ, which is represented in the figure somewhat to the left of the median line. It consists of a smaller dorsal or upper chamber-the atrium or auricle, and a lower larger chamber-the ventricle. The auricle shows two openings by which it communicates with the right auricle. The upper of these, F.ov, is the foramen ovale; the lower is the so-called interatrial foramen, For.ia. The small pulmonary vein, V.pul, opens directly into the auricle. The ventricle has a trabecular or sinusoidal structure. The cavity in the drawing is that of the left side. It communicates with the cavity 
of the right ventricle by means of the interventricular foramen, For.iv. Above the ventricle is seen the main trunk of the aorta, which divides and gives rise to three aortic arches, Ao.III, Ao.IV, Ao.V. The first and second arches are still present, but are very slender. Their common stem, Car.ex, forms the future stem of the external carotid. From the dorsal end of the third arch, Ao.III, there runs forward the vessel, Car.in, which joins the first and second arches, and itself becomes part of the stem of the internal carotid. The aortic arches form on each side of the descending aorta, Ao.D, which unites with its fellow just above the level of the stomach to form the main median dorsal aorta, Ao. The aorta gives off a series of intersegmental vessels which mount dorsalward between the spinal cord and the outer surface of the embryo. The main aorta gives off on its ventral side a vessel, Co.ax, which becomes the coeliac axis of the adult; and lower down, three vessels, A.o.m, which anastomose in several places. In their.course to the yolk-sac they cross to the right of the intestine and give off branches to the mesentery. The two upper roots atrophy later, but the lower persists to form the stem of the superior mesenteric artery. Just before reaching the cloaca the aorta gives off vessels, $A . i l$, which run to the hind leg, and develop into the external iliac of the adult. Just beyond, the aorta gives off a small branch, A.caud, to the tail; and at the same time bifurcates to form the umbilical arteries, A.um, which take a sinuous course alongside the allantois, $A l l$, and ultimately ramify outside of the body of the embryo in the walls of the allantois. The allantois itself springs from the cloaca as a narrow canal which makes a sharp bend and runs to the umbilicus, gradually expanding. Outside of the embryo, the allantois forms; in the pig-as in all ungulates - a very large vesicle. In man and the primates, on the contrary, the allantois is rudimentary. This is one of the most striking differences between the pig and the human embryo.

Of the veins little is shown in this figure, but the omphalo-mesaraic and portal veins are included. The former, V.om, arises on the surface of the yolk-sac, passes through the umbilicus into the mesentery of the intestine, and extends parallel with the ileum until it crosses it, a little posterior to the pancreas. It is there joined by another vein, V.s.m. the superior mesenteric, which is of about the same caliber. The common trunk formed by the union of these two veins is the portal vein, V.port, which divides into two branches, extending to the right and left of the omphalo-mesaraic vessels. The left branch is small. It passes on the left of the dorsal pancreas and extends to the liver. The right branch passes between the two pancreatic anlages, then bends to the right and enters the liver, into the sinusoids of which it discharges its blood-stream.

\section{Pig Embryo of $12.0 \mathrm{~mm}$. General Anatomy.}

Dissection of the Viscera (Fig. I70). -The specimen figured measured 12.7 $\mathrm{mm}$. Both limbs and the body-wall on the left side have been removed, displaying the organs in situ. The umbilical cord has been cut lengthwise so as to display its 
internal structure. The body proper is divided by the diaphragm, Dia, into an upper smaller pericardial chamber and a lower larger abdominal chamber. The diaphragm is a thin membrane, which extends from the level of the base of the fore leg, F.L, to the ventral wall of the body. The body seems filled chiefly by three large organs: the heart, $A u, V e n$, above the diaphragm; and the liver, $L i$,

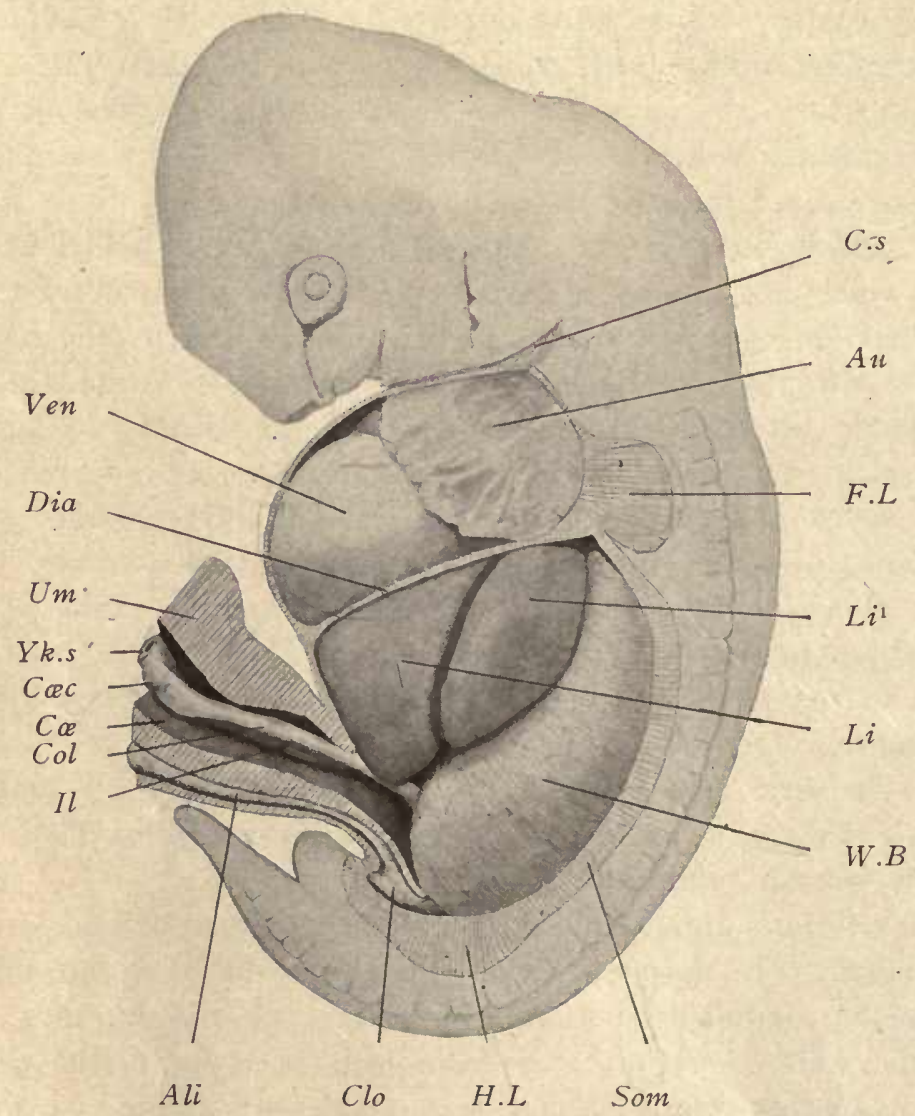

Fig. 170.-Pig of 12.7 ma. Dissection of the Viscera by Richard E. Schamon.

All, Allantois. $A u$, Auricle of the heart. Cac, Cæcum. Clo, Cloaca. Ca, Colom. Col, Large intestine. C.s, Cervical sinus, nearly obliterated. Dia, Diaphragm. F. L, Front limb. H.L, Hind limb. Il, Small intestine. $L i$, Ventral, $L i^{1}$, dorsal lobe of liver. Som, Cut surface of somatopleure. Um, Wall of the umbilical cord. Ven, Ventricle of the heart. W.B, Wolffian body. Yk.s, Yolk-stalk. $\times 8$ diams.

$L i^{1}$, and Wolffian body, W.B, below the diaphragm. The heart shows its large auricle, $A u$, the walls of which are thin and translucent. 'It entirely conceals the veins, which enter the heart through the diaphragm, and the aorta, which runs from the ventricle toward the pharynx. The ventricle, Ven, is much larger than the auricle; its walls are not translucent; its rounded apex points away from the auricle. The liver lies close against the diaphragm and shows two lobes: the larger ventral lobe, 



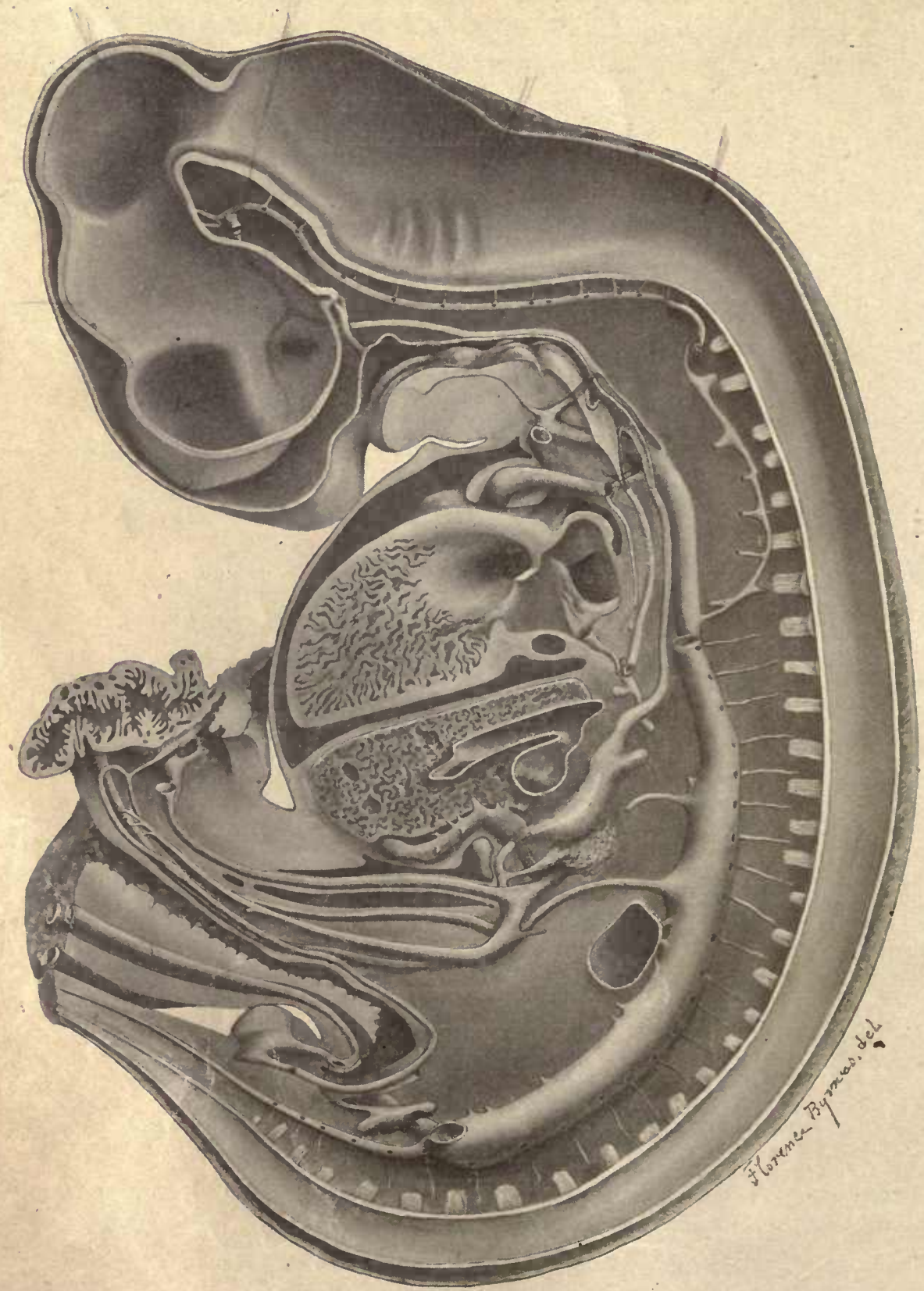

FIG. I7I. 


\section{Fig. I7 2.-Pig Embryo of I2.0 mi. Reconstruction from the Transverse Sections, Series 5.}

For the most part the organs represented are in or near the median plane. The drawing illustrates especially the disposition of the alimentary tract, the arterial system, and the heart. A.bas, Basilar artery, formed by the union of the two vertebral arteries, $A, v$, and joined, under the mid-brain, by the anterior ends of the internal carotids. A.cau, Caudal artery, the small median prolongation of the dorsal aorta. All, The allantois; it is joined by the Wolffian duct, and empties into the cloaca. an.pl, The cloacal membrane. Ao, Median dorsal aorta. Ao.d, Right descending aorta; a small vessel connecting the dorsal ends of the third (carotid) and fourth aortic arches. Ao.D, Main right descending aorta, passing downward to join its fellow and form the median aorta. A.s, Subclavian artery. Au, Left auricle of the heart. A.um, The umbilical artery which runs in the mesodermic wall of the allantois and joins the caudal end of the rlorsal aorta. A.v, Vertebral artery. A.vi, The vitelline artery, which becomes the superior mesenteric artery of the adult." $c$, Artery known as the coeliac axis in the adult. car.e, External carotid artery, arising from the base of the third aortic arch. car.i, Internal carotid artery. $\mathrm{Cbl}$, Cerebellum. $d$, Left duct of Cuvier. Dien, Diencephalon. $D . V$, Ductus venosus Arantii. ep, Small plug of entodermal epithelium in the rectum; there are several small irregular passages through the plug. Epgl, Anlage of the epiglottis.' $f$, Interventricular foramen, opening into the space $a, b, c$, of Fig. I 76 . F. $M$, Foramen of Monro. f.o, Foramen ovale, between the cardiac auricles. 6. Fa"-bladder. In, Entodermal wall of the intestine. is, One of the series of intersegmental arteries. rac blind end of the ureter, the anlage of the renal pelvis. Lar, Anlage of the larynx, consisting (2) thal plate. $L i$, Liver. $L u$, Entodermal portion of the lung. $M . b$, Mid-brain: $M d . o b$, N., A spinal nerve. Neu, Neuromeres of the hind-brain. Oe, Esophagus. op, Stalk. s slk is the anlage of the optic nerve. P.A, Pulmonary artery. pan, Dorsal anlage vart of which becomes the duct; behind the duct is the anlage of the ventral panLiowth from the duct of the gall-bladder. p.c, Pericardial cavity. P.V, Pul= St, Stomach; the letters are placed beside the diverticulum which is charis in man. $t$, Posterior portion of the tongue, an eminence on the floor of the ventral ends of the third and fourth gill-arches. T.i, Tuberculum 7 of the tongue. Tra, Entodermal trachea. Ug.si, Cloaca. Um.d, icle, the letters being placed on the septum inferius. Y.mes, Superior . $v$, Vitelline vein. W.I), Wolffian duct. $x$, Anastomosis between ans. $Y k$, Yolk-sac. Yk.s, Entodermal yolk-stalk, connecting the -(Drawn by F. T. Lewis.) 


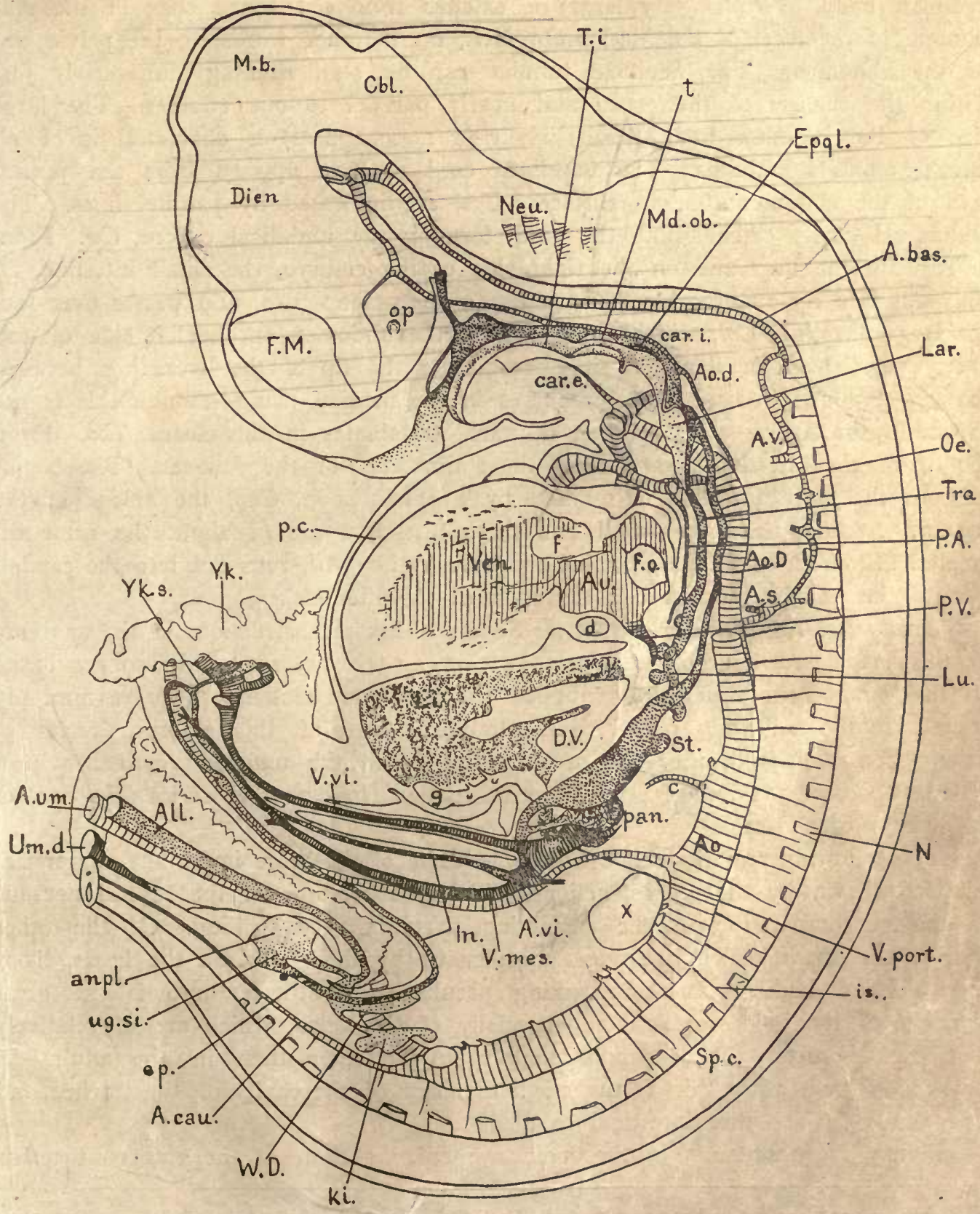

FIG. 172. 
$L i$, extends to the umbilicus; the smaller dorsal lobe, $L i^{1}$, abuts against the Wolffian body. In the fresh specimen the liver is conspicuous by its dark color. The Wolffian body, W.B, is very large; it extends from the dorsal edge of the diaphragm to the level of the hind limb, H.L, or, in other words, to the pelvic end of the abdomen. The Wolffian tubules can be seen running transversely just within the surface of the organ and nearly parallel to one another. The large size of the Wolffian body (fetal kidney or mesonephros) is characteristic of all known amniote embryos. The umbilical cord projects upward from the ventral wall of the abdomen; its cut surfaces, $U m$, are indicated by parallel lines. The abdominal cavity extends into the cord, forming the umbilical cœlom, Cos. From underneath the liver and on the right side of the embryo, the small intestine, $I l$, runs out into the umbilical cœlom, makes a sharp loop turn, and passes over into the large intestine, Col, which runs back to the abdomen on the left side of and nearly parallel to the ileum, $I l$; it passes under the tip of the liver, then between the two Wolffian bodies, where it curves in the median plane-though this is not shown in the figure-and, bending tailward, terminates in the cloaca, Clo. From the tip of the intestinal loop springs the stalk, Yk.s, of the yolk-sac. The beginning of the large intestine is marked by a small knob, Cac, the anlage of the cæcum. At this stage the small and large intestines are of about the same diameter. From the cloaca, Clo, a hollow prolongation, $\mathrm{All}$, runs out into the caudad wall of the umbilical cord; it is the stalk of the allantois.

Anatomical Reconstructions from the Sections.-Six reconstructions of the anatomy of this stage are figured.* Figures I7I, I73, I74, I75, I77, and 179 are based on the same series which has supplied the transverse section of the $12 \mathrm{~mm}$. pig figured in the following pages. The umbilical cord of this embryo (Series 5) having been damaged, the loop of the intestine in the umbilical cord has been added to figures $\mathrm{I} 7 \mathrm{I}$ and $\mathrm{I} 74$ by a reconstruction from another series (No. $5^{\mathrm{I}}$ ) of an embryo of the same size.

The following remarks call attention to some of the more important anatomical relations shown by the reconstructions. The great volume of the central nervous system as compared with the remaining parts is very striking. Of the other organs, the three which are most conspicuous by their size are the heart, liver, and Wolffian bodies. Another striking peculiarity of the embryo is the great diameter of the blood-vessels, and especially of the veins, which are of relatively enormous diameter, being proportionately much larger than in the adult. In marked contrast with this is the small diameter of the cavity of the trachea and lungs and of the entire intestinal canal.

Figure 172 represents in the main a median section of the embryo together

* Figures 171 , I75, I77, and 179 were made by Dr. F. T. Lew $\varsigma$; figure 174 by Mr. F.P. Johnson; figure 173 is from a wax reconstruction by Dr. John L. Bremer. In all cases the reconstructions were made with special reference to their present use. It gives me much pleasure to acknorledge my obligations to these menbers of our laboratory staff. 
with the organs of the right side, but with two exceptions, first, the floor of the pharynx is represented as if cut through considerably to the left of the median plane; second, the heart is cut to the left of the median plane. The brain and spinal cord are drawn as if opened to show the modeling of the inner surface of the medullary tube. The pharynx is so drawn as to indicate something of the modeling of its floor surface. The opening of the veins into the heart and of the auricle into the ventricle, and the interventricular orifices, are shown. Of the digestive canal only the entoderm is represented, so that the figure displays the entodermal walls of the œesophagus, stomach, and intestine, and shows the two pancreatic anlages. Similarly only the entodermal portion of the trachea and

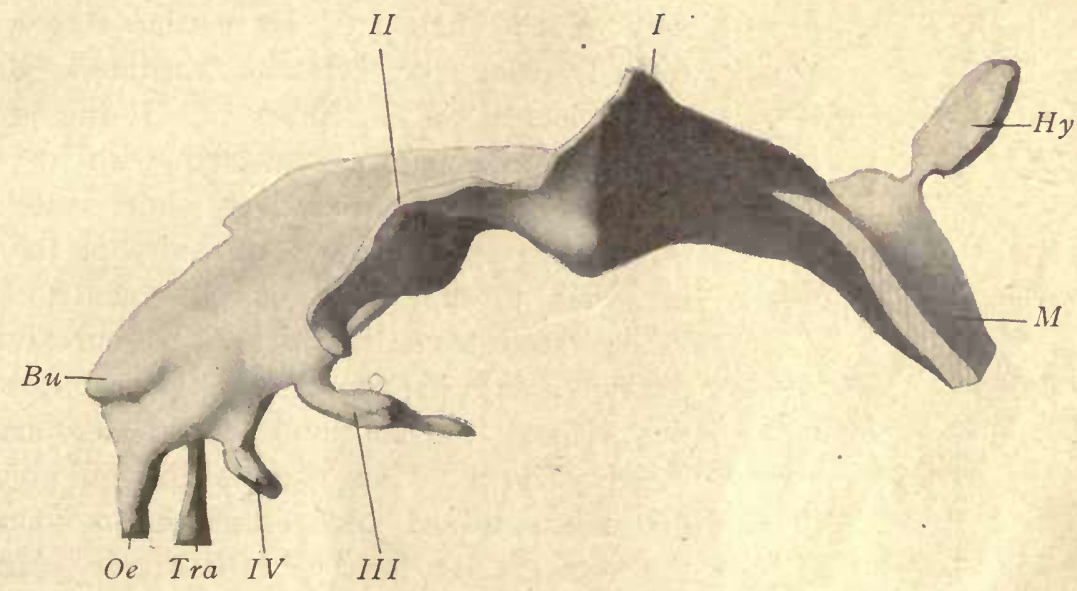

Fig. 173.-Pig, i2 mi. Sagittal Series 7. Wax Reconstruction by J. L. Bremer.

$B u$, Pharyngeal bursa. Hy, Hypophysis. M, Mouth-cavity. Oe, Esophagus. Tra, Trachea. I, II, III, IV, Gill-pouches, developed as lateral pouches of the pharynx. $\times 40$ diams.

lungs is included, and the same is true of the caudal end of the Wolffian duct and of its outgrowth, which forms the anlage of the kidney. The same is further true of the gall-bladder, of which only the epithelial portion is represented. In this figure the arterial system is fully displayed. The pulmonary artery and the aortic trunk are completely separated. A small artery from the pulmonary arch to the lungs is included, and the figure shows the entire system of branches from the main aorta.

Figure $\mathrm{r} 75$ is in many respects similar to figure 172 , and is intended to show chiefly the disposition of the veins. There are also included in this figure the Wolffian body and its duct. The pharynx and the heart are supposed to have been cut through, well to the right of the median plane. This makes it possible to indicate in the figure the origin of the pulmonary aorta and of the true aorta. The following are the most important veins: the umbilical, which passes around the umbilical opening and entars the liver; the portal vein, which receives the 
blood from the abdominal viscera, and also delivers it to the liver. In this specimen there is quite a wide and free connection within the liver between the portal and umbilical veins. In other embryos of this size such a connection does not always exist. The large vena cava inferior is on the right side of the embryo,

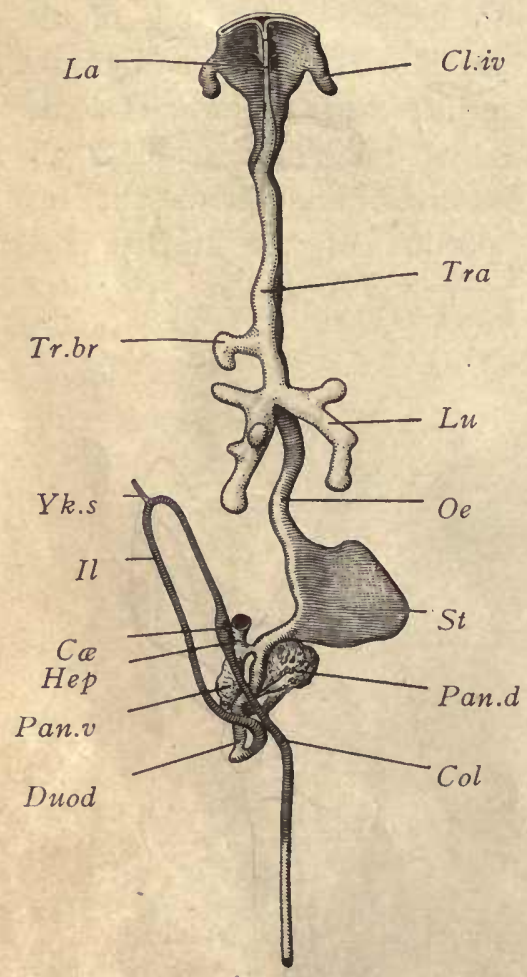

Fig. 174.-PIG Enbryo of 12.0 MM. RECONSTRUCTION FROM SERIES 5, to SHOW the ENtodermal Canal, Viewed from the Ventral. Side.

$\mathrm{C} \propto$, Cæcum. Cl.iv, Fourth gill-pouch. Col, Colon. Duod, Duodenum. Hep, Hepatic duct. $I l$, Ileum. $L a$, Larynx. Lu, Lung Oe, Gisophagus. Pan.d, Dorsal pancreas. Pan.v, Ventral pancreas. St, Stomach. Tra, Trachea. Tr.Br, Tracheal bronchus. I $k . s$, Yolksac. $X \mathrm{I}_{4}$ diams. - (Draw'n by F.P. Johnson.) from the pharyngeal entoderm, but from the ectoderm of the mouth-cavity proper. Figure $\mathbf{I 7 4}$ is inserted in order to give a clear idea of the entodermal canal, as viewed from the ventral side. Only the posterior end of the pharynx is included, and the cloaca and the allantois are omitted. The figure represents only the entoderm without any of the surrounding mesiderm. and passes through the liver, which thus receives blood directly from the Wolffian bodies and the cardinal veins. From the upper side of the liver the hepatic vein goes directly to the heart, uniting with the common cardinals, which receive the jugular veins from the head and the post-cardinal veins from the body. The cardinal veins are now very much changed. In earlier stages they extended from the common cardinals almost the entire length of the embryo. Of this great vessel there now remains connected with the common cardinal only a comparatively short vessel.

Figure 173 gives a lateral view of the pharynx, in order to show the four gill-pouches, I, II, III, IV, as seen from the side. The curvature of the pharynx, and its passage at its posterior end into the ventral trachea and dorsal œesophagus, are clearly shown. As regards the gill-pouches, the first rises upward and terminates in a sharp apex; the second lies nearly in the same plane as the portion of the pharynx from which it arises and has a prolongation toward the third pouch, and the end of the prolongation bends ventralward; the third is narrow as it parts from the pharynx, then bends downward and forward and has a prolongation, the anlage of the thymus gland, which extends toward the root of the aorta; the fourth also begins with a narrow stalk and has an expanded end, one apex of which extends outward (to join the cervical sinus), the other inward and downward, the latter being the anlage of the postbranchial body. On the dorsal side projects the pedunculate hypophysis, which is developed, not 


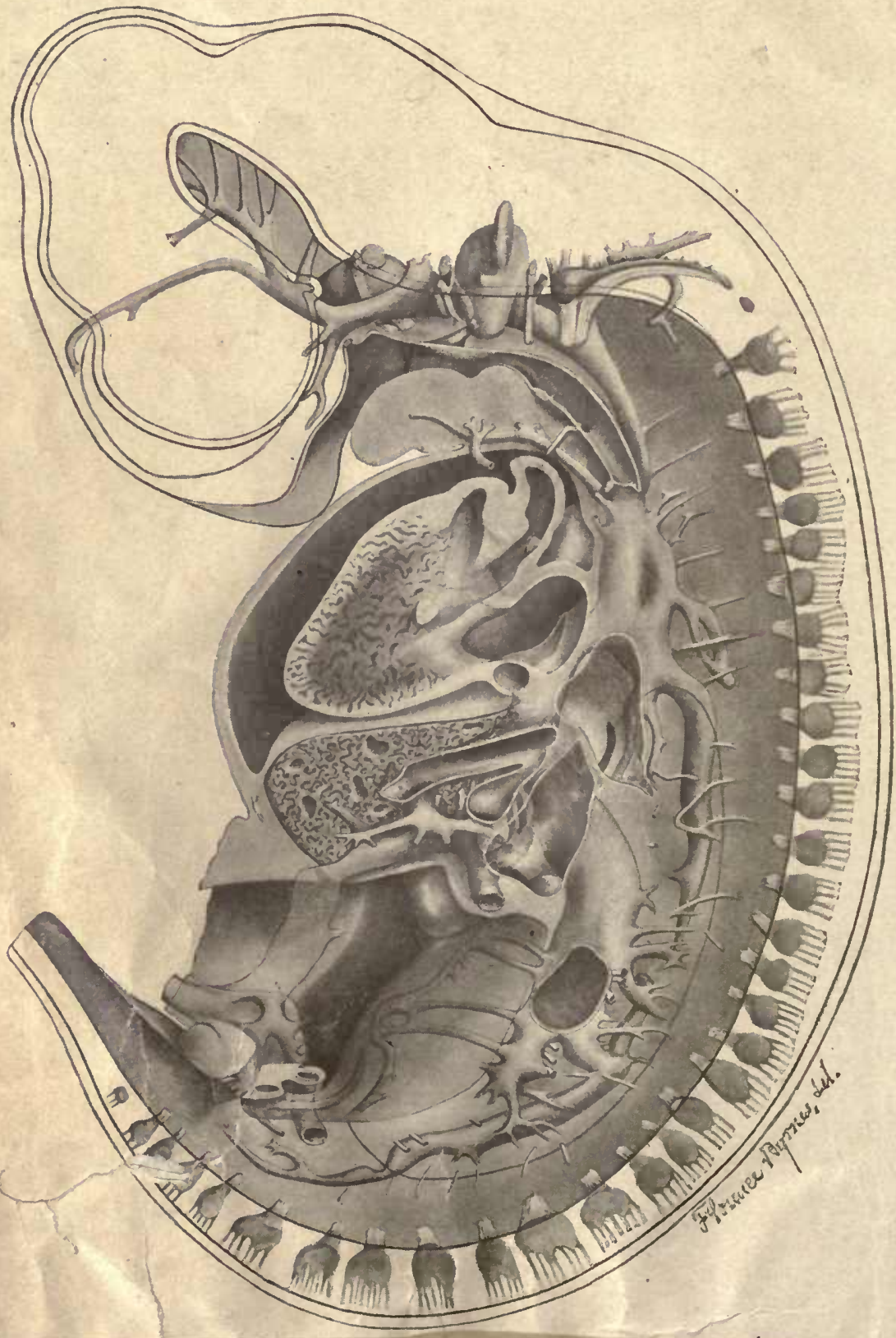

FIG. 175 


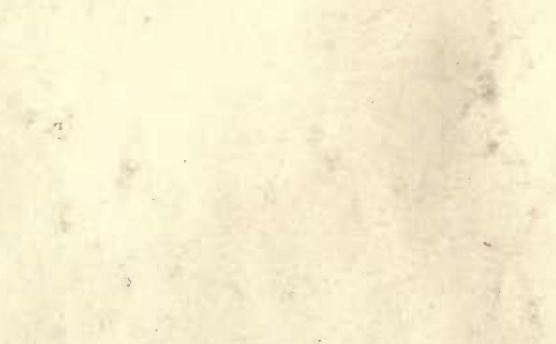

$-$

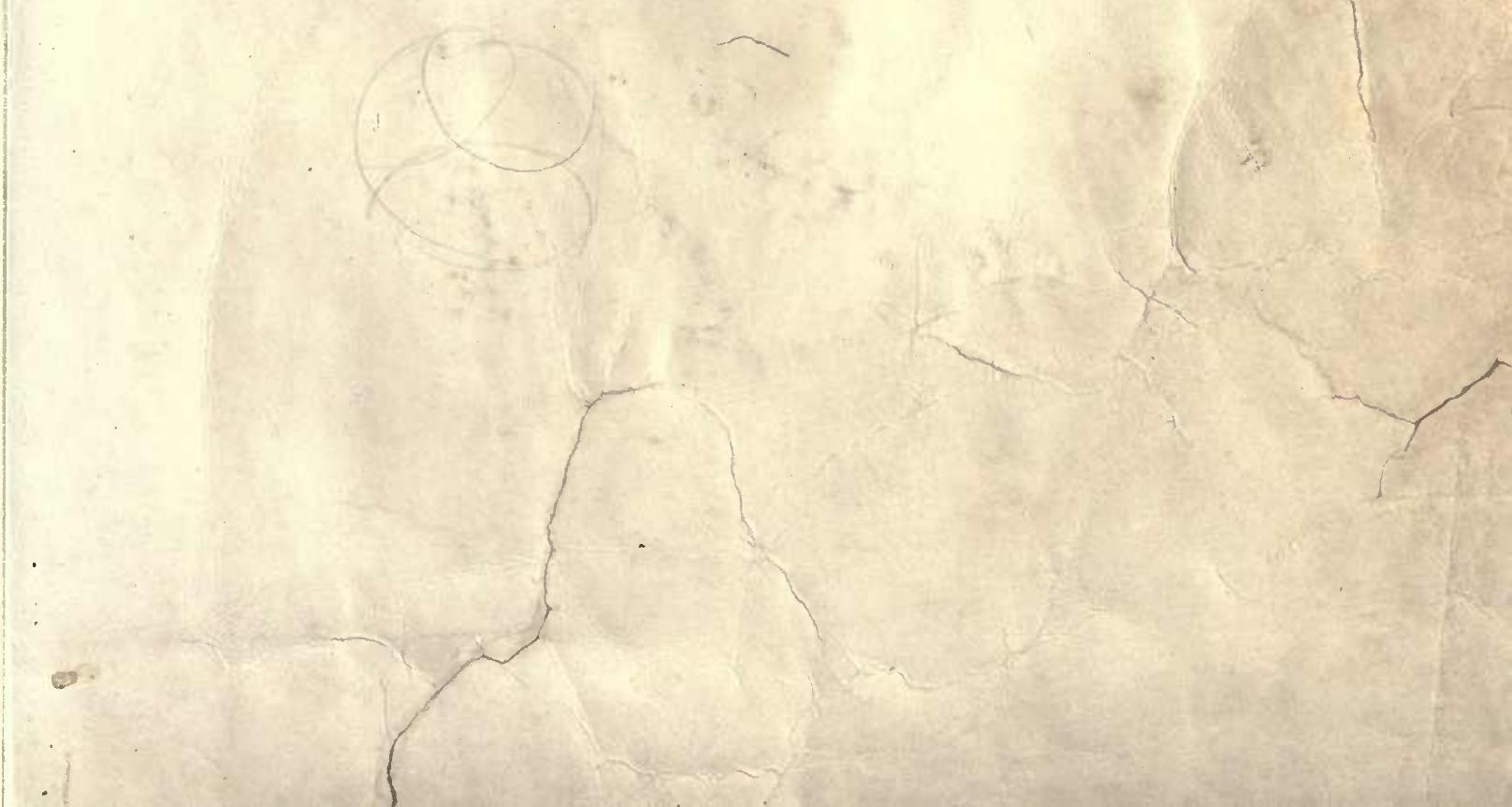



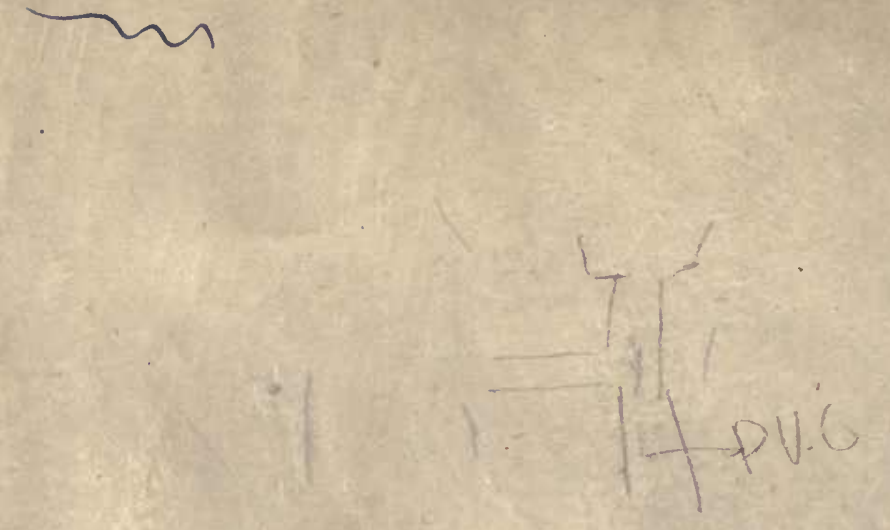

Fig. i76.-Pig Embryo of i 2.0 mm. Reconstruction from the Transverse Sections, Series 5.

The figure illustrates chiefly the veins of the right side, and shows the right auricle and ventricle of the heart. $A$, The right umbilical artery, only a small portion being drawn as it curves around the Wolffian duct, W.D. $a$, Tip of aortic septum, which divides the aortic limb of the heart into the pulmonary aorta, $P$, and main aorta, $A o$; by a growth of the cardiac tissue, $a, b$, and $c$ of the figure become joined, shutting off the space around the base of the aorta; this space communicates by the interventricular foramen with the left ventricle, and serves as the permanent or adult channel of communication between the true aorta, $A o$, and the left ventricle. All, Allantois. Ao, Aortic division of the aortic limb of the heart. $A u$, Right auricle. $b$, See " $a$." $\quad c$, See " $a$. ." $c a r d$, Superior part of the cardinal vein (the anlage of the azygos). card", Inferior part of the posterior cardinal vein. $c l . \mathrm{x}$, Opening of the first gill-pouch into the pharynx, the pharyn $\mathrm{x}$ being indicated by dotted shading. $c l .2$, Opening of the second gill-pouch into the pharynx. $c l .3, c l .4$, Entodermal portions of the third and fourth gill-clefts. c.om, Dotted outline of the omental or lesser peritoneal cavity. $d$, Left duct of Cuvier. D.C, Right duct of Cuvier, the main venous trunk entering the heart from the right side. D.V, Ductus venosus Arantii. F.H, Foramen epiploicum (of Winslow), drawn in black. F.pp, Foramen, drawn in black, between the pleural and peritoneal cavities. The foramen is bounded by the lung, liver, and Wolffian bodly; the figure shows the pleural side of the opening. If we pass through the foramen as drawn we reach the abdominal cavity. The outline of the pleural cavity is nuarked by a dotted line. Gen, Genital tubercle, represented as somewhat displaced from the median line, which it really occupies. G.R, Genital rilge. $J u g^{\prime}, J u g^{\prime \prime}$, Jugular or anterior cardinal vein. $L i$, Liver. $l . s$, Anlage of the lateral venous sinus. $m x$, Vein of the inferior maxilla or manclible. P, Pulmonary division of the aortic limb of the heart. p.c, Pericardial cavity. Pl, Dotted outline of pleural cavity. Rec, Rectuns. Sc, Sub-cardinal vein, which is derived from the cardinal and on the right side of the body forms part of the vena cava inferior. Scl, Subclavian vein. sls, Anlage of the superior longitudinal venous sinus, which is formed by the union of the veins, l.s., from the sicles to make it single vessel between the rerebral hemispheres. Ur, Ureter. Um.l. Right umbilical vein. $v$, Valve of the sinus verusus. Fen, Right ventricie of the heart. V.H.C, Vena lepatica communis. vop, Ophthalmic vein. Y.P, I'oral vein. $W . b$, Woltfian body. $W^{r} . D$, Wolffian duct. $x$, Anastomosis betweoi the cardinal venous systems of the right and left sides. $X_{14}$ diams.-

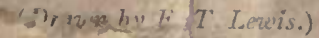




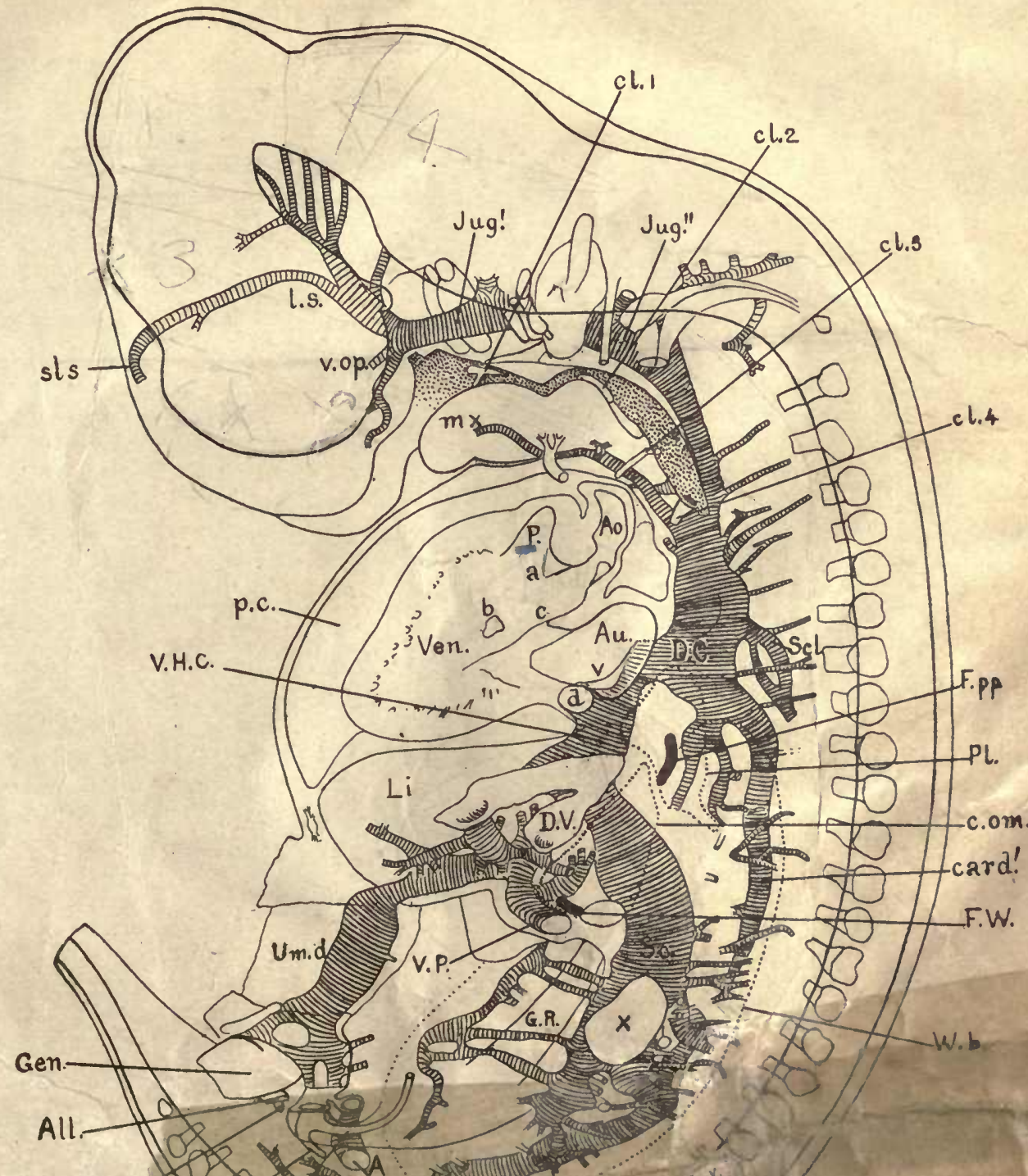

Rec 
STUDY OF PIG EMBRYOS.

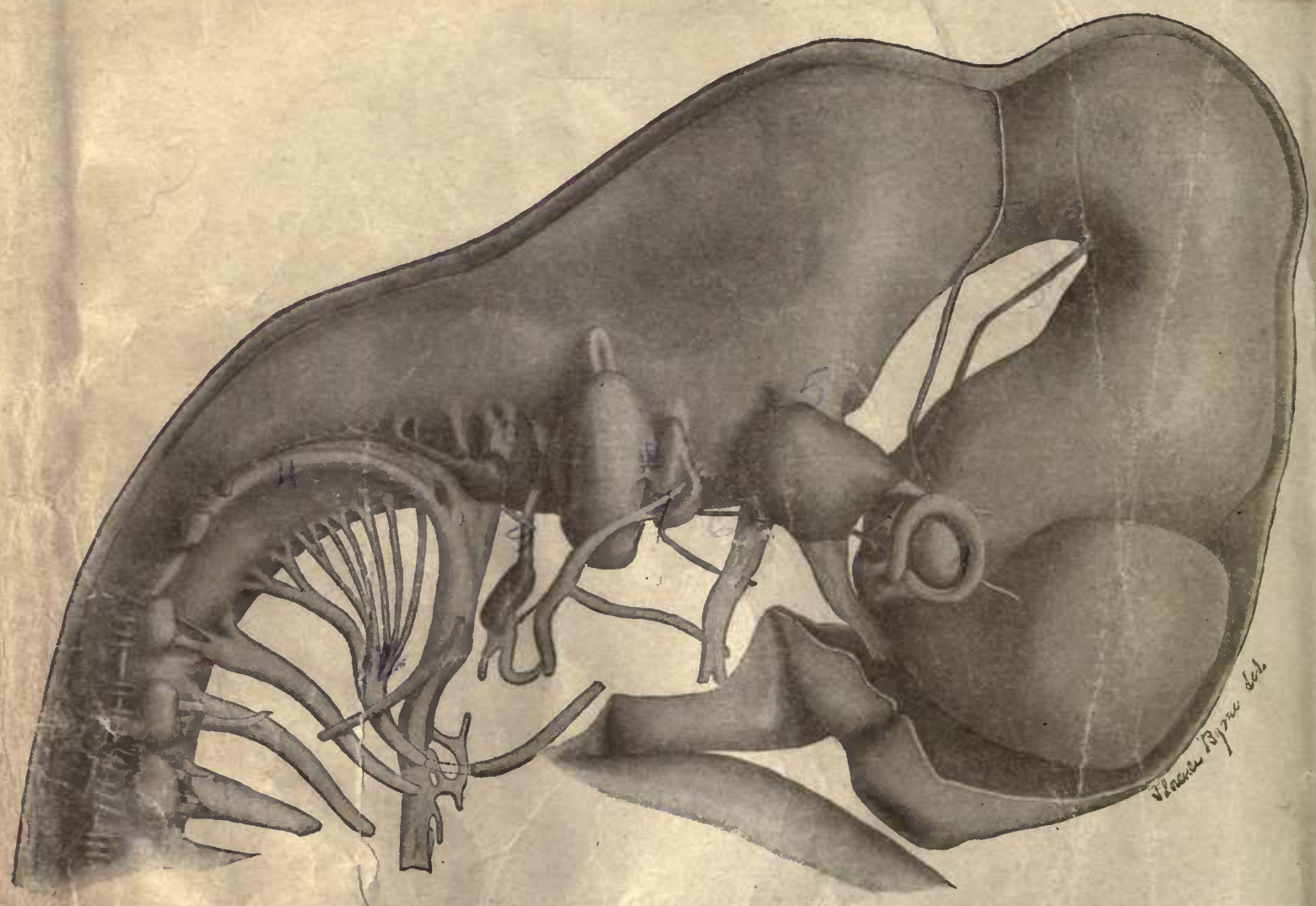

FIG. 177 . 


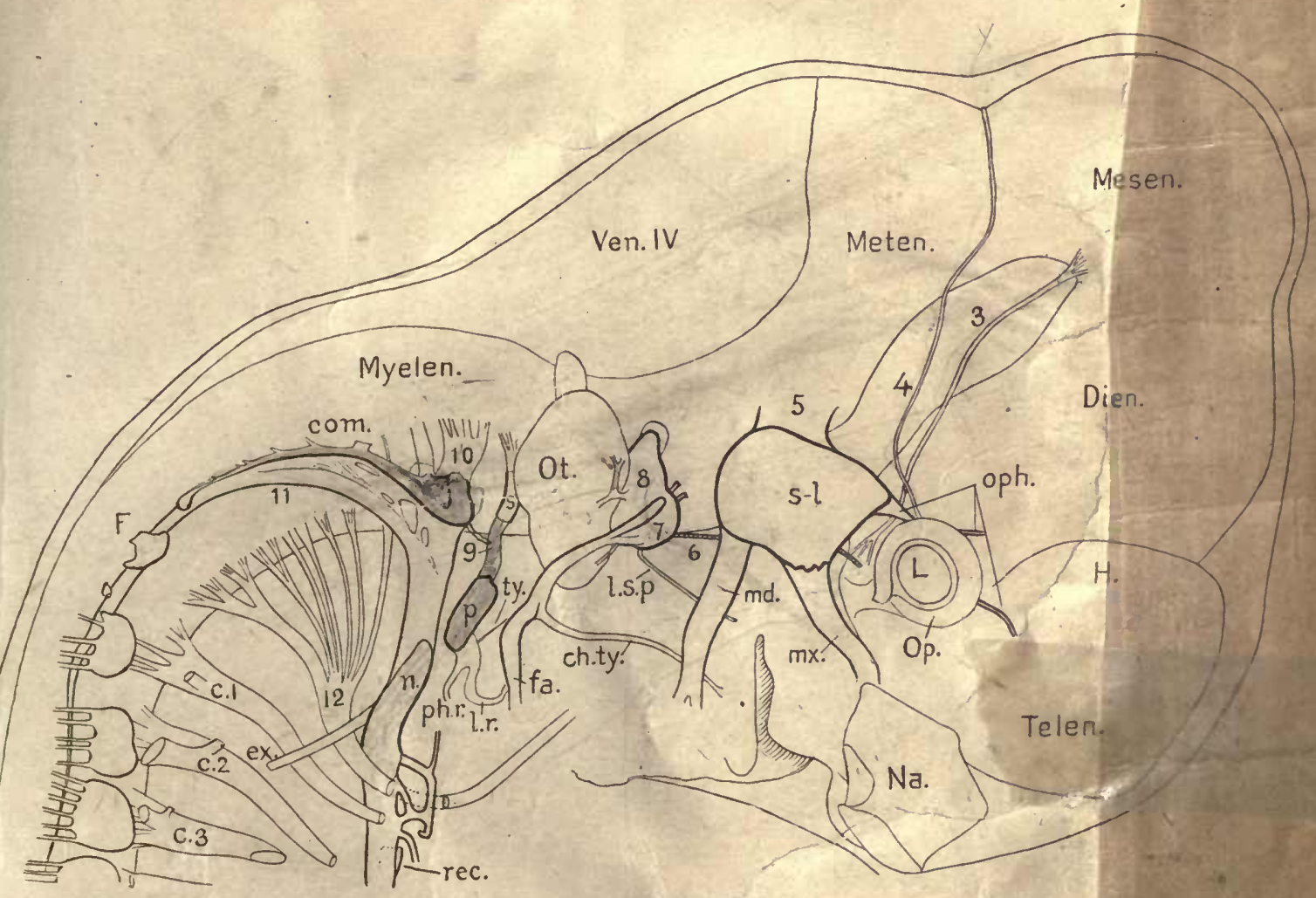

Fig. 178.-Pig Embryo of i2.o mm. Reconstruction from the Transverse Sections, Stries 5.

To show especially the cephalic nerves. c.I, c.2,c.3, Cervical nerves. ch.ty, Chorda tympani. com, Gangliontic commissure connecting with the jugular ganglion, $j$. Dien, Diencephalon. ex, External hranch of the spinal accessory nerve. $F$, Froriep's ganglion, which in man completely disappears. ja, Facil l nerve. $H$, Cerebral hemisphere. $j$, Jugular ganglion, from which the small auricular nerve runs forward the glosso-pharyngeus. L, Lens. l.r, Laryngeal branch of the glcsso-pharyngeal nerve. i.s.p. ficial petrosal nerve. mid Mandibular branch of the triceming cephalon. $m x$, Maxillary branch of the trigeminal. Myelen, Myelencephailon. $n$, Nodosal gangitun. Na, Nasal pit. $O p$, Optic cup. oph, Ophthalmic branch of the trigeminal. Ot, Otwe st." p, Petrosa! ganglion. ph.r, Pharyngeal branch of the glosso-pharyngeal nurve. rec. Recurrent laryngeal nerve. $s$, Superior ganglion. s-l, Semilunar ganglion. Telen, Trelencephalun. ty, Tympanir nerye. Ten.II Roof of the fourth ventricle. 3 Oculomotor nerve. 4, Trochlear n-ve. 5, Trigeminal nerve. 6, Abdurens nerve. 7 , Geniculate ganglion. 8, Acoustic ganglion. 9, Glo sso-pharyngeal nerve. ro, Yagus nerre. - II, Spinal accessory nerve. I2, Hypoglossal nerve. $\chi_{20}$ diar:s.-(Drawn by F. T. Lewis. 


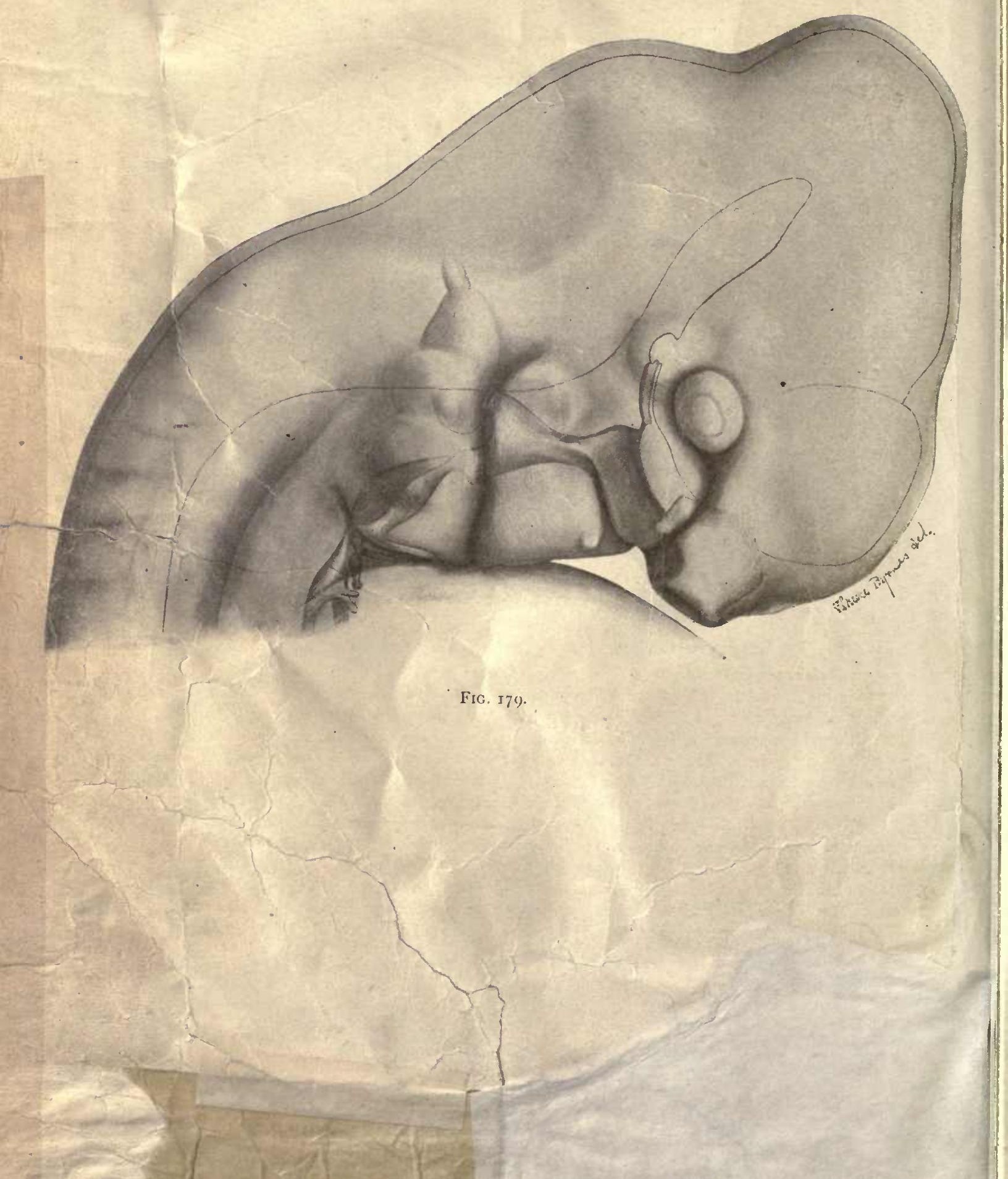


Figure 178 shows the disposition of the cephalic and upper cervical nerves and also the position of the nasal cavity, the eye, and the otocyst.

Figure 180 gives an outline of the head and combines an indication of the external modeling of the gill-arches, with a representation of the shape of the pharynx.

\section{Pig Embryo of $6 \mathrm{~mm}$. Studied in Sections.}

Of this stage three transverse sections are figured in order to give more exact notions as to the structure of neuromeres, of the pharynx, and of the secondary segments.

Transverse Section through the Fourth Ventricle (Fig. 18I).-The section is taken through the level of the head, and may be directly compared with figure 189 . The relations are so closely similar that it is unnecessary to describe the present section (Fig. I8I) in detail. The explanation of the figure is sufficient for the identification of the parts. The otocyst is large and conspicuous, and the arrangement of the nerves is essentially similar to what we find in the older embryos. The neuromeres, however, are very distinct, especially those upon the left side of the embryo, $N$. I, 2, 3, 4. Of these, the third is perhaps the most characteristic. Each neuromere is separated from its fellow by an internal sharp ridge, so that the inner boundary of each neuromere toward the cavity of the fourth ventricle is a small arc of a circle. The cells are elongated and are placed radially to the inner curved surface of the neuromere. A thin but distinct layer of ectoglia is present. The light line, which marks the boundary between the adjacent neuromeres, is produced by the comparative absence of nuclei. As to the number of neuromeres our knowledge is still defective; nor have we yet succeeded in making sure of their exact relation to the nerves of the head, though such a relation evidently exists. Thus we find, for example, that the facial nerve is always connected with neuromere 2 of our figure, and the glosso-pharyngeal nerve with neuromere 4 .

Transverse Section through the Region of the Branchial Arches (Fig. 182). - The branchial arches are much more conspicuous at this stage than in later ones, being separated from one another by deep ectodermal depressions, figure $29, I, I I, I I I$, $I V$; and, although $I I I$ and $I V$ are already being turned in, preparatory to the formation of the cervical sinus, they are still distinct and their order in the series is evident. The section (Fig. I82) shows on the dorsal side the spinal cord, in which we can already recognize the subdivision into dorsal zone, D.Z, and rentral zone, $V: Z$. To the dorsal zone is appended the dorsal root; from the middle of the ventral zone comes off the ventral root of a cervical nerve, $N$. Just between the dorsal root and the wall of the spinal cord can be seen the section of the accessory nerve. The secondary somite, $M y$, is sharply defined and has a distinct growing edge showing at its upper limit in the sioction. The inner leaf of the secondary somite is stained more lightly than the neighboring tissue, corresponding to the modifications which the cells are undergoing preparatory to their change 
into young muscle-fibers. In the $12 \mathrm{~mm}$. pig in this region the cells of the muscleplate have already broken apart and no distinct plate can any longer be recognized. Below the muscle-plate follows the section of the anterior cardinal vein, Card. Lower down and in the median line we have the section of the pharynx, $P h$,

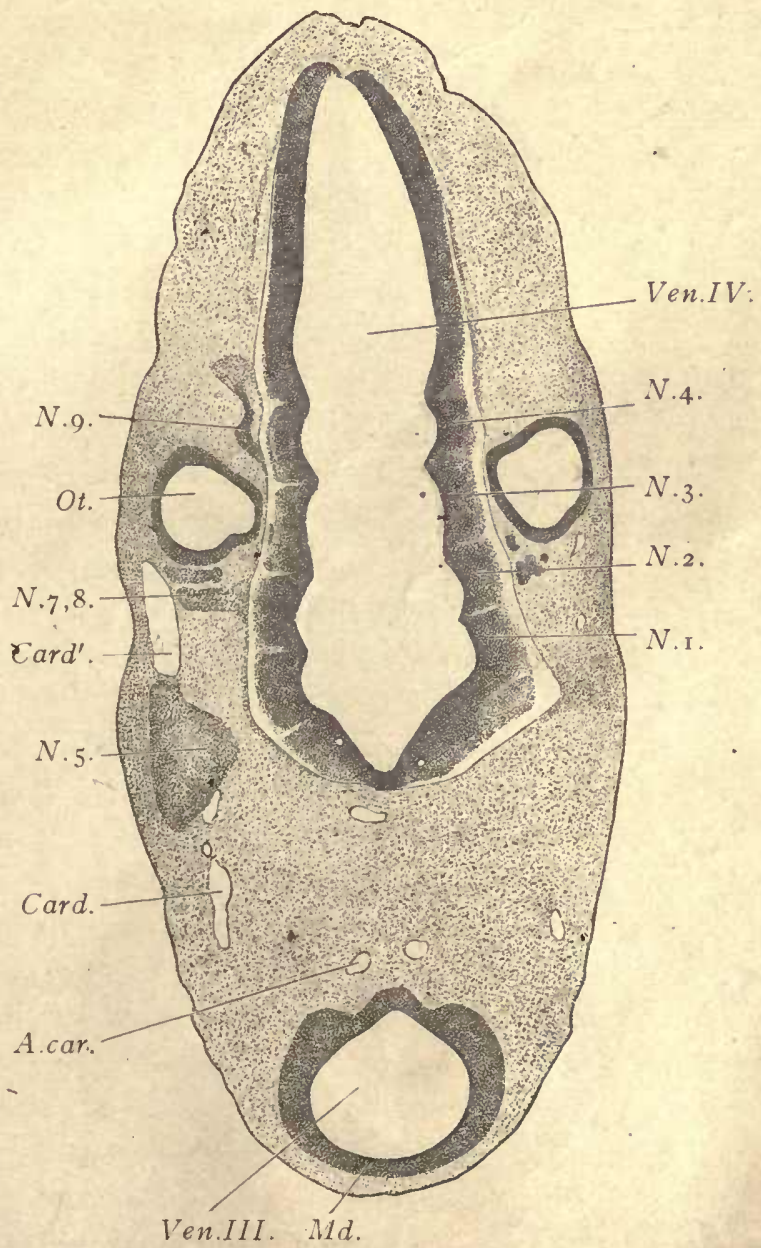

Fig. i8t.-Pig, 6.o mm. Transverse Series 9, Section 90.

A.car, Carotid artery. Card, Card', Anterior cardinal vein. Md, Medullary wall of the fore-brain. N.I, N.2 N.3, N.4, Neuromeres of the hind-brain. N.5, Trigeminal ganglion. N.7, 8, Acustico-facial ganglion. N.9, Root of the glosso-pharyngeal nerve. Ot, Otocyst. Ven.III, Third ventricle or cavity of the fore-brain. $V e n . I V$, Fourth ventricle. $\times 35$ diams.

lined by the epithelial entoderm. The pharynx is surrounded by the rery large aortic vessels, which start from the ventral side of the pharynx, and pass upward along its sides to join the descending aorta, Ao.d.4, at about the level of the jugular veins. The vessels shown are the fourth aortic arches. Their symmetry and 
their relations to the pharynx are beautifully demonstrated in this section. Below the aorta we find a section of the third internal gill-cleft, cl.III, a narrow, slitlike cavity lined by entoderm. By following the series of sections, the connection of this cavity with that of the pharynx can be traced, thus demonstrating that the

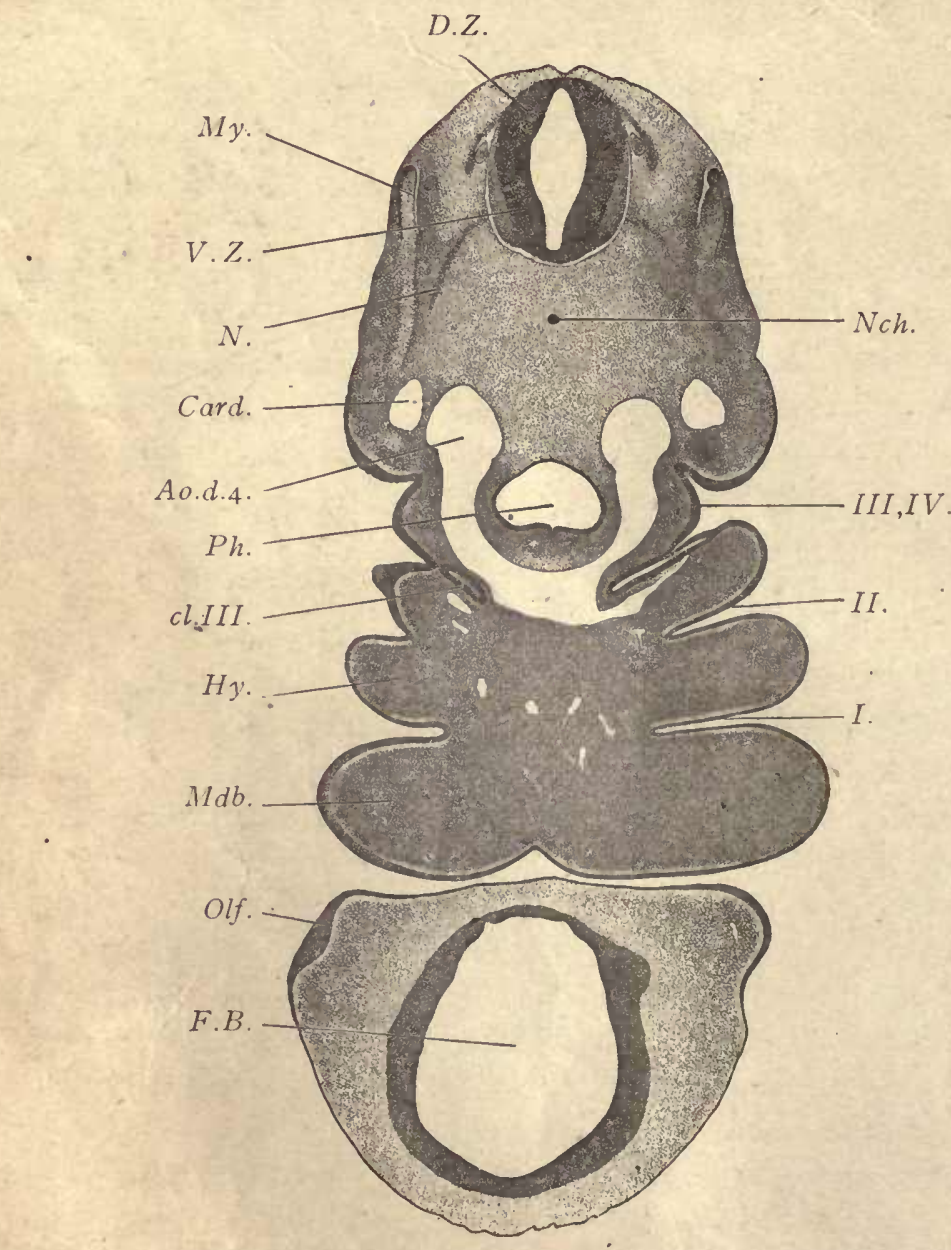

Fig. 182.-Pig, 6.0 m. Transverse Series 9, Section i7 I.

Ao.d.4, Descending aorta receiving the right fourth aortic arch. Card, Anterior cardinal vein. cl.III, Third entodęrmal gill-cleft. D.Z, Dorsal zone of spinal cord. F.B, Fore-brain. $H y$, Hyoid branchial arch. $M d b$, Mandibular branchial arch. $M y$, Muscle-plate. $N$, Nerve. $N c h$, Notochord. Olf, Olfactory plate. Ph, Pharynx. V.Z, Ventral zone of spinal cord. I, II, III, IV, First to fourth ectodermal gill-clefts. $\times 35$ diams.

cleft is an evagination of the pharynx, as are all the gill-clefts. On the left-hand side of the embryo the junction of the entoderm of the internal pouch with the ectoderm is shown. The two germ-layers have united to form a typical closing plate. Above the third gill-cleft, the outline of the embryo shows a deep depres- 
- sion, $I I I, I V$, which is due to the commencing formation of the cervical sinus. From the upper end of this depression runs upward the ectodermal fourth cleft, and from its lower part extends downward the ectodermal third cleft. Between the third and fourth clefts the external surface of the embryo protrudes somewhat. This protuberance corresponds to the so-called fourth branchial arch. Between the third external cleft and the second, $I I$, is a still greater protuberance on the outside of the embryo. This marks the third branchial arch. The third aortic arches are somewhat imperfectly shown, but the connection of the left third arch with the central aorta appears. Between the second and first external clefts we have the second or hyoid branchial arch, $H y$; and, similarly, between the first or auditory cleft, $I$, and the oral fissure, which separates the head from the body of the embryo, we have the very large and protuberant mandibular arch, $M d b$. The head of the embryo is completely separated in this section from the body. It shows the cavity of the fore-brain, F.B, bounded by the ectoderm of the medullary wall, and on one side also shows the thickening of the epidermis, Olf, which forms the olfactory plate or plakode, which is to become the lining of the nasal pit.

Transverse Section of the Lower End of the Embryo (Fig. 183).-Our third section is very near, the end of the series. Owing to the curvature of the posterior end of the body of younger embryos (compare Fig. I65; pig, $7.5 \mathrm{~mm}$.), sections taken in the plane which we call transverse strike the lumbar region so as to give longitudinal sections of the spinal cord and primitive segments. Figure $\mathrm{I}_{3}$, therefore, shows the

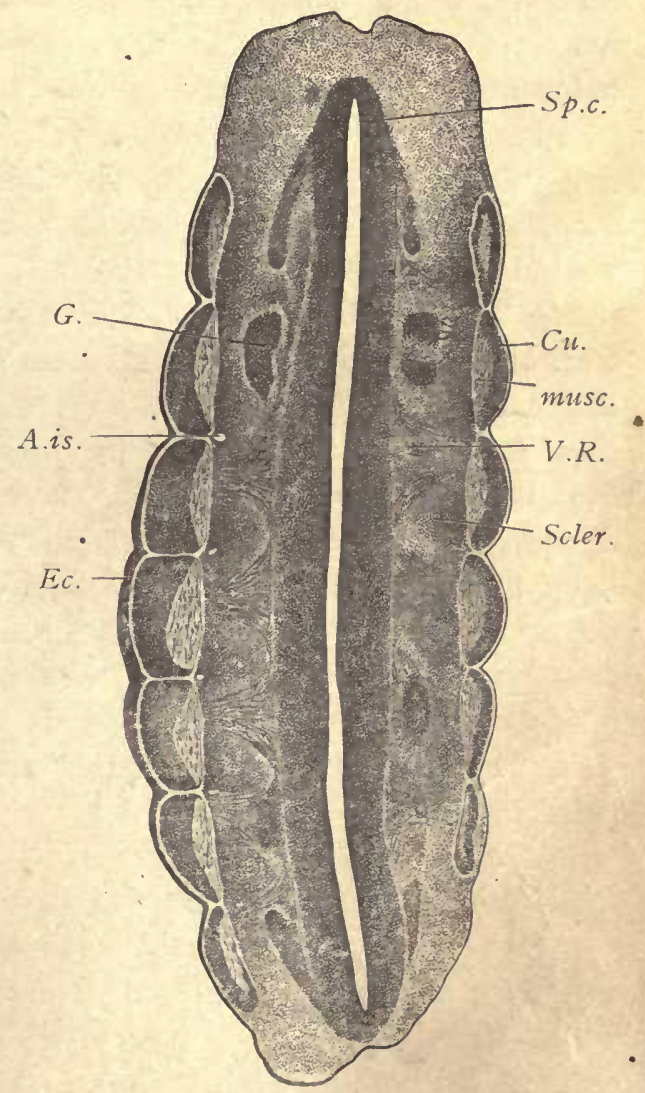

Fig. 183.-Pig, 6.0 mu. Transverse Series 9, Section $5^{\text {I } 9}$.

$A . i s$, Intersegmental artery. $C u$, Cutis plate. $E c$, Ectoderm. G, Ganglion. musc, Muscle-plate. Scler, Sclerqtome, auct. Sp.c; Spinal cord. $V . R$, Ventral nerve-root. $\times 50$ diams.

cavity of the spinal cord, Sp.c, cut for a very long distance. At the upper and lower ends of the section, the dorsal side of the spinal cord is cut, and accordingly we see at these levels sections of the ganglia, $G$, on either side of the spinal cord. In the middle of our section the ventral portion of the spinal cord is cut, and here, therefore, the ventral roots, V.R, of the nerves are displayed. The somites are clearly marked by the external configuration of the embryo, the 
ectoderm, $E c$, forming an arch over the outside of each segment. Each mesodermic somite shows three distinct parts: next to the ectoderm the broad, epithelioid cutis plate, within which comes the spindle-shaped section of the inner portion of the somite, musc, the anlage of the skeletal muscles; and, third, an expanding mass of mesenchyma, Scler, which is sometimes termed the sclerotome. This term, however, is not wholly felicitous, because this mesenchyma forms not only the segments of the skeleton, but the connective tissue of the whole region about the spinal cord in the dorsal part of the embryo. The figure shows very clearly that the ganglia and ventral nerve-roots are arranged in exact conformity to the segments, and it can be easily observed, by following through the series of sections, that for each somite there is one ganglion and one ventral root. It also shows that the ventral roots reach directly to the muscle-plate. The muscle-plate is histologically partly differentiated, for its cells have already elongated in a direction parallel with the longitudinal axis of the embryo, and their nuclei also have become much larger than any other nuclei in the neighboring parts of the embryo, being perhaps three times as large as the mesenchymal nuclei of the sclerotome. They are oval in form, contain many fine, and usually one or two somewhat larger granules, the larger ones being deeply stained; but the nuclei, as a whole, are stained more lightly than their neighbors. Each somite is very clearly separated from its neighbors, and between the ends of the adjacent muscle-plates there is a small clear space entirely free from cells and extending outward to the epidermis. Just inside of this space in every case is a small blood-vessel, the intersegmental artery, A.is. The intersegmental arteries are small branches which arise in symmetrical pairs from the dorsal aorta.

\section{Pig Embryo of $9 \mathrm{~mm}$. Studied in Sections.}

Pig embryos of this stage supplement very instructively those of $12 \mathrm{~mm}$. It will, of course, be advantageous for the student to prepare serial sections himself. When that is not possible, there should at least be sections prepared for the laboratory which the student may. examine. Four sections are illustrated and described below. They have been chosen to supplement the descriptions of the sections of the pig of $12 \mathrm{~mm}$., and they will be found to illustrate certain fundamental morphological relations in the embryo more clearly than older stages.

Sagittal Section to the Right of the Median Plane (Fig. I84).--In the accompanying figure 184 the cephalic end of the embryo is omitted; a portion of the heart, the entire length of the Wolffian body, and the tail are included. The dorsal outline; of the embryo forms a characteristic curve. A long series of spinal ganglia, $G$, is shown arranged in regular succession and following the curvature of the back. The ganglia are easily recognizable by their dark staining; each of them is so large as to occupy at least four fifths of the length of the segment to which it belongs. The boundaries between the adjacent primitive segments are indicated by the positions of the intersegmental arteries, A.is: Even when their 


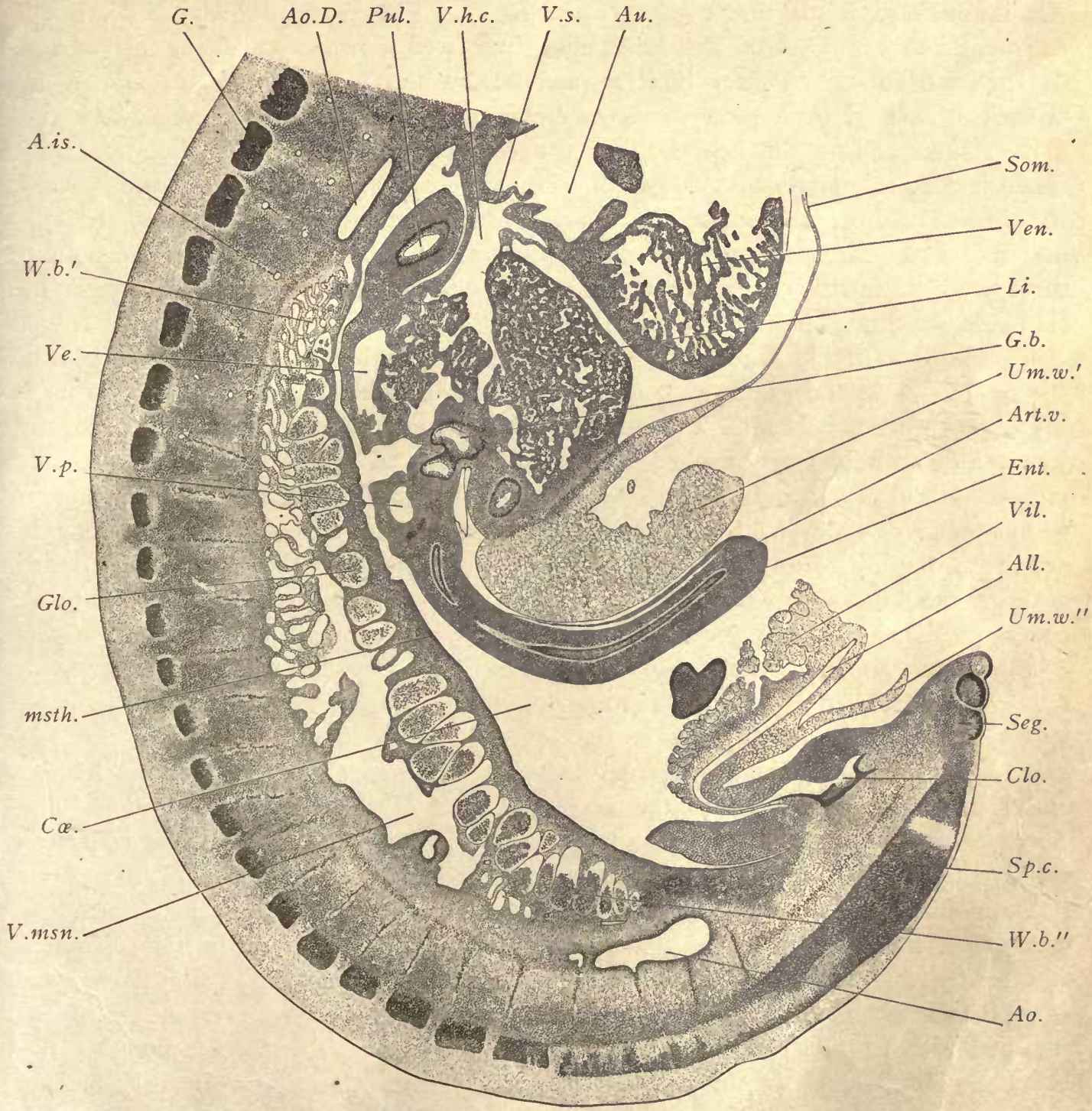

Fig. 184.-Pig, 9.0 mir. Sagrttal Series 53, Section 213.

A.is, Intersegmental artery. All, Allantois. Ao, Median aorta. Ao.D, Descending aorta. Art.v, Arteria vitellina. Au, Auricle. Clo, Cloaca. Co, Cœlom. Ent, Entoderm. G, Ganglion. G.b, Gall-bladder. Glo, Glomerulus. Li, Liver. msth, Mesothelium. Pul, Lung. Seg, Segment. Som, Somatopleure. Sp.c, Spinal cord. Um.w', Upper wall of umbilicus. Um.w", Lower wall of umbilicus. Ve, Vein in liver. Ven, Ventricle of heart. V.h.c, Vena hepatica communis. Vil, Villus. V.msn, Vena sub-cardinalis. V.p, Portal vein. V.s, Valvula sinistra. $W \cdot b^{\prime}, W \cdot b^{\prime \prime}$, Wolffiın body. $\quad \times 22$ diams. 
cavities do not show, the position of these vessels is marked by the darker line of tissue. The origin of one of these intersegmental vessels from the dorsal aorta, $A o$, is indicated in the lower part of the figure. The Wolffian body, $W \cdot b^{\prime}, W \cdot b^{\prime \prime}$, extends from the level of the lungs and liver well down toward the pelvic end of the embryo. Its ventral limit is marked by the body-cavity, $C \alpha$, and it is, of course, covered by a layer of mesothelium, msth, which here, as everywhere and at all stages, forms the boundary of the cœlom. In the Wolffian body we distinguish readily numerous sections of the epithelial Wolffian tubules, and toward the ventral side of the organ the characteristic glomeruli, Glo. Between the glomeruli and the mesothelium there is a layer of mesenchyma, but between the tubules there is little tissue, the intertubular spaces being almost entirely occupied by sinusoids developéd from the cardinal vein. The larger sinusoid or venous space, V.msn, is due to the section of the venous trunk which joins the lower end of the vena cava inferior, and is known as the sub-cardinal vein. In the upper part of the figure we encounter a section of the descending aorta, Ao.d, and of the lungs, $P u l$, or pulmonary anlage. The latter consists of a ring of entoderm bounding the central cavity and enclosed by a thicker layer of mesenchyma, which, again, is bounded by a layer of mesothelium. The space or cœlom about the lung is shown in the figure to be continuous with the cœlom of the abdominal region. On the ventral side we have the heart partly shown, the ventricle, Ven, being so cut as to exhibit the trabecular structure of the network of the sinusoidal spaces. The auricle, $A u$, is without sinusoids. The great venous trunk, vena hepatica communis, V.h.c, opens into the auricle, the opening being guarded by two valves, that on the dorsal side of the opening in the figure, V.s, being the left valve. The vein receives blood from the liver, $L i$, and from the Wolffian bodies, and it persists in the adult as the uppermost part of the vena cava inferior. The ductus venosus Arantii, which is so large in the human fetal liver, is less conspicuous in the pig; the ductus is the venous trunk formed by the union of the portal and umbilical veins within the liver; it joins the vena cava inferior to form the vena hepatica communis. The liver, $L i$, consists of liver cells or hepatic cylinders and numerous sinusoids of many diameters. On the lower side of the liver there is a considerable accumulation of mesenchyma by which the liver is united on the one end to the body-wall, Som, to the umbilical wall, Um.w', and to the mesentery by which the intestine is suspended from the liver. In this mesenchyma is lodged the gall-bladder, which is cut thrice. The reference line G.b runs to the uppermost of the three sections. The diameter of the gall-bladder is "several times that of the entodermal intestine, and its lining epithelium is thicker than any other epithelium of the embryo at this stage. The section of the bladder nearest the portal vein, V.p, corresponds to the beginning of the ductus cysticu Underneath the liver in the section of the mesentery is situated the portal vein, $V . p$. From the mesentery extends out the intestine (duodenum). It is a somewhat cylindrical tube which curves over ventralward and passes out through the opening 
of the umbilicus. It consists of a very small tube of entoderm, Ent, with only a small internal cavity (compare Fig. I86, Rect.). The thickness of the intestinal wall is due chiefly to the considerable development of the mesenchyma. The external covering of the intestine is a layer of mesothelium which becomes the peritoneal epithelium of the adult. In the tissue of the organ we distinguish the narrow vitelline artery, Art.v. The umbilical opening is very wide and is bounded both above and below by a prolongation, Um.w', Um.w', of the somatopleure of the embryo. The wall on the upper side is much thicker than on the lower. The umbilical opening is partly occupied by the duodenum. Appended to the inferior wall of the umbilicus is the allantois, All, which arises from the enlarged caudal end (cloaca), Clo, of the intestine. It passes out first inward, then makes an acute but rounded angle, and extends outward through the umbilical opening. It may, therefore, be said to consist of two limbs, one within the body of the embryo joining the cloaca, and the other passing out through the umbilical opening. The limb arising from the cloaca is completely united with the body-wall, and is, of course, upon the side toward the cœlom covered in by mesothelium. The lining of the allantoic cavity is an epithelium, and is a portion of the entoderm. Along the second limb of the allantois the mesothelium on the side toward the cavity of the umbilicus forms a series of clumsy projections, Vil, the mesothelial zilli of the allantois. They are smallest toward the embryo and increase in size distally. With higher power one can see that the mesothelium of the villi is very thin and the mesenchyma in their interior of quite loose texture. In later stages the mesothelium grows, the mesenchyma in large part disappears, and the villi then seem hardly more than small bags of mesothelium with but little contents, save some coagulum. They continue to enlarge until the embryo is 17 or $18 \mathrm{~mm}$. long, after which they begin to abort. In these older stages the villi extend far into the abdomen and are packed in between the abdominal viscera, presenting curious appearances in section. As the tail of the embryo is bent to one side, it offers us a section of a portion of the spinal cord, Sp.c, and at its tip a glimpse of three primitive somites, Seg.

Frontal Section through the Mid-brain and Fore-brain (Fig. 185).-Comparison with figure 165 (pig, $7.5 \mathrm{~mm}$.) will make it clear that in a frontal serics we shail obtain a few sections of the head which include only mid-brain and fore-brain and show no other special cephalic structures. The mid-brain, M.B, is somewhat rounded in form and passes over into the fore-brain, which is quite long and which already shows traces of its subdivision into two parts, the diencephalon, Dien, which lies nearest to the mid-brain, and the prosencephalon, Pros, which constitutes the terminal portion of the brain and which produces the lateral expansions which are to form the cerebral hemispheres. The expanding prosencephalon is separated by a constriction from the diencephalon, which in its turn is similarly separated from the mid-brain. The diencephalon and prosencephalon together represent the fore-brain. They are subdivisions of the primary first cerebral vesicle. 
It is important to note that they do not correspond to complete subdivisions, and have not the same morphological value as the three primary vesicles. The histological development is much less advanced than in the pig of $12 \mathrm{~mm}$. The ectoderm is very thin, consisting for the most part of a single layer of cells, but here and there the formation of a second layer is seen to be beginning. The mesoderm is very simple in character and almost uniform in appearance, but there is a distinct difference between the mesenchyma around the brain and that underneath the epidermis, the former having cells farther apart. This is almost the first stage in

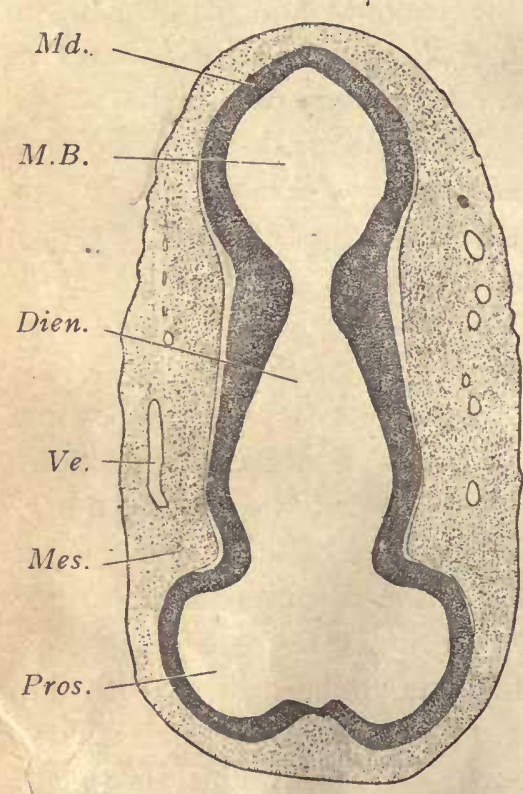

Fig. I85.-Pig, 9.0 mi. Frontal Series 54, Sectión 194 .

Dien, Diencephalon. M.B, Mid-brain. $M d$, Medullary wall of brain. mes,

Mesenchyma. Pros, Prosencephalon. "P, Vein. $\times 22$ diams. the differentiation of the arachnoid zone around the brain. The pia mater, however, though quite thin, is well defined by the condensation of the mesenchymal cells and by the somewhat numerous small blood-vessels in it. The medullary wall is everywhere quite thick and crowded with nuclei. In the region of the diencephalon the ectoglia is distinctly formed, but elsewhere has hardly begun its differentiation. On the inside of the medullary wall, close to the surface, there are everywhere very numerous mitotic figures.

Frontal Section through the Umbilical Opening (Fig. 186).-The illustration is part of the same section in the series from which figure 185 is taken. For convenience of comparison the position has been reversed so as to bring the dorsal side of the embryo uppermost in figure 186 . It results from this that right and left sides of the embryo are reversed in the engraving as compared with the other figures of transverse and frontal sections. By examining figure $\mathrm{I} 66$ (pig, Io $\mathrm{mm}$.) the student will see that sections in the frontal plane, owing to the curvature of the posterior end-of the body-wall, furnish transverse sections of the spinal cord of the pelvic region. Therefore, the section here figured, although part of a frontal series, is directly comparable to a transverse section of the body. In the upper part of the figure we have the spinal cord, Sp.c, and on one side of that the ganglion, $G$. Owing to the spiral twist of the embryo the section is not symmetrical, so that the posterior limb, P.L, appears only on one side of the section. Laterad from the nerve shown in the figure is the large muscle-plate, $M y$, the cells of which are already beginning to change into muscle-fibers. On the dorsal side of the plate we find its growing edge, m.pl, where the tissue of the muscle-plate proper bends over and passes continuously into the external wall of the somite. From this growing edge the cells are added to the muscle-plate by which it extends upward. 
The similar edge on the ventral side provides for the extension of the muscle-plate downward. In the median line, below the spinal cord is the small notochord, Nch, and the large median dorsal aorta, Ao. In the ventral portion of the embryo appears the large body-cavity into which protrude the Wolffian bodies and the intes-

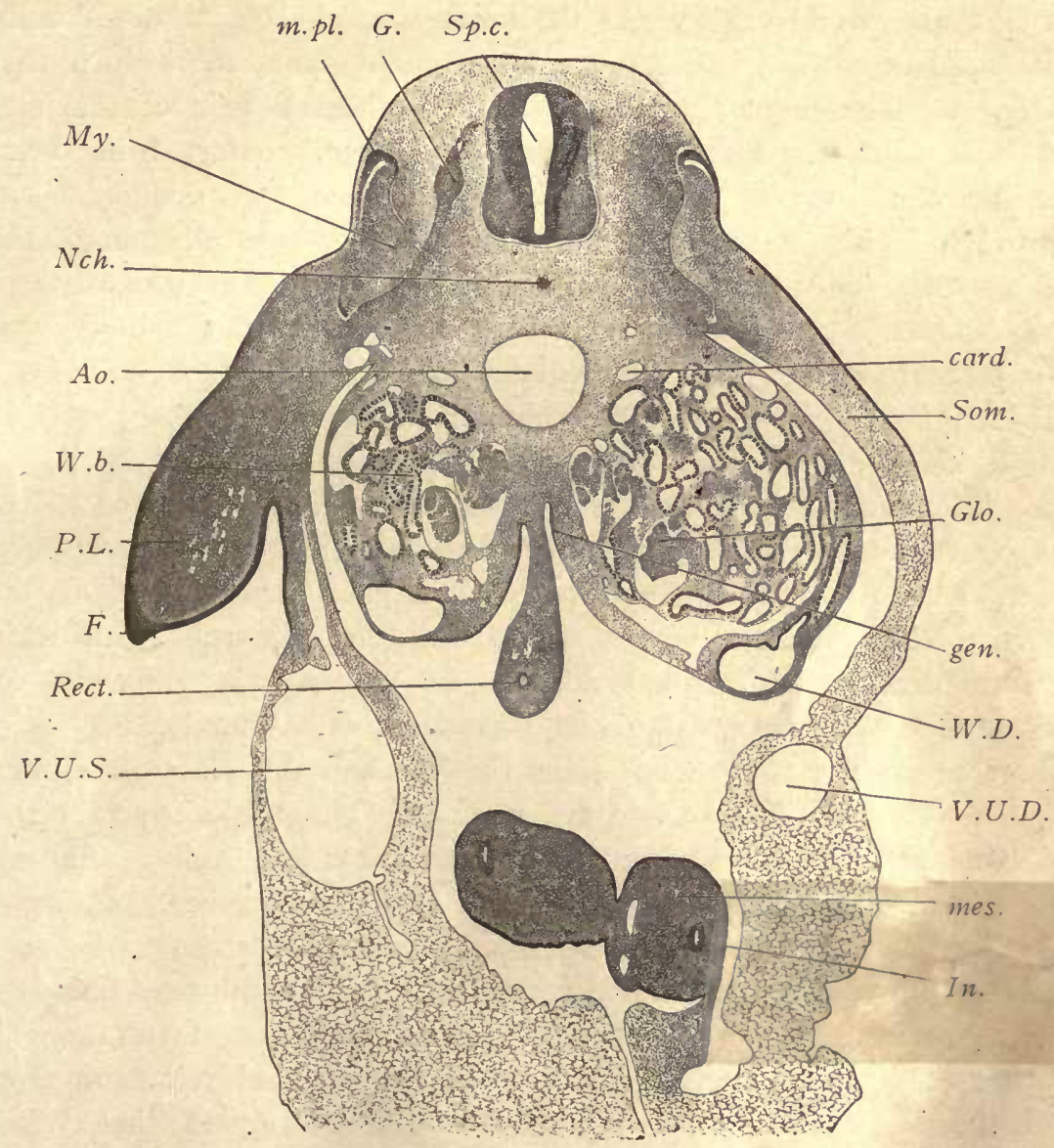

Fig. r86.-Pig, 9.0 mim. Frontal Series 54, Section r94.

Ao, Aorta. card, Cardinal vein. F, Ectodermal fold at the border of the limb-bud. G, Ganglion. gen, Genital ridge. Glo, Glomerulus. In, Small intestine (jejunum). mes, Splanchnic mesoderm (of the intestinal wall). m.pl, Dorsal growing edge of the muscle-plate. $M y$, Muscle-plate of secondary segment. Nch, Notochord. P.L, Posterior limb. Rect, Large intestine. Som, Somatopleure. Sp.C, Spinal cord. V.U.D, Right umbilical vein. V.U.S, Left umbilical vein. W.b, Wolffian body. W.D, Wolffian duct. $\times 35$ diams.

tine. The cœlom also has a downward prolongation into the beginning of the umbilical cord, and in this prolongation lies the so-called extra-embryonic loop of the intestine, In. The coelom is bounded everywhere by the layer of mesothelium represented in the engraving as a continuous line. With a higher power the meso- 
thelium is seen to consist of a single layer of cells, but varying somewhat in thickness in different regions. By following the contour of the mesothelium the student will recognize at once that all of the viscera are, in the anatomical sense, outside of the colom. The Wolffian bodies, $W . b$, are voluminous organs projecting from below the aorta on either side of the large intestine, Rect, and extending far into the abdominal cavity. At the lower ventral edge of the Wolffian body appears the Wolffian duct, W.D, a wide, longitudinal canal into which the Wolffian tubules open. The large size of the duct is characteristic of this stage. In later stages it is smaller. The tubules are very large, contorted in their course, and appear, therefore, variously cut. They are formed by a cuboidal epithelium and are provided with a sinusoidal circulation. The endothelium of the blood spaces can generally be seen fitting closely against the epithelium of the tubules. Here and there, however, there is some mesenchyma between the blood spaces and the walls of the tubules. On the median side of the Wolffian body are the glomeruli, which are of large size, and similar in structure to the glomerulus of the permanent kidney, though differing from the renal glomeruli in their proportions and in the details of their structure. It is not difficult to make a reconstruction of the course of a single tubule by following it through a few neighboring sections. The general course of a tubule is in the transverse plane, but it is much contorted. Each tubule begins at one of the glomeruli, with which it is in open communication. It then bends so as to make a somewhat irregular S-shaped figure, and finally opens into the Wolffian duct. After leaving the glomerulus it widens somewhat, but before it joins the Wolffian duct it again diminishes in diameter. The changes in diameter are gradual. The blood spaces or sinusoids of the Wolffian body are derived from the posterior cardinal veins. The veins and tubules, when the latter first become distinct, lie near together. As development coninnes both enlarge and encroach upon one another's territory; hence there is an intimate intercrescence of the blood-vessels and of the tubules, resulting in the formation of sinusoids. The whole of the Wolffian body might from one point of view, therefore, be regarded as a modification of the cardinal vein, and morphologically all of the blood spaces between the tubules belong to that vein. There remain typically two portions of the cardinal vein which are more or less open and distinct. The one on the dorsal side of the Wolffian body, card, may be conveniently regarded as representing the original cardinal vein. The other, on the ventral side of the Wolffian body, is at first not a very distinct channel, but gradually becomes more and more so, and is known by the distinctive name of sub-cardinal vein. It is a vessel of great morphological importance, since on the right side of the embryo it acquires a connection with the liver which renders it possible for the blood of the right sub-cardinal vein to pass through the blood spaces of the liver directly to the heart. This makes a very direct channel, a more direct one than existed previously, when the blood from the sub-cardinal came to join that of the cardinal, passing up to the common cardinal and then back to the heart. The new 
channel through the liver rapidly enlarges and becomes recognizable as the vena cava inferior. This important venous trunk is a combined vessel, comprising, first, a part of the sinus venosus of the heart; second, the vena hepatica communis; third, a large channel developed from the sinusoids of the liver; fourth, the upper part of the right sub-cardinal vein; and, fifth, the lower part of the right cardinal. The vena cava inferior has already been developed in the pig embryo of $9 \mathrm{~mm}$. Between the two Wolffian bodies hangs down the large intestine, Rect, suspended by its mesentery in the median line. The entodermal portion is a very small circle of epithelium with an extremely minute lumen, which in the section is scarcely larger than a single nucleus. The mesentery and intestine are covered by a welldefined mesothelium and have a considerable amount of mesenchyma, in which there is no distinct histological differentiation beyond the presence of a number of small blood-vessels. At this stage the large intestine runs nearly in the median plane to the pelvic end of the body. In the opposite direction, toward the head, it bends to the left of the embryo, making a loop which passes over into the end of the ileum. The ileum forms the continuation of the loop and extends into the cœlom at the base of the umbilical cord. There it bends back and returns toward the dorsal side of the embryo to pass over into the duodenum and join the stomach. Owing to the fact that the small intestine extends into the extra-embryonic cœlom of the umbilical cord, there makes a loop, and returns to the embryonic region, we get typically a double section of the intestine as shown in the figure, one of each limb of the loop. The entoderm, In, in these loops forms a small ring, which, however, is much larger than the entodermal ring of the large intestine at this stage. Each loop contains a large amount of mesenchyma, mes, the ceits of which are somewhat crowded, so that the tissue appears dark in the stained-section. The boundary between the body of the embryo and the tissue of tixe umbilical corrd is marked by the position of the two umbilical veins, that of the left sin I.U.S. being very much larger than that of the right side, V.U.D. By following ao. the somatopleure, Som, of the embryo, it will be seen that these veins are lodged therein, and that the continuation of the somatopleure beyond these veins forms the substance of the umbilical cord. The limb-bud, P.L, is a large mass of rather dense mesenchyma, entirely without muscles or nerves and covered by ectoderm. At the edge of the limb-bud the ectoderm shows a special thickening, $F$. The theory has been advanced that this thickening is homologous with the ectodermal fold which produces the fin of fishes, or at least that portion of the fin in which the fin-rays are developed.

Frontal Section through the Second and Third Gill-Clefts (Fig. 187). Is preparation the section hits the posterior wall, $O t$, of the otocyst and is just anterior to the origin of the glosso-pharyngeal nerve. The appearance of the section oi the hind-brain is characteristic for this region of young cmbryos. The deck-plate has grown gradually in size and forms a wide membrane, epen, the ependymal roof of the fourth ventricle. Owing to this growth of the 
deck-plate, the upper or dorsal limits of the dorsal zones, D.Z, are brought far apart and the cavity of the hind-brain is thus enlarged. The dorsal zone is divided by an angle in the interior and by the point of entrance of the nerve-roots. on the exterior from the ventral zone, V.Z. On their dorsal side the dorsal zones thin out and pass over gradually into the ependyma. The ependyma consists of a single layer of cells. In the dorsal zone the differentiation of the three primary layers of the medullary wall has scarcely begun, but in the ventral zones the three layers are already distinguishable, though not far advanced in their differentiation.

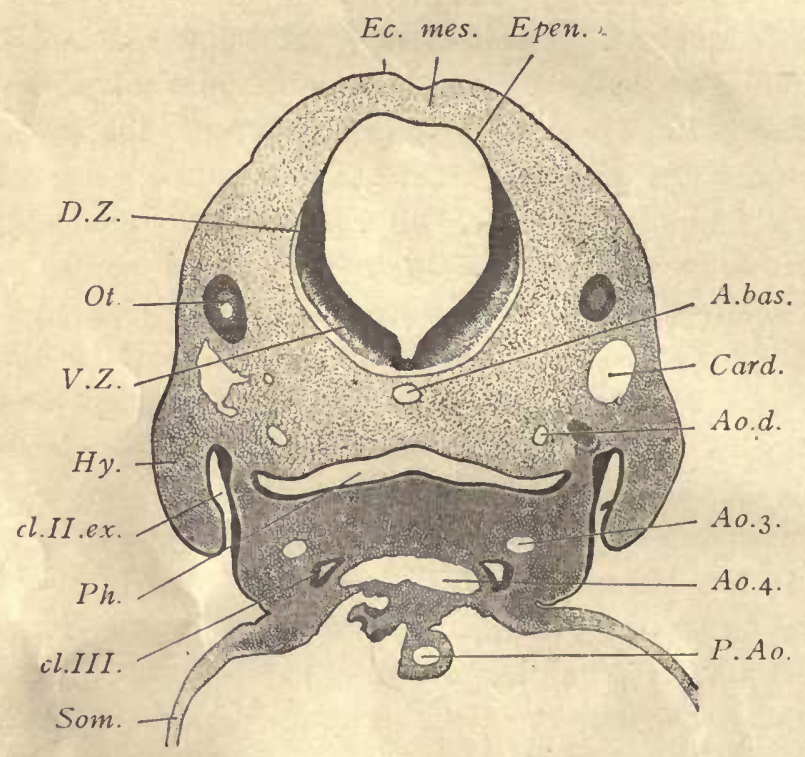

Fig. I87.-Pig, 9.0 mar. Frontal Series 54, Section 459.

Lisbas, Basilar artery. io.d, Descending aorta of the ieft side. A0.3, Third aortic arch. Ao.4, Fourth aortic arch arising from the median ventral aorta. Card, Anterior cardinal vein. cl.II.ex, External portion of the second gill-cleft. cl.III, Third gill-cleft. D.Z, Dorsal zone of the medulla oblongata. Ec, Ectoderm. Epen, Ependymal roof of the hind-brain. Hy, Hyoid arch. mes, Mesenchyma. Ot, Posterior wall of the otocyst. P.Ao, Pulmonary aorta. Ph, Pharynx. Som, Somatopleure. V.Z, Ventral zone.' $X 22$ diams.

In the floor-plate there are two layers. Below the medullary tube lies the basilar artery, A.bas, and below that, not far from the upper wall of the pharynx, lies the small round notochord in the midst of loose mesenchymal cells, which have not yet begun to condense themselves about the notochord. The pharynx is a wide space of rather small dorso-ventral diameter, and having a much thinner layer of cutoden $n$ on its dorsal than on its ventral side. Above the pharynx on either side lies the section of the descending aorta, Ao.d. The reference line to this vessel crosses a dark mass of cells which belong to the ganglion nodosum of the tenth nerve. Below the pharynx the section shows the third aortic arch, Ao.3, and the fourth aurtic arch, A0.4, just springing off from the median aortic frunk above the heart, so that the two fourth arches are connected across the median 
ne. Between the third and fourth aortic arches on either side is a small cavity ined by entoderm, cl:III, a diverticulum from the third gill-cleft. Immediately below the otocyst is the anterior cardinal vein, Card. From a point below the cardinal there extends a prolongation, $H y$, which may be taken as a portion of the hyoid or second branchial arch. It extends downward and consists of a mass of mesenchyma covered by ectoderm. It encloses a space, cl.II.ex, which may be regarded as the external portion of the second gill-cleft. In a neighhoring section (455) the prolongation of the pharynx shown in figure 187 can be traced still farther until it opens into this space, cl.II.ex. The second cleft is open upon both sides of the embryo, the first and third have closing membranes, the fourth cleft is not yet so far developed that its entoderm has come in contact with the epidermis of the embryo. The second cleft probably always becomes open, differing in this respect from all the others. Why it has this peculiarity we do not know. The opening does not persist, but the exact history of its closure is at present unknown. The process, $H y$, described as shutting in the external portion of the second gill-cleft has sometimes been termed the operculum, because it covers a gill-cleft opening, as does the operculum of a bony fish. In the lower part of our figure a portion of the somatopleure, Som, is shown where it extends ventralward to form the wall of the pericardial cavity. There is also included in the drawing a part of the pulmonary aorta, P.Ao.

\section{Pig Embryo of $12 \mathrm{~mm}$. Studied in Sections.}

A pig embryo of $12 \mathrm{~mm}$. has been selected as the center of study in this book because its anatomical relations are such that they may be readily grasped by the student who has already studied the anatomy of an adult mammal, human or other. At the same time the development of the organs is so advanced that their fundamental relations may be observed. From an embryo of this site the transition to the study of younger embryos is, even for the beginner, comparatively easy. It is not necessary that the embryo should be of this exact size; indeed, it may be somewhat advantageous for the student to have an embryo a milifineter larger, or one, two, or even three millimeters smaller, since the figures and explanations referring to the $\mathrm{I} 2 \mathrm{~mm}$. slage will enable him to identify all the struotures to be found and yet call upon him for the exercise of care and judgment in fying, from the data given in the following pages, the various parts in the what different stage he may be studying. Of $\mathrm{r} 2 \mathrm{~mm}$. pigs the author has $\mathrm{h}$ his disposal five good series belonging to the Harvard Embryological Collection

The transverse series is the most important, and should form the ba the study, and accordingly most of the sections figured are from such $A$ Next in importance comes the sagittal series, but it is desirable that every should have a series in the three standard planes at his disposal for sturly In the practical laboratory study each student should be required to make a series of accurate camera lucida drawings of carefully selected typial sections; then 
name correctly all the parts shown in each section and to identify the distribution of the three germ-layers in every case.* A sufficient number of high-power drawings ought to be added to illustrate the character of the various tissues.

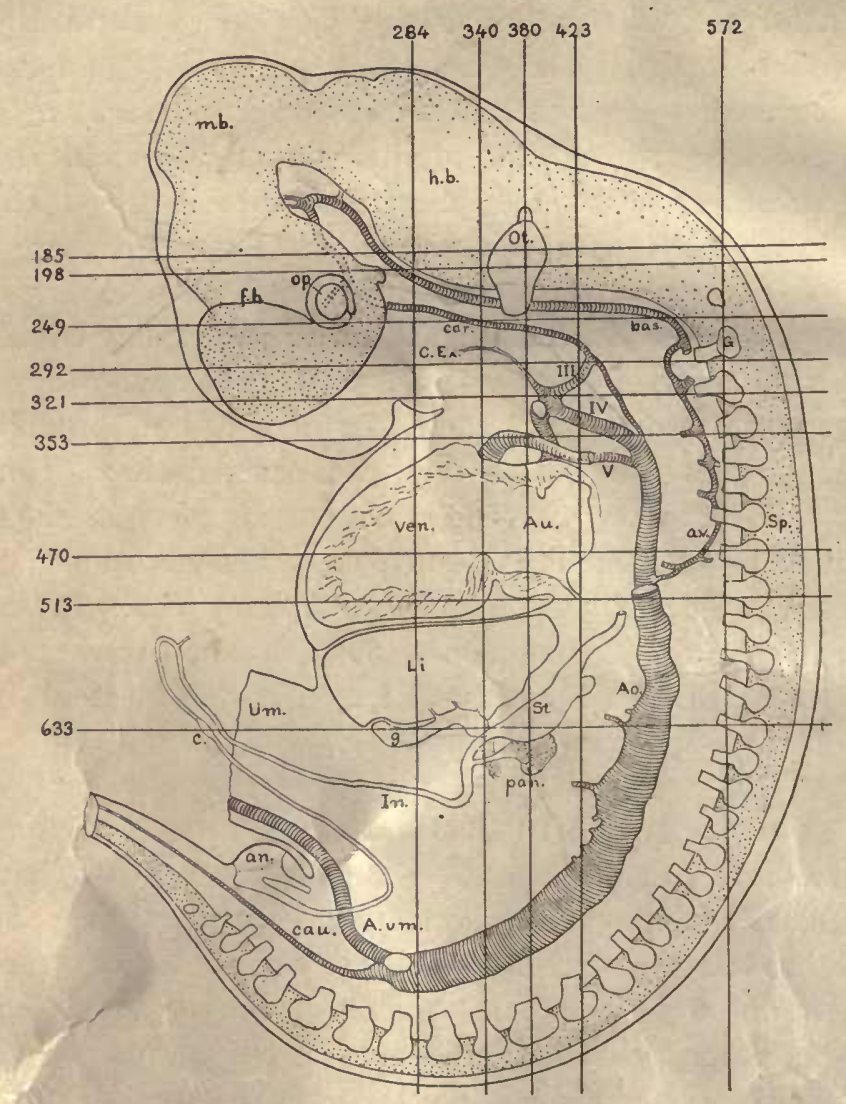

Fic. 188.-Reconstruction of a Pig Embryo of 12.0 Mm. With Indications of the Planes of SECtions FigURED.

un, Cloacal membrane. Ao, Aorta. Au, Auricle. A.um, Umbilical artery. a.z', Vertebral artery. bas, Basilar artery. c, Anlage of cxcum. car, Internal carotid. cau, Caudal artery. C.Ex, External carotid artery. $f, b$, Fore-brain. $G$, Spinal ganglion. $g$, Gall-bladder. $h . b$, Hind-brain. $I n$, Intestine. $L i$, Liver. $m . b$, Mid-brain. op, Optic vesicle. Ot, Otocyst. pan, Pancreas. Sp, Spinal cord. St, Stomach. Um, Umbilical opening. Ven, Ventricle of heart. $I I I, I V, V$, Aortic arches.
185 Transverse section, Fig. I 89
284 Frontal section, Fig. 203
198 Transverse section, Fig. I9I
340 Frontal section, Fig. 204
249 Transverse section, Fig. 192
380 Frontal section, Fig. 205
292 Transverse section, Fig. I93
423 Frontal section, Fig. 206
321 Transverse section, Fig. 194
572 Frontal section, Fig. 207

35. Transverse section, Fig. 195

470 Transverse section, Fig. I 96

513 Transverse section, Fig. 107

633 Transverse section, Fig. 198

* For making camera lucida drawirgs, a 3 -inch objective will be found convenient. An Abbe camera is recommended. Compare the directions for drawing in Chapter VIII. 
The accompanying forure 188 represents the outline of the pig embryo which was cut into the series of transverse sections from which figures 189 to 198 have been made. The student can casily identify the parts in the figure by comparison with that of the pig of $10 \mathrm{~mm}$. (Fig . 66), aided by the accompanying description of the same. The sections of this embryo are ro $\mu$ in thickness, and are 966 in number, not I200, as the student might expect. The discrepancy is due to the shrinkage of the embryo when imbedded in paraffin. The shrinkage is always very great, and in the case of embryos causes a loss of almost 20 per cent in the length; but as it seems to take place uniformly throughout the embryo, it causes no distortion, so that the embryo in paraffin is an exact though greatly reduced copy - so to speak - of the living embryo. It should be remembered that no correct measurements of the size of organs or cells can be obtained from sections made by the paraffin method. This limitation upon the use of sections is too often forgotten. The horizontal lines indicate approximately the levels at which the sections here figured belong. For convenience the direction and position of the frontal sections represented in figures 203 to 207 are also indicated approximately on the same outline, although, of course, the frontal series was from another embryo.

\section{Pig Embryo of $12.0 \mathrm{~mm}$. Study of Transverse Sections.}

The figures and descriptions, here presented of ten sections have been selected as illustrating the most important structures, with the exception of the umbilical opening and of the kidney, which can be better represented in sections from older or younger stages.

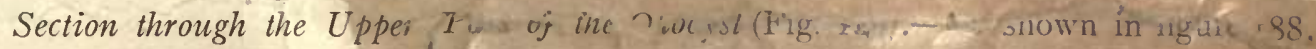
by the line 185 , this section is taken from a level about half-way between the eyc and the apex of the otocyst, ()t. It passes, therefore, through the fore-brain, F.b. and the fourth ventricle, V $\operatorname{m} . I V$, or cavity of the hind-brain. The section is bounded by a thin layer of epidermis, between which and the brain-wall there is a large amount of mesenchymal tissue. Alongside the hind-brain lies a series of important structures imbedded in the mesenchyma, which are identical upon the twin ciras. although they difer somewhat in the section, as the plane of cutting was not symmetrically transverse for the head. These structures are in the following order: $N$, ne trigeminal ganglior, $N \cdot 7,8$, the acustico-facial ganglion complex; $O t$, the otocyst, an oval vesicle with very distinct epithelial walls. Next the ninth or glosso-pharyngeal nerve (scarcely appearing in the section on the right side of the embryo) is shown by the upper portion of its ganglion on the exe (the right in the igure); the close to the me

posteri thr -nifloped 
to the ganglionic commissure which extends above the min of the hypoglossal nerve. On the left the continuity of this commissure is better shown than on the right, where it offers two parts, one, com, entirely ganglionic, and another, com.II, which comprises both the ganglionic portion and fibers which share in the forma-

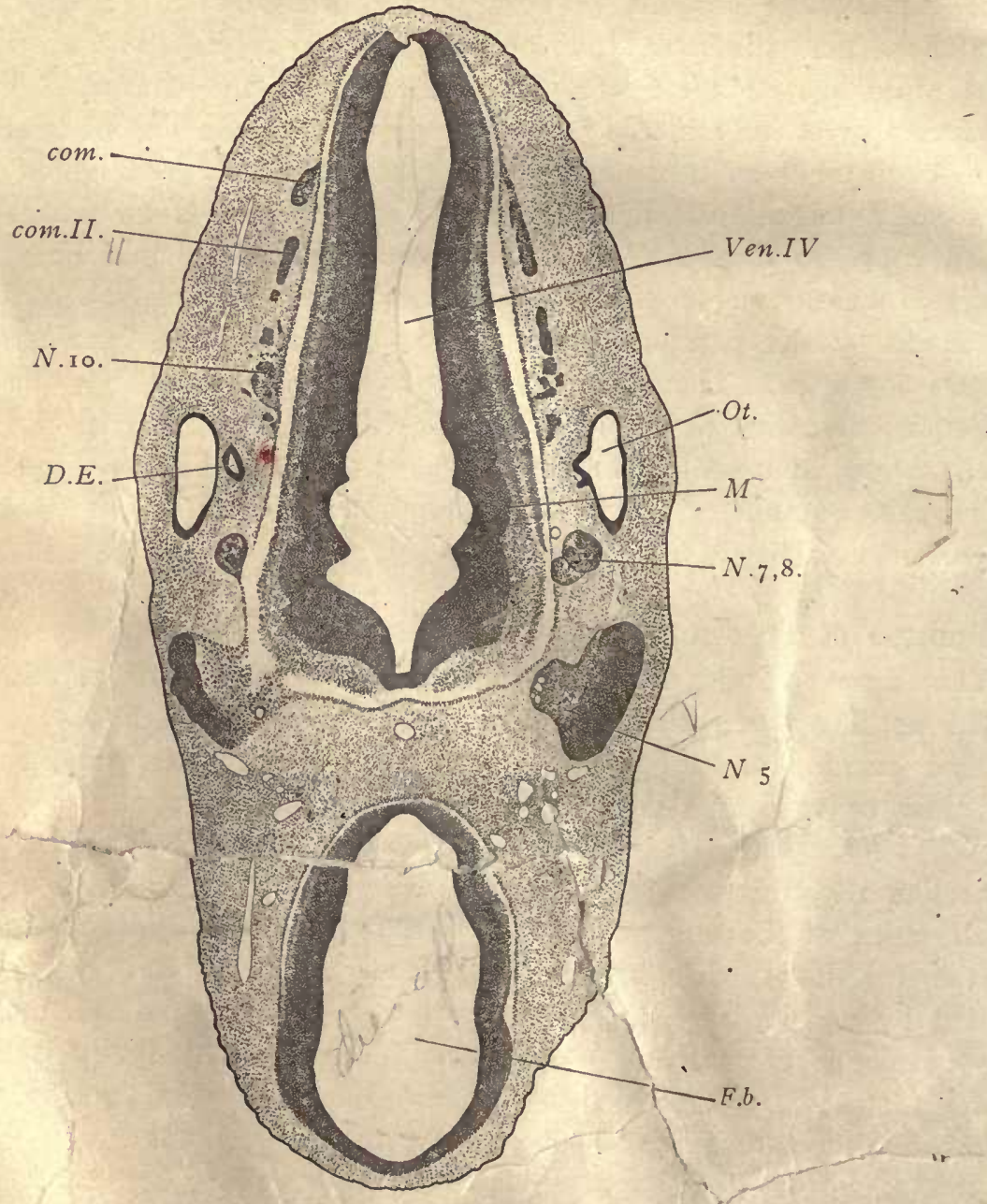

Fig. 189.-Pig, I2.0 mim. Transverse Series 5, Section 185.

com, Ganglionic commissure between hypoglossal and vagus nerves. com.II, Ganglionic commissure with fibers of the root of the spinal accessory nerve. D.E, Ductus endolymphaticus. F.b, Fore-brain. Md, Medulla 'lonuta. N.5, Trigeminal ganglion. N.7,8, Acustico-facial ganglion. N.ro, Jugular ganglion of the Of, Otocyst. Ten.IV, Fonurth ventrini- $Y ? 2$ diams. 
marks in the study of the topography of the embryonic head. The nerve-cells of the ganglion are grouped, for the most part, on the side toward the ectoderm, where they are closely crowded together, making a deeply staining mass. Nearer the brain-wall the tissue of the ganglion is much less condensed, is somewhat penetrated by small blood-vessels, and contains a considerable number of nervefibers, which are gathered into small bundles. Toward the brain-wall the bundles become distinct, and on the right side of the embryo the passage of the nervefibers into the brain can be readily seen. The nerve-fibers at this stage are merely neuraxons; that is to say, thread-like prolongations of the bodies of the nerve-cells (neurones). The fibers are entirely without sheaths. They stain very lightly, and hence, in the preparation, may be detected by their light appearance. The nerve-fibers may be conveniently rendered conspicuous by counterstaining the sections with Lyons blue. The nerve-fibers of the trigeminus, which enter the wall of the hind-brain, form in part a bundle of fibers, which extends along past the acustico-facial ganglia within the medullary wall. These fibers represent the commencement of the ascending trigeminal tract of anatomists. The other ganglia associated with the hind-brain are not well shown in this section. The otocyst (compare Fig. 42, p. 79) has a very sharply defined epithelial wall and is imbedded in loose. mesenchymal tissue. On the right side of the embryo we have the ductus endolymphaticus, D.E, the opening of which into the main cavity of the otocyst is shown on the left side. The epithelial wall of the ductus is thicker than that of the greater part of the otocyst proper. The wall, $M d_{1} \ldots$ of the hing brain exhibits already characteristic differentiations, for it shows clearly the inree primitive lavers; the outer neuroglia layer (éctoglia) is thin, and appears light in the section - use it takes the stain slightly. It is in this outer neuroglia layer (ectoglin) that the entire sensory nerve-fibers are primarily distributed, and, there fore, it is is a portion of this sver that we find the ascending trigeminal tract situated. Next to the ectoglia comes the middle layer, in which

the medullary wall are situated, and which is, therefore, termed the neurone gray layer (cinerea), easily recognizable under the microscope by its brighter color, which is due principally to the fact that the nuclei in this layer, though numerous, are much less crowded than in the innermost of the three layers, or primitive ependymal láyer, which at this stage is quite thick. Owing to the presence of nuclei, the gray layer is, of course, stained much more than the ectoglia. The nuclei of the brain-wall show as yet very little diferentiation. There are numerous mitotic figures which are situated exclusively close to the inner surface of the brainwall in the fore-brain. The structure the for -brain is similar, but the develor ment is less advanced; the differentiation of the neurone layer is only inn ning, and it has acquired little thickness. In the d-brain we ser mer along the region between the otocysts, a sea scalloped outline to the wall. nex incth one of the spac 
a neuromere. 'The neuromeres correspond in number and position to the neighboring primitive segments, and are, therefore, to be designated as segmental structures. They also bear an evident relation to the development of the nerves, and the accepted hypothesis is that from each neuromere springs a single nerve. The attempts which have been made to verify this hypothesis have met with very serious difficulties, for the relations are extremely complicated, and until the matter shall have been much more thoroughly investigated than at present, we must remain in the dark as to the precise morphological value of the neuromeres. But, inasmuch as they appear with the greatest constancy in the embryos of all vertebrates, we cannot help accepting the view that they are really structures of fundamental importance. At the stage we are studying the neuromeres have already begun to lose their distinctness, and in slightly older pigs can be traced only with difficulty. In younger stages their primitive characteristics are better shown (compare page 246). As regards the blood-vessels in the present section: there are small branches of the veins, which show outside of the ganglionic commissure, com; parts of the cardinal vein appear in close proximity to the trigeminal ganglion, and again at the side of the head. In the median line between the fore-brain and hind-brain, or nearer to the layer, appears a section of the basilar artery. Near the fore-brain on either side is the loop of the carotid artery. There are several important points to be observed in the region between the trigeminal ganglia and the fore-brain. In order to show these more clearly, a separate illustration (Fig. I90), on a larger scale, of this portion of the section is given. The trigeminal ganglion, the wall of the fore-brain, and the wall of the hind-brain will be at once identified, so that the correspondence with the general figure is easily followed. Between the trigeminal ganglion and the fore-brain are four veins, two of . which, Card' and Card"', are larger and are parts of the main cardinal stem passing from the region $n f$ the hind-brain to that of the fore-brain, while the iwo smaller ones, Card", are merely 'jranches of the same vessel. Close to the section, Card"', of the cardinal nearest the fore-brain lie the very small sections of the fourth, N.4, and third, N.3, cerebral nerves. The fourth nerve is minute in size and lies just behind the vein. The third nerve, though somewhat larger, is also very small and lies anterior to the vein somewhat on its medial side. Both of these nerves, owing to their small dimensions, are somewhat difficult to observe with the low power. The detailed figure brings out more clear/other points. It shows very clearly the junction of the trigeminal, $N .5$, and ar stico-facial, $N .7,8$, ganglia with the wall of the find-brain, and also the division of that wall into its trree primary layers, the Tin Ec.gl, the gray layer. Cin and ie inner or ependymal layer, Epen, and in floor-plate $R a_{2}$. Immediately below it is the basilar side ain is the section for the loop of the carotid, antericue nd of the basilar artery, which -ixhally symmetrical vertebral orresponds 
designated in the adult as the posterior communicating branch, by which the end of the carotid proper anastomoses with the basilar artery. At the side of the forebrain appears a blood-vessel, Card.L, which might be called the lateral cardinal. It is a branch of the main cardinal stem and passes over the side of the fore-brain

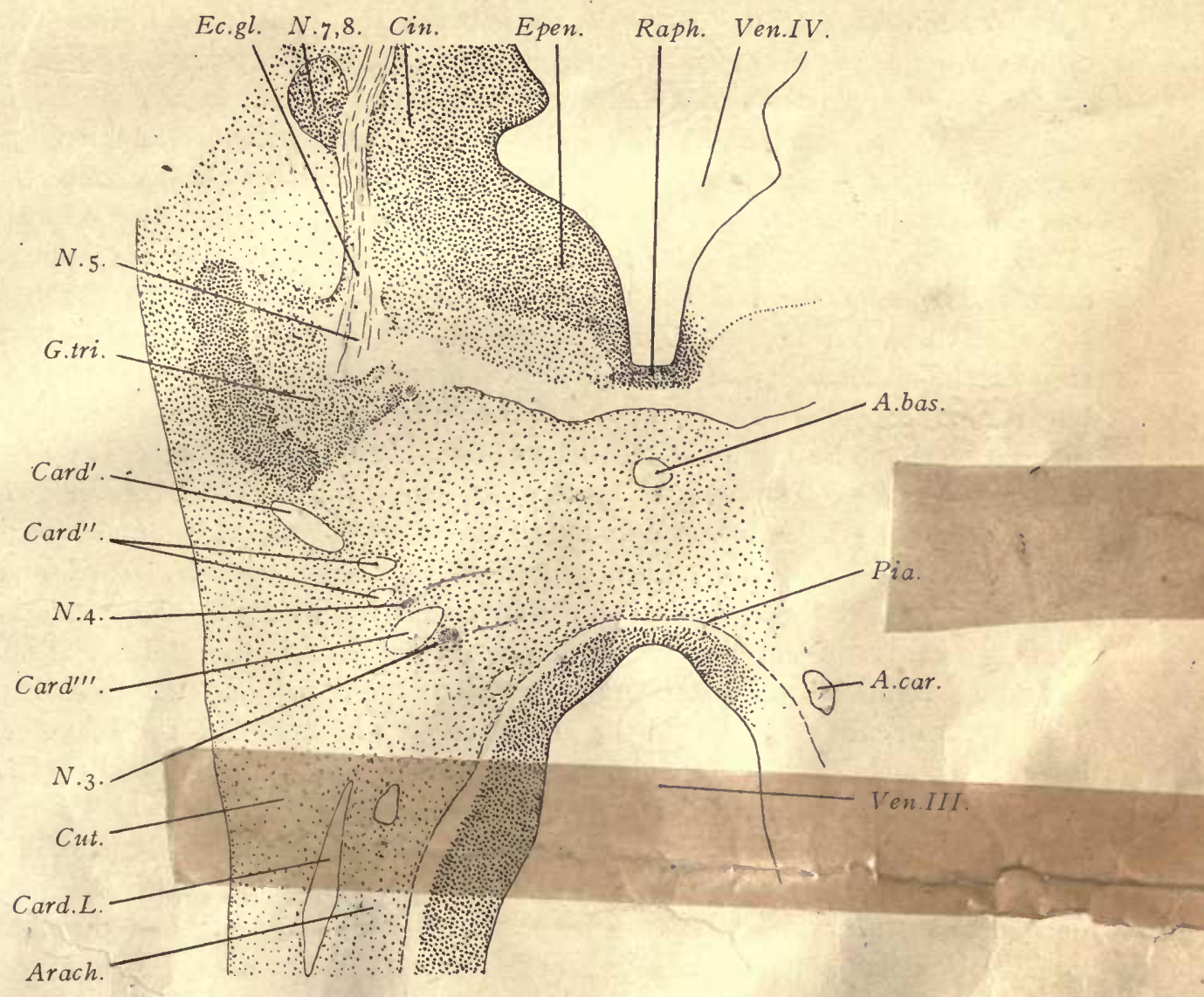

Fig. igo.-Portion of Figure i89 More Highly Magnified.

A.bas, Basilar artery. A.car, Internal carotid artery. Arach, Arachnoid zone. Cin, Neurone layer of medulla. Cut, Cutis layer, Card', Card", Card"', Anterior cardinal vein. Card.L, Lateral branch of the cardinal vein. Ec.gl, Ectoglia. Epen, Ependymal layer. G.tri, Trigeminal ganglion. N.3, Oculo-motor nerve. N.4, Trochlear nerve. N.5, Sensory root of trigeminus. N.7,8, Acustico-facial ganglion. Pia, Pia mater. Raph, Raphe of the medulla oblongata. Ven.III, Third ventricle of the brain. Ven.IV, Fourth ventricle of the brain. $\times 50$ diams.

toward the median dorsal surface thereof, where it meets the correspondir of the opposite side, with which it then unites to form a single mer: This vessel ultimately acquires great siz ad is known as the su' dinal simus. It is hown in figure 172 . in the median line, pursist to form 
in the embryo are all small branches of the veins when they first appear. Their great enlargement does not occur until comparatively advanced stages. Finally, attention should be paid to the following important modifications in the mesenchyma. Already there has been a rich development of a plexus of fine blood-vessels over the surface of both the fore- and hind-brain which has been accompanied by a slight condensation of the mesenchyma between the blood-vessels, thus marking a distinct membrane, in which we can easily recognize the pia mater, Pia. Outside of the pia mater comes a relatively broad zone; Arach, in which the cells are widely separated from one another and are connected by slender and long processes, so that the intercellular spaces are very extensive. This broad zone is the anlage of the arachnoid membrane. It is much more differentiated around the ventral portion of the brain than around the dorsal side. Between the arachnoid zone and the external epidermis the mesenchyma is somewhat more condensed and the cells are elongated in form, in part almost spindle-shaped, forming a layer, $\mathrm{Cut}$, which we may consider the anlage of the cutis, and perhaps, also, of the subcutaneous tissue, but this is doubtful. Between the arachnoid zone and the cutis zone, so placed that they cannot be quite said to belong to either one or the other, appear numerous blood-vessels. These form a more or less distinct vascular layer, which appears with remarkable constancy in all classes of vertebrates, and over a large part of the body. It may, therefore, be called the panchoroid. It is unquestionably of very great morphological importance, but its history is imperfectly known.

As regards the histological condition of the tissues, the student should make careful observations. Attention may be directed especially to the following points: The epidermis at the sides of the section is two-layered and consists of an inner layer of cuboidal cells, the anlage of the Malpighian layer of the adult, and of an outer layer of very thin cells, the epitrichium, the nuclei of which are flattened and appear darkly staired. Toward the mediutin line, above the hind-brain and Delow tiu fore-brain, the epidermis becomes gradually one-layered and much thinner. The mesenchyma exhibits three principal forms of cells: First, those which are equally branched in all directions, and represent a primitive form of the tissue. Such may be found in the neighborhood of the basilar artery. Second, the elongated cells of the cutis zone; and, third, the cells of the arachnoid zone above described. The blood-vessels have very distinct endothelial walls which are very thin, being thickened only to furnish space tor the nuclei, which, unlike those of the adult, project not only into the lumen of the vessel, but also against the surrounding mesenchyma. The red blood-corpuscles are rounded cells, someis oval, not infrequently son.ewhat distated. Their nuclei are nearly spherical intain a number of fine granules. Mitotic figures are quite frequent. A few. clei are beginning to charge $y$ becoming smaller and taking the stain icompare page 94). In nervous system the differentiation of the "hrain is more at than in the fore-brair but even in the in tion hotwe? irerve-cells and the young neuroglia 
cells (neuroblasts and spongioblasts) is not very clear. The nuclei are only just beginning to acquire distinct nucleoli, such as would be characteristic of later stages. The nuclei of the tissues differ markedly from those of the earliest embryonic stages, but can scarcely be said to have assumed in any of the tissues adult characteristics

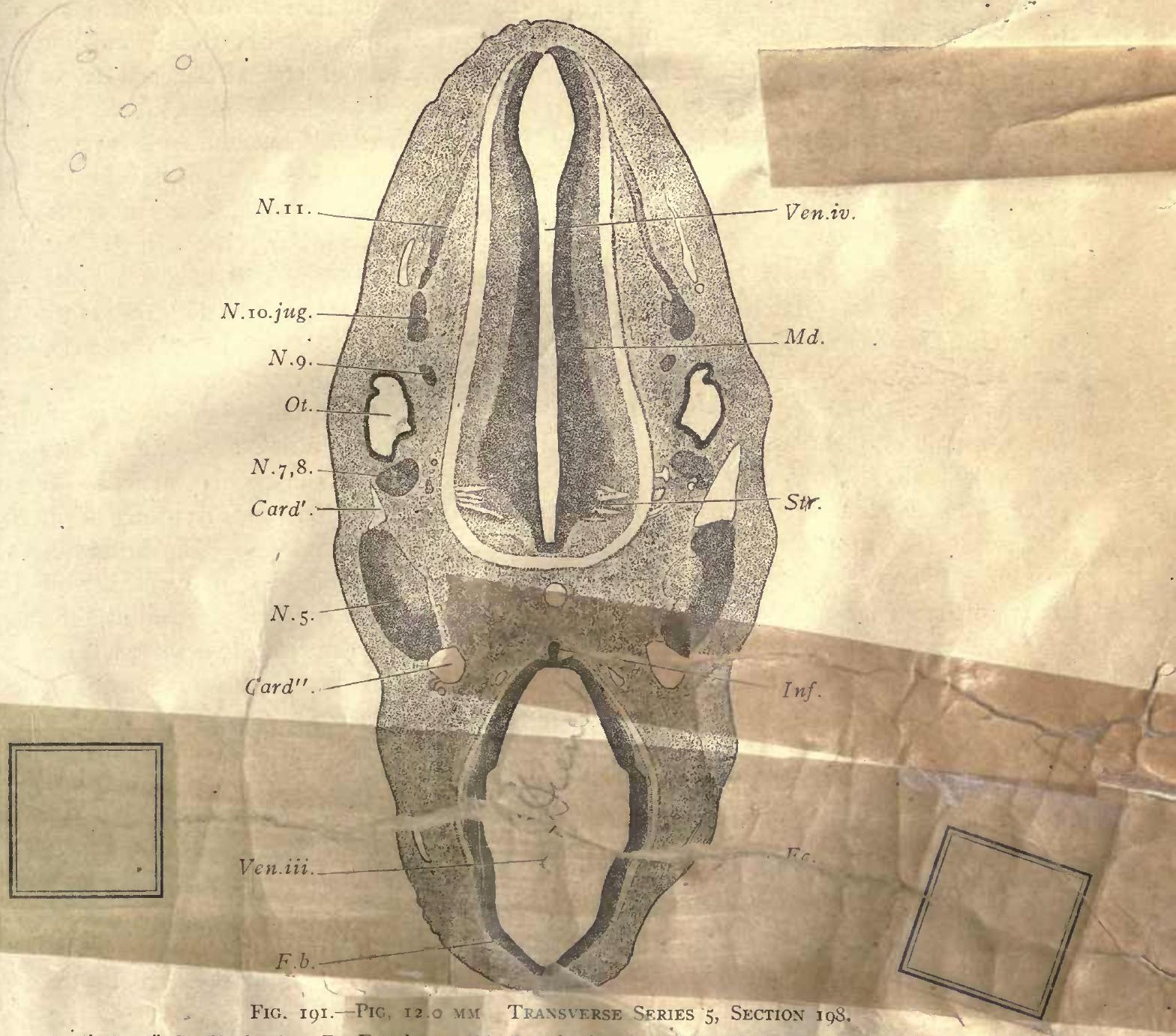

Card', Card", Cardinal vein. Ec, Ectoderm. F.b, Fore-brain. Inf, If fundibular gland. N.5, 'Trigeminal ganglion. $N \cdot 7,8$, Acustico-facial ganglion. N.f, Ganglion nodosum of the glosso-pharyngeal nerve. N.ro jug, Jugular ganglion of the vagus nerve. N.r., Root of th, spinal accessory nerve. Md, Medulla gata. Ot, Otocyst. Str, Strite acusio - Ken.iii, Xhird ve itricke. Ven.iv', Fourth ventricle.

Section through the Lner Parl of the Otocyst (Fig. section 19s, and, therefore, th sections belo. bring out three points not showe in the 
spinal accessory nerve, N.II, which arises from the cervical (in the figure upper) end of the hind-brain and runs forward to join the vagus ganglion, N.ro jug, the jugular ganglion of the adult. $2^{\circ}$, the characteristic relations of the anterior cardinal vein to the trigeminal ganglion, N.5. The vein is cut twice, Card and Cark", for it curves around the ganglion, passing on the inside of the ganglion between it and the wall of the brain. The original vein persists throughout life in this position, and enlarges into the cavernous simus of the adult. Inside or mesially of the seventh to twelfth nerves the cardinal vein is obliterated, and is replaced by a new vessel produced by "island formation" outside these nerves, and designated as the vena capitis lateralis. It is, as it were, interpolated in the course of the original vein, and this interpolation is the principal factor in transforming the embryonic anterior cardinal into the adult jugular vein. In the 12 mm. pig the vena capitis Jateralis is formed outside the otocyst and of the seventh and eighth nerves. Later it extends by more island formations outside the ninth to twelfth nerves also. The jugular, therefore, is to be defined as.the anterior cardinal vein which, by successive island formations, has migrated to a new position outside of the otocyst and cephalic ganglia. $3^{\circ}$, to show the infundibular gland, Inf, a small evagination from the ventral floor of the fore-brain, $F . b$. The evagination is really hollow, but the cavity does not appear in the section figured. It enters into very close relations with another hollow evagination, which springs from the dorsal roof of the oral cavity and is known as the hypophysis. The infundibular gland and the. hypophysis become intimately associated with one another in their further development and give rise to the pituitary body of the adult, the gland becoming the posterior lobe, the hypophysis the anterior lobe of that organ. The hypophysis may be best studied in sagittal sections (see page 292). The present. section, figure 191 , being at a lower level than figure 189 , passes through 1.ie ventral portion of the hind-brain and shows only a narrow part of the cavity of the fourth ventricle, Ven.iv. The three lavers in the wall, $M d$, of the hind-brain

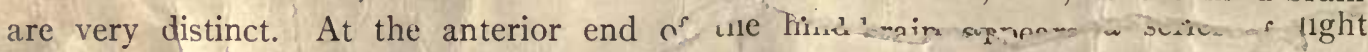
lines, Str, which are cumed by nem-e-fibers. These lines have been identified as the stria armovace. They need to be more accurately studied, however, for they seem resher to be fibers of the lateral root of the facial nerve. Close to the anteIIor section of the cardinal vein, Card", appear the minute fourth and third nerves, which, however, are not indicated in the figure. Both-lie close to the wall of the vein on the side away from the trigeminal ganglior. The fourth nerve lies nearer the ousside of the embryo, the third nerve nearer the median plane. At about me level as this part of the jugular vein, and very close to the wall of the in, is situater tha loop of the internal cirrotic. Lower down, but not close 1] of the gematic in, is the section of the lateral jugular.

orst Gill-Cleft and Optic ingginntions (Fig. 192). - The upper cervical fegion of the spinal cord, on tha ontion rives. In this and the three 
sections next following the complicated pharynx appears in various forms. The general shape of the pharynx has been described with the aid of a figure of a wax model of the pharynx made from the same series of sections from which these

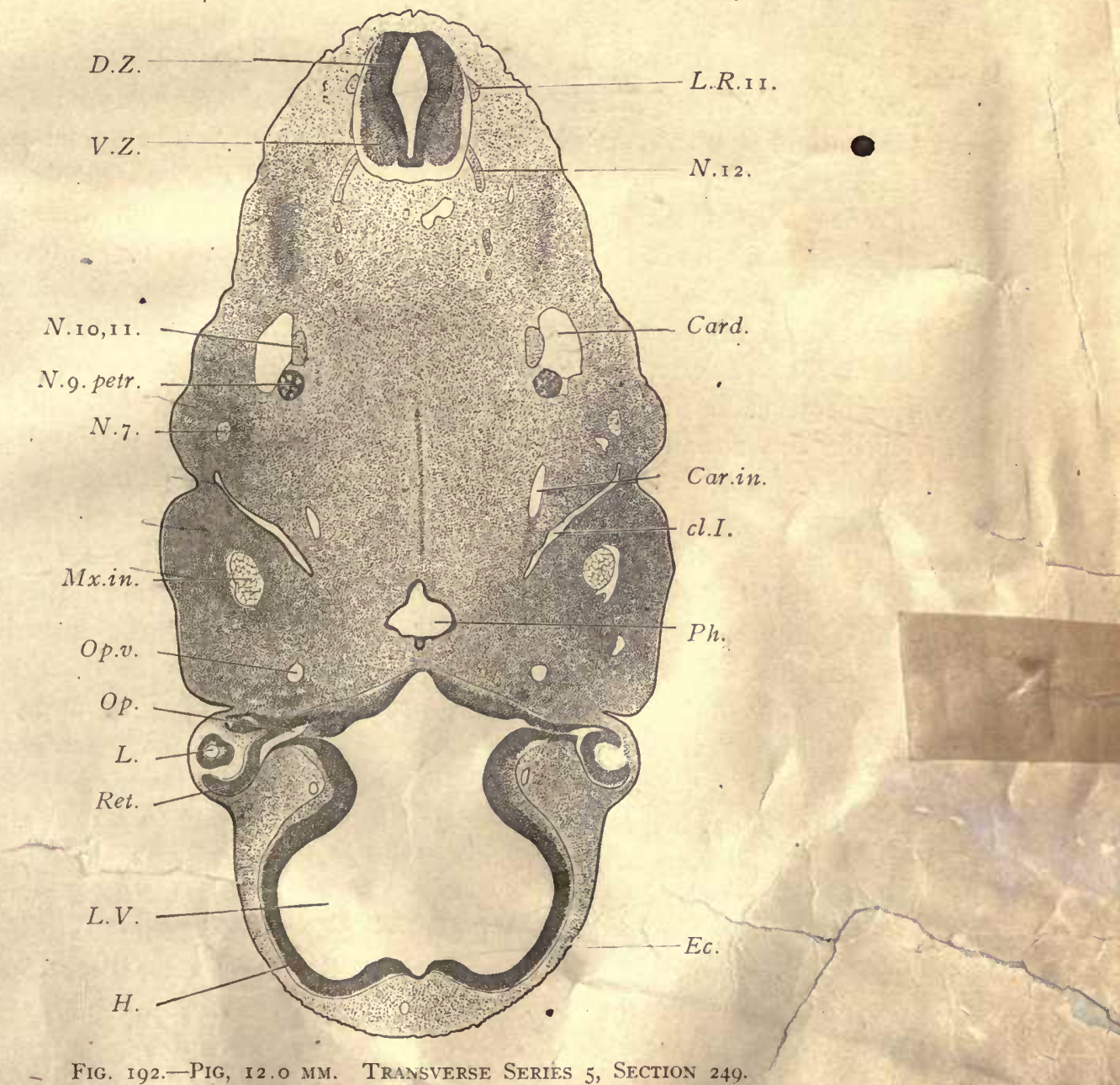

Card, Anterior cardinal vein. Car.in, Internal carotid artery. cl.I, First or auditory gill-fleft. D.Z, Dorsal zone of spinal cord. Ec, Ectoderm. H, Anlage of cerebral hemisphere. L, Lens. L.R.rr, Lateral rout of the eieventh nerve. L.V, Lateral ventricle. Mx.in, Inferior maxillary nerve. $N_{. j}$, Facial nerve! N.9.petr. Petrosal ganglion of the ninth nerve. N.10,1 I, United vagus and spinal accessory nerve. N.12, Hypo glossal nerve. $O p$, Stalk of the optic evagination. Op.v, Ophthalmic vein. Ph, Pharynx. Ret, Retina. $\checkmark . \angle$, Ventral zone of spinal cord. $\times 22$ drams.

figures are taken (compare Fig. I73, p. 237). The shape of the pharynx and of its four pairs of latel colies at this stage is remarkably constant, so that the st a dent is not likely to encounter any serious difficulty in dentiffiris the spinal cond is oval in the sertion. Its cavity has exprnded in the $m^{i}$ 
lateral walls are quite thick, the median ventral wall is thinner, and the median dorsal wall (deck-plate) is very thin. The three primitive layers of the medullary tube are very clearly marked out, the ectoglia appearing light, the ependymal layer appearing dark. The differentiation is much more advanced on the ventral side of the spinal cord than on the dorsal side, and, indeed, it is only in the ventral part that the three layers are perfectly differentiated. In the median ventral line we have the floor-plate, in which we can distinguish only two zones, while in the deck-plate there is no differentiation of layers whatever. The spinal cord is clearly divided into a dorsal zone, D.Z, and a ventral zone, V.Z, on each side. The two dorsal zones are connected across the median line by the thin deck-plate, and the ventral zones similarly by the thin floor-plate. The lower or ventral limit of the dorsal zone is marked by the entrance of the dorsal or ganglionic root and by the fibers, which represent the outgoing lateral roots. In the actual section figured, the lateral roots, L.R.ri, are those which enter into the formation of the eleventh nerve. The true dorsal root does not appear in the figure. Internally the division between the two zones is marked by the lateral angle of the central cavity shown in the section. In the dorsal zone the differentiation of the three layers has made slight progress. In the ventral zone, however, the development is far more advanced. The most characteristic feature of this movement is the growth of the inerea or neurone layer, which increases in a twofold manner: first, by encroaching upon the inner or ependymal layer; and, second, by the growth of its contituent elements. Examination with a high power shows at once that the cells lave grown very much. Their nuclei are larger, granular in appearance, rarely with any indication of a distinct nucleolus. Most of the cells are neuroblasts and have well-marked protoplasmic bodies, finely granular in texture. They have-many f them already produced long, slender outgrowths which we can identify as the suraxons. In order to study the distribution of the neuraxons and the form of he nerrablasts, it is necessary to apply the Golgi rapid method, by. which it can - drmonswated that a portion of the neuraxons is distributed entirely within the edullary vall, while another portion passes out to form ventral roots, one of which, T.I2, forming part of the hypoglossal nerve, is shown in the figure. A third brtion of the neuraxons, at least in the upper cervical region, as also in the inedulla oblongata, passes out to form the lateral roots. The positions of the exits these two bundles of nerve-fibers are constant and characteristic. The ventral not always passes out from the middle of the ventral zone about half-way between the median floor-plate and the dorsal limit of the zone. The lateral root alvays passes out at the $\mathrm{u}_{\mathrm{r}}$ er dorsal limit of the ventral zone and immediately below the point of entance of the true dorsal root. Formerly the lateral roots were distinguished from the dorsal roots. Following downward in the figure we 70 section of the cardinal vein, Card, just inside of which lies the common $\checkmark$ of the united tenth and eleventh. or vagus and accessorius norves, the lower part of the petrosal ganglion, N.9.petr, of the glosso- 
pharyngeal nerve. Lower down and nearer the ectoderm lies the facial nerve, $N .7$, situated in what is called the hyoid arch or mass of tissue intervening between the first and second gill-clefts. The hyoid arch is further marked by a bulge in the external outline of the section, which leads down into a deep groove beyond which the outline of the section again rises and arches forward to the eye. This groove is the external depression of the first gill-cleft and ultimately is transformed into the external auditory meatus. The position of this groove is well shown in figure I66, $A u$, on page 223. Just inside the auditory groove appears the outer end of the first or auditory internal gill-pouch, cl.I. It is a long, oblique slit, quite narrow, and is lined by a layer of entoderm. If we follow it along through several sections, we shall find that higher up its outer end comes in contact with the ectoderm at the bottom of the auditory groove, and there the two germ-layers, entoderm and ectoderm, unite to form a single membrane, the closing plate of the gill-pouch. Following through the section downward in the series, we can trace the cleft to its connection with the pharynx, $P h$. On the posterior side of the cleft we find the internal carotid, Car.in. Only the roof of the pharynx, $P h$, is cut, so that it occupies but a small area in the section. On its anterior side it shows a small knob-like projection toward the floor of the fore-brain. This is a part of the stalk of the hypophysis: Below the first gill-cleft appears the very large and conspicuous inferior maxillary nerve, $M x$.in, and beneath that the section of the small ophthalmic vein, $O p . v$. The fore-brain is quite complicated in shape, having two lateral expansions, $L . V$, of its cavity which are destined to form the lateral ventricles. The walls; $H$, of the lateral ventricles are the anlages of the cerebral hemispheres. From the ventral (in the figure upp $r$ ) part of the fore-brain spring on either side the optic stalks, $O p$. These are hollow prolongations of the brain, which expand at their distal ends to form the retina of the eyen Ret, and the pigment layer. The expanded distal ends constitute each a sort of cup, of whin the optic stalk is the stem. The cup is two-layered, the space between the two layers being a prolongation of the central cavity of the brain. The inner of the two layers forms the retina proper and is considerably thickened. The outer layer is quite thin and is already quite abundantly laden with pigment granules. At the edge of thcup the pigment layer passes over uninterruptedly into the thick retina layer. In the cavity of the optic cup lies the vesicular lens, $L$, which arose from an evagination of the overlying ectoderm. The vesicle is, however, now completely separated from the layer which produces it. It has at this stage a very large cavity, and in adiacent sections it can be readily seen that the innce side or that toward the brait is already thickening and changing its character so as to form the nuain part of the adult lens. The thickening depends chiefly upon the rapid and encrmous 1 manation of the epithelial cells of this part of the vesicle, so that they are transformed inw, the so-called fibers of the adult lens, each adult fiber

Section through the Second Gill-Cleft and Oral Fissure (Fig. 19.2).-The level of his section is such that th head is cut separately and appears in section without 


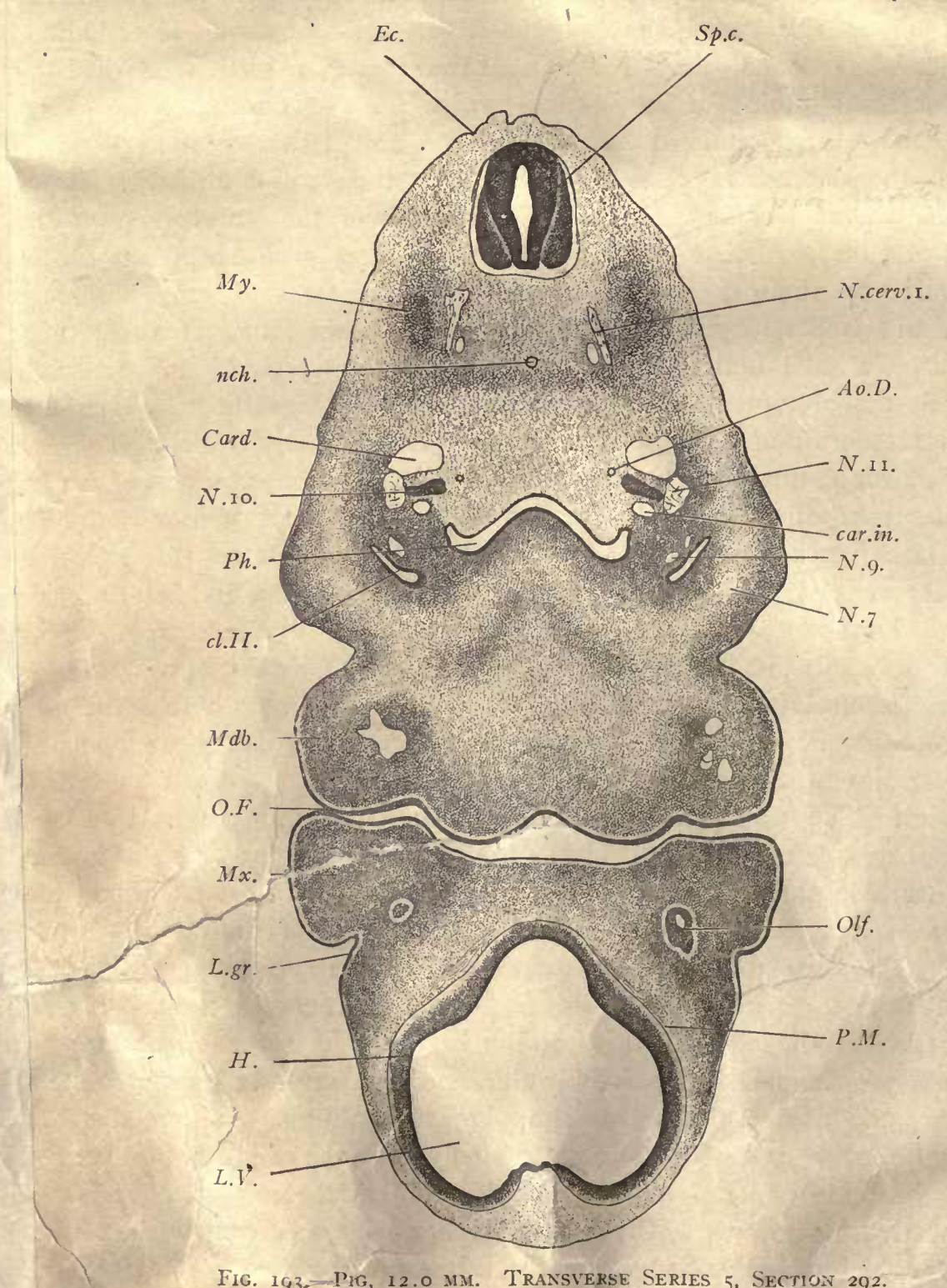

An. (1), Deseending aorta. Card, Anterior cardinal vein. car.in, Interna! carotid artery, cl.II, Second gill-cleft Ec, Ectoderm. H, Anlage of cerebral hemispheres. L.gr, Lachrymal groove. L.V, Lateral ventricle of brain. Mdn, Maddibular arch. $M x$, Maxillary process. My, Myotome. nch, Notochord N.cerv.1, first cervical nerve. $N .7$, Facial nerve. $N .9$, Glosso-pharyngeal nerve. $N$.Io, Vagus nerve. N.II, Spinal accessory nerve. O.F, Oral fissure or space petween the head and mandibles. Olf, Olfactory pit. Ph, Iharyns P.M, Pia mater. Sp.c, Spinal cord. $\times 22$ diams. 
connection with the body of the embryo. The space O.F, between the head piece and body piece, may be designated as the oral fissure, since it is into this space that the mouth opens. In general there is considerable resemblance between this and the section last described, but in the present section the eyes have disappeared and we get the first indications of the nasal pits, Olf. That on the left side of the body shows a trace of the cavity of the pit. The posterior part of the pharynx, $P h$, is cut in the section, instead of the anterior part as in figure 192. The first gill-cleft does not show, but the second cleft, cl.II, does. It lies posterior to the first cleft and therefore appears higher up in the figure. The spinal cord, Sp.c, shows the same general structure as in the previous section. On either side of it may be seen the small and inconspicuous root of the eleventh or accessory nerve. It could not be properly represented in the figure. Some distance below the cord lies the small circular section of the notochord, which differs so slightly in staining from the surrounding mesenchyma that it cannot be well made out without the use of a higher magnifying power. It is enclosed by a distinct membrane which is thick enough to produce a double outline, and contains a considerable number of scattered nuclei, which are, however, nowhere much crowded. The nuclei are round in form, decidedly larger than those of the surrounding mesenchyma, granular, and containing each several more conspicuous, dirkly staining granules. There is a very slight gathering. of mesenchymal cells about the notochord, as if to form the anlage of a sheath. Just below the notochord there is a broad band of somewhat darker staining, due to a greater condensation of the mesenchyma in that region, and this condensation represents the beginning of the formation of the vertebral structures. On either side we find the transformed myotome, $M y$, or anlage of the striated muscular tissue. This tissue is produced from the secondary somites of earlier stages. The cells have now separated from one another, have lost their distinctly segmental grouping, and begun to elongate into true muscle-fibers. All that can be distinguished with the low power is the somewhat darker appearance of this part of the section, due to the great crowriling of the nuclei. Between the muscular anlage and the notochord ". crition shows a portion of the first cervical nerve, N.cerv.I, and just w. nerve is a small blood-vessel not represented in the figure. There is a similar blood-vessel symmetrically placed on the opposite side. They are the small vertebral arteries. The anterior cardinal veins, Card, are large and con picuous vessels, but despite their size they have merely endothelial walls and there is no condensation of the mesenchymal cells around them, although such a condensation is to take place later to form the anlages of the muscular and cornect ive-tissue coats (media and adventitia) of the adult. On the dorsal side of he cardinal vein and close to it is a light spot in which can be easily distingu with the high power, several more ar less distinct bundles of nerve-fibers whic stparated from one another by mesenchymal cells. For this reason it is what difficul to recognize this nerve with the low power or to repiesent it 
figure. On the ventral side of the vein there appears a darkly stained mass, $N$.ro, the nodosal ganglion of the vagus nerve, and outside of this ganglion is the section of the spinal accessory nerve. Immediately below the nodosal ganglion we have the internal carotid artery, car.in. A little to the inside of the jugular is a small vessel, Ao.D, of great morphological importance. The corresponding vessel appears on the opposite side. Although. very small, this vessel has a distinct coat of condensed mesenchyma around its endothelium. The two vessels are the descending aorté, which have almost completely aborted, and in slightly older specimens will be found to have disappeared altogether. The descending aortæ are the longitudinal trunks by which the dorsal ends of the five aortic arches of early stages are connected together. The portion shown in this section is the part of the descending aorta between the tops of the third and fourth aortic arches. The relations are shown in the reconstruction (Fig. 172). The pharynx, $P h$, is narrow in its dorsal ventral diameter, but wide transversely, and offers the very characteristic yoke-shaped figure in the section. The distal portions of the second gill-clefts are shown, and they appear disconnected from the pharynx, the connection occurring in sections higher up. Each cleft is somewhat slit-like, so that its cavity is an oblique fissure and somewhat parallel in position to the first cleft (Fig. I92). Both the pharynx and the gill-clefts are, of course, lined throughout by entoderm, which forms a sharply defined layer crowded everywhere with nuclei, which are of about the same size as those of the surrounding mesenchyma. In the pharynx the entoderm is somewhat thinner on the dorsal than on the ventral side. In the clefts it is thicker than in the pharynx proper, and especially in the clefts it may be observed that the mitotic figures always occupy a superficial position. On the dorsal side of the cleft is a very small blood-vessel, near which, with a higher power, one may see a small nerve, and nearby, but more dorsalward, a second nerve. Both of these are branches of the glossopharyngeus, and lie behind the cleft. They are, therefore, termed the post-trematic branches. Below the cleft and somewhat on its median side is a similar third nerve-branch, the pre-trematic of the glosso-pharyngeus, runnirg in front of the cleit. The outline of the embryo forms a rounded eminence outside of the second cleft; it represents in part the hyoid arch. In the midst of the mesoderni this appears a-light area with a few nerve-fibers, the end of the facial nerve, $N$... The mandibular arch or process, $M d b$, is very distinct and prominent. It is separated from the hyoid arch by a deep external notch, which corresponds to the external first or auditory cleft. In the interior of the mandibular process there are light spaces differing in their exact distribution on the two sides of the spcrilmen. These spaces contain Ifrve-fibers and they represent the inferior maxilerves. We now come to the oral fissure, O.F, which separates the body he head. In the head portion of the section we have the maxillary process, hich is separated in part from the rest of the head by the deep lachrymal L.gr. On either side thure shows a shaving from the epithelium of tie 
olfactory chamber, Olf. The fore-brain has expanded laterally, L.V, to form the lateral ventricles, the walls of which, $H$, are the anlages of the cerebral hemispheres. On the dorsal side, which is the lower side in the figure, the hemispheres project somewhat, leaving a median space between them. This median space is filled with mesenchyma, which may already be regarded as the anlage of the falx. In the tissue of the falx are two very small blood-vessels, the forward prolongations of the lateral jugulars, which are to unite to form the median superior longitudinal sinus. In the previous section these vessels also reappear, but are already united (Fig. 192). In the median dorsal line the wall of the fore-brain is thin and shows a characteristic notch. Close to the surface of the fore-brain there is a very distinctly marked vascular layer, the commencing pia mater, P.M, and with a high power it can be easily seen that the differentiation of the arachnoid zone has already begun.

Section through the Third Gill-Cleft and Nasal Pits (Fig. I94).-In this section the head is clearly separated by a considerable space from the rest of the section. The transverse diameter of the embryo is here much less than higher or lower, so that the section as a whole seems somewhat narrow. It shows the entire length of the third gill-cleft, cl.iii, exhibiting, on one hand, its connection with the median pharynx, and, on the other hand, its dorsal extremity, where its entoderm joins the ectoderm. The external outline of the embryo makes a deep depression outside the end of the third cleft. This depression is the cervical sinus (compare Fig. I66, C.S; pig of 1o $\mathrm{mm}$.). In the section the cervical sinus displays a narrow downward prolongation. If followed through in the series of sections, this prolongation, which is on the inside of the hyoid arch, $H y$, will be found to connect with the second cleft. The spinal cord, Sp.c, presents essentially the same structure as in figures. II 6 and iI7. Our section passes through the roots of the second cervical rarve, N.ceris, and shows both the dorsal ganglion and the ventral root ariging foom the rentral zone. These two roots join and form the nerve-trunk, Nv.2, which almus immediately divides, sending one branch vertically upward into a mass of denser crowded cells (the aniage of the dorsal musculature), and a ventral branch which descends almost vertically toward the pharynx. Just inside of this ventral branch we have in section of the vertebral artery, Art.v. Between the dorsal summit of the ganglion and $t$ cord there is a minute bundle of nerve-fibers not shown in the figure. These fibers constitute the commissural trunk of the eleventh nerve. The third gill-isfl, cl.iii, is cut almost symmetrically, and extends from the median line to the cdge of the section. It is lined throughout by the entoderm, which at the end of the cleft on each side has met and fused with the ectoderm to form the epithelial membrane, the closing plate. The membrane apparently no mally renains intact in mammals. In the ichthyopsida the membrane becomes ruptetitod during embryonic lifs and the gill-cleft is opened to the exterior. At the end of the cleft the entoder ? bas undergone a special growth forming a distinct mass. Wod, on 
the side of the cleft toward the head. This structure is the anlage of the nodulus thymicus and is already penetrated by small blood-vessels which are. perhaps not capillaries, but sinusoids. The fate of nodulus is uncertain; it probably forms the head of the thymus, and not the carotid gland as some

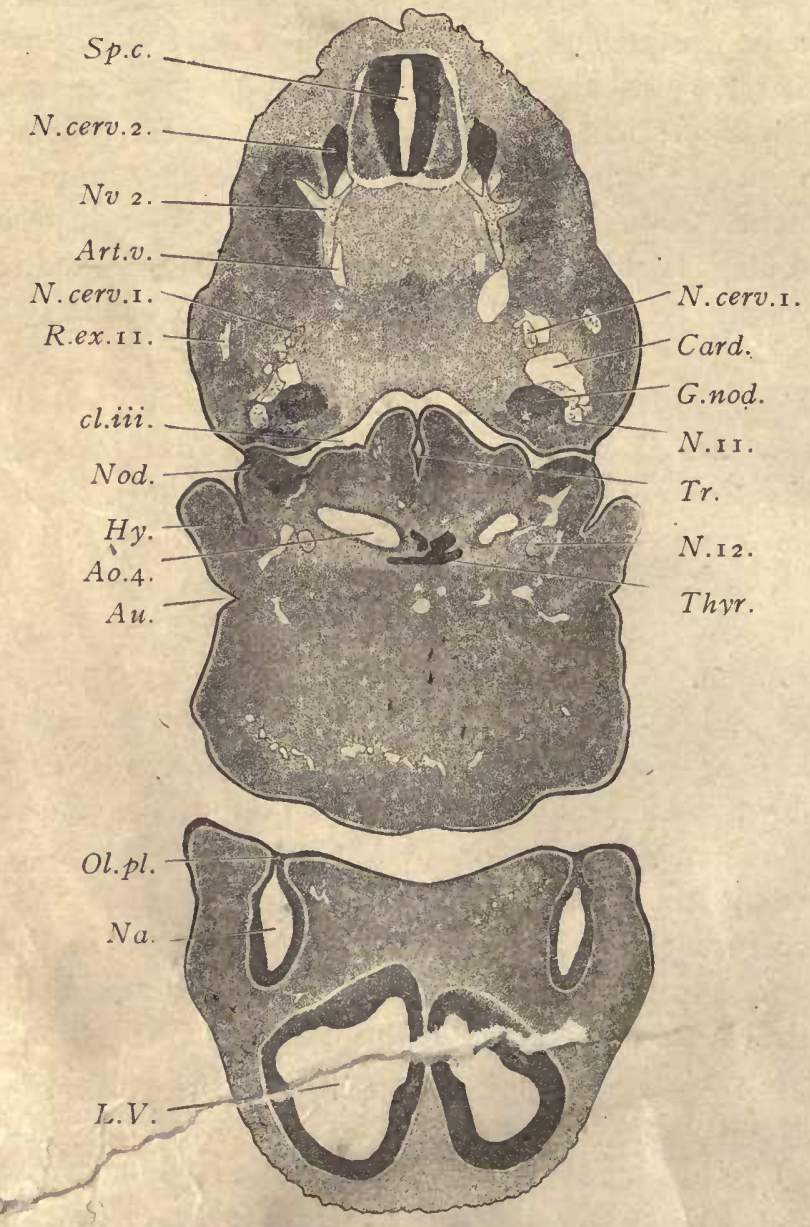

Fig. I94.-Pig, I2.0 Mm. Transverse Series 5, Section 321.

Au.4, Fourth aortic arch. Art.v, Vertebral artery. Au, External auditory cleft. Card, Anterior cardinal vein. cl.iii, Third internal gill-cleft. G.nod, Ganglion nodosum. Hy, Hyoid arch. L.V, Lateral ventricle. Na, Nasal pit. N.cerv.I, First cervical nerve. N.cerv.2, Second cervical nerve. N.I , Spinal accessory nerve. N.I2, Hypoglossal nerve. Nod, Nodulus thymicus. Nv.2, Main trunk of second cervical nerve. Ol.pl, Olfactory plate. R.ex.rI, Ramus externus of the spinal accessory. Sp.c, Spinal cord. Thyr, Thyroid. Tr, Trachea. $\times 22$ diams.

have suggested. The student should clearly understand that the median region of the third gill-cleft is really the pharynx proper. From its median ventral line arises the beginning of the trachea, $T r$, which should, perhaps, be ditre de designated as the anlage of the larynx. The entoderm fxtends down 
in the median line for a considerable distance, making a figure which, in the section, is shaped somewhat like an inverted spear-head. In the center of the section appears a small cavity. Farther down toward the lungs we have only an epithelial plate with no cavity observable in it (Fig. 195, Tra), the entoderm of the trachea at this stage forming a solid cord. Ventrad from the trachea, in the median region and between the two aortic arches, is a small, irregular, deeply stained mass of cells, Thyr, the anlage of the thyroid gland. These cells are entodermal, the anlage having been developed by a downgrowth of the epithelium of the floor of the pharynx, although at the present stage the original connection with the pharynx has been lost. The anlage is now isolated from its parent germ-layer and is imbedded in mesenchyma. It is solid, for the cavities of the thyroid follicles are not developed until considerably later. Just above the third gill-cleft may be seen a large, darkly stained mass, G.nod, the ganglion nodosum of the vagus nerve. Immediately above it is a section of the anterior cardinal vein, Card. Between the ganglion and the vein is a bundle of nerve-fibers representing the twelfth or hypoglossal nerve, which reappears again in the section below the pharynx, at N.I2. The reason for this double appearance of the hypoglossal nerve may be seen readily by examination of the reconstruction (Fig. I78). Close to the ganglion on its outcr side is the section of the spinal accessory nerve, N.rI. A little above the jugular vein is the section of the first cervical nerve, N.cerv.I, laterad from which is the external branch, R.ex.II, of the spinal accessory nerve. This branch in the adult innervates the sternocleidomastoid and trapezius muscles.

The lower part of the figure represents the section of the head and shows the two nasal pits, Na, closed toward the mouth side by the olfactory plate, Ol.pl, the epithelial membrane somewhat resembling the closing plate of a gill-cleft, but it is formed by a fusion of the ectoderm on the two sides of the opening of the nasal pits. When the nasal pits are first formed, they are open throughout their whole extent. The formation of the olfactory plate is the first step toward the separation of the two nasal cavities from the nral cavity. In later stages this plate disappears, and its forward portion is replaced by meenchyna, so that the separation of the nasal and oral cavities is permanent, ut the posterior portion of the membrane becomes first very thin, and finally disappears altnether, the establishing a secondary connection between the nose and mouth lor onch frasal chamber. On the dorsal side of the nasal pits (below in Fig. 194), the criebrai hemispheres are cut separately, their darkly stained walls bounding on each side the large lateral ventricle, L.V.

Section through the Fourth Gill-Cleft (Fig. I95).-Oi the entodermal gill-clefts or -pouches the fourth is by far the smallest, and as it appears in sections ( $r$.IV) is inconspicuous. The section figured differs by two striking features from those of the series above described: first, because the head is no longer included; and, second, because the cardiac structures are beginning to show. On the dorsal side the spinal cord is cut at the level of the ganglion, G.3, of the third ceryical nerve. 
The dorsal root of the ganglion joining the spinal cord, Sp.c, is shown on both sides of the section, and the nerve itself also appears, being best shown on the left side of the embryo, where a short piece, R.D.3, of the ramus dorsalis is included and a much longer piece, R.V.3, of the ramus ventralis. Just inside of the nerve at the level of the notochord, $N c h$, is the cross-section of the vertebral artery. On the right side of the embryo the section passes through a portion of

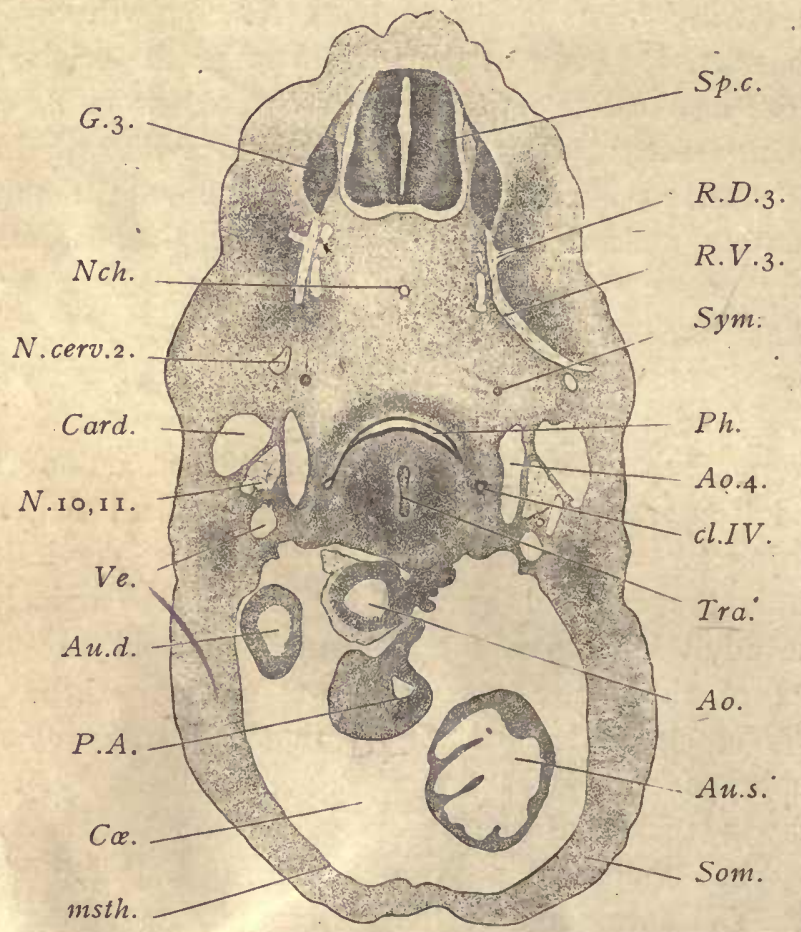

Fig. I95.-Pig, te.0 ma. Transverse Series 5, Section 353.

Ao, Aorta. Ao.4, Fourth aortic an.h. Au.d, Right auricle. Au.s, Left auricle. Card, Anterior cardinal vein: Cœ, Cœlom. cl.IV, Fourt gill-pouch. G.3, Ganglion of third cervical nerve. msth, Mesothelium. $N$. cerv.2, Second cervical nerve. Nch, Notochord. N.1o,1 I, United vagus and spinal accessory nerves. P.A, Pulmonary artery Ph, Pharynx. R.D.3, Dorsal ramus of the third cervical nerve. R.V.3, Ventral ramus Sthe cervical nerve. Som, Somatopleure. Sp.c, Spinal cord. Sym, Sympathetic nerve chain. Tra, Trachea. Ve, Vein to lower jaw. $\times 22$ diams.

the second cervical nerve, N.cerv.2. The anterior cardinal, Card, is a very large vessel. Close to its ventral wall appear a few fibers which represent the first cervical nerve, but they are too indistinct to be represented in the figure. They may easily be found with the higher power. In the median plane is the crescent-shaped section of the pharynx, Ph. Between the jugular vein and the pharynx lies the fourth atrtic arih, A0.4. The right and left arches are at this stage about equal in size, althugh the left arch is destined to form the main aortic arch of the body, 
and only a portion of the right arch will persist to form a portion of the stem of the pulmonary artery. The figure indicates the manner in which these aortic arches pass up from the heart below on either side of the pharynx. A little above the aortic arch on either side may be seen a small, round spot, Sym, which is somewhat conspicuous on account of its deeper staining. It is a section of the cervical sympathetic. Examination with a higher power shows that it consists of somewhat crowded cells, some of which have larger nuclei. These are the neuroblasts. The mesenchymal cells immediately around the anlage are disposed about it in somewhat concentric lines. Between the cardinal vein and the aortic arch is situated the large, conspicuous nerve-trunk, N.Io,Ir, constituted by the united vagus and spinal accessory nerves. Below this double nerve is a blood-vessel, $V e$, a branch of the cardinal vein. This vessel drains the tongue and facial region of the embryo. It is homologous with the inferior jugular vein of lower vertebrates, and in mammals gives rise to the lingual and facial veins of the adult, and in some species forms the external jugular, but the human external jugular is a secondary anastomosis between the linguo-facial and the junction of the internal jugular and subclavian veins. The homologies between this vein and those of the adult have not yet been worked out. Returning now to the pharynx, $P h$ : on the right side the prolongation of the pharynx to join the fourth cleft can be clearly followed; on the left side of the embryo, the right of the figure, the fourth cleft; cl.IV, does not display its connection with the pharynx, but is a separate, small, epithelial cavity lined by a cylinder epithelium. Underneath the pharynx appears a vertical plate, Tra, formed by the entoderm of the trachea. This plate is thinnest in the middle, somewhat wider toward the top and bottom of the section. It is qu solid, except for a minute cavity at its dorsal end. This minute cavity may be traced from the opening of the glottis through the series of sections down until it becomes connected with the comparatively large cavities of the developing bronchi of the lung. Below the pharyngeal region descends the thick somatopleure, Som, which encloses the pericardial cnelom, $C \alpha$, in which the heart is lodged. The inner surface of the somatopleure is covered by the thin mesothelium, $m s t h$. Of the cardiac structures we note first the section of the main aorta, Ao, and of the pulmonary aorta, $P . A$, and finally small sections of the uppermost part of the two auricles, Au.d and Au.s. More of the left auricle is included in the section than of the right.

Section through the Anterior Limbs and Heart (Fig. I96).- The section figured is much lower in the series than the last and was selected in order to illustrate the anterior limb-buds, the common cardinals, and the heart. The position and shape of the limb-buds are sufficiently shown in figure $\mathrm{I} 66$. The section demonstrates that the limb-bud is formed chiefly by a dense mass of mesoderm covered by a thin layer of ectoderm. The mesoderm connsists of very much crowded cells in which it is very difficult to recognize any distinct differentiations, yet it is probable that here are mingled both true mesenchymal cells and cells which originally belonged to 
the muscle-plates, but which have now broken apart and are developing singly into muscle-fibers. In certain amphibia the cells from the muscle-plate can be distinguished from the mesenchymal cells of the limb, and what we know of the development of the muscles in amniota confirms the view that striated muscles and mesenchyma are genetically entirely distinct. No skeletal elements whatever have yet arisen in the limb. We have here a striking illustration of the fact that the skeleton is very late in its development, and, embryologically speaking, is in no sense the framework upon which the body is built up, but rather a late supplementary development. The main morphological features in all parts of the embryo are entirely fixed by the soft tissues before the skeletal structures arise. Both nerves and bloodvessels have grown into the limb. The nerves are the ventral branches of the spinal nerves. Several of these unite together and form the brachial plexus, one part of which, $B r: P l x$, is shown in the section. In the present embryo this nervetrunk includes fibers derived from both the sixth and seventh cervical nerves. Just above the nerve-trunk is the section of the subclavian or axillary vein, which is a branch from the jugular. The dorsal region of the embryo is relatively larger at the level of this section than higher up, owing chiefly to the great development of the mesoderm. The spinal cord, Sp.c, resembles that in figure I95, but is both larger and more differentiated. On the left side of the embryo the fundamental morphological characteristics of the spinal nerve are well illustrated in this section. The dorsal root, D.R, bears the ganglion, $G$, which joins the dorsal zone of the spinal cord. The fibers of this root are produced from the cells of the ganglion and grow from the ganglion into the spinal cord. Other fibers from the same cells grow out ir the opposite direction and form the nerve-trunk or root which descends from the ganglion in a nearly vertical direction. The ventral root, $V . R$, arises from the ventral zone, takes an oblique course, and joins the dorsal root a little below the level of the spinal cord to form a single nerve-trunk, which, however, soon subdivides into its two primary branches. The first or dorsal branch, R.D, bends at an acute angle upward and outward. The second or ventral branch, ramus ventralis, continues downwayd and curves into the limb. Owing to this curvature, in order to follow its course the nerve must be traced through adjacent sections. If this is done, the ver $\&$ ral ramus will be found to take part in the formation of the brachial plexus. Some distance below the spinal cord is the small notochord. Farther down, but also in the median line, appear two small rings of epithelium. Of these, the smaller upper one, $E$, is the entodermal lining of the cesophagus, and the larger lower one is the entodermal lining of the trachea. Around each of these rings there has already occurred a slight condensation of the mesenchyma, the first step toward the ultimate differentiation of the submucous and muscular coats of the œsophagus and trachea. The entoderm of both the œsophagus and trachea is a moderately thick layer composed of elongated cells, the nuclei of which are distributed at various levels, but so as to leave the superficial portion of the layer comparatively free. It is in this superficial portion that the mitotic figures always occur. On the ven- 
tral side of the trachea and somewhat toward the left, but quite close to it, appear two small blood-vessels, the pulmonary arteries. They are so small that their lumina scarcely show in the figure. Two sections nearer the head, the two vessels are found united in a single stem. Opposite the arteries below the trachea

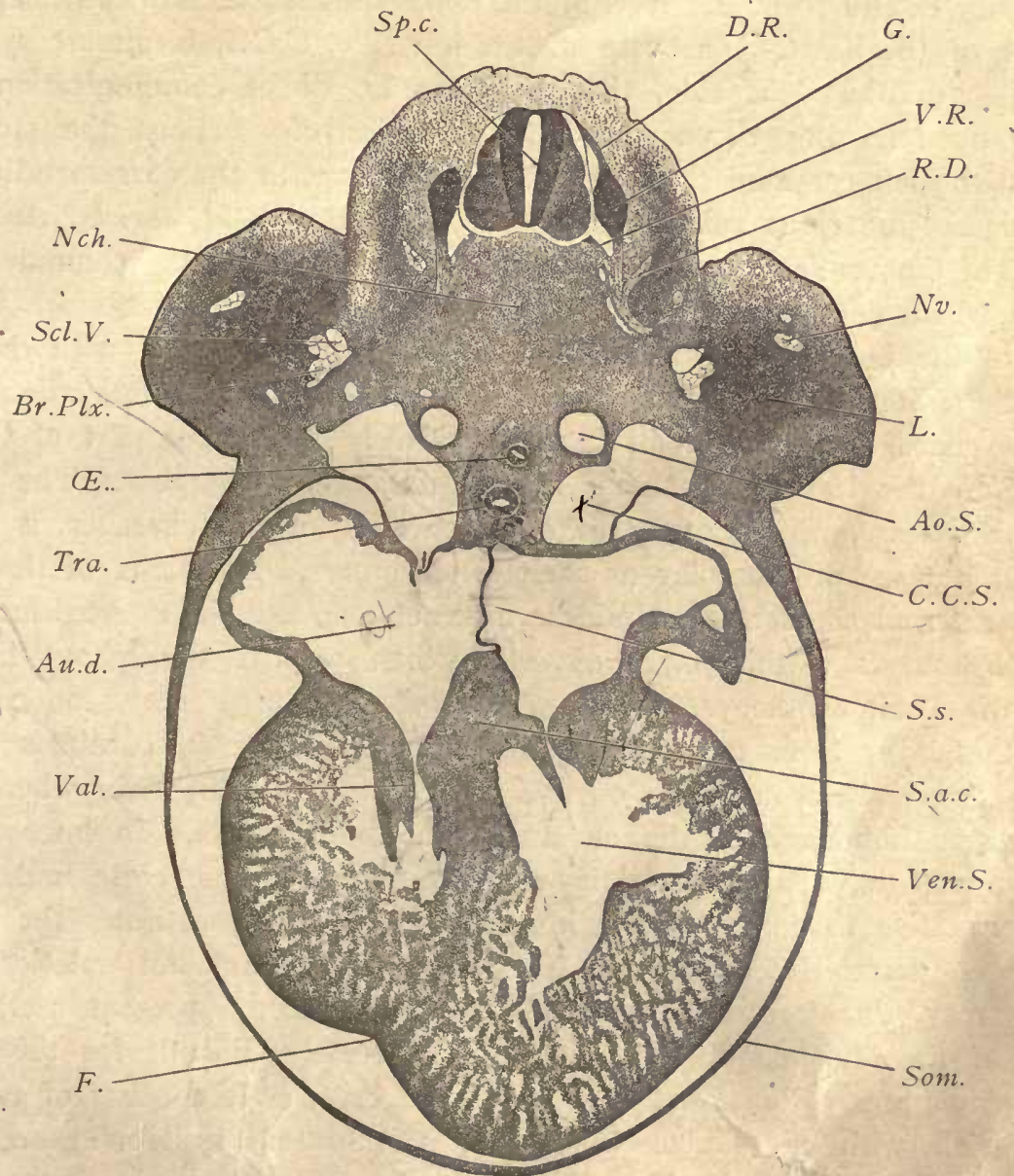

Fig. 196.-PIG, I2.0 m. Transverse Series 5, Section 470.

Ao.S, Left descending aorta. Au.d, Right auricle. Br.Plx, Brachial plexus. C.C.S, Vena cardinalis communi sinistra. D.R, Dorsal root of spinal nerve. $F$, Cardiac fissure. $G$, Spinal gang!ion. $L$, Anterior limb-bud $N c h$, Notochord. $N v$, Branch of brachial plexus. $C$, Esophagus. R.D, Ramus dorsalis of spinal nerve. S.a.c, Septum of the auricular canal. Scl.V, Subclavian vein. Som, Somatopleure. Sp.c, Spinal cord. S.s, Septum superius. Tra, Trachea. Val, Auriculo-ventricularvalve. Ven.S, Left ventricle of the heart. $V . R$, Ventral root of spinal nerve. $\times 22$ diams.

a minute opening, not shown in the figure, marks the tip of tb ural chamber. To the right and left of the cesophagus appear t of the two descending aortce, of which the left, Ao.S, is alread the right. Ultimately the greater part of the right 
of the adult being formed from the left aorta. Lower down in the series the two descending aortæ unite to form the single median dorsal aorta. The common cardinals, C.C.S, are two enormous venous trunks which deliver the blood to the heart. They lie symmetrically placed to the right and left of the œsophagus and trachea. They extend from the level of the descending aortæ downward and inward to the level of the heart. The vein of the left side, C.C.S, is almost symmetrical with its fellow of the right side, though it has no direct communication with the heart; but by following down through the series of sections the student can observe that the left common cardinal connects across with the corresponding vein of the right side. The right vein opens directly into the right auricle, Au.d, of the heart. All of the venous blood is collected at this stage by the common cardinals, except that which comes through the liver. The common cardinals are formed by the union of the jugular or anterior cardinal vein from the head with the posterior cardinal vein from the body. The opening of the right vein into the auricle of the heart is guarded by two small flaps or valves. The lower part of the section is occupied by the large heart lying in the pericardial chamber. The body-wall, Som, or somatopleure, which forms the outer covering of this chamber, is quite thin and without a trace of muscular or skeletal structures. It consists of three distinct layers - the external ectoderm, the middle mesenchyma, and the internal mesothelium. The mesothelium is a thin layer of cells which persists throughout life and is known in the adult as the pericardial epithelium. In the present section it is easy to follow this layer from the somatopleure past the common cardinals on to the heart and completely around the outside of the heart itself. Everywhere it forms the covering or boundary of the cœlom of the pericardium. In later stages this mesothelium will have an especial layer of connective tissue close under it:. The layer of connective tissue, together with the mesothelium, constitutes the pericardial. membrane of descriptive anatomy. The essential fundamental relations - of this membrane may, therefore, be easily understood from the present section. From the study of the adult conditions alone it is extremely difficult for the student to grasp these relations. The heart is a very large organ. It consists of two auricles and a ventricle with two limbs. The auricles have thin walls and are separated from one another by a very thin membrane, the septum superius, S.s. The right auricle, $A u . d$, receives upon its dorsal side the opening of the right vein or common cardinal, the opening being guarded by valves. Of these valves, the one toward the median line disappears, but the other, toward the right of the embryo, persists to form both the Eustachian and Thebesian valves of the adult. As stated above, the left common cardinal delivers its blood to the right vein, and so indirectl (2) heart. The ventricles of the heart are much larger than the auricles, 7. entricular limb or future left ventricle, Ven.S, is already larger than The external groove, $F$, which marks the boundary between the shown by the section. The trabecular structure of the affords a diagnostic mark by which the ventricles, 
if they are cut, may be easily recognized in sections. The development of the trabeculæ corresponds to the formation of blood sinusoids of the heart. The trabeculæ consist of young muscle-cells, and each bundle of cells is closely invested by the endothelium of the heart. The blood thus circulates freely between the trabeculæ, but remains, as in every blood-channel, separated by the endothelium from the neighboring tissue. The tissues of the heart are thus enabled to get their nourishment from the blood circulating through the organ. The sinusoidal type of circulation which we here encounter appears in the heart of all vertebrate embryos, and is the permanent form of circulation in the frog. In mammals, on * the other hand, although the sinusoidal circulation is kept throughout life and the ventricles always have their trabecular structure, yet we find, in addition, the development of a true capillary circulation to supplement the sinusoidal. This capillary circulation is supplied by the coronary arteries, and develops comparatively late. Between the auricles and the ventricles the heart is narrow. This constricted region is known as the auricular canal. A broad partition, S.a.c, divides the cavity of the auricular canal into right and left channels, forming open vessels between the auricles and ventricles. From the lower edges of these channels flaps of tissue project into the ventricles. The flaps are the anlages of the atrioventricular valves.

Sect?ons through the Anterior Limbs to Show the Brachial Plexus (Fig. 197).Figure 197 was drawn from a single section, except that the nerves in the limbs represent a reconstruction from several adjacent sections. The limb-bud, A.L, projects freely from the side of the body, is covered by ectoderm, $E c$, and filled with a very dense tissue, the cells of which show no very clear histological differentiation. The spinal cord, Sp.c, is fairly well advanced in its development at this level, and shows a darker, inner layer, Epen, a middle gray layer, cin, and an outer neuroglia, Ec.gl. The cord is completely surrounded by the developing pia mater, which is quite thin, but highly vascular. The ganglia are cut almost symmetrically on the two sides and show their dorsal roots. The descending trunk from each ganglion is joined by the ventral roots, V.R, which arise from the ventral zone of the cord in several bundles which unite about the same time with both one another and the dorsal root to form the main nerve-trunk, N.8, which enters into the formation of the brachial plexus. Just after the junction of the two roots the nerve gives off a branch which runs obliquely dorsalward into the anlage of the dorsal muscles, Musc. This branch is, of course, the dorsal ramus. The trunk, $N$, which runs toward the limb is the ventral ramus. Below the spinal cord is the notochord, Nch, which is completely surrounded by a dense mass of mesenchymal cells, Vert, the anlage of an intervertebral disc. T arr - down in the section are the two descending aorta, $A o$, which are $n t$ uniting to form the single median dorsal aor:. On either s of darker cells from the aorta upward toward the later vertebral disc. The dark cells belong to the sympathe 


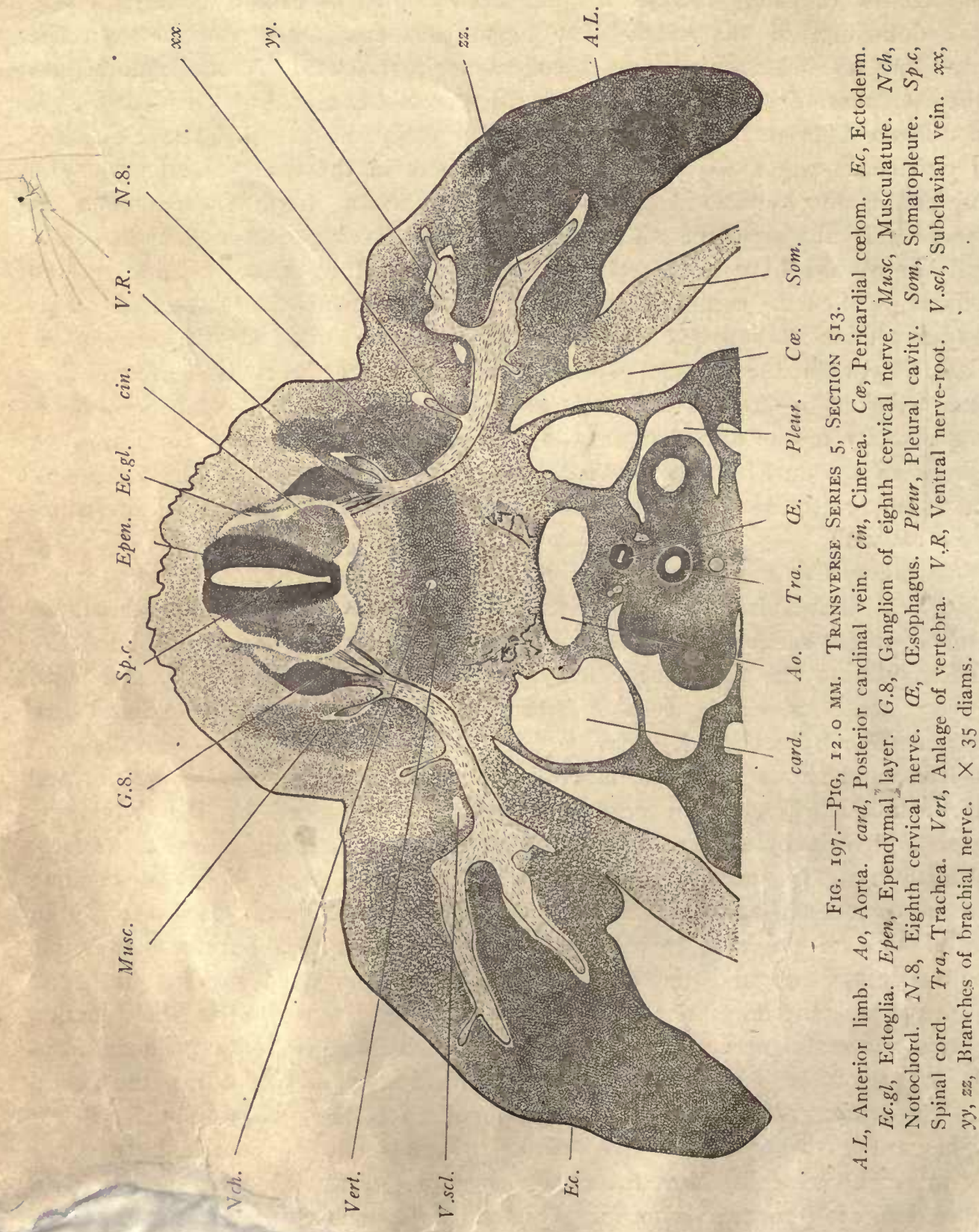


accompanied by nerve-fibers. Below the aorta runs a ring of epithelium, $E$, representing the entoderm of the cesophagus, and farther ventralward a second layer of epithelium, Tra, the entodermal lining of the trachea. Both of these rings of epithelium are surrounded by somewhat condensed mesenchyma, the differentiation of which about the asophagus is more advanced than about the trachea. Around the œsophagus next to the epithelium is a thin, looser layer of mesenchyma, the anlage of the mesodermic portion of the future mucous membrane, and perhaps also of the submucosa. Outside of this looser mesenchymal envelope is a second denser layer in which the cells appear elongated, having begun their differentiation into smooth muscle-cells. To the right and.left of the œsophagus and at a slightly lower level lie the sections of the vagus nerves, the right nerve being situated a little higher than the left. To the right and the left of the aorta appear the very large posterior cardinal veins, card. From the sides of the trachea project lobes of tissue which represent the anlages of the lungs. These lobes of tissue are each covered by a layer of mesothelium, and protrude, as it were, into the cœlom of the pleural cavities, Pleur. Farther to one side the cœlom, Co, of the abdominal cavity is also in part shown. It is bounded externally by the body-wall, Som, of the embryo. Below the trachea in the median line is a small blood-vessel, a section of the pulmonary vein. As regards the great nerve of the limb, N.8, it must be remembered that it forms a portion of the brachial plexus and is joined by other cervical nerves. From the voluminous trunk thus developed there arise three principal branches; the first, $x x$, is at the base of the limb, is small, and runs off dorsally. The other two represent a terminal forking of the nerve-trunk, one, $y y$, running to the dorsal side of the limb, the other, $z z$, to the ventral side.

Section through the Stomach and Liver (Fig. I98).-We now pass to a section well below the heart in order to study the characteristics of the Wolffian body, stomach, and liver. At this level, as comparison with. figures 194 and 196 will show, the body of the embryo has its greatest dimensions. The upper edge, Um, of the umbilical cord appears in this section. The spinal cord with its ganglia and nerves presents essentially the same features as in figure 196 . The notochord, $N c h$, forms a small circle in section and is surrounded by the anlage of an intervertebral disc, which appears as an area relatively large, over which the mesenchymal cells are more crowded or condensed than elsewhere. At its periphery the anlage merges without divisional boundary into the surrounding mesenchyma. It is more expanded laterally than ventrally. In the median line below the notochord is the large dorsal aorta, Ao, which is formed by the union of the two descending aortæ shown in figure 196 , and which extends through the abdominal region of the embryo to the pelvic region, where it forks 16 form the two allantoic arteries, which, passing on either side of the intestine, continue their course along the side of the internal allantois or future bladdor, until they reach the umbilicus, where they enter the umbilical cord to sur the stra-embryonic or placental 
circulation. The aorta is surrounded by mesenchyma, and to this are, so to speak, appended the large Wolffian bodies, W.B, one on each side. From the dorsal region of the embryo to the umbilical cord extends the somatopleure or body-wall, Som, which, like that around the pericardial chamber, consists of an

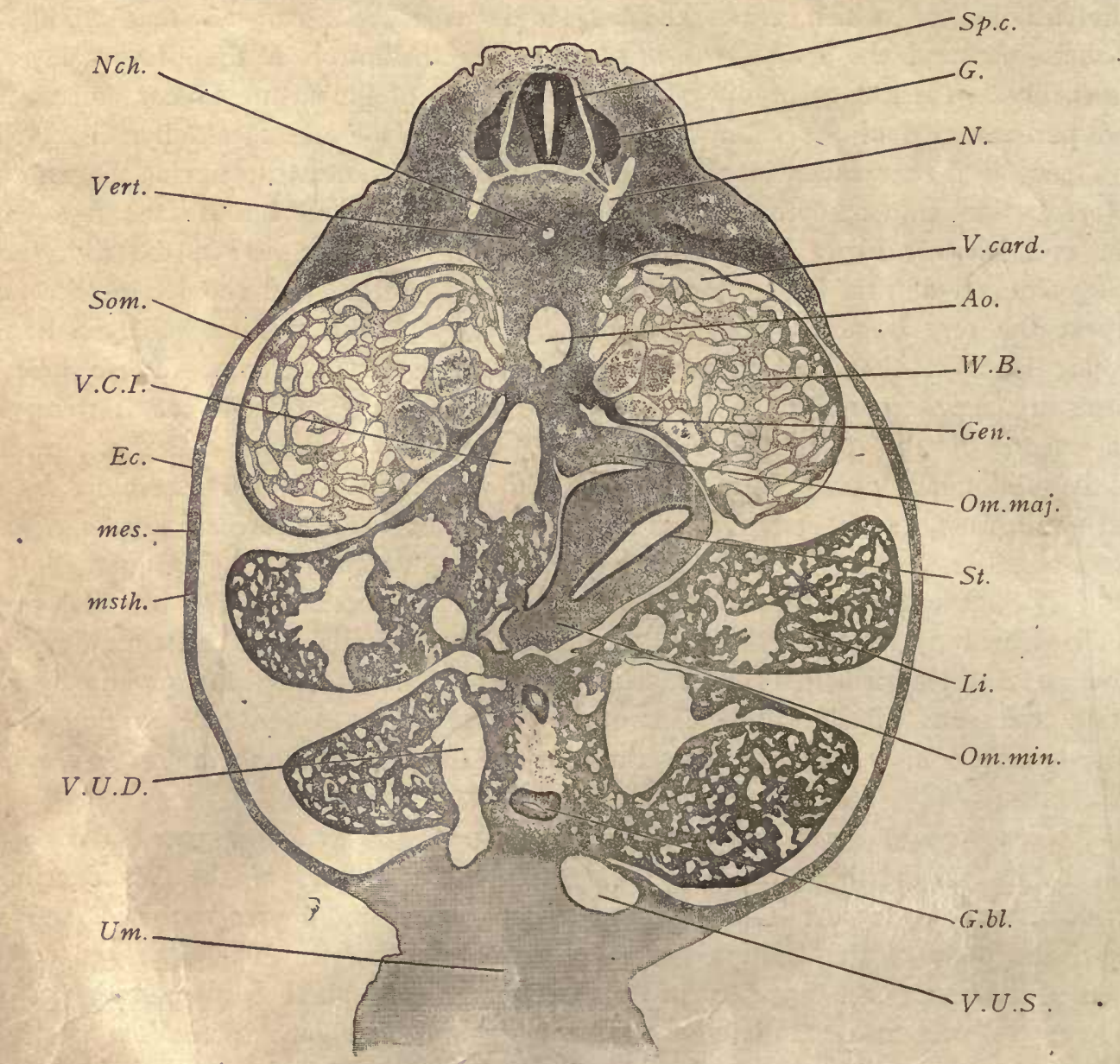

Fig. ig8.-Pig, i2.0 mir. Transverse Series 5, Section 63.3.

Ao, Dorsal aorta. Ec, Ectoderm. G, Spinal ganglion. G.bl, Gall-bladder. Gen, Anlage of genital gland. $L i$, Liver. mes, Somatic mesen hyma. msth, Somatic mesothelium. $N$, Spinal nerve. Nch, Notochord. Om.maj, Omentum majus. Om.min, Omentum minus. Som, Somatopleure. Sp.c, Spinal cord. St, Stomach. Um, Unbilical cord. V.card, Posterior cardinal vein. V.C.I, Vena cava inferior. Vert, Anlage of ve:bs. V.U.D, Right umbilical vein. V.U.S, Left umbilical vein. W.B, Wolffian body. $\times 22$ diams.

external ectoderm, Ec, a middle mesenchyma, mes, and an internal mesothelium, inst. It is important for the student to understand the arrangement of the germleyers in the somatopleure. The mesothelium is commonly known in the descriptive anatomy of the adult as the peritoneal epithelium. The peritoneal membrane 
consists of this epithelium and of the underlying connective tissue. In sections like that figured it can be readily followed not only over the inner surface of the bodywall, but over the surface of the Wolffian body and liver, and upon the left side of the body also over the surfaces of the greater omentum, stomach, and lesser 1 omentum. The relations of the abdominal viscera to the peritoneum, which are so perplexing to the student of adult anatomy, are here shown diagrammatically, as it were, by the section of the actual embryo. It is evident from such a section that the abdominal cavity (splanchnocele) is completely bounded by mesothelium, and that all the abdominal viscera are outside of the cavity. This conception, which is so important yet so difficult to the student of anatomy, is easily mastered by the study of embryonic relations. The Wolffian body, W.B, is the fetal or embryonic kidney, and is also termed the mesonephros (compare page Iog). Relatively to other parts, it is much larger in the pig than in man or the rabbit. It consists of numerous epithelial tubules very much contorted with blood spaces between them, of glomeruli which always lie toward the median and inferior side of the organ, and, finally, of a single longitudinal canal, the Wolffian duct, into which all of the tubules open. The tubules are formed by the cuboidal epithelium. The glomeruli resemble in their structure those of the kidney. Each is a bunch ' of blood-vessels covered in by a layer of epithelium which forms one boundary of the space into which the glomerulus projects. The opposite side of the space is also bounded by epithelium, which at the stalk of the glomeru'is hecomes continuous with the covering of the glomerulus itself, the whole structure resembling closely that of a Malpighian corpuscle of the true kidney. The space around each glomerulus is really the beginning of a Wolffian tubule. The spaces between the tubules are almost entirely blood-channels, and are lined by endothelium, which, for the most part, is closely fitted against the epithelium of the tubules. Uc-acionally a small amount of mesenchyma can be found between the tubules, or betwees the tubules and the nearest endothelium. We have, aceordingly, in these organs a typical simusoidal circulation. The blood spaces of the Wolffian body really belong to the posterior cardinal veins into which the Wolffian tubules in the course of their development have, as it were, penetrated, although without destroying the continuity of the vascular endothelium It is by the intercrescence of the tubules and of the endothelium that the sinusoidal condition is establisher $A$ portion uf the original channel remains on the dorsal side of the Wolffian body, mu. less free, V.card. We thus learn that, owing to the development of the Wolfian body, the posterior cardinal veins as such disappear. The Wolffian duct is always on the ventral side of the organ, and can casily be traced through as a continuous tube from section to section. In the figure it may be easily found in the left mesonephros, it being there the lowermost of the cavities drawn in the organ. On the median lower surface of the Wolffian body, underneath the glomeruli, is an accumulation of tissue, Gen, the anlage of the gerital gland, which is yet very slightly advanced. Below the ?atis on the right side of the embryo is a 
large trunk of the vena cava inferior, V.C.I, on its way. past the right dorsal lobe of the liver. Near the aorta on the left is the mesogastrium, Om.mai, or future great omentum, by which the stomach is suspended from the median dorsal wall of the abdomen. The stomach, St, is entirely upon the left side of the body and is directly connected with the liver by means of the anlage of the lesser omentum, Om.min. The walls of the stomach are constituted by the splanchnopleure, and, therefore, comprise a layer of thickened entoderm, which bounds the cavity of the organ, and a relatively thick layer of mesoderm which forms the greater

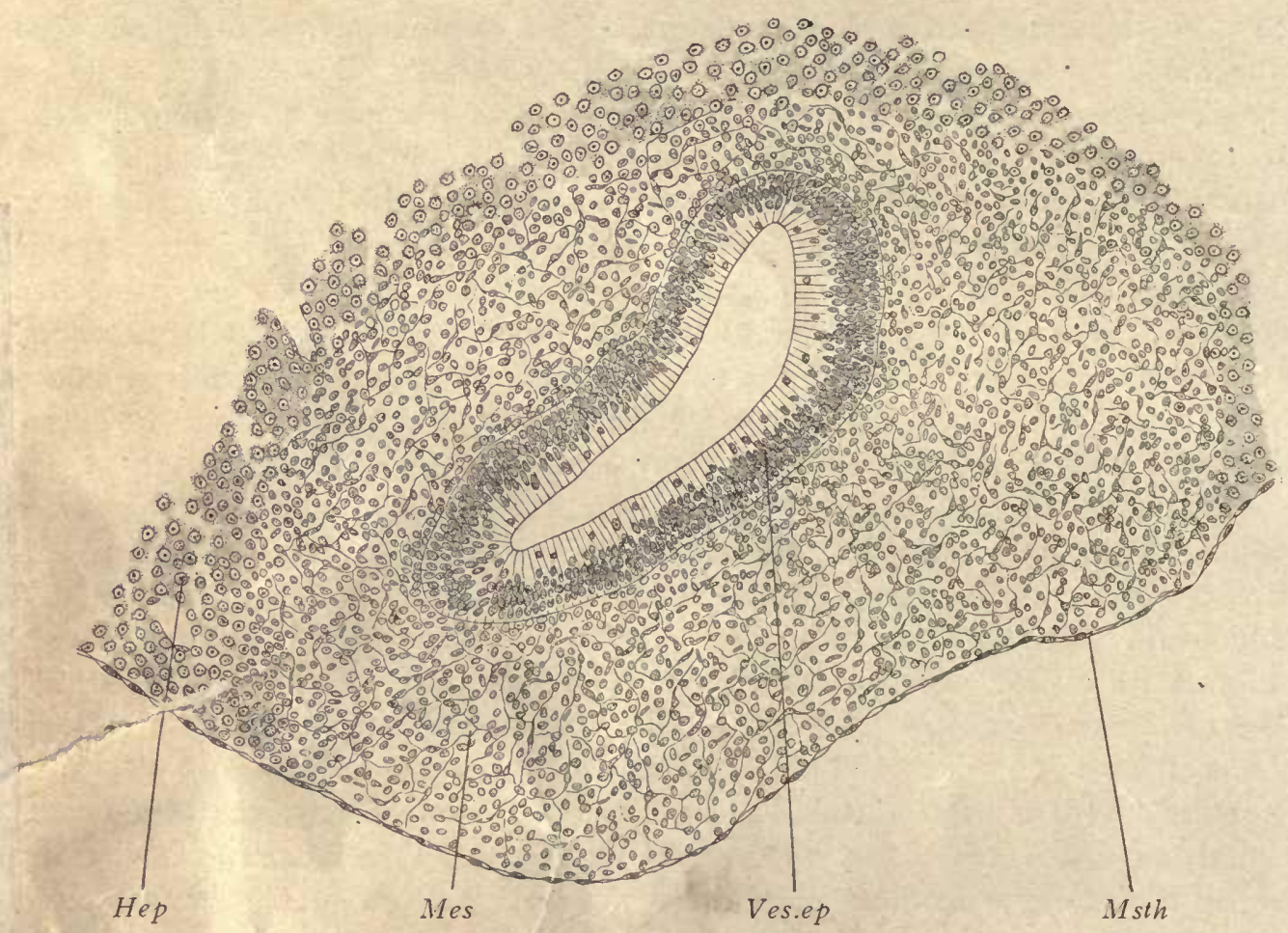

Fig. i99.-Section of Gall-bladder of a 14.0.Mm. Pig. Frontal Series 67, Section i7 I.

Hep, Hepatic cells. Mes, Mesenchyma. Msth, Mesothelium. Ves.ep, Epithelium of gall-bladder.

art un the wall, and the very thin superficial mesothelium. The entoderm is a smooth layer of moderate thickness composed of elongated epithelial cells. It forms no folds and shows no trace of differentiation into gastric glands. In the mesenchyma there are some capillary blood-vessels. The mesothelium is thicker than over the liver and somatopleure, and contains crowded, more or less nearly spherical nuclei. The liver, $L i$, is by far the largest organ of the body. It takes up nearly half the section. It is divided into four main lobes, the two dorsal and two ventral; two on the right and two on the left. The reference line, $L i$, runs to the left dorsal lobe. The liver consists of a complicated network of relatively 


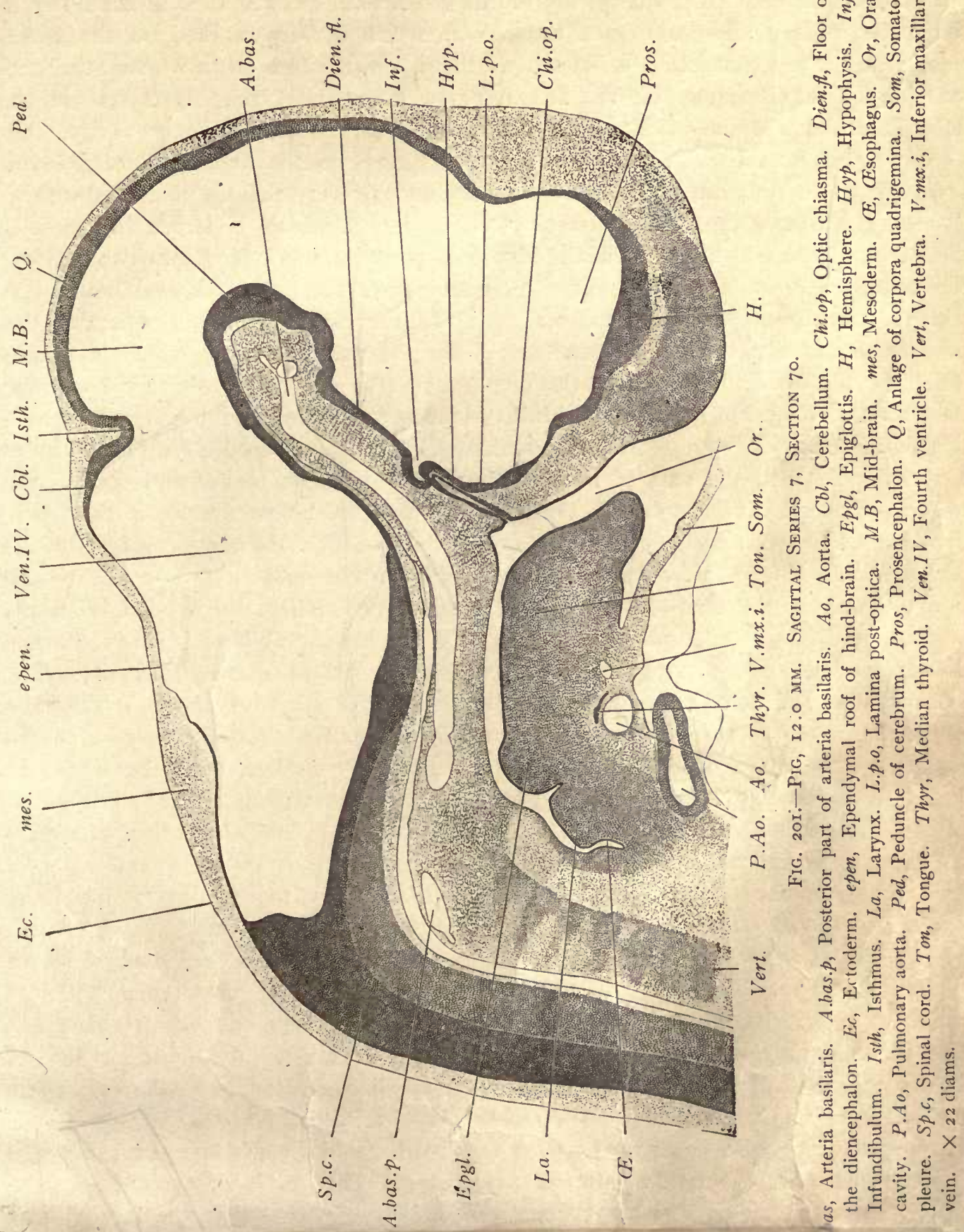


of its various parts to one another. The hind-brain begins at the spinal cord, $S p . c$, and has a very large cavity, the fourth ventricle, Ven.IV. It is separated from the region of the mid-brain by a constriction which is very marked on the dorsal side, Isth. The constriction is known as the isthmus: It is always from the dorsal side of the isthmus that the fourth nerve takes its origin. It is one of the fixed landmarks of the brain. The mid-brain, $M . B$, also has a large cavity, and, as a whole, forms the great arch which corresponds to the head-bend of the embryo. It passes forward and downward, without any very definite line of demarcation at this stage, into the fore-brain, the cavity of which is larger in diameter than that of the mid-brain. The fore-brain is partially subdivided into two regions; the anterior, Pros, is the prosencephalon and gives rise to the lateral outgrowths which form the cerebral hemispheres. Already the deep depression separates this part of the fore-brain on its dorsal side from the posterior part, which is termed the diencephalon. The limits of the diencephalon at this stage are very indistinct; later its boundary against the mid-brain becomes clearly marked by the differentiation of the epiphysis and posterior commissure. The spinal cord, Sp.c, forms almost a right, angle with the axis of the hind-brain. This angle marks and corresponds to the neck-bend of the embryo. On its dorsal side the hind-brain has a thin ependymal roof, epen, which, however, toward the isthmus thickens considerably to produce the anlage, $\mathrm{Cbl}$, of the median portion of the cerebellum. On the rentral side the wall of the hind-brain varies in appearance. Where the section is exactly median, it displays the raphe or floor-plate of the region. Where it is off the median plane, it shows instead the thicker, lateral wall of the medulla oblongata. The walls of the mid-brain on the dorsal side, $Q$, are almost uniform in thickness and texture. They are, however, later to be differentiated into the corpora quadrigemina. The ventral side of the mid-brain, $P e d$, is considerably thicker than the dorsal, and forms a strongly marked arch. It is represented in the adult essentially by a part of the peduncle of the cerebrum. The floor, Dien.fl, of the diencephalon is a thin membrane of which the part nearest to the mid-brain will produce the mammary bodies, and the part farther from the mid-brain the tuber cinereum. It has already formed a special outgrowth, Inf, the anlage of the infundibular gland, which extends out from the brain and arches over the end of the hypophysis, Hyp. The hypophysis is an outgrowth from the ectodermal lining of the mouth, Or. Its method of development can be clearly made out at this stage. The infundibular gland in older embryos extends farther on the posterior side of the hypophysis. Meanwhile the hypophysis loses all connection with the epithelium of the oral cavity, somewhat as does the otocyst with the overlying epidermis which produces it. The hypophysis proper and the infundibular gland undergo their further development in intimate association. The result of their differentiation is the pituitary body, which is really a duplex organ. Below the infundibular gland the wall of the brain shows a thickening, Chi.op, which can be followed through in the series laterally until it connects with the optic stalk. This thicken- 
ing of the brain-wall in later stages furnishes the passage for the fibers of the optic nerve, and is, therefore, the anlage of the optic chiasma. Between the infundibular gland and the optic chiasma extends the post-optic lamina, L.p.o. On the opposite side of the chiasma follows the lamina terminalis, which leads us forward to the wall of the hemispheres, $H$. Underneath the hind-brain extends the large basilar artery, A.bas; at its posterior end, A.bas.p, the basilar artery is joined by the two vertebral arteries from the fusion of which it is really produced. Underneath the fore-brain we have the opening of the mouth, Or, from the dorsal side of which springs the elongated evagination of the hypophysis. The oral cavity runs into the pharynx, the floor of which is formed in part by the anlage of the tongue, Ton, and of the epiglottis, Epgl, a rounded eminence very different in shape at this stage from the adult epiglottis. The pharynx can be followed along until it passes over into the œsophagus, E:, which, however, is not well shown, as the section passes through it away from the true median plane. Between the œesophagus and the anlage of the epiglottis is a mound of tissue, $L a$, which represents the lateral wall of the developing larynx. The mound is separated from the anlage of the epiglottis by a deep notch. In the median plane the mound is filled with entoderm which forms a wide plate through which there is only a narrow opening leading down into the trachea. Finally, we see from the base of the mandible the somatopleure, Som, extending off to form the boundary of the pericardial chamber. The figure also includes a presentation of the inferior maxillary vein, $V . m x . i$, and of the thyroid gland, Thyr, which immediately overlies the main trunk of the ventral aorta. This aorta gives off on either side of the pharynx three principal branches, of which the smallest is the base of the carotid and corresponds to the third aortic arch. The second and third branches are much larger and correspond to the third and fourth aortic arches. The pulmonary aorta, P.Ao, is already separated from the main aorta of the body.

Sagittal Section of the Head through the Principal Ganglia (Fig. 202). - The section is to one side of the median plane. It exhibits the optic nerve, the trigeminal, acustico-facial, petrosal, jugular, and nodosal ganglia; but, on the other hand, exhibits little of the brain, there being only a shaving from the lateral wall of the fore-brain, $H$, and a section of the widest part of the hind-brain which shows the cavity or lateral recess, R.L, of the fourth ventricle. The auditory vesicle is cut, Ot. It is formed by a layer of epithelium derived from the ectoderm, although now not connected with the overlying part of the epidermis by the invagination of which the octocyst is developed. It shows a narrow, upward prolongation, the anlage of the ductus endolymphaticus (compare Fig. 42). The epithelial otocyst lies in a line with the great cephalic ganglia and occupies its invariable and permanent position behind the acustico-facial ganglion, Ac.F, and in front of the glosso-pharyngeal, G.petr. The position of the otocyst makes it an invaluable landmark in the study of sections of the head. Only the lateral portion of the pharynx, $P h$, appears. It forms a wide, almost slit-like diverticulum, from 
which extend farther laterally the first and second entodermal gill-pouches. In the figure can be seen a small depression extending downward from the œesophageal or posterior end of the pharynx. This depression marks the beginning of the second cleft. Nothing is seen of the third and fourth clefts in this section, as they both lie nearer the median plane. The pocket or diverticulum of the cervical sinus,

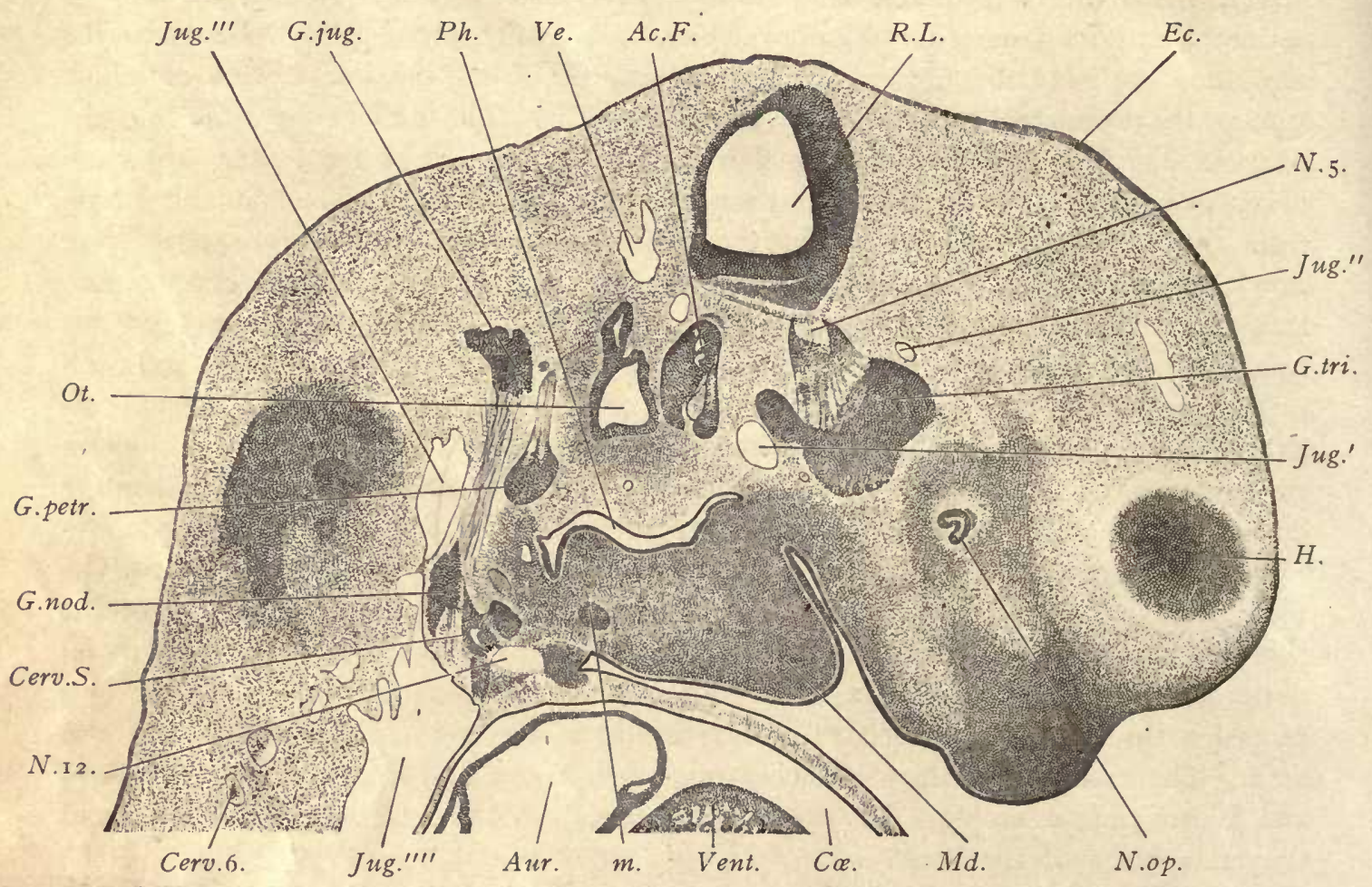

Fig. 202.-Pig, 12.0 m. No. 7. Sagittal Section 25.

Ac.F, Acustico-facial ganglion complex. Aur, Auricle of the heart. Cerv.S, Diverticulum of the cervical sinus, just in front of which shows the anlage of the thymus, which is deeply stained. Cerv. 6, Sixth cervical nerve. $C \propto$, Cœlom around the heart or pericardial cavity. Ec, Ectoderm. G.jug, Ganglion jugulare of the vagus nerve. G.nod, Ganglion nodosum of the vagus nerve. G.petr, Ganglion petrosum of the glosso-pharyngeal nerve. G.tri', Ganglion of the trigeminus nerve. $H$, Lateral wall of the cerebral hemisphere. Jug $g^{\prime}-J u g^{\prime \prime \prime \prime}$, Jugular vein $\left(J u g^{\prime}\right.$, Behind the trigeminus. Jug", Branch in front of the trigeminus. Jug"', Main stem behind the vagus. Jug'"', Main stem descending to join the duct of Cuvier). m, An undetermined structure, probably the anlage of a lingual muscle. $M d$, Mandible. $N .5$, Root of the fifth or trigeminal nerve. N.op, Optic nerve. N.I 2, Twelfth or hypcglossal nerve. Ot, Otocyst. Ph, Pharynx. R.L, Recessus lateralis of the fourth ventricle. Ve, Small branch of the jugular vein. Vent, Ventricle of the heart. $\times 22$ diams.

Cerv.S, lies near the ganglion nodosum, G.nod. From its appearance it might easily be mistaken for the section of a gill-cleft, but it is in reality lined not by entoderm but by ectoderm, and its cavity can be easily traced through the series of sections of the exterior of the embryd where the epithelium lining the sinus becomes continuous with the epidermis. Cephalad from the sinus, but close to it, lies a small 
dark rounded mass, the anlage of the nodulus thymicus (compare Fig. I94, Nod). The nodulus anlage is produced by proliferation of the entodermal cells on the anterior side of the third cleft, and is penetrated by blood-vessels which seem to be sinusoids, although their history has not been worked out. The great vein of the head, which for convenience we may term the jugular,-although the application of this name to the vein in its present condition is somewhat inexact,- is cut several times, owing to its irregular course. Its main stem, Jug'"'", arises nearly vertically through the cervical region and is, relatively to the size of the embryo, of huge diameter. It continues upward, $J u g^{\prime \prime \prime}$, along the dorsal side of the vagus to about half-way between the ganglion nodosum and ganglion jugulare. At that point the vessel curves inward and forward, and therefore is not encountered again in this section until, having bent upward again, it shows, $J u g^{\prime}$, on its way past the trigeminal ganglion. A branch of the jugular, $J u g^{\prime \prime}$, is cut just above the ganglion, and another small and probably not very important branch is shown at $V e$.

The nerves are shown as follows: The optic nerve, N.op, still has its central cavity, which, nearer the median plane, opens into the third ventricle of the brain, and in the section resembles in shape an inverted $U$. On the side of the nerve toward the mouth there is a deep notch-the section of the choroid fissure. The trigeminal ganglion, G.tri, is very largc, and its trilobate form is clearly indicated by the figure. The lobe to which the reference line, G.tri, runs gives off the ramus ophthalmicus; the lobe nearest the jugular gives off the ramus maxillaris inferior, while the middle lobe gives off the ramus maxillaris superior. From the ganglion the fibers and nerve-cells extend upward to form the root, N.5, which joins the hind-brain at a characteristic point-namely, at the summit of the Varolian bend and where the hind-brain is widest (compare Figs. 189 and 203). By its great size and by its topographical association with the lateral apex of the recessus lateralis of the fourth ventricle, the trigeminal ganglion may always be readily identified in sections of embryos. The acustico-facial ganglia, Ac.F, may also be readily determined by their typical position immediately in front of the otocyst, Ot. But it is quite difficult to identify the four components of this complex structure; namely, $\mathrm{I}^{\circ}$, the motor root of the facial nerve; $2^{\circ}$, the facial or geniculate ganglion; $3^{\circ}$, the vestibular ganglion; $4^{\circ}$, the cochlear ganglion. In figure 202 three divisions are shown. The large, darkly stained division, to which the reference line, Ac.F, runs and which lies nearest to the otocyst, is the vestibular portion of the acoustic ganglion; the small, light area occupying a middle position in the inferior part of the complex is the motor division of the seventh herve, or lateral root of the facial; it can be followed to the brain, which it enters as four bundles of fibers; its path of entrance is shown better in frontal sections (Fig. 204, t.m). Just in front of the facial motor root lies a second smaller dark mass, the geniculate ganglion of the facial, with an upward prolongation, the sensory root. The ninth or glosso-pharyngeal nerve is represented by the ganglion petrosum, G.petr, and its 
ascending sensory root. This nerve may be quickly identified because it is the first behind the otocyst. The upper ganglion of this nerve, the so-called Ehrenritter's ganglion, is represented by an accumulation of cells in the upper part of this root. As regards the tenth nerve, or vagus, both its ganglia and the fibrous trunk connecting them are shown. The upper or jugular ganglion, G.jug, is nearly on a level with the otocyst, while the lower or nodosal ganglion, G.nod, lies near the cervical sinus. To the nerve-trunk between the two ganglia are adjoined the fibers of the eleventh or spinal accessory nerve, which does not otherwise appear in this section. A small piece only of the hypoglossal nerve can be seen, N.ri2. The space occupied by this nerve is blank in the engraving; in the specimen it shows horizontal fibers.

\section{Pig Embryo of $12.0 \mathrm{~mm}$. Study of Frontal Sections.}

The frontal series has special value for the study of the hind-brain and associated structures, as the plane of the section is approximately at right angles to the axis of the hind-brain. It also furnishes instructive pictures of the vena cava inferior and of the relations of developing vertebræ and nerves.

Portions of three sections illustrating the structure of the hind-brain and associated parts are given below. The following remarks on the hind-brain are intended to make clearer the significance of these sections. The wall of the hindbrain is, of course, produced by the development of the wall of the medullary tube. Its most striking peculiarity is the enormous expansion of the deck-plate, which forms the very wide epithelial layer, (Fig. 203, epen), the so-called ependymal roof of the fourth ventricle. It starts from the upper edge of the dorsal zone, $D . Z$, and forms a wide arch which is covered in externally, by a rather thin layer of mesoderm, mes, and the nearby epidermis, $E c$, of the embryo. The covering is so slight in development at this stage that in the fresh specimen the roof of the fourth ventricle, including its coverings, appears as a translucent membrane through which we can readily distinguish the great cavity of the fourth rentricle itself. The expanse of the ependymal arch is greatest at the region of the trigeminal root. From there backward toward the spinal cord its expanse gradually diminishes. In correspondence with the growth of the deck-plate the lateral walls of the medullary tube become bent outward and downward, so that, though they remain near together on their ventral side, where they are united by the floor-plate or median raphe (Fig. 205, raph), yet their upper dorsal edges are far apart. In consequence of this change of their position the original lateral walls appear as the floor of the hind-brain, and we recognize in them the anlages of the medulla oblongata. We diștinguish here, as everywhere in the medullary wall, the dorsal and ventral zones. The ventral zone is intimately united with its fellow by the short median raphe. Between them is a deep fissure (Fig. 204, $f$ ), which is never wholly obliterated. The floor-plate undergoes a great development in later stages and is transformed into the median raphe of the adult medulla. The lateral or 
morphologically dorsal limit of the ventral zone is marked by the exit of the lateral roots (Fig. 203, L.R). The ventral limit of the dorsal zone is marked by the entrance of the sensory or ganglionic fibers (Fig. 203, G.tri; Fig. 204, Fac). Toward the dorsal side the dorsal zone gradually thins out and passes over into the ependyma, epen. The great development of the lateral roots is perhaps the most important single characteristic of the medulla oblongata. They furnish the principal motor or efferent nerve-tracts of the brain and form an important constituent part of four nerves: first, the trigeminal or fifth; second, the facial or seventh; third, the glosso-pharyngeal or ninth; and fourth, the vagus or tenth. There are no lateral roots known to occur anterior to the medulla oblongata, unless possibly the fourth nerve, the relations of which in many respects are peculiar, should turn out to be a lateral root. In the spinal cord we find lateral roots in the upper cervical region, and it is not improbable that they may yet be found associated with the dorsal roots of spinal nerves lower "down. But even in the cervical cord the lateral roots attain but a slight development. The contrast with other portions of the central nervous system makes the great development of the lateral roots in the medulla oblongata all the more striking. The dorsal zone of the hind-brain lags considerably behind the ventral zone in its development, and at all stages the ventral zone forms a larger proportion of the medulla th $\mathrm{n}$ does the dorsal zone.

Section 'through the Trigeminal Roots (Fig. 203). - The section passes through the widest part of the hind-brain, the cavity of which is enormously distended. It is bounded on the dorsal side only by the very thin ependymal roof, epen, which

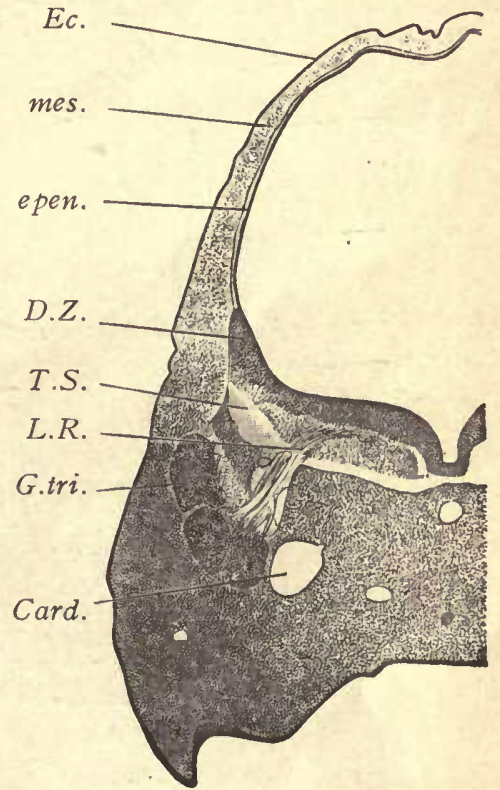

Fig. 203.-PIG, I 2.0 MiN. Frontal Series 6, Section 284.

Card, Anterior cardinal vein. D.Z, Upper portion of the dorsal zone of His. Ec, Ectoderm. epen, Ependymal roof of the fourth ventricle. G.tri, Ganglion trigemini. L.R, Lateral root of the trigeminal nerve. mes, Mesenchyma. T.S, Tractus solitarius of W. His. $\times 22$ diams.

does not form any part of the true nervous structure, although it passes into and is directly continuous with the dorsal zone, $D . Z$, which is thus seen to be only a thickened portion of the wall of the neural tube, just as the ependyma is the attenuated deck-plate. The trigeminal ganglion, G.tri, is very large and sends its sensory fibers upward into the dorsal zone to form there a distinct bundle of nerve-fibers which persists throughout life and is known in the adult as the trigeminal tract, T.S. The entering sensory fibers fork; their ascending branches form the relatively short ascending tract, their descending branches the much longer descending tract, which gradually grows through the length of the medulla oblon- 
gata well outside the tractus solitarius, which, however; it joins just before the spinal cord is reached. The other root of the nerve, L.R, is lateral. It lies below the ganglion near the median plane. Its fibers arise from neuroblasts in the ventral zone and gather together as a distinct bundle which starts near the median line, takes a curving course through the ventral zone, and makes its exit from the

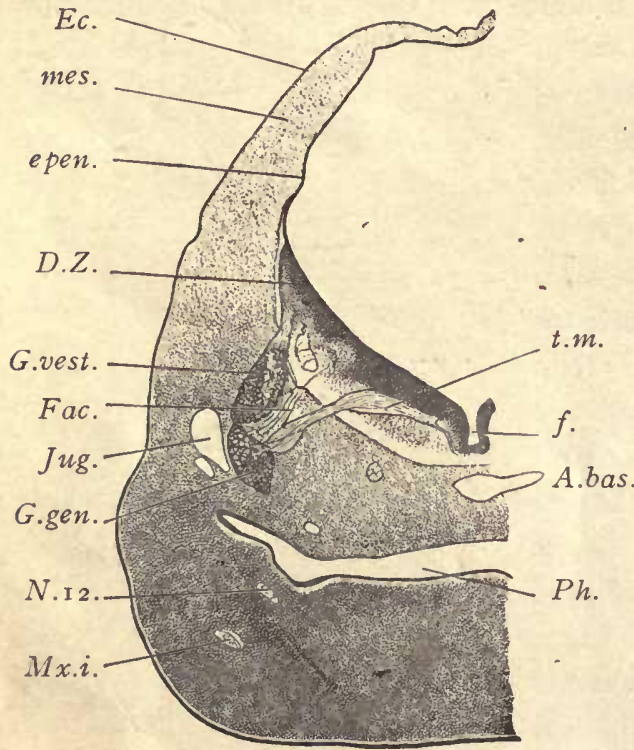

Fig. 204.-Pig, I2.0 MM. Frontal Series 6, SECTION 340.

A.bas, Arteria basilaris. D.Z, Dorsal zone of the medulla oblongata. Ec, Ectoderm. epen, Ependymal roof of the fourth ventricle. $f$, Median fissure of the medulla oblongata. Fac, Sensory root of the facial nerve. G.gen, Geniculate ganglion of the facial nerve. G.vest, Ganglion vestibuli of the acoustic nerve. Jug, Lateral vein. mes, Mesenchyma. Mx.i, Inferior maxillary branch of the trigeminal nerve. $N$.I2, Hypoglossal nerve. Ph, Pharynx. $t . m$, Motor tract of facial nerve. $\times 22$ diams. medullary wall at the dorsal limit of the zone. It has a striking resemblance to the root of the facial nerve. We do not yet know' whether such a course of the fibers is characteristic of all lateral roots or only of the trigeminal and facial roots. 'On the medial side of the trigeminal ganglion is a large vein, Card, the anterior cardinal vein. In the median line in the mesenchyma immediately below the raphe is the section of the basilar artery, and considerably. below that is the small section of the notochord which it is very difficult to distinguish with a low power. Between the notochord and the cardinal vein is the section of the carotid artery.

Section through the Acustico-facial Ganglion (Fig. 204).-In this section the thickened ventral wall of the hind-brain (i. e., the anlage of the medulla oblongata) is not spread out nearly horizontally, as in the trigeminal region, but rises obliquely on either side from the median line. The right and left sides of the medulla are divided from one another by a deep median fissure, $f$. In the median line we see also the basilar artery, A.bas, and still lower the wide, slit-like pharynx, $P h$, the outer portion of which ascends obliquely toward the lateral vein, Jug. The ascending lateral part of the pharynx is a portion of the first gill-pouch or future Eustachian tube, and is quite clearly marked off from the pharynx proper by its oblique direction. Of the acustico-facial ganglion complex the section shows four parts: the ganglion vestibuli, G.vest; the geniculate ganglion, G.gen; the sensory root, Fac, of the facial nerve arising from the geniculate ganglion and entering the brain to form there a distinct fiber-tract which is oval in the section and lies just below the entering vestibular fibers, and is clearly indicated in the drawing; and, finally, the motor tract, t.m, of the facial nerve. This tract is a very dis- 
tinctly marked bundle of nerve-fibers which arise from neuroblasts of the ventral zone, traverse that zone almost horizontally, then bend downward and pass out from the brain-wall, appearing as the lateral root of the facial nerve. The root runs first toward and then past the geniculate ganglion. The cardinal vein originally was inside the ganglia; by island formation it has migrated outside the ganglia, forming the lateral vein, Jug. In the mandible below the pharynx appear two nerves. Of these, the upper is the hypoglossal, N.r2, which lies near the angle formed by the junction of the first gill-cleft with the pharynx. The lower of the two nerves, $M x . i$, is the inferior maxillary.

Section through the Otocyst (Fig. 205).The figure is from a section not far from the last. The hind-brain has narrowed considerably; its thickened floor, $M d . o b l$, the anlage of the medulla oblongata, rises steeply from the median line. Its ependymal roof, epen, is less expanded than in figures 204 and 205. It forms a sharp angle in the dorsal median line. The median ventral fissure between the two sides of the medulla is deeper than farther forward. The pharynx, $P h$, is wide and has expanded laterally into the common beginning of the first and second gill-pouches. Between the pharynx and the raphe the basilar artery, A.bas, has been cut transversely. Below it and near the pharynx is the small notochord, which, however, can be clearly recognized only with the higher power, and is, therefore, not represented in this or the preceding figure. The otocyst is a large

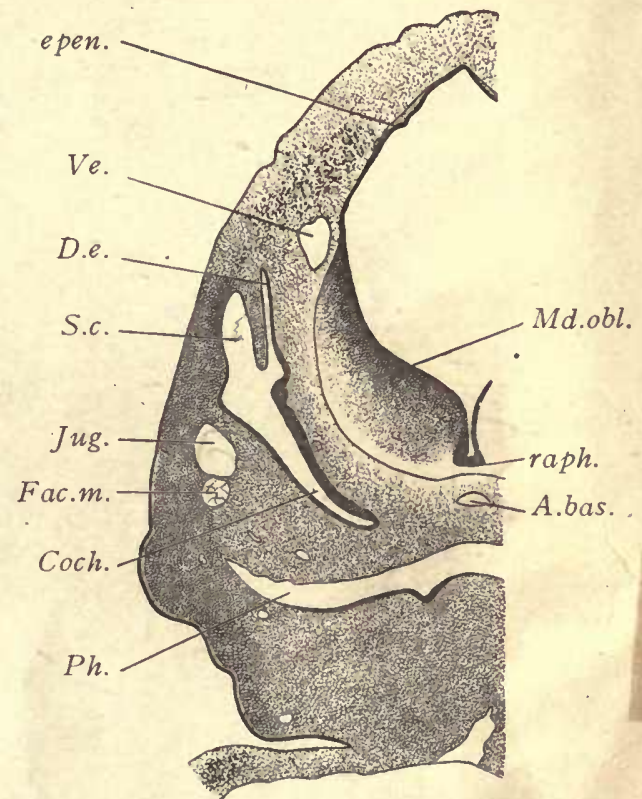

Fig. 205.-Pig, I2.0 Mm. Frontal Series 6, SeCtion 380.

A.bas, Basilar artery. Coch, Cochlea. D.e, Ductus endolymphaticus. epen, Ependyma. Fac.m, Motor division of the facial nerve. Jug, Vena lateralis capitis. Md.obl, Medulla oblongata. $P h$, Pharynx. raph, Median raphe of the medulla oblongata. S.c, Anlage of the semicircular canals. $V e$, Vein. $\times 22$ diams.

epithelial vesicle with three well-marked divisions: First, the common chamber, S.c, out of which the three semicircular canals are to be differentiated. Second, a slender canal, D.e., which one easily -identifies as the anlage of the ductus endolymphaticus. It lies between the semicircular canal and the wall of the medulla oblongata. Third, the long, curving, but not spiral cochlea, Coch. The common chamber formed by the union of these divisions is later subdivided to form the upper utriculus and lower sacculus. Outside the cochlea lies the cross-section of the vena lateralis capitis, Jug, which appears in the adult as part of the internal jugular. Just below the lateral vein is the section of the motor portion, Fac.m, of the facial nerve. The sensory portion of the facial nerve at this stage is 
much smaller, and runs only a short distance downward from the geniculate ganglion and is entirely separate from the motor portion. The morphological constitution of the facial nerve is still very obscure, and a satisfactory account of its development is, for the present, impossible.

Section through the Vena Cava Inferior (Fig. 206). - The section displays the huge vena cava inferior cut through most of its length. For the composition of

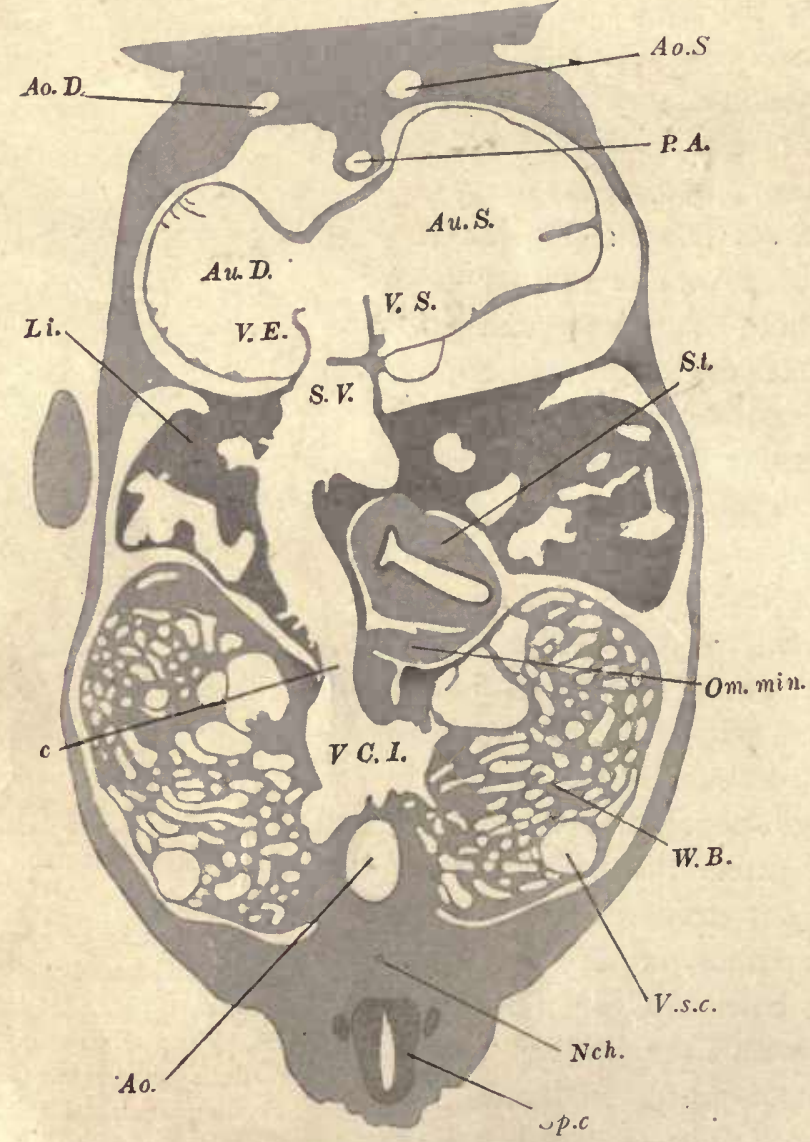

Fig. 206.-Pig of i2.0 mi. Frontal Series 6, Section 423.

Ao, Main dorsal aorta. Ao.D, Right descending aorta. Ao.S, Left descending aorta. Au.D, Right auricle. $A u . S$, Left auricle. $c$, Vena cava passing through the caval ligament. Nch, Notochord. Om.min, Omentum minus. P.A, Pulmonary aorta. Sp.c, Spinal cord. St, Stomach. S.V, Sinus venosus. V.C.I, Vena cava inferior. V.E, Valvula Eustachii. V.S, Valvula sinistra. V.s.c, Vena subcardinalis. W.B, Wolffian body. $\times x_{5}$ diams.

the vein see page 257. In the section it starts between the Wolffian bodies, $W . B$, as a large vessel, V.C.I, formed by the median union of the two subcardinal veins of the Wolffian bodies. It passes upward, $c$, through a thin band of tissue, the caval ligament, to the right of the lesser omentum, Om.min, into the substance of the liver, $L i$, through which it takes a slightly sinuous course. Several junctions 
of the hepatic veins with the main vessel are cut in the section. The liver is attached to the diaphragm. Above the diaphragm the cava is continued, with thin walls, for a short stretch, S.V, which is the modified sinus venosus of the heart, and which opens directly into the right auricle, Au.D. The opening is guarded by two valves, the valvula sinistra, V.S, on the left, and the valvula Eustachii, $V . E$, on the right, which together prevent the back-flow of the blood from the heart into the vein. Above the heart appear the pulmonary aorta, P.A, and the two descending aortæ, Ao.D, Ao.S. The main dorsal aorta, Ao, shows in the lower part of the section. The stomach, $S t$, lies on the left side and is closely attached to the liver by the short. and thick anlage of the great omentum, and is attached to the caval ligament by the longer band of the lesser omentum, $O m$. min. The space bounded by the stomach, the lesser omentum, and the liver is the lesser peritoneal cavity (bursa omentalis). In the Wolffian body the sub-cardinal vein, V.s.c, is easily identified, and with a higher power the intertubular sinusoids reveal their characteristics clearly, the sinusoidal epithelium being fitted closely to the surface of the Wolffian tubules. The division of the calom by the diaphragm into an upper pericardial and a lower abdominal chamber is perfectly demonstrated by this section. The student should observe that the mesothelium forms for both chambers the absolutely unbroken boundary of the cœlom.

Section through the Dorsal Vertebre (Fig. 207). - Owing to the curvature of the embryo the spinal cord is cut twice; once, $S p . c^{\prime}$, toward the head end of the embryo, and again, $S p . c^{\prime \prime}$, lower down toward the tail end. Alongside the sections of the spinal cord appear the large, darkly stained masses of the ganglia, $G$. The section also passes through the bases of the anterior limbs, A.L, in one of wh: he can see one of the branches, N.br, of the brachial plexus. Between the wo pieces of the spinal cord of the section the plane passes on the ventral side of ate

cord and shows the series of vertebral formations, together with the nerve-roots, $N^{\prime}, N^{\prime \prime}$, the intersegmental arteries, A.i.s, and the segmental veins, small vessels which lie close to the intersegmental arteries. The nerves are sections of the dorsal root below the ganglia. Each nerve has a distinct outline and is partly penetrated by ingrowing mesenchymal cells which subdivide the nerve into rounded fiber bundles. In each bundle the nerve-fibers appear as fine dots, which, however, by the use of the fine adjustment can be followed up and down through the section, and thus identified as fibers. The single fibers are more or less isolated from one another, and between them are delicate threads, the nature of which is not known. Between the adjacent rounded bundles of fibers there is often a distinct space. The anlages of the intervertebral disc, Iv.D, are formed entirely from condensed mesenchyma, and therefore stand out somewhat conspicuously in the section owing to their darker staining. Each anlage is bowshaped, the concavity of the bow facing toward the tail of the embryo. The end. of the bow pass behind the nerve-trunk of the segment to which the anlage belongs. The anlages extend completely across the median line, and by following 


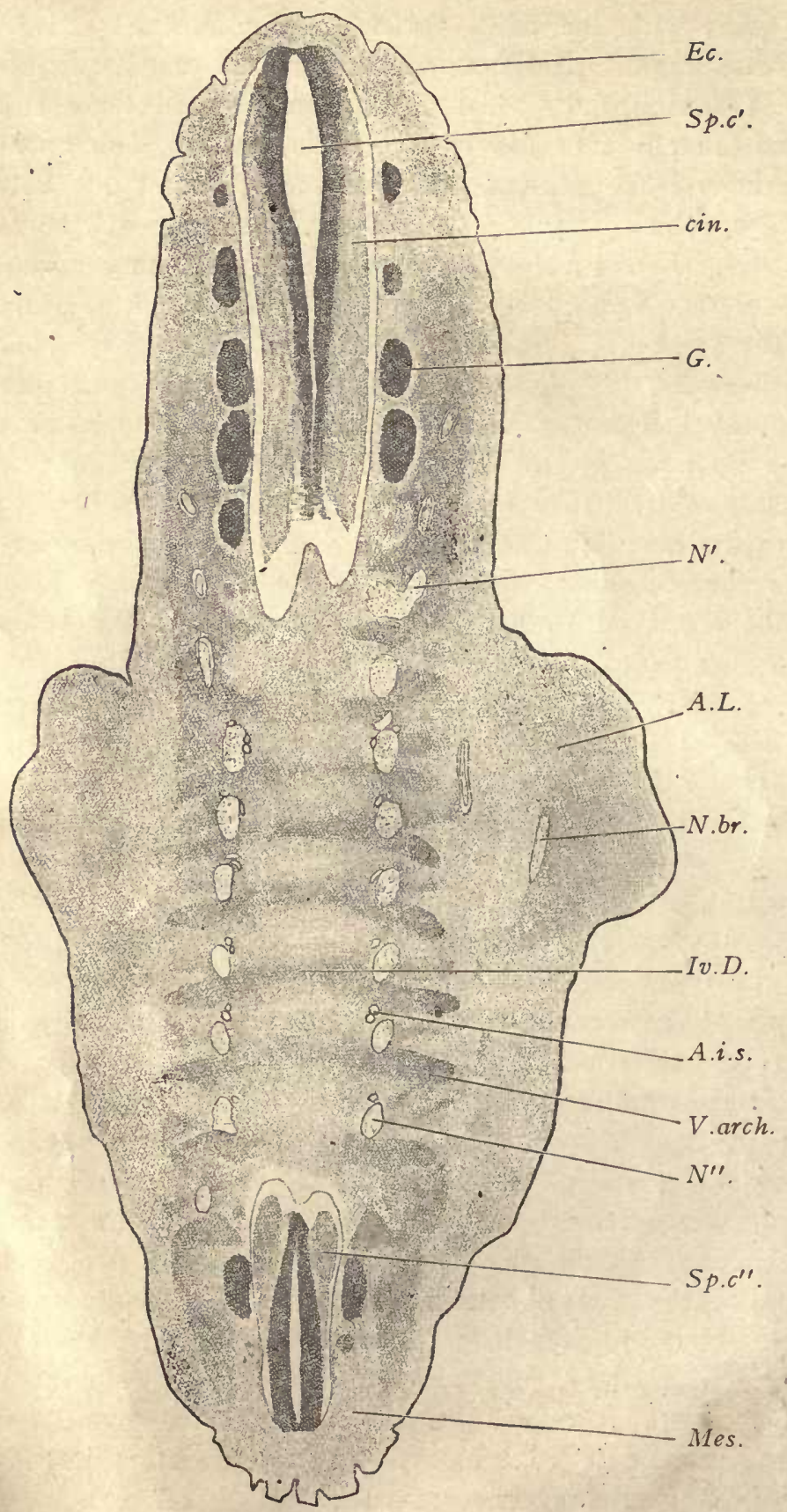

Fig. 207.-Pig, 12.0 mu. Frontal Series 6, Section 572.

i.s, Intersegmental artery. A.L, Anterior limb. cin, Cinerea of spinal cord. Ec; Ectoderm. G, Ganglion. Iv.D. Intervertebral disc. Mes, Mesoderm. $N^{\prime}, N^{\prime \prime}$, Nerves. N.br, Nerve-branch of brachial plexus. $S p \cdot c^{\prime}$, Cephalad portion of spinal cord. $S p \cdot c^{\prime \prime}$. Caudad portion of spinal cord. V.arch, Anlage of arch of vertebra. $X 22$ diams. 
through in the series of sections, it may be found that the condensed mesenchyma surrounds the notochord, which, therefore, passes through the central portion of each intervertebral anlage. The bodies of the vertebræ at this stage consist merely of the loose mesenchyma between the intervertebral discs, are entirely without any distinct limitation, and merge into the surrounding loose mesenchyma. Near the anterior border of each nerve-trunk, and usually somewhat toward the median side of it, lie-the intersegmental vessels, which are of small size and vary greatly in their exact position and number, according as they are more or less branched. Between the ends of the vertebral bows outside of the nerve-trunks can be seen with higher power clusters of elongated cells with developing muscle-fibers which are here still segmentally arranged between the processes of the developing vertebræ.

\section{Pig Embryo of $17 \mathrm{~mm}$. Study of Sections.}

Since the pig of $\mathrm{I} 2 \mathrm{~mm}$. contains the anlages of perhaps every important part of the body sufficiently. advanced in development to be clearly recognized, we find in the immediate subsequent development that we have to do not so much with an introduction of new parts as with the differentiation of those which have already commenced. Embryos of $17 \mathrm{~mm}$. are convenient for the study of the differentiations referred to. Particularly important for the student to note are the advances in the development of the vertebræ, of the lungs, of the Wolffian bodies and genital glands, and of the kidneys. These points are illustrated in figures 208 to 2 Io, representing portions of three transverse sections of a I7 $\mathrm{mm}$. embryo.

Transverse Section through the Lungs (Fig. 208).-The epidermis of the embryo has become more distinct owing to its growth in thickness, which is . . umpli.hed by the increase of the number of layers of cells. The growth is very marked as the sides of the section about the level of the vertebra. At these points it can be clearly seen that upon the outside the epidermis has a very thin layer of flattened the nuclei of which are themselves also somewhat flattened. This single layo of cells is known as the epitrichium, because the hairs are developed entirely derneath it. Where the epidermis is thickest, one can observe that the layers 8 W s mext to the mesoderm are closely packed together with round nuclei. They repr commencing formation of the basal layer of the adult epidermis. Between the layer and the epitrichium the cells are more loosely placed, forming the in stage of the mucous layer. The mesenchyma is very much developed and on a large territory in the dorsal region of the embryo. It carries the nerves as blood-vessels and shows at various points accumulations of more darkly stained $\mathrm{s}$, which are of two kinds: first, groups of mesenchymal cells proper, the anlage. thelial muscle-cells, the various skeletal muscles. There is little differentiation otherwise 
in it: (I) The anlage of the vertebra, Vert, which is now quite well defined; around the edge of it the cells have assumed an elongated form and have elongated nuclei; the elongation is parallel with the surface of the anlage. These cells result from the commencing differentiation of the perichondrium, which at this stage merges on the one side into the anlage of the vertebræ, and on the other into the surrounding mesenchyma. The cells of the vertebra have changed into young cartilage-cells. They are now distinctly separated from one another by a well-devel-

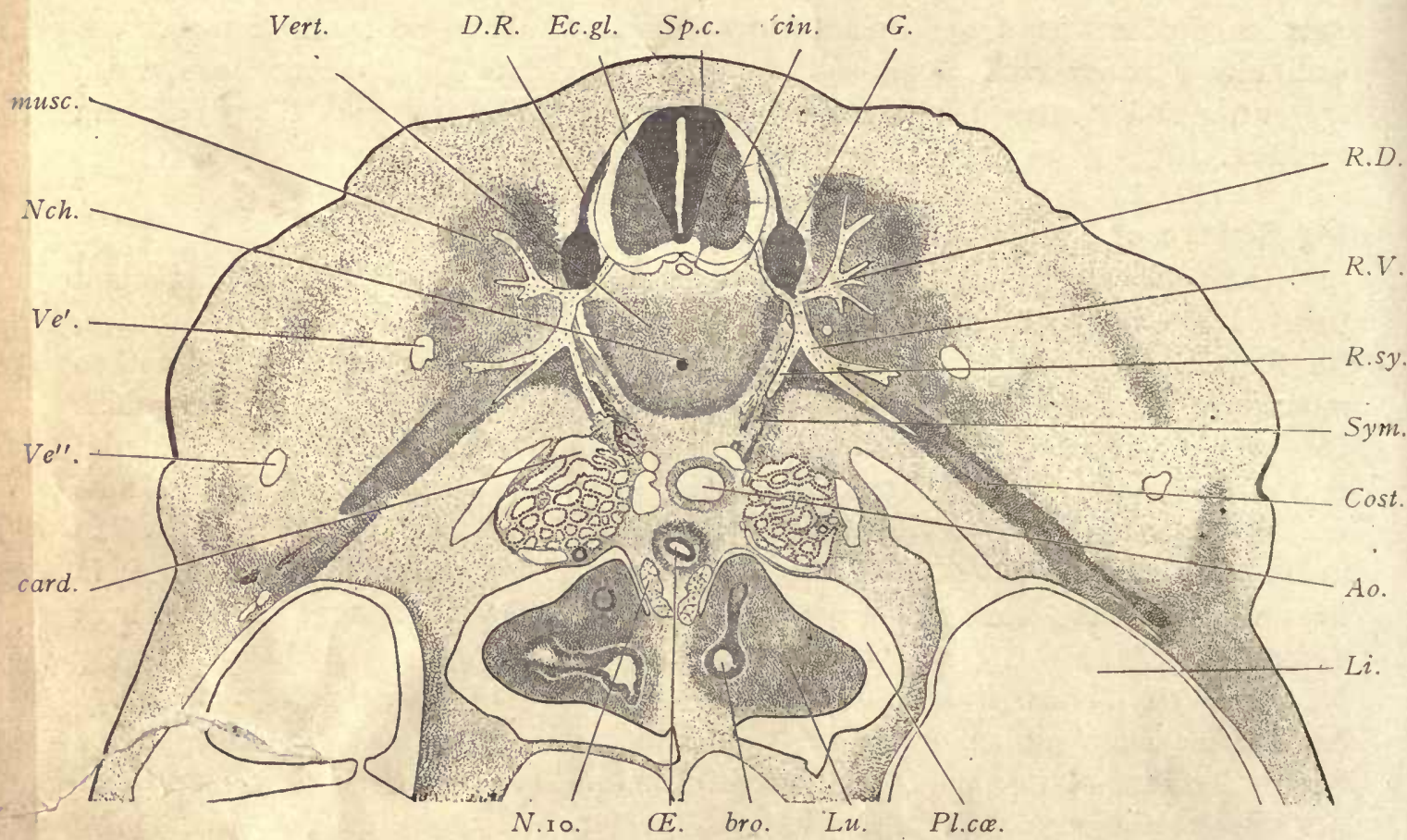

Fig! 208.-Pig, 17.0 mm. Transverse Series 5i, Section 464.

Ao, Aorta, bro, Entodermal bronchus. card, Posterior cardinal vein. cin, Neurone layer (cinerea) of spinal cord, Cost, Anlage of ribs. D.R, Dorsal root. Ec.gl, Ectoglia. G, Ganglion. Li, Liver. Lu, Lung. musc, Dorsal musculature. N.ro, Vagus nerve. Nch, Notochord. $E$ E, Esophagus. Pl.ce, Pleural cœlom. R.D, Ramus dorsalis. R.V, Ramus ventralis. R.sy, Ramus sympathicus. Sp.c, Spinal cord. Sym, Sympathetic ganglion. $V e^{\prime}, V e^{\prime \prime}$, Branches of the subclavian vein. Vert, Vertebra. $\times 22$ diams.

oped matrix. Each cell occupies a separate space or capsule in the matrix. The protoplasm of the cell, having changed to a transparent substance and being unstained, seems to have disappeared, but the nucleus remains distinct, for it stains readily, has a sharp outline, and contains a number of dark granules, one or two of which are conspicuous by their greater size and irregular shape. The nucleus itself, in most of the cells, is somewhat irregular in outline, as if distorted by shrinkage. Toward the center of the anlage the cytomorphosis is most advanced. 'Toward its outen surfice the cells are less changet. lik neares together, and have 
more, regularly shaped nuclei. In the center of the vertebra lies the round notochord, Nch, the sheath of which has increased considerably in thickness, and, being unstained, appears as a clear space between the cells of the notochord and those of the enclosing vertebra. The nuclei in the notochord are numerous and somewhat crowded together. (2) The costal processes, Cost, of the vertebra, which are rod-like and extend quite far down into the somatopleure. The histogenetic changes in these processes are similar to those in the vertebra, but less advanced. They have progressed somewhat more in the proximal than in the distal portion of the rib. (3) Around the central nervous system the pia mater has become more distinct, and the arachnoid membrane is indicated by the wide separation of its cells and the length of the processes connecting them. Its differentiation is most easily recognized at the sides of the spinal cord. The outer limit of the arachnoid is shown by a slight condensation of the mesenchyma which marks the first step in the differentiation of the dura mater, the anlage of which is further defined by the elongated form of the mesenchymal cells, by which they differ from the mesenchymal cells on both sides. (4) There is a distinct layer of condensed mesenchyma around the aorta, Ao. The layer thus formed consists of elongated cells, and perhaps corresponds only to the muscular coat of the vessel. (5) About the cesophagus, $\mathscr{E}$, the mesenchyma forms two distinct layers. The inner, next to the epithelium, is of looser texture, and is the anlage of both the mucous and submucous layers of the adult. The outer layer is denser and consists chiefly of young smooth muscle-cells, which are merely modified mesenchymal cells, characterized by the greater development of their protoplasm and by their elongated form. Traces of the differentiation of the outer layer into the inner circular muscular coat and the outer longitudinal coat of the adult are clear in the section.

The spinal cord, Sp.c, has changed its outline as seen in section, being brodest in the ventral zones, which have also begun to expand ventralward so that the outline of the cord shows on its ventral side a concavity, the first indication of the ventral fissure. The three layers of the spinal cord are yery distinct. The change in form, however, it can be clearly seen, is due chiefly to the growth of the gray layer, cin, especially in the ventral zone. The gray layer in the dorsal zone is still very slightly developed. From the dorsal zone descends on either side the dorsal nerve-root, D.R, which presently joins the ganglion, $G$. The ganglion now occupies a much lower position than in the earlier stages (compare Fig. I98, G). From the ventral zone springs the ventral root which unites with the dorsal at the lower tip of the ganglion. From the nerve-trunk thus formed there is given off almost immediately the dorsal branch, $R . D$, which soon ramifies in the midst of a dark mass of tissue, the anlage of the dorsal musculature, musc. The main nerve-trunk descends ventralward and sends off at the level of the vertebra a sympathetic branch, R.sy, which runs obliquely downward and inward toward the aorta, and there terminates in the anlage of the 
sympathetic chain, Sym, which consists partly of nerve-fibers, partly of ganglion cells which have migrated along the nerve and taken up their position at its end. These cells are easily recognized by their very dark staining. Their nuclei are a little lighter than those of the neighboring mesenchymal cells, but the cells, owing to their deep coloration, are conspicuous even when the section is examined only with the low power. The sympathetic anlage comes in close contact with a portion of the cardinal vein, card, near the aorta. The main nerve-trunk, R.V, continues obliquely downward and presently forks into an upper and a lower branch. The cardinal veins, card, lie on either side of the aorta, but they are almost completely obliterated by the ingrowth of the Wolffian tubules, which subdivide the vein into numerous smaller channels or sinusoids. The section also shows two branches, $V e^{\prime}$, and $V e^{\prime \prime}$, of the subclavian vein. The identity of these branches has not yet been determined. Beneath the aorta, Ao, follows the œsophagus, $E$, the lumen of which is much smaller than that of the aorta. Its epithelium has the general characteristics of the epithelial entoderm at this stage, being a rather thick cylinder epithelium. As above mentioned, the differentiation of the mucous. and muscular layers of the œsophagus shows clearly. Below the œesophagus lie the two large vagus nerves, N.Io, and then follow the sections of the two lungs, $\mathrm{Lu}$. Each lung is a lobe of tissue connected with its fellow across the median line of the embryo and projecting laterally far into the pleural cavity, Pl.coe. The lung consists chiefly of a large accumulation of dense mesenchyma in which the epithelial bronchi, bro, ramify. Every bronchus has a central lumen and its walls are formed by a moderately thick layer of cylinder entodermal cells. The surface of each lung is covered by mesothelium, which is shown as a distinct line in the engraving. The mesothelium can be followed to the root of the lung, where it is reflected on to the outer wall of the pleural chamber. The pleural cavity, Pl.coe, is thus everywhere bounded by mesothelium which persists throughout life, being known in the adult as the pleural epithelium.

Section through the Wolffian Body and Genital Gland (Fig. 209).- The general characteristics of the ectoderm, mesenchyma, and nervous system are nearly the same as in the section last described. On one side the section shows a thickening of the ectoderm, the anlage of a mammary gland, mam (compare. page 320 ). The branches of the nerves are not so well shown in this section as in the previous one. The level of our section corresponds to the lower end of the vena cava inferior, which is marked at this stage by the two large mesonephric veins, V.msn, which come from the Wolffian bodies and by their union constitute the lower end of the vena cava. The mesonephric veins are, strictly speaking, portions thereof. The Wolffian bodies are the most conspicuous structures shown in the section. They consist chiefly of a great number of tubules, W.t, very much crowded together. On the median side of the organ appear the large glomeruli, Glo, and-on their ventral side we have the section of the longitudinal Wolffian duct, W.D. The tubules of the Wolffian body are formed by a more or less nearly cuboidal 
epithelium, the nuclei of which are decidedly larger than those of the mesenchymal cells. The nuclei themselves stain deeply, have well-marked outlines, and very distinct granules in their interior. The protoplasm of the cells also stains somewhat with cochineal, carmine, hematoxylin, etc. There is very little mesenchyma

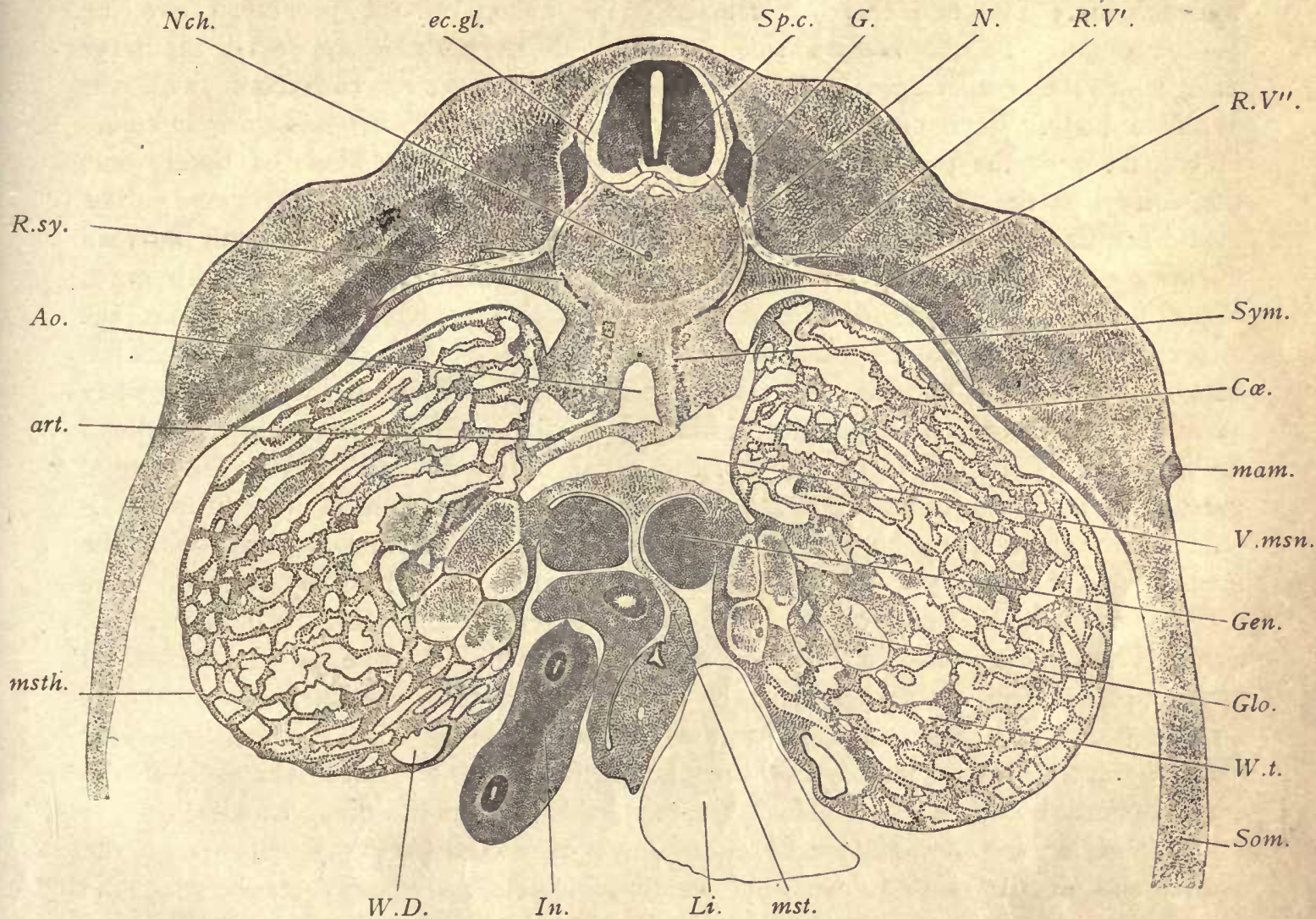

Fig. 209.-Pig, 17.0 mis. Transverse Series 5i, Section 651.

Ao, Dorsal aorta. art, Glomerular artery. Ce, Coelom. ec.gl, Ectoglia. G, Ganglion. Gen, Genital gland. Glo, Glomerulus of Wolffian body. In, Intestine. Li, Liver. mam, Mammary anlagè. mst, Mesentery. msth, Mesothelium. N, Ventral nerve. Nch, Notochord. R.sy, Ramus sympathicus of nerve. R.V', R.V', Branches of the ventral ramus of the spinal nerve. Som, Somatopleure. Sp.c, Spinal cord. Sym, Sympathetic ganglion. V.msn, Vena mesonephrica. W.D, Wolffian duct. W.t, Wolffian tubule. $\times 22$ diams.

in the organ, but each tubule is closely invested by vascular endothelium; hence the tubules are separated from one another only by blood spaces, which, morphologically speaking, are portions of the cavity of the cardinal vein. These blood spaces are highly characteristic and are typical sinusoids. The intertubular circulation of the Wolffian body is, so far as known, always sinusoidal. The aorta, Ao, is seen in the figure to give off a small branch, art, which runs toward the Wolffian body. There are numerous such branches, each one of which may be traced to a 
glomerulus of the mesonephros. Each glomerulus has a capillary circulation, and the blood on leaving the glomerulus is supposed to be emptied into the venous sinusoids. More exact investigation of this point is needed. The mesonephros is covered by a layer of mesothelium, msth, underneath which is a thin layer of mesenchyma. The two together constitute the anlage of the peritoneal covering of the organ. To the median side of the Wolffian body is appended the large anlage of the genital gland, Gen, which has a constricted connection with the Wolffian body. Each gland is covered by mesothelium and extends until it comes in contact with the mesentery, mst. The gland contains two kinds of tissue, one, the anlage of the medullary, the other of the cortical portion of the gland. The medullary tissue resembles the neighboring mesenchyma and occupies only a small territory about the stalk of the organ. The cortical tissue contains cells with much larger nuclei and clearly developed protoplasmic bodies. It occupies by far the larger part of the gland. Comparison with figure 198 will show that the genital anlage at this stage occupies the same topographical relation to the Wolffian body as at earlier stages. It differs now from the earlier condition chiefly by its growth in size and by its advancement in histological differentiation. Below the genital gland the intestinal canal is cut several times. One portion of the intestine is seen in the section to be connected by means of the mesentery, mst, with the median dorsal tissues of the embryo. The intestine is formed by a small tube of entoderm with a small cavity. The entoderm is a rather thick cylinder epithelium. The greater part in bulk of the walls of the intestine is constituted by mesenchyma. The external surface is covered by a thin mesothelial layer. The mesenchyma is beginning to show the differentiation of the external muscular from the internal mucous coat. There is at this stage no trace whatever of the development of any folds or glands on the inside of the intestinal canal.

Section through the Kidney (Fig. 210).-This section being much nearer the caudal end of the embryo, we find, as throughout all the early stages, that the differentiation of the tissues is less advanced than nearer the head. We have accordingly, so to speak, an earlier stage in the development of the spinal cord, Sp.c, of the nerves, and of the vertebra. In the median line is the large aorta, Ao, about which the mesenchyma is only slightly condensed. Near the aorta are the conspicuous anlages of the sympathetic system, Sym, which appear at this level in a very characteristic hook-shaped pattern. At the dorsal end of the hook the nerve-fibers are much more numerous than in the ventral portion of the anlage. The sympathetic cells themselves are extremely conspicuous, owing to the depth of their stain. On either side is situated the anlage of the permanent kidney, Ki. Each anlage consists of an irregularly branching space bounded by a thick layer of epithelium, which has somewhat the appearance of the intestinal entoderm at this stage. If the series of sections be followed through farther toward the tail of the embryo, the epithelial space will be seen to contract to a relatively small tube, the ureter, which opens into the Wolffian duct of the same side. The ex- 
panded portion of the cavity shown in our figure corresponds in part to the pelvis of the adult organ. Its irregular shape is due to the fact that it is forming a series of outgrowths, which are to give rise to the collecting tubules. Around the ends of the branches of the renal pelvis is a darker tissue, in which the cells are very much crowded. It is the material out of which the glomeruli and convoluted tubules of the kidney are to be differentiated. By a secondary process these tubules become united with the branches from the renal pelvis, the branches forming the collecting tubules only of the adult organ (compare page IIo). The

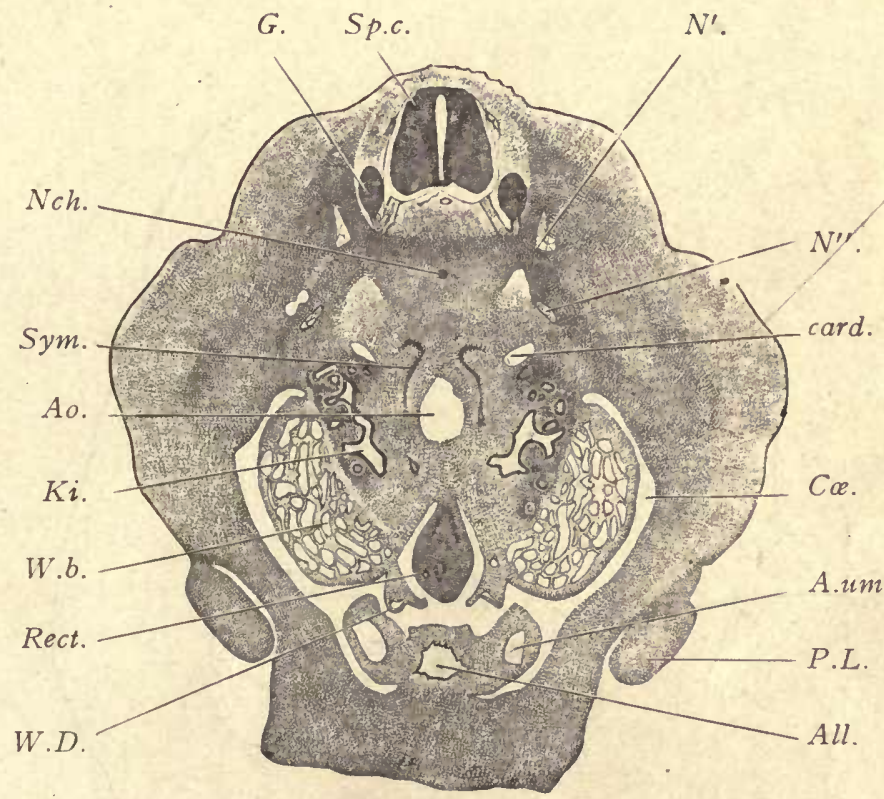

Fig. 210.-Pig, 17.0 mu. Transverse Series 5i, Section 759.

All; Allantois. Ao, Aorta. A.um, Umbilical artery. card, Branch of cardinal vein. Cœ, Colom. G, Ganglion. $K i$, Kidney. $N^{\prime}, N^{\prime \prime}$, Nerves. Nch, Notochord. P.L, Posterior limbs. Rect, Large intestine. Sp.c, Spinal cord. Svm, Sympathetic ganglion. W.b, Wolffian body. W.D, Wolffian duct. $\times 17$ diams.

origin of the renal anlage may easily be followed in earlier stages. It is found that from the pelvic end of each Wolffian duct there develops a dorsal outgrowth, which is lined by epithelium. This outgrowth elongates in a headward direction. Its end expands; the narrow portion is the ureter, the expanded portion the anlage of the pelvis. The pelvis becomes irregular in shape and forms outgrowths. Around it appears the condensed tissue just referred to. On the ventral and lateral sides of the kidneys in our section appear the ends of the Wolffian bodies, $W . b$. From the ventral and inner edge of each Wolffian body is a projecting lobe of tissue in which the Wolffian duct, W.D, is lodged. The walls of the Wolffian duct are a rather thin, cuboidal epithelium, surrounded by mesenchyma in which there is no very clear evidence of specialization. Between the Wolffian bodies 
is suspended the large intestine. It has a small canal formed by entoderm and very thick mesodermic walls.

Attached to the ventral side of the body-wall of the embryo is the allantois, All, the cavity of which is quite large, somewhat irregular in shape, and lined by a cuboidal epithelium, a portion of the entoderm. By following through the sections it can be seen that the allantois and large intestine join at the cloaca. The entodermal allantois is surrounded by mesenchyma, which is very much looser in texture than that of the intestine proper. On either side of the allantois is a projecting lobe of tissue in which the umbilical artery, A.um, is lodged. The

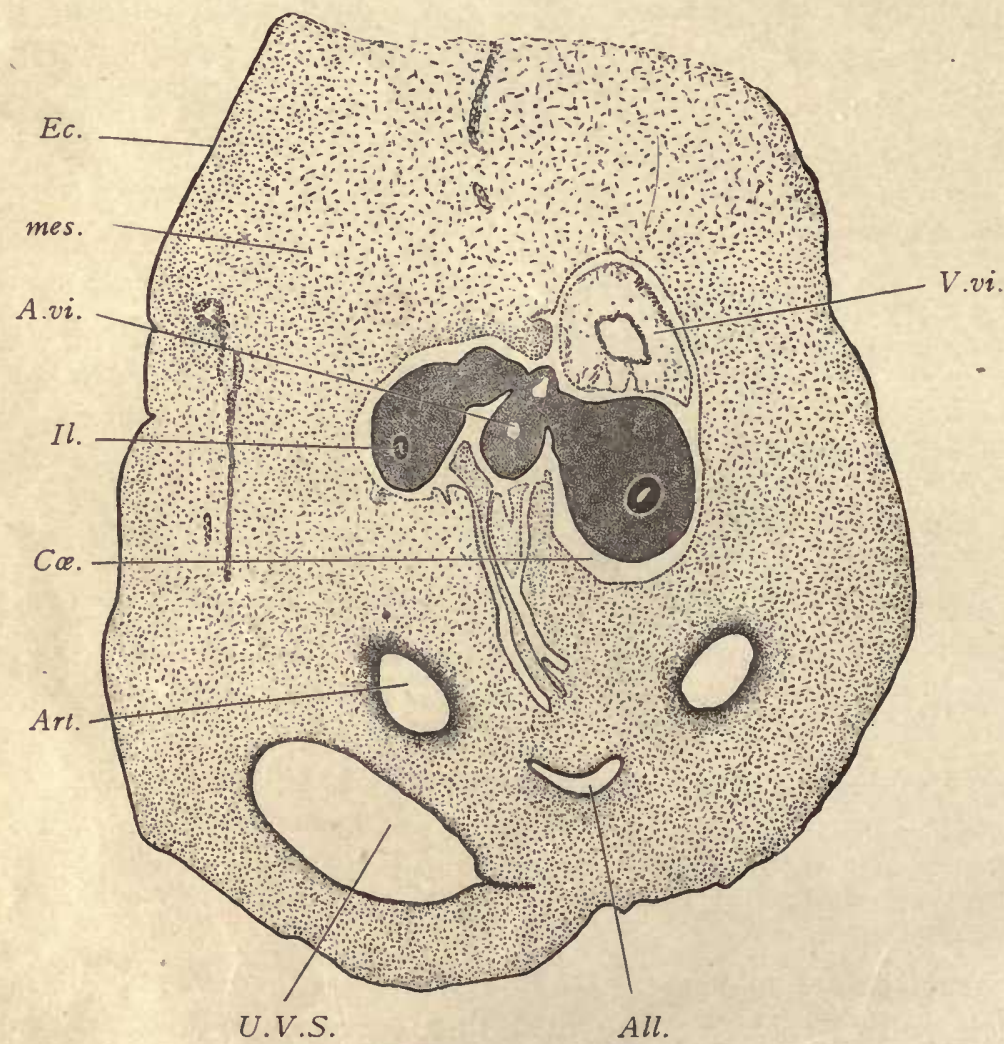

Fig. 2i1.-Pig, i7.o Mm. Frontal Series 39, Section 64.

$A l l$, Allantois. Art, Umbilical artery. A.vi, Vitelline artery. Co, Colom. Ec, Ectoderm. Il, lleum. mes, Mesenchyma. U.V.S, Left umbilical vein. V.vi, Vitelline vein. $\times 35$ diams.

two arteries pass upward to the umbilicus, then outward to the placenta. Downward they continue to the level of the cloaca, there pass to the dorsal side of the embryo, and unite with the end of the median dorsal aorta.

Frontal Section of the Umbilical Cord (Fig. 211).-We get in frontal series of the embryo sections of the umbilical cord which are more or less nearly transverse. The major part of the area of such sections is occupied by mesenchyma, 
Mes. On the ventral side of the cord is the cavity of the allantois, All, lined by a thin layer of entoderm, and with no marked condensation of the mesenchyma around it. A little lower is the large umbilical vein, U.V.S, which appears as a prolongation of the left umbilical of the body proper, but the part within the cord is probably the product. of the fusion of the two original veins. A little higher are the two umbilical arteries, Art, which lie symmetrically as regards the allantois. All three vessels are strengthened by walls of condensed mesenchyma, which is much more prominent around the arteries than around the vein. The center of the cord is occupied by a large irregular space, $C \propto$, a prolongation of the body cavity. In this umbilical coelom are lodged the loop of the intestine and the cord containing the vitelline vein. The intestine is cut twice, the section on the left passing through the ileum, $I l$, and on the right through the jejunum, which is much larger than the ileum, having both a larger entodermal portion and a thicker mesodermal part. The two segments of the intestine are joined together, and in the part between them are two blood-vessels, one, the inferior, is the vitelline artery, A.vi, which extends beyond the intestinal loop, to ramify upon the yolksac; the other vessel is the superior mesenteric vein, which does not extend beyond the intestine. The mesenchyma of the intestines and of the bit of mesentery between them consists of very crowded cells, so that the tissue appears darkly stained. Above the loop lies the cord in which is situated the vitelline vein, V.vi. The vein is centrally placed; the cord forms a thick wall of very loose mesenchyma covered by a thin mesothelial layer. The cord is a very characteristic embryonic structure; it arises from the mesentery of the duodenum and extends through the umbilical opening to the yolk-sac. The vein which it contains is thought to arise by the fusion of the two original vitelline or omphalo-mesaraic veins. Its union with the superior mesenteric vein to form the portal vein is shown in figure Ioo. All the surfaces of the colom are covered by a distinct mesothelium. The main tissue of the umbilical cord is a typical loose mesenchyma, Mes. The ectoderm, $E c$, is the direct prolongation of the embryonic epidermis, and consists for the most part of a single layer of cells, although the formation of a second outer layer seems to be beginning.

\section{Pig Embryo of $20 \mathrm{~mm}$. Study of Sections.}

Nine sections of this stage are figured. In the practical laboratory work embryos a little larger or smaller may serve equally well to illustrate the developmental conditions of this stage.

TransverseSection through the Snout (Fig. 2I2). - The parts shown are the same as in figure 219 , to the description of which reference is made. The present figure $2 \mathrm{I} 2$ is added to illustrate the development of the palate shelf, Pal. The palate shelf is a large protuberance on the inner side of the maxillary process. Its inner edge abuts against the tongue, Ton, its upper edge underlies the maxillo-turbinal fold, max.tb, and its lower edge forms part of the roof of the oral cavity, Or. 
At this stage it consists of a large mass of undifferentiated mesenchyma, covered by a layer of epithelium. The two palate shelves continue to grow toward one another until they meet in the median line below the nasal septum, Sept. As they approach one another the tongue descends. Ultimately the two palate shelves unite with one another and with the overlying nasal septum. The epithelium of the two shelves concresces and forms for a time a partition, which marks the point of union of the two shelves, both with one another and with the nasal septum. This partition persists for a short time only, for it soon disappears by resorption.

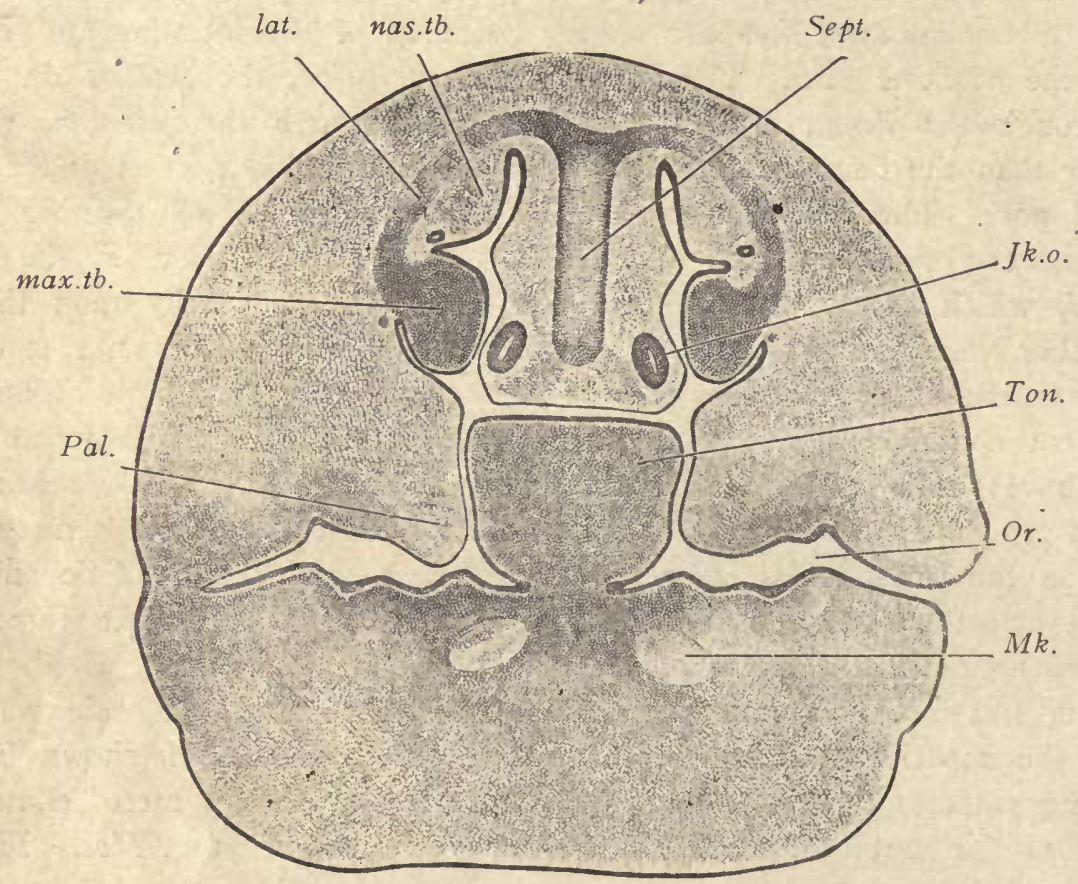

Fig. 21 2.-Pig, 20 mis. Transverse Series 59, Section 522.

$J k . o$, Jakobson's organ. lat, Lateral ethmoid cartilage. max.tb, Maxillo-turbinal fold. Mk, Meckel's cartilage. nas.tb, Naso-turbinal fold. Or, Oral cavity. Pal, Palate shelf. Sept, Cartilage of nasal septum. Ton, Tongue. $\times 22$ diams.

The union of the palate shelves separates definitely the nasal and oral cavities from one another. Their union is gradual, beginning in front and gradually extending backward. It is a not infrequent anomaly that the palate shelves fail to unite perfectly. When this occurs, there results the condition known as cleft palate.

Transverse Section through the Lower Part of the Neck (Fig. 213).-The spinal cord, Sp.c, shows a very great enlargement of the ventral zones, which now project downward so as to enclose between them a distinct groove in the median ventral line, which can be identified as the commencing anterior fissure of the cord. In this groove runs a small, longitudinal blood-vessel, the arteria sulci, which from 


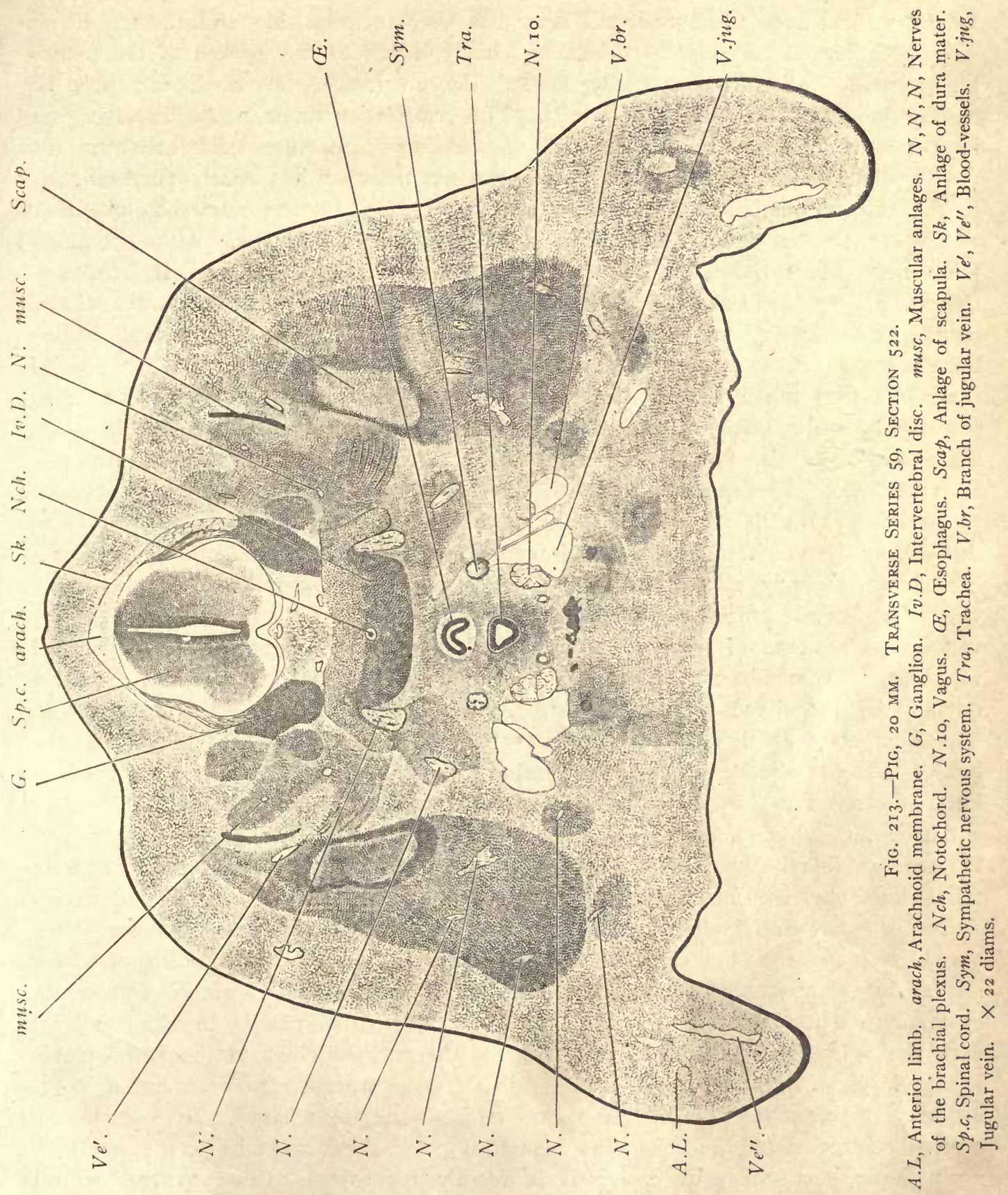


time to time gives off small branches, which enter the substance of the spinal cord. In the ventral zone the ependymal layer has become quite thin and the middle or gray layer has acquired great thickness, chiefly owing to the growth of the neuroblasts, many of which, especially toward the outside of the cord, can now be readily identified as young nerve-cells. The ectoglia or outer neuroglia layer has increased in thickness. Many of the processes of the neuroglia cells can be readily distinguished, running, for the most part, more or less nearly perpendicular to the surface of the cord. Between the neuroglia fibers are numerous fine dots which are the cut ends of the nerve-fibers running longitudinally. Although about these nerve-fibers there are as yet no medullary sheaths developed, it is, nevertheless, proper to speak now of the ectoglia as the external white matter of the cord. Immediately beneath the entrance of the dorsal root the external outline of the cord shows a concavity which disappears in later stages. The dorsal zones are very much smaller than the ventral. The differentiation of their three primary layers is being completed by the development of a distinct middle layer. The ectoglia of the dorsal zone resembles that of the ventral zone in structure and thickness. The spinal ganglia, $G$, have descended from their original position, so that they now lie on a level with the lower edge of the spinal cord, and the nerve-root, by which each ganglion is connected with the dorsal zone of the cord, has correspondingly elongated. The lower edges of the ganglia come in contact with the lateral processes of the vertebra. Between the spinal cord and the vertebra is an area of loose mesenchyma which may be regarded as a portion of the arachnoid membrane. Close to the upper surface of the vertebra, bounded dorsally by the tissue just mentioned, are two symmetrically placed blood-vessels. The intervertebral ligament, Iv.D, is only partially cut. Above it appears the lighter tissue of the next following vertebra, which is shown better several sections lower down. The vertebra is distinctly cartilaginous, though not yet fully differentiated, and is surrounded by a distinct fibrous layer, the perichondrium. In the median line below the vertebra lie the osophagus, $\mathscr{E}$, and trachea, Tra, both tubes lined by entoderm. The cavity of the osophagus is somewhat crescentshaped, that of the trachea triangular. About the œsophagus the mesoderm forms two layers, an inner lighter layer and an outer muscular layer, the cells of which are already elongated. The mesenchyma about the trachea is more condensed, especially on the sides and below, and the condensed tissue is in close contact with the epithelium. On the dorsal side of the trachea close to the entoderm is a thin layer of transversely elongated cells. The sympathetic nervous system, Sym, appears symmetrically placed near the trachea and œsophagus. In section the sympathetic is round and contains numerous nerve-fibers and characteristic young sympathetic nerve-cells, by which it is readily recognized. Close to the ventral side of the sympathetic is the section of the large jugular vein, V.jug, a branch of which, V.br, lies laterad from the main vessel. This branch receives blood-vessels from the facial region. Between the main jugular and its branch are some 
lymphatic spaces, somewhat irregular in form, and lined by a thin endothelium so that they present a close resemblance to veins in their structure; if followed up toward the head the lymphatics are found to unite with the large jugular lymphsac (Fig. 6o, s.l.j). Close to the medial wall of the jugular vein is situated the large trunk of the vagus nerve, N.Io. At a little lower level than the vagus nerves and in the median line lies the anlage of the thyroid gland, which, owing to its darker staining, is somewhat conspicuous. The cells of the thyroid form an irregularly shaped branching mass. The spaces between the branches are chiefly occupied by small endothelial blood-vessels. The arrangement of these cavities and the relation of their endothelium to the cells of the organ recall the blood sinusoids of the liver and of the suprarenal capsule. The thyroid cells are compactly arranged without distinct cell-boundaries, but with protoplasm which stains somewhat and with nuclei of rounded form, distinct outline, and granular appearance, the granules being decidedly more conspicuous than the granules in the nuclei of the neighboring mesenchymal cells. Just ventral to each jugular vein is a small darker body, consisting of closely compacted cells, resembling in appearance those of the thyroid. The body has a very distinct external outline and is actively growing, for several of its nuclei are in mitosis. The bodies in question are the parathyroid glands. The rest of the section is mainly occupied by mesenchyma and numerous darker masses, musc, the anlages of the various muscles of the neck and throat. On each side is shown a small piece of the cartilaginous scapula, Scap. At the lower corner of the section is an indication of the anterior limb, A.L, and of its vein, $V e^{\prime \prime}$.

Section through the Lungs (Fig. 214). - The spinal cord shows very clearly in the differentiation of the three primary layers of the medullary wall. Its structure is similar to that shown in figure 208 , and need not be again described. The vertebra, Vert, is now distinctly young cartilage. On its ventral side its boundary is quite distinct, the formation of the perichondrium having there begun. Laterally it merges into a dense mesenchyma, by which it is united without demarcation with the $r i b$, cost', and indirectly with the vertebral arch, V.ar, both of which are cartilaginous. The cells of the vertebral cartilage occupy rounded cavities, each of which is marked by a distinct capsule. The matrix between the capsules is homogeneous, stains slightly, and has acquired a greater density than in earlier stages. The cells themselves exhibit traces of their protoplasmic bodies and have deeply stained nuclei which are quite irregular in shape and very granular. Immediately around the notochord the spaces occupied by the cells are the largest, the capsules most distinct, and the nuclei most altered. Proceeding toward the periphery of the cartilage, the cells appear in successively earlier and earlier stages, until at the very periphery we have normal nuclei and a transition to mesenchyma. The notochord has contracted, leaving a space between the notochordal cells and the vertebral cartilage. Immediately below the vertebra are the conspicuous anlages of the sympathetic system, Sym. They overlie the sections of the posterior cardinal 


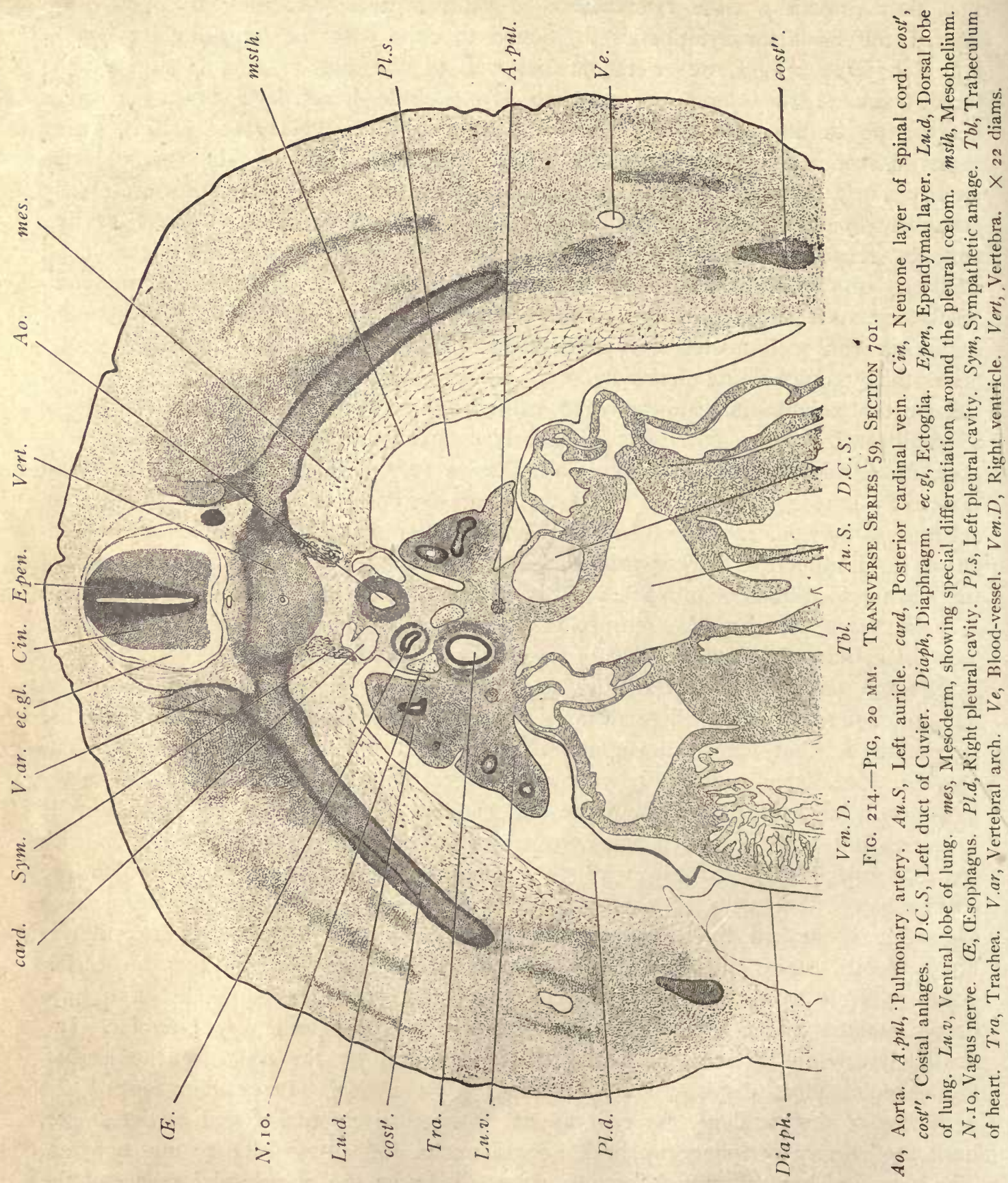


veins, card. These are now quite small vessels, the vena cava inferior having become the main channel for the return of the blood from the abdominal region to the heart. The two cardinal veins are not quite symmetrically placed, that on the left side lying a little lower than that on the right. Between them is situated the median aorta, $A o$, with a relatively thick and well-developed muscular coat, the deeper staining of which makes it conspicuous even with low powers. The asophagus, $\mathcal{E}$, and trachea, Tra, are not in the median line, but are both displaced toward the right of the embryo. As compared with earlier stages, both structures show an advance, first, by the growth of the entoderm, and, second, by the differentiation of the surrounding mesenchyma. In both œsophagus and trachea the entoderm is a ring of cylinder epithelium, the tracheal ring being much larger than the œsophageal. The mesenchyma about the œesophagus forms two distinct layers, an inner looser layer and an outer denser muscular layer. Around the trachea the. mesoderm is much condensed. On the dorsal side of the trachea the cells form next to the epithelium a special layer characterized by the elongated form of the cells. Between the osophagus and trachea are situated the vagus nerves, that of the right side, N.Io, occupying a higher position than that on the left, so that the nerves are not symmetrically placed. The cardinal veins, the aorta, the œsophagus, the vagus nerve, and the trachea are all imbedded in mesenchyma, which, together. with these structures, forms the so-called mediastinum by which the right and left pulmonary cavities, Pl.d, Pl.s, are separated from one another. On its ventral side the mediastinum joins on to the veins entering the heart. On either side of the mediastinum at the level of the trachea may be seen the projecting lung. That on the left side shows clearly the division of the organ into a dorsal lobe, Lu.d, and a ventral lobe, Lu.v. Each lung consists at this stage chiefly of mesenchymal tissue and is covered by a layer of mesothelium which forms the boundary of the pleural cœlom. Within the mesenchyma appear several sections of the branches of the entodermal bronchi. Each bronchus is lined at this stage by a rather thick entodermal layer of cylinder cells. The union of the lung with the mediastinum constitutes the so-called root of the lung. In the root of the lung is seen the small pulmonary artery, A.pul. The two arteries join a little nearer the head and on the left side of the embryo to form a single trunk, the main pulmonary artery. Originally the pulmonary arteries arise symmetrically as branches from the fifth aortic arches. They soon unite, however, throughout the greater part of their extent, forming a single vessel. The two arteries shown in our figure represent the two original symmetrical vessels where they are about to enter the lungs. On the ventral side of the section various cardiac structures are shown, but so cut that the picture is not very instructive. It will suffice to refer to the explanation of the figure for the identification of the parts.

Sections through the Müllerian Ducts (Fig. 215).-The female or Müllerian ducts are remarkable for their late development. In the $\mathrm{I} 2 \mathrm{~mm}$. pig the small funnel-shaped in aginations of the mesothelium, which represent the first 
anlages of the ducts, can just be recognized on the lower mesial surface of the Wolffian body near the cephalic end of the organ. In the $20 \mathrm{~mm}$. pig the funnels have lengthened into tubes, which run a short distance caudad, close underneath the Wolffian duct. Figure $215 \mathrm{~A}$ is a section through the right Müllerian funnel, $F$. The mesothelium near the funnel is considerably thickened, forming the socalled tubal band, Ep. The funnel is lodged in a small ridge, Rid, which projects downward from the mesonephros. The Wolffian duct does not appear in the section as it does not extend so far headward. Figure $215 \mathrm{~B}$ is a section farther caudad, to show the oval Müllerian duct, $M . D$, which immediately underlies the Wolffian duct, $W . D$, and causes a small protuberance on the surface of the mesonephros.

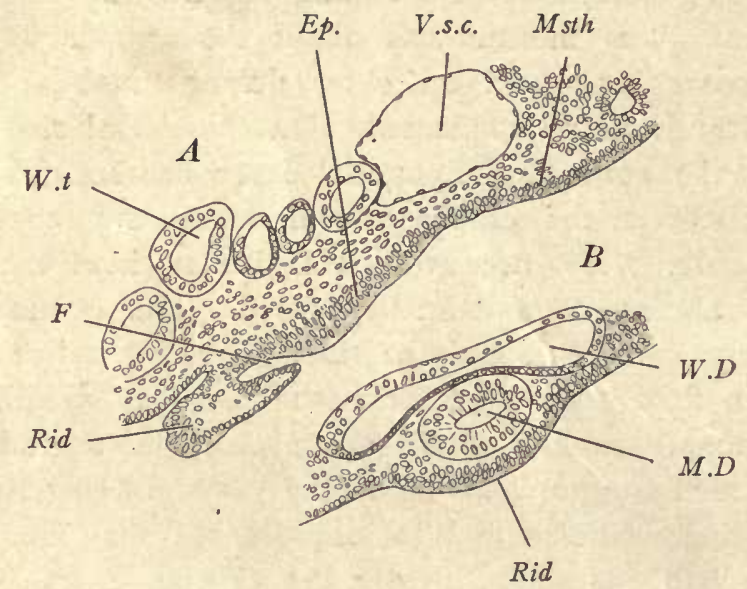

Fig. 215.-Pig of 20 mu. Transverse Series 59, A, Section $85 x$; B, Section 887.

$E p$, Tubal band of thickened mesothelium. F, Funnel-shaped opening of the Müllerian duct. M.D, Müllerian duct. Msth, Mesothelium. Rid, Ridge containing the Müllerian funnel. V.s.c, Sub-cardinal vein. W.D, Wolffian duct. W.t, Wolffian tubules. $\times$ r 50 diams.

A short distance farther on the duct ends in a blind point. It is destined to continue its backward elongation until it reaches and joins the neck of the allantois. At just what stage the junction occurs in the pig is undetermined.

Section through the Posterior Limbs (Fig. 216).-Although this section is from a transverse series, yet, owing to the curvature of the body, it shows the spinal cord cut very obliquely. The three layers of the cord, the ependymal, epen, the cinerea or neurone layer, $\mathrm{Cin}$, and the ectoglia are well marked. Something of the dorsal root, D.R, and of the ganglia, $G$, of a lumbar nerve are also shown in the section. The nerves have already joined together to form a very complex lumbar plexus, sections of portions of which appear at various points. These are all indicated by the reference letter $N$ in the figure, it being thought not desirable to attempt an identification of each component of the plexus. The plexus is more or less symmetrically placed on the right and left, at about the level of the intestine, Rect. The limbs are large projections extending dow:ward and containing in 

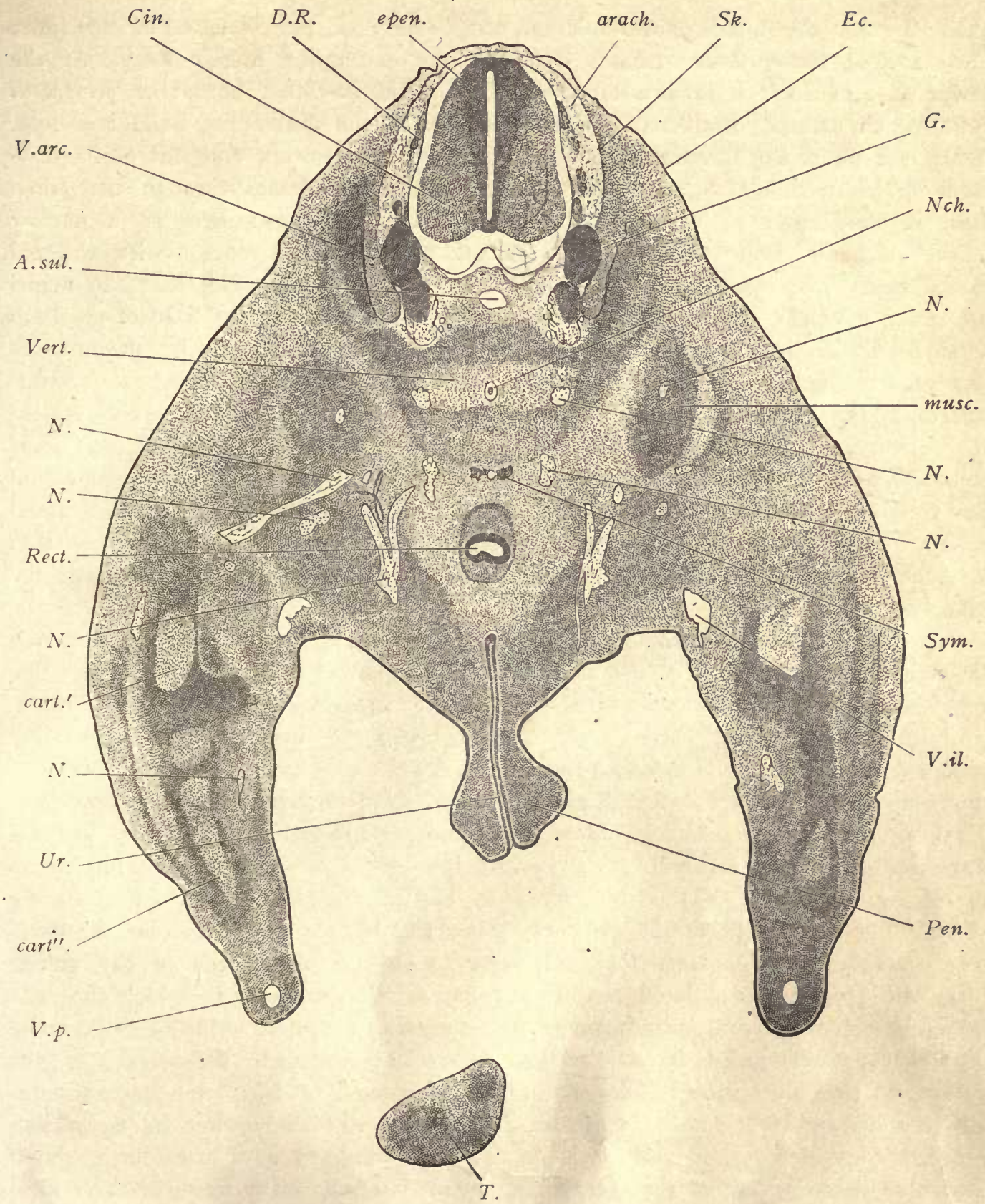

Fig. 216.-Pig, 20 mar. Transverse Series 59, Section 1253.

arach, Arachnoid membrane. A.sul, Arteria sulci. cart', $\operatorname{cart}^{\prime \prime}$, Cartilaginous anlages of elements of the skeleton of the limb. Cin, Neurone layer of spinal cord. D.R, Dorsal root. Ec, Ectoderm. epen, Ependymal layer of spinal cord. $G$, Spinal ganglion. musc, One of the muscular anlages. $N, N, N$, Nerves of the lumbar plexus. Nch, Notochord. Pen, Penis. Rect, Rectum. Sk, Anlage of the dura mater. Sym, Sympathetic nerve. $T$, Tail. U.r, Urethra. V.arc, Vertebral arch. Vert, Vertebra. V.il, Iliac vein. V.p, Border vein of the limb. $\times 22$ diams. 
their interior the cartilaginous anlages, cart', cart", of the skeleton of the limb, and, around these, darker masses of tissue, the developing muscle-fibers. At the lower edge of each limb is a blood-vessel, V.p, the so-called border or peripheral vein, which extends completely around the edge of the developing hand and foot. When the digits are developed, this vein becomes broken up, and out of its divisions are formed the digital vessels. The section also passes through the penis, $P e n$, in the center of which is the urethra, Ur. It shows here as a narrow epithelial band without any cavity, except a very small one at its external dorsal end. The band is lighter in the center, owing to the fact that the nuclei are grouped chiefly close to the two surfaces of the band. At the base of the limb is situated the irregularly shaped section of the iliac vein, V.il. In the median line may be noted the following structures. Immediately underneath the nervous system is the arteria sulci, A.sul. The vertebra, Vert, and notochord, Nch, resemble corresponding structures in the section described on page $3^{1} 5$, except that their cytomorphosis is slightly less advanced. Below the vertebra lie the paired anlages of the sympathetic nervous system, Sym, between which is the small median caudal artery. The intestine, Rect, has its transverse diameter somewhat increased, so that it appears oval in the section. Around it is beginning the differentiation of the mucosa and muscularis.

Section through the Mammary Anlage (Fig. 217).-The figure represents a section through the somatopleure of the embryo in the region of a mammary gland. The ectoderm, $E c$, covers the external surface of the somatopleure, as does the mesothelium, $m s t h$, the inner surface, the space between the two covering layers being occupied by various mesodermic structures. The ectoderm consists of two or three layers of cells, the external one of which, Eptr, the epitrichium, is very thin. To form the mammary anlage, Mam, the ectoderm suddenly thickens and projects somewhat outward and still more inward into the mesoderm. The epitrichium passes continuously over the thickening, in the production of which it takes no share. The inner edge of the ectoderm is marked by a very distinct line or basement membrane, $b$, against the underlying mesoderm. The cells of the anlage form two groups, one a band next to the basement membrane, in which the cells present a somewhat radial arrangement, and the other a central group of cells, many of which are elongated in a direction somewhat parallel to the surface of the anlage, so that they form curving lines. The elongated cells in later stages gradually cornify and fall out, so that the anlage becomes hollow, but its excavation proceeds very slowly, and in man is not usually completed until after birth. Soon after the hollowing out of the anlage has begun, it sends out a series of buds from its inner surface. These buds become elongated, somewhat twisted cords of cells, and offer at this stage resemblance to embryonic sweat-glands. The outgrowths subsequently branch and develop central cavities, and are ultimately transformed into 'the secretory portion of the gland.

Figure 217 also illustrates some irnportant points in regard to the differentia- 
tion of the somatopleure. Parallel to the ectoderm, and some distance from it, is a layer, Pan, which is marked out by numerous blood-vessels. This is the panchoroid layer. There is a slight but unmistakable difference in the mesoderm within and without this layer, for in the region between the panchoroid and the ectoderm the cells are somewhat more crowded. They probably represent the anlage of

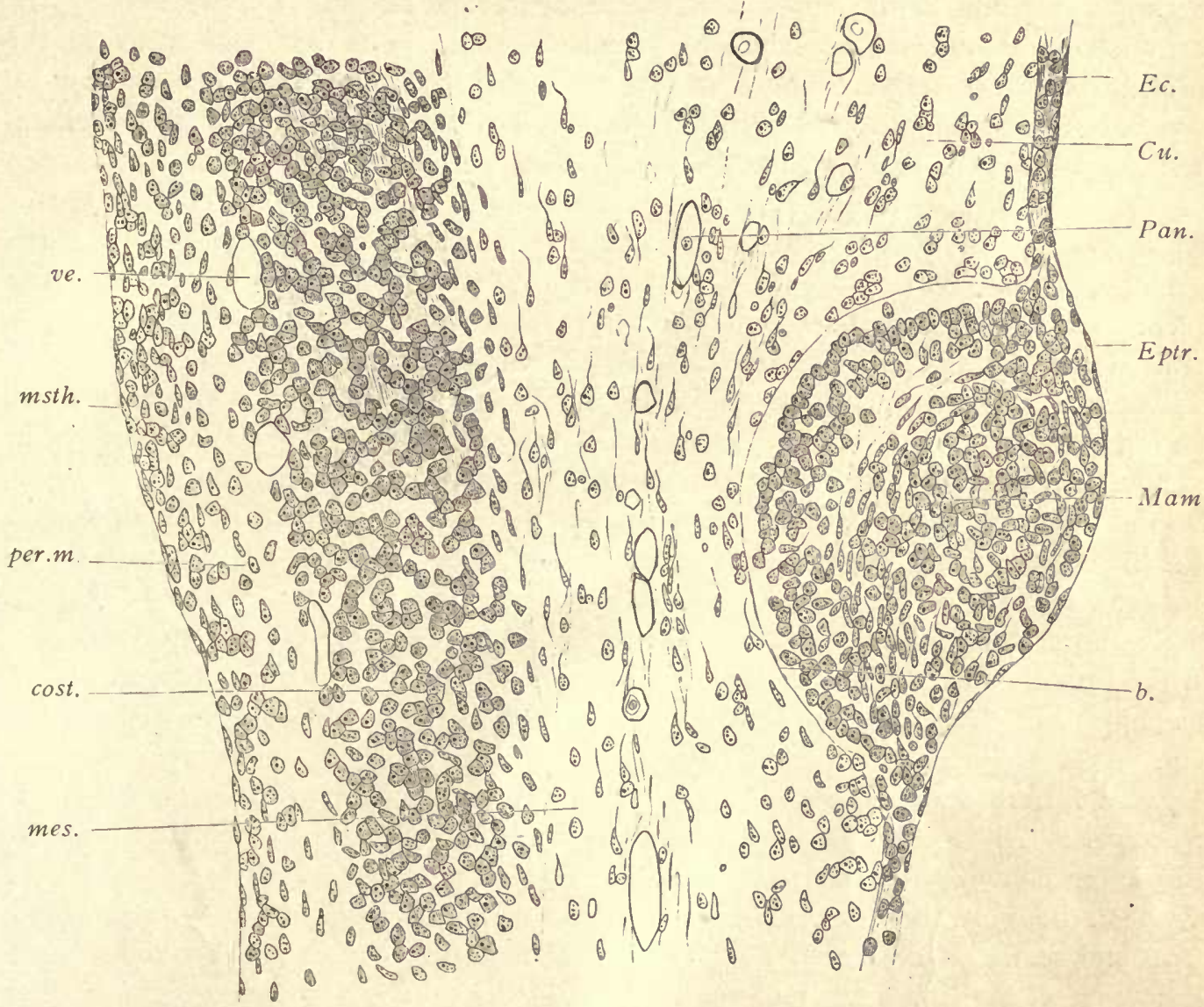

Fig. 2i7.-Pig, 20.0 Mm. Transverse Series 59, Section 1043.

$b$, Basement membrane of epidermis. -cost, Costal anlage. Cu, Cutis. Ec, Ectoderm. Eptr, Epitrichium, Mam, Mammary anlage. mes, Mesenchyma. msth, Mesothelium. Pan, Tunica panchoroidea. per.m.

Peritoneal mesoderm. ve, Blood-vessel. $\times 250$ diams.

the cutis, $\mathrm{Cu}$, and of the cutis only. Within the vascular layer the mesodermic cells, mes, are not so near to one another, and the processes, by which they are connected, are more slender. Toward the mesothelium is a broad band of denser tissue, cost, the rudiment of a rib, the inner boundary of which is further marked by several blood-vessels, ve. Between the costal anlage and the mesothelium is a layer of embryonic connective tissue, the cells of which are more crowded toward 
the mesothelium, so that we may say that two layers of mesenchyma are already imperfectly differentiated within the rib. The denser layer next the mesothelium is destined to become still more marked and to transform itself into the connectivetissue layer of the peritoneum. With the overlying mesothelium it develops into the peritoneal membrane of descriptive anatomy.

Sagittal Section through the Right Lung and Kidney (Fig. 218).- The lungs occupy a position in the upper part of the figure and are easily recognized by the conspicuous-entodermal bronchi, bro, which resemble in microscopic structure the bronchi of earlier stages. The branches are widely separated from one another by the voluminous mesenchyma of the organ. The lung is covered by mesothelium, $m s t h$, and projects into the pleural cavity, Pleu.c, which is lined by a continuation of the mesothelium of the lung itself. The pleural cavity can be followed downward past the Wolffian body, $W \cdot b^{\prime}$, and liver, and from there past the genital gland, Gen, and so on to the lowest part of the abdominal cavity, Ab.co. The pleural cavity at this stage is entirely separated from the pericardial, but it is still directly continuous with the abdominal cavity. On the ventral side (in the figure, to the right) of the pleural cavity are the great veins, the common cardinal, $C . C$, descending from above, and the ductus venosus, $D u . v$, rising from below. The pleural cavity is separated from the common cardinal by a lamina of the mesoderm, $x$, and from the ductus venosus by a similar but thinner lamina, $Y$. Both laminx are bounded on the pleural side by the mesothelium, and on the venous side by the endothelium of the vessel. The opening of the veins into the right auricle, Au.d, does not appear in this section, though a small bit of the left valve, v.s, which guards this opening is shown. The Wolffian body is divided into two parts, an upper, $W \cdot b^{\prime}$, on a level with the liver, and a lower, $W \cdot b^{\prime \prime}$, toward the pelvic end of the abdomen. The lower part is larger than the upper. The two parts are separated from one another chiefly by the mesonephric vein, V.msn, which is the principal vessel to take the blood from the Wolffian body. It delivers the blood to the lower end of the vena cava inferior. The separation of the two parts of the Wolffian body is, however, further accented by the position of the genital gland, Gen, and of the kidney, Ki. The structure of the latter organ does not differ much from that of earlier stages, but the diameter of the tubules has increased, and there has been an advance in the differentiation of the convoluted tubules and of the glomeruli. The genital gland (testis) is remarkable for its large size. It is covered by a layer of mesothelium, underneath which is a rather broad layer of elongated

Fig. 218.-Pig, 20.0 Mm. Sagittal Series 6o, Section 213.

Ab.co, Abdominal colom. All.vi, Mesothelial villi of the allantois. Au.d, Right auricle. bro, Entodermal bronchus. C.C, Common cardinal. Co $e^{\prime}, C e^{\prime \prime}$, Coelom. Diaph', Diaph", Diaphragm. Du.v, Ductus venosus. G.bl, Gall-bladder. Gen, Genital gland. $I n^{\prime}, I n^{\prime \prime}, I n^{\prime \prime \prime}$, Intestine. $K i$, Kidney. $L i$, Liver. Mes, Mesenchyma. msih, Pleural mesothelium. P.cee, Pleural cœlom. Pleu.c, Pleural cavity. Ve. hep, Ve.hep', Hepatic veins. V.msn, Vena mesonephrica. v.s, Valvula sinistra. $W . b^{\prime}, W . b^{\prime \prime}$, Wolffian body. $x$, Partition separating the pleural cavity from the duct of Cuvier. $Y$, Partition separating the pleural cavity from the ductus venosus. $\times 22$ diams. 


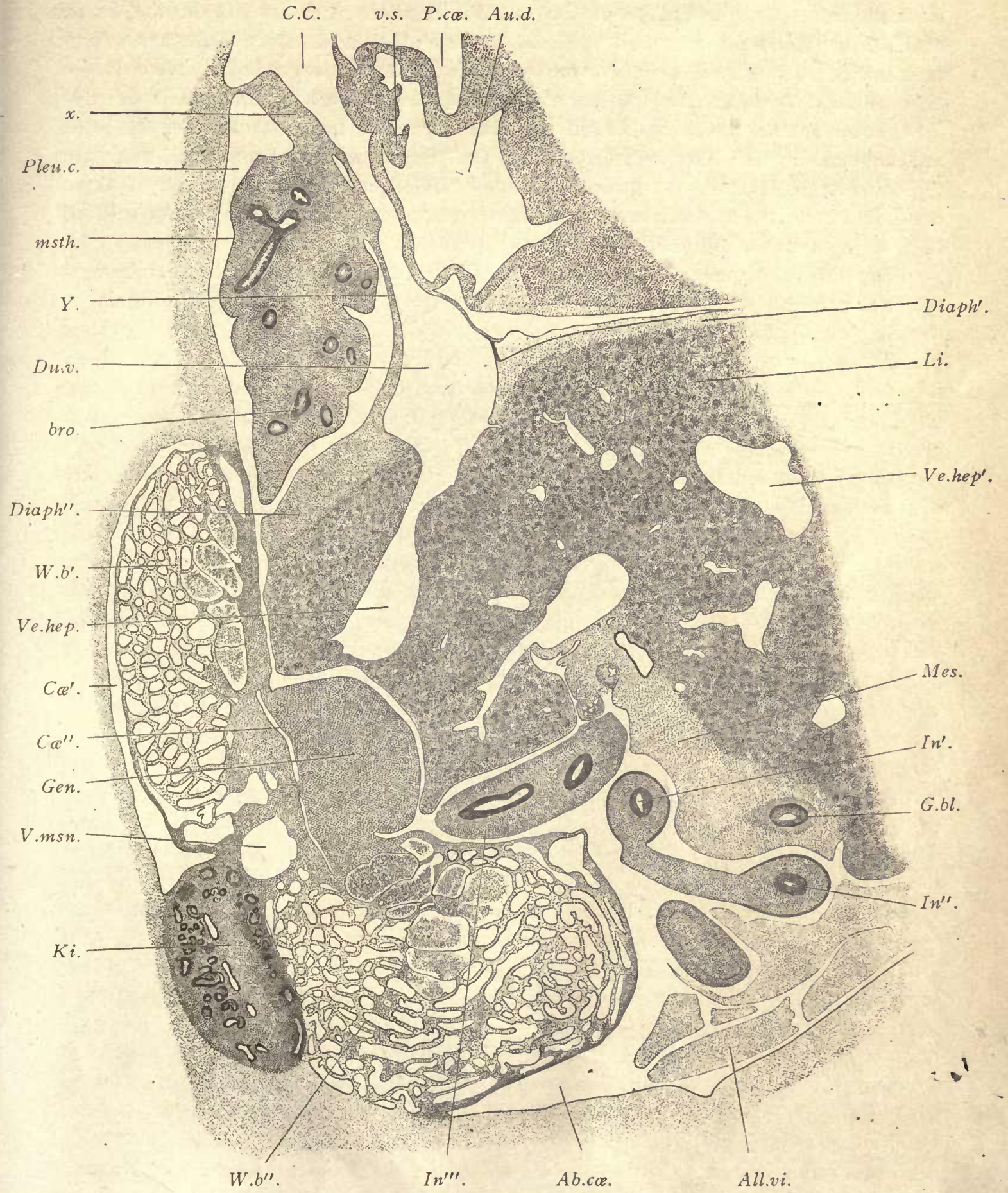

FIG, 218 . 
mesenchymal cells, the anlage of the tunica albuginea. The interior of the organ contains a number of contorted epithelioid cords of cells in which there are a certain number of so-called primitive ova, cells which are distinguished by their larger size, rounded form, greater transparency, and spherical nuclei. The bands of cells are known as the sexual cords, and they are separated from one another by loose mesenchymal tissue. The cords frequently anastomose with one another. They are the solid anlages of the seminiferous tubules. The question of the origin of these cords has been much debated, but cannot be considered as yet settled. As to both the origin and the ultimate fate of the primitive ova in the mammalian testis we have only incomplete information. The cords remain solid throughout embryonic life, not acquiring a central cavity until after birth. The liver is a very voluminous organ permeated everywhere by sinusoidal blood-vessels which offer the greatest possible variety in size. In the figure only the larger of these blood-vessels have been drawn in, A large proportion of the smaller sinusoids are crowded with nucleated red blood-corpuscles, the nuclei of which are small and deeply stained; hence each cluster of corpuscles stands out as a darker spot in the liver, for the liver cells themselves stain lightly and have nuclei which, though three or four times the size of the nuclei of the blood-corpuscles, yet appear relatively pale in the stained specimen. The blood-corpuscles which form the clusters in the liver differ somewhat from those in the active circulation, for they are smaller, and show less of the characteristic hemoglobin color. It has been demonstrated that the liver at this stage furnishes sites for the multiplication of the blood-corpuscles, and the clusters, which are so conspicuous in the organ, correspond not to blood-corpuscles in active circulation, but rather to corpuscles which have found a lodging-place in the liver and are there proliferating. Our knowledge of the blood-forming function of the embryonic liver is imperfect. Above the liver is the septum transversum or diaphragm, Diaph', Diaph", which is formed chiefly by mesenchyma. On the lower side of the liver is another broad accumulation of mesenchyma, Mes, in which is lodged the gall-bladder, a small section, G.bl, of the entodermal lining of which is included. The intestine, $I n^{\prime}, I n^{\prime \prime}, I n^{\prime \prime \prime}$, is cut several times, because at this stage the intestinal canal forms several coils in the abdominal cavity below the liver and on the ventral side of the Wolffian bodies. Below the intestines appear the curious mesothelial villi, All.vi, of the allantois (compare page 253). At this stage the villi are little more than large vesicles of mesothelium, which contain in their interior some coagulum and a very few mesenchymal cells, associated with which are a few fibers-whether true connective-tissue fibers or not is undetermined. The mesothelium of the villi is a very thin layer of flattened cells.

Frontal Sections of the Head.-The three sections to be described are from a special series of the head. The plane of the series was made as nearly as possible transverse and at right angles to the plane of the roof of the mouth. They illustrate some important points in regard to the development of the facial region and of the fore-brain. In all of the sections the differentiation of the mesoderm 
around the brain is clearly demonstrated. The pia mater is very distinct. In those parts of the sections where the brain-wall is cut obliquely, it can be distinguished only by a somewhat careful observation, as the tissues of the pia mater and of the brain overlap. All about the brain is the broad zone of the arachnoid (Figs. 220 and $22 \mathrm{I}$, arach), easily distinguishable even with a low power by its light coloration. It consists of widely separated cells connected together by very distinct processes, and is permeated by a number of small blood-vessels running in various directions through the layer. Its external boundary is now very distinct, being marked by a layer, $S k$, of somewhat crowded, elongated cells which merge on the side

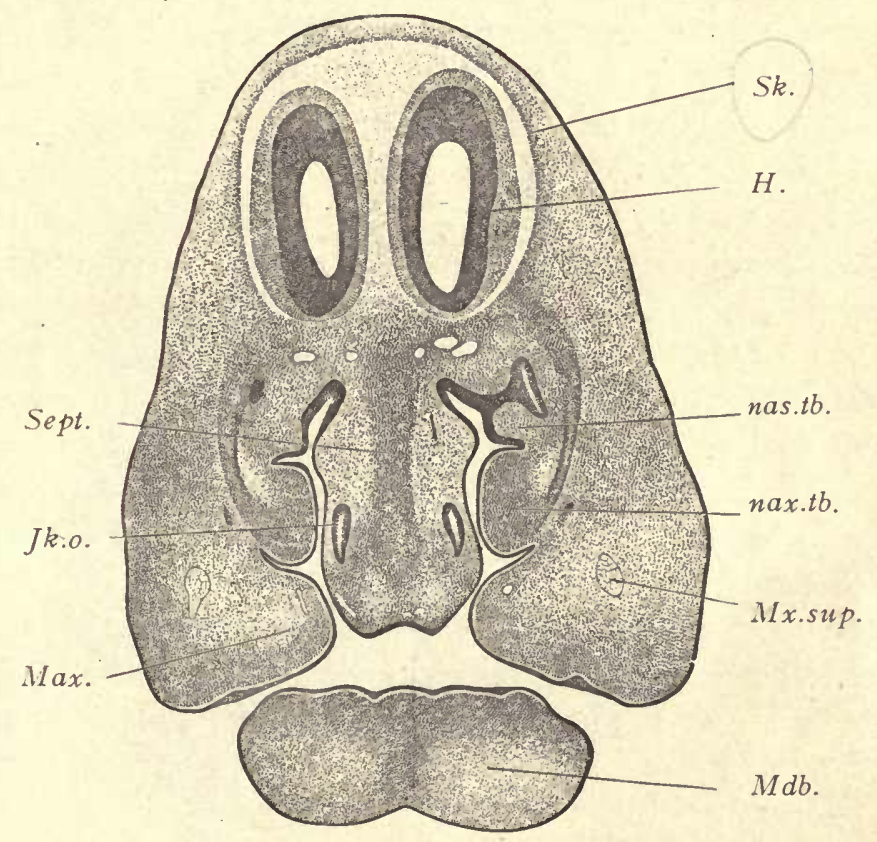

Fig. 219.-Pig, 20.0 mi. Frontal Section of Head. Serifs 40, Section 68.

$H$, Cerebral hemisphere. Jk.o, Jakobson's organ. Max, Maxillary process. max.tb, Maxillo-turbinal fold. $M d b$, Mandible. Mx.sup, Superior maxillary nerve. nas.tb, Naso-turbinal fold. Sept, Nasal septum. $S k$, Mesenchymal anlage of the dura mater and skull. $\times 18$ diams.

toward the ectoderm into the general surrounding mesenchyma. Out of this denser layer (Figs. 219 and 220, $S k$ ) arise both dura mater and the membrane bones of the skull.

Section through the Anterior Part of the Snout (Fig. 219).-On the dorsal side appear the two cerebral hemispheres, $H$, cut separately and each showing the cavity of its lateral ventricle. On the ventral side the mandible, $M d b$, is cut separately and is separated by the oral fissure from the rest of the section. The maxillary processes, Max, are large, and each is furnished with an inward prolongation extending toward the median line. From the oral fissure there extend upward 
two irregular cavities, the nasal chambers. The two cavities are separated from one another by a broad mass of tissue, the nasal septum, the ventral edge of which at this stage forms a portion of the roof of the mouth-cavity. In the center of the nasal septum is a broad band, Sept, of denser mesenchymal tissue, the anlage of the cartilaginous septum of the nose. On either side of the nasal septum is the irregularly shaped nasal cavity, which opens into the mouth between the ventral edge of the nasal septum and the inner edge of the maxillary process. The

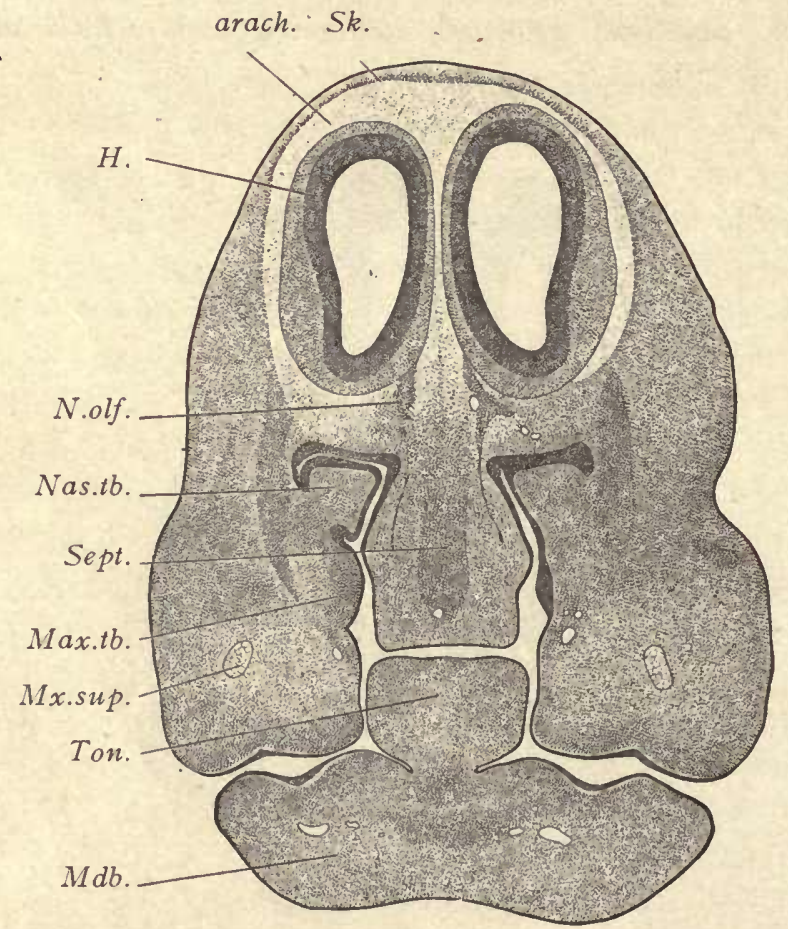

Fig. 220.-Pig, 20.0 mi. Frontal Section of Head. Series 40, Section 84.

arach, Arachnoid membrane. $H$, Cerebral hemispheres. Max.tb, Maxillo-turbinal fold. $M d b$, Mandible. $M x$. sup, Superior maxillary nerve. Nas.tb, Naso-turbinal fold. N.olf, Olfactory nerve. Sept, Cartilaginous septum of the nose. $S k$, Mesenchymal anlage of the dura mater and skull. Ton, Tongue. $\times$ r 8 diams.

medial side of each nasal cavity is comparatively regular, but the external side shows two prominences, each of which is formed by a mass of mesenchymal tissue covered by epithelium. The upper of these projections, nas.tb, is the anlage of the naso-turbinal fold, and the lower, max.tb, the anlage of the maxillo-turbinal fold. In the nasal septum itself are two oval rings of epithelium, sections of Jakobson's organs. This organ is an evagination of the epithelial lining of the nasal cavity, which opens anteriorly and extends backward some distance in the nasal septum. In the maxillary process may be observed the superior maxillary nerve, Mx.sup. The number of cells in the nerve has increased, and consequently 
the division of the nerve-fibers into distinct bundles has become more marked as compared with the pig embryo of $\mathrm{I} 2 \mathrm{~mm}$.

Section through the Middle of the Snout (Fig. 220). - The relations are very similar to those described in the previous section, so that it will suffice to note the three most important differences: first, the absence of Jakobson's organ; second, the appearance of the tongue, Ton, and third, of the olfactory nerve, N.olf. The tongue is a protuberance attached to the lower jaw, $M d b$. Its connection with the jaw is rather narrow and corresponds to the frenum. The tongue extends upward between the maxillary processes until it is almost or quite in contact with the lower edge of the nasal septum. It is formed by a somewhat dense mass of tissue in which there is no very evident histological differentiation, and is covered by a layer of epithelium of moderate thickness and which is probably entirely derived from the entoderm, for the tongue first appears as a small median protuberance on the ventral floor of the pharynx, between the first gill-pouches. The olfactory nerve, N.olf, can be seen joining the lower part of the inner side of the brain-wall and extending down toward the nasal cavity and branching. Under the part of the nerve near the brain-wall numerous cells are mingled with the fibers, and by their crowding render the nerve conspicuous in stained sections. The fibers of the olfactory nerve differ from all other nerve-fibers known in vertebrates. They arise as prolongations of certain of the epithelial cells of the olfactory region of the nose and grow from these cells into the brain, where they have their termination in the glomeruli of the olfactory bulb. All other nervefibers arise from nerve-cells either of the central nervous system or of the ganglia. Morphologically, therefore, the olfactory nerve takes a unique place, and is not directly comparable with any other nerve of the brain. The cells which accumulate in the course of the olfactory nerve do not, so far as known, have any direct share in the production of the nerve-fibers; nor do they result in the formation of the medullary sheaths, as they do in other nerves, the olfactory nerve-fibers remaining naked, as it is termed, throughout life.

Section through the Fore-brain and Eyes (Fig. 22I). - The section passes behind the nasal cavities, no part of which is shown. The maxillary and mandibular processes are united and the pharynx, $P h$, appears as a closed cavity. On the dorsal side of the section the fore-brain stands out conspicuously, both from its dark staining and from being surrounded by the lightly stained broad zone of the arachnoid, arach. The cavity of the fore-brain has two lateral expansions, L.V, the lateral ventricles, which extend outward and upward. The walls, $H$, of the lateral ventricles are much thinner than the walls of the lower part of the forebrain and are the anlages of the cerebral hemispheres. In the median plane the hemispheres include between themselves a partition, $F x$, of mesodermic tissue which may be designated as the embryonic falx, since within it, though considerably later, the adult falx will be differentiat $d$. In the adult, the falx appears as a prolongation of the dura mater. From the bottom of the falx there extends on 
each side a fold, $P l x$, which projects into the cavity of the lateral ventricle. This fold contains in its interior a prolongation of the mesodermic tissue of the falx, and it is covered by a continuation of the wall of the hemispheres. The covering layer of the fold is much thinner than any other portion of the brain-wall shown in the section, and shows no differentiation into layers. It retains throughout

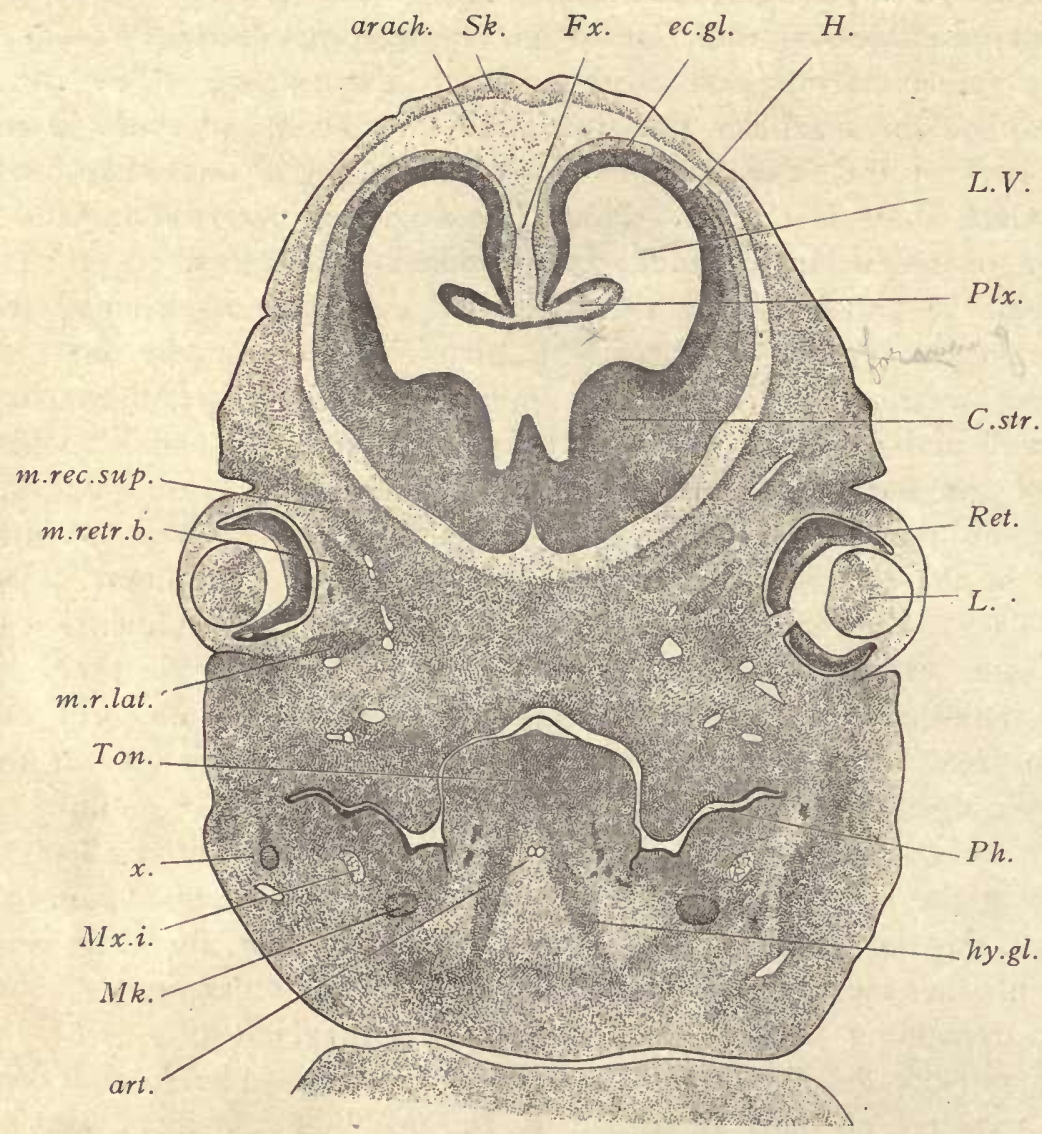

Fig. 221.-Pig, 20 mis. Frontal Section of Head. Series 40, Section 123.

arach, Arachnoid zone. art, Lingual arteries. C.str, Corpus striatum. ec.gl, Ectoglia. Fx, Falx cerebri. $H$, Cerebral hemisphere. hy.gl, Hyoglossal muscle. L, Lens. L.V, Lateral ventricle. M $k$, Meckel's cartilage. m.rec.sup, Musculus rectus superior. m.retr.b, Musculus retractor bulbi. m.r.lat, Musculus rectus lateralis ( $c f$. text). $M x . i$, Inferior maxillary nerve. $P h$, Pharynx. Plx, Plexus shoroideus lateralis. Ret, Retina. $S k$, Anlage of membranous skull. Ton, Tongue. $x$, Unidentified structure. $\times$ i 8 diams.

life an epithelial character and is already to be termed ependyma. The ependyma of the two folds is connected across the median line, and it forms the median dorsal boundary of the cavity of the fore-brain. The two folds are the anlages of the lateral choroid plexus. They are destined to grow much in size and in complexity of form, but they always remain morphologically what they now are, 
vascularized mesenchyma covered by ependyma. The choroid plexus protrudes into the cavity of the brain in the same way in which the viscera may be said to protrude into the abdominal cavity. The cavity of the brain is bounded by the brain-wall or ependyma, just as the abdominal cavity is bounded by the peritoneum. The vascular tissue of the choroid plexus is outside of the cavity of the brain, in the same way that the tissue of the kidney is outside the cavity of the abdomen. Throughout life the choroid plexus springs, as it does from the start, from the medial wall of the hemispheres, and it is only at that point that it can receive its blood-supply. The lateral walls of the hemispheres, $H$, gradually thicken as they continue ventralward, and on the ventral side of the brain form in part the lateral boundary of the medial portion of the brain-cavity, as an especial thickening of the brain-wall which projects far into the cavity. The thickening, C.str, is the corpus striatum. Between the summit of the corpus striatum and the choroid plexus is an open passage through which we may pass from the median portion of the brain-cavity into the lateral ventricle, L.V. The passage is the foramen of Munro, which we learn from this section is bounded above by the choroid plexus, and below by the corpus striatum. On the dorsal and middle sides of the hemispheres, the ectoglia, ec.gl, is already clearly differentiated. There is, however, at this stage, no clear indication of the cortex cerebri, although in the slightly older stages it will begin to develop by the accumulation of neuroblasts immediately beneath the ectoglia. The notochord does not appear between the brain and the pharynx, the section being too far forward. The notochord stops near the hypophysis. The eyes are not cut quite symmetrically. They show the lens, $L$, and retina, Ret, clearly and the left eye of the embryo shows also the entrance of the optic nerve. On the right side of the embryo, near the eye, are three areas which are somewhat more darkly stained than the surrounding mesenchyma. These are the anlages of the muscles of the eye. They have not yet been studied sufficiently to make their identification certain, but it seems probable that the uppermost of these anlages, m.rec.sup, is the rectus superior, that the middle one, m.retr.b, is the retractor bulbi, and that the lowest one, m.r.lat, is the rectus lateralis. Until a reconstruction is made these identifications can be recorded as tentative only. The pharynx, $P h$, appears as a yoke-shaped slit lined throughout by entoderm. From its median ventral floor rises the great mass of the tongue, Ton, over which the dorsal roof of the pharynx forms a closely fitting arch. A portion of the epithelium of the tongue is loosened from the underlying tissue, probably owing to defective preservation. Upon the lower side of the tongue extend downward the anlages of the hyoglossal muscles, hy.gl, between which are situated the lingual arteries, art. On either side, in the part of the section corresponding to the mandible, appears Meckel's cartilage, $M k$, a somewhat conspicuous and easily identified structure, owing to its dark staining. Meckel's cartilage is the primitive skeletal element of the mandibular arch, and is homologous with the cartilaginous jaw of the lower fishes. It is, for the greater part, 
a purely embryonic structure, the mandible of the adult being a secondary bone. By referring to figure 166 (pig, ro $\mathrm{mm}$.), it can be seen that the mandibular arch extends upward toward the otocyst and forms the boundary of the first gill-cleft, the outer division of which becomes the meatus auditorius externus. In other words, the upper portion of the mandibular arch is in close proximity to the otocyst and the anlage of the tympanum or middle ear. Meckel's cartilage is a

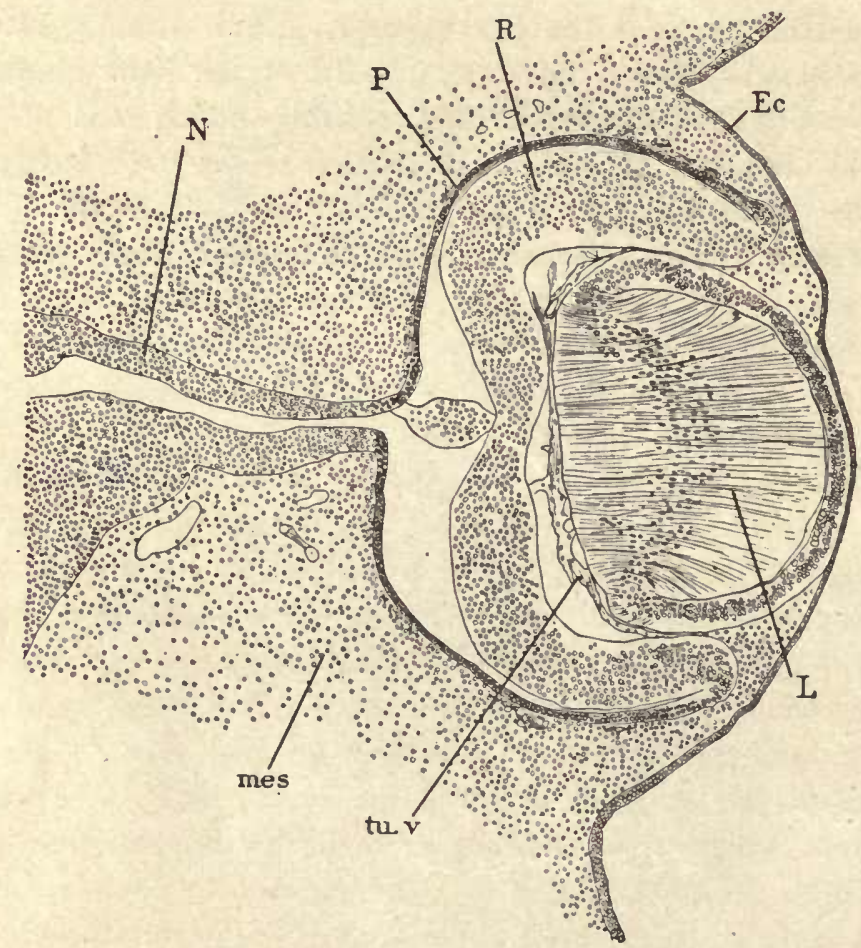

Fig. 222.-Rabbit Embryo of Thirteen Days; Section of the Eye.,

$E c$, Epidermis. L, Lens. mes, Mesenchyma. $N$, Anlage of optic nerve. $P$, Pigment layer. $R$, Retina. $t u . v$, Tunica vasculosa lentis.

rod-like structure extending the entire length of the arch. Its upper end is, therefore, close to the future tympanum. While the greater part of Meckel's cartilage disappears during later development, the upper end persists and takes a direct share in the formation of the malleus. A little outside of Meckel's cartilage in our 'section is the inferior maxillary nerve, $M x . i$, and still farther lateralward is a small, darkly stained body, $x$, which has not yet been identified with certainty.

\section{Pig Embryo of $24 \mathrm{~mm}$. Study of Sections.}

Section through the Eye (Fig. 223). - In the pig of $24 \mathrm{~mm}$. the anlages of all the parts of the adult eye may be said to be present, with the exception of the pigment layer of the iris, which arises somewhat later by a forward growth of the 
retina and pigment layer. The origin of the retina and lens is illustrated by the chicken embryo (Figs. I53, I54), and in a more advanced stage by the pig of I $2 \mathrm{~mm}$. (Fig. 192). There is added here figure 222, from a section of the eye of a rabbit embryo of thirteen days, in order to facilitate the comparison between the $12 \mathrm{~mm}$. stage and the $24 \mathrm{~mm}$. stage of the pig. In figure 222 the ectoderm, $E c$, forms an arch over the eye and indicates the commencing formation of the cornea, the layer of ectoderm being destined to become the external epithelium of the cornea. Between the lens and the retina there has been an ingrowth of tissue accompanied by blood-vessels, which forms a more or less distinct covering over the surface of the lens and constitutes the so-called tunica vasculosa, tu.v. The space between the retina and lens will increase during the following stages and will become occupied by a very clear tissue containing a minimal number of cells. This clear tissue is the commencement of the vitreous humor. Between the lens and the overlying ectoderm the mesenchyma has begun to penetrate. This mesenchyma will ultimately furnish the connective tissue of the cornea and of the iris. About the eyeball as yet there is no distinct condensation of tissue such as will appear later to develop the anlages of the choroid and scleral coats of the eyeball.

In the pig of $24 \mathrm{~mm}$. (Fig. 223) we encounter a marked advance in the differentiation of all parts of the eye. Above and below the eye the anlages of the eyelids, L.sup, L.inf, have appeared. The anlage is at this stage merely a projecting fold of the ectoderm filled with mesenchyma and extending a short distance over the projecting cornea. The folds will continue to grow until the eyelids meet in the middle of the eye, covering it completely. The ectoderm of the two lids where they meet unites. The union of the two lids occurs in all mammals, and in some cases they do not separate again until after birth, in which case the animals are said to be "born blind." The ectoderm, $E c$, of the cornea describes a wide, high arch, underneath which is a broad band of embryonic connective tissue, corn, which forms the main thickness of the cornea. Between the connective tissue of the cornea and the anterior surface of the lens is a clear space, an.ch, which we can identify as the anterior chamber of the eye, which in the adult is filled only with the aqueous humor. On the corneal side the anterior chamber is bounded by a distinct layer of cells, $E p$, the internal epithelium of the cornea. This layer is, however, formed from the mesenchyma, the cells of which develop into the internal epithelioid covering of the cornea. At the upper and lower edge of the cornea there is a separate forward growth, Ir, of the connective tissue between the cornea and the lens. It is the anlage of the connective-tissue layer of the iris. In later stages it will grow still farther over the lens from all sides, leaving a central opening, the pupil, and it will acquire a special pigmented layer on its side nearest the lens. At the base of the iris anlage is a small blood-vessel, Schl, which is commonly designated in the adult as the canal of Schlemm. The retina has increased in thickness and is closely covered by a pigment layer, Pig. The separation which appears on the inner side of the eyeball between the retina and pig- 
ment layer in figure 223 is probably artificial, the result of shrinkage during the preservation of the specimen. At its outer edge the retina, Ret, suddenly thins out and passes over into the external pigment layer, which is heavily loaded with dark, uniform, pigment granules, especially crowded together on the side of the layer

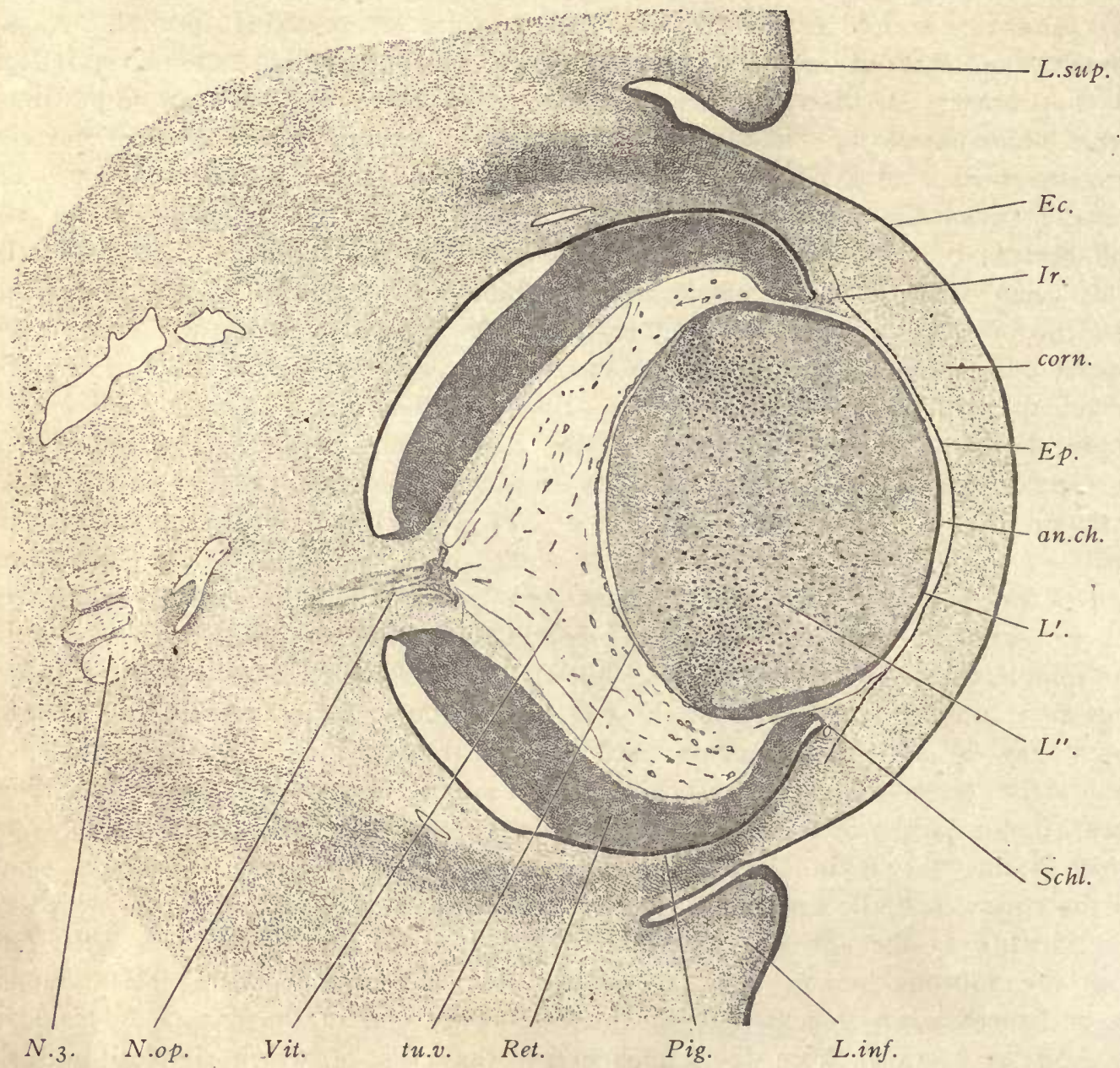

Fig. 223.-Pig, 24.0 mm. Transverse Series 62, Section 428.

an.ch, Anterior chamber of eye. corn, Corneal mesoderm. Ec, Ectoderm (epidermis). Ep, Inner epithelium of cornea. Ir, Mesodermal anlage of iris. $L^{\prime}$, Outer layer of lens. $L^{\prime \prime}$, Inner layer of lens. L.inf, Inferior eyelid. L.sup, Superior eyelid. N.3, Oculo-motor nerve. N.op, Optic nerve. Pig, Pigment layer. Ret, Retina. Schl, Canal of Schlemm. tu.v, Tunica vasculosa lentis. Vit, Vitreous humor." $\times 50$ diams.

nearest the retina. In later stages the pigment layer grows forward on the inner side of the iris, making a fold, so that the iris is covered on the inside by a double layer of pigmented epithelium, the uvea. The retina resembles closely in structure the brain-wall in an early stage, for it has on its outer surface a thin 
layer corresponding to the ectoglia, and within a broad, nucleated zone. The mitotic figures are found only next to the surface of the retina nearest the pigment layer. Since the space between the pigment layer and the retina corresponds to the cavity of the brain, it is evident that the position of the mitotic figures is homologous with their position in the medullary wall elsewhere. The section of the lens clearly reveals its vesicular structure. The external wall of the lens vesicle, $L^{\prime}$, is a comparatively thin epithelial layer which stains quite readily and therefore stands out clearly in the section. Toward the edges of the lens the outer layer slightly thickens and then passes over quite abruptly into the inner layer of the vesicle, $L^{\prime \prime}$, which is very thick and constitutes by far the greater part of the bulk of the organ and gives to the lens its characteristic shape. The outer and inner walls of the lens are in close contact so that there is no actual cavity present. The epithelial cells of the inner wall have elongated enormously, so much that they might perhaps already be.termed "fibers." Each cell is supposed to extend through the entire thickness of the inner wall. The nuclei are placed somewhat irregularly in the middle portion of the long cells so that they constitute a more or less distinct band in the section. Toward the edge of the lens the nuclear band becomes more distinct, and where the inner wall merges into the outer, the band becomes narrow and the nuclei are much crowded together. The nuclei of the lens fibers are oval, being slightly elongated in the same direction as the fibers, and each nucleus contains usually a distinct nucleolus. Between the lens and the retina is the vitreous humor, Vit, which has become quite voluminous. It contains a few mesenchymal cells and a few small blood-vessels, and when examined with a high power it is seen to be permeated by a fine network which is probably to be interpreted as a modification of the protoplasmic threads of the mesenchyma. There are also a very few cells of rounded form and distinct outline, with a single small granular nucleus, which are probably leucocytes. Against the surface of the lens there is a delicate homogeneous hyaloid membrane, which can usually be better seen where by shrinkage it has been loosened from the surface of the lens, as is apt to occur. Against the hyaloid membrane are a number of small blood-vessels, more numerous than those elsewhere in the vitreous humor, and forming a fairly distinct vascular membrane around the lens. The membrane, tu.v, is called the tunica. vasculosa lentis. The blood-vessels of the vitreous humor are chiefly, possibly at this stage exclusively, brariches of the central artery of the retina. The artery enters the eye through the optic nerve, and sends branches throughout the vitreous humor. The space originally occupied in the humor by the stem of the central artery persists, and is called the hyaloid canal. The muscles of the eye are already differentiated, but their relations cannot be properly understood without a reconstruction.

Median Sagitlal Section (Fig. 224).-The section figured is very nearly median for the region of the head, but in the body it passes to the left of the median plane. The area occupied in the section by the neck and head of the embryo is 


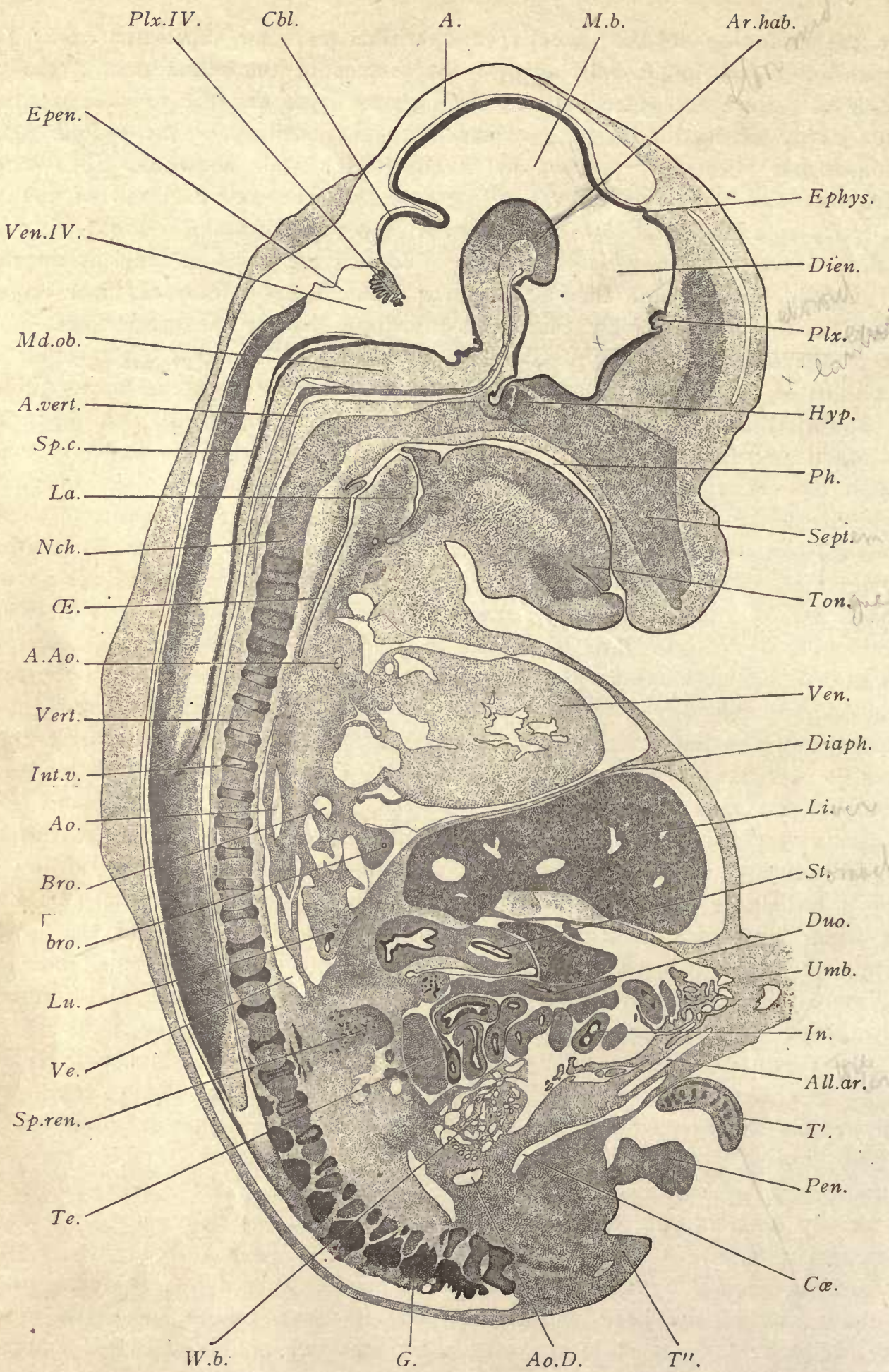

FIG. 224. 
almost as great as that occupied by the rest of the body. The great size of the head at this stage is characteristic. Attention is especially directed to the sharp angle which the medulla oblongata, Md.ob, makes with the spinal cord, Sp.c, and to the very great bend formed by the floor of the mid-brain, Ar.hab, in consequence of which the floor of the hind-brain above the medulla oblongata and the floor of the fore-brain are brought quite close together and run in almost parallel directions. The cavity of the brain is very large. Its walls in the median plane are, for the most part, thin. From the roof of the diencephalon, Dien, there runs off a small evagination, Ephys, a shallow pocket or diverticulum of the medullary wall. It is the anlage of the epiphysis or pineal organ of the adult. It is an important landmark in the topography of the brain, for its position is always at the extreme posterior limit of the fore-brain. In the wall of the mid-brain, behind the epiphysis, for some distance the ectoglia shows considerable thickening and contains a very large number of nerve-fibers running transversely. They constitute the posterior commissure, which morphologically belongs to the mid-brain. In later stages the opening of the epiphysis and the anterior boundary of the posterior commissure are separated by a narrow band of ependyma. Immediately in front of the epiphysis; close to the external surface of the medullary wall, is another tract of nerve-fibers which is very small and is known as the superior commissure. The superior commissure develops much later than the posterior, and is much smaller in size. The two commissures are found in vertebrates of all classes and are exceedingly constant anatomical features of the brain. Anterior to the epiphysis the dorsal roof of the diencephalon forms a broad arch which descends in the figure vertically until it ends in a small inward projection, $P l x$, of the brainwall, the anlage of the choroid plexus. Below this point the brain-wall is continued, forming the lamina terminalis. It then makes a bend almost at right angles and runs in a horizontal direction toward the dorsal side of the embryo. This portion of the brain-wall shows a considerable thickening, the optic chiasma. Behind the optic chiasma the brain-wall forms a short evagination, the infundibular gland, which bends over so as to lie close to the dorsal side of the hypophysis, Hyp. The hypophysis, which in earlier stages appears clearly as an evagination of the oral

Fig. 224.-Pig, 24.0 mm. Sagittal Serifs 63, Section 30.

A, Arachnoid space, in this specimen containing extravasated blood. A.Ao, Arch of the main aorta. All.ar, Allantoic artery. Ao, Dorsal aorta. Ao.D, End of dorsal aorta. Ar.hab, Habenular arch (floor of midbrain). A.vert, Vertebral artery joining its mate to form the basilar artery. Bro, Main bronchus of lung. bro, Branch bronchus within the lung. $\mathrm{Cbl}$, Cerebellum. Ca, Coelom. Diaph, Diaphragm. Dien, Diencephalon. Duo, Duodenum. Epen, Ependymal roof of hind-brain. Ephys, Epiphysis. G, Spinal ganglion. $H y p$, Hypophysis. In, Intestine. Int.v, Anlage of intervertebral ligament. La, Lateral wall of larynx. $L i$, Liver. Lu, Lung. M.b, Mid-brain. Mḋ.ob, Medulla oblongata. Nch, Notochord. $E$, Esophagus. Pen, Penis. Ph, Pharynx. Plx, Choroid plexus of fore-brain. Plx.IV, Choroid plexus of hind-brain. Sept, Cartilaginous nasal septum. Sp.c, Spinal cord. Sp.ren, Suprarenal capsule. St, Stomach. $T^{\prime}, T^{\prime \prime}$, Tail. $T e$, Testis. Ton, Anterior portion of tongue. Umb, Umbilical cord. Ve, Cardinal vein. Ven, Ventricle of the heart. Ven.IV, Fourth ventricle, or cavity of the hind-brain. Vert, Vertebra. W.b, Wolffian body. $\times 8$ diams. 
epithelium (Fig. 20r), is now entirely separated from the mouth, and is an epithelial vesicle with an irregular cavity. The epithelium has sent out, especially on its anterior side, a number of solid outgrowths. The infundibular gland and hypophysis constitute the pituitary body of the adult. They are surrounded by loose mesenchymal tissue. The sella turcica, in which the pituitary body of the adult is lodged, is marked out, because the chondrification, which is to form the sphenoidal cartilages, has already begun about these structures. The sphenoidal cartilage is continuous, on the one hand, with that of the nasal septum, Sept, and, on the other, with that of the vertebral column, Vert. From the opening of the infundibular gland the brain-wall ascends and joins the habenular arch, where it suddenly thickens. The arch forms the floor of the mid-brain. The roof of the mid-brain, $M . b$, is quite thin, and forms the large arch in which the differentiation of the anterior and posterior corpora quadrigemina is not yet shown. At its posterior boundary the wall of the roof of the mid-brain bends inward, marking the constriction of the so-called isthmus. We now reach the cavity, Ven.IV, or fourth ventricle, of the hind-brain. This cavity is subdivided into an anterior and a posterior portion. The boundary is marked on the dorsal side by the inward projection of the ependymal roof of the ventricle to form the choroid plexus, Plx. $I V$, and on the ventral side by the angle formed by the union of the medulla oblongata, Md.ob, with the vertical peduncles of the brain. The peduncles, continuing upward, join the habenular arch. In front of the choroid plexus the arch. ing brain-wall, $\mathrm{Cbl}$, represents the median anlage of the cerebellum. The lateral portions of the cerebellum are much thicker. Behind the choroid plexus the roof, Epen, of the fourth ventricle is very thin: The medulla oblongata, Md.ob, is a thick mass of tissue which passes over abruptly into the spinal cord. The spinal cord is cut, as a whole, somewhat obliquely: In its upper part, where the reference line, $S p . c$, is placed, the section is almost exactly median, and shows, therefore, the floor-plate or raphe of the spinal cord. In front of the cord is the vertebral artery, A.vert, which joins its fellow to form the basilar artery which runs in the median line the entire length of the hind-brain. The vertebral column is in the cartilaginous stage. It is an absolutely continuous uninterrupted rod of cartilage which merges at the neck with the cartilaginous skull. The entire continuous cartilaginous structure is termed the chondrostyle, for the study of which comparison with the neighboring sections is indispensable. Out of it both the cartilaginous skull and the vertebræ are differentiated. More or less nearly in the center of the chondrostyle are found the remnants of the notochord, which, however, never extends anterior to the pituitary body, $H y p$. The division of the chondrostyle into separate vertebræ is indicated by the segmental flexures of the notochord and by the commencing differentiation of the intervertebral ligaments. The space occupied by the notochord expands in the region corresponding to the division between each two vertebræ. The notochord in the intervertebral expansions is expanded and partly degenerated, forming an enlarged mass of irregular strands of cells, which becomes 
the nucleus pulposus of the adult. From each such mass goes off a narrow extension of the notochord, through what is to become the body of the vertebra. Sometimes this extension is continuous with the intervertebral portions of the notochord, but more usually it forms a series of isolated fragments, for the notochord in the parts corresponding to the bodies of the vertebræ is already in process of resorption. The diameter of the chondrostyle is nearly uniform in the vertebral region, but is a little smaller in the part corresponding to each body of a vertebra and a little wider in the parts corresponding to the intervertebral ligaments. The cartilage of the body of the vertebra continues past the intervertebral expansion of the notochordal cavity, but the external portion of the chondrostyle opposite each such expansion exhibits a modification of its cells, for they have become lengthened out in a direction parallel with the vertebral axis. The tissue thus produced is the anlage of the intervertebral ligaments. The mouth and pharynx, $P h$, form a narrow cavity, the floor of which is constituted by the tongue, Ton, the tip of which has already become free. The surface of the tongue forms a long arch, at the posterior end of which lies the epiglattis, a projecting fold of tissue which covers the opening of the trachea. The side of the trachea is marked by the longitudinal fold, $L a$, which separates the trachea proper from the upper end of the œsophagus, $C E$. At the upper end of the osophagus there is a small dorsal diverticulum. If the reference line $E$ were continued a short distance past the osophagus, it would lead to the section of the main aorta. A little lower down is the section of the arch of the aorta, A.Ao. The heart shows chiefly its large ventricle, Ven. The section is not favorable for an exhibition of its structure or for that of the lungs, $L u$. It does, however,- since in this part of the embryo the section passes to one side of the median plane,--show the main bronchus, Bro, coming off from the trachea to the lung, and some of the smaller entodermal bronchial branches, bro, in the lung itself. The heart and lung are separated from the abdominal cavity by the diaphragm, Diaph. It is only to the dorsal part of this diaphragm that the liver, $L i$, is attached. In earlier stages the liver is connected with the whole of the diaphragm (septum transversum). We now have a portion of the diaphragm without connection with the liver. Below the liver is the section of the stomach, $S t$, the entoderm of which is cut twice. Below the stomach lies the duodenum, Duo, extending from the dorsal side of the embryo and running toward the umbilicus. At the dorsal end of the duodenum is a group or cluster of darkly stained cells, marking the position of the anlage of the pancreas. Below the duodenum the loops of the intestine, In, are cut repeatedly. On the dorsal side of these loops is the section of the genital gland, in this specimen, testis, Te. Dorsalward from the genital gland is the complicated anlage of the suprarenal capsule, Sp.ren, which is really a double organ, having one part derived from the sympathetic nervous system and another from a modification of mesenchymal cells. In a sagittal series the connection of the anlage with the sympathetic nervous chain of the abdomen can be readily made out. In the anlage the nerve-fibers and the sympathetic cells 
are irregularly distributed, although the cells are more or less grouped together. The sympathetic tissue constitutes the dorsal part of the anlage and gives rise to the so-called medulla of the adult organ. The ventral portion of the anlage, as seen in the section, consists of bands or cords of cells separated from one another by venous sinusoids. The cells are much more closely compacted in this portion of the anlage than in the sympathetic, and they are further distinguished by having nuclei which stain much less deeply. The cords of cells, here seen, develop into the cortex of the adult organ. The fate of the medulla or sympathetic portion of the suprarenal in man is not known. The section passes through the side of the allantois, and, therefore, shows only one of the lateral arteries, All.ar, but the allantois still bears a number of degenerating mesothelial villi (compare page 253). At the pelvic end of the abdomen a small bit of the Wolffian body, $W . b$, is displayed. 


\section{CHAPTER VII.}

\section{STUDY OF THE UTERUS AND THE FETAL APPENDAGES OF MAN.}

\section{Histology of the Uterus.}

In most mammals the uterus is double. This is the case in the pig, the rabbit, and the mouse, the three species which furnish material for the practical study as planned in this book. In these animals each uterus is a long, more or less cylindrical tube. In primates the double uterus exists only during very early embryonic stages, after which the two are found united into a single median uterus. The mammalian uterus is always lined by a mucous membrane, consisting of a superficial epithelium which forms glands, and of a deeper layer of reticulate connective tissue, in which there are lymph spaces, nerves, and a fairly abundant blood supply. The mucous membrane is subject to very marked periodic changes in structure. It is enclosed by the muscular layers of the organ, the muscle-fibers being of the smooth type. In animals with double uteri the musclefibers form two distinct layers, an inner circular and an outer longitudinal layer. In the primate "uterus the disposition of the fibers is far more complicated, and the two distinct layers cannot be identified. The surface of the uterus, wherever it is free, is covered by a layer of peritoneum which consists of a layer of flattened epithelial cells and a thin underlying layer of fibrillar connective tissue.

The human uterus at birth has a mucosa which is about $0.2 \mathrm{~mm}$. thick. The mucosa is soft, pale gray or reddish gray in color; it consists of a covering of ciliated epithelium and a connective-tissue layer. It is without glands, the glands not appearing usually until the third or fourth year, and developing very slowly up to the age of puberty.

The development of the human uterine glands is accompanied by remarkable and complex changes of the epithelium.* The adult glands, as shown by figure 225 , are much branched, and the branches occasionally anastomose with one another. The model, from which the figure is taken, demonstrates that the conception of the character of the uterine glands, which has hitherto prevailed, is very inadequate.

\section{Menstruation.}

The function of menstruation involves great changes in the mucosa of the body of the uterus. We distinguish three periods: (I) tumefaction of the mucosa, with accompanying structural changes, taking five days, or, according to Hensen,

\footnotetext{
* Unpublished investigations by C. A. Hedblom, to whom I am indebted for the privilege of inserting figure 225 .
} 
ten days; (2) menstruation proper, about four days; (3) restoration of the resting mucosa, about seven days. The times given are approximate only. The whole

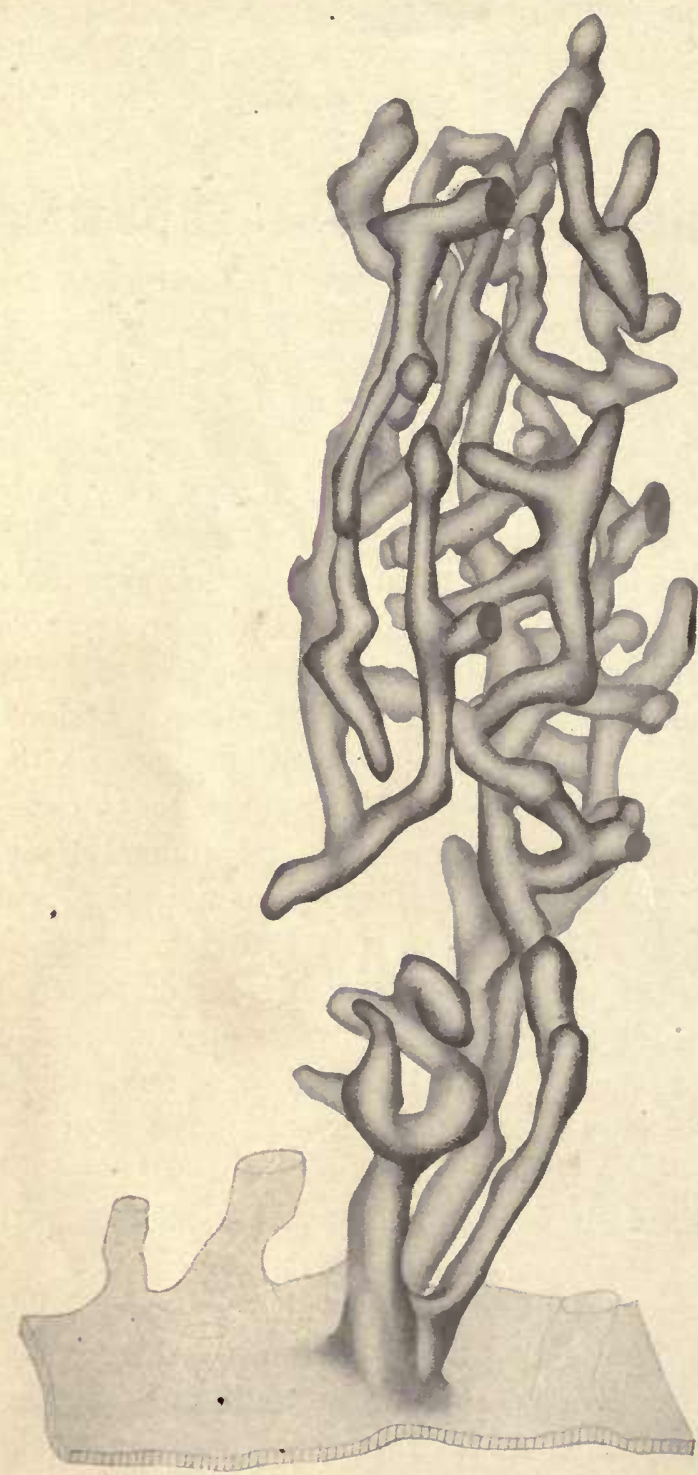

Fig. 225. - Uterine Gland of a Virgin OF Eighteen Years, with a Portion of the SURface Epithelium. WAX Reconstruction by C. A. HEDBLOM. $\times 5$ o diams. cycle of changes covers about sixteen days. Since the monthly period is about four weeks, the period of rest, as thus calculated, is only about twelve days.

r. Tumefaction. - A few days before the menstrual flow the mucosa gradually thickens; the surface becomes irregular; the openings of the glands lie in depressions. The connective-tissue cells are increased in number, and it is said by some authors in size, but the increase in size is doubtful; the number of round cells increases; the glands expand and become more irregular in their course; a short time before hemorrhage begins, the blood-ressels, especially the capillaries and reins, become greatly distended. We must assume that the connective-tissue cells proliferate, but we have no satisfactory observations upon their division. It was formerly asserted that the menstrual decidua contains decidual cells, but in all the specimens the author has studied there were none present.

2. Menstruation.-When the changes just described are completed, the decidua menstrualis is fully formed, and its partial disintegration begins. The process commences with an infiltration of blood into the subepithelial tissues. This infiltration has hitherto been commonly explained as due to the rupture of the capillaries; but as no ruptures at this period have been observed, we may justly regard this explanation as inadmissible, and account for the infiltration per diapedesin. It lasts for a day or two, and is apparently the immediate cause of a very rapid molecular disintegration of the superficial layers of the mucosa, which in consequence are lost; the superficial blood-vessels are now exposed, and, 
by rupturing, cause the well-known hemorrhagia of menstruation. By the disappearance of its upper portion the mucosa is left without any lining epithelium and is very much (and abruptly) reduced in thickness. Its surface is formed by connective tissue and exposed blood-vessels.

3. Restoration of the Mucosa.-At the close of menstruation the mucosa is 2 or $3 \mathrm{~mm}$. thick; the regeneration of the lost layers begins promptly and is completed in a variable time, probably from five to ten days. The hyperemia rapidly disappears; the extravasated blood-corpuscles are partly resorbed, partly cast off; the spindle-cell network grows upward, while from the cylinder epithelium of the glands young cells grow and spread up and out so as to produce a new epithelial covering; new subepithelial capillaries appear. The details of these changes are imperfectly known; they effect the return of the mucosa to its resting-stage.

Decidua Menstrualis.--Specimens from the first day of menstruation are the most instructive. They should be preserved in Zenker's fluid; sections may be made perpendicular to the decidual surface from blocks io to $I_{5} \mathrm{~mm}$. cube, and stained with alum hematoxylin and eosin. The use of Mallory's triple connectivetissue stain will demonstrate the fibrillar tissue in the decidua and the very large amount of the same in the muscularis.

The accompanying illustration (Fig. 226) is from a uterus in active menstruation. The decidual membrane is from I.I to $I .3 \mathrm{~mm}$. thick; its surface is irregularly tumefied; the gland openings lie for the most part in the depressions. In the cavity of the uterus there was a small blood-clot. The demarcation between the decidua and the muscularis is sharp. The upper fourth, $d$, of the decidua is broken down and very much disintegrated; its cells stain less readily than those of the deep portion of the membrane; the tissue is divided into numerous more or less separate-small masses. Some of the blood-vessels are ruptured. The superficial epithelium, $e p$, is loosened everywhere; in places fragments of it have fallen off, and in some parts it is gone altogether; it stains readily with alum hematoxylin, differing in this respect from the underlying connective tissue. The deeper layer of the decidua is dense with crowded well-stained cells, which lie in groups and are probably proliferated connective-tissue cells. They have small oval or elongated darkly stained nuclei, with very small granular protoplasmatic bodies. There is no indication of any enlargement of the cells, such as occurs in the production of true "decidual" cells. There are very few leucocytes. The glands are enlarged somewhat, and are lined by a normal cỳlinder epithelium, which offers no obvious change as compared with that of the glands of the resting uterus.

\section{The Pregnant Uterus: the Two Stages.}

When the ovum implants itself in the uterine wall, it becomes covered by a growth of the mucous membrane or decidua which we know as the decidua reflexa. For an account of this process see pages I24 to I27, where also proper 


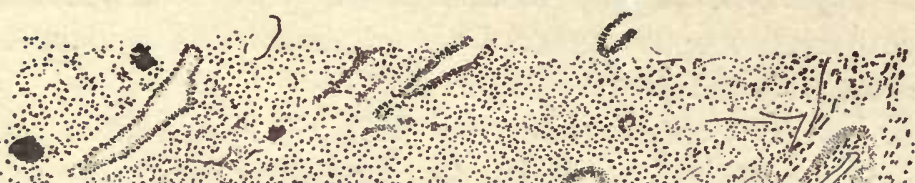

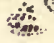

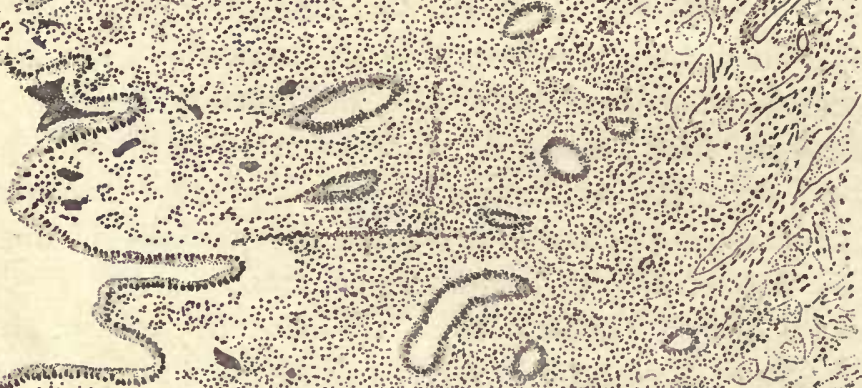
W. 0.10

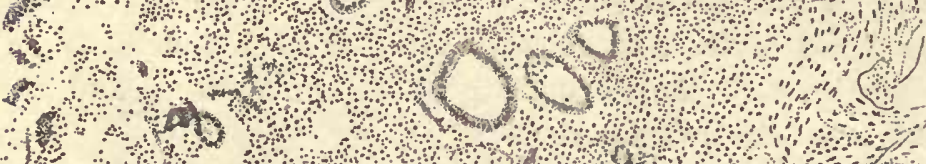

1 a or and (3)

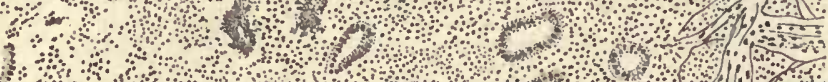
?

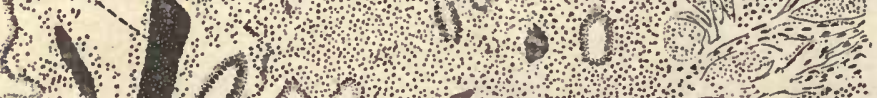

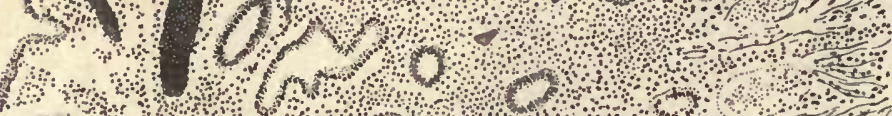

of A

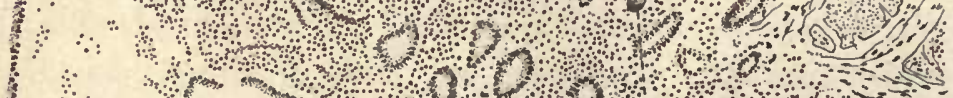


definitions of the terms decidua reflexa, serotina, and vera are given. -As the ovum increases in size the decidua reflexa also increases, and gradually becomes thinner and thinner, until it ultimately disappears. The exact date of its disappearance is not known; it falls somewhere within the fifth month. Accordingly, we may divide the period of pregnancy into two phases or stages, each comprising about half of the whole period. During the first stage the decidual reflexa is present (Fig. 227); during the second stage it is absent, so that the chorion læve comes into direct contact with the decidua vera. In the following sections a typical uterus of the first and second stages' each is described.

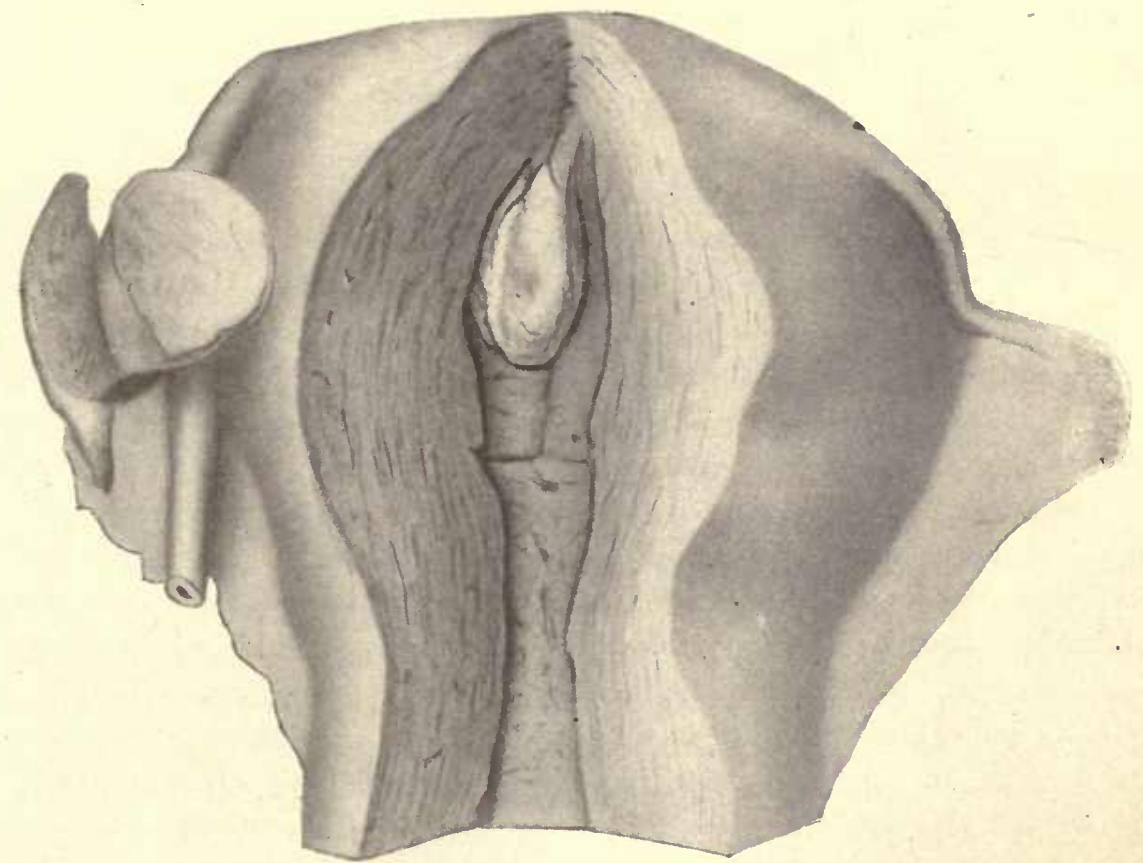

Fig. 227. - Human Uterus about One Month Pregnant. Natural Size. (The Uterus has been Opened by an Incision along Its Median Line, so as to Display the small. Oval Bag formed by the Decidua REFLEXA.)

\section{Human Uterus Three Months Pregnant.}

The uterus measures about $3 \frac{1}{2}$ inches in transverse diameter, and shows well-marked venous sinuses on its external surface. It should be opened by a crucial incision on the anterior side; its walls will-be found about an inch or more in thickness; it contains a grayish red bag (decidua reflexa), which nearly fills the cavity of the uterus and encloses the embryo, so that upon opening the womb we do not encounter the fetus directly. The inner bag has a smooth surface, but shows a few small pores; it is without blood-vessels and is attached to the dorsal wall of the uterus. The inner surface of the uterus shows a rich network 
of blood-vessels, many of which are large, irregular sinuses. The uterine walls consist of an outer muscular layer, and an inner decidual layer; the latter takes up nearly half the thickness of the wall, and is known as the decidua vera. Comparison with the seventh month uterus shows that the proportion of the layers changes, because during gestation the muscular layer increases and the decidual layer diminishes in thickness. The inner bag, when opened, shows the large cavity in which the embryo lies floating in amniotic fluid. The bag is formed by three very distinct membranes, of which the outermost, the decidua reflexa, is opaque and the thickest; the two inner ones are thin and transparent; the innermost is the delicate amnion; the middle membrane is the chorion, and is quite distinct from -both the amnion and reflexa; to the latter it is connected by a number of small branching villi scattered at some distance from one another over the surface; the villi adhere firmly to the reflexa by their tips. The embryo (Fig. 109) resembles a child in its general appearance; the length of the head and rump together is nearly $8 \mathrm{~cm}$., and the head is approximately equal in bulk to the rump. The umbilical cord is from 5 to $7 \mathrm{~mm}$. in diameter and usually about I $2 \mathrm{~cm}$. long. From its distal end the blood-vessels spread out over the placental area, and around the edge of the area rises the decidua reflexa, which does not extend on to the placenta. Floating in the amniotic fluid is a pearshaped vesicle, the yolk-sac, which is about $7 \mathrm{~mm}$. in diameter; it has a fine network of blood-vessels upon its surface, and is connected at its pointed end with a long, slender pedicle, the yolk-stalk, which runs to the placental end of the umbilical cord, there enters the cord itself, and runs through its entire length to its attachment to one of the coils of the intestine of the embryo.* Over the whole of the placental area the chorion gives off large villous trunks, each of which has numerous branches, with ramifications of the fetal vessels; the villi fill a space about I $\mathrm{cm}$. wide between the membrane of the chorion frondosum and the surface of the uterine decidua serotina, to which the tips of some of the villi are attached. With care the villi may be separated from the decidua, which is seen, when it is thus uncovered, to be cavernous; the caverns are rounded in form and part of them may be followed, on the one hand, until they connect with the blood sinuses of the uterus, and, on the other, until they open into the intervillous spaces, which therefore receive a direct supply of blood from the mother.

The principal difference to be noted between the uterus before and that after the fifth month in the relations of parts is the presence or absence of the decidua reflexa as a distinct membrane. During the fourth month the reflexa stretches as the membranes expand, and becomes thinner and thinner until by the end of the fourth month it is as delicate and transparent as the chorion and lies close against the decidua vera.

* At this stage a large part of the yolk-stalk within the umbilical cord has degenerated and usually disappeared by resorption. 


\section{Human Uterus Seven Months Pregnant.}

If we examine a pregnant uterus at any time during the sixth to ninth month of gestation, we find essentially the same relations of the parts-the most marked difference being in the size of the uterus, which increases with the duration of gestation, to correspond to the growth of the fetus. A description of a uterus seven months after conception will suffice, therefore, for our present purpose.

Such a uterus is a large, rounded bag with muscular walls, and measures 7 or 8 inches in diameter. Examined externally, it is remarkable especially for the numerous large sinus-like blood-vessels; its surface is smooth; the texture of the walls is firm to the touch, but the walls yield to pressure, so that the position of the child can be felt. As the placenta is situated normally upon the dorsal side, it is usual to open the uterus by a crucial incision of the ventral wall. The walls are about one half of an inch thick, sometimes more, sometimes less, and as soon as they are cut open'we enter at once into the cavity of the uterus containing the fetus and nearly a pint of serous liquid-the amniotic fluid. The fetus normally lies on one side, has the head bent forward, the arms crossed over the chest, the thighs drawn against the abdomen, and the legs crossed (compare Fig. III). It resembles closely the child at birth, but is smaller; its head is, relatively to the size of the body, larger; the abdomen is more protuberant, and the limbs proportionately smaller. The inner surface of the uterus is smooth and glistening; if it is touched with the finger, it is found to be covered by a thin but rather tough membrane, called the amnion, which is only loosely attached. Examination of the uterine wall, where it has been cut through, shows that its thickness is formed principally by the muscular layer, which is made up by numerous laminæ of fibers, between which are the large and crowded blood sinuses, similar to those distinguishable on the external surface of the uterus. About one fifth or less of the wall inside the muscularis has a different texture and can be partly peeled off as two distinct membranes, the innermost of which is the amnion already mentioned, and the outer is the chorion united with the decidua. The amnion and chorion are appendages of the embryo; the decidua is the modified mucous membrane of the uterus. Let us return to the embryo. From its abdomen there springs a long, whitish cord, known as the umbilical cord; it is usually from about one third to one half an inch in diameter and $40 \mathrm{~cm}$. long, but its dimensions are extremely variable; it always shows a spiral twist, and contains three large blood-vessels, two arteries and one vein, all of which can be distinguished through the translucent tissue. The distal end of the cord is attached to the wall (placenta) of the uterus usually near the middle of the dorsal side of the organ. It is easily seen that the blood-vessels of the umbilical cord radiate out from its end over the surface of the uterus underneath the amnion, branching as they go; they spread, however, only over a circumscribed area, the placental, where the wall of the uterus is very much thickened. A vertical section through the placental area shows that the amnion and chorion are widely separated from the decidua and muscularis by a 
spongy mass soaked with maternal blood. This mass consists of numerous trees of tissue, which spring with comparatively thick stems from the chorion and branch again and again. In these stems and branches are to be found the final ramifications of the vessels of the umbilical cord; the trees are known as chorionic or placental villi. Some of their end-twigs are very closely attached to the surface of the decidua. In the center of the placental area the villi form a mass about three fourths of an inch thick, but toward the edge of the area the mass gradually thins out until at the very edge the chorion and decidua come into immediate contact. The mass of villi, together with the overlying portions of the chorionic and amniotic membranes and the underlying portion of the decidua, constitutes what is known as the placenta. The decidua of the placental area is called the decidua serotina; the chorion of the placenta is known as the chorion frondosum. When birth takes place, the whole placenta is expelled after the delivery of the child; the placenta of the obstetrician is, therefore, partly of fetal, partly of maternal, origin.

\section{Decidua Vera of the First Stage in Section.}

Specimens may be preserved in Zenker's or Tellyesnicky's fluid, or they may be preserved with less good results in Müller's or Parker's fluid or in picro-sulphuric acid. Sections may be made of the, entire wall in celloidin, or, if it is desired to get thinner sections, in paraffin, in which case it is advantageous to remove as much as possible of the muscular coat so as to cut only the decidual membrane.

The following description is based upon a uterus one month pregnant. Figure 228 was obtained from a vertical section of the decidua, by drawing the outlines of the glands or gland spaces, $G l$, and by dotting the entire area occupied by the connective tissue. The blood-vessels are indicated by double outlines. The artery, Art, owing to its spiral course, is cut repeatedly. The figure demonstrates very clearly that the gland cavities are so arranged that the decidua is divided into an upper compact layer, Comp, and a lower cavernous layer, Cav; the difference being due to the size and number of the gland cavities. The amount of epithelium to be observed at this stage varies greatly. It is sometimes wholly absent from the surface, in other cases absent or present in patches. In the glands the epithelium has. undergone many modifications. In some parts the original cylinder epithelium of the glands is well preserved in patches, and such patches of epithelium are found at every stage until after delivery. It has been observed that these patches serve to regenerate the epithelium of the glands, and, by spreading from the glands on to the surface, to regenerate also the epithelial covering of the uterine mucosa. But for the most part the glandular epithelium is considerably altered. We find places in which the cells, though attached to the surrounding connective tissue, are separated from one another by small fissures. In other places the cells are a little larger (Fig. 229), each for the most part cleft from its fellow, and some of them loosened from the wall and lying free in the cavity. Apparently the cells which are thus freed become swollen, probably by imbibition, both the 


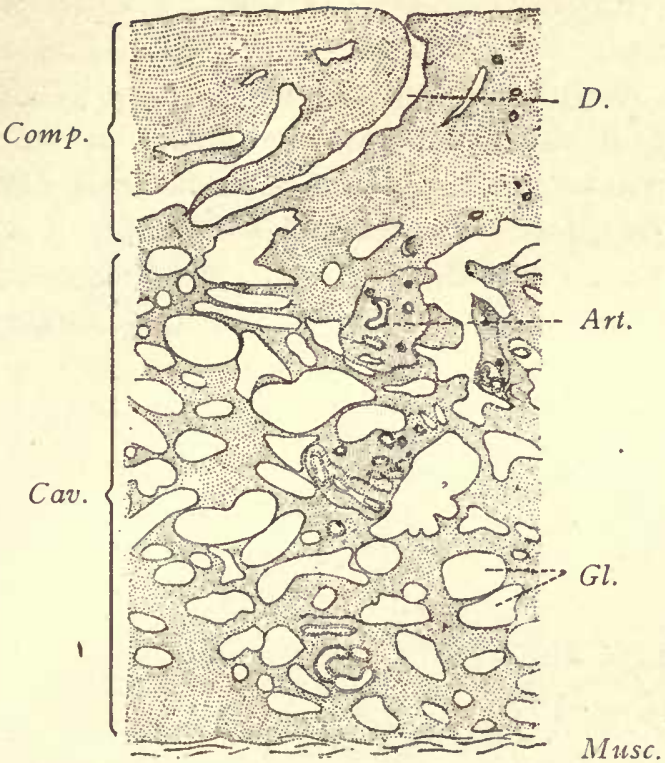

Fig. 228.-Vertical Section of a Humad Uterus

(Decidua vera), One Month Pregnant.

Comp, Compact layer. Cav, Cavernous layer. $D$,

- Gland-duct. Art, Spiral artery. $G l$, Spaces occupied by epithelial glands. Musc, Muscularis. (For clearness all the glandular epithelium has been omitted from the drawing.)

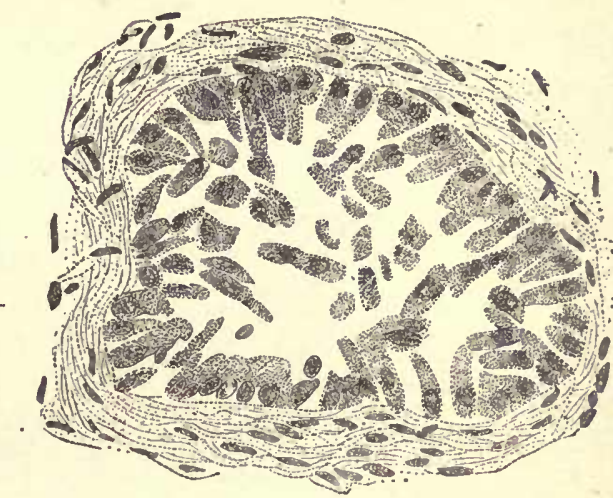

Fig. 229.-Human Uterus, One Month PregNant. Section of Gland from the Cavernous LAyer, With the EPITHeltum PaRtLy Adherent to the Walls. $\times 445$ diams.

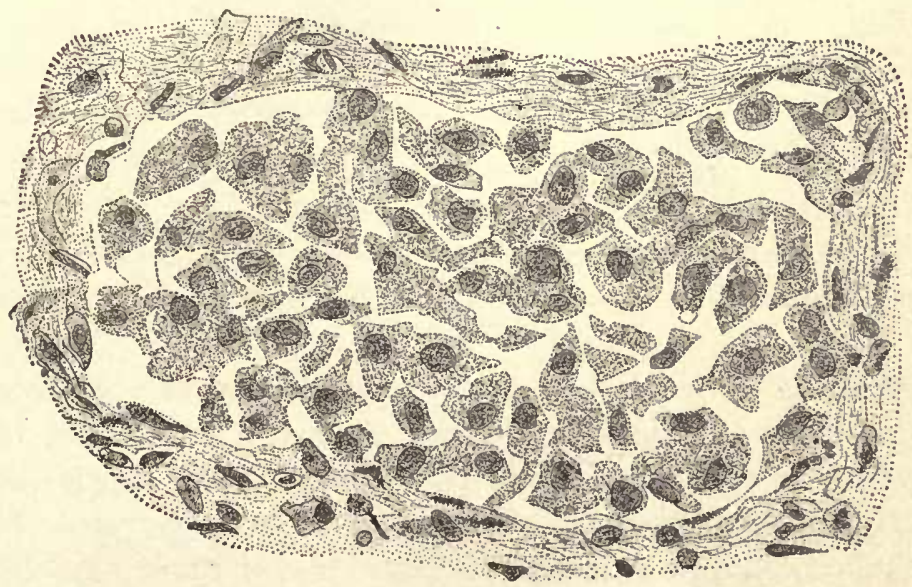

Fig. 230.-Human Uterus, One Month Pregnant. Section of a Gland from the Cavernous Layer with the Epithelium Loosened from the Walls. The Epithelial Cells are Swollen. 
protoplasm and the nuclei becoming enlarged (Fig. 230). The cells lie separately and almost completely fill the gland cavity. They are no longer cylindrical in shape, but irregular. Their protoplasm is finely granular and stains rather lightly. The nuclei are rounded, granular, and with sharp outlines. In somewhat older stages one finds the cells. replaced by a granular material. The obvious interpretation of the appearances described is that the glandular epithelium is breaking down and disintegrating, or, in other words, passing through a special form of degeneration which is highly characteristic. In later stages some of the broken-down

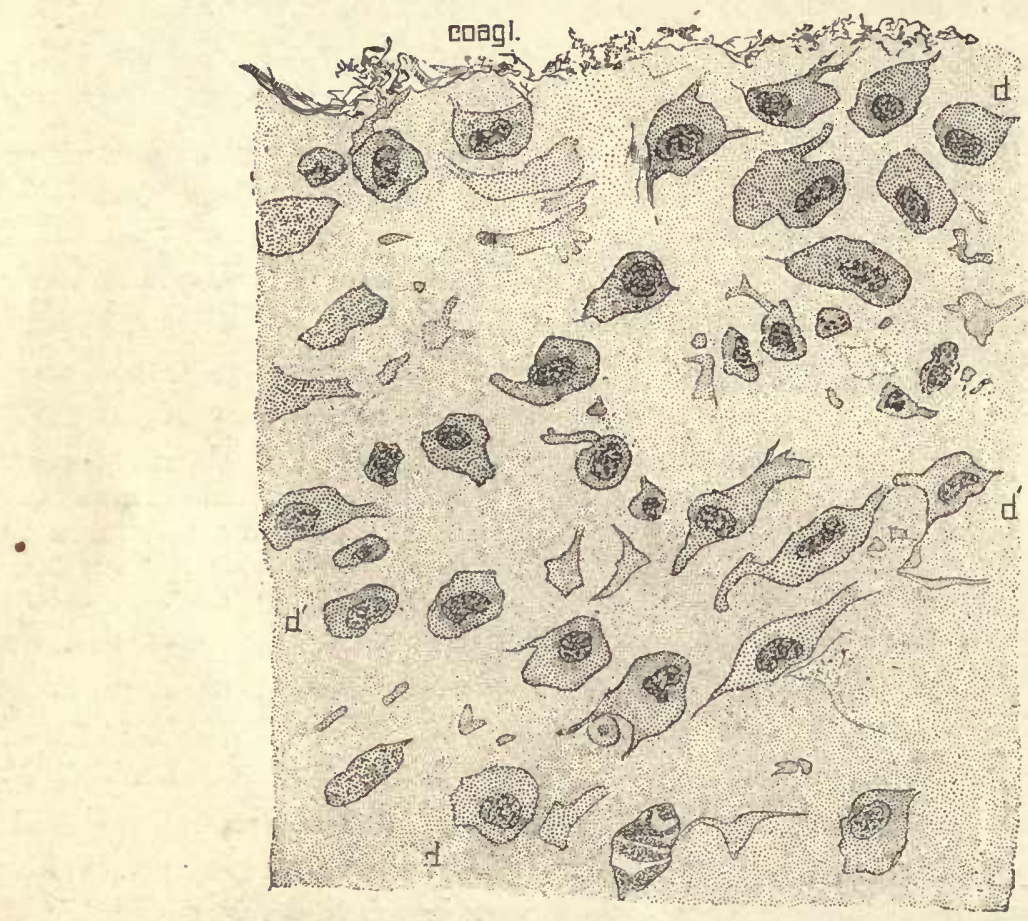

Fig. 23r.-Uterus One Month Pregnant; Portion of the Compact Layer of the Decidua Seen in Vertical Section.

Coagl, Coagulum upon the surface. $d, d^{\prime}$, Decidual cells. $\times 445$ diams.

material forms hyaloid rounded concretions, which, owing to their deep staining, are somewhat conspicuous. The concretions usually include a number of spherical vacuolcs.

The formation of decidual cells has already begun in the upper or compact layer (Fig. 23I). They are modified connective-tissue cells, which have grown in size and altered their structure. Their bodies stain deeply. with eosin; the nuclei are round, oval, slightly irregular in shape, coarsely granular, and sharp in outline. The cells themselves, though irregular and variable in shape, are all more or less provided with processes running off in various directions. Scattered between the cells are many sections of the processes. Occasionally it may be seen that two 
cells are connected. Later on the decidual cells acquire smoother and more rounded outlines, and appear to lose altogether their connections with one another. In the cavernous layer there are no decidual cells.

\section{Decidua Reflexa of the First Stage.}

The decidua reflexa may be preserved in Zenker's fluid, Parker's fluid, or picrosulphuric acid. It should be hardened with the portions of the chorion and chorionic villi adherent to it. It may be imbedded in celloidin and the sections stained with alum hematoxylin and eosin, with Beale's carmine, or with a so-called fibrin stain.

As stated above (page 343), the presence of the decidua reflexa distinguishes the first stage of pregnancy from the second, in which the reflexa is absent, having disappeared by degeneration and absorption. To observe this process of the disappearance of the reflexa, membranes from the second and third months should be examined.

Section of Decidua Reflexa of Two Months.-At this time the reflexa starts from the edge of the placental area as a membrane of considerable thickness, but it rapidly thins out, the very thinnest point being opposite the placenta. Examination of sections shows that the entire reflexa is undergoing degeneration which is found to be more advanced the more remote the part examined is from the placenta. The chorion læve lies very near the reflexa, being separated only by the villi, which are already very much altered by degeneration. In the

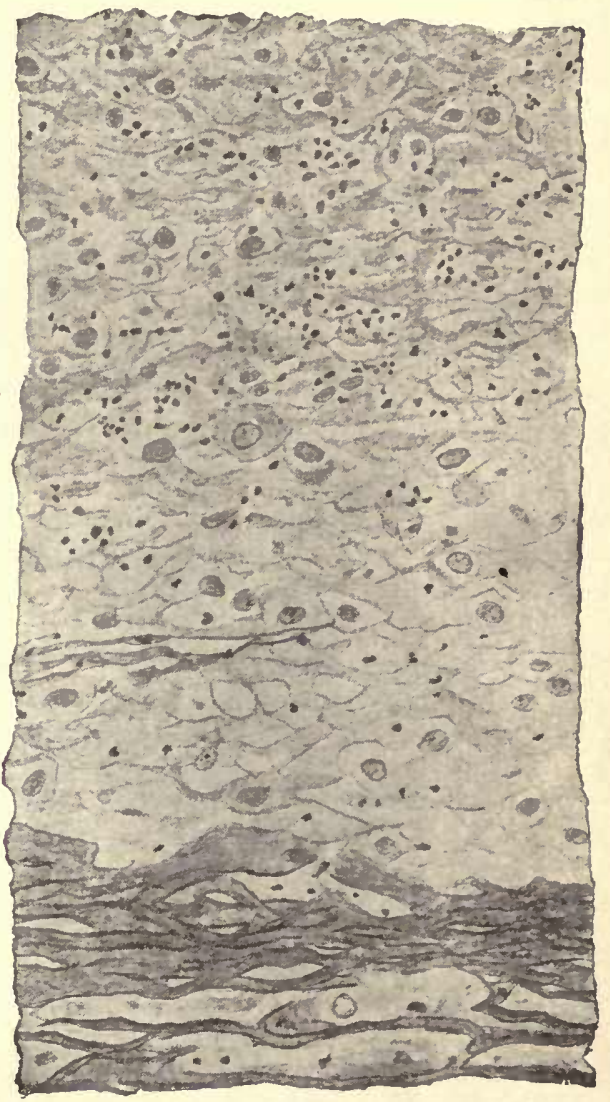

Fig. 232.-Section of Human Decidua Reflexa at Two Months. region halfway between the base and the apex of the reflexa the tissue (Fig. 232) shows only vague traces of its original structure. Only here and there can a distinct cell with its nucleus be made out. Most of the cells have broken down and fused into irregular hyaline masses without organization. Ramifying through the fused detritus appear strands and lines, which are more darkly stained by both carmine and hematoxylin. On account of their fibrous appearance, these strands are often spoken of as fibrin, although they are presumably not the same as the true fibrin from the blood. The fibrin is much 
more developed upon the inner or chorionic side than upon the outer side of the reflexa. On the inner side it forms a dense network, which fuses with the degenerated ectoderm of the chorionic villi wherever the villi are in contact with the decidua. It also ramifies nearly halfway through the decidua, the ramifications being followed easily, owing to the dark staining of the substance. Over the outside of the decidua the fibrin forms a much thinner layer or may be only indistinctly formed.

In a decidua reflexa of three months the conditions are essentially the same, except that the degeneration is further advanced and the membrane thinner. Traces of cellular structure are still more vague and the fibrin is more developed. In all parts of the membrane there appear leucocytes which are particularly numerous and conspicuous in the neighborhood of the placenta. It is natural to assume that they are concerned in the resorption of the reflexa. There is an inner thicker layer of fibrin and a thinner outer layer, which is now always present and distinct. Between these two layers is a stratum in which the remains of the cells may be seen. Occasionally there is an appearance which suggests surviving decidual cells, and, indeed, in sections taken from parts close to the placenta true decidual cells may be identified.

The origin of the chorion læve by the disappearance of its villi is described on page 367 . The sections of the decidua reflexa will enable the student to see also some of the phases of the degeneration of the villi. They are very much altered. Their ectoderm undergoes a hypertrophic degeneration and becomes hyaline tissue, which stains darkly. The degenerated ectoderm of adjacent villi fuses more or less extensively. The mesoderm of the villi shows a partial loss of its primitive cellular organization.

\section{Decidua Vera and Chorion Læve of the Second Stage.}

Pieces of the decidua vera of from six to nine months with the chorion and amnion carefully preserved in situ may be hardened in Müller's or 'Tellyesnicky's fluid. Blocks half an inch or less in size may be imbedded in celloidin, and sections made perpendicularly to the surface may be stained with alum hematoxylin and 'eosin, or with Heidenhain's hematoxylin and orange $G$, or with picro-carmine.

The decidua reflexa having been resorbed, the chorion (Fig. 233, Cho) has come into contact with the surface of the uterus, and the chorionic epithelium, $c$, is closely adherent to the surface of the decidua, from which the original epithelium has completely disappeared. The amnion is loosely connected with the chorion by a few strands or threads, which are represented in the figure and the nature of which is not known. Both the amnion, $A m$, and the chorion, Cho, being developed from the original somatopleure (compare page 82), consist of a mesodermic and an ectodermal layer. The ectoderm of the amnion is a single layer of cuboidal cells placed on the side of the membrane toward the embryo and away from the uterus. The ectoderm, $c$, of the chorion, on the contrary, is next the uterus. Hence it will be noticed that the mesodermic layers of the amnion 
and chorion are adjacent. Both membranes are quite thin. The decidua is a relatively voluminous membrane containing blood-vessels, $v$, which for the sake of distinctness have been filled in with black in the drawing. It also contains a series of elongated spaces, which represent sections of the glands. These spaces, $g l$, are present only in the inferior half of the decidua. Owing to their absence from the superior half, that portion has a more compact structure, and is, therefore, designated as the compact layer; the lower portion, being broken up and

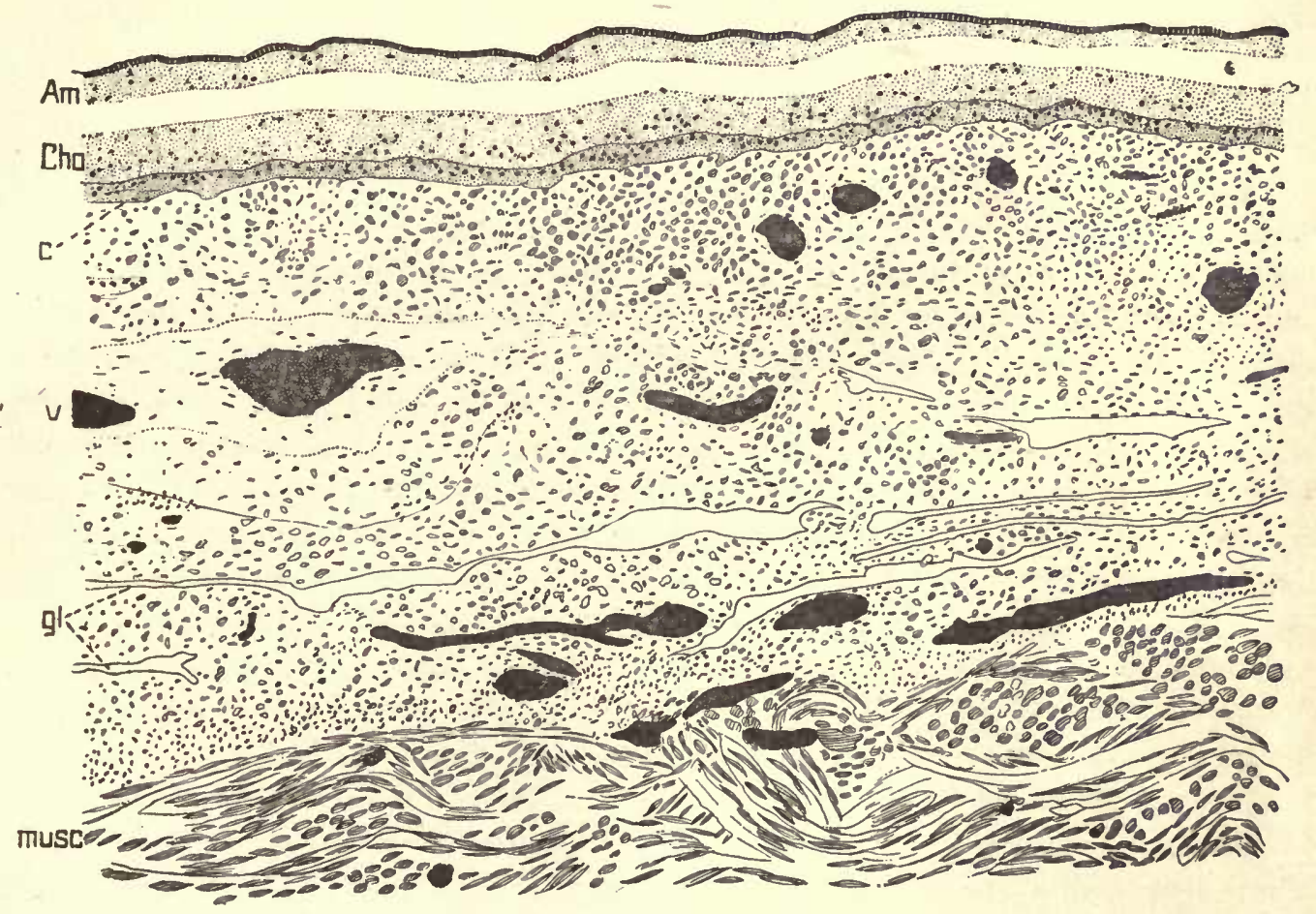

Fig. 233.-Human Uterus about Seven Months Pregnant. Vertical Section of the Decidua Vera with the Fetal Membranes in situ.

Am, Amnion. Cho, Chorion. c, Chorionic epithelium. v, Blood-vessel. gl, Glands. musc, Muscularis. $\times 40$ diams.

made loose in texture by the somewhat numerous gland cavities, is called the cavernous layer, the caverns, of course, corresponding to the gland spaces. The gland spaces are now very much stretched out, a condition which results simply. from the general expansion of the uterus during pregnancy. In the gland spaces appear patches of epithelium still intact, and in the cavities themselves isolated cells in various phases of degeneration and disintegration, similar to the phases which may be observed in the decidua vera of one month; but the degeneration is, on the whole, considerably more advanced than in the early stage. Around 
some of the larger blood-vessels there is connective tissue only slightly modified, and the original structure of the mucous membrane is more or less, but not perfectly, preserved in the deep portion of the decidua. The majority of the cells, especially in the compact layer, have grown in size and become transformed into true decidual cells. In the ectoderm of the chorion, $c$, the cells lie two or three deep. They have distinct walls, a very coarsely granular protoplasm, and nuclei which stain darkly. By these characteristics they are éasily distinguished from the neighboring decidual cells, to which, however, they offer a slight superficial resemblance.*

\section{The Placenta in Situ.}

The placenta in its natural position in the uterus follows the curvature of the uterine walls, hence its free or amniotic surface is slightly concave. Its decidual surface is strongly convex. It is thickest in the center and thins out gradually toward its edge. The uterus should be obtained in the freshest possible condition and be opened by a crucial incision on the ventral side. The embryo should then be removed, the umbilical cord cut through, care being taken to bring as little pressure as possible on the uterus or the placenta, and the whole organ placed in the preservative, which should be either Tellyesnicky's or Müller's fluid. In view of the large size of the organ, it is very necessary to use large quantities of the preserving fluid, and this fluid must be changed several times in order to insure good histological preservation. When the hardening is completed, columns about one-half inch square may be cut out so as to pass vertically from the inner to the outer surface of the placenta, preserving the amniotic and chorionic membranes in place. The blocks are to be imbedded in celloidin and ought to remain at least three days in thin and three days in thick celloidin, so as to insure a thorough penetration of the imbedding material into the intervillous spaces. Make the sections so that they pass vertically through the placenta. Stain with alum hematoxylin and eosin.

Placenta at Seven Months. - A section made according to the method just described is represented in figure 234. The thin amnion, $A m$, covers the upper (or inner) surface of the chorionic membrane, Cho. This membrane is. separated from the decidua, $D$, by a dense forest of villi, of which innumerable sections appear. In younger placentas the distance between the chorion and the decidua is considerably less, and the number of sections of villi is smaller, but the average size of those sections larger. In the present specimen the distance between the chorion and the decidua is nearly twice as great as the diameter of the muscular coat, $M c$, of the uterus. The ends of some of the villi touch the decidual tissue, and are imbedded in it. Their imbedded ends are without, covering epithelium, but their connective tissue is immediately surrounded by hyaline substance which

* It should perhaps be noted that in some comparatively recent text-books the chorionic ectoderm has been described as the decidua reflexa, an error which is much to be regretted. 


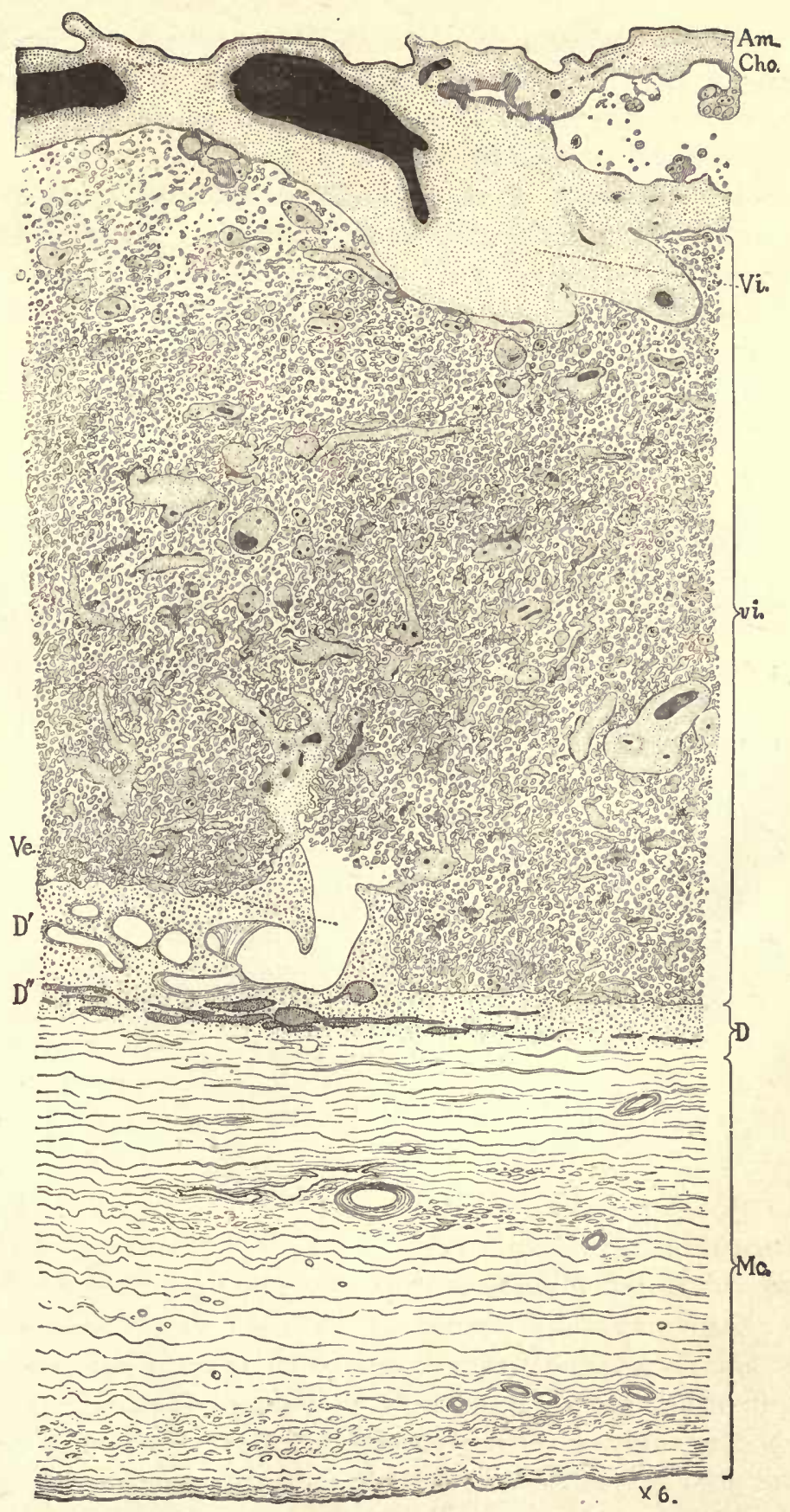

Fig. 234.-Human Placenta in situ, about Seven Months. Vertical Section.

$A m$, Amnion. Cho, Chorion. Vi, Villous trunk. vi, Sections of villi in the substance of the placenta. $D^{\prime}, D^{\prime \prime}$, Decidua serotina. $M c$, Muscularis. $V e$, Uterine artery, opening into the placenta; the fetal blood-vessels are drawn black; the maternal blood-vessels are white; the chorionic tissue is stippled, except the canalized fibrin, which is shaded by lines. The remnants of the gland cavities in the decidua are stippled dark. $\times 6$ diams. 
is probably degenerated epithelium. The decidua serotina is plainly divided into an -upper compact, $D^{\prime}$, and a lower ravernous layer, $D^{\prime \prime}$. The section figured passes through an arterial vessel, $V e$, which makes an abrupt turn so as to discharge its blood into the intervillous spaces.

The histological structure of all the parts should be carefully studied.

(As regards the structure of the amnion, see page 370 .)

The chorion consists of two layers, the outer ectodermic and inner mesodermic. Over the chorionic membrane proper the ectoderm offers a great variety of appearances. In some places it may be seen to have still its primitive organization, a single inner layer of distinct cells and an outer syncytial layer, more or less similar

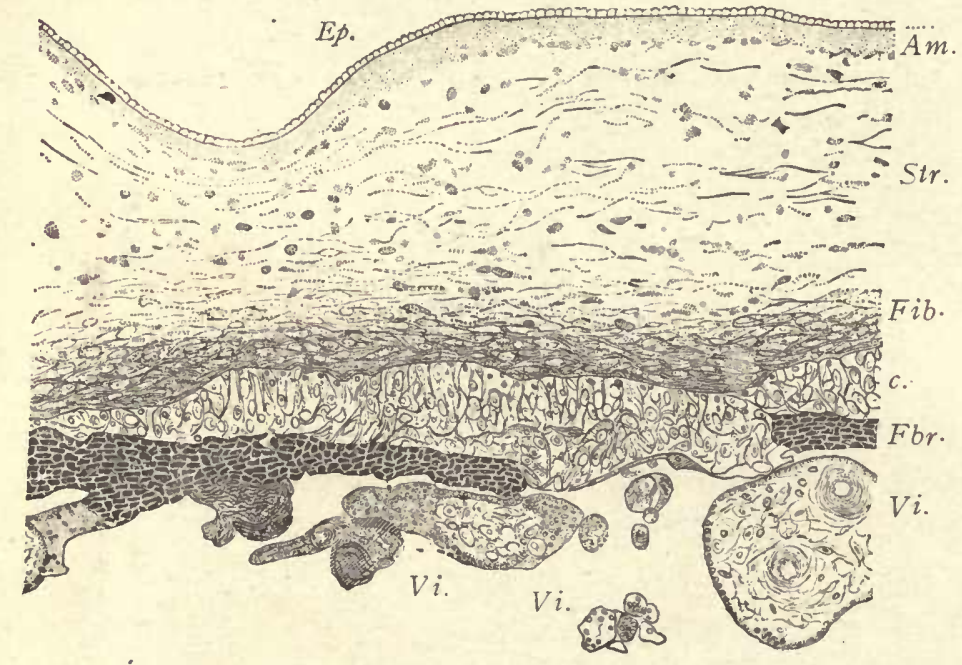

Fig: 235--Human Placental Chorion and Amnion of the fifth Month.

Ep, Amniotic epithelium. Am, Amnion. Str, Stroma. Fib, Fibrillar layer. Fbr, Fibrin layer. $c$, Chorionic cellular layer of ectoderm. $V i$, Chorionic villi. $\times 7 \mathrm{I}$ diams.

to those represented in figure 243 . For the most part, however, the chorionic ectoderm has been considerably modified from its primitive condition. The inner or cellular layer exhibits irregular, thickened patches, which present every possible degree of variation as to their size. A cell patch from a somewhat younger stage is represented in figure 235 as seen with a low magnification, and another patch of the age we are studying is represented in figure 236. The patches vary in appearance; the cells are more distinct in the small patches, less so in the large patches, in which there are often parts more or less degenerated. The cell-bodies stain lightly; their nuclei are granular, not very sharply defined, and variable in size and shape. The cellular layer is always sharply defined against the mesoderm. Toward the outside the patches offer varying relations. In some cases a part of 
a cell patch may form the whole thickness of the ectoderm, as shown in figure 235, or the whole of a cell patch may do so. More commonly, however, the cellular patch is covered more or less completely by a special substance, which is termed canalized fibrin, and which is believed to represent the original outer syncytial layer in a degenerated condition. The fibrin is a constant, normal, and very remarkable constituent of the placenta. Its formation seems to begin always in the outer or syncytial layer of the chorionic ectoderm, but it may also spread into

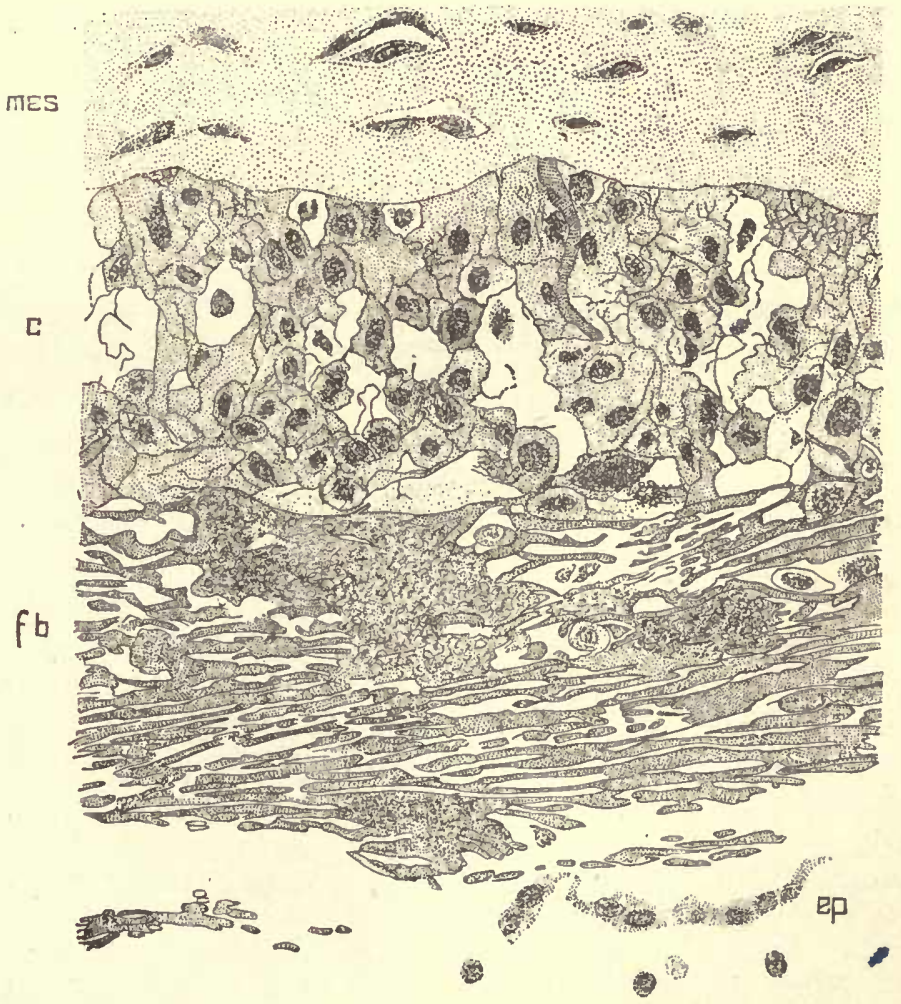

Fig. 236.-Human Chorion of Seven Months' Placenta.

$c$, Cellular layer. $f b$, Fibrin layer. $e p$, Remnants of epithelial layer. mes, Mesoderm. $\times 445$ diams.

the cellular layer, which then becomes replaced by fibrin, so that this last alone represents the ectoderm of the chorion. The fibrin layer consists of a very refringent substance permeated by numerous channels (Fig. 236, $f b$ ). T The substance has a violent affinity for carmine and hematoxylin, and hence is always deeply colored in sections stained with either of these dyes. The channels tend to run more or less parallel to the surface of the chorion, and are connected by numerous short cross-channels. Some of the channels contain cells. or nuclei. The appearances, however, are very variable; the fibrin often sends long outshoots into the 
cellular layers. To summarize, we may say that the ectoderm of the chorionic membrane undergoes patchwise manifold changes. It exists in three general forms: the nucleated protoplasm or syncytium, the cellular layer, and the canalized fibrin. A patch of the ectoderm may consist of any one of these modifications or any two, or of all three. But they have fixed relative positions, for when the syncytium is present, it always covers the free surface of the chorion; when the cellular layer is present, it always lies next the mesoderm; and when all three forms are present over the same part, the fibrin is always the middle stratum.

The mesoderm of the chorion in early stages has a homogeneous matrix, which about the ninth week begins to change its appearance. In the frondosum, in our specimen, the matrix has acquired a distinctly fibrous structure. Usually the production of fibers is much greater in the immediate neighborhood of the ectoderm,

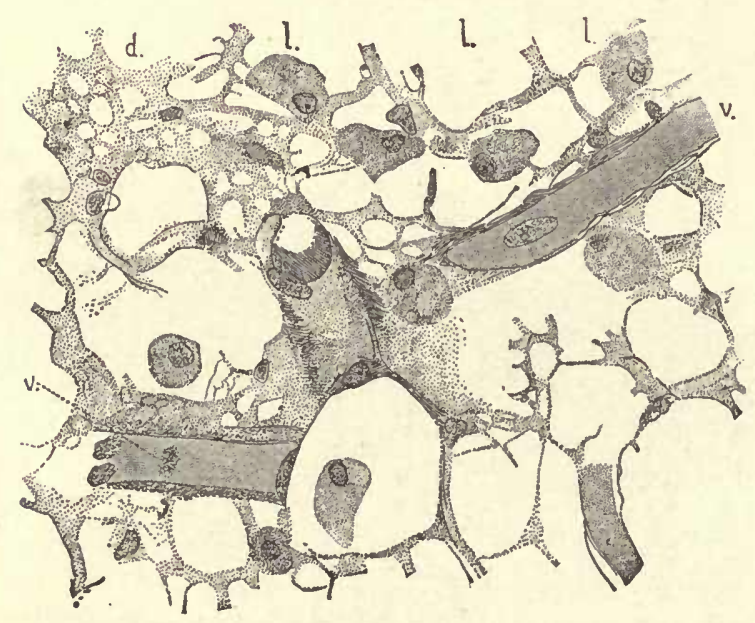

Fig. 237.-Adenoid Tissue from a Villus of a Human Placenta of Four Months.

$l, l, l$, Degenerating blood-cells. $v, v$, Capillary blood-vessels. $d$, Finer meshwork surrounding a capillary. $\times 35^{2}$ diams.

and this may go so far as to mark out a more or less distinct subectodermal fibrillar layer (Fig. 235, Fib). There appears to be no mesothelial layer upon the chorion at this stage, but it seems possible that its presence might be revealed by the application of proper special methods.

In the villi the ectoderm differs from that of the chorionic membrane in several respects: (I) The cellular layer after the first month becomes less and less conspicuous, and after the fourth month is present only in a few isolated patches, which have been termed the cell-knots. (2) For the most part the villi remain covered by the syncytial layer, which in many places is thickened. In later stages these thickenings are small and numerous, constituting the so-called proliferation islands with many nuclei. Many of the little thickenings appear in sections of 
the villi, and here and there are converted into canalized fibrin. (3) The proliferation islands are converted into canalized fibrin and at the same time grow and fuse, forming larger patches, particularly on the larger stems. In this manner are produced the large areas and columns of fibrin such as appear in our section. Over the tips of the villi, where they are imbedded in the decidua serotina, the epithelium apparently degenerates and becomes hyaline tissue, but without canalization. The mesoderm exists in two principal forms, adenoid tissue and fibrillar tissue around the blood-vessels. The adenoid tissue (Fig. 237) may be considered as the proper tissue of the villus. It consists of a network of protoplasmic threads, which start from nucleated masses. There are many large meshes, which are partly occupied by the very large, coarsely granular cells, $l, l$, which generally are widely scattered, but sometimes are present in large numbers. These free cells are extravasated blood-corpuscles, which have increased in size. Probably they are dead or at least dying and have swollen by imbibition. They undergo disintegration, their protoplasm becoming vacuolated; the vacuoles increase in size as the protoplasm is dissolved, until finally the cell-body entirely disappears. About the capillary blood-vessels, $v$, the network is more finely spun. Around the larger blood-vessels the mesoderm has a distinct intercellular substance with a tendency to fibrillar differentiation in quite a wide zone around the blood-vessels. In this zone the cells become elongated or irregularly fusiform. Around the larger vessels the cells are grouped in laminæ, and apparently are contractile, so that they must be looked upon as an imperfectly differentiated form of smooth muscular tissue.

\section{Decidua Serotina at Seven Months.}

Specimens may be treated as described for the placenta in situ (page 352). If, however, the best results are desired, the whole of the uterus should be cut through and the placenta divided into smaller pieces from I to $2 \mathrm{~cm}$. in diameter, so as to allow a freer penetration of the preserving fluid. Either Zenker's or Tellyesnicky's fluid is recommended. In a normal uterus about seven months pregnant we find the following relations: The serotina is about $1.5 \mathrm{~mm}$. thick, and contains an enormous number of decidual cells (Fig. 238); the cavernous, $D^{\prime}$, and compact, $D^{\prime \prime}$, layers, are very clearly separated; the mucosa is sharply marked off from the muscularis, although scattered decidual cells have penetrated between the muscular fibers. The muscularis is about ro $\mathrm{mm}$. thick and is characterized by the presence of quite large and numerous venous thrombi, especially in the part toward the decidua. The decidua itself contains ferw blood-vessels. Upon the surface of the decidua can be distinguished a special layer of denser decidual tissue, which in many places is interrupted by the ends of the chorionic villi which have penetrated it, as is well shown in the accompanying figure. The gland cavities of the spongy layer, $D^{\prime}$, are long and slit-like; they are filled for the most part with fine granular matter, which stains light blue with hematoxylin; 
they also contain a little blood, and sometimes a few decidual cells. There also occur in them hyaloid concretions-oval bodies several times larger than any of the decidual cells, and presenting a vacuolated appearance. In uteri over two months pregnant they are probably invariably present. In many places the glandular epithelium is perfectly distinct; its cells vary greatly in appearance, neighbors being often quite dissimilar; nearly all are cuboidal, but some are flattened out; of the former, a number are small with darkly stained nuclei, but the majority of the cells are enlarged, with greatly enlarged, hyaline, very refringent nuclei.

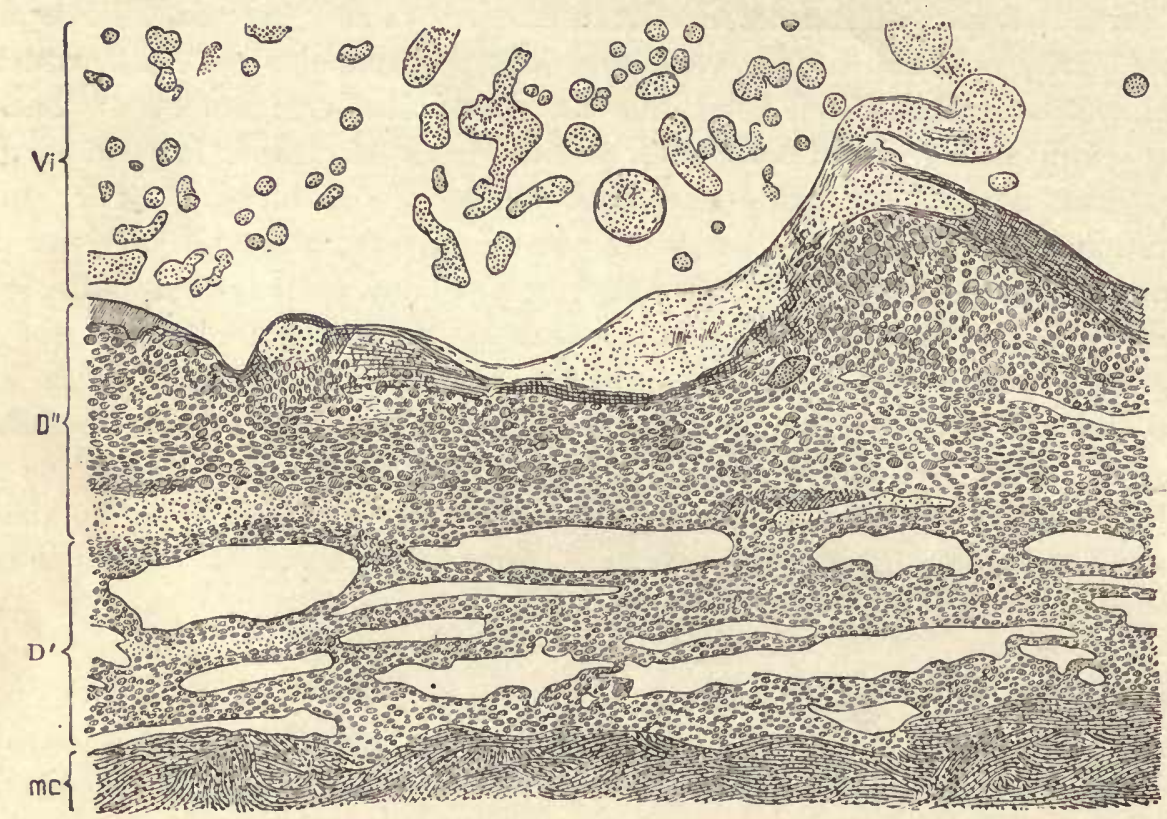

Fig. 238,- The Human Decidua Serotina at Seven Months. The Section is Taken from near the Margin of the Placenta.

$V i$, Chorionic villi; the intervillous spaces were filled with maternal blood, which is not represented in the figure. $D^{\prime}$, Cavernous layer of the decidua. $D^{\prime \prime}$, Compact layer of the decidua. $m c$ Muscularis.

There are also in many of the gland spaces isolated enlarged cells which have detached themselves from the wall, and in some cases the detached cells nearly fill the gland cavity, very much as in figure 230 .

The decidual cells of the cavernous layer (Fig. $238, D^{\prime}$ ) are smaller and more crowded than most of those of the compact layer. The largest cells are scattered through the compact layer, but are most numerous toward the surface. They extend around the margin of the placenta and have penetrated the chorion, in the cellular layer of which they are very numerous; the immigration imparts to the chorionic layer in question somewhat the appearance of a decidual membrane. Misled by this peculiarity, some authors have held this layer to be maternal in 
origin, and accordingly have described it as a "decidua subchorialis." The decidual cells exhibit great variety in their features (Fig. 239). They are nearly all oval discs, so that their outlines differ according as they are seen lying in the tissue turned one way or another; they vary greatly in size; the larger they are, the more nuclei they contain; the nuclei are usually more or less elongated; the contents of the cell granular. Some of the cells present another type, $c$; these are more nearly round, are clear and transparent; the nucleus is round, stains lightly, and contains relatively few and small chromatin granules; such cells are most numerous about the placental margin.

\section{The Human Placenta.}

Specimens of the fresh normal human placenta may be obtained without diffculty from maternity hospitals. The placenta should be thoroughly examined in the fresh state by the student and all the points in the description below

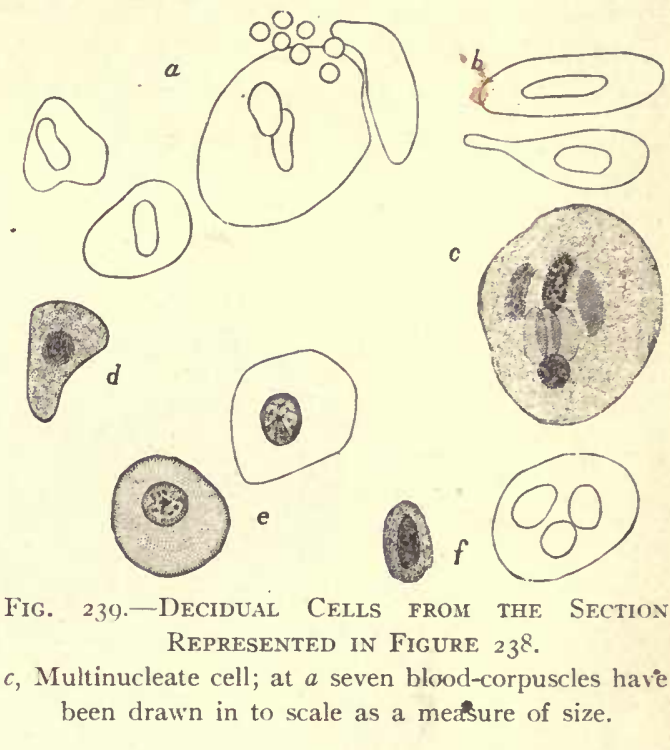
verified by him. To make an injected specimen either the starch injection mass or the colored gelatin mass may be used according as it is desired to demonstrate only the coarser or all the branches of the vessels. The injection should be made through one of the arteries of the umbilical cord. As there is almost invariably a cross-anastomosis between the two arteries close to the placenta, it is sufficient to inject one of them in order to fill the entire system of vessels. The starch mass may be injected in the cold specimen: If the gelatin mass is used, the specimen must be submerged in warm water until it is sufficiently heated to keep the gelatin mass melted during the process of injection. After the gelatin injection is com- pleted, the placenta may be preserved in 70 per cent alcohol, to every roo c.c. of which 2 c.c. of hydrochloric acid have been added. After twenty-four hours replace the acidulated alcohol by fresh alcohol of 70 per cent, which should be again changed after another twenty-four hours. Specimens will then keep indefinitely. Such specimens may be used either for sections of the placenta to be made from pieces imbedded in celloidin, or for the study of isolated fragments of the villi, which are pulled out of the placenta by forceps.

The human placenta is a disc of tissue to which the umbilical "cord of the child is attached by its distal end. As a result of normal labor the amnion and chorion, by which the fetus in utero is surrounded, are ruptured; the child is then expelled, but by means of the long umbilical cord remains attached to the uterus; after an interval the placenta, with which the cord retains its connection, 
is loosened from the uterine wall and expelled, together with the fetal envelopes and portions of the decidual membranes (uterine mucosa) of the mother; the parts thus thrown off secondarily constitute the so-called after-birth of obstetricians.

The placenta at full term, as thus obtained by natural expulsion, is a moist mass, containing a great deal of blood, spongy in texture, about 7 inches in diameter, but very variable in size, being roughly proportionate to the bulk of the child; usually oval, sometimes round, but not infrequently irregular in shape. One surface is smooth and covered by a pellucid membrane (the amnion), and reddish gray in color; to this surface the umbilical cord is attached, and it shows the arteries and veins branching out irregularly from the cord over the surface of the placenta (Fig. 240). The opposite surface is rough, lacerated, and usually covered irregularly with more or less blood, which is often dark and clotted. When the blood is removed, the surface is seen to be crossed by a system of grooves which divide the placental tissue into irregular areas, each perhaps an inch or so in diameter; these areas are called cotyledons. The placenta is about 25 or $30 \mathrm{~mm}$. thick, but thins out rapidly at the edges, and its tissue passes over from the margin of the placenta.

When in situ, the placenta is fastened to the walls of the uferus by its rough or cotyledonary surface; its smooth, amniotic surface faces the cavity in which the fetus lies.

A more detailed examination of the gross appearance of a placenta discharged at term leads to the following additional observations: The color is a reddish or purplish gray, varying in tint according to the condition of the blood, and mottled between the divaricating blood-vessels by patches and networks of pale yellowish or flesh color. The light pattern is produced by the tissue of the villi shining through the membrane of the chorion. These appearances are less distinct when the placenta, as is usually the case, is covered by the thin amnion. The amnion, however, is very easily detached as far as the insertion of the umbilical cord, to the end of which it is firmly attached, but it cannot be traced farther because on the cord itself there is no amnion. The blood-vessels run out in all directions from the end of the cord; each vessel produces a ridge upon the placental surface, so that its course is readily followed. The arteries and veins are more easily distinguished after double injection, as is shown in figure 240.

The two kinds of vessels do not run together; the arteries lie near the surface, just above the veins; the arteries fork repeatedly, until they are represented only by small branches and fine vessels; some of the small branches disappear quite suddenly by dipping down into the deeper-lying tissue in order to pass into the villi. The veins (Fig. 240) are considerably larger than the arteries; they branch in a similar manner, but some of the trunks disappear from the surface more abruptly than is the case with the arteries. There is the greatest possible variability in the vessels of the placenta; one never sees two placentre with vessels alike.

The insertion of the cord is always eccentric; the degree of eccentricity is 
variable and is easily seen to be related to the distribution of the vessels. The insertion may ever be entirely outside the placenta, which yet may otherwise be normally developed. Such insertions are called velamentous. The usual type is shown in figure 240. The arteries come down together from the cord; they usually, but not always, anastomose by a short transverse vessel, which lies about half an inch above the surface of the placenta; it could not be shown

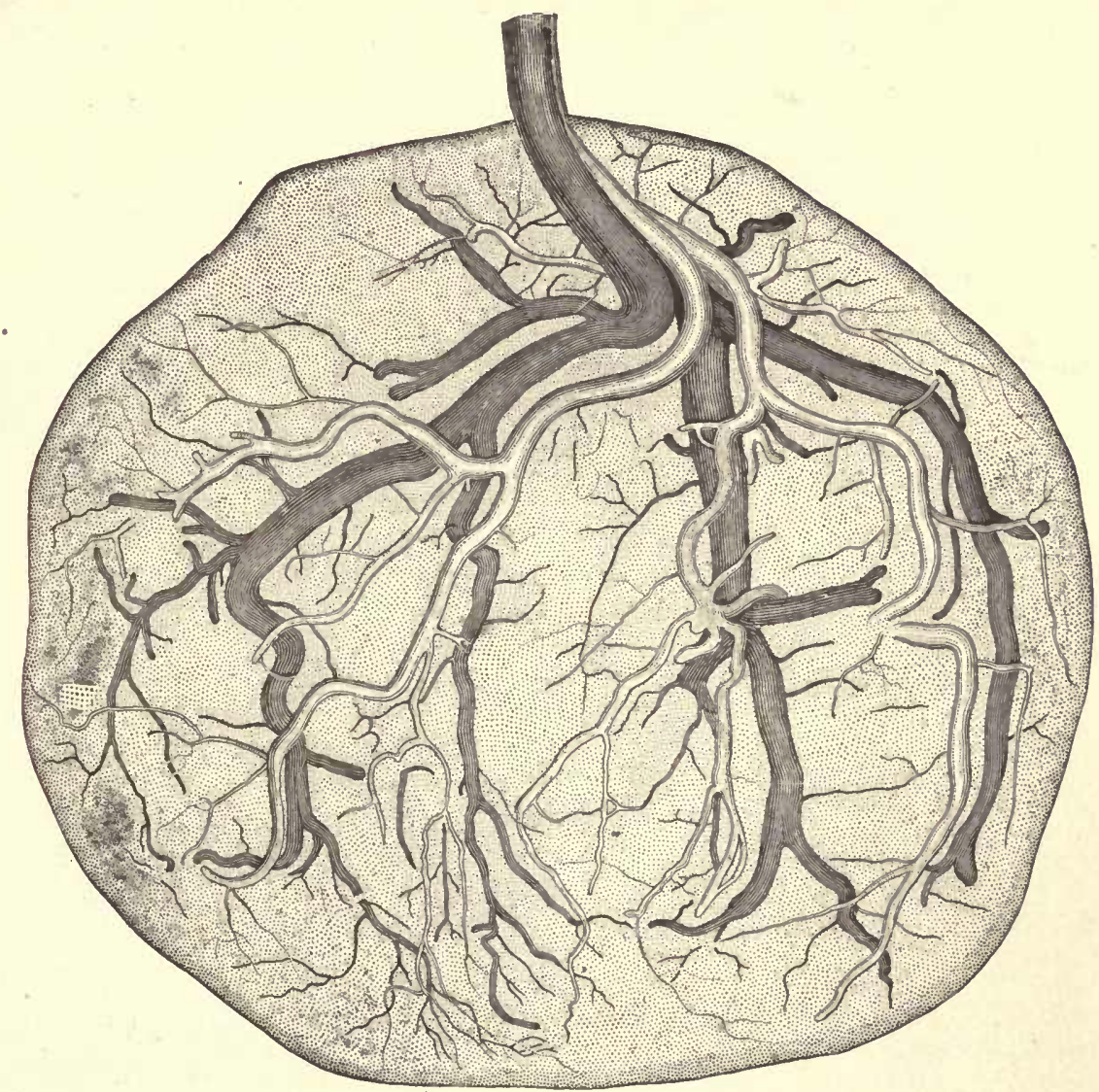

Fig. 240.-Human Placenta at Full Term, Doubly Injected to Show the Superficial Distribution of THE BLOOD-VESSELS.

The veins are drawn dark and lie deeper than the arteries. One half natural size.

in the figure. Very rarely, if ever, are there any arterial or venous anastomoses on the surface of the placenta. The arteries there spread out in a manner which may be described as roughly symmetrical. The veins partially follow the course of the arteries. When the cord is inserted near the margin the symmetry of the placental vessels is greater, when the insertion is near the center the symmetry is less, than in the figure. 
The reverse or uterine surface of the placenta is rough and divided into numerous rounded, oval, or angular portions termed lobes or cotyledons, as stated above. These vary from half an inch to an inch and a half in diameter. The whole of this surface consists of a thin, soft, somewhat leathery investment by the decidual membrane, which dips down in various parts to form the grooves that separate the cotyledons from each other. This layer is a portion of the decidua serotina, which, as long as the parts are in situ, constitutes the boundary between

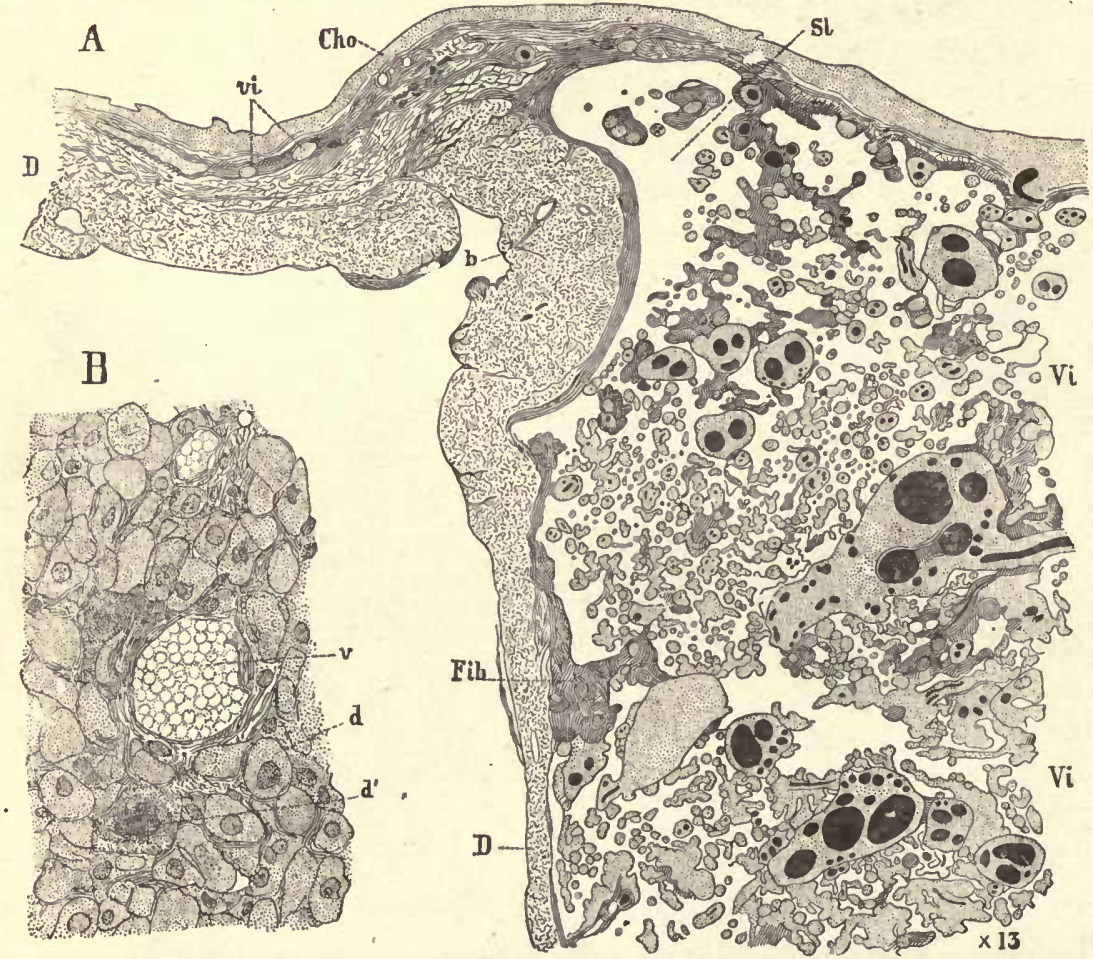

Fig. 241.-Human Placenta after Delivery at Full Term.

A, Vertical section through the margin: $D$, decidua; vi, aborted villi outside the placenta; Cho, chorion; $S i$, sinus; $V i$, placental villi; Fib, fibrin. B, Portion of A more highly magnified to show the decidual tissue near $b$ : $v$, blood-vessel; $d$, decidual cell with one nucleus; $d^{\prime}$, decidual cell with several nuclei.

the placenta and the muscular substance of the uterus, but which at the time of labor becomes split asunder, so that, while a portion is carried off along with the placenta and constitutes its external membrane, the rest remains attached to the inner surface of the uterus. If a placenta is cut through, it is found to consist of a spongy mass containing a large quantity of blood and bounded by two membranes, each less than a millimeter thick; the upper one is the chorion, covered by the still thinner amnion, and greatly thickened where the vessels lie in it; the lower one is the decidual tissue, together with the ends of the villi imbedded in 
it (cf. especially page 357 and Fig. 238 ); it represents only a portion of the decidua, the other portion having remained upon the uterine wall. The spongy mass is found upon examination to consist of an immense number of tufts of fine rods of tissue, which are irregularly cylindrical in shape. Further examination shows that they are twigs (Fig. 248) with rounded ends and springing from branchlets which in their turn árise from branches, and so on until a large main stem is found, which starts from the chorion. This branching system is richly supplied with blood from the fetal vessels on the surface of the placenta. The villi are interwoven so that the twigs of one branch are interlaced with those of another, and apparently separate twigs may grow together and their vessels anastomose; but on this point we are unable to speak positively. The villous twigs next the surface of the decidua penetrate that tissue a slight distance.

The intervillous spaces are filled, or nearly so, with blood; they form a complex system of channels. The intervillous blood is maternal. Farre says, in his article in Todd's "Cyclopædia" (Vol. V, page 7 16 ), in reference to the placental decidua: "Numerous valve-like apertures are observed upon all parts of the surface. They are the orifices of the veins which have been torn off from the uterus. A probe passed into any one of these, after taking an oblique direction, enters at once into the placental substance. Small arteries, about half an inch in length, are also everywhere observed embedded in this layer. After making several sharp spiral turns, they likewise suddenly open into the placenta"; and on page 7 I9 he adds: "These venous orifices occupy three situations. The first and most numerous are scattered over the inner side of the general layer of decidua which constitutes the upper boundary of the placenta; the second form openings upon the sides of the decidual prolongations or dissepiments, which separate the lobes [cotyledons] from each other; while the third lead directly into the interrupted channel in the margin, termed the circular sinus." The circular sinus (Fig. 24I, Si) is merely a space at the edge of the placenta which is left comparatively free from the villi. It is not a continuous channel, but is interrupted here and there. Subsequent writers have gone but little beyond Farre's account, which has been entirely overlooked by most recent investigators, who, accordingly, have announced as new discoveries many facts known to Farre. Under these circumstances it is -interesting to direct renewed attention to Farre's masterly article.

\section{Histology of the Human Chorion.}

The chorion may be preserved in Zenker's or Tellyesnicky's fluid or in Bouin's picro-formalin fluid. Pieces may be stained in toto with alum cochineal or borax carmine and transverse sections cut in paraffin. The sections may be advantageously counterstained with eosin or orange G.

For the general history of the chorion see page 82 . As it is formed by the somatopleure, it comprises an outer ectoderm and an inner mesoderm, which latter comprises mesenchyma and mesothelium. 
The ectoderm undergoes a very precocious growth producing a very large number of cells, which form the thick trophodermic layer as described on page 365 . Then follows the stage in which, by degeneration, spaces are produced in the trophoderm into which the blood of the mother enters and circulates; and at the same time prolongations of the chorionic mesoderm extend into the trophoderm. The ectodermal cells arrange themselves as a covering for these mesodermic outgrowths and so complete a villus. The trophoderm between the developing villi entirely disappears. The ectoderm, which covers both the villi and the chorionic membrane proper, consists of two layers; an inner cellular and an outer syncytial layer. Much of the trophoderm may still remain for awhile around and beyond the tips of the villi, but it disappears rapidly, probably during the third week, so that the villi alone are left. The two-layered stage of the ectoderm is only partially preserved during the later development. Many parts of it become thinned out so as to contain only one layer of cells, while other parts thicken and degenerate. These changes may be studied in sections of older placentas (see Fig. 234).

The mesoderm of the chorion consists at first of mesenchymal cells with a homogeneous matrix and a layer of mesothelium. In later stages the mesenchymal tissue becomes partly fibrillar, and it is doubtful whether the mesothelium persists or not. During the third week we find the chorion vascular. .. Around the larger blood-vessels the mesoderm forms a more or less distinct coat in which the cells are somewhat more crowded together in laminæ. After the perivascular coats have acquired a certain thickness the cells of their inner portions become more elongated, more regularly spindle-shaped, and more closely packed than those of the outer layer. The transition from the denser to the looser tissue is gradual. We are perhaps entitled to call the denser, inner layer the media, and the outer, looser layer the adventitia, although neither of the layers has by any means the full histological differentiation characteristic of the like-named layers of the blood-vessels of the adult. The histogenetic changes in the chorion frondosum go further than in the chorion læve, which may be said to be, as it were, arrested in its development.

\section{The Chorion with Trophoderm.}

When the chorionic vesicle has an internal diameter of from 3 to 6 or $7 \mathrm{~mm}$., it will be found to exhibit well-developed trophodermic layers. Such a vesicle may be hardened in Zenker's fluid or, better, in Flemming's or Hermann's fluid, as these produce at the same time a differential color (Fig. 242). The chorionic membrane is quite thin, and consists chiefly of mesoderm, mes, with a covering of ectoderm, Ec, consisting of two layers of cells. The mesoderm extends down to form the core of the villi shown. These villi are much branched and are also covered by a layer of ectoderm. At the denser ends of the villi the ectoderm is very much thickened, forming a great mass of cells, so that the ectoderm con- 
nected with one villus is fused with that of adjacent villi, the whole constituting a large irregular mass of cells, Tro, the trophoderm. In many places it has already disappeared, so that there are spaces, lac, in the trophoblastic mass. On the edges of these spaces the trophoblast is undergoing degeneration, deg, and where that is occurring it is marked in the figure by the deeper staining of the degenerated material. Upon examination with a higher power (Fig. 243) it will be noted that the mesodermic cells are stained much more deeply than the matrix.

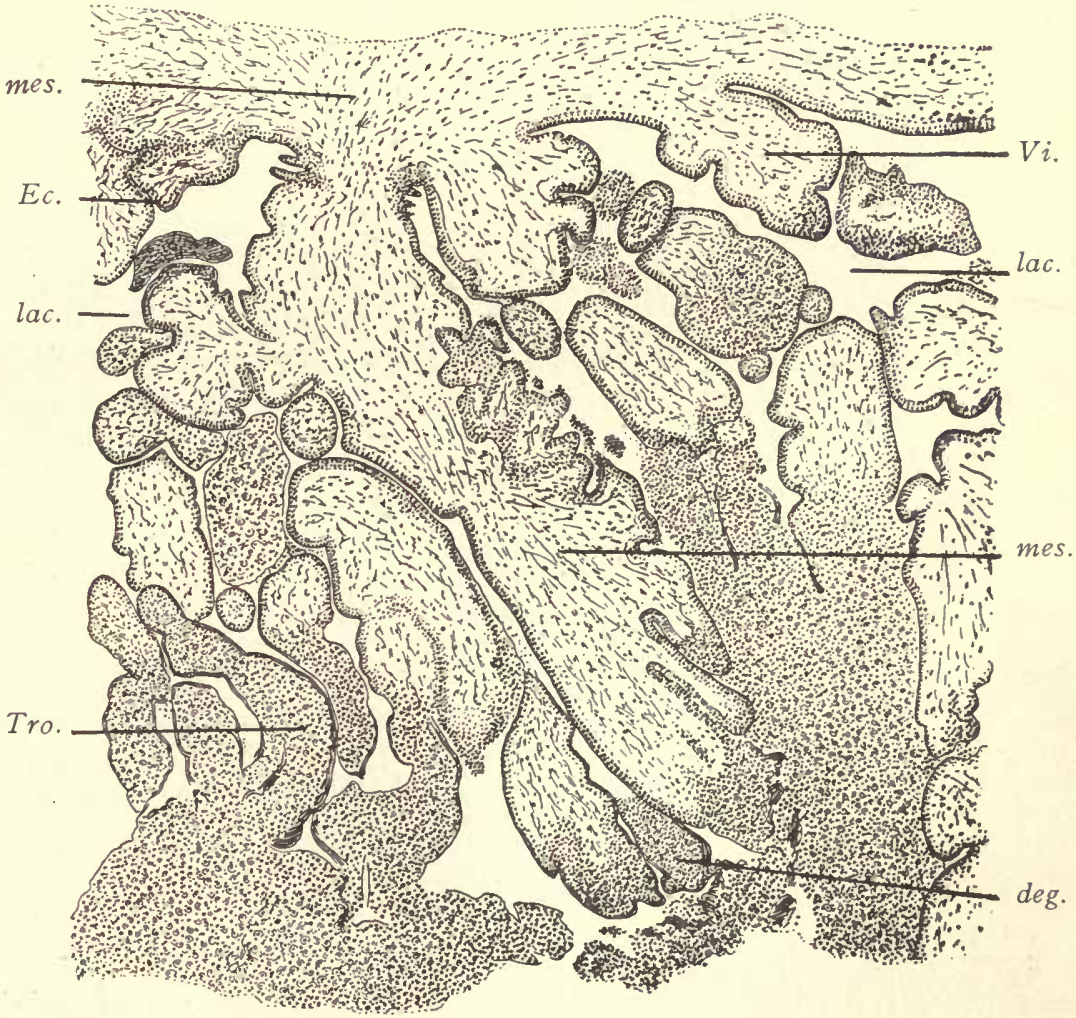

Fig. 242.- Section of a Very Young Human Chorion.

deg, Degenerating ectoderm. Ec, Epithelial ectoderm. lac, Lacuna for maternal blood. mes, Mesoderm. Tro, Trophoderm. Vi, Villi. $\times 50$ diams.

They have an elongated form and run in various directions, more or less parallel to the epithelium, $E c^{\prime}$. Many of them are cut transversely or obliquely. Aside from the trophoderm, the ectoderm is everywhere two-layered. The inner layer is distinctly cellular, the outlines of the cells being very sharply marked and the nuclei being relatively large. In the outer layer, which is stained more darkly, there are no cell boundaries to be recognized, the structure being syncytial. The nuclei are smaller and more deeply stained than those of the inner layer. In 
the trophoderm we find great masses of cells somewhat similar to those of the cellular layer upon the chorionic membrane and over the surface of the villi, but they are larger and more lightly stained. They lie closely packed together; their nuclei are rounded in form, but vary considerably in size and shape. Many of them contain one or two distinct spots, which, however, are sometimes absent. On the edges of the spaces which have been formed, and sometimes apparently in the interior of the mass of trophoderm, we find bands and lines of degenerative

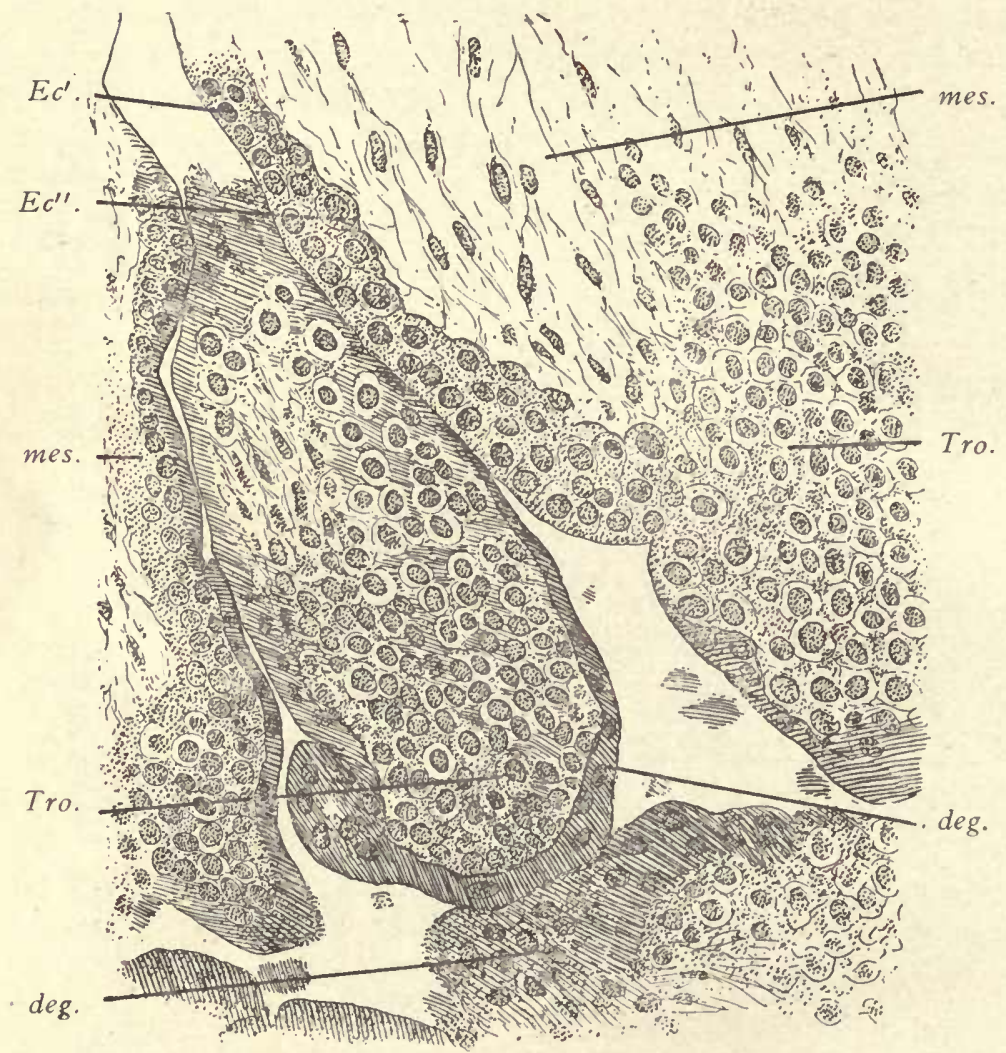

Fig. 243.-Portion of the Preceding Figure More Highly Magnified.

$d e g$, Degenerating ectoderm. $E c^{\prime}$, Outer syncytial layer of ectoderm. $E c^{\prime \prime}$, Inner cellular layer of ectoderm. mes, Mesoderm of villus. Tro, Trophoblast. $\times 350$ diams.

material in which we can find nuclei, but no distinct cell boundaries. The substance between the nuclei is more or less uniformly granular in texture and stains quite deeply. The nuclei of the degenerative material vary extremely in appearance. In some cases they are small and stain rather deeply, and are then found to be present in more or less considerable numbers. Occasionally, however, the nuclei are much larger, and more rarely one sees a nucleus of exceptionally great diameter. 
Our knowledge of the human trophoderm being still very imperfect, its full history is partly a matter of supposition. The appearances described indicate that the trophoderm undergoes a rapid degeneration, during which the cells fuse, while their protoplasm becomes a hyaline material. - We must then further suppose that the degenerated substance is resorbed and disappears altogether. Finally, we must assume that the entire trophoderm does not disappear, but that enough is preserved to form the permanent covering of the villi.

It may be noted that the specimen on which the above description is based agrees essentially with the specimen described by Siegenbeek van Heukelom, which is regarded as normal.

\section{The Chorionic Villi.}

The villi may be obtained in connettion with the preparations of the uterus and placenta. In order to see the youngest stages of the first villi it is necessary to have the chorionic membrane of the second or early part of the third week. At this stage the trophoderm is present and the first villi are appearing (compare page II5). To study the growth and form of the villi, single villi or pieces of villi should be snipped off from the chorion at various stages. Such pieces may. be examined as opaque. objects in alcohol, or they may be stained and mounted as permanent preparations. To obtain injected villi it is best to inject the placenta through one of the arteries of the umbilical cord, using as the injecting mass gelatin colored with carmine or Prussian blue. Such injections are very easily made.

Branching of the Villi.- The formation of a branch is usually initiated by an outgrowth of the ectoderm. Branches grow very rapidly; the outgrowth which forms the branch occurs with every degree of participation of the mesoderm. The two extremes are, first, the bud consisting wholly of epithelium, which may become a process with a long, thin pedicle and a thickened free end remaining sometimes entirely without mesoderm; later the mesoderm penetrates it and completes the structure. Second, a thick bud with a well-developed cord of connective tissue and having a nearly cylindrical form. Between these extremes every intermediate stage can be found. The tips of the branches are for the most part free, but some of them come in contact with the surfaces of the decidua and penetrate it for a short distance. By this means the villi of the embryo are attached to the decidua of the mother. The villi do not penetrate the glands of the uterus at any period, as was at one time supposed. The ectoderm on the tip of the villi, where it is in contact with decidual tissue, undergoes a hyaline degeneration.

The shape of the villi varies according to the part of the chorion and the age of the embryo. Over the chorion læve there is first an arrest of development and a subsequent slow degeneration of the tissues which lose all recognizable organization of the protoplasm, and to a large extent of their nuclei also. At the same 
time the villi alter in shape (Fig. 244), becoming more and more filamentous. By the fourth month only a few tapering threads with very few branches remain. The villi disappear almost completely from the chorion læve, except near the edge of the placenta. The villi of the chorion frondosum or placental region, on the contrary, make an enormous growth. At first they are short, thick-set bodies of irregular shape, as shown in figure 245. At twelve weeks their form is extremely characteristic (Fig. 246). The main stem gives off numerous branches at more or less acute angles; and these again other branches, until at last the terminal twigs are reached. The branches are extremely irregular and variable, though in general club-shaped and constricted at the base. The branches may be bigger than the trunk which bears them, or of any less size. In older stages

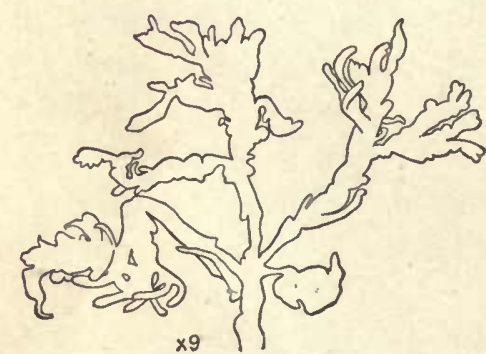

Fig. 244.-Aborting Villus from the Human Chorton Lete of the Second Month.

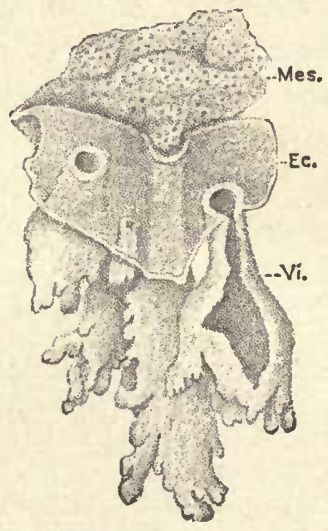

Fig. 245.-Fragment of the Chorion of Figure 84, Highly Magnified.

Ec, Ectoderm. Mes, Mesoderm. Vi, Villus formed wholly by ectoderm.

there is a progressive change. During the fifth month we find the irregularity of shape, though still very marked, decidedly less exaggerated (Fig. 247). The branches tend to come off at more nearly right angles. One finds very numerous free ends, as of course- only a small portion of the branches touch the decidual surface. The branches, too, are less out of proportion to the stems, less constricted at their bases, less awkward in form. The gradual changes continue until at full term, as shown by figure 248 , the branches are long, slender, and less closely set as well as less subdivided than at early stages. They have nodular projections like branches arrested at the beginning of their development. There are numerous spots upon the surfaces of the villi. Microscopic examination shows that these spots are proliferation islands, as we may call them, or little thickenings of the ectoderm with crowded nuclei. Not all the villi, however, have changed to the slender form, for some still preserve the earlier, clumsier shapes. In sections 


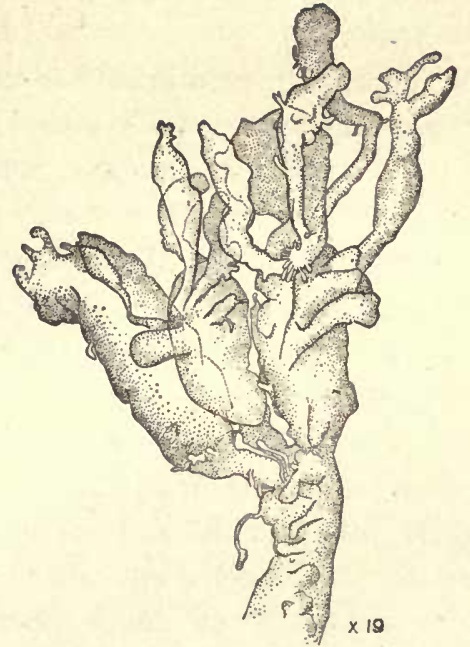

Fig. 246.-Isolated Terminal Branch of a . Villus from a Human Chorion of Twelve WEEKS.

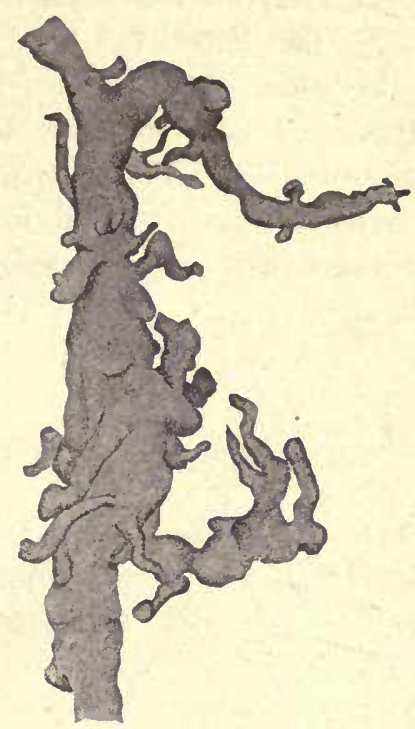

Fig. 247.-Villous Stem from a Human Placenta of the Fifth Month. $\times 9$ diams.

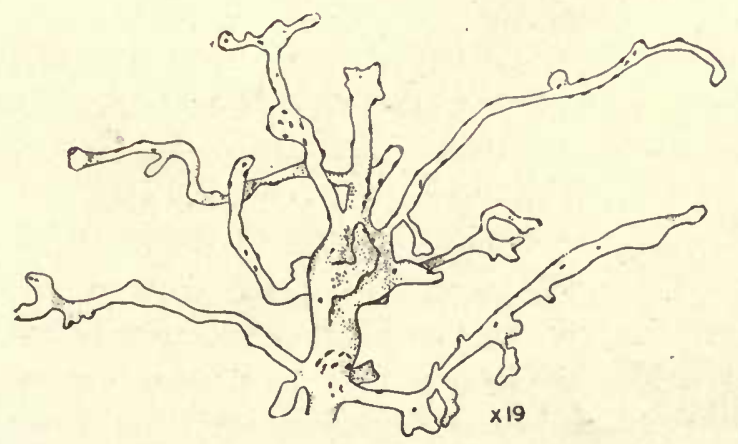

Fig. 248.-Terminal Branches of a Vil.LUs from a Human Placenta at Full Term.

The little spots indicate proliferation islands of the covering epithelium.

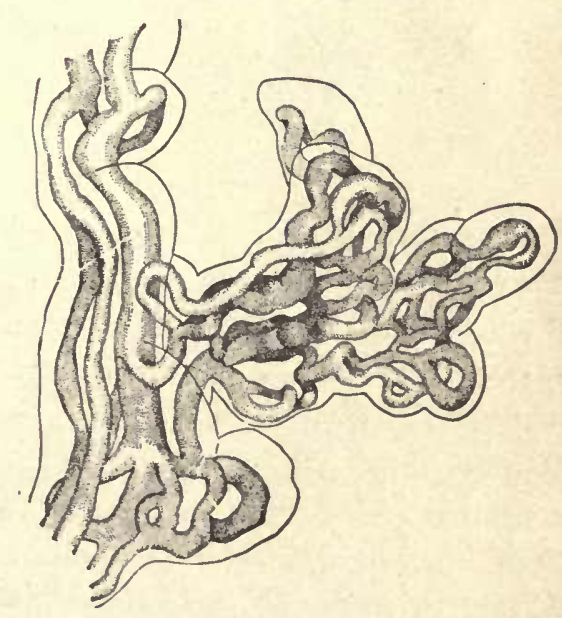

FIG. 249.-PORTION OF AN INJECTED VILIUS from a Placenta of about Five Months. $\times 2$ Io diams. 
of placentas of different ages the villi offer characteristic differences; for the younger the stage, the fewer the total number of branches and the larger their average size. The older the placenta, the more numerous and smaller are the branches as they appear in sections (Fig. 234).

Injected Villi.-The arteries and veins of the chorionic membrane enter the villi. After a short course in the main stalk of a villus, the vessels give rise to many branchlets, and gradually the character of the circulation changes, until in

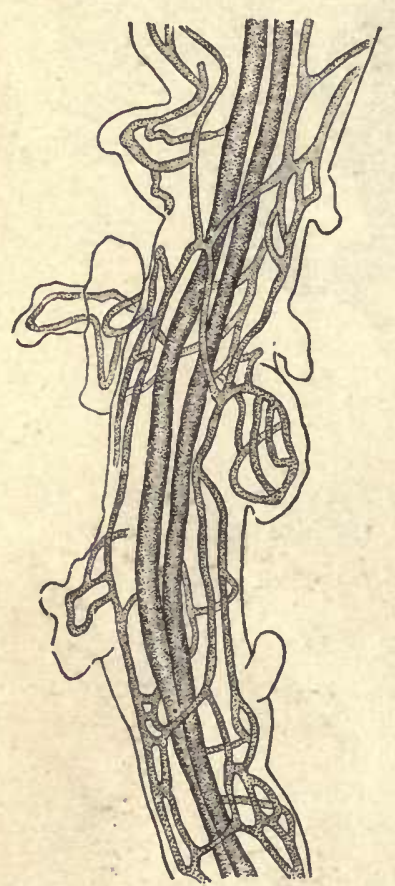

FIG. 250.-PORTION, OF A Small injected Villous Stem from a Placenta of ABOUT Five Months. $X$ ro5 diams. the smallest villous twigs there are capillaries only (Fig. 249). The capillaries are remarkable for their large size, and on this account have been interpreted as arteries and veins by some of the older writers. Their caliber is often sufficient for the passage of from two to six blood-corpuscles abreast. They are very variable in diameter, and also peculiar. in exhibiting sudden constrictions and dilatations. In the short knob-like branches there is often only a single capillary loop, but as the branch becomes larger the number of loops increases and they form anastomoses. In the branches large enough to admit of it, there are two (or sometimes only one) longitudinal central vessels, the artery and vein of a superficial network of capillaries (Fig. 250). The formation of loops and the large size of the capillaries are not especially characteristic of the villi, but of the fetal bloodvessels in general.

The histology of the villi is described in the section on the placenta in situ, page 356 .

\section{The Structure of the Amnion.}

The structure of the amnion may be studied in sections, such as will be obtained by the student in connection with the sections of the chicken and pig embryos. These preparations will show the early stages. When the amnion is first formed, it consists of two layers of cells, both very thin, and with somewhat widely separated nuclei in each layer. Sometimes the nuclei lie in small groups. Between the two layers is a distinct space. The layer facing the embryo is a continuation of the embryonic ectoderm, and is more regular and better defined than the second or mesodermal layer, which is less regular and sends at intervals protoplasmic processes across the space between the two layers to attach themselves to the ectoderm.

Human Amnion at Two Months.-A section is shown in figure 25I. The ectoderm, $E c$, is still very thin, but where the nuclei are placed the layer is a little thicker. The. mesoderm, on the other hand, has become quite thick, and is 
readily seen to be separated into two parts, a thin mesothelial layer, $M$ sth, covering the surface of the amnion toward the chorion, and a mesenchymal layer, Mes, which makes up the greater part of the membrane. Traces of fibrillar structure in the mesenchyma are observable. No blood-vessels, lymphatics, or nerves have been found.

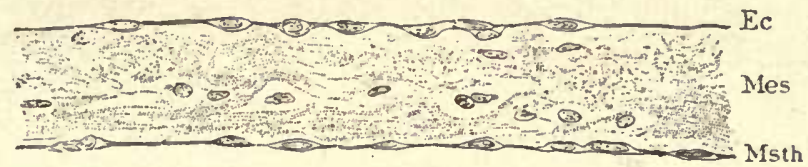

Fig. 251.-Transverse Section of a Human Amnion of Two Months.

Ec, Ectoderm. Mes, Mesenchymal mesoderm. Msth, Mesothelium. $\times 250$ diams.

Human Amnion after the Fifth Month. - This should be studied both in sections and in surface views of the whole membrane, small pieces being mounted with the ectodermal side up. The preparation may be stained with alum hematoxylin and eosin. Sections show that the ectoderm (Fig. 252, ect) has grown somewhat
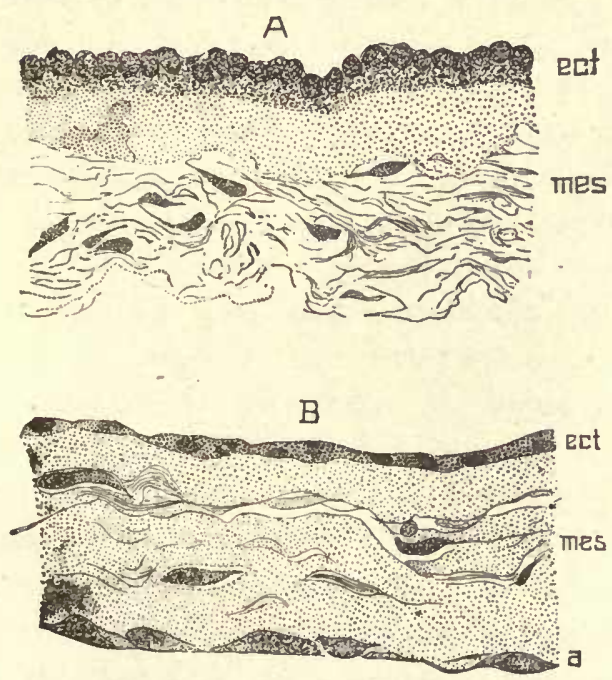

Fig. 252.-Two Sections of the Human Amnion.

A, From an embryo of eight months; B, at term. ect, Ectoderm. mes, Mesoderm. a, Mesothelium. $\times 340$ diams.

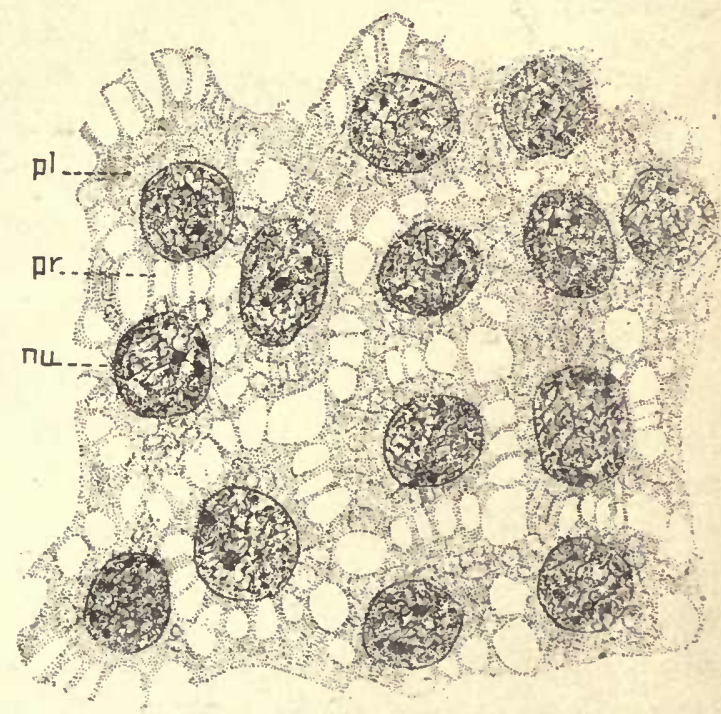

Fig. 253:- Surface View of the Human Amniotic Epithelium of the Fourth MoNTH.

$p l$, Protoplasm. pr, Intercellular processes. $m u$, Nucleus. $\times 1225$ diams.

in thickness. Usually the cells are cuboidal (Fig. 252, A), each with a rounded top in which is situated the more or less nearly spherical nucleus. Sometimes, however, the nuclei lie deeper down. Less frequently the epithelium is thin (Fig. 252, B), and its nuclei, which are transversely elongated, lie farther apart. As regards the mesoderm, it will be noticed that there is usually, perhaps always, 
a layer of nearly homogeneous basal substance or matrix immediately underneath the ectoderm and remarkable for containing no cells. Sometimes the remaining portion of the mesoderm is broken up so as to offer a fibrillar structure (Fig. 252, A), and when that is the case we can no longer make out a distinct mesothelial layer. At other times the more or less homogeneous matrix can be seen through the whole thickness of the amnion (Fig. 252, B), and when this is the case the mesothelium, $a$, can be readily identified.

In surface views the amniotic ectoderm is seen to consist of more or less regularly distributed nuclei with cell-bodies connecting with one another by intercellular bridges of protoplasm (Fig. 253). The nuclei, $m u$, are relatively large,

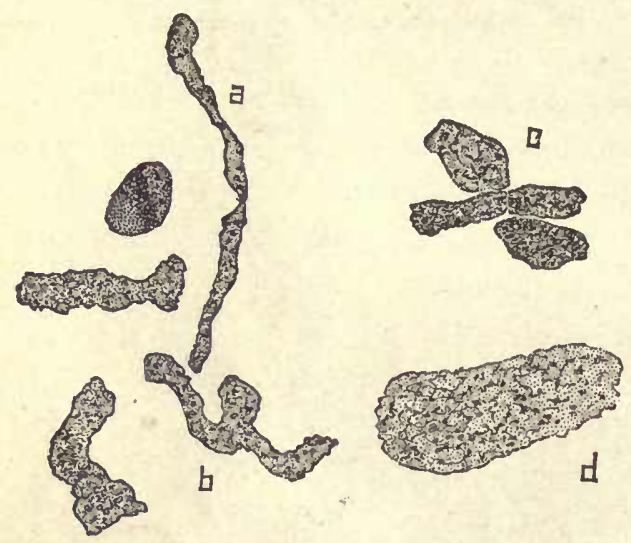

Fig. 254.-Natural Group of Nuclei from the Mesoderm of the Human Amnion of the Fifth Month. (For lettering see text.) $\times 1225$ diams. rounded, and with distinct outlines. They have a more or less well-marked intranuclear network with thickened nodes and a small number of deeply stained granules which are probably chromatin. Each nucleus is surrounded by a cell-body, $p l$, and the adjacent cell-bodies are separated from one another by clear spaces which are crossed by threads of material, pr, stretching as bridges between the neighboring cells. The protoplasm is vacuolated. The whole picture thus leads to the view that the epithelium is a sponge-work of protoplasm somewhat condensed around each nucleus. As regards the mesoderm, it is very difficult to obtain clear pictures of the cells, though the nuclei can be readily observed. They vary greatly in appearance, being sometimes fairly regular and uniform, though always far less so than the nuclei of the mesenchyma of the embryo proper. In other cases (Fig. 254) the nuclei are exceedingly irregular; some are large with a distinct network, $d$; others are smaller and differ but slightly from the normal. Some are very irregular, $b$, others slightly irregular, $c$, and others again strangely elongated and narrow, $a$. Many other forms besides those represented in figure 254 may be found. It has been suggested that these varied shapes of the nuclei indicate degenerative changes, and, in fact, many of the nuclei are actually breaking down, for in some specimens every stage between a.nucleus and scattered granules can be observed, and one may find nuclei with distinct membranes, without membranes, masses of granular matter stained, clusters of granules crowded together, and, finally, other clusters more or less scattered.

\section{The Umbilical Cord.}

The umbilical cord may be well preserved in Zenker's or Tellyesnicky's fluid. Transverse sections may be prepared in paraffin and stained with alum hematoxylin 
and eosin, or with Heidenhain's iron hematoxylin and orange G; or, if it is desired to study the development of the connective-tissue fibrillæ, with Mallory's triple connective-tissue stain.

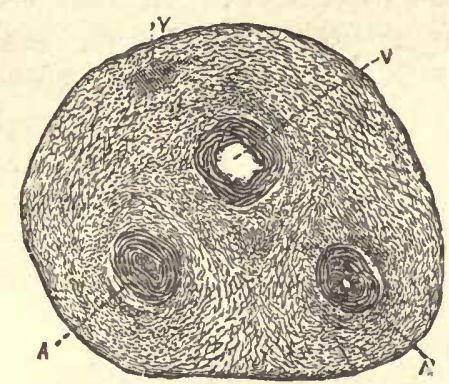

Fig. 255.-Cross-Section of a Human Umbilical CORD AT TERM.

$A, A^{\prime}$, Umbilical arteries much contracted. $V, \mathrm{Um}-$ bilical vein. $Y$, Remnant of allantois. $X \mathrm{I} 2$ diams.

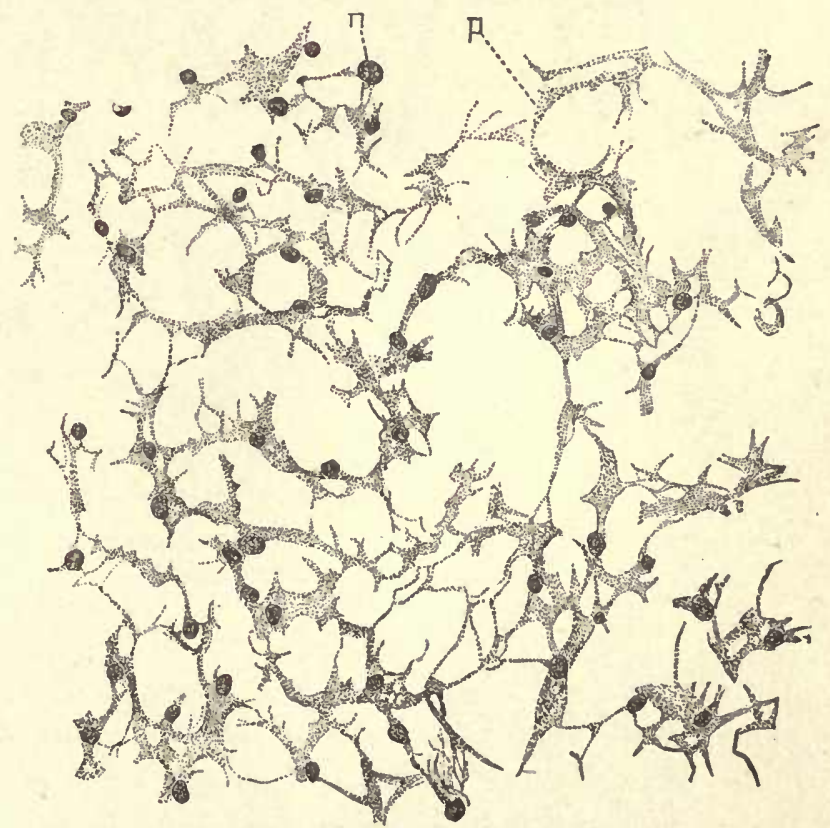

Fig. 257.-Connective Tissue from the Umbilical Cord of a Human Embryo of 2I mM. Stained with Alum Cochineal and Eosin.

$n$, Nucleus. $p$, Protoplasmic network. $\times 540$ diams.

A general description of the umbilical cord has been given, pages II 5 to II6, and two sections (Fig. 66) are there represented showing the structures which appear in sections of the umbilical cord. At full term some of these structures 
are still present but somewhat modified (Fig. 255), while others have been partly or wholly obliterated. As contrasted with the early stages, we find that the cœlom is entirely obliterated, that the yolk-stalk has usually been completely resorbed, and that only traces of the allantois can be seen, $Y$. The blood-vessels have grown; there are two arteries, $A, A^{\prime}$, and a single vein, $V$. Around each of these is a well-developed muscular coat produced by differentiation of the surrounding mesenchymal cells, which have assumed an elongated form and con-

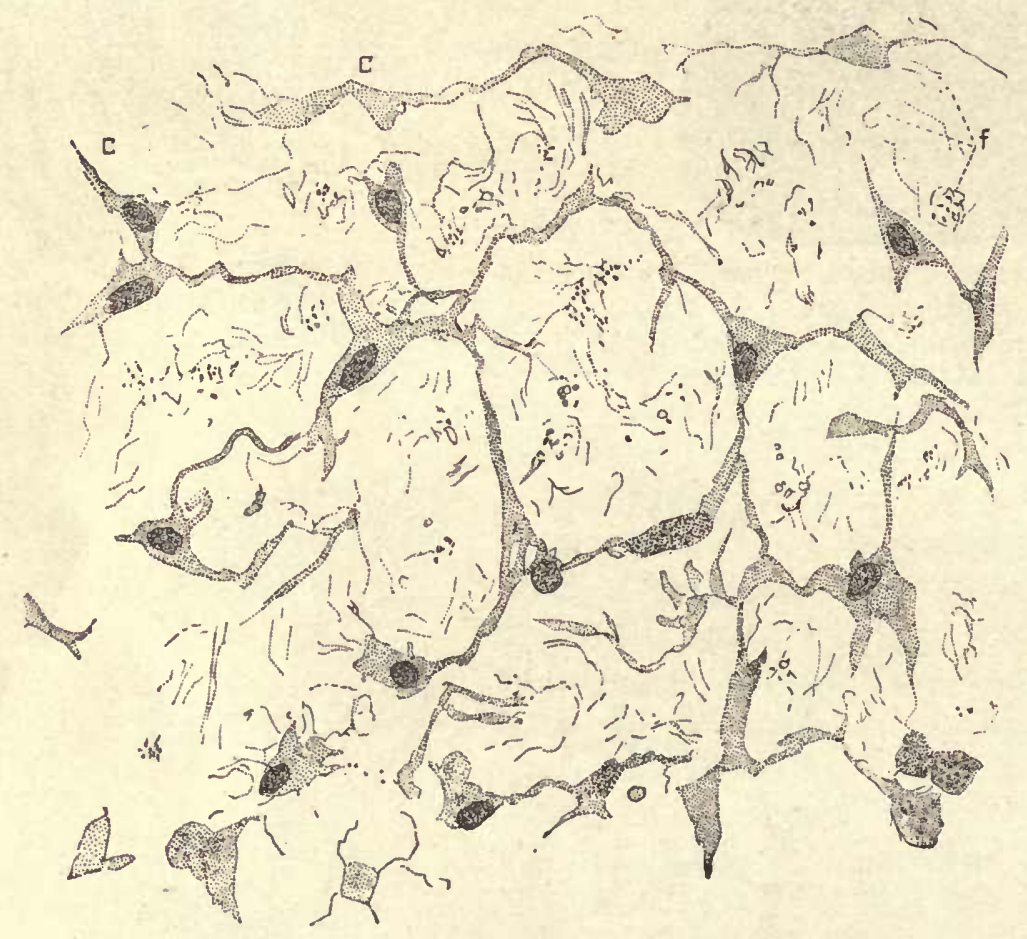

Fig. 258.-Connective Tissue from the Umbilical Cord of a Human Embryo of Three Months, Stained with Alum Cochineal and Eosin. $\times$ 5 I I diams. $c, c$, Cells. $f$, Fibrillæ.

tractile function. It will be remembered that the allantois in man is primitively a very narrow tubular diverticulum which extends originally nearly to the chorion (compare Fig. 87). As the umbilical cord lengthens the allantois fails to lengthen equally. During the second month it increases very little in diameter. After the second month it appears in sections as a small group of epithelioid cells (Fig. 256) with distinct walls, irregularly granular contents, and round nuclei; the group may or may not show a remnant of the original central cavity. Around the cells, ent, there is a slight condensation of the connective tissue, mes, to form, as it were, an envelope.

The mesoderm varies in appearance according to the age of the specimen. 
Its growth and differentiation are rapid. During the second month it consists merely of numerous cells (Fig. 257) imbedded in a clear substance. The cells form a complex network of which the filaments and meshes are extremely variable in size. The nuclei are oval, granular, and do not always have accumulations of protoplasm about them forming main cell-bodies. (Compare description of first stage of the mesenchyma, page 89.) By the end of the third month the cells have assumed nearly their definite form (Fig. 258). Their protoplasm is increased in amount and forms a large body around each nucleus. The network has become simpler and coarsèr, the meshes bigger, and the filaments fewer and thicker. In the matrix are numerous connective-tissue fibrillæ, not yet disposed in bundles.

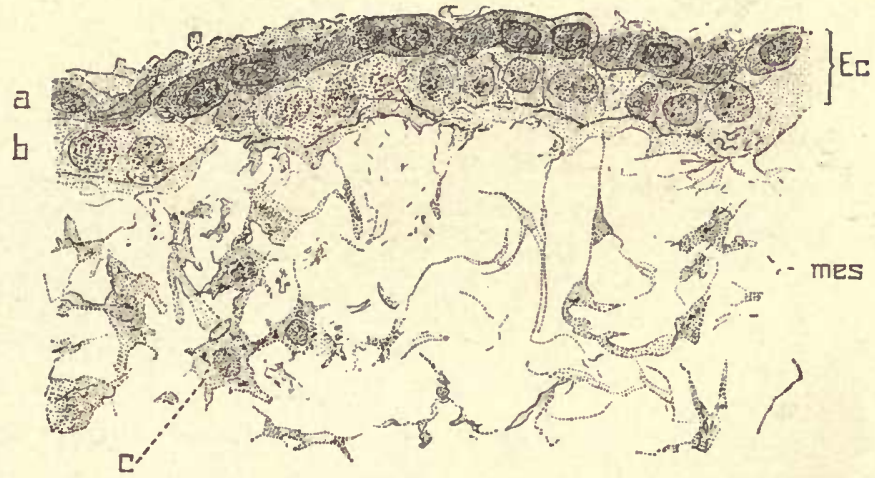

Fig. 259.-Ectoderis of an Umbilical Cord of a Human Embryo of Three Months.

$E c$, Ectoderm. mes, Mesoderm. $c$, Mesenchymal cell. $a$, Outer layer of ectoderm. $b$, Inner layer of ectoderm. $\times 545$ diams.

In older cords there is an obvious increase in the number of fibrillæ and they form wavy bundles. In the cord of yet older stages the matrix also contains mucin which may be stained by alum hematoxylin. In such cords so stained the blotch of color appears in the intercellular spaces.

The ectoderm is at first a single layer of cells, as it is also over the body of the embryo, and as it remains permanently over the amnion. At three months we find the ectoderm to be two-layered, corresponding to the second stage of the epidermis of the embryo. In still older stages there is slight increase in the number of layers of the ectoderm, but it never passes much beyond the stage of the embryonic epidermis at the fourth month. Figure 259 is from a cord at three months. The outer layer, $a$, of ectodermal cells is granular and stains much more darkly than the inner layer, $b$, in which also cell bundles are more distinct.

\section{The Structure of the Human Yolk-sac.}

The human yolk-sac may be preserved in Zenker's or-Tellyesnicky's fluid, stained in toto with alum cochineal, imbedded in paraffin, and cut in transverse sections. Yolk-sacs of the second month are preferable for study. 
The general history of the yolk-sac is described on pages $6_{3}$ and 66 . It becomes a pear-shaped vesicle which in man attains its maximum diameter about the end of the fourth week. It then measures from 7 to II $\mathrm{mm}^{\text {. From its }}$ pointed end runs the long stalk by which it is connected with the intestine. In very early stages the stalk is hollow and its cavity is lined by entoderm. But this condition is soon obliterated, the stalk becoming solid and the entoderm disappearing. In this condition we found the yolk-stalk in an embryo of $2 \mathrm{I} \mathrm{mm}$. (Fig. 66, A). The sac itself remains hollow (Fig.

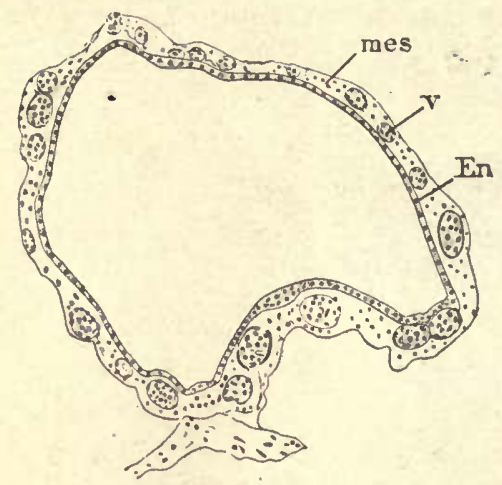

Fig. 260.-Section of THE YolK-SAC of a Very Young Human Embryo.

En, Entoderm. mes, Mesoderm. v, Blood-vessel.-(After Fr. Keibel.) 260). It has a lining of entodermal cells, En, and a thicker layer of mesoderm, mes, containing bloodvessels, $v$. The network of the vessels imparts a characteristic appearance to the external or mesodermic surface of the yolk-sac. In the earliest stages observed the entoderm consisted of a single layer of cuboidal cells.

Transverse Section of a Yolk-sac of about Two Months.-The contents of the fresh yolk-sac are fluid, but coagulate when the organ is hardened. In the coagulum are found some stained bodies which are supposed to be yolk material. The entoderm has undergone proliferation and thickening. These cells are more or less irregular and disposed in two or three layers. Many of the superficial cells are stained deeply and have small nuclei, while the deeper lying cells are larger, more lightly stained, and have larger nuclei and more distinct cell boundaries. The mesoderm consists chiefly of somewhat crowded mesenchymal cells, the nuclei of which are smaller than the entodermal cells, and a well-marked layer of mesothelium, which forms the external covering of the yolk-sac. In the mesoderm appear relatively large blood-vessels, which are usually found filled with blood-corpuscles. The blood-vessels have distinct endothelial walls and lie in the part of the mesoderm toward the. mesothelium, so that they are separated somewhat from the entoderm and seem often to lie immediately underneath the mesothelium. They are so large that each vessel causes a protuberance upon the yolk-sac. 


\section{CHAP'TER VIII.}

\section{METHODS.}

\section{Measuring Length of Embryos.}

Owing to the many changes during development in the curvature of the-longitudinal axis of the mammalian embryo, it is impracticable to measure that axis or to employ any one system of measurements to obtain comparable results for all ages. For this reason the best practice is to measure in all cases the greatest length of the embryo in its natural attitude along a straight line. The limbs are not to be included in such measurements. This greatest length in young stages will not include the head (compare, for example, Fig. 95), but in most stages the head would be included. Many German authors employ the measurement introduced by His, which he calls the Nackenlänge, which corresponds to the distance in a straight line from the neck-bend to the caudal bend. As it is impossible to measure this distance in later stages, it seems best not to use it at all. The length of an embryo, as given by German authors, is often indicated by the abbreviation NL., and is, of course, often different from the measures used in this work.

\section{Dissection of Embryos.}

Vertebrate embryos may be dissected without serious difficulty. Specimens hardened in Zenker's fluid are particularly favorable for this purpose. Dissection should be more extensively practiced than is at present usual in embryological work, since by it all the viscera, the central nervous system and even the main nerve trunks may be demonstrated advantageously.

By the following simple method the embryo may be securely attached to the bottom of the dish in which the dissection is to be made, best in 80 per cent alcohol. Place the specimen in 95 per cent alcohol for half an hour, then in a mixture of equal parts of alcohol and ether for fifteen minutes. Put a few drops of celloidin dissolved in alcohol and ether on the bottom of the dish; put the specimen in place; allow the celloidin to stand for two or three minutes until a film is formed and then cover the specimen with 80 per cent alcohol, which will set the celloidin. After the dissection is finished the specimen may be freed by dissolving the celloidin with a mixture of alcohol and ether in equal parts.

\section{Methods of Hardening and Preserving.}

The three most generally useful methods for preserving embryos are with Zenker's or Tellyesnicky's fluids and to per cent formalin. Good results may be had 
with the other reagents. Specimens preserved with Bouin's fluid have the advantage of staining sharply. To study the medullary sheaths of nerve-fibers, as is necessary to follow the development of the fiber tracts in later stages, the specimens may be preserved in Müller's fluid. Flemming's, Hermann's, and Bouin's fluids are valuable, especially for cytological study, but are applicable only to small pieces.

\section{. I. ZenKer's Fluid.}

Formula: Corrosive sublimate

Potassium bichromate. . . . . . . . . .

Sodium sulphate $\ldots \ldots \ldots \ldots \ldots \ldots \ldots \ldots \ldots \ldots \ldots \ldots \ldots \ldots \ldots \ldots \ldots \ldots$ I $\mathrm{gm}$.

Water ................................. roo c.c.

Add 5 c.c. of glacial acetic acid to the fluid immediately before using.

The fluid does not have great penetrating power, but may be used for embryos of $25 \mathrm{~mm}$. The amount of fluid should be from ten to twenty times the volume of the specimen, and better results are obtained if the fluid is changed after a few hours. Chicks of the first and second days are hardened in from two to four hours; embryos of from 6 to $8 \mathrm{~mm}$. in from eight to ten hours; embryos of $12 \mathrm{~mm}$. in twentyfour hours; larger embryos in from thirty to forty hours. After the proper interval in Zenker's fluid the specimens must be removed and washed in running water for from twelve to twenty-four hours. Transfer to 50 per cent alcohol for from one to three hours, then to 60 per cent, 70 per cent, and 80 per cent. It is indispensable to remove now the excess of corrosive sublimate by adding sufficient tincture of iodine to give the alcohol the color of port wine; if the iodine disappears, it must be renewed. After from one to three days, according to the size of the specimen, transfer it to fresh 80 per cent alcohol, which must be changed until it no longer extracts any iodine from the specimen.

\section{Tellyesnicky's Fluid.}

Formula: Bichromate of potassium

$3 \mathrm{gm}$.

Water ..............................

Immediately before using add 5 c.c. glacial acetic acid.

This reagent is employed in the same manner as Zenker's fluid, except that the treatment with iodine is omitted.

3. Formalin.

Formula: Formalin

Water.

Io c.c.

90 c.c.

Specimens are placed in the fluid, which should be changed in a few hours. On account of its extreme simplicity this method is used especially for human embryos. If the fluid is used in large quantity embryos even of $80 \mathrm{~mm}$. may be well preserved. They may be kept indefinitely in the solution, and transferred to alcohol when needed for sectioning. The method has the disadvantage that the transfer to alcohol, even if made very gradually, always causes a considerable shrinkage, 
for the prevention of which no satisfactory method has been devised. Fortunately, the shrinkage usually produces no distortion.

4. BouIN'S FluID.

Formula: Picric acid, saturated aqueous solution $\ldots \ldots \ldots \ldots \ldots \ldots \ldots \ldots \ldots \ldots$. 225 c.c.

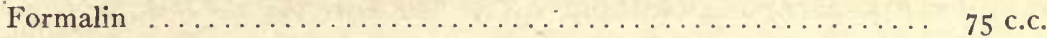

Glacial acetic acid ............................... I5 c.c.

Specimens are kept in the fluid from two to seven days, not longer, according to their size; transfer to 30 per cent alcohol for one hour, to 50 per cent alcohol for from one to two hours, to 60 per cent alcohol for twelve hours, and finally to 70 per cent alcohol, which must be changed daily until it no longer shows even a trace of yellow discoloration by picric acid.

5. Muller's Fluid.

Formula: Bichromate of potassium ........................ $20 \mathrm{gm}$.

Sulphate of sodium $\ldots \ldots \ldots \ldots \ldots \ldots \ldots \ldots \ldots \ldots \ldots \ldots \ldots \ldots \ldots \ldots$ io $\mathrm{gm}$.

Water ..................................... I000 c.c.

Müller's fluid is a valuable reagent, and for the study of the later stages of the nervous system indispensable. The objections to its use are that it requires a long time to act, that it renders the specimens brittle, and makes them somewhat difficult to stain. It must be used in large quantities and be frequently changed, and allowed to act on the specimens from three to eight weeks, according to their size. The appearance of a film or scum indicates that the fluid needs to be changed.

6. Parker's Fluid.

Formula:* 7o per cent alcohol.............................. 100 c.c.

Formaldehyde $\ldots \ldots \ldots \ldots \ldots \ldots \ldots \ldots \ldots \ldots \ldots \ldots \ldots \ldots \ldots \ldots \ldots \ldots \ldots$ I c.c.

Very convenient when a simple and expeditious preservative is necessary. The specimens are placed in the fluid, which ought to be renewed in a few hours. They may be kept permanently in the fluid, but it is desirable, before using them for study, to remove the formaldehyde by treating them with fresh 70 per cent alcohol.

7. Flemming's Fluid.

Formula: I per cent solution of chromic acid................... 5 o c.c.

2 per cent solution of osmic acid...................... $f_{2}$ c.c.

Glacial acetic acid .............................. 3 c.c.

This fluid must be used freshly mixed, and must not be kept in the dark. The specimens must be of small size and as fresh as possible. The amount of fluid used should be not less than ${ }_{5}$ times the volume of the specimen. Specimens are kept in the fluid from twenty-four to forty-eight hours, washed in running water from four to twenty-four hours, and then transferred to alcohols of gradually increasing strength. The fluid is useful chiefly for cytological work. 
8. HermanN's Fluid.

Formula: $\quad$ y per cent platinum chloride in distilled water $\ldots \ldots \ldots \ldots \ldots \ldots \ldots$ c.c.

2 per cent osmic acid in distilled water............... 8 c.c.

Glacial acetic acid...................... 4 c.c.

Used in the same manner and with the same precautions as No. 7 .

\section{Preservation in Alcohol.}

When a specimen is to be preserved with alcohol alone, it should be put first in 30 or 50 per cent alcohol for an hour or more, then into 60 per cent for several hours, 70 per cent for from twelve to twenty-four hours, and finally into 80 per cent, in which it should be kept until required for use. If the specimen is to be sectioned, it must be placed in 95 per cent alcohol, which must be renewed at least once, and be allowed to act for twenty-four hours or more, unless the specimen is very small, when a somewhat shorter time may suffice.

\section{Directions for Imbedding Specimens to be Microtomed.}

A. To Imbed in Paraffin:

I. Stain in toto. (See page 382 .)

2. Dehydrate in alcohol from three to twenty-four hours.

3. Place in, oil of cloves and turpentine (equal parts), one to twenty-four hours.

4. Place in fresh cloves and turpentine for one to twenty-four hours.

5. Place in soft paraffin at $54^{\circ} \mathrm{C}$. for thirty to ninety minutes.

6. Place in hard paraffin at $54^{\circ} \mathrm{C}$. for thirty to ninety minutes.

7. Warm a glass plate to about $70^{\circ} \mathrm{C}$.; place on it a paper tray or metal imbedding frame; fill the box with hard paraffin at $54^{\circ} \mathrm{C}$. Warm a spatula and with it remove the specimen to the tray or frame, and arrange it in a proper position. As soon as the paraffin has set, chill it rapidly with cold water, otherwise the paraffin is likely to crystallize and therefore to cut badly.

B. To Imbed in Celloidin:

I. Dehydrate the mass thoroughly in 95 per cent alcohol, four to twentyfour hours.

2. Place mass for twenty-four hours in alcohol and ether, equal parts.

3. Place mass in thin syrupy solution of celloidin in equal parts of ether and alcohol for at least twenty-four hours. (If the specimen contains cavities several days are necessary to allow the celloidin to penetrate and fill them.)

4. Place mass in thick viscid solution of celloidin in equal parts of ether and alcohol for twenty-four hours.

5. Set mass on block of vulcanite, compressed fiber, or maple-wood, and as soon as a film has formed over the surface of the celloidin (two to five minutes) -

6. Immerse in 80 per cent alcohol for twenty-four hours.

7. Cut. 


\section{Method of Mounting Paraffin Sections.}

The student is advised to. use for serial sections slides $40 \times 76 \mathrm{~mm}$. and from $\mathrm{I} .75$ to $2 \mathrm{~mm}$. thick. The thick slides are much better than the thin ones recommended by dealers. The cover-glasses ought to be 0.17 to $0.18 \mathrm{~mm}$. thick. A generally convenient size is $35 \times 50 \mathrm{~mm}$.

The serial order of the sections should be preserved with the utmost care, and time spent in arranging the sections in straight rows will be found to be time saved.

The albumen-glycerin method of fastening the sections to the slide will be found satisfactory.

Formula: Take the white of one fresh egg, beat slightly until equally fluid, filter it (it will take about twenty-four hours), and add an equal amount of glycerin. To this fluid add a small piece of camphor.

I. Clean the slide thoroughly.

2. Put on a small drop of albumen solution.

3. Spread it out very thin with the finger.

4. Add five or six drops of distilled water, which must flow evenly over the coating of albumen.

5. Place the sections on slides in regular rows.

6. Warm the slides gently over an alcohol flame, to allow the sections to flatten. The paraffin must not melt.

7. Drain off the water.as completely as possible, and arrange the sections in straight rows.

8. Place the slides in oven for twelve to twenty-four hours to evaporate the water completely.

9. Dissolve off the paraffin in turpentine or xylol.

Io. Put in absolute alcohol for three to five minutes.

II. Clear in turpentine or chloroform.

I2. Mount in damar varnish. (Canada balsam is undesirable, because it becomes much discolored with age.)

\section{Methods of Staining.}

In embryological work the specimens are usually stained in toto before imbedding, either with alum cochineal or with borax carmine, the former being the more generally useful stain. Staining on the slide is also much used either to secure a counterstain after the in toto coloration or to secure some special result. For counterstains eosin, Lyons blue, and orange $\mathrm{G}$ are particularly recommended. A few of the most important special stains are given below.

\section{Alum Cochineal.}

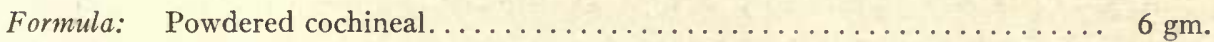

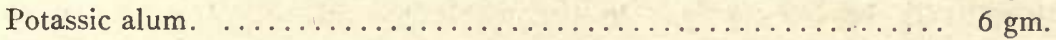

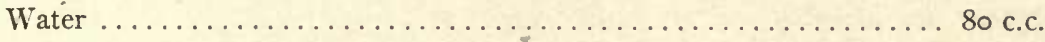


Boil vigorously for twenty minutes; allow the fluid to settle; decant the clear fluid; add more water and boil again; filter the entire solution and evaporate the filtrate to 80 c.c.; add a small piece of thymol or camphor to prevent the growth of fungi. Alum cochineal is, on the whole, the best reagent for in toto staining, as it will penetrate quite large objects and color them uniformly throughout, and gives a good differentiation of the tissues.

For in toto staining place the specimen in water until it sinks; then transfer it to the cochineal for twenty-four hours, or for large specimens longer; the depth of the stain will depend upon the strength of the solution; transfer to clean water for fifteen to twenty minutes to extract the alum, which otherwise will crystallize in the tissues when the specimen is placed in alcohol; the object must not be left too long in water, because it extracts the color alsa; put in 50 per cent alcohol for one hour, then successively in 70 per cent, 80 per cent, and 95 per cent, when the specimen will be ready for imbedding.

\section{Borax Carmine.}

Formula: Best carmine...

Borax $3 \mathrm{gm}$.

Water

$2 \mathrm{gm}$.

$5 \circ$ c.c.

Boil for twenty minutes; allow the solution to cool; add water enough to restore that lost by evaporation, then add 50 c.c. of 70 per cent alcohol, let the solution stand twenty-four hours; filter. Borax carmine gives a good nuclear stain and may be advantageously supplemented by counterstains.

For in toto staining place the specimen in water until it sinks; transfer to the carmine for twenty-four hours, or longer for large specimens; wash in water for five minutes; then place it in 70 per cent alcohol, to every roo c.c. of which 2 c.c. of hydrochloric acid have been added; after one hour transfer to fresh 70 per cent alcohol, which must be renewed in an hour or two, and finally transfer to 80 per cent and 95 per cent alcohol, and the specimen will be ready to imbed.

\section{Alum Hematoxylin.}

Formula: I. Dissolve ro grms. hematoxylin in 60 c.c. absolute alcohol.

2. Dissolve 15 grms. ammonia alum in Ioo c.c. warm water, and allow the solution to cool.

3. Mix the two solutions and allow the stain to "ripen" in a shallow open dish exposed to the light for three days.

4. Filter, then add 25 c.c. pure glycerin and 25 c.c. methyl alcohol.

5. After three days filter.

The solution thus prepared will keep indefinitely. It is one of the best nuclear stains known. Eosin may be used with it as a counterstain giving beautiful preparations.

r. Place sections from water into a filtered diluted solution of the stain for from fifteen minutes to twenty-four hours, according to the strength of the staining solution. (Slow staining gives the best results.) 
2. Wash thoroughly in water until the section is blue.

3. Dehydrate and mount.

4. Counterstains are used either with celloidin sections treated singly, or with paraffin sections after they have been fastened on the slide. The three here recommended are alcoholic solutions, and the method of using is the same for all. If the sections are dried on the slide in an oven for three days, they will adhere to the glass much more securely during the manipulations of counterstaining. Lyons blue is less permanent than eosin and orange G.

For staining paraffin sections on the slide it is convenient to have eight jars or dishes large enough to hold a slide. The slide is transferred from jar to jar in the order below, being allowed to remain in each jar a few minutes. The very most scrupulous care is necessary to keep all the fluids clean, and it is indispensable to filter them frequently; the sections on the slide catch and hold the particles floating in the reagents when they are not clean.

Order of jars: I. Xylol.

2. Xylol.

3. Xylol and absolute alcohol, equal parts.

4. Absolute alcohol.

5. Counterstain.

6. Alcohol of 95 per cent.

7. Absolute alcohol.

8. Xylol.

Eosin Formula: $\quad 2$ per cent in 95 per cent alcohol.

Orange G. Formula: i per cent in 95 per cent alcohol.

Lyons blue Formula: I per cent in 95 per cent alcohol.

\section{Heidenhain's Iron Hematoxylin.}

Formula I: Iron alum $2 \mathrm{gm}$.

Distilled water 100 c.c.

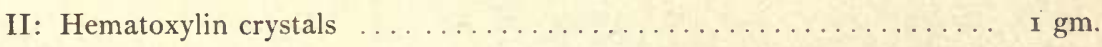

95 per cent alcohol . . . . . . . . . .

Distilled water, to be added after the hematoxylin is dissolved in the

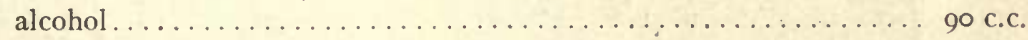

(If the stain does not work, add 0.5 grm. "lithium bicarbonate.)

I. Place sections in the iron solution. for from six to ten hours. (Specimens hardened with Flemming's or Hermann's fluid require longer than specimens from Zenker's or Tellyesnicky's fluid.)

2. Wash quickly in water.

3. Transfer to the hematoxylin solution for from twtlve to eighteen hours.

4. Wash in tap-water.

5. Decolorize in the iron solution.

6. Wash thoroughly in tap-water.

7. Dehydrate and mount. 
This stain is useful for cytological work, the study of cell division, etc. The preparations are often improved by counterstaining with orange $\mathrm{G}$.

\section{Beale's Carmine.}

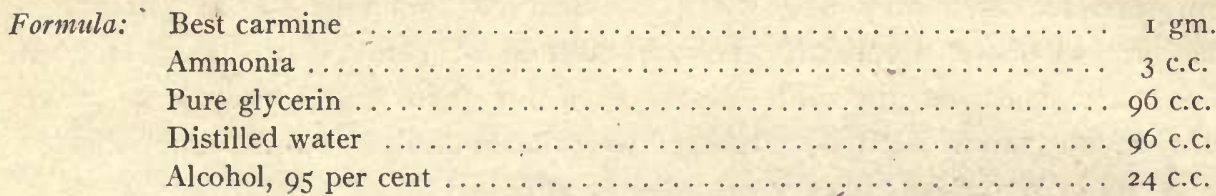

Dissolve in ammonia plus part of the water, add the rest of the water, and allow the solution to stand in an open dish until the ammonia is nearly all driven off. Then add the alcohol and glycerin. For use dilute with an equal part of glycerin. Stain for twenty-four hours in an open dish, which, together with a second open dish containing acetic acid, is placed under a bell-jar; wash the sections thoroughly in water and then in very weak hydrochloric acid (I c.c. to 500 c.c. water), and again in water.

Beale's carmine is especially valuable for the study of the central nervous system and of the placenta.

\section{Weigert's Copper Hematoxylin.}

Formula I: Copper solution:

Acetate of copper in saturated aqueous solution.

II: Hematoxylin solution:

Hematoxylin crystals ...................... 2 gm.

95 per cent alcohol .......................... 20 c.c.

Distilled water ........................... 80 c.c.

(If the stain does not work, add $0.5 \mathrm{grm}$. of lithium bicarbonate.)

III: Iron solution:

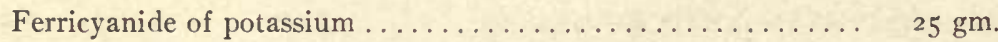

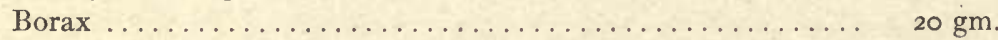

Water ............................... 2000 c.c.

This stain is indispensable for the study of the nervous system after the medullary sheaths have begun to develop; the specimens must be preserved in Müller's fluid. The method is also valuable for the study of the placenta and uterus.

I. Place the sections in water.

2. Place the sections in the copper solution for twenty-four hours.

3. Wash quickly in water.

4. Put them in the hematoxylin solution for five to ten minutes. The sections should turn a deep blue-black.

5. Wash thoroughly in water.

6. Decolorize in the iron solution; the section must be gently moved about to secure an even decolorization. When part of the section shows a brown color, it should be removed and examined. 
7: Wash thoroughly in water to remove the iron solution, no trace of which can be left without ruining the specimen. (Unless the washing is very thorough, the sections will gradually fade out after the final mounting.)

8. Dehydrate with alcohol and mount at once in damar.

8. Mallory's Triple Connective-tissue Stain.

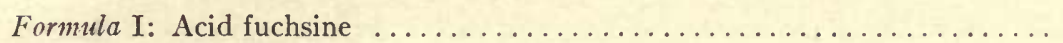

Distilled water

I. O gm.

II: Distilled water

1000.0 c.c.

Phosphomolybdic acid, to be added before other ingredients

100.0 c.c.

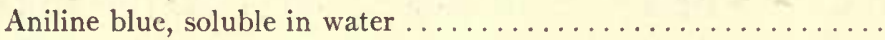

I. $0 \mathrm{gm}$.

Orange $\mathrm{G}$

$0.5 \mathrm{gm}$.

$2.0 \mathrm{gm}$.

I. Preserve in corrosive sublimate or Zenker's fluid.

2. Stain the sections in the fuchsine solution one to three minutes.

3. Wash in water very quickly.

4. Place in the phosphomolybdic solution two to twenty minutes.

5. Wash off excess stain in water.

6. Dehydrate in 95 per cent alcohol and mount in damar.

This method gives a perfect differential stain of connective-tissue fibrils, and it is to be used whenever the fibrils are to be especially studied.

\section{Methods of Reconstruction.}

It is often important to obtain definite plastic conceptions of the anatomy of embryos or parts of embryos too small for dissection. To secure these in the best form, it is necessary to reconstruct either drawings or models from sections. The methods employed for these two forms of reconstruction, being different, must be described separately.

Reconstruction of Drawings from Sections.-To make these reconstructions satisfactory, it is indispensable to have an accurate outline of the embryo representing it in the point of view to be used for the reconstruction and enlarged to the precise scale upon which the reconstruction is to be made. This drawing must, of course, be made before the embryo is imbedded and sectioned. It is further necessary to know accurately the plane of the sections and their thickness, and, finally, the total number of sections in the series must be counted. A convenient scale for the reconstruction of the anatomy of mammalian embryos is a magnification of from $I_{5}$ to 30 diameters.

Let us suppose that a pig of $\mathrm{I} 2 \mathrm{~mm}$. has been drawn in a side view magnified 20 diameters; that the embryo has been cut into 900 transverse sections and the approximate plane of the sections is known. It may be more exactly determined by the study of the sections themselves; for instance, it may be determined what section is the last to pass through the surface of the head in the region of the fore-brain and the last to pass through the border of the anterior limb. Then it 
can be further ascertained through which dorsal segments these two sections pass. By these data the plane of the two sections can be accurately fixed. Over the outline of the embryo is now drawn a series of lines which represent the position of the sections. It is generally sufficient to put in lines which represent only every second, third, or even fourth section. If at any point where the structure is complicated more details are needed, lines for the additional sections can be interpolated. In our supposed case, our lines representing every fourth section, there would be 225 parallel lines, and these should be numbered to correspond to the sections which they represent.

The outlines of the actual sections corresponding to the numbered lines in the diagram must now be made with the camera lucida. In regard to these great care is necessary, especially if, as is likely to be the case, the sections are from embryos imbedded in paraffin, because when an embryo is so imbedded it always shrinks, and after imbedding is smaller than before. The shrinkage seems to be uniform throughout and not to disturb the topographical relations even of the finest structures. Unfortunately the shrinkage is not constant, but varies from specimen to specimen, hence a camera drawing made from the sections and magnified 20 diameters will not be of the right size to fit in the diagram, and these drawings, must, therefore, be corrected. This may be done either, as is best, by making the original camera lucida drawings of the right magnification for direct use in reconstruction, or they may be made nearly the right magnification and when they are measured off the necessary correction may be introduced by measuring them with proportional dividers.

From the camera lucida drawings of the single sections the measurements are taken to fix the position of the parts in the reconstruction.

For a given section the exact position in the reconstruction is fixed by the line on the outline drawing of the embryo corresponding to the number of the section. On the drawing of the section the distance of the organ to be reconstructed from the point in the section corresponding to the outline of the embryo is measured off, and then marked upon the proper line of the reconstruction diagram. A similar measurement is then taken from the next section and transferred to the diagram in the same manner, and so on . with successive sections until a series of dots is obtained which mark the outline of the organ. These dots are then connected by a continuous line, which will indicate the form and correct position of the organ. Simple reconstructions may be easily made by these means. When, however, more complicated reconstructions are attempted, much judgment and skill are necessary in the selecting of parts which may be successfully represented in a single drawing, bearing in mind always the point of view which is assumed for the reconstruction, so that organs may be correctly represented in their relative positions, nearer or farther from the observer as he looks at the drawing. After the outlines are completed the shading of the parts must be added, and this often requires a special degree of skill and a considerable faculty of plastic imagination. As examples of 
complicated reconstructions, the student is referred to figures 169 and 172 , pages 229 and 235.

Oftentimes simpler reconstructions are very helpful in which only a few sections are combined, as, for example, to show the course and branches of the spinal nerves in young embryos. In such a case the outline of the middle section of the series proposed to be combined may be selected to give the outline of the reconstructed drawing. Camera lucida drawings of this and the neighboring sections to be included should be made of the desired magnification. The reconstruction itself may be made upon tracing paper, which is laid successively over the drawings of the sections and the parts required from each can be added upon the tracing paper, which will thus combine in a single drawing the parts intended to be represented. Reconstructions of this kind are easily made by students and are often very instructive.

Reconstruction with Wax Plates by Born's Method.-The basis of this method is to make in wax a magnified reproduction of the single sections, representing in the wax such portions of the section as it is desired to reproduce in plastic reconstruction. To this end wax plates must be made which represent a definite magnification of the thickness of a section. For working by this method it is usually advantageous to employ rather thick sections, say, of $20 \mu$. If the magnification chosen is fifty times, which is practically often convenient, then the wax plates should be made fifty times $20 \mu$ in thickness, or I $\mathrm{mm}$. The most convenient plates to work with are those from I to $2 \mathrm{~mm}$. thick. Upon a wax plate of the requisite thickness a camera lucida drawing is made. This may be done with a lithographic crayon or with a fine steel point. The drawings must be of exactly the right magnification; in the-illustration chosen, 50 diameters. Next, the wax plate is put upon a glass or a metal surface where it lies perfectly flat, and with a sharp thin-bladed knife or scalpel the outlines of the organs which it is intended to reconstruct are cut out as may be desired. Our bit of wax then represents a model of the parts selected from the section, and equally magnified in the three dimensions of space. Wax plates made from successive sections are then piled up, one on top of the other, in the proper order. If they are rightly superimposed, an operation which often requires skill and judgment, and always requires the utmost care, then the pile of plates will correctly represent the form of the parts included in the reconstruction. To fasten the plates together it is only necessary to pass a warm metal instrument over the edges of the plates, enough to melt the wax a little. With proper care this may readily be accomplished without destroying the surface modeling of the reconstruction.

The simplest method of making wax plates is to have a large tin pan with vertical sides. This is filled with very hot water, and melted beeswax is poured on the surface of the water and allowed to cool. Plates of sufficiently exact and even thickness may be cast in this way, provided the operation is carried out in a quiet place so that the surface of the water is not disturbed while the wax is hardening. 
It will be found convenient to have a large plate of iron, not less than one eighth of an inch in thickness, which may be placed upon supporters. The tin pan should be set upon this plate and the plate heated by lamps below in order to keep the water hot enough to allow the wax to spread evenly over the surface of the water. The water must be freed from air before the wax is poured in, but must not be allowed to boil after the wax has been added. If bubbles appear in the wax plate, they may be removed while the wax is still hot by directing the blue flame from a Bunsen burner down upon them. If the pan is heated directly without the iron plate, it is sure to warp and become unfit for use. Thin iron plates are also liable to be warped.

To determine the thickness of the plates cast as described we proceed empirically. A weighed quantity of wax is melted and poured into the pan. After the plate has solidified it is removed by cutting it free from the edges of the pan, and the thickness of the plate is then measured at various points by micrometer callipers. From these data it is easy to calculate exactly what thickness of plate one gram of beeswax represents. To get accurate results it is advisable to cast several plates of varying thickness and determine the average for one gram in that way. Having determined what one gram represents in thickness, it becomes thereafter only necessary to weigh out the proper number of grams in order to obtain any desired thickness of wax plate. It will be found advantageous to filter the wax before using it. This may easily be done by a double hot-water filter. Such a filter may be made of copper. It is desirable to connect it with a Mariotti's flask to maintain a constant water level.

Directions for Orienting Serial Sections of Embryos. (NOTE: The lower edge of the ribbon is the one to the left, when the observer has the object between himself and the knife.)

I. Transverse Series.

Normal thickness: $10 \mu$.

Dorsal surface to be toward the lower edge of the ribbon.

Series to begin with the head.

In cutting, the left side of the embryo must strike the knife first. 2. Sagittal Series.

Normal thickness: Small embryos, זо $\mu$.

$\begin{array}{lll}\text { Medium “ “ } & \text { " } 5 \mu \text {. } \\ \text { Large } & 20 \mu \text {. }\end{array}$

The head of the embryo to be toward the lower edge of the ribbon.

Series to begin with the right side.

In cutting, the ventral side of the embryo must strike the knife first. 3. Frontal Sections.

Normal thickness: Small embryos, Iо $\mu$.

$\begin{array}{lll}\text { Medium “ } & \mathrm{I} 5 \mu . \\ \text { Large } & 20 \mu .\end{array}$


The head of the embryo is to be toward the lower edge of the ribbon.

The series is to begin with the ventral side.

In cutting, the left side of the embryo must strike the knife first.

In mounting leave space for the label at the left-hand end of the slide. Keep the sections in the order cut. Arrange them on the slides in the sequence of ordinary written lines.

\section{Microtomes.}

There are many forms of microtome which may be used with good results and which will work very satisfactorily for making sections of small objects. The cutting of larger objects, such as pig embryos of from $\mathrm{I}_{5}$ to $20 \mathrm{~mm}$., and of pieces

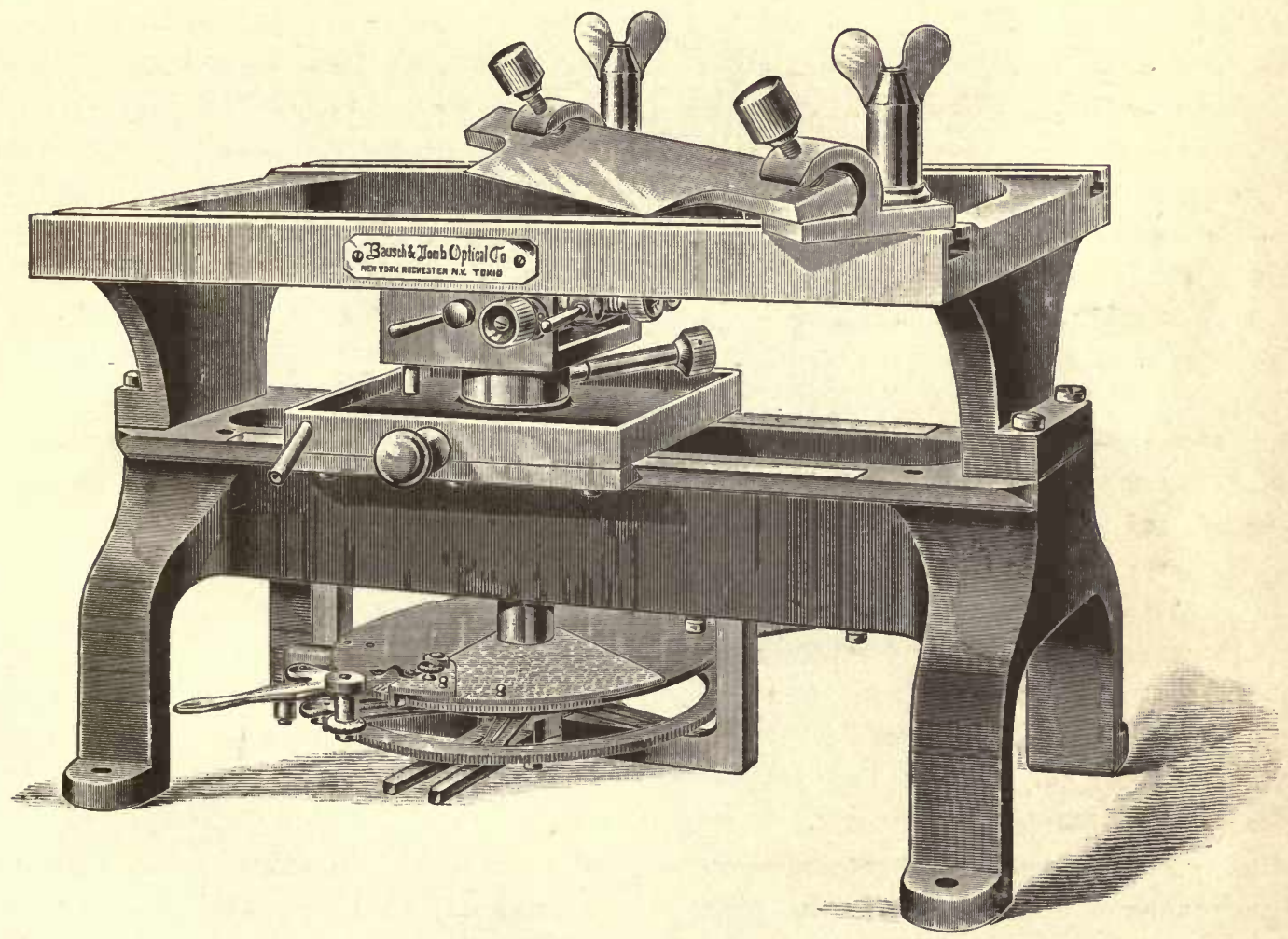

Fig. 26i.-The Precision Microtome.

of the uterus or other organs, is more difficult, and microtomes which work satisfactorily with small objects often fail to give good even sections of more difficult objects. For embryological work a microtome ought, therefore, to be selected which will give perfectly regular sections in long series of any desired thickness from $\mathrm{I} \mu$ up to $25 \mu$. It is also desirable for economy of time to have a microtome which works automatically. These considerations lead the author to recom- 
mend for embryological use especially two forms of microtome made by Messrs. Bausch \& Lomb, of Rochester, N. Y., and designated by them as the "precision" and "rotary" microtomes.

The precision microtome (Fig. 26I) consists, first, of an upper square form upon which the knife may be clamped in any desired position; second, of two horizontal ways upon which moves the carriage which bears the object-holder; and, third, of a micrometer screw with an automatic feeding contrivance on the under side of the movable carriage. The construction is very solid and great rigidity of the parts is secured. The microtome may be used for either paraffin or celloidin cutting. According to the author's experience, this microtome considerably surpasses all other types in the accuracy of the work which may be done

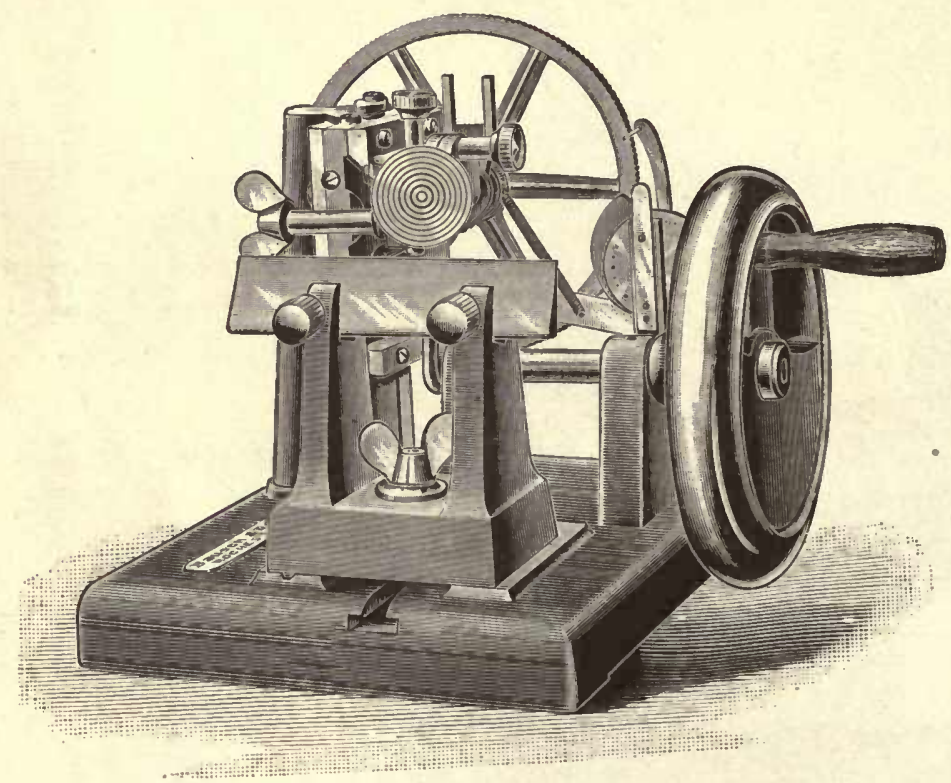

Fig. 262.-The Automatic Rotary Microtome.

with it. The rotary microtome was originally made in Germany, and various patterns have been put upon the market by German, French, English, and American manufacturers. The new pattern recently introduced by Messrs. Bausch \& Lomb embodies a considerable number of improvements, which render the instrument (Fig. 262) very desirable for general laboratory use. It works with accuracy, is very easy to manipulate, and cuts sections with extreme rapidity. It is adapted only for paraffin work. For the general use of students, in elementary courses especially, this microtome is to be preferred to the "precision," as it requires less care and works more rapidly. A single rotary microtome will be found sufficient for a class of from twenty to thirty students in embryology. 
The microtome is an instrument of precision, which implies that it must be treated with extreme delicacy and kept most scrupulously clean. It will be found usually, when complaint is made against the microtome; that the complaint is misdirected, and ought to be not against the machine, but against the owner. The modern microtome necessarily has several adjustments, every one of which must be exact and secure. If any one of them is imperfect or insecure, if any of the movable parts is allowed to become corroded, or gummed up with oil, or loose, or clogged with dust or dirt of any kind, the microtome will not and cannot work as an instrument of precision.

The knife used for cutting ought to be regarded as an integral part of the microtome and as its most delicate and easily injured part. A perfect knife-edge is the greatest treasure of the microtomist. To sharpen the knife satisfactorily for fine section cutting is a really serious difficulty. A skillful person, however, may get a good edge by using the very finest grade of oil-stone. No oil should be used, but instead a mixture of equal parts of glycerin and water. Before the knife is honed it must be made as clean as possible. The oil-stone itself also must be cleaned with equal care, and the mixture of glycerin and water should, if necessary, be filtered before using to keep it free from dirt. A single particle of dirt may be the cause of making many microscopic notches in the edge of a knife. A knife is well sharpened when its edge appears smooth and straight under a magnifying power of twenty-five diameters. The microtome knife should be as unlike a razor as possible. It must have a very thick back and be as heavy and rigid as practicable, so that the actual cutting-edge may be as steady and inflexible as it can be made. Knives of suitably heavy construction are now furnished with all the best microtomes. 


\section{N D E X .}

Abdominal cavity, origin, 87

Abozzo, 9

After-birth, defined, 360

Alcohol, 380

Allantois, chick, 214

general account, I Io

human, 136, 142, 374

in umbilical cord, 374

origin, 83

in primates, 123

pig, $9.0 \mathrm{~mm}$., 253

I $7.0 \mathrm{~mm} ., 3$ ro, 3 I

and chorion in ungulates, $\mathrm{I} I 3$

Alum cochineal, 38 I

hematoxylin, 382

Amiurus, $5 \mathrm{I}$

Amnio-cardiac vesicles, chick, I80, I 86

Amnion, general account, II 7

human, at two months, 370

at three months, 344

after fifth month, $37 \mathrm{r}$

at seven months, 345

structure, $37^{\circ}$

origin, $8_{3}$

in primates, 122

raphe, chick, 203, 2 ro

Amniota, 7

Anal plate, 58,59

origin of, 54

Anamniota, 7

Angioblast, chick, 187

development, 9 I

early history, 80

first appearance, 66

Anlage, defined; 9

Annelids, 9

Anterior cavity, 88

Anura, 8

Anus, origin of, 54

Aorta, chick, 191, 192, 200, 202, 205
Aorta, descending, pig, $6.0 \mathrm{~mm} ., 247$ I $2.0 \mathrm{~mm}$., 274, 28 I

dorsal, pig, $9.0 \mathrm{~mm} ., 255$ I $2.0 \mathrm{~mm} ., 282,283,285$ I $7.0 \mathrm{~mm} ., 305$

first appearance, 93

Aortic arches, chick, 184, 202

general account, roo

human, I42, $144,145,146$

pig, $6.0 \mathrm{~mm}$., 247

I $2.0 \mathrm{~mm} ., 278,293$

Zimmermann's, Ioo

Aortic system, general account, 99

Appendages, embryonic, absence in lower vertebrates, 82

Arachnoid, pig, 9.0 mm.,. 254

$$
\begin{aligned}
& 12.0 \mathrm{~mm} ., 266 \\
& 17.0 \mathrm{~mm} ., 305 \\
& 20.0 \mathrm{~mm} \text {., } 325
\end{aligned}
$$

Archenteron, general account, 57

origin of, 54

Area, germinal, 96

opaca, defined, 64 first appearance, 97

pellucida, defined, 64 first appearance, 97

vasculosa, 50, 66, 9I

first appearance, 97

of rabbit, 98

vessels of, 97

vitellina, 65

Arteries, basilaris, pig, $9.0 \mathrm{~mm}$., $25^{8}$

$$
\text { r } 2.0 \mathrm{~mm} ., 264,293
$$

carotid, pig, $\mathrm{r} 2 \mathrm{~mm}$., 235, $27 \mathrm{I}, 274$

loop, pig, 264

central of retina, 333

intersegmental, pig, $6.0 \mathrm{~mm} ., 249$

$$
\begin{aligned}
& 9.0 \mathrm{~mm} ., 250 \\
& 12.0 \mathrm{~mm} ., 3 \text { or }
\end{aligned}
$$

lingual, 329 
Arteries, posterior communicating branch, 265 pulmonary, pig, $12.0 \mathrm{~mm} ., 28 \mathrm{x}$

$$
20.0 \mathrm{~mm} \text {., 3 } 7
$$

sulci, 3 I 2,320

umbilical, pig, $17.0 \mathrm{~mm} ., 3 \mathrm{ro}, 3 \mathrm{II}$

vertebral, pig, $12.0 \mathrm{~mm}$., 273

vitelline, 3 II

$$
24.0 \mathrm{~mm} \text {., } 336
$$

chick, 2 I 1

Ascending trigeminal tract, 263

Astral figures in ovum, 40

Atriozoa, 9

Auricle, pig, $12.0 \mathrm{~mm} ., 282$

Bacilliform bodies, $\mathrm{I} 68$

Beale's carmine, $3^{84}$

Biogenetic law, 3 I

Bladder, origin, I I 4

Blastodermic vesicle, 45

formation of, 44

in primates $_{2} \mathbf{1}_{2} 2$

in monkey, second stage, $\mathbf{I} 28$

in rabbit, 166

Blastopore, 53

$$
\text { study of, } 167-173
$$

division of, 54

Blood, degeneration in chorion, 357

origin, 90

pig, $12.0 \mathrm{~mm}$., 266

$$
20.0 \mathrm{~mm} ., 324
$$

Blood-corpuscles, 93

red, general account, 93 ichthyoid, 94

sauroid, 94 mammalian, 94

Blood-islands, 9I

chick, I 79

Blood-plates, 94

Blood-vessels, definition, 90

development in chick, $9 \circ$ in mammals, 92

first appearance, 66

growth into embryo, 93

origin, 90

primitive course, man, 142

Body-cavity of vertebrates, 5

Body-stalk, defined, I I I

origin in primates, 123

vessels of, I I 2

and umbilical cord, II 5

Borax carmine, $3^{82}$

Born's method, 387

Brain, 73
Branchial arches, defined, 62

pig, $6.0 \mathrm{~mm}$., 246, 249

$$
\begin{aligned}
& 7.8 \mathrm{~mm} ., 222 \\
& 9.0 \mathrm{~mm} ., 258 \\
& 10.0 \mathrm{~mm} ., 224 \\
& 12.0 \mathrm{~mm} ., 274
\end{aligned}
$$

Canal, auricular, 283

digestive, 59

hyaloid, 333

neurenteric, origin of, 54,68

notochordal, 53

of Schlemm, $33 \mathrm{I}$

Capsule, periotic, 78

Carnivora, 8

Carotids, origin, 100

Cartilage, first appearance, 304

Meckel's, 329

Cavity, abdominal, origin, 87

anterior, 88

head, 87

hyoid, 87

mandibular, 87

pericardial, chick, 181,209 origin, 87

pleural, 87

premandibular, 87

Cells, death of, 15

germ, 25

lutein, 35

removal of, $\mathrm{x} 6$

somatic, 28

Cephalochorda, 9

Cerebellum, pig, I $2.0 \mathrm{~mm} ., 292$

Cervical sinus, human, 147-149

Cheiroptera, 8

Chick embryo, method of obtaining, I 74

preservation, I 76

study of, $174^{-218}$

with eight segments, I 76

comparison with rabbit, 179

longitudinal section, 180

transverse sections, I 8 I

with twenty-four segments, $x 97$

with twenty-eight segments, differentiation in, 216

studied in sections, $199^{-2} 16$

Chondrostyle, 56

pig, $24.0 \mathrm{~mm}$., 336

Chorda dorsalis. See Notochord.

Chorion, chick, 203, 2 I I

defined, 64

frondosum, defined, $\mathbf{2} 27$ 
Chorion, general account, i 7

human, at three months, 344

degeneration of blood in, 357

ectoderm of, 364

histology of, $3^{6} 3$

læve, second stage, $35^{\circ}$

mesoderm of, 356,364

placental, 354

trophoderm of, 365

villi, 367

læve, defined, 127

origin, 83

relation to uterus in ungulates, I $_{3}$

Chromosome, accessory, 28 number in segmentation nucleus, $4 \mathrm{I}$

Cinerea, 263,270

defined, 74

Cisterna chyli, 105

Cochlea, origin, 78

Colom, defined, i 8

double, 8 I

embryonic, 84

of the head, 87

origin, $8 \mathrm{I}$

pig, $9.0 \mathrm{~mm} ., 255$

I $2.0 \mathrm{~mm}$., $30 \mathrm{I}$

umbilical, 3 I I

ventral, 87

vertebrates, 5

Commissure, ganglionic, 262

posterior, 335

superior, 335

Copper hematoxylin, 384

Cord, umbilical, pig, r $7.0 \mathrm{~mm} ., 3$ ro

sexual, 322

Corona radiata, 34

Corpora quadrigemina, pig, $12.0 \mathrm{~mm} ., 292$

Corpus luteum, 35

striatum; 329

Costal processes, 305,32 I

Counterstains, $38 \mathrm{I}$

Cutis, anlage, 266

pig, $20.0^{\circ} \mathrm{mm} ., 32 \mathrm{I}$

Cutis-plate, 86

Cytomorphosis, I I

Decidua caduca, defined, I 24

cavernous layer of, $35 \mathrm{I}$

compact layer of, $35 \mathrm{I}$

defined, 124

reflexa, at three months, 343

at four months, 344

atrophy, I 27
Decidua reflexa, defined, I 24

disappearance, 343

first stage, 349

serotina, at seven months, 345,357 defined, 24

subchorialis, 359

vera, at three months, 344

defined, I 24

first stage, 346

second stage, 350

Decidual cells, development, 348 mature, 359

Deck-plate, 72

pig, $9.0 \mathrm{~mm}$., 257

Degeneration, hypertrophic, 15

Dermatome, 86

Development, arrest of, 9 I

embryonic, 16

larval, 16

summary of, 10

Diaphragm, pig, $24.0 \mathrm{~mm} ., 337$

Diencephalon, origin, 74

Differentiation, I 3 two types of, 14

Digestive canal, general account, 59 of vertebrates, 5

Dipnoi, 8

Discus proligerus, 34

Diverticulum, Meckel's, 57

Dorsal furrow, 68 roots, 270,283

Ducts, Müllerian, I I 0, 3 I 7 urogenital, I Io

Wolffian, I 10

chick, 2 I 2

pig, I $2.0 \mathrm{~mm} ., 287$

I $7.0 \mathrm{~mm} ., 309$

$20.0 \mathrm{~mm} ., 3 \mathrm{I}$.

Ductus arteriosus, 9I

defined, I 0 I

Cuvieri, defined, $\mathrm{IO}_{3}$

endolymphaticus, 299

origin, 78

thoracicus, 105

venosus Arantii, pig, $9.0 \mathrm{~mm} ., 252$

Dyads, 37

Ebauche, 9

Ectoderm, chick, 2 I 7

defined, 18

Ectoglia, $263,3 \times 4$

defined, 74

Elasmobranchs, 8 
Embryo, Amiurus, $5 \mathrm{I}$ appendages of, 49 arising from embryonic shield, 50 dissection, 377 growth of, 49 human, I 8 imbedding, 380 measuring, 377 preservation, 377 separation from yolk, 49

Embryonic shield, 44, 47 rabbit, $170-\mathrm{r} 73$

Entoderm, chick, 2 I 7 defined, $\mathrm{r} 8$ earliest growth, mammals, 46 origin of permanent, 54

Eosin, 383

Ependyma, defined, 74

Ependymal layer, primitive, 263

Epidermis, pig, I $2.0 \mathrm{~mm}$., 266

$$
\text { I } 7.0 \mathrm{~mm} ., 3 \circ 3
$$

Epiglottis, 337 pig, $12.0 \mathrm{~mm} ., 293$

Epiphysis, 335

Epithelial bodies, 63

Epitrichium, 303, 320

Eustachian tube, origin, 63

Excretory organs, general account, 108

Eye, general account, 76 pig, $12.0 \mathrm{~mm}$., $27 \mathrm{x}$

$$
20.0 \mathrm{~mm} ., 329
$$$$
24.0 \mathrm{~mm} ., 33 \mathrm{r}
$$

Eyelids, 331

Facial motor tract, 298

Falx, pig, $12.0 \mathrm{~mm}$., 275

$$
20.0 \mathrm{~mm} ., 327
$$

Fertilization of the ovum, $3^{8}$

Fibrin, in chorion, 355 in decidua reflexa, 349

Filum terminale, 75

Fin compared with limb, 257

Floor-plate, 72

Fluid, Bouin's, 379

Flemming's, 379

Hermann's, 380

Müller's, 379

Parker's, 379

Tellyesnicky's, 378

Zenker's, 378

Foramen epiploicum, 290

of Monro, 329

defined, 74
Fore-brain, chick, 180,201 differentiation, 74 origin, 72 pig, $9.0 \mathrm{~mm} ., 253$ I 2.0 mm., $27 \mathrm{r}, 292$

Fore-gut, chick, $x 80, x 8 \mathrm{r}, \mathrm{r} 87$ general account, 60 origin, 57

Formalin, 378

Fovea cardiaca, chick, r 90 origin, 57

Furrow, dorsal, 68

Gall-bladder, pig, r $4.0 \mathrm{~mm} ., 288$

Ganglia, auditory, division of, 79 origin, 72

pig, $9.0 \mathrm{~mm} ., 250$

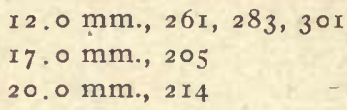

Ganglion, acustico-facial, 293, 295, 298 jugular, 293, 296 nodosum, 277,293 petrosal, 293 trigeminal, 262, 293, 295, 297

Ganglionic crest, 72 chick, $180,185,188$, r $9 \mathrm{r}$

Ganoids, 7

Genetic restriction, I4

Germ-cells, general account, 25 of Acanthias, 26

Germ-layers, general account, 18 specific quality, I9 tissues from, I 9

Germinal area, 96 wall, 65

Gibbon, ovum in third stage, $I_{3} \mathrm{I}$

Gill-clefts, 62 chick, first, $20 \mathrm{I}$ second, 202 third, 205

human, $140-147$

pig, $6.0 \mathrm{~mm}$., 248 $9.0 \mathrm{~mm} ., 257,259$ I 2.0.mm., 271, 273, 275, 277

Gill-pouches, origin, 62

Glands, classification, 23 general account, 2 I genital, pig, I $2.0 \mathrm{~mm} ., 287$

$$
\begin{aligned}
& \text { I } 7.0 \mathrm{~mm} ., 308 \\
& 20.0 \mathrm{~mm} ., 322
\end{aligned}
$$

Globules, polar, $37,161,162$

Gray layer, 263,270

defined, 74 
Groove, primitive, 48

Growth, law of unequal, 24 of embryo, 49

Head-bend, 223

Head-process, 53

Heart, chick, 18 $6,187,188,203,205,206$ general account, 189

origin, 96 .

pig, I $2.0 \mathrm{~mm} ., 282$

Heidenhain's hematoxylin, 382

Hemispheres, cerebral, pig, r $2.0 \mathrm{~mm}$., 275

origin, 74

$20.0 \mathrm{~mm} ., 325,329$

Hensen's knot, 48, I 70

Heredity, theory of, 28

Hermaphrodites, 27 pseudo-, 32

Hind-brain, chick, 180, 190, 191, 203

differentiation, 74

origin, 72

pig, $9.0 \mathrm{~mm}$., $25^{8}$

I $2.0 \mathrm{~mm}, 263,292,296$

Hind-gut, general account, $6 \mathrm{r}$

origin, 57

Hormone theory of sex, 27

Human embryo, i 8

age, calculation of, II 8

classification of stages, 1 I 9

Coste, 138

Dandy, 58, 137

Eternod, 136

Frassi, I 9

His, E, 136

Lg, ${ }_{4}$ I

Sch, I 4 I

$\mathrm{SR}, \mathrm{I}_{3} 6$

2. I $5 \mathrm{~mm}$., I 4 I

2. $6 \mathrm{~mm}$., 143

$3.2 \mathrm{~mm} ., 145$

$4.0 \mathrm{~mm}$., $\mathrm{I} 47$

$4.2 \mathrm{~mm}$., I 44

Kollmann, 137

Mall, 147

Peters, 128

Spee, I .54.mm., I 35

stages, classification of, I I 9

first, I I 9

second, I I 9, I 28

third, I 19

fourth, I I 9, I 34

fifth, I 20, I 36

sixth, I 20,137
Human embryo, stages, seventh, I 21,140

eighth, I 2 I, I 4 I

ninth, I $21, \mathrm{I} 43$

tenth, $12 x, 146$

eleventh, I 2 I, 147

four weeks to four months, $148-159$

relations to uterus, 124

Hyoid cavity, 87

Hypophysis, pig, $12.0 \mathrm{~mm} ., 292$

$24.0 \mathrm{~mm}$., 335

vertebrates, 5

Ichthyopsida, 7

Impregnation of the ovum, 38

Infundibular gland, pig, I $2.0 \mathrm{~mm} ., 268,292$ $24.0 \mathrm{~mm}$., 335

Inner man in segmentation, 44,47

Insectivora, 8

Intermediate cell-mass, 85

Intervertebral discs, pig, r $2.0 \mathrm{~mm} ., 30 \mathrm{r}$ $20.0 \mathrm{~mm} ., 3$ I 4

Intestine, caudal, 2 I 3

open in chick, I 91,2 IO, 2 I 2

pig, $9.0 \mathrm{~mm} ., 252,255,257$

I $7.0 \mathrm{~mm} ., 308,3 \mathrm{II}$

Iris, 33 I

Iron hematoxylin, 382

Isthmus of brain, origin, 74

pig, $12.0 \mathrm{~mm}:, 292$

Iter, defined, 74

Jakobson's organ, 326

Kidney, origin of renal anlage, 309

pig, $17.0 \mathrm{~mm} ., 308$

$20.0 \mathrm{~mm}$., 322

Knot, Hensen's, 48, r 70 primitive, 48

Kopffortsatz, 53

Lachrymal groove, 222,224

Lamina terminalis, 335

Larynx, anlage of, 276, 293

Lateral roots, 270,297

Layer, subzonal, 44

Lens of eye, chick, $20 \mathrm{I}$

origin, 78

pig, I $2.0 \mathrm{~mm} ., 27 \mathrm{I}$

$20.0 \mathrm{~mm} ., 329$

24 . $0 \mathrm{~mm}$., 333

Lesser peritoneal space, $290,30 \mathrm{I}$ 
Leucocytes, general account, 96

Limbs, pig, $9.0 \mathrm{~mm} ., 257$

$$
\text { 12.0 mm., } 279
$$$$
20.0 \mathrm{~mm} \text {., } 3 \mathrm{I} 8
$$

vertebrates, 4

Liver, chick, 207

general account, to

pig, $9.0 \mathrm{~mm} ., 252$

$$
\begin{aligned}
& \text { I } 2.0 \mathrm{~mm} ., 288 \\
& 20.0 \mathrm{~mm} ., 324 \\
& 24.0 \mathrm{~mm} ., 337
\end{aligned}
$$

vertebrates, 5

Lungs, pig, 9.0 mm., 252

$$
\begin{aligned}
& 12.0 \mathrm{~mm} ., 285 \\
& \text { 1 } 7.0 \mathrm{~mm}, 306 \\
& 20.0 \mathrm{~mm}, 3_{17}, 322
\end{aligned}
$$

Lutein, 35

Lymph-glands, 105

Lymph-sacs, 105

Lymphatic spaces, pig, $20.0 \mathrm{~mm} ., 3{ }_{5}$ system, 105

Lyons blue, $3^{8} 3$

Mnlleus, $33^{\circ}$

Mallory's stain, $3^{8} 5$

Mammary anlage, 320 bodies, 292

Mandibular cavity, 87

Marsipobranchs, 7

Marsupials, 8

Mass and surface, 20

Maxillary process, 224 pig, 20.0 mm., 325

Maxillo-turbinal fold, 311,326

Meatus, external auditory, $27 \mathrm{I}$

Meckel's cartilage, 329 diverticulum, 57

Mediastinum, 3 I 7

Medulla óblongata, pig, I $2.0 \mathrm{~mm} ., 296$ $20.0 \mathrm{~mm}$., 336

Medullary canal, differentiation, 7 I origin, 69 stratification, 74 structure, 69

groove, chick, I 78, I 80, I 94 human, ${ }^{3} 6$ origin, 68

plate, human, 134 origin, 67

Membrana serosa, 64

Menstruation, 339

Mesectoderm, i 85

Mesencephalon, chick, 180
Mesenchyma, defined, I 8 histogenesis, 89 pig, I $2.0 \mathrm{~mm}$., 266 $17.0 \mathrm{~mm} ., 303$

Mesoderm, chick, 2 I 8 defined, 18 early history, 79 origin, $5 \mathrm{I}$ somatic, defined, $8 \mathrm{I}$ splanchnic, 192 defined, $8 \mathrm{I}$

Mesonephros, defined, rog

Mesothelium, origin, 8 I

Metamerism, 2

Metanephros, origin, I 10

Metencephalon, origin, 74

Microtomes, 389 knives for, 39 1

Mid-brain, chick, 180, I 84 differentiation, 74 origin, 72 pig, $9.0 \mathrm{~mm} ., 253$

$$
\text { I } 2 \text {. } 0 \mathrm{~mm} \text {., } 292
$$

Milk-line, 225,226

Monkey, ovum in second stage, I 27

Monotremes, 8

Mouth of vertebrates, 5

Müllerian ducts, pig, $20.0 \mathrm{~mm}$., 3 I 7

Muscle-plate, 86 pig, $9.0 \mathrm{~mm} ., 254$

Muscles, hyoglossal, 329 of eye, 329

$$
\text { origin, } 89
$$

Myelencephalon, origin, 74

Myotomes, pig, $12.0 \mathrm{~mm} ., 273$

Nasal pits, 76 pig, I $2.0 \mathrm{~mm} ., 277$

Naso-turbinal fold, 326

Neck-bend, 223, 292

Necrobiosis, I 5

Nephrotome, chick, I93, 2 I 2 differentiation, 86 origin, 85

Nerve fibers, pig, $12.0 \mathrm{~mm}$., 263 roots, origin of, 270,280

Nerves, acoustic, 295, 298 cervical, $273,275,278$ facial, pig, I $2.0 \mathrm{~mm} ., 27 \mathrm{r}, 295,298$ fourth, pig, $12.0 \mathrm{~mm}, 264,268$ glosso-pharyngeal, 274, 295 hypoglossal, 277,296 inferior maxillary, 274 
Nerves, olfactory, 76,327

optic, 77,295

origin, 72

spinal, 280

spinal accessory, $268,277,296$

superior maxillary, 326

third, pig, I $2.0 \mathrm{~mm} ., 264,268$

trigeminal, 295, 297

vagus, $285,296,3$ I 7

Nervous system, origin, 67 vertebrates, 4

Neuraxons, 270

Neuroblasts, $72,73,270$

Neuroglia layer, outer, 263

Neuromeres, pig, $6.0 \mathrm{~mm}$., 246

$$
\text { I } 2 \text {. } 0 \mathrm{~mm} ., 264
$$

Neuropore, anterior, 69, I 78

Nodulus thymicus, 276,295 origin, 63

Notochord, anlage of, 55

growth, 55

pig, $12.0 \mathrm{~mm} ., 273$

r $7.0 \mathrm{~mm} ., 305$

$20.0 \mathrm{~mm}$., 3 I 5

$24.0 \mathrm{~mm} ., 336$

relation to axial mesoderm, 56

tltimate fate, 55

vertebrates, 4

Notochordal canal, 53

Nuclei pulposi, 56, 337

(Esophagus, origin, 57

pig, $12.0 \mathrm{~mm} ., 280,285$

I $7.0 \mathrm{~mm} ., 305,306$

$20.0 \mathrm{~mm}$., 3 3 4; 3 7

Olfactory nerves, origin, 76

plate, pig, $6.0 \mathrm{~mm} ., 249$

$$
\text { I } 2.0 \mathrm{~mm} ., 277
$$

Omentum, pig, $12.0 \mathrm{~mm} ., 288,290$

Operculum, pig, 259

Optic chiasma, 292, 335

vesicles, chick, I33, I80, I83, 202 differentiation, 77

Oral plate, 58

$$
\text { chick, } 184
$$

human, ${ }_{142}$

Orange G, $3^{8} 3$

Organs, constitution of, 20

Otocyst, chick, r99, 200 general account, 78

pig, 1 $2.0 \mathrm{~mm}, 261,263,267,293,299$

Ova, primitive, 26

Ovulation, 35

mouse, I6I
Ovum, before maturation, 34

constitution, I 2

fertilization, mouse, 163

gibbon, third stage, I3I

holoblastic, 10

human, 34

second stage, I 28

Peters's, I 28

impregnation, $3^{8}$

isotropism of, 12

maturation, 36

meroblastic, ro

monkey, second stage, I 27

mosaic theory of, $\mathrm{x} 2$

segmentation, 42

mammals, 160

mouse, I 60, I 85

Palate, 3 I I

cleft, 9 I, 3 I 2

Panchoroid, 266, 32 I

Pancreas, general account, 107 pig, I $2.0 \mathrm{~mm}$., 290

$24.0 \mathrm{~mm}$., 337

Pangenesis, 29

Parablast, 64

Parathyroid glands, 63, 315

Penis, pig, $20.0 \mathrm{~mm}$., 320

Pericardial cavity, chick, 181,209 origin, 87

Pericardial epithelium, pig, I $2.0 \mathrm{~mm} ., 282$

Perichondrium, origin of, 304

Peritoneal membrane, pig, $12.0 \mathrm{~mm}$., 286 $20.0 \mathrm{~mm} ., 322$

Peritoneum, pig, $20.0 \mathrm{~mm}$., 322

Pharynx, chick, I 84, 205

general account, $6 \mathrm{r}$

origin, 57

pig, I 2.0 mm., $237,238,269,274$

vertebrates, 3

Pia mater, pig, $9.0 \mathrm{~mm}$., 2.54

$$
\begin{aligned}
& \text { I } 2.0 \mathrm{~mm} ., 266 \\
& \text { I } 7.0 \mathrm{~mm} ., 305 \\
& 20.0 \mathrm{~mm} ., 325
\end{aligned}
$$

Pig embryo, anatomy, general, 228

$7.8 \mathrm{~mm}$. stage, 228

I $2.0 \mathrm{~mm}$. stage, $23 \mathrm{I}$

form, external, 22 I

$$
\begin{aligned}
& 7.5 \mathrm{~mm} ., 22 \mathrm{I} \\
& \text { I } 0.0 \mathrm{~mm} ., 223 \\
& \text { I } 5.0 \mathrm{~mm} ., 225 \\
& 20.0 \mathrm{~mm} ., 226
\end{aligned}
$$

methods of obtaining, 219 
Pig embryo, sections, diagram of, 260 selection of stages, $22 \mathrm{I}$ serial sections, 220 studied in sections, $6.0 \mathrm{~mm}$., 246

$$
\begin{aligned}
& 9.0 \mathrm{~mm} ., 250 \\
& 12.0 \mathrm{~mm} ., 259 \\
& 17.0 \mathrm{~mm} ., 303 \\
& 20.0 \mathrm{~mm} ., 31 \mathrm{r} \\
& 24.0 \mathrm{~mm} ., 330
\end{aligned}
$$

viscera dissected, $23 \mathrm{I}$

Pituitary body, 268, 292, 336

Placenta, allantoic, defined, I I 3

at seven months, $345,35^{2}$

chorionic, defined, I 3

cotyledons of, 362

general description, 359

in situ, $35^{2}$

intervillous spaces, 363

vessels of, 360

Placentalia, 8

Plakodes, chick, 2 I 7 general account, 76

Plate, closing of gill-cleft, $27 \mathrm{x}$

Pleural cavity, 322 origin, 87

Pleuro-peritoneal space, 87

Plexus, brachial, 280,283 lateral choroid, 328 lumbar, 318

Polar globules, 37 mouse, first, 16 I second, 162

Post-branchial bodies, 63

Premandibular cavity, 87

Primates, 8

Primitive axis, 52 groove, chick, 178,197 streak, $5^{\circ}$

Pro-amnion, defined, 80

Prochorion, 45

Pronephros, defined, 108

Pronuclei, fusion of, $4 \mathrm{I}$ mouse, 164

Pronucleus, female, 37

male, 39 mouse, 163 mouse, 163

Prosencephalon; chick, 180 .

Protovertebræ, defined, 84

Pupil of eye, $33 \mathrm{r}$

Rabbit embryo with eight segments, I 79

Recapitulation, law of, 29
Reconstructions, by drawings, $3^{8} 5$ by wax plates, 387

Reduction division, 37

Regression, 15

Restriction, law of genetic, i4

Rhombencephalon, chick, 180

Rodents, 8

Sauropsida, 7

Sclerotome, pig, $6.0 \mathrm{~mm}$., $25^{\circ}$

Sections, orienting, 388 paraffin, mounting of, $38 \mathrm{I}$ staining, $38 \mathrm{I}$

Segmental vesicle, 86 zone, 178

Segmentation nucleus, $4 \mathrm{I}$ of the ovum, $4^{2}$ in $\operatorname{Limax}, 43$

spindle, 42

Segmented animals, 2

Segments, chick, third, 192 general morphology, 2 occipital, 180 primitive, 84

Sella turcica, 336

Sense-organs, 5

Septum, nasal, 312, 326 transversum, chick, 190, 209 relation to cœlom, 87

SSex, 27

cause of, 28

cells, 26

Sexual characteristics, secondary, 27 cords, 322

Shield, embryonic, 44,47

Sinus, cavernous, 268

cervicalis, 222,223 human, 147-149 pig, $6.0 \mathrm{~mm} ., 249$

lateral, 265

$$
\text { I } 2.0 \mathrm{~mm} ., 275
$$

rhomboidal, 69 chick, I 78,180

superior longitudinal, 265,275

terminalis, of chick, 91, 97

venosus, origin, 98

Sinusoids, of heart, 283

of liver, $252,289,324$

of suprarenals, 338

of Wolffian body, 256,287,306, 307 origin in liver, 209

Skull, anlage of, 325

Somatic cavity. See Splanchnocele. 
Somatopleure, 7,82

chick, I87, 2 I I

pig, I 2 . ० mm., 282,286

$20.0 \mathrm{~mm}$., 318

Somites, chick, 192, 207, 212

defined, 84

differentiation, 85

general morphology, 2

origin, 84

secondary, 86.

pig, $6.0 \mathrm{~mm} ., 246,25^{\circ}$

Spermatozoon, 33

entrance into ovum, 38

mouse, I 63

Spinal cord, 73

chick, 205

differentiation, 75

pig, $6.0 \mathrm{~mm} ., 246$

$$
\begin{aligned}
& \text { I } 2.0 \mathrm{~mm} ., 269 \\
& \text { I } 7.0 \mathrm{~mm} ., 305 \\
& 20.0 \mathrm{~mm} ., 3 \text { I } 2
\end{aligned}
$$

Splanchnocele, 84,87

pig, $12.0 \mathrm{~mm}, 287$

Splanchnopleure, 7,82

chick, I $87,203,2$ I I

pig, $12.0 \mathrm{~mm} ., 288$

Spongioblasts, 73

Staining, methods of, $38 \mathrm{I}$

Stigma of Graafian follicle, 35

Stomach, origin, 57

pig, I $2.0 \mathrm{~mm} ., 288$

Stomodæum, I 84

Streak, primitive, 48

rabbit, $170-173$

Striæ acusticæ, 268

Subzonal layer, 44

Suprarenal capsule, pig, $24.0 \mathrm{~mm}$, 337

Surface and mass, 20

Sympathetic system, cervical, 279, 283

pig, $17.0 \mathrm{~mm} ., 305,308$

$20.0 \mathrm{~mm}$., $3 \mathrm{I} 4$

Telencephalon, origin, 74

Teleosts, 8

Testis, 322

Tetrads, 36

Thyroid gland, origin, 63

pig, $12.0 \mathrm{~mm} ., 277$

$20.0 \mathrm{~mm} ., 315$

Tissue, classification of, 19

Tongue, pig, $12.0 \mathrm{~mm}$., 293

$20.0 \mathrm{~mm} ., 3$ I , 327

Tonsil, origin, 63

26
Trachea, pig, I $2.0 \mathrm{~mm} ., 276,279,280$ $20.0 \mathrm{~mm} ., 314,317$

Trigeminal tract, 263,297

Trophoblast, (footnote) 44

Trophoderm, degeneration, 366 early stage described, 364

general account, I I 4 origin, 47

Tubal band, $3 \mathrm{I} 8$

Tuber cinereum, 292

Tunica albuginea, 322 vasculosa lentis, 33 I, 333

Tunicata, 9

Umbilical cord, amnion from, 1 I 5 general account, I I 5 human, at seven months, 345 ectoderm of, 375 mesoderm of, 374 study of, $37^{2}$

opening, pig, $9.0 \mathrm{~mm}$., 253

Umbilicus, pig, $9.0 \mathrm{~mm}$., 254

Unguiculates, 8

Ungulates, 8

Urodela, 8

Urogenital ducts, 5 general account, 1 io ridge, of vertebrates, 5

Uterus, general histology, 339 human, pregnant, two stages of, I 24 menstrual changes, 339 pregnant, two stages of, 34 I at three months, 343 at seven months, 345

Uvea, 332

Valves, Eustachian, 282, 301 sinistral, $30 \mathrm{r}$

Thebesian, 282

Veins, anterior cardinal, origin, 102 pig, $12 . \circ \mathrm{mm}$., 268

cardinal, chick, 200, 207, 210 pig, I 2 . 0 mm., 264, 268, 285 I $7.0 \mathrm{~mm} ., 306$ $20.0 \mathrm{~mm}, 317$

common, origin of, $9^{8}, 1 \circ 3$ pig, I 2 . $0 \mathrm{~mm}$., 282 primitive arrangement, $9^{8}$

iliac, 320

inferior cava, origin, 104, 257 jugular, 279

maxillary, 293 
Veins, jugular, pig, $12.0 \mathrm{~mm} ., 268$

$$
20.0 \mathrm{~mm} \text {., } 3 \mathrm{I} 4
$$

lateral cardinal, 265

of the head, 268

lingual, 279

omphalo-mesaraic, chick, I 78, I 80, I90, I 9 I, 208, 2 ro

origin, 93

primitive arrangement of, 98,103

ophthalmic, $27 \mathrm{I}$

peripheral (of limb), 320

portal, pig, $9.0 \mathrm{~mm} ., 252$

I $2.0 \mathrm{~mm} ., 290$

posterior cardinal, origin, 103

pulmonary, 285

subcardinal, 256

superior longitudinal sinus, 265 mesenteric, 3 I I

umbilical, origin, 104

pig, $9.0 \mathrm{~mm} ., 257$

$$
\text { I } 2.0 \mathrm{~mm} ., 289
$$

I $7.0 \mathrm{~mm} ., 3$ I

vitelline, pig, $9.0 \mathrm{~mm} ., 253$

$$
\text { I } 7.0 \mathrm{~mm} \text {., } 3 \text { I I }
$$

Velum transversum, origin, 74

Vena capitis lateralis, 268,299

cava inferior, 300

development, 257

hepatica communis, pig, $9.0 \mathrm{~mm} ., 252$

Venous system, 102

Ventral roots, 270,283

Ventricle, fourth, pig, $6.0 \mathrm{~mm} ., 246$

$$
\text { I } 2.0 \mathrm{~mm} ., 293
$$

lateral, pig, $\mathrm{x} 2.0 \mathrm{~mm}$., 275

$$
20.0 \mathrm{~mm}, 3^{27}
$$

of heart, pig, $12.0 \mathrm{~mm} ., 282$

Vertebræ, pig, $12.0 \mathrm{~mm}$., 303

$$
\begin{aligned}
& \text { I } 7.0 \mathrm{~mm} ., 304 \\
& 20.0 \mathrm{~mm} ., 314,315 \\
& 24.0 \mathrm{~mm} ., 337
\end{aligned}
$$

Vertebrate type, 2

fundamental characteristics of, 3

modifications of, 7
Vesicles, amnio-cardiac, 87 chick, 180,186 cerebral, origin, $7 \mathrm{r}$ optic, origin, $7 \mathrm{I}$ segmental, 86

Villi, branching, 367

histology of, 356

of allantois, 253,324

of chorion, 367

shape of, 367

vessels of, 370

Weigert's hematoxylin, 384

Weismannism, 29

Wolffian body, general account, 109

pig, $9.0 \mathrm{~mm} ., 252,256$

$$
\begin{aligned}
& \text { I } 2.0 \mathrm{~mm} ., 287 \\
& \text { I } 7.0 \mathrm{~mm} ., 306 \\
& 20.0 \mathrm{~mm} ., 322
\end{aligned}
$$

duct, chick, 2 I 2

tubules, origin, 86

Yolk, absorption of, 65

Yolk-cavity, 53

Yolk-sac, angioblast of, 66

entoderm of, 64

formed by splanchnopleure, 82

general morphology, 63

human, 66

structure, 375

in umbilical cord, 374

mesoderm of, 66

Zimmermann's arch, Io I

Zona pellucida, 34

radiata, 34

Zones, dorsal, 72

of His, 72

parietal, defined, 84

segmental, I 78, I 93,2 I 3

defined, 84

ventral, 72 
、 
( 

-
.

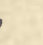

.

-

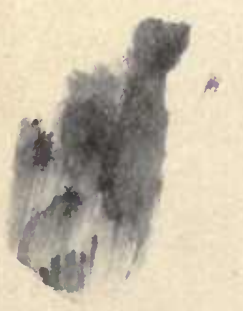





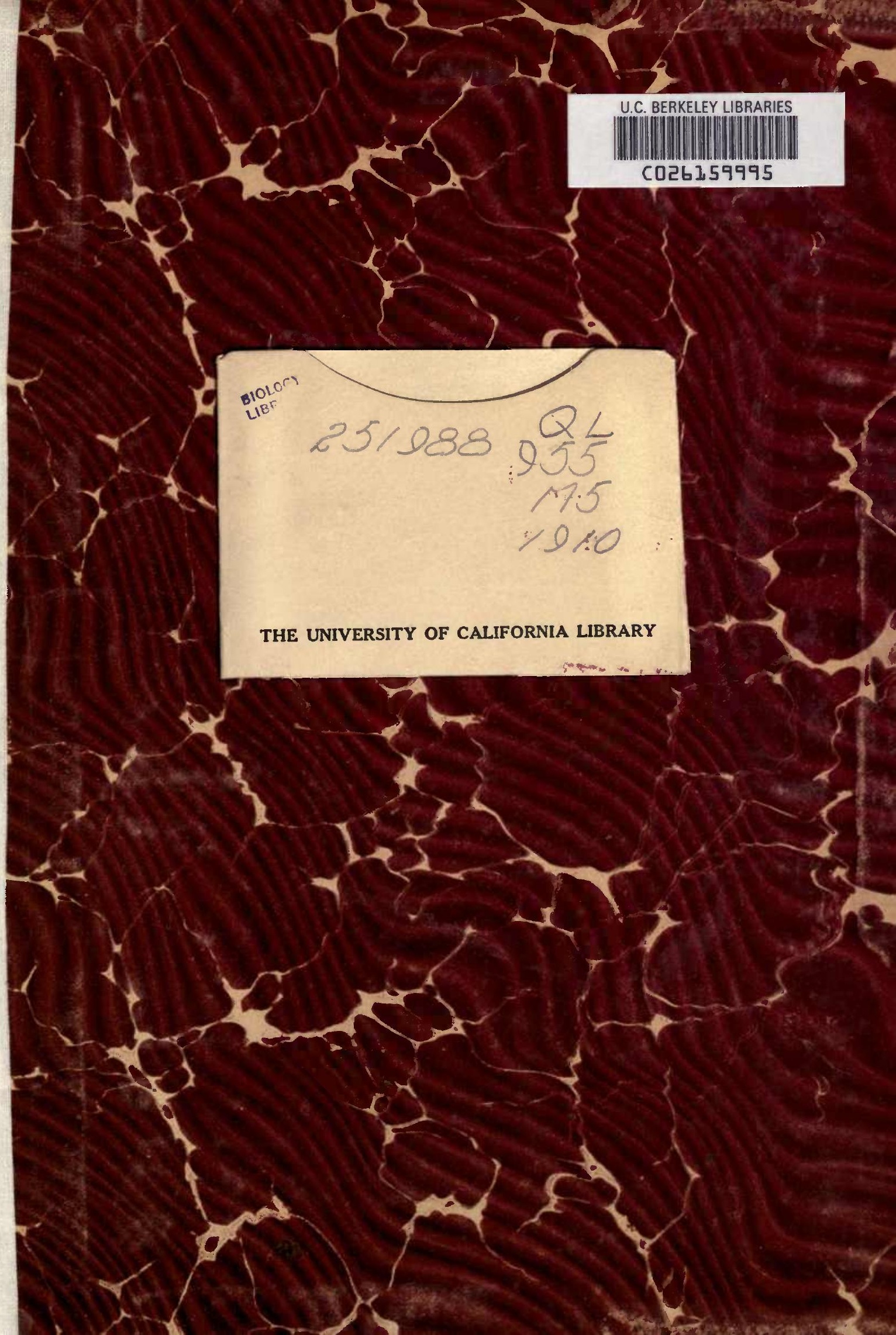


8 \% W. W.

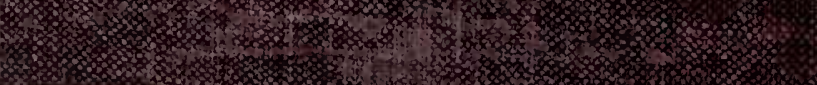

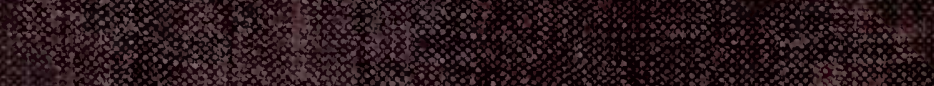

H. W w w w w w w \%

F.

$$
\begin{aligned}
& \text { F, }
\end{aligned}
$$

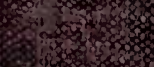$$
\text { Vin }
$$$$
\text { W }
$$$$
\text { \% }
$$

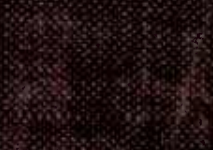

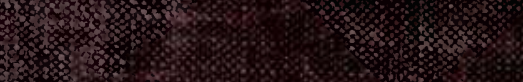

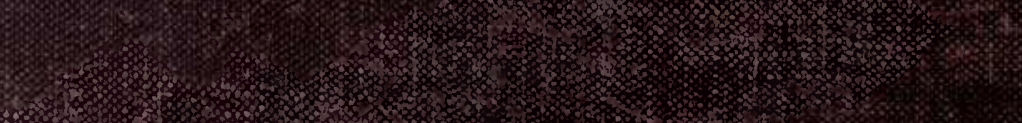
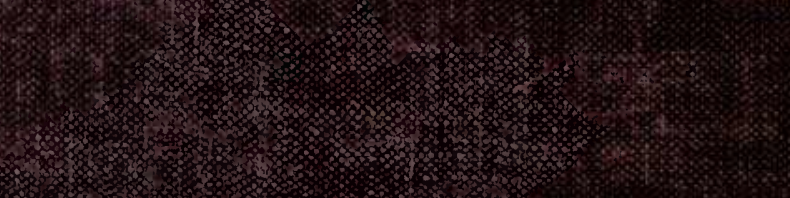

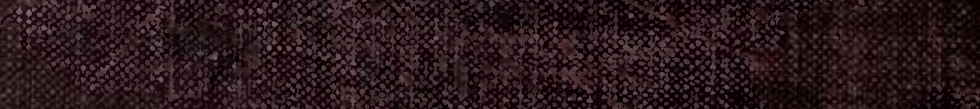

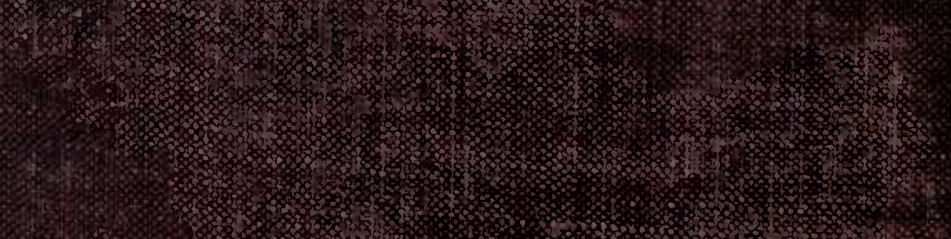
W

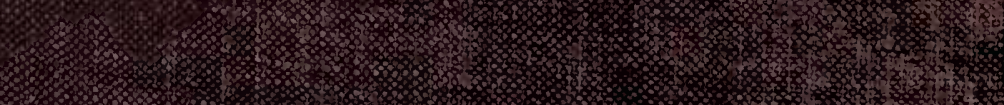

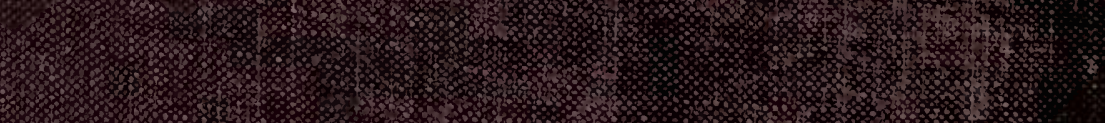

